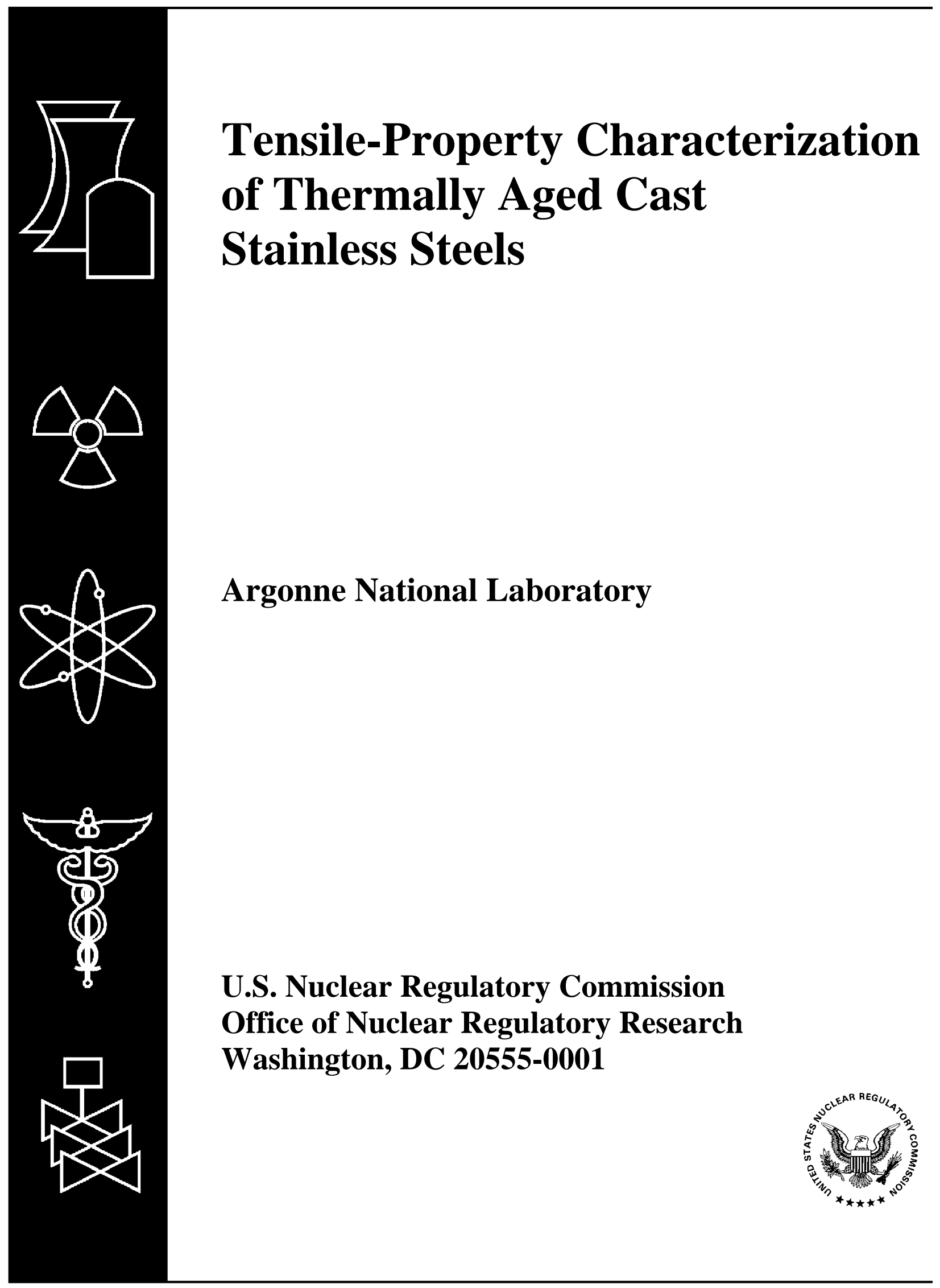




\section{Tensile-Property Characterization of Thermally Aged Cast Stainless Steels}

Manuscript Completed: January 1994

Date Published: February 1994

Prepared by

W.F. Michaud, P.T. Toben, W.K. Soppet, O.K. Chopra

Argonne National Laboratory

9700 South Cass Avenue

Argonne, IL 60439

Prepared for

Division of Engineering Office of Nuclear Regulatory Research U.S. Nuclear Regulatory Commission Washington, DC 20555-0001

NRC FIN A2243

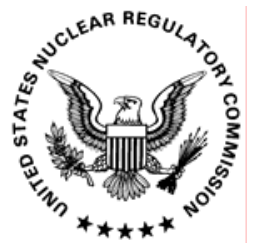


Previous Documents in Series

Initial Assessment of the Mechanisms and Significance of Low-Temperature Embrittlement of Cast Stainless Steels in LWR Systems, NUREG/CR-5385, ANL-89/17 (August 1990).

Estimation of Fracture Toughness of Cast Stainless Steels During Thermal Aging in LWR Systems, NUREG/CR-4513, ANL-90/42 (June 1991).

ii 


\title{
Tensile-Property Characterization of \\ Thermally Aged Cast Stainless Steels
}

\author{
by \\ W. F. Michaud, P. T. Toben, \\ W. K. Soppet, and O. K. Chopra
}

\begin{abstract}
The effect of thermal aging on tensile properties of cast stainless steels during service in light water reactors has been evaluated. Tensile data for several experimental and commercial heats of cast stainless steels are presented. Thermal aging increases the tensile strength of these steels. The high-C Mo-bearing $\mathrm{CF}-8 \mathrm{M}$ steels are more susceptible to thermal aging than the Mo-free CF-3 or CF-8 steels. A procedure and correlations are presented for predicting the change in tensile flow and yield stresses and engineering stress-vs.-strain curve of cast stainless steel as a function of time and temperature of service. The tensile properties of aged cast stainless steel are estimated from known material information, i.e., chemical composition and the initial tensile strength of the steel. The correlations described in this report may be used for assessing thermal embrittlement of cast stainless steel components.
\end{abstract}




\section{Contents}

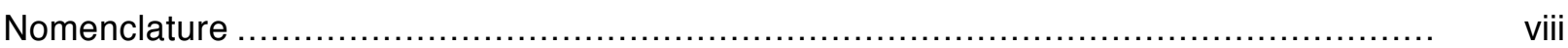

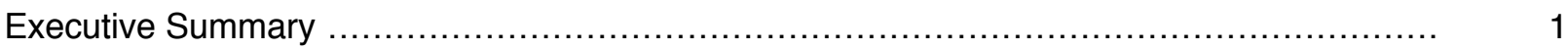

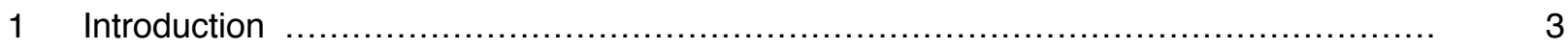

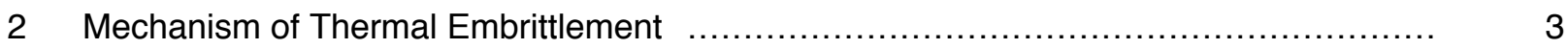

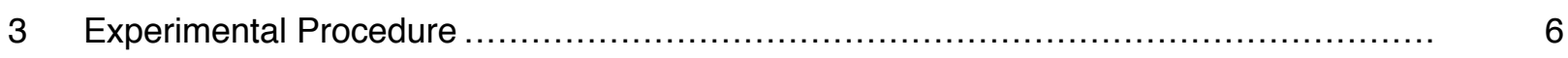

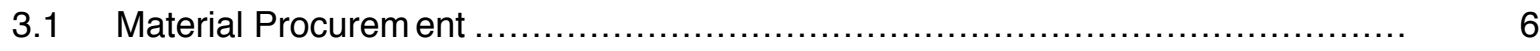

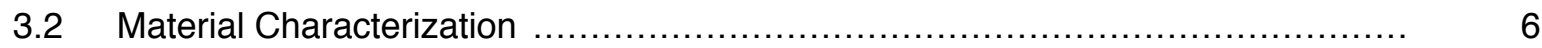

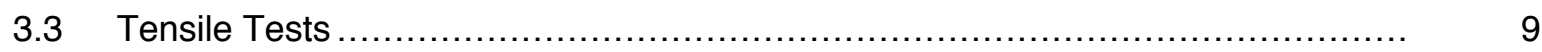

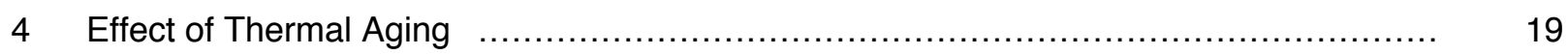

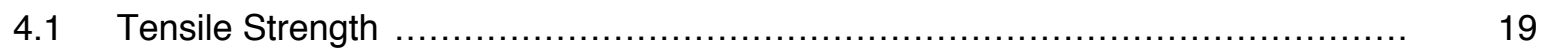

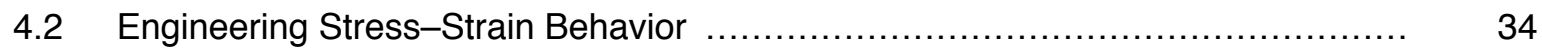

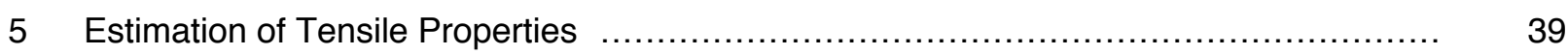

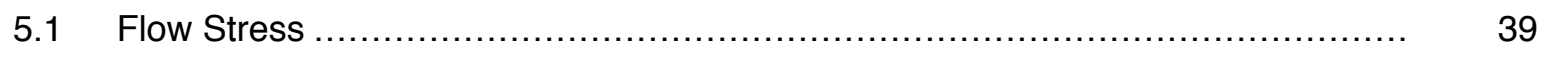

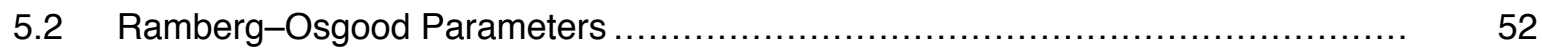

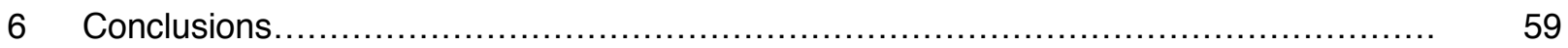

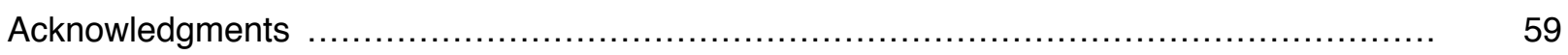

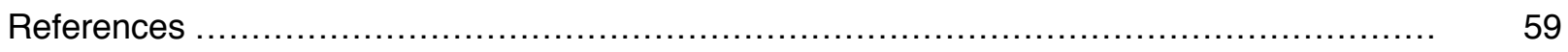

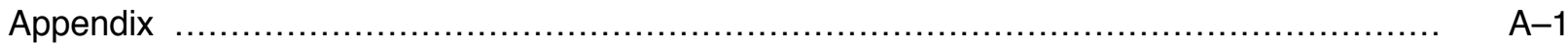

\section{List of Figures}

1. Decrease in Charpy-impact energy for various heats of cast stainless steels

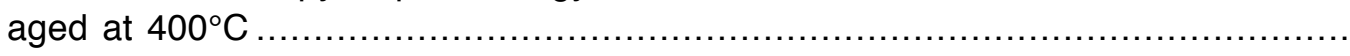

2. Grain structure along the axial and circumferential sections of centrifugally cast pipes P1 and P2. 
3. Grain structure of various static-cast slabs .

4. Ferrite morphology from three locations across the thickness of static-cast slabs of CF-3 (Heat 69), CF-8 (Heats 73 and 68), and CF-8M (Heats 74, 70, and 75).

5. Ferrite morphology along the three orientations of the centrifugally cast pipes (Heats P1, P2, and P3) and the static-cast pump impeller (Heat I)

6. Orientation and location of the mechanical-test specimens taken from (a) and

(b) pipe sections and (c) slabs

7. Orientation and location of the mechanical-test specimens taken from KRB pump cover plate.

8. Configuration of tensile test specimen

9. Effect of thermal aging on engineering stress vs. strain curves for Heat 69

10. Effect of ther mal aging on engineering stress vs. strain curves for Heat P2

11. Effect of thermal aging on engineering stress vs. strain curves for Heat 68

12. Effect of thermal aging on engineering stress vs. strain curves for Heat P1

13. Effect of thermal aging on engineering stress vs. strain curves for Heat 74

14. Effect of thermal aging on engineering stress vs. strain curves for Heat 75

15. Ratio of yield and ultimate stress of aged and unaged cast stainless steel as a function of the aging parameter $P$

16. Fracture surface of tensile specimens of unaged and aged Heat P1 tested at room temperature and $290^{\circ} \mathrm{C}$

17. Fracture surface of tensile specimens of unaged and aged Heat 68 tested at room temperature and $290^{\circ} \mathrm{C}$

18. Fracture surface of tensile specimens of unaged and aged Heat 75 tested at room temperature and $290^{\circ} \mathrm{C}$

19. Values of yield and ultimate stress estimated from Charpy-impact data and those obtained from tensile tests for Heats $69,68,74$, and 75

20. Examples of ROFIT analysis of tensile stress-vs.-strain curves at room temperature and $290^{\circ} \mathrm{C}$ for cast stainless steels using flow stress as the reference stress. 
21. Flow stress ratio of aged cast stainless steels at RT and $290^{\circ} \mathrm{C}$ as a function of the normalized aging parameter. The solid lines represent correlations obtained by subtracting $\sigma$ from the best-fit curve.

22. Experimental and estimated flow stress of aged cast stainless steel at $290^{\circ} \mathrm{C}$ and RT

23. Experimental and estimated yield stress of aged cast stainless steel at $290^{\circ} \mathrm{C}$ and RT

24. Correlation between the Ramberg/Osgood parameter $\alpha_{1}$ and flow stress at RT and $290^{\circ} \mathrm{C}$ for cast stainless steels.

25. Engineering stress-vs.-strain curve at RT and $290^{\circ} \mathrm{C}$, estimated from the chemical composition and initial tensile strength and determined

experimentally for thermally aged $\mathrm{CF}-3, \mathrm{CF}-8$, and $\mathrm{CF}-8 \mathrm{M}$ steels

\section{List of Tables}

1. Product form, chemical composition, hardness, and ferrite morphology of various heats of cast stainless steel

2. Ferrite content and grain structure of various cast stainless steel pipes $\quad \ldots \ldots \ldots \ldots . . . .7$

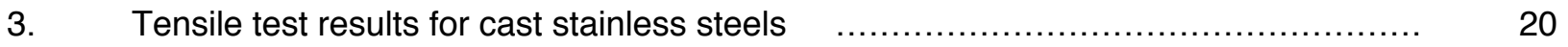

4. Best-fit values of the Ramberg-Osgood constants for unaged and aged cast stainless steels at room temperature.

5. Best-fit values of the Ramberg-Osgood constants for unaged and aged cast stainless steels at $290^{\circ} \mathrm{C}$

6. Values of constants in Eq. 8 for estimating tensile flow stress of aged cast stainless steels

7. Values of constants in Eq. 10 for estimating tensile yield stress of aged cast stainless steels.

8. Values of Ramberg/Osgood parameter $n_{1}$ and the constants in Eq. 11 for estimating parameter $\alpha_{1}$ for aged cast stainless steels 


\section{Nomenclature}

b Uncracked ligament of Charpy-impact specimen (mm).

B Thickness of Charpy-impact specimen (mm).

E Modulus of elasticity (MPa).

P Aging parameter, i.e., the log of the time of aging at $400^{\circ} \mathrm{C}$.

$\mathrm{P}_{y} \quad$ Yield load for an instrumented Charpy-impact test (N).

Q Activation energy for the process of thermal embrittlement ( $\mathrm{kJ} / \mathrm{mole})$.

n Ramberg-Osgood parameter.

$R_{f} \quad$ Ratio of the tensile flow stress of aged and unaged cast stainless steel.

$\mathrm{R}_{\mathrm{y}} \quad$ Ratio of the tensile yield stress of aged and unaged cast stainless steel.

t $\quad$ Service or aging time $(h)$.

$\mathrm{T}_{\mathrm{S}} \quad$ Service or aging temperature $\left({ }^{\circ} \mathrm{C}\right)$.

W Width of Charpy-impact specimen (mm).

$\alpha \quad$ Ramberg-Osgood parameter.

$\varepsilon \quad$ Engineering strain.

$\varepsilon_{0} \quad$ Reference strain in Ramberg-Osgood equation.

$\theta \quad$ Aging behavior at $400^{\circ} \mathrm{C}$, i.e., the log of the time to achieve $\beta$ reduction in impact energy at $400^{\circ} \mathrm{C}$.

$\sigma \quad$ Engineering stress (MPa).

$\sigma_{f} \quad$ Engineering flow stress (MPa).

$\sigma_{0} \quad$ Reference stress in Ramberg-Osgood equation (MPa).

$\sigma_{\mathrm{y}} \quad$ Engineering yield stress (MPa).

SI units of measure have been used in this report. Conversion factors for measurements in British units are as follows:

$\begin{array}{lll}\text { To convert } & \text { to } & \text { multiply by } \\ \text { in. } & \mathrm{mm} & 25.4 \\ \mathrm{~J}^{\star} & \mathrm{ft} \cdot \mathrm{lb} & 0.7376 \\ \mathrm{~kJ} / \mathrm{m}^{2} & \text { in. }-\mathrm{lb} / \mathrm{in}^{2} & 5.71015 \\ \mathrm{~kJ} / \mathrm{mole} & \mathrm{kcal} / \mathrm{mole} & 0.239\end{array}$

* When impact energy is expressed in $\mathrm{J} / \mathrm{cm}^{2}$, first multiply by 0.8 to obtain impact energy of a standard Charpy-V notch specimen in $\mathrm{J}$. 


\section{Executive Summary}

Cast duplex stainless steels used in light water reactor (LWR) systems for primary pressure-boundary components are susceptible to thermal embrittlement at reactor operating temperatures. Thermal aging of cast stainless steels at these temperatures causes an increase in hardness and tensile strength and a decrease in ductility, impact strength, and fracture toughness of the material and the Charpy transition curve shifts to higher temperatures. Investigations at Argonne National Laboratory have shown that thermal embrittlement of cast stainless steel components may occur within the reactor design lifetime of $40 \mathrm{yr}$. Various grades and heats of cast stainless steel exhibit varying degrees of thermal embrittlement. In general, the low-C CF-3 steels are the most resistant to thermal embrittlement, and the Mobearing, high-C CF-8M steels are the least resistant. An assessment of mechanical-property degradation due to thermal embrittlement is therefore required to evaluate the performance of cast stainless steel components during prolonged exposure to service temperatures, because rupture of the primary pressure boundary could lead to a loss-of-coolant accident and possible exposure of the public to radiation.

This report presents tensile-property data on several heats of cast stainless steels aged up to $58,000 \mathrm{~h}$ at temperatures between 290 and $450^{\circ} \mathrm{C}\left(554\right.$ and $\left.752^{\circ} \mathrm{F}\right)$. The tensile data are analyzed to establish the effects of thermal aging on tensile strength and engineering stress-strain behavior (represented by the Ramberg-Osgood equation) of cast stainless steels. A procedure and correlations are presented for predicting the change in tensile flow and yield stress, and in the engineering stress-vs.-strain curve of cast stainless steel components due to thermal aging during service in LWRs. The tensile properties of aged cast stainless steel are estimated from information that is readily available from certified material test records for the component, i.e., chemical composition and the initial tensile strength of the unaged material. The correlations described in this report may be used for assessing thermal embrittlement of cast stainless steel components. 


\section{Introduction}

Cast duplex stainless steels used in light water reactor (LWR) systems for primary pressure-boundary components, such as valve bodies, pump casings, and primary coolant piping, are susceptible to thermal embrittlement at reactor operating temperatures, i.e., $280-320^{\circ} \mathrm{C}$ $\left(536-608^{\circ} \mathrm{F}\right)$. Thermal aging of cast stainless steels (i.e., ASTM Specification A-351 for Grades $\mathrm{CF}-3, \mathrm{CF}-3 \mathrm{~A}, \mathrm{CF}-8, \mathrm{CF}-8 \mathrm{~A}$, and $\mathrm{CF}-8 \mathrm{M}^{*}$ ) at these temperatures causes an increase in hardness and tensile strength and a decrease in ductility, impact strength, and fracture toughness of the material and the Charpy transition curve shifts to higher temperatures. An assessment of mechanical-property degradation due to thermal embrittlement is therefore required to evaluate the performance of cast stainless steel components during prolonged exposure to service temperatures, because rupture of the primary pressure boundary could lead to a loss-ofcoolant accident and possible exposure of the public to radiation.

Investigations at Argonne National Laboratory (ANL) ${ }^{1-10}$ and elsewhere ${ }^{1-16}$ have shown that thermal embrittlement of cast stainless steel components occurs during the reactor design lifetime of $40 \mathrm{yr}$. Various grades and heats of cast stainless steel exhibit varying degrees of thermal embrittlement. In general, the low-C CF-3 steels are the most resistant to thermal embrittlement, and the Mo-bearing, high-C CF-8M steels are the least resistant.

A procedure and correlations have been developed at ANL for estimating mechanical properties of cast stainless steel components under LWR operating conditions from material information readily available in certified material test records. 5 These correlations were later updated and optimized with an expanded data base and mechanical-property results obtained from cast stainless steels that were aged up to $\approx 58,000 \mathrm{~h}$ at $290-350^{\circ} \mathrm{C}\left(554-662^{\circ} \mathrm{F}\right) \cdot 6,7$ The results are expressed in terms of engineering fracture toughness parameters that can be used in fitness-for-service analyses, e.g., "NRCPIPE" computer code for analyzing elastic-plastic fracture mechanics of nuclear power plant piping. However, correlations for estimating tensile stress-strain behavior of thermally aged cast stainless steels have not been developed.

This report presents tensile-property data on several heats of cast stainless steels aged up to $58,000 \mathrm{~h}$ at temperatures between 290 and $450^{\circ} \mathrm{C}\left(554\right.$ and $\left.752^{\circ} \mathrm{F}\right)$. The results are analyzed to establish the effects of thermal aging on tensile strength and engineering stress-strain behavior, represented by the Ramberg-Osgood (RO) equation, of cast stainless steels. A procedure and correlations are presented for estimating tensile flow and yield stress and RO parameters of aged cast stainless steel from known material information, i.e., composition and initial tensile strength of the unaged material.

\section{Mechanism of Thermal Embrittlement}

Thermal embrittlement of cast duplex stainless steels results in brittle fracture associated with either cleavage of ferrite or separation of the ferrite/austenite phase boundary. Thermal aging of cast stainless steels at temperatures $<500^{\circ} \mathrm{C}\left(932^{\circ} \mathrm{F}\right)$ leads to precipitation of additional

\footnotetext{
* In this report, Grades CF-3A and CF- $8 A$ are considered equivalent to CF-3 and CF-8, respectively. The A designation represents high tensile strength. The chemical composition of $\mathrm{CF}-3 \mathrm{~A}$ and $\mathrm{CF}-8 \mathrm{~A}$ is further restricted within the composition limits of $\mathrm{CF}-3$ and $\mathrm{CF}-8$, respectively, to obtain a ferrite/austenite ratio that results in higher ultimate and yield strengths.
} 
phases in the ferrite, e.g., formation of a $\mathrm{Cr}$-rich $\alpha^{\prime}$ phase by spinodal decomposition; nucleation and growth of $\alpha^{\prime}$; precipitation of a $\mathrm{Ni}-$ and $\mathrm{Si}-$ rich $\mathrm{G}$ phase, $\mathrm{M}_{23} \mathrm{C}_{6}$ carbide, and $\gamma_{2}$ austenite; and additional precipitation and/or growth of existing carbides at the ferrite/austenite phase boundaries.1,2,8-10,17-21 Thermal aging has little or no effect on the austenite phase. The formation of $\mathrm{Cr}$-rich $\alpha^{\prime}$ phase by spinodal decomposition of ferrite is the primary mechanism for thermal embrittlement. The $\alpha^{\prime}$ phase strengthens the ferrite matrix, i.e., it increases strain hardening and local tensile stress, so that the critical stress level for brittle fracture is achieved at higher temperatures.

The degree or extent of thermal embrittlement is controlled by the amount of brittle fracture, which depends on both material and aging conditions. In some cast stainless steels, a fraction of the material may fail in brittle fashion but the surrounding austenite provides ductility and toughness. Such steels have adequate impact strength even after long-term aging. Predominantly brittle failure occurs when either the ferrite phase is continuous, e.g., in cast material with a large ferrite content, or the ferrite/austenite phase boundary provides an easy path for crack propagation, e.g., in high-C or high-N steels that contain phase-boundary carbides or nitrides. Consequently, the amount, size, and distribution of ferrite in the duplex structure and phase-boundary precipitates are important parameters that control the extent of thermal embrittlement. The decrease in room-temperature (RT) Charpy-impact energy during thermal aging at $400^{\circ} \mathrm{C}\left(752^{\circ} \mathrm{F}\right)$ of various heats of cast stainless steel ${ }^{4-6,11,15,16}$ is shown in Fig. 1. The results indicate that all the materials reach a "saturation" RT impact energy, i.e., a minimum value that would be achieved by the material after long-term aging. The actual value of the saturation RT impact energy for a specific cast stainless steel is independent of aging temperature but depends strongly on the chemical composition of the steel; it is lower for the Mo-bearing CF-8M steels than for the Mo-free CF-3 or CF-8 steels, and decreases with an increase in ferrite content or the concentration of $\mathrm{C}$ or $\mathrm{N}$ in the steel.

The time to reach saturation, i.e., the kinetics of thermal embrittlement, depends on both material and aging parameters. Figure 1 indicates that the time for aging at $400^{\circ} \mathrm{C}$ for a given decrease in impact energy varies by more than two orders of magnitude for the various heats,

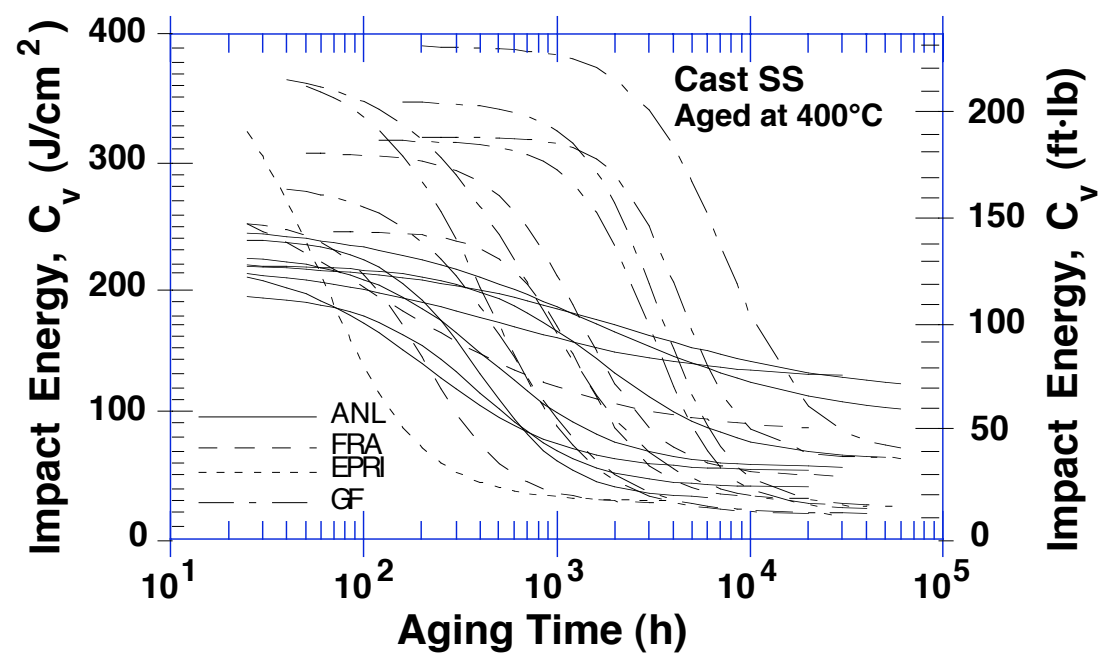

Figure 1. Decrease in Charpy-impact energy for various heats of cast stainless steels aged at $400^{\circ} \mathrm{C}$. FRA = Framatome, EPRI= Electrical Power Research Institute, GF= Georg Fischer Co. 
ANL and Framatome (FRA) heats, and 10,000-30,000 $\mathrm{h}$ for the Georg Fischer Co. (GF) heats. Activation energies of thermal embrittlement range from 65 to $230 \mathrm{~kJ} / \mathrm{mole}$ (15 to $55 \mathrm{kcal} / \mathrm{mole}) .4-6,11,13-15,22$ These values are well below the $202 \mathrm{~kJ} / \mathrm{mole}(48 \mathrm{kcal} / \mathrm{mole})$ value associated with $\mathrm{Cr}$ bulk diffusion in the $\mathrm{Fe}-28 \mathrm{Cr}$ alloy. 23 Small changes in the constituent elements of the material can cause the kinetics of thermal embrittlement to vary significantly. The logarithm of the aging time at $400^{\circ} \mathrm{C}$ for a $50 \%$ reduction in RT Charpy-impact energy has been shown to be an important parameter for characterizing the kinetics of thermal embrittlement. 5,6

Activation energy is high for steels that show fast embrittlement at $400^{\circ} \mathrm{C}$ and low for those that show slow embrittlement at $400^{\circ} \mathrm{C}$. Also, materials with the same chemical composition but differing heat treatment show different kinetics of embrittlement. ${ }^{11}$ Microstructural examination of aged cast stainless steels suggests that slow embrittlement at $400^{\circ} \mathrm{C}$ and low activation energy are associated with clusters of $\mathrm{Ni}-\mathrm{Si}, \mathrm{Mo}-\mathrm{Si}$, and $\mathrm{Ni}-\mathrm{Si}-\mathrm{Mo}$ in the ferrite matrix. ${ }^{8-10}$ These clusters are considered precursors of G-phase nucleation and precipitation. Cast stainless steels with low activation energy and slow embrittlement at $400^{\circ} \mathrm{C}$ show $\mathrm{G}$-phase precipitation after aging, and steels with high activation energy and fast embrittlement at $400^{\circ} \mathrm{C}$ do not contain a $\mathrm{G}$ phase. ${ }^{8-10,17,18}$ The presence of $\mathrm{Ni}-\mathrm{Si}-\mathrm{Mo}$ clusters in the ferrite matrix of an unaged material may be considered a signature of steels that are potentially sensitive to thermal embrittlement, i.e., steels with $\mathrm{Ni}-\mathrm{Si}-\mathrm{Mo}$ clusters in the ferrite matrix show low activation energy for thermal embrittlement but take longer to embrittle at $400^{\circ} \mathrm{C}$.

The kinetics of thermal embrittlement of cast stainless steels are controlled primarily by the kinetics of ferrite strengthening, e.g., activation energy determined from ferrite hardness measurements shows very good agreement with that obtained from the Charpy-impact data.13,14,22 Microstructural characterization and annealing studies on thermally aged cast stainless steel show that strengthening of ferrite is caused primarily by spinodal decomposition of ferrite to form the $\mathrm{Cr}$-rich $\alpha^{\prime}$ phase.4,9,10 Consequently, the kinetics of thermal embrittlement should be controlled by the amplitude and frequency of $\mathrm{Cr}$ fluctuations produced by spinodal decomposition, i.e., by the size and spacing of the $\alpha^{\prime}$ phase. The low activation energies of thermal embrittlement are most likely caused by variations in the spacing of $\mathrm{Cr}$ fluctuations; atom probe field-ion microscopy studies indicate that the spacing between $\mathrm{Cr}$ fluctuations decreases with decreasing temperature.17,21 During thermal aging, production heat treatment and possibly the casting process, both of which affect ferrite composition and microstructure of unaged material, can influence microstructural evolution and, therefore, the kinetics of embrittlement.

For most materials, the kinetics of thermal embrittlement vary with aging temperature. For a specific heat of cast stainless steel, activation energy of thermal embrittlement is not constant over the temperature range of $290-450^{\circ} \mathrm{C}\left(554-842^{\circ} \mathrm{F}\right)$, but increases with decreasing temperature. ${ }^{1-3,13}$ The increase is particularly significant between 400 and $450^{\circ} \mathrm{C}$ $\left(752\right.$ and $\left.842^{\circ} \mathrm{F}\right)$. In addition, materials aged at $450^{\circ} \mathrm{C}$ show precipitation of phase-boundary carbides (also nitrides in high- $\mathrm{N}$ steels) and a decrease in ferrite content of the steel. ${ }^{1,2}$ At reactor temperatures, such processes either do not occur or their kinetics are extremely slow. Consequently, data obtained after $450^{\circ} \mathrm{C}$ aging do not reflect the mechanisms that are active under reactor operating conditions, and extrapolation of the $450^{\circ} \mathrm{C}$ data to predict the extent of thermal embrittlement at reactor temperatures is not valid. The activation energy for thermal embrittlement may be represented by an average value in the temperature range of $290-400^{\circ} \mathrm{C}$ $\left(554-752^{\circ} \mathrm{F}\right)$. 


\section{Experimental Procedure}

\subsection{Material Procurement}

Material was obtained from various experimental and commercial heats of CF-3, CF-8, and CF-8M (ASTM Specification A 351) cast stainless steels in differing product forms and section thicknesses. Six experimental heats of cast stainless steel were procured in the form of 76-mm-thick slabs. Materials from the commercial heats included sections of four different centrifugally cast pipes (CF-8 and CF-8M grades), a pump impeller (CF-3 grade), and a pump casing ring ( $\mathrm{CF}-8$ grade). The outer diameter and wall thickness of the cast pipes ranged from 0.6 to $0.9 \mathrm{~m}$ and 38.1 to $76.2 \mathrm{~mm}$, respectively.

A cover plate assembly from the recirculating pump of the $K R B$ reactor in Gundremmingen, Germany was also procured. The cover plate assembly was in service for approximately eight effective full power years (efpys) at a service temperature of $284^{\circ} \mathrm{C}\left(543^{\circ} \mathrm{F}\right)$. The plate assembly was decontaminated and samples were obtained from the inner 25-mmthick region of the plate. The average temperature of the samples was estimated to be $280^{\circ} \mathrm{C}$.

\subsection{Material Characterization}

The various cast materials were characterized to determine their chemical composition, hardness, grain structure, and ferrite content and distribution. All castings were examined in the three orientations as well as at different locations, namely, material near the center and near the inner and outer surfaces of the pipes, and top and bottom regions of the slabs. A ferrite scope (Auto Test FE, Probe Type FSP-1) was used to measure the ferrite content of the castings. The instrument was calibrated with several weld metal standards (ferrite numbers between 2.4 and 28.1) obtained from the British Welding Institute. The distribution of ferrite was determined by the intercept method, i.e., by counting the number of ferrite/austenite phase boundaries intersected by a superimposed outline. The chemical composition, hardness, and ferrite content and distribution of the cast materials are given in Table 1. For each material, the ferrite content calculated from the composition with Hull's equivalent factor ${ }^{24}$ are also listed in the table.

Some differences in hardness and ferritic content were observed for material from various locations in the castings. The ferrite content was lower and the hardness slightly higher toward the top of the static-cast slabs or the inner surface of the centrifugally cast pipes. This behavior appears to be related to compositional variations, particularly changes in Ni content. In general, the hardness of the cast material increased with an increase in ferrite content. For the same ferrite content, the hardness of $\mathrm{CF}-8$ and $\mathrm{CF}-8 \mathrm{M}$ material is comparable, whereas the hardness of CF-3 material is lower. An increase in N content increases the hardness of cast stainless steels of all grades.

The grain structures of the various castings were examined along the axial, circumferential, and radial sections. Descriptions of the structures observed in the four centrifugally cast pipe sections and the pump casing ring are given in Table 2. Two castings, Heats P1 and P2, contain equiaxed grains across the entire thickness of the pipe. Grain size and distribution in the various orientations do not differ significantly, as shown in Fig. 2. The equiaxed grains were probably produced intentionally by a low pouring temperature or by shear between the 
Table 1. Product form, chemical composition, hardness, and ferrite morphology of various heats of cast stainless steel

\begin{tabular}{|c|c|c|c|c|c|c|c|c|c|c|c|c|c|c|}
\hline \multirow[b]{2}{*}{ Heat } & \multirow[b]{2}{*}{ Grade } & \multicolumn{9}{|c|}{ Chemical Composition (wt.\%) } & \multicolumn{2}{|c|}{$\begin{array}{c}\text { Ferrite }^{a} \\
(\%)\end{array}$} & \multirow{2}{*}{$\begin{array}{c}\text { Hard- } \\
\text { ness } \\
\mathrm{R}_{\mathrm{B}} \\
\end{array}$} & \multirow{2}{*}{$\begin{array}{c}\text { Ferrite } \\
\text { Spacing } \\
(\mu \mathrm{m})\end{array}$} \\
\hline & & $\mathrm{Mn}$ & $\mathrm{Si}$ & $\mathrm{P}$ & $\mathrm{S}$ & Mo & $\mathrm{Cr}$ & $\mathrm{Ni}$ & $\mathrm{N}$ & C & Calc. & Meas. & & \\
\hline \multicolumn{15}{|c|}{$\underline{76-\mathrm{mm} \text { Slabs }}{ }^{\mathrm{b}}$} \\
\hline 69 & CF-3 & 0.63 & 1.13 & 0.015 & 0.005 & 0.34 & 20.18 & 8.59 & 0.028 & 0.023 & 21.0 & 23.6 & 83.7 & 35 \\
\hline 73 & CF-8 & 0.72 & 1.09 & 0.028 & 0.016 & 0.25 & 19.43 & 8.54 & 0.053 & 0.070 & 7.0 & 7.7 & 78.8 & 253 \\
\hline 68 & CF-8 & 0.64 & 1.07 & 0.021 & 0.014 & 0.31 & 20.64 & 8.08 & 0.062 & 0.063 & 14.9 & 23.4 & 84.6 & 87 \\
\hline 70 & CF-8M & 0.55 & 0.72 & 0.021 & 0.016 & 2.30 & 19.17 & 9.01 & 0.049 & 0.066 & 14.2 & 18.9 & 86.5 & 96 \\
\hline 74 & CF-8M & 0.54 & 0.73 & 0.022 & 0.016 & 2.51 & 19.11 & 9.03 & 0.048 & 0.064 & 15.5 & 18.4 & 85.8 & 90 \\
\hline 75 & CF-8M & 0.53 & 0.67 & 0.022 & 0.012 & 2.58 & 20.86 & 9.12 & 0.052 & 0.065 & 24.8 & 27.8 & 89.5 & 69 \\
\hline \multicolumn{15}{|c|}{$\underline{\text { Reactor Components }}^{\mathrm{C}}$} \\
\hline P3 & $\mathrm{CF}-3$ & 1.06 & 0.88 & 0.017 & 0.014 & 0.01 & 18.89 & 8.45 & 0.168 & 0.021 & 2.8 & 1.9 & 82.2 & - \\
\hline $\mathrm{P} 2$ & $\mathrm{CF}-3$ & 0.74 & 0.94 & 0.019 & 0.006 & 0.16 & 20.20 & 9.38 & 0.040 & 0.019 & 12.5 & 15.6 & 83.8 & 69 \\
\hline I & $\mathrm{CF}-3$ & 0.47 & 0.83 & 0.030 & 0.011 & 0.45 & 20.20 & 8.70 & 0.032 & 0.019 & 20.4 & 17.1 & 81.0 & 65 \\
\hline $\mathrm{C} 1$ & CF-8 & 1.22 & 1.18 & 0.033 & 0.008 & 0.65 & 19.00 & 9.37 & 0.040 & 0.039 & 7.8 & 2.2 & 79.5 & - \\
\hline $\mathrm{P} 1$ & CF-8 & 0.59 & 1.12 & 0.026 & 0.013 & 0.04 & 20.49 & 8.10 & 0.056 & 0.036 & 17.7 & 24.1 & 84.9 & 90 \\
\hline 205 & CF-8M & 0.93 & 0.63 & 0.019 & - & 3.37 & 17.88 & 8.80 & - & 0.040 & 21.0 & 15.9 & - & 79 \\
\hline 758 & CF-8M & 0.91 & 0.62 & 0.018 & - & 3.36 & 17.91 & 8.70 & - & 0.030 & 24.2 & 19.2 & - & 62 \\
\hline \multicolumn{15}{|c|}{$\underline{\text { Reactor-Aged }}^{d}$} \\
\hline KRB & CF-8 & 0.31 & 1.17 & - & - & 0.17 & 21.99 & 8.03 & 0.038 & 0.062 & 27.7 & 34.0 & - & - \\
\hline
\end{tabular}

a Calculated from the composition with Hull's equivalent factor.

Measured by ferrite scope AUTO Test FE, Probe Type FSP-1.

b Static Cast Slabs: Foundry ESCO; Size 610 x 610 x 76 mm.

c Centrifugally Cast Pipes:

P3 Foundry SANDUSKY; Size $580 \mathrm{~mm}$ O.D.,76 mm wall.

$P 2$ Foundry FAM, France; Size $930 \mathrm{~mm}$ O.D., $73 \mathrm{~mm}$ wall.

$P 1$ Foundry ESCO; Size $890 \mathrm{~mm}$ O.D., $63 \mathrm{~mm}$ wall. 205 Size $305 \mathrm{~mm}$ O.D., $25 \mathrm{~mm}$ wall.

Static Cast:

Elbow 758: Size $305 \mathrm{~mm}$ O.D., $30 \mathrm{~mm}$ wall.

Pump Impeller l. Foundry ESCO; Size $660 \mathrm{~mm}$ diameter.

Pump Casing C1: Foundry ESCO; Size $600 \mathrm{~mm}$ O.D., $57 \mathrm{~mm}$ wall.

d KRB Reactor Pump Cover Plate: Foundry GF; Size $890 \mathrm{~mm}$ diameter.

Table 2. Ferrite content and grain structure of various cast stainless steel pipes

\begin{tabular}{|c|c|c|c|c|c|c|c|}
\hline \multirow[b]{2}{*}{ Heat } & \multirow{2}{*}{$\begin{array}{l}\text { o.d. } \\
(\mathrm{m})\end{array}$} & \multirow{2}{*}{$\begin{array}{l}\text { Wall } \\
(\mathrm{mm})\end{array}$} & \multirow[b]{2}{*}{ Process } & \multirow[b]{2}{*}{ Grade } & \multicolumn{2}{|c|}{ Ferrite Content $(\%)^{a}$} & \multirow[b]{2}{*}{ Grain Structure } \\
\hline & & & & & o.d. & i.d. & \\
\hline $\mathrm{C} 1$ & 0.60 & 57.1 & Static & CF-8 & 2.3 & 1.7 & $\begin{array}{l}\text { Banded, columnar/equiaxed radial to } \\
\text { axial growth near ends }\end{array}$ \\
\hline P1 & 0.89 & 63.5 & Centr. & CF-8 & 27.6 & 19.5 & Equiaxed across thickness \\
\hline P3 & 0.58 & 51.6 & Centr. & CF-3 & 2.5 & 0.9 & $\begin{array}{l}\text { Banded, radially oriented columnar one } \\
\text { equiaxed band }(\approx 4 \mathrm{~mm} \text { deep) near i.d. }\end{array}$ \\
\hline $\mathrm{P} 2$ & 0.93 & 73.0 & Centr. & CF-3 & 15.9 & 13.2 & Equiaxed across thickness \\
\hline
\end{tabular}




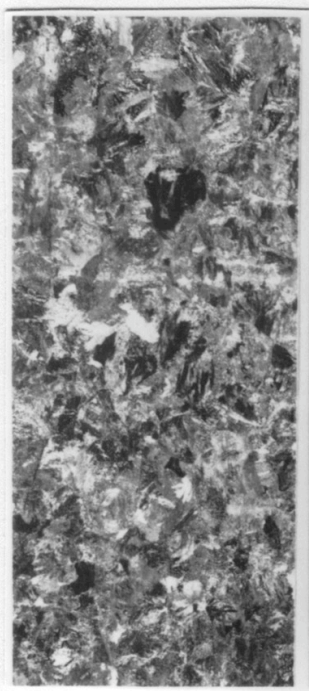

Axial Heat $\mathbf{P} 1$

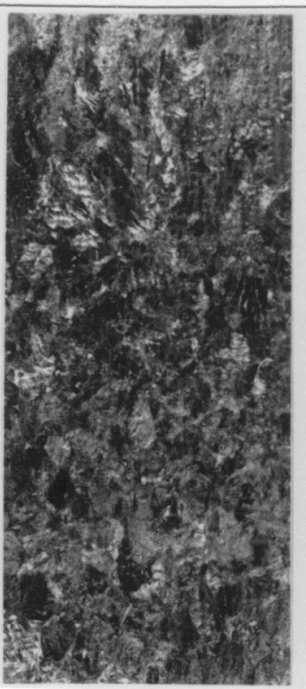

Circum.

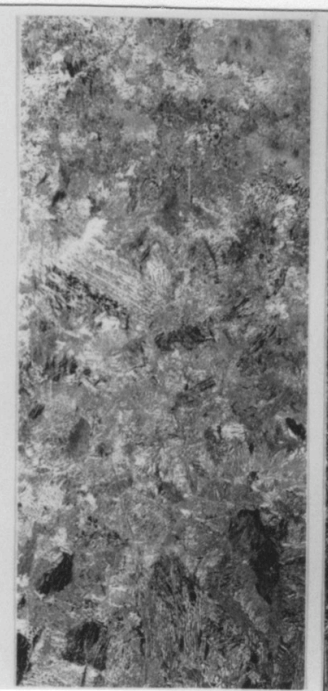

Axial

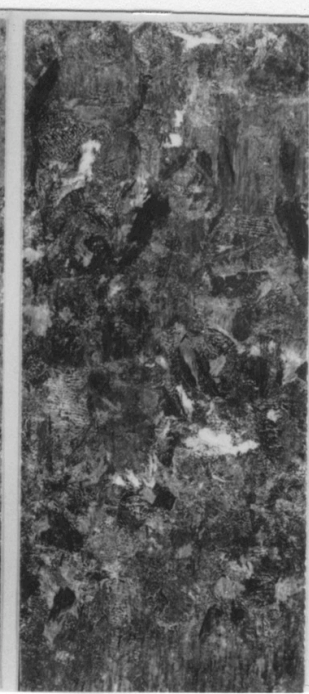

Circum.

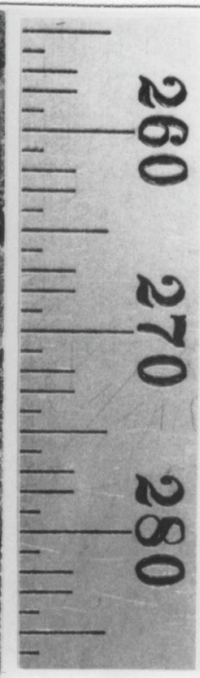

Heat P2

Axial

Circum.

Heat P1

Circum.

Heat P2

Figure 2. Grain structure along the axial and circumferential sections of centrifugally cast pipes P1 and P2

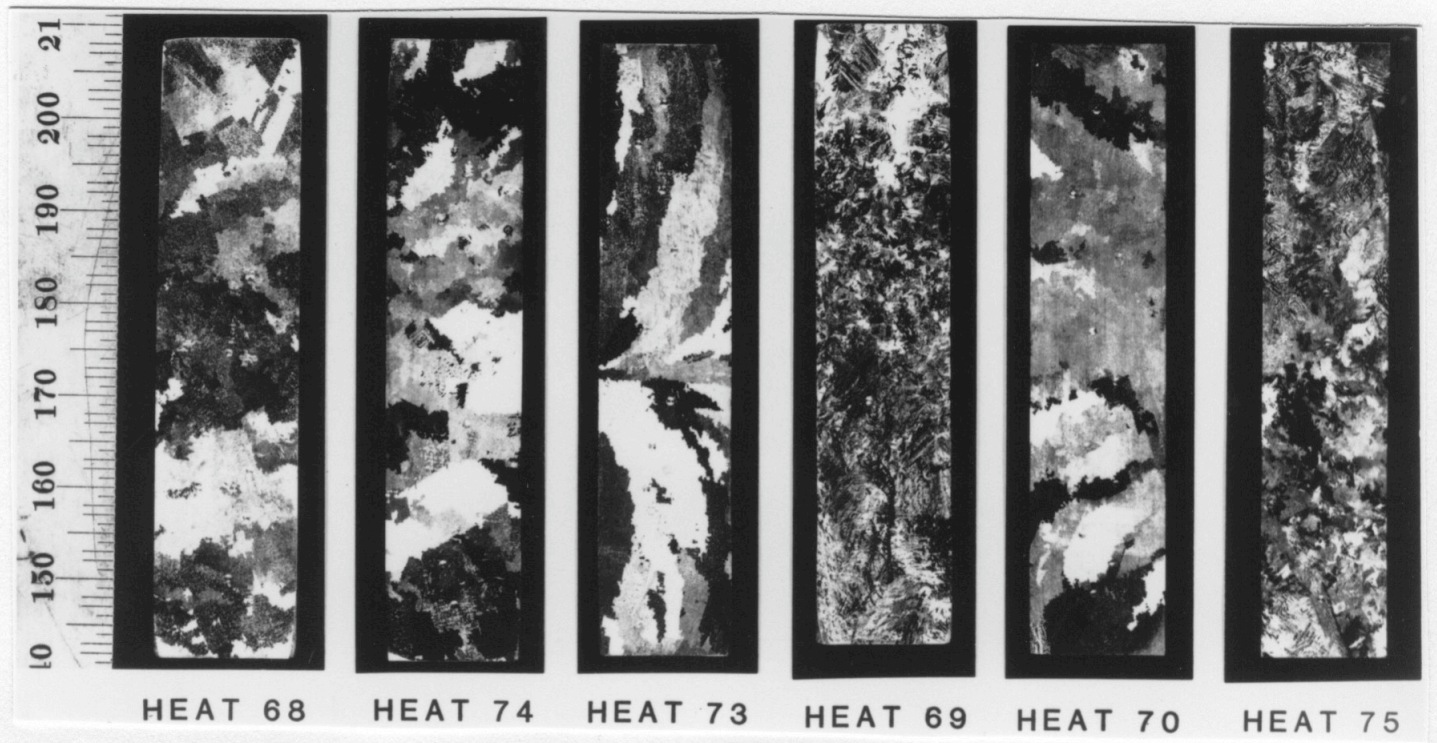

Figure 3. Grain structure of various static-cast slabs

liquid and solid; the shear could cause dendrite arms to break and disperse in the liquid-solid region.

The static-cast slabs and the pump casing ring showed a mixed structure of columnar and equiaxed grains. The grain structures of the cast slabs are shown in Fig. 3. A change from horizontal to vertical growth of the columnar grains was observed near the edges of the cast slabs, e.g., Heat 73. The size of the equiaxed grains in the mixed structure is generally smaller than that of the columnar grains. 
The ferrite morphology of the various materials varied with ferrite content, chemical composition, and size of the casting. The ferrite morphology was globular for materials containing $<5 \%$ ferrite. Some differences in morphology were observed between the different grades of cast stainless steel containing $>5 \%$ ferrite. The CF-8 and CF-8M steels exhibited a lacy morphol ogy, i.e., an interlaced network of ferrite islands, whereas the CF-3 cast steel showed a mixture of lacy and acicular ferrite. The acicular morphology is characterized by fine needle-like ferrite that is distributed in the austenite matrix. All morphologies were randomly arranged within the casting. Typical microstructures for the static-cast slabs and pump impeller and centrifugally cast pipes are shown in Figs. 4 and 5.

\section{3 Tensile Tests}

Tensile tests were conducted at ANL and at Materials Engineering Associates (MEA). The orientation and location of the mechanical-test specimens from pipe sections, slabs, and the KRB pump cover plate are shown in Figs. 6 and 7. The specimen blanks from the experimental and commercial heats were aged at $290,320,350,400$, and $450^{\circ} \mathrm{C}(554,608,662,752$, and $842^{\circ} \mathrm{F}$ ) for times up to $58,000 \mathrm{~h}$. Details of the test procedure and results for the tests at MEA have been presented earlier.25,26

Tests at ANL were performed according to ASTM Specification E 8 and E 21 in an Instron tensile test machine with a maximum loading capacity of $90 \mathrm{kN}$ (20 kips). Cylindrical specimens with a diameter of $5.0 \mathrm{~mm}(0.2 \mathrm{in}$.) and a gauge length of $20.3 \mathrm{~mm}(0.8 \mathrm{in}$.) were used for all the tests, Fig. 8. An axial extensometer, with an initial gauge length of $20.3 \mathrm{~mm}(0.8 \mathrm{in}$.), was used for continuous measurement of strain during room-temperature tests. An IBM computer was used to digitize load, crosshead movement, and axial displacement data, and to store the data on floppy disks. Analog traces of load-vs.-crosshead displacement and load-vs.-extensometer displacement were also obtained for each test.

The true stress-strain data were calculated up to the maximum load using the constantvolume criterion, which assumes a homogeneous distribution of strain along the gauge length. However, most specimens showed inhomogeneous deformation because of relatively large grain structure. The specimen surfaces along the gauge length were irregular, and the fracture cross sections were often elliptical. These factors created some uncertainty in the true stress-strain data. True fracture stress was obtained from the fracture load and cross-sectional area at fracture. The strain at fracture, i.e., total elongation, was determined from extensometer displacements. Total elongation was also measured from crosshead displacements; the values obtained from the extensometer were $\approx 64 \%$ of the values determined from crosshead displacement. Correlations between crosshead displacement and total strain in the specimen gauge length were also developed from the room-temperature tests. These correlations were used to correct the data for the elevated-temperature tests.

The tests at $290^{\circ} \mathrm{C}\left(\approx 550^{\circ} \mathrm{F}\right)$ were conducted in a forced-air recirculating furnace. Thermocouples were mounted above and below the specimen gauge length to monitor and control the temperature within $\pm 2^{\circ} \mathrm{C}$. For the tests on samples aged for $<10,000 \mathrm{~h}$, an axial extensometer was not used for the elevated-temperature tests. Total strain in the specimen gauge length was determined from correlations developed from the room-temperature tests. The total elongation was determined from the crosshead displacement multiplied by 0.64 . 

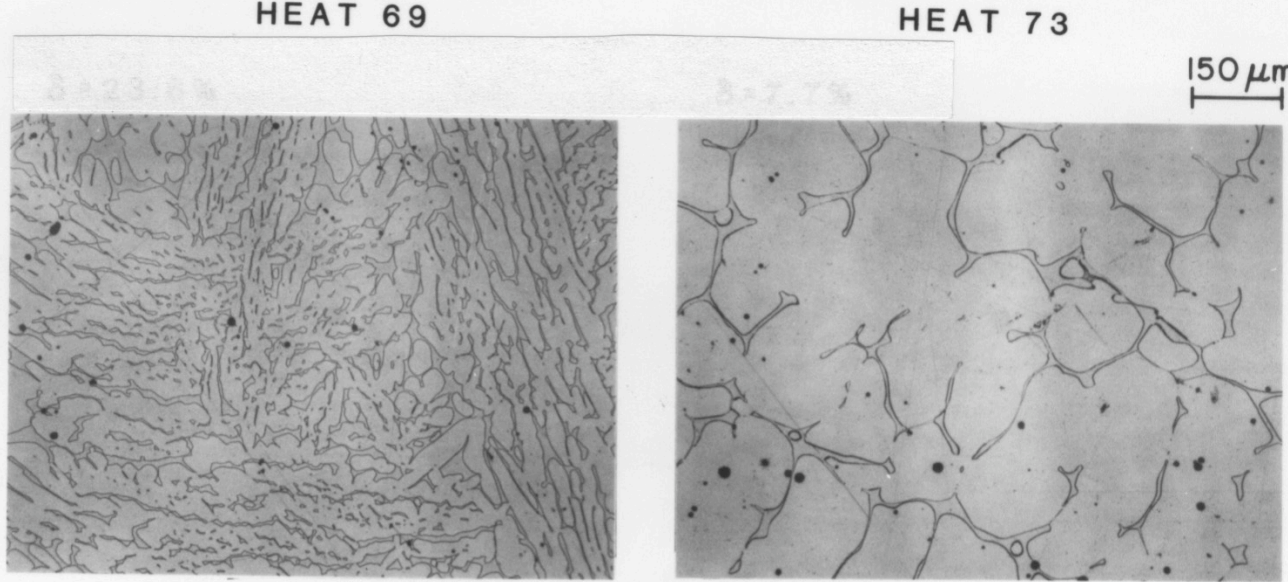

TOP SECTION
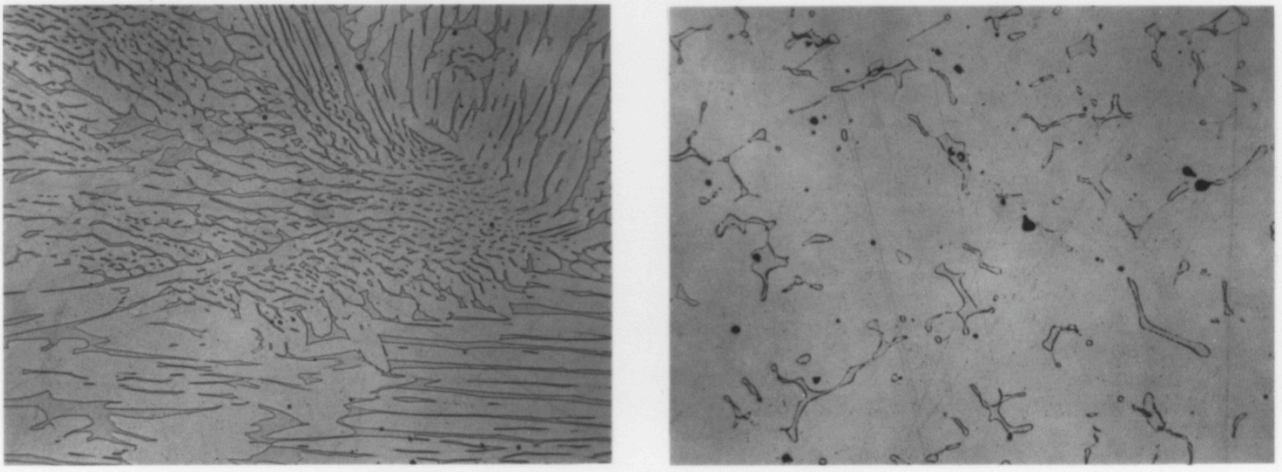

MIDDLE SECTION
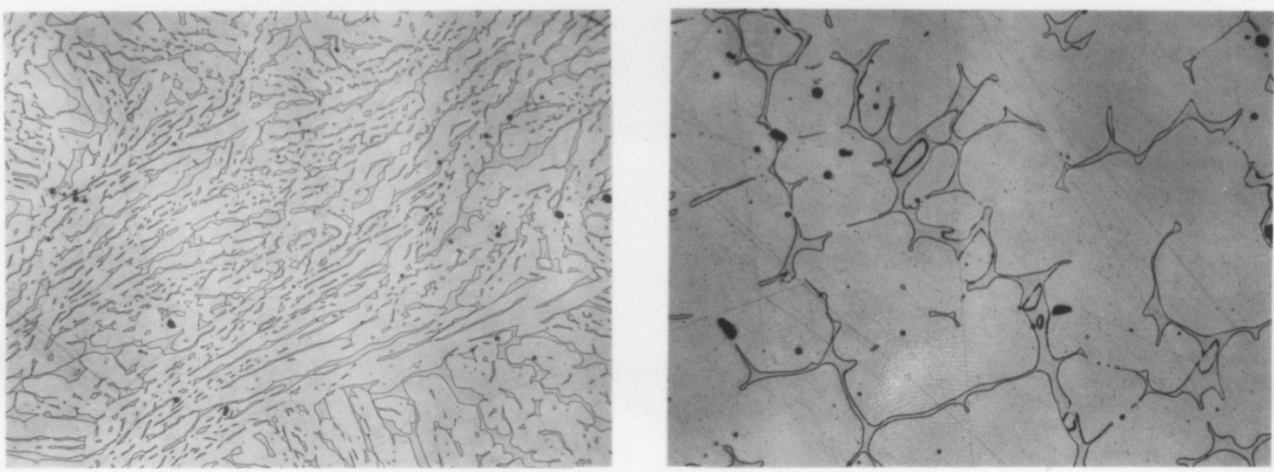

BOTTOM SECTION

Figure 4. Ferrite morphology from three locations across the thickness of static-cast slabs of $\mathrm{CF}-3$ (Heat 69), $\mathrm{CF}-8$ (Heats 73 and 68), and $\mathrm{CF}-8 \mathrm{M}$ (Heats 74, 70, and 75) 
HEAT 68

HEAT 74
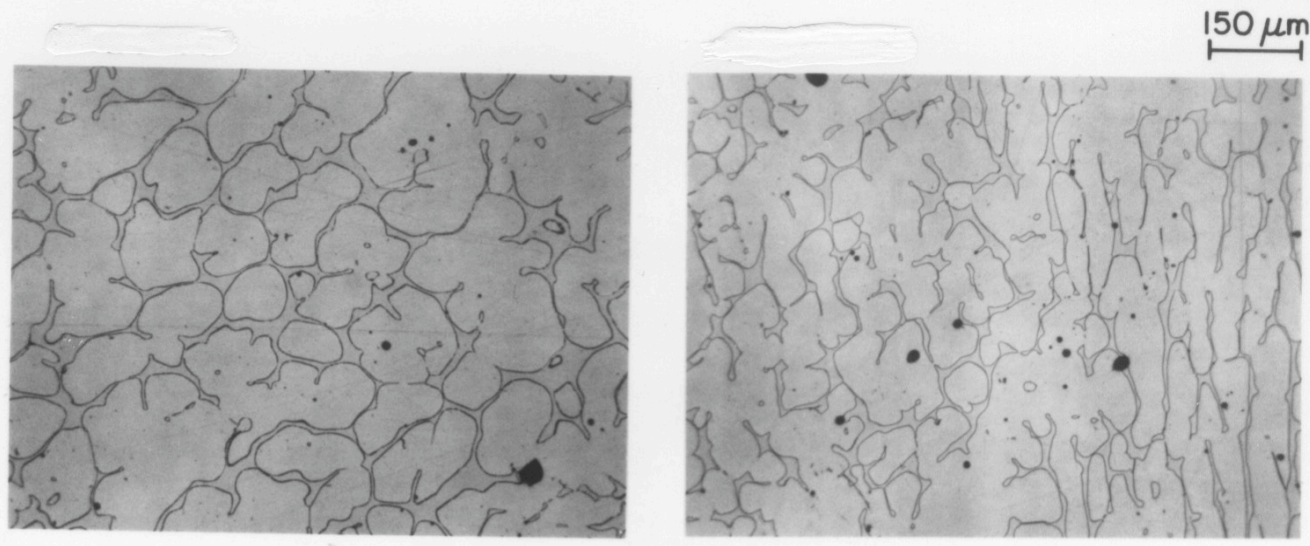

TOP SECTION
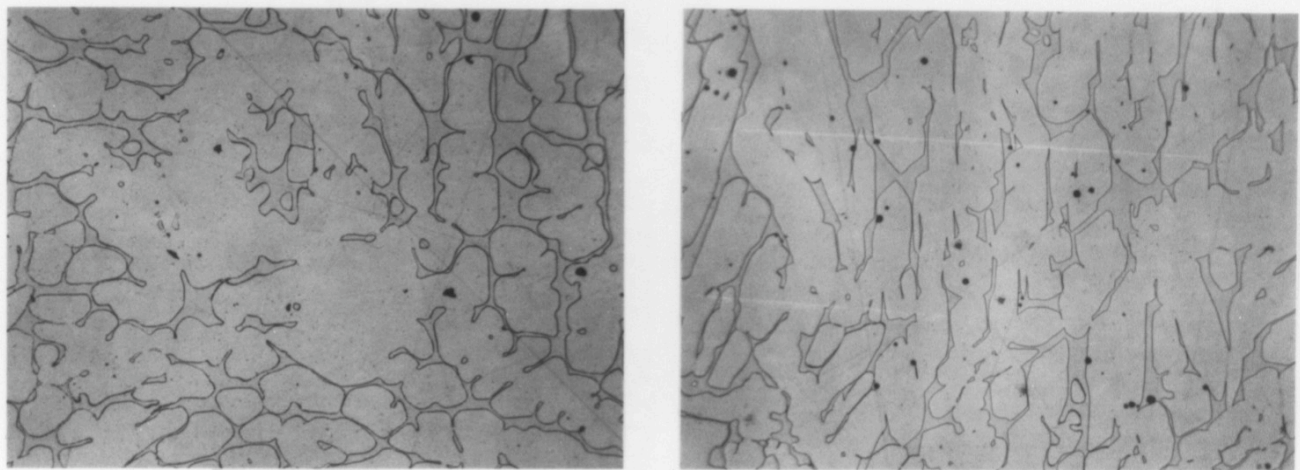

MIDDLE SECTION
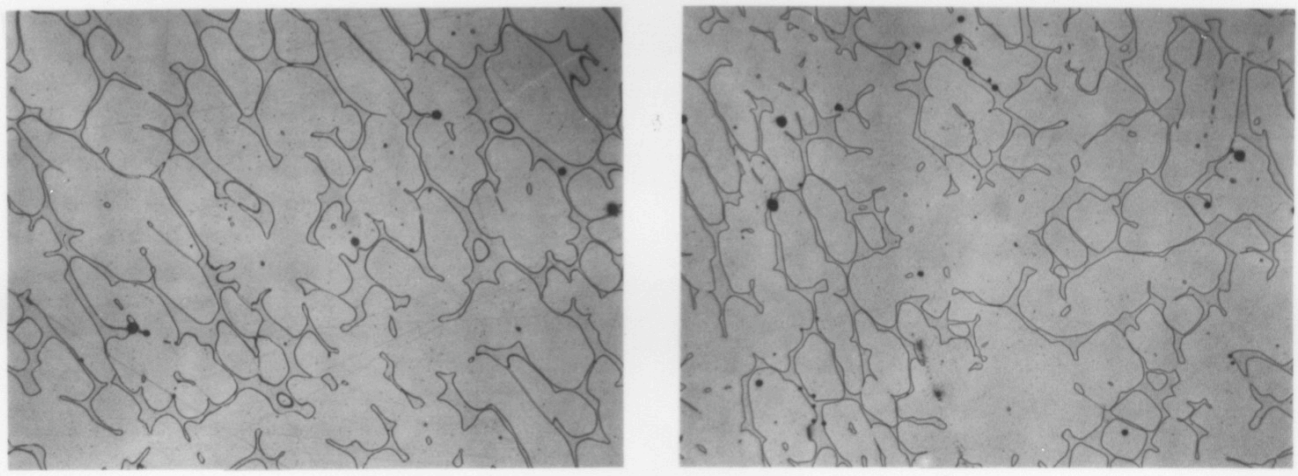

BOTTOM SECTION

Figure 4. (Contd.) 

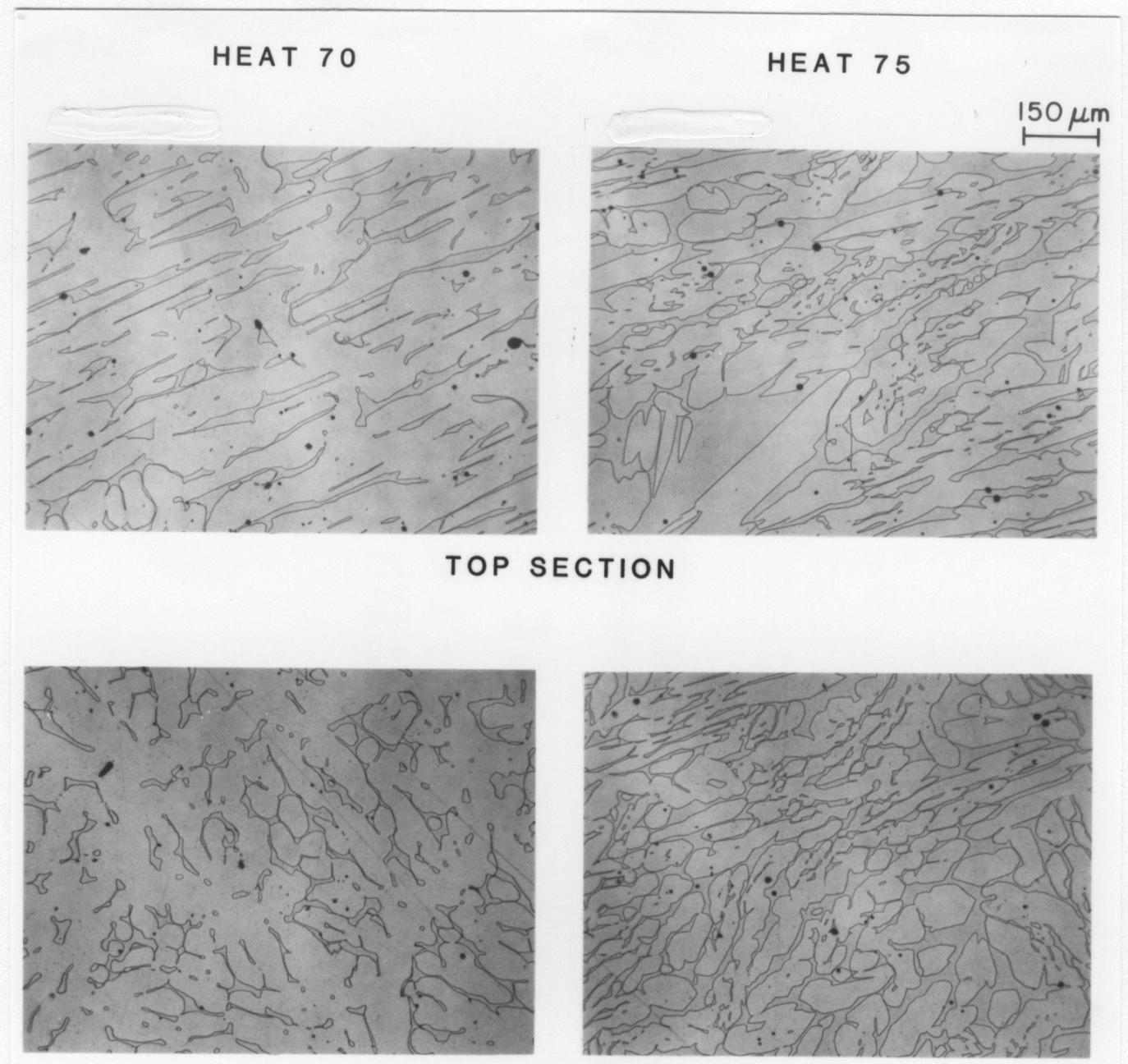

MIDDLE SECTION
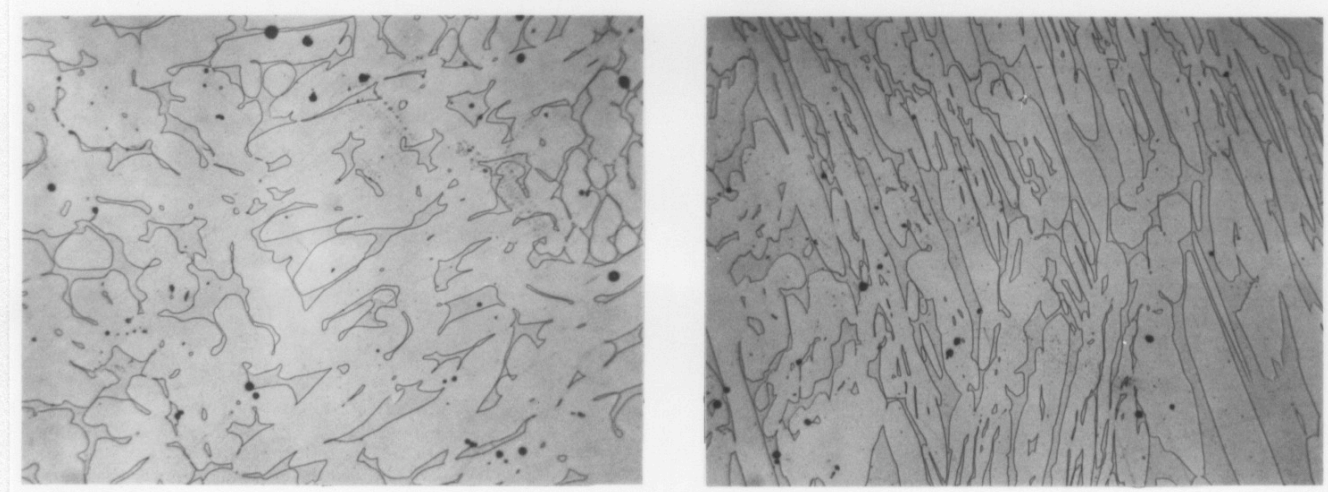

BOTTOM SECTION

Figure 4. (Contd.) 
HEAT P 1
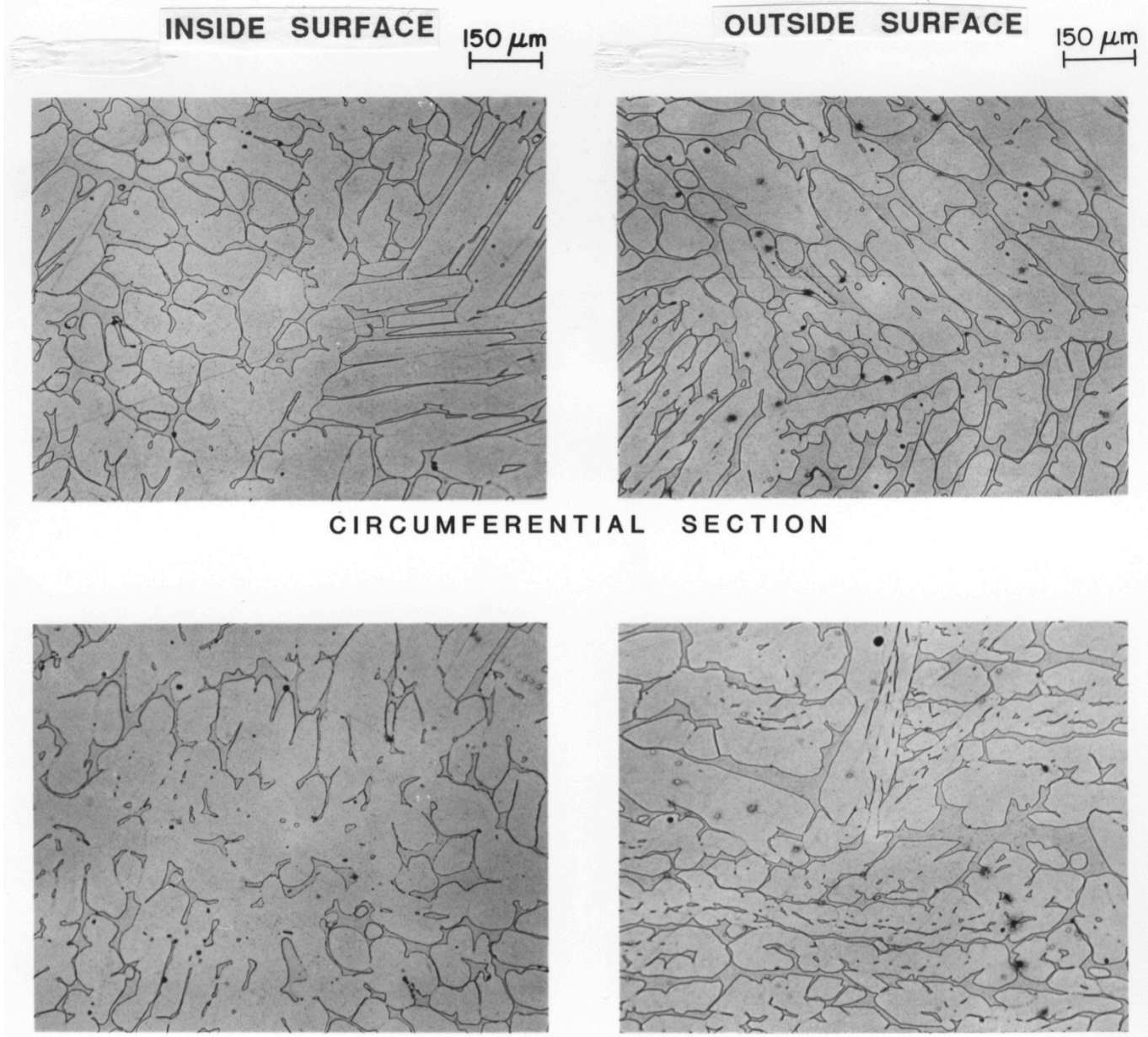

LONGITUDINAL SECTION
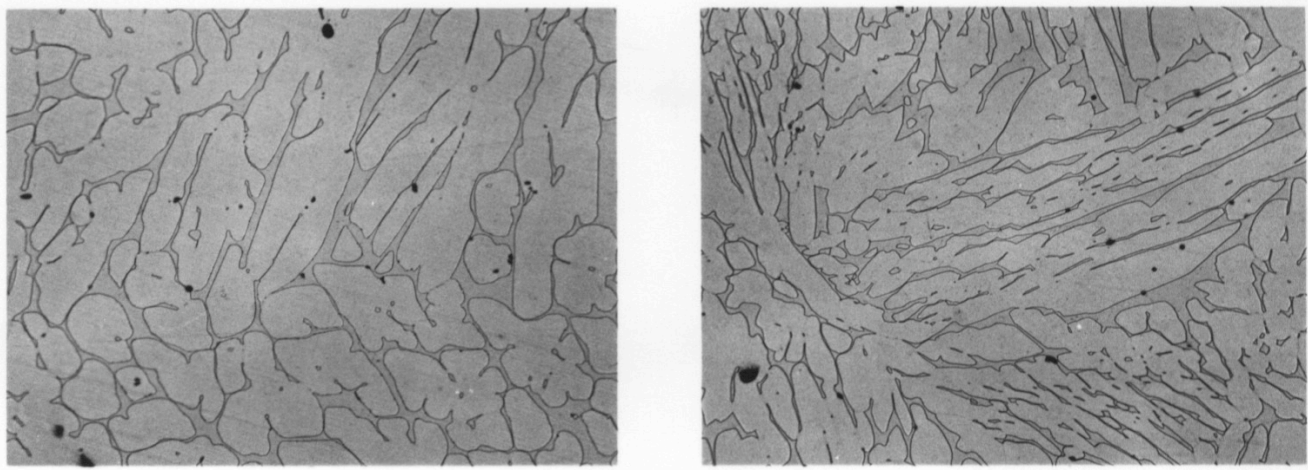

RADIAL SECTION

Figure 5. Ferrite morphology along the three orientations of the centrifugally cast pipes (Heats P1, P2, and P3) and the static-cast pump impeller (Heat I) 

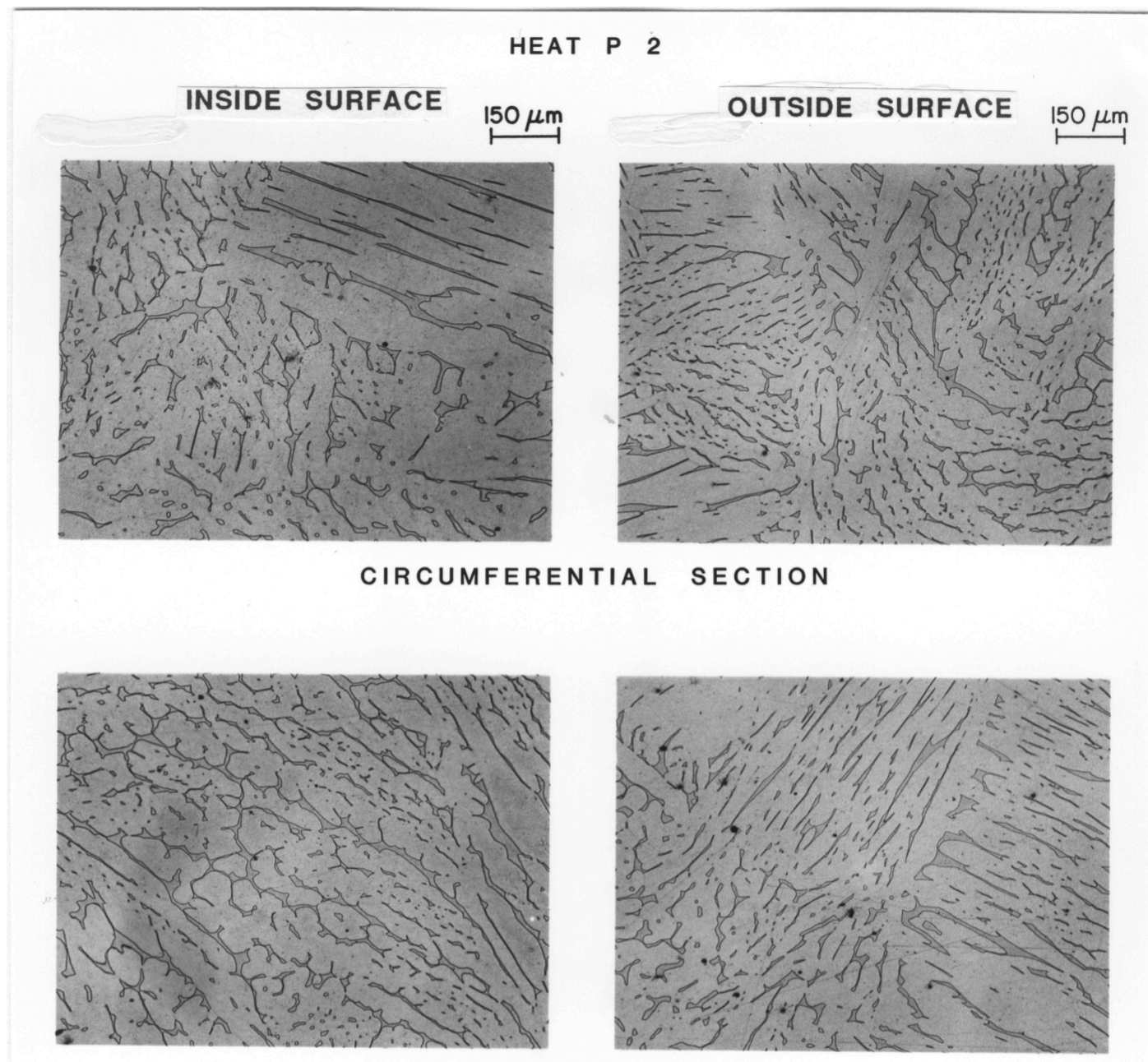

LONGITUDINAL SECTION
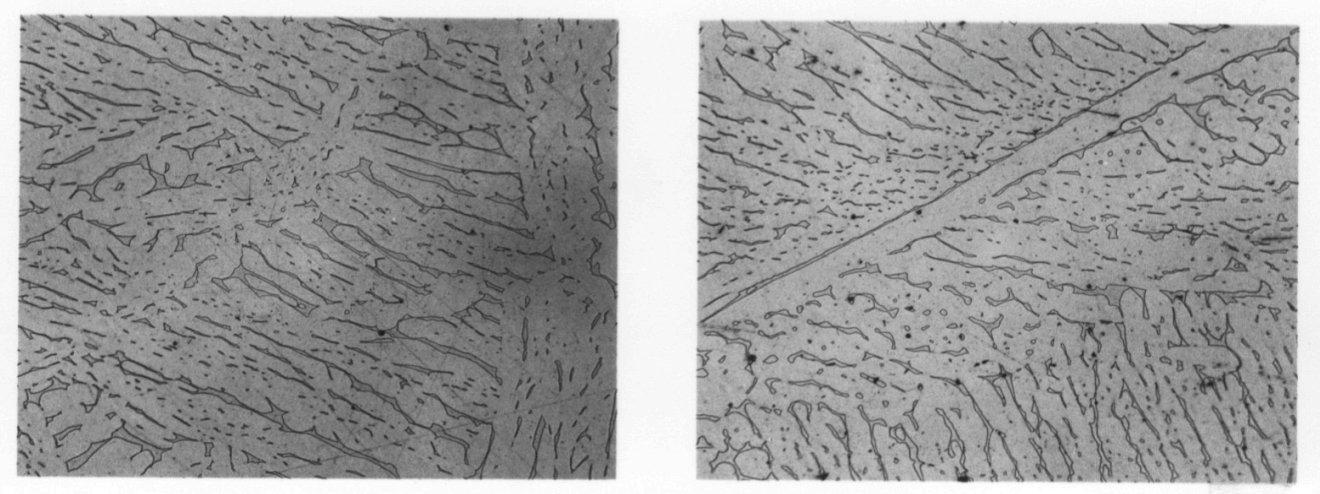

RADIAL SECTION

Figure 5. (Contd.) 
HEAT P 3
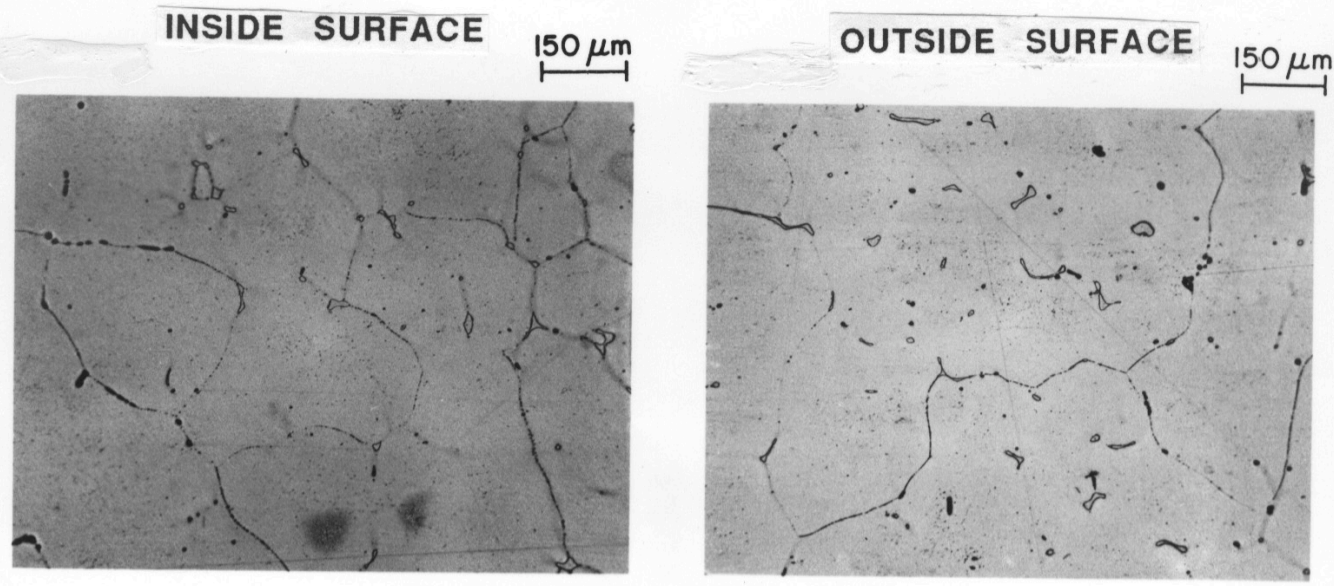

CIRCUMFERENTIAL SECTION
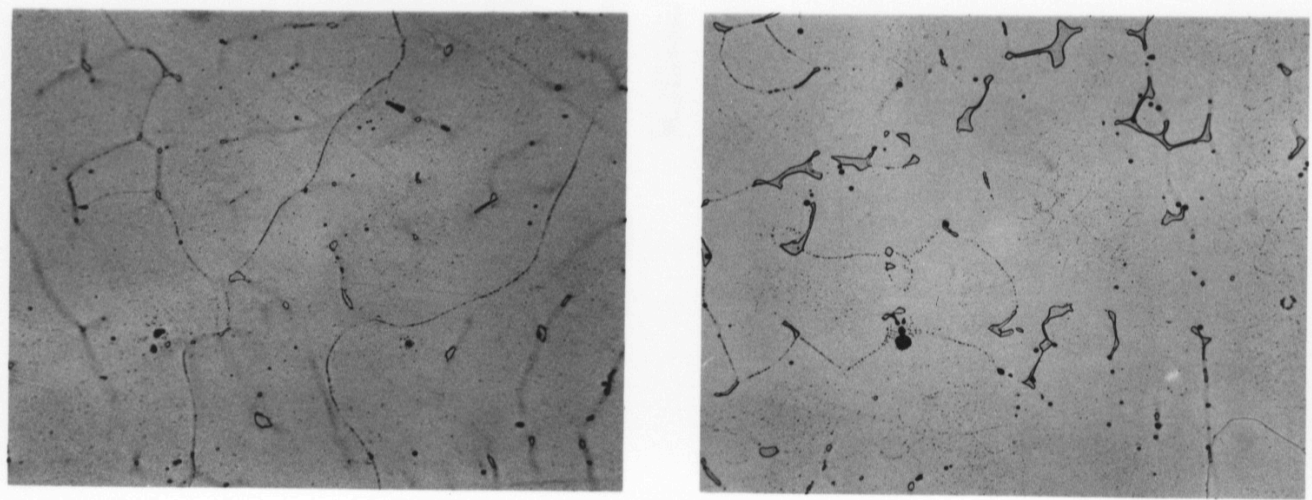

LONGITUDINAL SECTION
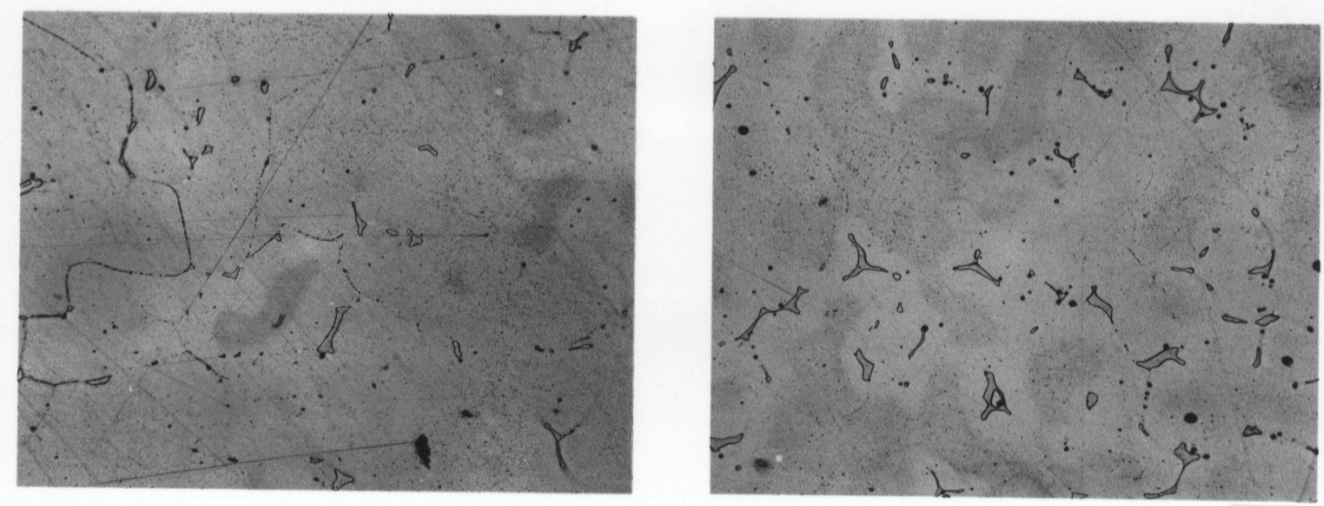

RADIAL SECTION

Figure 5. (Contd.) 


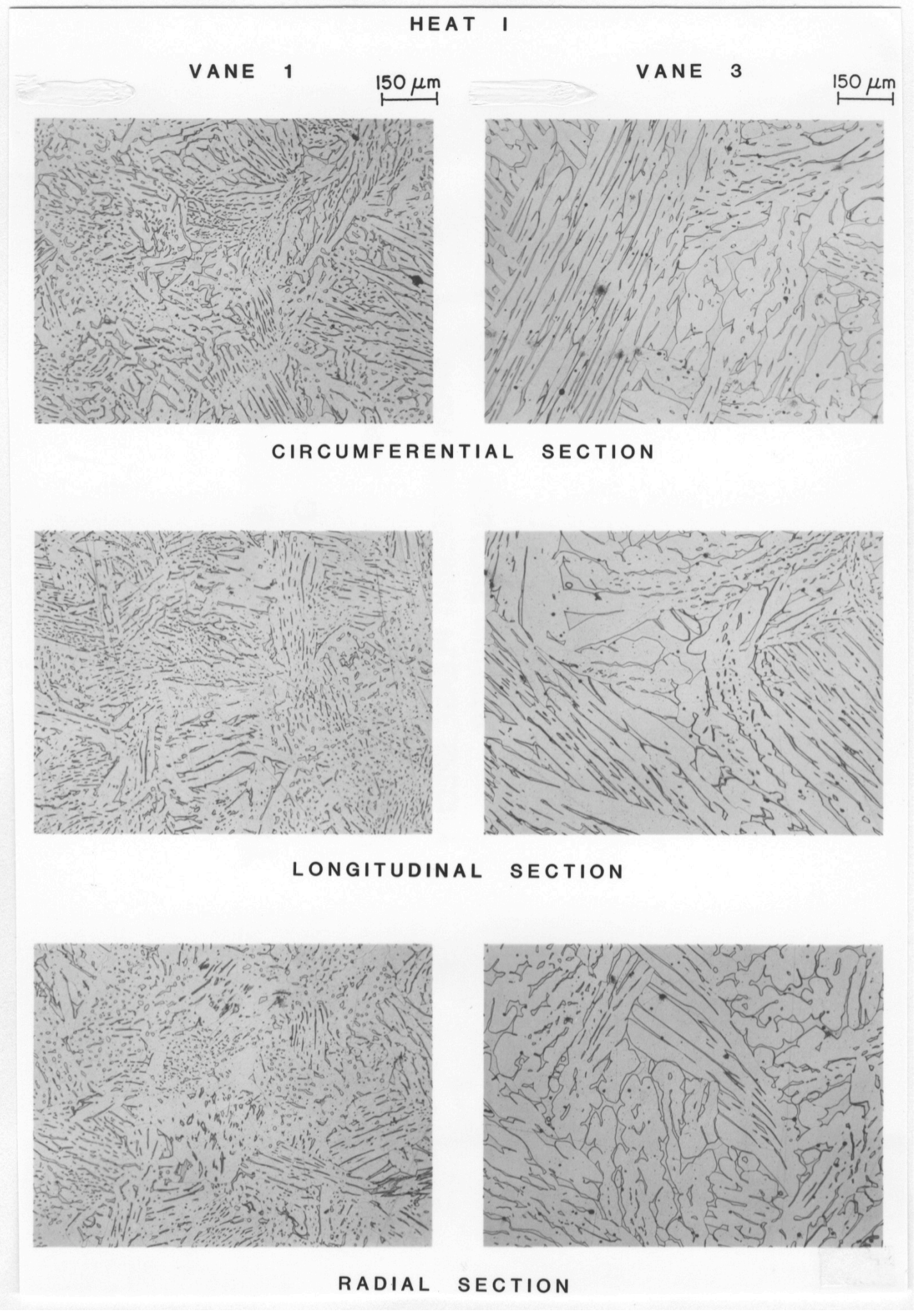

Figure 5. (Contd.) 

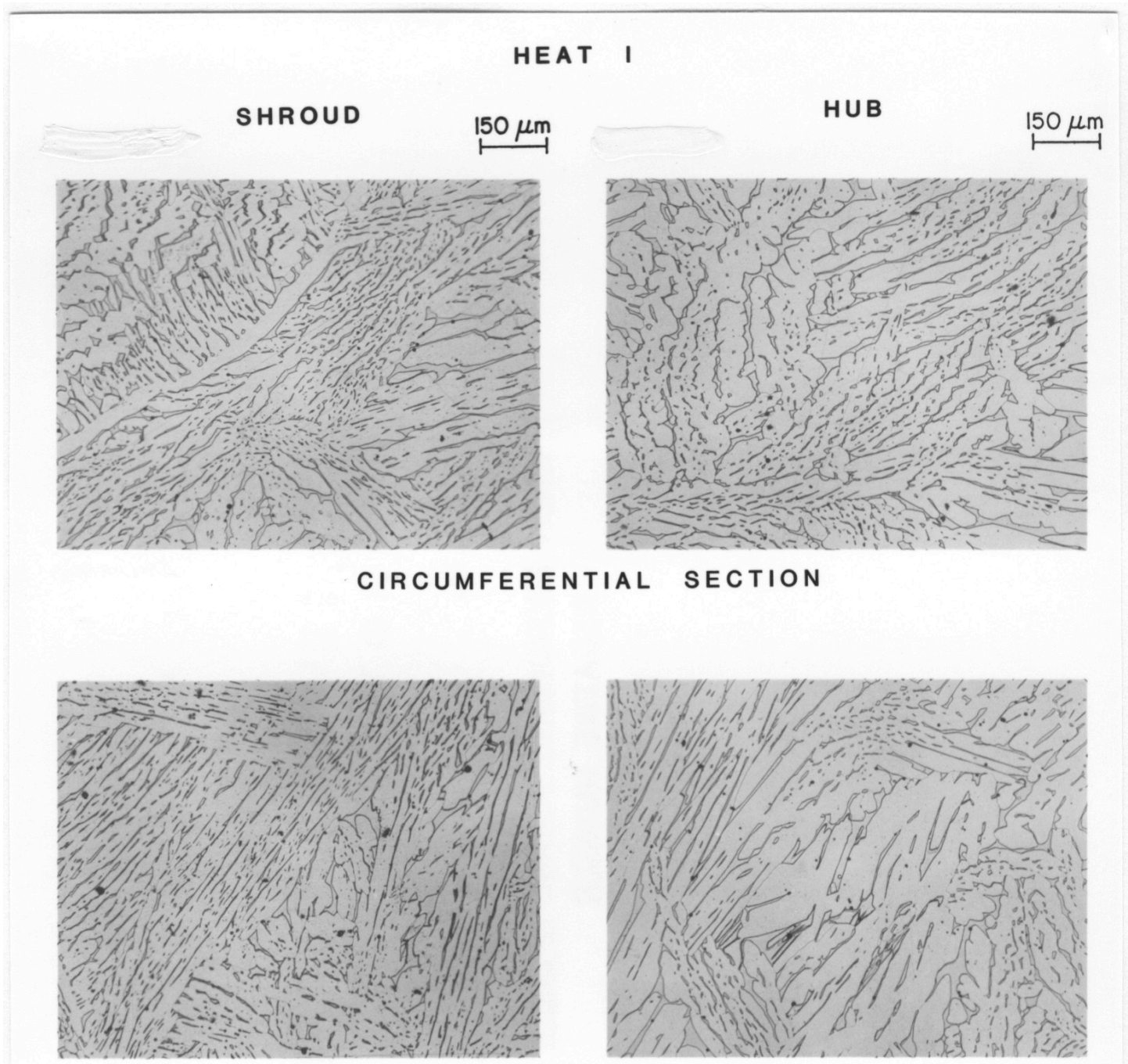

LONGITUDINAL SECTION
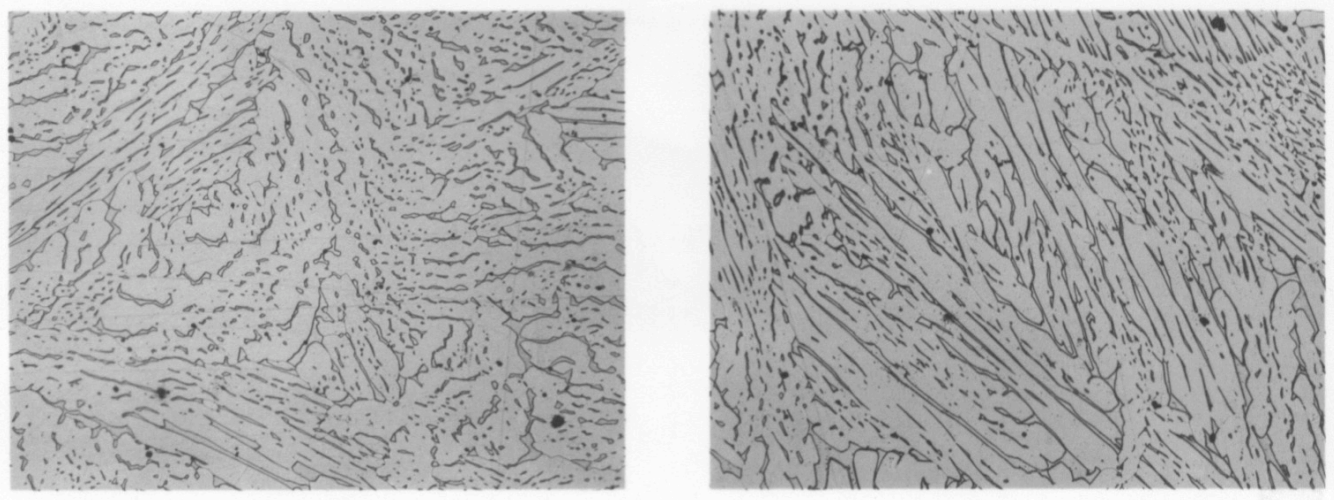

RADIAL SECTION

Figure 5. (Contd.) 


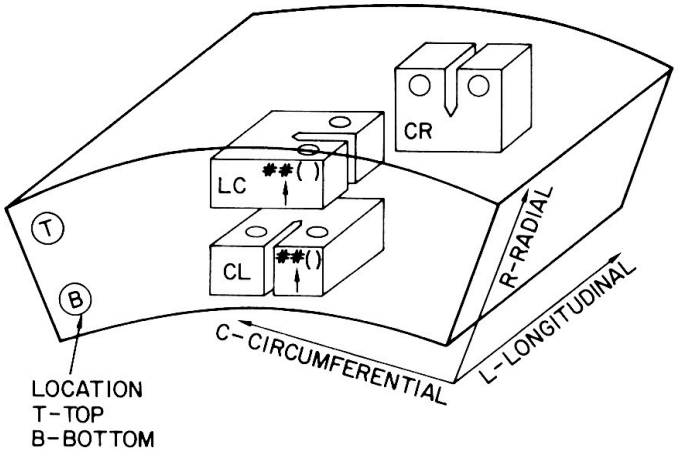

COMPACT-TENSION SPECIMENS

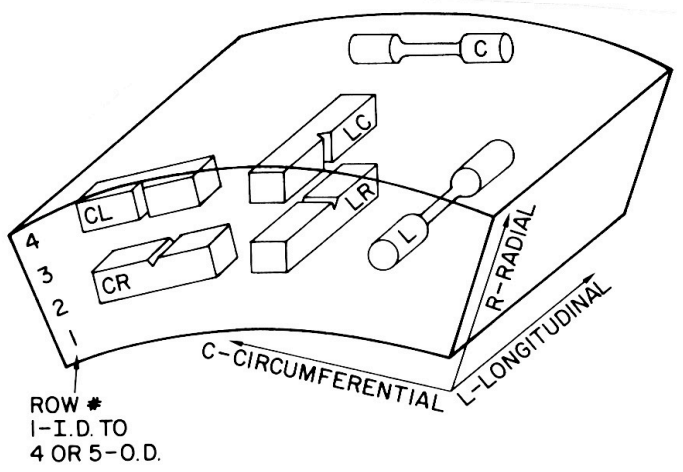

CHARPY IMPACT AND TENSILE SPECIMENS

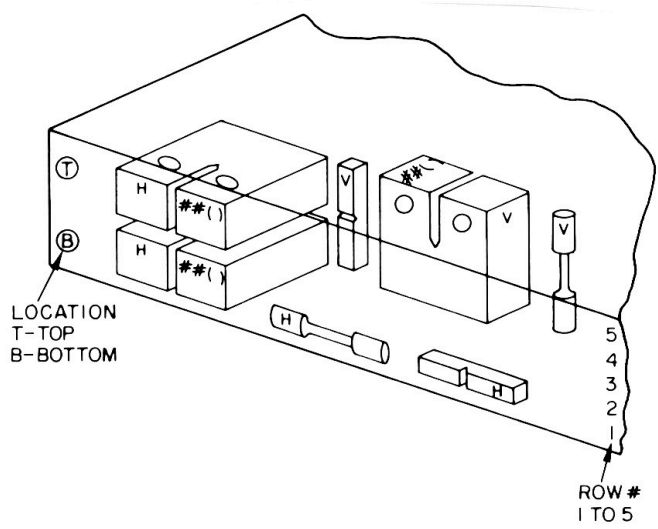

MECHANICAL TEST SPECIMENS

Figure 6. Orientation and location of the mechanical-test specimens taken from (a) and (b) pipe sections and (c) slabs

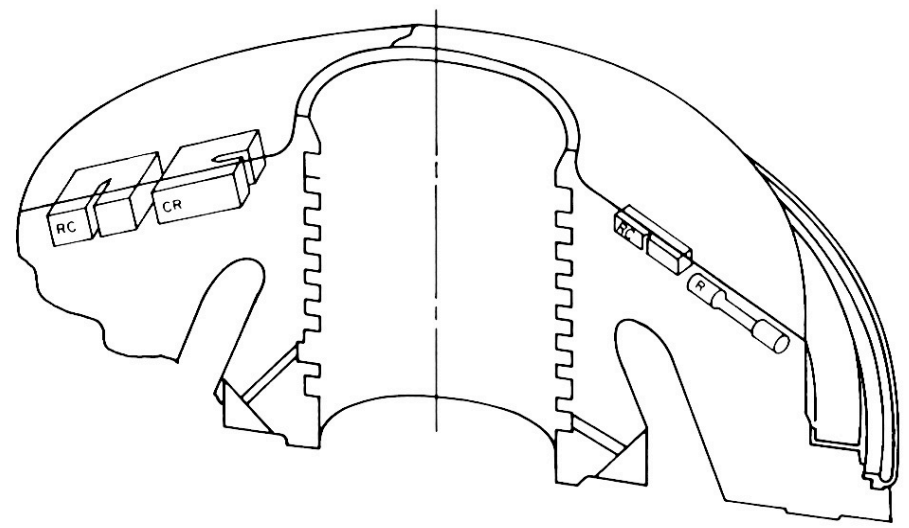

Figure 7. Orientation and location of the mechanical-test specimens taken from KRB pump cover plate 


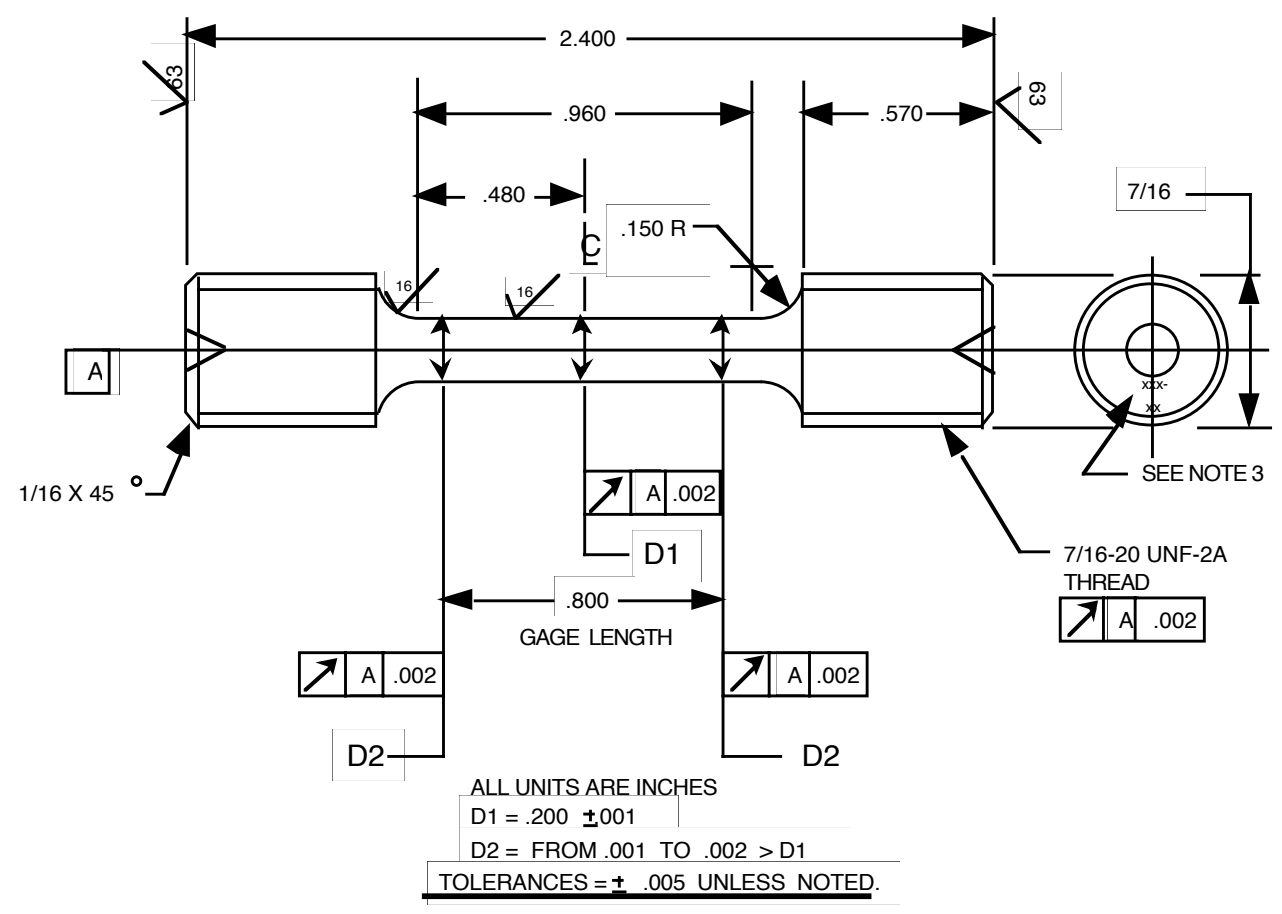

\footnotetext{
1. THE .800 INCH GAGE LENGTH TO HAVE UNIFORM SLOPE FROM MAX. DIA. D2 TO MIN. DIA. D1. UNIFORM SLOPE MUST NOT EXCEED .004 CHANGE IN DIA. PER 1 INCH CHANGE IN LENGTH.

2. ALL RADII MUST BLEND WITHOUT UNDERCUTS OR STEPS.

3. DIMENSIONS AND SPECIFICATIONS TYPICAL BOTH ENDS.

4. ALL SURFACES TO BE FREE OF BURRS.
}

\section{Figure 8. Configuration of tensile test specimen}

Elevated-temperature tests on the long-term-aged samples were conducted with a clip gauge mounted on the specimen grips. Total strain in the specimen gauge length was determined from correlations developed from room-temperature tests conducted with clip gauge attached to the specimen grips and the extensometer mounted on the specimen gauge length.

The results for ten commercial and experimental heats aged up to $58,000 \mathrm{~h}$ at temperatures between 290 and $450^{\circ} \mathrm{C}$ are given in Table 3; results from the tests at MEA are also included in the table.

\section{Effect of Thermal Aging}

\subsection{Tensile Strength}

Tensile tests were conducted at room temperature and $290^{\circ} \mathrm{C}\left(554^{\circ} \mathrm{F}\right)$ on three commercial and five experimental heats aged up to $58,000 \mathrm{~h}$ at $290-450^{\circ} \mathrm{C}\left(554-842^{\circ} \mathrm{F}\right)$ and on serviceaged material from the KRB pump cover plate. The results from the tests, which were conducted at $\mathrm{ANL}^{27}$ and MEA, 25,26 are given in the Appendix. The engineering stress-vs.-strain curves for six of the heats of CF-3, CF-8, and CF-8M steel aged up to $50,000 \mathrm{~h}$ at 320,350 , and $400^{\circ} \mathrm{C}$ and tested at room temperature and $290^{\circ} \mathrm{C}$ are shown in Figs. 9-14. At both test 
Table 3. Tensile test results for cast stainless stee/s

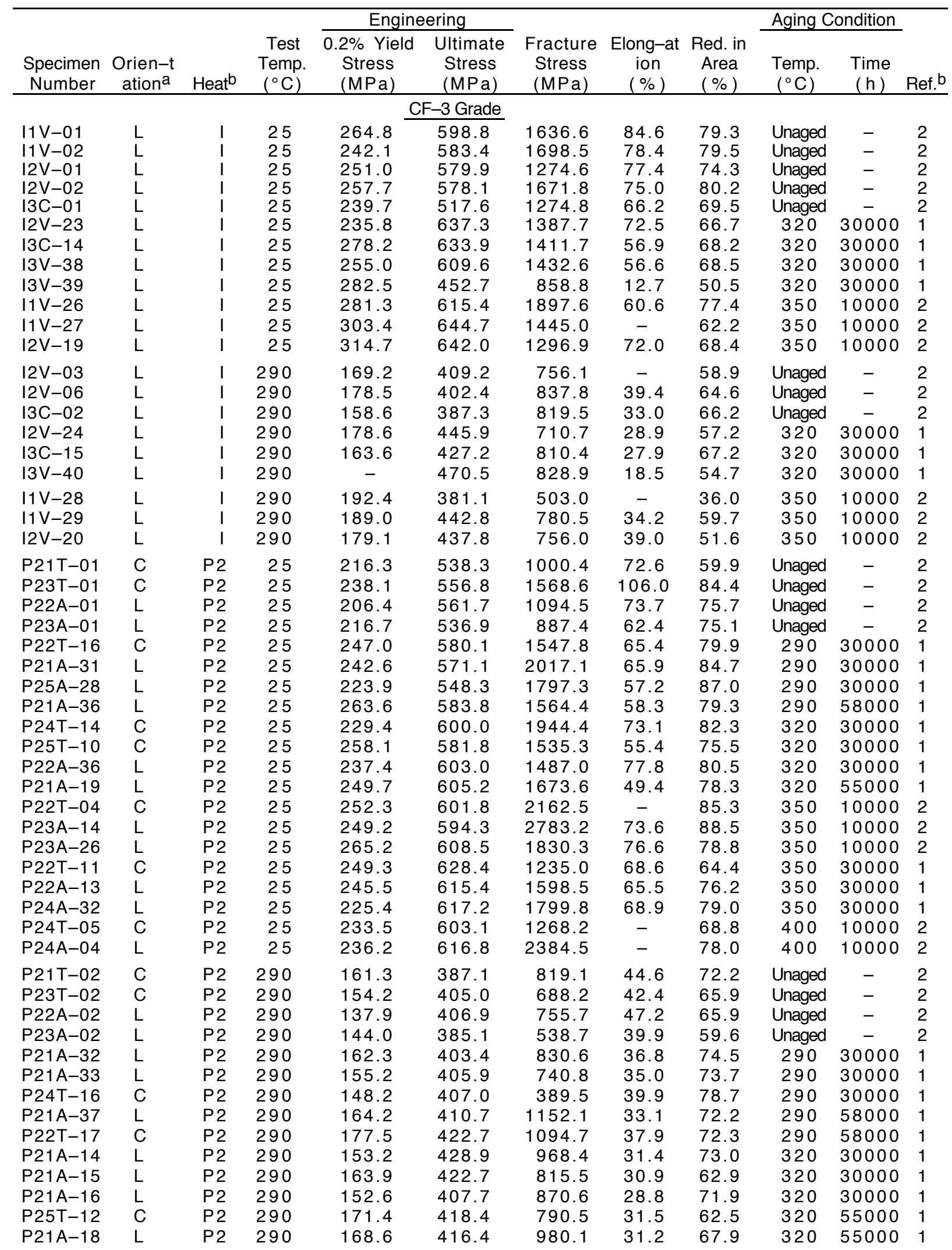


Table 3. (Contd.)

\begin{tabular}{|c|c|c|c|c|c|c|c|c|c|c|c|}
\hline \multirow[b]{2}{*}{$\begin{array}{c}\text { Specimen } \\
\text { Number }\end{array}$} & \multirow[b]{2}{*}{$\begin{array}{c}\text { Orien-t } \\
\text { ationa }^{a}\end{array}$} & \multirow[b]{2}{*}{ Heat } & \multirow[b]{2}{*}{$\begin{array}{l}\text { Test } \\
\text { Temp. } \\
\left({ }^{\circ} \mathrm{C}\right)\end{array}$} & \multicolumn{2}{|c|}{ Engineering } & \multirow[b]{2}{*}{$\begin{array}{c}\text { Fracture } \\
\text { Stress } \\
(\mathrm{MPa}) \\
\end{array}$} & \multirow[b]{2}{*}{$\begin{array}{c}\text { Elong-at } \\
\text { ion } \\
(\%) \\
\end{array}$} & \multirow[b]{2}{*}{$\begin{array}{l}\text { Red. in } \\
\text { Area } \\
(\%)\end{array}$} & \multicolumn{2}{|c|}{ Aging Condition } & \multirow[b]{2}{*}{ Ref. $^{b}$} \\
\hline & & & & $\begin{array}{c}0.2 \% \text { Yield } \\
\text { Stress } \\
(\mathrm{MPa}) \\
\end{array}$ & $\begin{array}{c}\text { Ultimate } \\
\text { Stress } \\
(\mathrm{MPa}) \\
\end{array}$ & & & & $\begin{array}{l}\text { Temp. } \\
\left({ }^{\circ} \mathrm{C}\right)\end{array}$ & $\begin{array}{c}\text { Time } \\
(\mathrm{h})\end{array}$ & \\
\hline $\mathrm{P} 21 \mathrm{~T}-08$ & $\mathrm{C}$ & $\mathrm{P} 2$ & 290 & 155.6 & 423.5 & 876.3 & 49.8 & 70.7 & 350 & 10000 & 2 \\
\hline P22T-05 & $\mathrm{C}$ & $\mathrm{P} 2$ & 290 & 153.8 & 399.0 & 696.5 & 43.0 & 66.9 & 350 & 10000 & 2 \\
\hline P23A-15 & $\mathrm{L}$ & $\mathrm{P} 2$ & 290 & 161.0 & 415.4 & 900.5 & - & 69.7 & 350 & 10000 & 2 \\
\hline P23A-27 & $\mathrm{L}$ & $\mathrm{P} 2$ & 290 & 154.1 & 419.4 & 970.7 & 39.6 & 72.9 & 350 & 10000 & 2 \\
\hline $\mathrm{P} 22 \mathrm{~T}-12$ & $\mathrm{C}$ & $\mathrm{P} 2$ & 290 & 167.0 & 436.2 & 740.2 & 28.2 & 56.0 & 350 & 30000 & 1 \\
\hline P24A-30 & $\mathrm{L}$ & $\mathrm{P} 2$ & 290 & 142.4 & 432.1 & 828.0 & 38.5 & 60.9 & 350 & 30000 & 1 \\
\hline P24A-33 & $\mathrm{L}$ & $\mathrm{P} 2$ & 290 & 149.1 & 425.7 & 463.7 & 41.5 & 62.6 & 350 & 30000 & 1 \\
\hline P24T-06 & $\mathrm{C}$ & $\mathrm{P} 2$ & 290 & 160.4 & 447.1 & 818.5 & 40.2 & 56.4 & 400 & 10000 & 2 \\
\hline P24A-05 & L & P2 & 290 & 146.6 & 430.6 & 655.0 & 40.2 & 53.8 & 400 & 10000 & 2 \\
\hline $693-40$ & $\mathrm{H}$ & 69 & 25 & 278.8 & 606.0 & 1070.1 & 57.1 & 48.9 & Unaged & - & 2 \\
\hline $693-41$ & $\mathrm{H}$ & 69 & 25 & 273.4 & 583.6 & 1093.4 & 54.1 & 54.4 & Unaged & - & 2 \\
\hline $694-30$ & $\mathrm{H}$ & 69 & 25 & 253.6 & 592.7 & 1430.6 & 50.1 & 71.8 & 290 & 30000 & 1 \\
\hline $694-31$ & $\mathrm{H}$ & 69 & 25 & 264.1 & 588.9 & 1714.0 & 51.1 & 76.4 & 290 & 30000 & 1 \\
\hline $69-135$ & V & 69 & 25 & 244.7 & 600.6 & 1617.5 & 62.8 & 74.5 & 290 & 30000 & 1 \\
\hline $694-21$ & $\mathrm{H}$ & 69 & 25 & 286.7 & 624.6 & 1757.0 & 52.2 & 76.1 & 320 & 10000 & 1 \\
\hline $694-25$ & $\mathrm{H}$ & 69 & 25 & 232.1 & 585.4 & 1428.3 & 70.5 & 75.2 & 320 & 10000 & 1 \\
\hline $692-40$ & $\mathrm{H}$ & 69 & 25 & 289.6 & 622.0 & 1516.1 & 43.6 & 72.3 & 320 & 30000 & 1 \\
\hline $692-41$ & $\mathrm{H}$ & 69 & 25 & 269.4 & 604.4 & 1623.8 & 38.0 & 75.2 & 320 & 30000 & 1 \\
\hline $69-245$ & V & 69 & 25 & 272.5 & 485.8 & - & 12.9 & 25.4 & 320 & 30000 & 1 \\
\hline $691-28$ & $\mathrm{H}$ & 69 & 25 & 291.6 & 637.9 & 1484.8 & 45.7 & 69.2 & 320 & 50000 & 1 \\
\hline $691-29$ & $\mathrm{H}$ & 69 & 25 & 287.7 & 650.5 & 1604.0 & 50.3 & 71.7 & 320 & 50000 & 1 \\
\hline $69-230$ & V & 69 & 25 & 298.8 & 669.4 & 1713.1 & 53.5 & 71.8 & 320 & 50000 & 1 \\
\hline $692-25$ & $\mathrm{H}$ & 69 & 25 & 285.4 & 633.4 & 1483.2 & 67.7 & 76.2 & 350 & 2570 & 2 \\
\hline $692-26$ & $\mathrm{H}$ & 69 & 25 & 302.6 & 648.2 & 1364.3 & 62.5 & 72.4 & 350 & 2570 & 2 \\
\hline $694-06$ & $\mathrm{H}$ & 69 & 25 & 292.1 & 652.0 & 1902.3 & 54.2 & 76.0 & 350 & 10000 & 1 \\
\hline $694-07$ & $\mathrm{H}$ & 69 & 25 & 266.2 & 635.5 & 1480.4 & 53.5 & 71.6 & 350 & 10000 & 1 \\
\hline $694-08$ & $\mathrm{H}$ & 69 & 25 & 246.8 & 619.9 & 1564.4 & 54.7 & 72.7 & 350 & 10000 & 1 \\
\hline $69-119$ & V & 69 & 25 & 249.6 & 617.3 & 1811.6 & 51.6 & 78.4 & 350 & 10000 & 1 \\
\hline $693-12$ & $\mathrm{H}$ & 69 & 25 & 270.9 & 674.0 & 1795.8 & 50.6 & 71.4 & 350 & 30000 & 1 \\
\hline $693-13$ & $\mathrm{H}$ & 69 & 25 & 259.2 & 655.1 & 1665.4 & 49.0 & 68.9 & 350 & 30000 & 1 \\
\hline $69-130$ & V & 69 & 25 & 258.4 & 661.2 & 1936.1 & 55.3 & 74.8 & 350 & 30000 & 1 \\
\hline $692-16$ & $\mathrm{H}$ & 69 & 25 & 253.6 & 638.8 & 1358.9 & 52.4 & 72.4 & 400 & 2570 & 2 \\
\hline $692-17$ & $\mathrm{H}$ & 69 & 25 & 300.9 & 683.2 & 1309.9 & 44.3 & 60.6 & 400 & 2570 & 2 \\
\hline $692-15$ & $\mathrm{H}$ & 69 & 25 & 287.8 & 673.9 & 1409.6 & 43.6 & 56.8 & 400 & 10000 & 1 \\
\hline $692-22$ & $\mathrm{H}$ & 69 & 25 & 278.6 & 699.1 & 1727.1 & 51.2 & 68.3 & 400 & 10000 & 1 \\
\hline $692-23$ & $\mathrm{H}$ & 69 & 25 & 260.5 & 664.7 & 1653.4 & 53.3 & 72.0 & 400 & 10000 & 1 \\
\hline $69-109$ & $\mathrm{~V}$ & 69 & 25 & 276.7 & 688.0 & 1796.6 & 65.7 & 73.5 & 400 & 10000 & 1 \\
\hline $691-04$ & $\mathrm{H}$ & 69 & 25 & 271.3 & 691.9 & 1476.9 & 36.5 & 71.4 & 450 & 2570 & 2 \\
\hline $691-05$ & $\mathrm{H}$ & 69 & 25 & 263.1 & 664.6 & 1528.2 & 56.6 & 73.2 & 450 & 2570 & 2 \\
\hline $693-42$ & $\mathrm{H}$ & 69 & 290 & 190.8 & 420.9 & 908.2 & 35.9 & 63.4 & Unaged & - & 2 \\
\hline $694-40$ & $\mathrm{H}$ & 69 & 290 & 177.0 & 417.0 & 809.0 & 33.6 & 59.7 & Unaged & - & 2 \\
\hline $694-32$ & $\mathrm{H}$ & 69 & 290 & 176.8 & 401.9 & 422.9 & 29.4 & 67.0 & 290 & 30000 & 1 \\
\hline $694-33$ & $\mathrm{H}$ & 69 & 290 & 173.0 & 386.7 & 543.7 & 21.5 & 51.3 & 290 & 30000 & 1 \\
\hline $69-236$ & $\mathrm{~V}$ & 69 & 290 & 194.1 & 440.5 & 818.0 & 30.8 & 61.9 & 290 & 30000 & 1 \\
\hline $694-26$ & $\mathrm{H}$ & 69 & 290 & 174.4 & 408.7 & 697.5 & 21.8 & 60.5 & 320 & 10000 & 1 \\
\hline $694-27$ & $\mathrm{H}$ & 69 & 290 & 183.5 & 422.8 & 973.7 & 20.9 & 65.3 & 320 & 10000 & 1 \\
\hline $692-42$ & $\mathrm{H}$ & 69 & 290 & 178.5 & 423.2 & 709.8 & 21.6 & 55.0 & 320 & 30000 & 1 \\
\hline $694-39$ & $\mathrm{H}$ & 69 & 290 & 168.2 & 413.8 & 692.4 & 21.3 & 51.7 & 320 & 30000 & 1 \\
\hline $69-246$ & $\mathrm{~V}$ & 69 & 290 & 165.5 & 423.0 & 921.8 & 27.8 & 70.6 & 320 & 30000 & 1 \\
\hline $692-28$ & $\mathrm{H}$ & 69 & 290 & 209.1 & 462.5 & 895.8 & 28.1 & 59.8 & 320 & 55000 & 1 \\
\hline $692-29$ & $\mathrm{H}$ & 69 & 290 & 212.3 & 464.4 & 911.6 & 27.9 & 58.5 & 320 & 55000 & 1 \\
\hline $69-130$ & V & 69 & 290 & 204.7 & 468.0 & 733.1 & 29.1 & 52.8 & 320 & 55000 & 1 \\
\hline $692-27$ & $\mathrm{H}$ & 69 & 290 & 173.1 & 451.3 & 752.6 & 32.3 & 53.8 & 350 & 2570 & 2 \\
\hline $694-09$ & $\mathrm{H}$ & 69 & 290 & 156.4 & 413.1 & 720.9 & 29.8 & 52.7 & 350 & 10000 & 1 \\
\hline $69-120$ & V & 69 & 290 & 179.8 & 429.8 & 875.1 & 23.2 & 68.8 & 350 & 10000 & 1 \\
\hline
\end{tabular}


Table 3. (Contd.)

\begin{tabular}{|c|c|c|c|c|c|c|c|c|c|c|c|}
\hline \multirow[b]{2}{*}{$\begin{array}{c}\text { Specimen } \\
\text { Number }\end{array}$} & \multirow[b]{2}{*}{$\begin{array}{c}\text { Orien-t } \\
\text { ationa }^{a}\end{array}$} & \multirow[b]{2}{*}{ Heat } & \multirow[b]{2}{*}{$\begin{array}{l}\text { Test } \\
\text { Temp. } \\
\left({ }^{\circ} \mathrm{C}\right)\end{array}$} & \multicolumn{2}{|c|}{ Engineering } & \multirow[b]{2}{*}{$\begin{array}{c}\text { Fracture } \\
\text { Stress } \\
(\mathrm{MPa}) \\
\end{array}$} & \multirow[b]{2}{*}{$\begin{array}{c}\text { Elong-at } \\
\text { ion } \\
(\%) \\
\end{array}$} & \multirow[b]{2}{*}{$\begin{array}{l}\text { Red. in } \\
\text { Area } \\
(\%)\end{array}$} & \multicolumn{2}{|c|}{ Aging Condition } & \multirow[b]{2}{*}{ Ref. $^{b}$} \\
\hline & & & & $\begin{array}{c}0.2 \% \text { Yield } \\
\text { Stress } \\
(\mathrm{MPa}) \\
\end{array}$ & $\begin{array}{l}\text { Ultimate } \\
\text { Stress } \\
(\mathrm{MPa}) \\
\end{array}$ & & & & $\begin{array}{l}\text { Temp. } \\
\left({ }^{\circ} \mathrm{C}\right)\end{array}$ & $\begin{array}{c}\text { Time } \\
(\mathrm{h})\end{array}$ & \\
\hline $693-14$ & $\mathrm{H}$ & 69 & 290 & 186.4 & 457.6 & 832.1 & 28.0 & 58.0 & 350 & 30000 & 1 \\
\hline $693-15$ & $\mathrm{H}$ & 69 & 290 & 195.8 & 444.0 & 639.2 & 32.5 & 46.9 & 350 & 30000 & 1 \\
\hline $69-270$ & V & 69 & 290 & 193.6 & 462.4 & 739.4 & 27.7 & 49.4 & 350 & 30000 & 1 \\
\hline $692-18$ & $\mathrm{H}$ & 69 & 290 & 163.9 & 444.8 & 556.4 & 21.8 & 33.2 & 400 & 2570 & 2 \\
\hline $692-24$ & $\mathrm{H}$ & 69 & 290 & 173.3 & 494.5 & 785.1 & 23.4 & 44.8 & 400 & 10000 & 1 \\
\hline $69-110$ & V & 69 & 290 & 196.9 & 481.6 & 802.3 & 26.1 & 51.5 & 400 & 10000 & 1 \\
\hline $691-06$ & $\mathrm{H}$ & 69 & 290 & 177.7 & 479.7 & 683.6 & 28.5 & 44.9 & 450 & 2570 & 2 \\
\hline $692-09$ & $\mathrm{H}$ & 69 & 290 & 175.7 & 477.1 & 793.4 & 27.4 & 51.0 & 450 & 2570 & 2 \\
\hline \multicolumn{12}{|c|}{ CF-8 Grade } \\
\hline $18-11$ & $\mathrm{C}$ & KRB & 25 & 306.2 & 554.9 & 1348.9 & 56.8 & 67.0 & 280 & 68000 & 1 \\
\hline $18-12$ & $\mathrm{C}$ & KRB & 25 & 288.5 & 555.9 & 1426.1 & 35.0 & 70.2 & 280 & 68000 & 1 \\
\hline $18-22$ & C & KRB & 25 & 291.9 & 568.9 & 1162.3 & 40.4 & 59.2 & 280 & 68000 & 1 \\
\hline $13-12$ & $\mathrm{C}$ & $\mathrm{KRB}$ & 25 & 317.3 & 567.4 & 1285.7 & 57.4 & 67.7 & Reannealed & - & 1 \\
\hline $13-21$ & $\mathrm{C}$ & KRB & 25 & 289.2 & 575.2 & 1209.1 & 43.4 & 67.0 & Reannealed & - & 1 \\
\hline $13-22$ & $\mathrm{C}$ & KRB & 25 & 287.7 & 531.2 & 1370.6 & 44.0 & 69.5 & Reannealed & - & 1 \\
\hline $15-11$ & $\mathrm{C}$ & KRB & 290 & 214.0 & 469.0 & 924.1 & 33.4 & 62.4 & 280 & 68000 & 1 \\
\hline $15-12$ & $\mathrm{C}$ & KRB & 290 & 208.4 & 444.5 & 774.1 & 28.9 & 51.1 & 280 & 68000 & 1 \\
\hline $15-21$ & C & KRB & 290 & 199.6 & 482.5 & 842.1 & 38.3 & 53.3 & 280 & 68000 & 1 \\
\hline $15-22$ & C & KRB & 290 & 180.2 & 434.0 & 710.1 & 35.7 & 51.1 & 280 & 68000 & 1 \\
\hline $16-21$ & C & KRB & 290 & 184.0 & 405.0 & 605.4 & 25.9 & 41.5 & Reannealed & - & 1 \\
\hline $17-21$ & C & KRB & 290 & 171.5 & 416.2 & 875.3 & 42.1 & 66.3 & Reannealed & - & 1 \\
\hline P13T-01 & $\mathrm{C}$ & P1 & 25 & 244.7 & 584.5 & 1221.5 & 56.5 & 68.8 & Unaged & - & 2 \\
\hline P13T-03 & $\mathrm{C}$ & P1 & 25 & 245.9 & 579.9 & 1206.6 & 54.6 & 65.9 & Unaged & - & 2 \\
\hline P11A-01 & $L$ & P1 & 25 & - & 584.9 & 1127.0 & 57.7 & 64.0 & Unaged & - & 2 \\
\hline P13A-01 & L & P1 & 25 & 248.5 & 584.5 & 1579.5 & 62.4 & 72.9 & Unaged & - & 2 \\
\hline P14T-09 & $\mathrm{C}$ & P1 & 25 & 266.2 & 596.2 & 1561.8 & 74.6 & 74.4 & 290 & 30000 & 1 \\
\hline P11A-25 & $\mathrm{L}$ & P1 & 25 & 285.7 & 595.2 & 1660.1 & 70.2 & 71.7 & 290 & 30000 & 1 \\
\hline P14A-26 & $L$ & P1 & 25 & 276.5 & 575.7 & 993.2 & 42.7 & 61.6 & 290 & 30000 & 1 \\
\hline P11A-28 & $\mathrm{L}$ & P1 & 25 & 263.5 & 536.0 & 1915.5 & 68.1 & 78.3 & 290 & 58000 & 1 \\
\hline P11A-29 & $\mathrm{L}$ & P1 & 25 & 266.1 & 562.0 & 415.05 & 63.5 & 51.9 & 290 & 58000 & 1 \\
\hline P11A-10 & $\mathrm{L}$ & P1 & 25 & 279.1 & 622.6 & 1625.9 & 62.5 & 70.7 & 320 & 30000 & 1 \\
\hline $\mathrm{P} 11 \mathrm{~T}-06$ & $\mathrm{C}$ & P1 & 25 & - & 569.3 & 1676.7 & 48.1 & 79.6 & 320 & 30000 & 1 \\
\hline P14T-08 & $\mathrm{C}$ & P1 & 25 & - & - & - & 47.7 & 78.3 & 320 & 30000 & 1 \\
\hline P11A-13 & $L$ & P1 & 25 & 299.0 & 646.8 & 1100.7 & 61.3 & 53.1 & 320 & 55000 & 1 \\
\hline P12A-25 & L & P1 & 25 & 287.6 & 688.3 & 1463.6 & 60.9 & 60.3 & 320 & 55000 & 1 \\
\hline P12T-05 & $\mathrm{C}$ & P1 & 25 & 276.8 & 610.8 & 1315.5 & 67.2 & 58.9 & 350 & 10000 & 2 \\
\hline P12T-06 & $\mathrm{C}$ & P1 & 25 & 263.4 & 608.7 & 864.7 & 87.2 & 76.1 & 350 & 10000 & 2 \\
\hline P12A-08 & $\mathrm{L}$ & P1 & 25 & 290.5 & 652.9 & 1625.9 & - & 75.2 & 350 & 10000 & 2 \\
\hline P12A-09 & $\mathrm{L}$ & P1 & 25 & 271.2 & 618.0 & 2322.0 & - & 80.2 & 350 & 10000 & 2 \\
\hline $\mathrm{P} 12 \mathrm{~T}-11$ & $\mathrm{C}$ & P1 & 25 & 266.6 & 617.0 & 1240.0 & 47.7 & 61.0 & 350 & 30000 & 1 \\
\hline P12A-13 & $\mathrm{L}$ & P1 & 25 & 285.0 & 637.0 & 1068.7 & 67.8 & 55.8 & 350 & 30000 & 1 \\
\hline $\mathrm{P} 12 \mathrm{~A}-14$ & $\mathrm{~L}$ & P1 & 25 & 263.5 & 634.9 & 1648.8 & 44.1 & 70.3 & 350 & 30000 & 1 \\
\hline P13T-07 & $\mathrm{C}$ & P1 & 25 & 285.0 & 660.0 & 1351.8 & 46.8 & 55.8 & 400 & 10000 & 2 \\
\hline P13A-07 & L & P1 & 25 & 286.3 & 677.7 & 1429.8 & 68.6 & 56.4 & 400 & 10000 & 2 \\
\hline P13T-02 & $\mathrm{C}$ & P1 & 290 & 148.5 & 408.8 & 642.2 & 33.3 & 43.9 & Unaged & - & 2 \\
\hline $\mathrm{P} 14 \mathrm{~T}-01$ & $\mathrm{C}$ & P1 & 290 & 157.0 & 437.5 & 622.3 & 32.6 & 46.4 & Unaged & - & 2 \\
\hline $\mathrm{P} 11 \mathrm{~A}-02$ & $\mathrm{~L}$ & P1 & 290 & 159.9 & 442.7 & 497.0 & 35.5 & 36.7 & Unaged & - & 2 \\
\hline P13A-02 & $L$ & P1 & 290 & 155.0 & 424.4 & 850.8 & 43.0 & 67.2 & Unaged & - & 2 \\
\hline $\mathrm{P} 14 \mathrm{~T}-10$ & $\mathrm{C}$ & P1 & 290 & 166.1 & 415.3 & 769.1 & 35.4 & 64.0 & 290 & 30000 & 1 \\
\hline P11A-26 & $\mathrm{L}$ & P1 & 290 & 160.3 & 394.6 & 605.7 & 51.3 & 73.6 & 290 & 30000 & 1 \\
\hline P14A-27 & $L$ & P1 & 290 & 167.8 & 421.6 & 812.5 & 29.4 & 60.1 & 290 & 30000 & 1 \\
\hline $\mathrm{P} 11 \mathrm{~A}-27$ & $\mathrm{~L}$ & P1 & 290 & 167.1 & 388.4 & 843.0 & 34.8 & 60.1 & 290 & 58000 & 1 \\
\hline$P 11 A-30$ & $\bar{L}$ & P1 & 290 & 179.3 & 445.5 & 683.6 & 39.5 & 46.6 & 290 & 58000 & 1 \\
\hline P11A-09 & $\mathrm{L}$ & P1 & 290 & 175.5 & 435.1 & 934.6 & 29.4 & 59.1 & 320 & 30000 & 1 \\
\hline P12A-19 & L & P1 & 290 & 174.8 & 456.9 & 731.1 & 27.4 & 51.6 & 320 & 30000 & 1 \\
\hline P12A-22 & L & P1 & 290 & 175.4 & 418.7 & 629.7 & 24.5 & 46.6 & 320 & 30000 & 1 \\
\hline
\end{tabular}


Table 3. (Contd.)

\begin{tabular}{|c|c|c|c|c|c|c|c|c|c|c|c|}
\hline \multirow[b]{2}{*}{$\begin{array}{c}\text { Specimen } \\
\text { Number }\end{array}$} & \multirow[b]{2}{*}{$\begin{array}{c}\text { Orien-t } \\
\text { ationa }^{a}\end{array}$} & \multirow[b]{2}{*}{ Heat } & \multirow[b]{2}{*}{$\begin{array}{l}\text { Test } \\
\text { Temp. } \\
\left({ }^{\circ} \mathrm{C}\right)\end{array}$} & \multicolumn{2}{|c|}{ Engineering } & \multirow[b]{2}{*}{$\begin{array}{c}\text { Fracture } \\
\text { Stress } \\
(\mathrm{MPa}) \\
\end{array}$} & \multirow[b]{2}{*}{$\begin{array}{c}\text { Elong-at } \\
\text { ion } \\
(\%)\end{array}$} & \multirow[b]{2}{*}{$\begin{array}{c}\text { Red. in } \\
\text { Area } \\
(\%)\end{array}$} & \multicolumn{2}{|c|}{ Aging Condition } & \multirow[b]{2}{*}{ Ref. ${ }^{b}$} \\
\hline & & & & $\begin{array}{c}0.2 \% \text { Yield } \\
\text { Stress } \\
(\mathrm{MPa}) \\
\end{array}$ & $\begin{array}{c}\text { Ultimate } \\
\text { Stress } \\
\text { (MPa) } \\
\end{array}$ & & & & $\begin{array}{l}\text { Temp. } \\
\left({ }^{\circ} \mathrm{C}\right)\end{array}$ & $\begin{array}{c}\text { Time } \\
(\mathrm{h})\end{array}$ & \\
\hline $\mathrm{P} 11 \mathrm{~A}-12$ & L & $\mathrm{P} 1$ & 290 & 188.2 & 424.8 & 865.0 & 39.5 & 56.6 & 320 & 55000 & 1 \\
\hline P12A-26 & L & P1 & 290 & 200.4 & 472.0 & 951.8 & 31.0 & 58.3 & 320 & 55000 & 1 \\
\hline $\mathrm{P} 12 \mathrm{~T}-08$ & $\mathrm{C}$ & $\mathrm{P} 1$ & 290 & 180.2 & 454.2 & 981.5 & - & 63.4 & 350 & 10000 & 2 \\
\hline P12A-10 & L & P1 & 290 & 173.7 & 451.3 & 919.6 & 43.4 & 62.8 & 350 & 10000 & 2 \\
\hline P12A-11 & L & P1 & 290 & 180.7 & 457.7 & 823.2 & 42.4 & 55.8 & 350 & 10000 & 2 \\
\hline $\mathrm{P} 12 \mathrm{~T}-12$ & $\mathrm{C}$ & $\mathrm{P} 1$ & 290 & 194.9 & 472.7 & 786.5 & 24.8 & 58.0 & 350 & 30000 & 1 \\
\hline P14A-22 & $\mathrm{L}$ & $\mathrm{P} 1$ & 290 & 185.3 & 462.3 & 719.2 & 25.3 & 44.5 & 350 & 30000 & 1 \\
\hline P14A-23 & L & $\mathrm{P} 1$ & 290 & 198.6 & 475.6 & 735.1 & 25.1 & 42.0 & 350 & 30000 & 1 \\
\hline P13T-08 & $\mathrm{C}$ & $\mathrm{P} 1$ & 290 & 167.3 & 502.2 & 791.0 & - & 49.6 & 400 & 10000 & 2 \\
\hline P13A-08 & $L$ & $\mathrm{P} 1$ & 290 & 162.9 & 485.6 & 881.0 & 35.6 & 51.0 & 400 & 10000 & 2 \\
\hline $683-40$ & $\mathrm{H}$ & 68 & 25 & 274.1 & 530.0 & 996.8 & 45.4 & 53.4 & Unaged & - & 2 \\
\hline $683-41$ & $\mathrm{H}$ & 68 & 25 & 279.4 & 517.2 & 811.9 & 36.4 & 49.2 & Unaged & - & 2 \\
\hline $683-33$ & $\mathrm{H}$ & 68 & 25 & 263.1 & 502.3 & 2242.4 & 55.7 & 83.2 & 290 & 30000 & 1 \\
\hline $684-31$ & $\mathrm{H}$ & 68 & 25 & 269.2 & 531.5 & 2050.9 & 42.3 & 79.7 & 290 & 30000 & 1 \\
\hline $68-145$ & V & 68 & 25 & 278.0 & 566.4 & 1903.0 & 42.1 & 78.9 & 290 & 30000 & 1 \\
\hline $684-21$ & $\mathrm{H}$ & 68 & 25 & 304.5 & 614.1 & 1291.8 & 67.8 & 61.9 & 320 & 10000 & 1 \\
\hline $684-22$ & $\mathrm{H}$ & 68 & 25 & 281.0 & 571.2 & 1880.1 & 76.6 & 80.7 & 320 & 10000 & 1 \\
\hline $682-41$ & $\mathrm{H}$ & 68 & 25 & 305.7 & 621.0 & 1544.6 & 40.8 & 71.2 & 320 & 30000 & 1 \\
\hline $684-39$ & $\mathrm{H}$ & 68 & 25 & 358.2 & 614.4 & 1716.9 & 43.4 & 75.4 & 320 & 30000 & 1 \\
\hline $68-264$ & V & 68 & 25 & 296.2 & 572.5 & 1903.8 & 65.3 & 75.6 & 320 & 30000 & 1 \\
\hline $681-28$ & $\mathrm{H}$ & 68 & 25 & 326.9 & 640.3 & 1130.7 & 51.0 & 66.6 & 320 & 50000 & 1 \\
\hline $681-29$ & $\mathrm{H}$ & 68 & 25 & 303.7 & 608.4 & 1666.1 & 50.7 & 71.1 & 320 & 50000 & 1 \\
\hline $68-230$ & V & 68 & 25 & 294.3 & 578.9 & 1696.8 & 47.0 & 75.8 & 320 & 50000 & 1 \\
\hline $682-25$ & $\mathrm{H}$ & 68 & 25 & 281.5 & 601.6 & 1647.2 & 64.1 & 75.2 & 350 & 5780 & 2 \\
\hline $682-26$ & $\mathrm{H}$ & 68 & 25 & 294.8 & 599.4 & 1620.7 & 62.0 & 73.5 & 350 & 5780 & 2 \\
\hline $684-06$ & $\mathrm{H}$ & 68 & 25 & 264.5 & 606.5 & 2014.0 & 53.7 & 78.3 & 350 & 10000 & 1 \\
\hline $684-07$ & $\mathrm{H}$ & 68 & 25 & 299.7 & 601.1 & 1953.4 & 47.4 & 76.7 & 350 & 10000 & 1 \\
\hline $684-08$ & $\mathrm{H}$ & 68 & 25 & 296.8 & 612.0 & 2084.7 & 52.6 & 78.5 & 350 & 10000 & 1 \\
\hline $68-129$ & V & 68 & 25 & 287.6 & 595.8 & 1688.6 & 58.5 & 73.5 & 350 & 10000 & 1 \\
\hline $684-10$ & $\mathrm{H}$ & 68 & 25 & 299.0 & 652.8 & 2072.9 & 44.6 & 74.9 & 350 & 30000 & 1 \\
\hline $684-11$ & $\mathrm{H}$ & 68 & 25 & 281.1 & 628.2 & 1342.3 & 55.0 & 57.4 & 350 & 30000 & 1 \\
\hline $68-139$ & V & 68 & 25 & 287.4 & 639.0 & 2090.6 & 44.5 & 77.0 & 350 & 30000 & 1 \\
\hline $682-16$ & $\mathrm{H}$ & 68 & 25 & 294.1 & 657.8 & 1534.6 & 75.0 & 64.0 & 400 & 2570 & 2 \\
\hline $682-17$ & $\mathrm{H}$ & 68 & 25 & 289.8 & 629.5 & 1313.0 & - & 64.6 & 400 & 2570 & 2 \\
\hline $682-15$ & $\mathrm{H}$ & 68 & 25 & 313.4 & 641.0 & 1526.3 & 36.8 & 64.4 & 400 & 10000 & 1 \\
\hline $682-22$ & $\mathrm{H}$ & 68 & 25 & 299.3 & 650.4 & 1019.0 & 43.9 & 49.4 & 400 & 10000 & 1 \\
\hline $682-23$ & $\mathrm{H}$ & 68 & 25 & 306.0 & 657.4 & 941.5 & 31.8 & 42.2 & 400 & 10000 & 1 \\
\hline $68-119$ & V & 68 & 25 & - & 624.3 & 1926.8 & 44.0 & 72.9 & 400 & 10000 & 1 \\
\hline $681-04$ & $\mathrm{H}$ & 68 & 25 & 291.3 & 653.1 & 1030.9 & 41.5 & 38.7 & 450 & 2570 & 2 \\
\hline $681-05$ & $\mathrm{H}$ & 68 & 25 & 309.4 & 632.9 & 1447.0 & - & 68.7 & 450 & 2570 & 2 \\
\hline $683-42$ & $\mathrm{H}$ & 68 & 290 & 162.7 & 397.3 & 815.4 & 29.2 & 57.8 & Unaged & - & 2 \\
\hline $684-40$ & $\mathrm{H}$ & 68 & 290 & 156.4 & 412.3 & 792.0 & 37.9 & 60.6 & Unaged & - & 2 \\
\hline $684-32$ & $\mathrm{H}$ & 68 & 290 & 149.5 & 333.2 & 513.0 & 27.7 & 62.2 & 290 & 30000 & 1 \\
\hline $684-33$ & $\mathrm{H}$ & 68 & 290 & 155.0 & 399.6 & 784.0 & 43.8 & 62.6 & 290 & 30000 & 1 \\
\hline $68-246$ & V & 68 & 290 & 170.6 & 390.7 & 522.1 & 28.4 & 51.5 & 290 & 30000 & 1 \\
\hline $684-23$ & $\mathrm{H}$ & 68 & 290 & 173.4 & 416.6 & 1048.7 & 26.8 & 72.7 & 320 & 10000 & 1 \\
\hline $684-24$ & $\mathrm{H}$ & 68 & 290 & 164.4 & 442.8 & 869.9 & 32.6 & 58.2 & 320 & 10000 & 1 \\
\hline $682-40$ & $\mathrm{H}$ & 68 & 290 & 174.3 & 440.8 & 853.5 & 36.6 & 62.3 & 320 & 30000 & 1 \\
\hline $682-42$ & $\mathrm{H}$ & 68 & 290 & 176.9 & 451.6 & 788.3 & 38.1 & 59.6 & 320 & 30000 & 1 \\
\hline $68-263$ & V & 68 & 290 & 165.6 & 450.3 & 758.2 & 37.2 & 48.0 & 320 & 30000 & 1 \\
\hline $682-28$ & $\mathrm{H}$ & 68 & 290 & 203.3 & 479.0 & 1132.6 & 32.6 & 64.0 & 320 & 50000 & 1 \\
\hline $682-29$ & $\mathrm{H}$ & 68 & 290 & 205.3 & 485.6 & 824.2 & 41.4 & 47.1 & 320 & 50000 & 1 \\
\hline $68-130$ & V & 68 & 290 & 201.3 & 475.2 & 699.1 & 38.9 & 46.2 & 320 & 50000 & 1 \\
\hline $682-27$ & $\mathrm{H}$ & 68 & 290 & 185.1 & 452.4 & 826.3 & 41.7 & 57.8 & 350 & 5780 & 2 \\
\hline $684-09$ & $\mathrm{H}$ & 68 & 290 & 164.3 & 500.3 & 765.7 & 30.5 & 51.9 & 350 & 10000 & 1 \\
\hline $68-130$ & V & 68 & 290 & 168.9 & 447.2 & 765.4 & 27.0 & 49.9 & 350 & 10000 & 1 \\
\hline
\end{tabular}


Table 3. (Contd.)

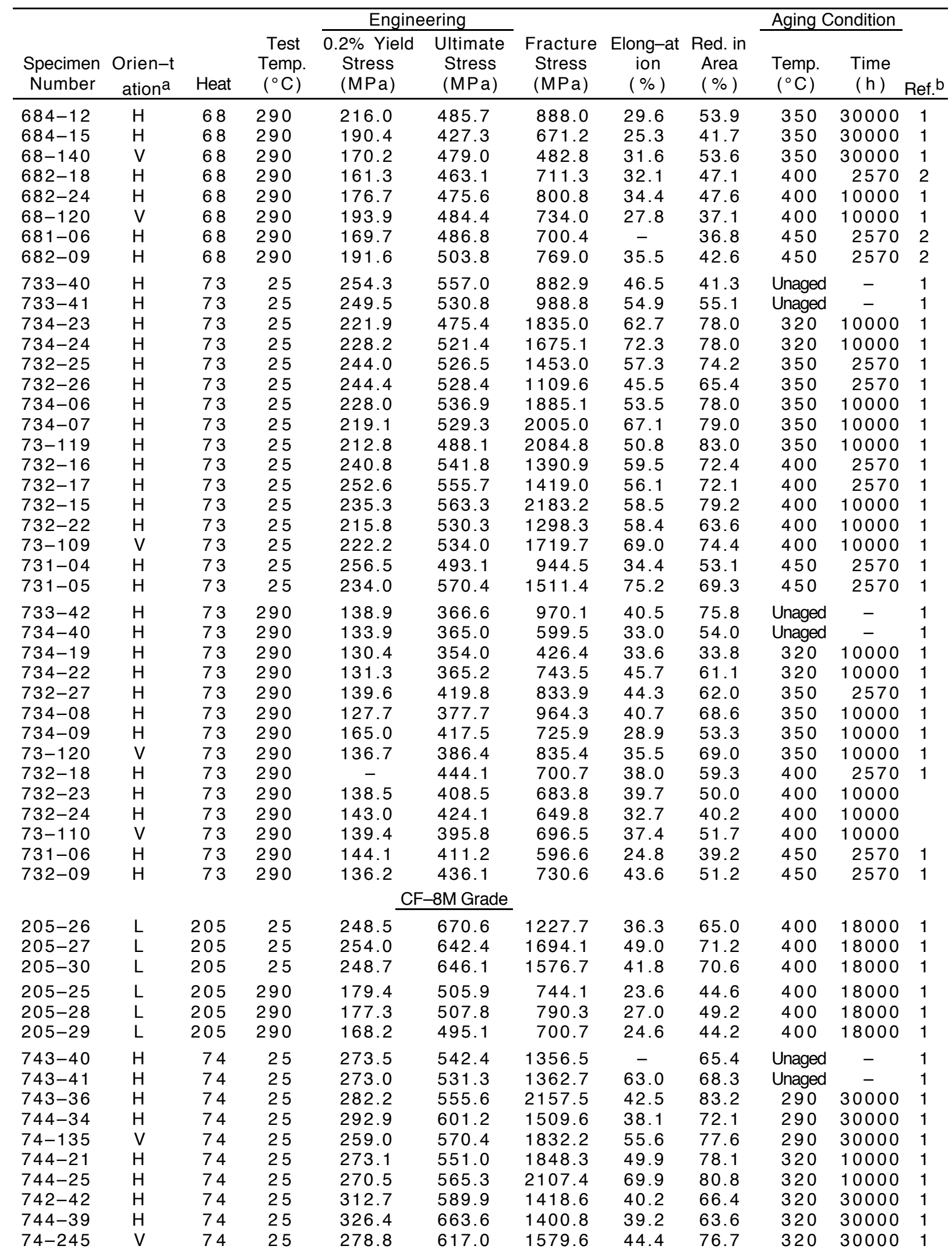


Table 3. (Contd.)

\begin{tabular}{|c|c|c|c|c|c|c|c|c|c|c|c|}
\hline \multirow[b]{2}{*}{$\begin{array}{c}\text { Specimen } \\
\text { Number }\end{array}$} & \multirow[b]{2}{*}{$\begin{array}{c}\text { Orien-t } \\
\text { ationa }^{a}\end{array}$} & \multirow[b]{2}{*}{ Heat } & \multirow[b]{2}{*}{$\begin{array}{l}\text { Test } \\
\text { Temp. } \\
\left({ }^{\circ} \mathrm{C}\right)\end{array}$} & \multicolumn{2}{|c|}{ Engineering } & \multirow[b]{2}{*}{$\begin{array}{c}\text { Fracture } \\
\text { Stress } \\
(\mathrm{MPa}) \\
\end{array}$} & \multirow[b]{2}{*}{$\begin{array}{c}\text { Elong-at } \\
\text { ion } \\
(\%) \\
\end{array}$} & \multirow[b]{2}{*}{$\begin{array}{l}\text { Red. in } \\
\text { Area } \\
(\%)\end{array}$} & \multicolumn{2}{|c|}{ Aging Condition } & \multirow[b]{2}{*}{ Ref. $^{b}$} \\
\hline & & & & $\begin{array}{c}0.2 \% \text { Yield } \\
\text { Stress } \\
(\mathrm{MPa}) \\
\end{array}$ & $\begin{array}{c}\text { Ultimate } \\
\text { Stress } \\
(\mathrm{MPa}) \\
\end{array}$ & & & & $\begin{array}{c}\text { Temp. } \\
\left({ }^{\circ} \mathrm{C}\right)\end{array}$ & $\begin{array}{c}\text { Time } \\
(\mathrm{h})\end{array}$ & \\
\hline $741-28$ & $\mathrm{H}$ & 74 & 25 & 309.1 & 668.1 & 1056.7 & 42.2 & 52.7 & 320 & 50000 & 1 \\
\hline $741-29$ & $\mathrm{H}$ & 74 & 25 & 304.5 & 638.1 & 1506.5 & 48.3 & 62.9 & 320 & 50000 & 1 \\
\hline $74-230$ & V & 74 & 25 & 299.4 & 631.6 & 1623.1 & 47.1 & 68.7 & 320 & 50000 & 1 \\
\hline $742-25$ & $\mathrm{H}$ & 74 & 25 & 269.0 & 575.1 & 1380.5 & 58.9 & 68.1 & 350 & 2570 & 1 \\
\hline $742-26$ & $\mathrm{H}$ & 74 & 25 & 261.0 & 536.8 & 1517.5 & 62.5 & 72.0 & 350 & 2570 & 1 \\
\hline $744-07$ & $\mathrm{H}$ & 74 & 25 & 281.7 & 606.1 & 1498.4 & 57.1 & 63.8 & 350 & 10000 & 1 \\
\hline $744-08$ & $\mathrm{H}$ & 74 & 25 & 278.2 & 619.2 & 1903.6 & 38.7 & 74.0 & 350 & 10000 & 1 \\
\hline $74-119$ & V & 74 & 25 & 267.5 & 587.8 & 2081.4 & 49.3 & 80.9 & 350 & 10000 & 1 \\
\hline $743-13$ & $\mathrm{H}$ & 74 & 25 & 265.8 & 647.2 & 1198.4 & 55.6 & 51.8 & 350 & 30000 & 1 \\
\hline $743-14$ & $\mathrm{H}$ & 74 & 25 & 260.3 & 613.5 & 1375.6 & 39.5 & 63.8 & 350 & 30000 & 1 \\
\hline $74-130$ & V & 74 & 25 & 272.3 & 624.8 & 1334.6 & 37.6 & 60.0 & 350 & 30000 & 1 \\
\hline $742-16$ & $\mathrm{H}$ & 74 & 25 & 268.6 & 602.3 & 1409.4 & 44.1 & 70.8 & 400 & 2570 & 1 \\
\hline $742-17$ & $\mathrm{H}$ & 74 & 25 & 267.7 & 579.7 & 1308.7 & 41.7 & 71.8 & 400 & 2570 & 1 \\
\hline $742-22$ & $\mathrm{H}$ & 74 & 25 & 269.8 & 626.3 & 991.8 & 40.7 & 69.1 & 400 & 10000 & 1 \\
\hline $742-23$ & $\mathrm{H}$ & 74 & 25 & 267.7 & 624.3 & 1170.2 & 46.4 & 72.6 & 400 & 10000 & 1 \\
\hline $74-110$ & V & 74 & 25 & 259.2 & 607.9 & 1429.6 & 48.5 & 69.6 & 400 & 10000 & 1 \\
\hline $741-04$ & $\mathrm{H}$ & 74 & 25 & 257.5 & 620.5 & 1293.5 & 43.5 & 60.5 & 450 & 2570 & 1 \\
\hline $741-05$ & $\mathrm{H}$ & 74 & 25 & 259.1 & 616.7 & 1176.2 & 58.8 & 52.9 & 450 & 2570 & 1 \\
\hline $744-40$ & $\mathrm{H}$ & 74 & 290 & 171.8 & 412.3 & 557.7 & 25.3 & 58.7 & Unaged & - & 1 \\
\hline $743-42$ & $\mathrm{H}$ & 74 & 290 & 164.6 & 442.8 & 674.7 & 35.4 & 42.1 & Unaged & - & 1 \\
\hline $744-35$ & $\mathrm{H}$ & 74 & 290 & 193.8 & 424.7 & 1061.2 & 42.8 & 69.9 & 290 & 30000 & 1 \\
\hline $744-36$ & $\mathrm{H}$ & 74 & 290 & 165.2 & 424.1 & 810.8 & 35.1 & 54.9 & 290 & 30000 & 1 \\
\hline $74-236$ & V & 74 & 290 & 179.9 & 436.3 & 1433.8 & 29.2 & 76.4 & 290 & 30000 & 1 \\
\hline $744-26$ & $\mathrm{H}$ & 74 & 290 & 192.7 & 440.1 & 890.9 & 36.3 & 62.6 & 320 & 10000 & 1 \\
\hline $744-27$ & $\mathrm{H}$ & 74 & 290 & 181.0 & 420.6 & 831.2 & 35.8 & 56.9 & 320 & 10000 & 1 \\
\hline $742-40$ & $\mathrm{H}$ & 74 & 290 & 153.7 & 452.7 & 915.5 & 31.5 & 57.3 & 320 & 30000 & 1 \\
\hline $742-41$ & $\mathrm{H}$ & 74 & 290 & 190.3 & 483.3 & 748.7 & 24.0 & 42.5 & 320 & 30000 & 1 \\
\hline $74-246$ & V & 74 & 290 & 184.4 & 481.7 & 839.4 & 33.5 & 52.5 & 320 & 30000 & 1 \\
\hline $742-28$ & $\mathrm{H}$ & 74 & 290 & 203.5 & 473.3 & 884.5 & 33.7 & 53.1 & 320 & 50000 & 1 \\
\hline $742-29$ & $\mathrm{H}$ & 74 & 290 & 215.2 & 474.4 & 743.6 & 24.3 & 41.2 & 320 & 50000 & 1 \\
\hline $74-130$ & V & 74 & 290 & 235.4 & 517.7 & 741.1 & 29.5 & 34.2 & 320 & 50000 & 1 \\
\hline $742-27$ & $\mathrm{H}$ & 74 & 290 & 174.9 & 454.3 & 787.2 & 31.5 & 65.9 & 350 & 2570 & 1 \\
\hline $744-06$ & $\mathrm{H}$ & 74 & 290 & 185.4 & 448.0 & 677.7 & 28.0 & 38.7 & 350 & 10000 & 1 \\
\hline $744-09$ & $\mathrm{H}$ & 74 & 290 & 197.8 & 507.2 & 889.6 & 34.2 & 52.4 & 350 & 10000 & 1 \\
\hline $74-120$ & V & 74 & 290 & 176.6 & 453.8 & 751.0 & 33.3 & 48.3 & 350 & 10000 & 1 \\
\hline $744-18$ & $\mathrm{H}$ & 74 & 290 & 228.5 & 510.5 & 791.9 & 27.6 & 40.3 & 350 & 30000 & 1 \\
\hline $743-15$ & $\mathrm{H}$ & 74 & 290 & 180.0 & 503.8 & 830.7 & 34.0 & 49.9 & 350 & 30000 & 1 \\
\hline $74-270$ & $\mathrm{H}$ & 74 & 290 & 182.2 & 495.0 & 729.9 & 31.2 & 40.2 & 350 & 30000 & 1 \\
\hline $742-18$ & $\mathrm{H}$ & 74 & 290 & 166.2 & 485.1 & 695.0 & 28.5 & 57.1 & 400 & 2570 & 1 \\
\hline $742-15$ & $\mathrm{H}$ & 74 & 290 & 178.9 & 516.0 & - & 33.7 & - & 400 & 10000 & 1 \\
\hline $742-24$ & $\mathrm{H}$ & 74 & 290 & 184.2 & 509.1 & 848.8 & 29.9 & 48.9 & 400 & 10000 & 1 \\
\hline $74-109$ & V & 74 & 290 & 172.0 & 476.8 & 546.4 & 33.3 & 48.7 & 400 & 10000 & 1 \\
\hline $741-06$ & $\mathrm{H}$ & 74 & 290 & 172.0 & 500.7 & 678.6 & 22.9 & 45.5 & 450 & 2570 & 1 \\
\hline $742-09$ & $\mathrm{H}$ & 74 & 290 & 170.3 & 485.2 & 697.5 & 21.3 & 35.8 & 450 & 2570 & 1 \\
\hline $753-40$ & $\mathrm{H}$ & 75 & 25 & 313.5 & 610.4 & 1056.6 & 47.4 & 67.9 & Unaged & - & 1 \\
\hline $753-41$ & $\mathrm{H}$ & 75 & 25 & 330.5 & 589.8 & 931.7 & 39.9 & 74.9 & Unaged & - & 1 \\
\hline $753-30$ & $\mathrm{H}$ & 75 & 25 & 315.6 & 609.0 & 1668.9 & 37.2 & 75.0 & 290 & 30000 & 1 \\
\hline $754-28$ & $\mathrm{H}$ & 75 & 25 & 321.7 & 619.2 & 1140.2 & 48.3 & 70.5 & 290 & 30000 & 1 \\
\hline $75-135$ & $\mathrm{~V}$ & 75 & 25 & 310.6 & 630.4 & 1448.9 & 48.3 & 69.7 & 290 & 30000 & 1 \\
\hline $754-21$ & $\mathrm{H}$ & 75 & 25 & 355.9 & 693.7 & 1822.9 & 58.5 & 75.0 & 320 & 10000 & 1 \\
\hline $754-25$ & $\mathrm{H}$ & 75 & 25 & 406.3 & 706.0 & 1763.9 & 46.4 & 71.2 & 320 & 10000 & 1 \\
\hline $752-41$ & $\mathrm{H}$ & 75 & 25 & 357.9 & 726.8 & 1224.7 & 38.8 & 52.4 & 320 & 30000 & 1 \\
\hline $754-39$ & $\mathrm{H}$ & 75 & 25 & 395.1 & 722.5 & 1203.2 & 39.2 & 52.5 & 320 & 30000 & 1 \\
\hline $75-245$ & $\mathrm{~V}$ & 75 & 25 & 323.1 & 691.3 & 1418.1 & 47.5 & 62.2 & 320 & 30000 & 1 \\
\hline $751-28$ & $\mathrm{H}$ & 75 & 25 & 334.7 & 689.5 & 1118.9 & 28.1 & 40.8 & 320 & 50000 & 1 \\
\hline $751-29$ & $\mathrm{H}$ & 75 & 25 & 352.0 & 729.8 & 1166.6 & 27.0 & 43.2 & 320 & 50000 & 1 \\
\hline
\end{tabular}


Table 3. (Contd.)

\begin{tabular}{|c|c|c|c|c|c|c|c|c|c|c|c|}
\hline \multirow[b]{2}{*}{$\begin{array}{c}\text { Specimen } \\
\text { Number }\end{array}$} & \multirow[b]{2}{*}{$\begin{array}{c}\text { Orien-t } \\
\text { ationa }^{\text {a }}\end{array}$} & \multirow[b]{2}{*}{ Heat } & \multirow[b]{2}{*}{$\begin{array}{l}\text { Test } \\
\text { Temp. } \\
\left({ }^{\circ} \mathrm{C}\right)\end{array}$} & \multicolumn{2}{|c|}{ Engineering } & \multirow[b]{2}{*}{$\begin{array}{c}\text { Fracture } \\
\text { Stress } \\
\text { (MPa) }\end{array}$} & \multirow[b]{2}{*}{$\begin{array}{c}\text { Elong-at } \\
\text { ion } \\
(\%)\end{array}$} & \multirow[b]{2}{*}{$\begin{array}{l}\text { Red. in } \\
\text { Area } \\
(\%)\end{array}$} & \multicolumn{2}{|c|}{ Aging Condition } & \multirow[b]{2}{*}{ Ref. ${ }^{b}$} \\
\hline & & & & $\begin{array}{c}0.2 \% \text { Yield } \\
\text { Stress } \\
(\mathrm{MPa})\end{array}$ & $\begin{array}{c}\text { Ultimate } \\
\text { Stress } \\
(\mathrm{MPa}) \\
\end{array}$ & & & & $\begin{array}{l}\text { Temp. } \\
\left({ }^{\circ} \mathrm{C}\right)\end{array}$ & $\begin{array}{l}\text { Time } \\
(\mathrm{h}) \\
\end{array}$ & \\
\hline $75-230$ & $\mathrm{~V}$ & 75 & 25 & 378.4 & 759.5 & 1179.8 & 32.7 & 38.6 & 320 & 50000 & 1 \\
\hline $752-25$ & $\mathrm{H}$ & 75 & 25 & - & 667.8 & 1677.6 & 37.5 & 68.4 & 350 & 2570 & 1 \\
\hline $752-26$ & $\mathrm{H}$ & 75 & 25 & 346.5 & 688.9 & 1522.3 & 37.6 & 64.6 & 350 & 2570 & 1 \\
\hline $754-06$ & $\mathrm{H}$ & 75 & 25 & 332.7 & 741.1 & 1543.1 & 41.4 & 59.6 & 350 & 10000 & 1 \\
\hline $754-07$ & $\mathrm{H}$ & 75 & 25 & 352.5 & 728.5 & 1159.6 & 39.5 & 54.2 & 350 & 10000 & 1 \\
\hline $754-08$ & $\mathrm{H}$ & 75 & 25 & 308.6 & 718.8 & 1455.1 & 42.3 & 56.1 & 350 & 10000 & 1 \\
\hline $75-119$ & V & 75 & 25 & 354.0 & 742.8 & 1437.7 & 44.4 & 53.6 & 350 & 10000 & 1 \\
\hline $753-10$ & $\mathrm{H}$ & 75 & 25 & 316.1 & 766.4 & 1256.0 & 31.5 & 41.4 & 350 & 30000 & 1 \\
\hline $753-11$ & $\mathrm{H}$ & 75 & 25 & 344.3 & 748.2 & 1051.8 & 26.3 & 31.6 & 350 & 30000 & 1 \\
\hline $75-130$ & V & 75 & 25 & 300.8 & 711.0 & 1061.3 & 30.5 & 34.9 & 350 & 30000 & 1 \\
\hline $752-16$ & $\mathrm{H}$ & 75 & 25 & 326.7 & 703.6 & 1531.0 & 38.5 & 64.3 & 400 & 2570 & 1 \\
\hline $752-17$ & $\mathrm{H}$ & 75 & 25 & 353.1 & 731.5 & 1441.4 & 33.5 & 64.0 & 400 & 2570 & 1 \\
\hline $752-15$ & $\mathrm{H}$ & 75 & 25 & 405.3 & 777.1 & 1305.1 & 36.4 & 45.0 & 400 & 10000 & 1 \\
\hline $752-22$ & $\mathrm{H}$ & 75 & 25 & - & 749.1 & 1093.5 & 28.1 & 37.6 & 400 & 10000 & 1 \\
\hline $752-23$ & $\mathrm{H}$ & 75 & 25 & 338.9 & 726.6 & 1328.9 & 26.7 & 53.6 & 400 & 10000 & 1 \\
\hline $75-109$ & V & 75 & 25 & 359.0 & 736.8 & 1171.8 & 38.1 & 46.3 & 400 & 10000 & 1 \\
\hline $751-04$ & $\mathrm{H}$ & 75 & 25 & 311.7 & 721.2 & 1094.2 & 25.1 & 38.4 & 450 & 2570 & 1 \\
\hline $751-05$ & $\mathrm{H}$ & 75 & 25 & 314.7 & 746.4 & 1217.2 & 32.0 & 41.6 & 450 & 2570 & 1 \\
\hline $754-40$ & $\mathrm{H}$ & 75 & 290 & 196.8 & 470.8 & 652.0 & 26.1 & 63.1 & Unaged & - & 1 \\
\hline $753-42$ & $\mathrm{H}$ & 75 & 290 & 191.5 & 474.8 & 620.7 & 30.5 & 45.0 & Unaged & - & 1 \\
\hline $754-29$ & $\mathrm{H}$ & 75 & 290 & 193.3 & 528.3 & 1051.1 & 33.5 & 60.5 & 290 & 30000 & 1 \\
\hline $754-30$ & $\mathrm{H}$ & 75 & 290 & 202.6 & 495.3 & 784.9 & 31.1 & 58.5 & 290 & 30000 & 1 \\
\hline $75-236$ & V & 75 & 290 & 211.8 & 486.2 & 924.1 & 32.0 & 58.2 & 290 & 30000 & 1 \\
\hline $754-26$ & $\mathrm{H}$ & 75 & 290 & 205.6 & 538.0 & 945.5 & 28.9 & 64.7 & 320 & 10000 & 1 \\
\hline $754-27$ & $\mathrm{H}$ & 75 & 290 & 212.2 & 534.3 & 957.8 & 29.2 & 64.6 & 320 & 10000 & 1 \\
\hline $752-40$ & $\mathrm{H}$ & 75 & 290 & 231.7 & 589.3 & 852.7 & 26.0 & 38.4 & 320 & 30000 & 1 \\
\hline $752-42$ & $\mathrm{H}$ & 75 & 290 & 268.5 & 575.8 & 893.6 & 22.9 & 46.0 & 320 & 30000 & 1 \\
\hline $75-246$ & V & 75 & 290 & 213.0 & 521.8 & 881.3 & 26.7 & 54.2 & 320 & 30000 & 1 \\
\hline $752-28$ & $\mathrm{H}$ & 75 & 290 & 258.9 & 587.9 & 933.6 & 24.4 & 43.2 & 320 & 50000 & 1 \\
\hline $752-29$ & $\mathrm{H}$ & 75 & 290 & 264.1 & 581.7 & 969.4 & 23.2 & 48.5 & 320 & 50000 & 1 \\
\hline $75-130$ & V & 75 & 290 & 239.6 & 557.8 & 889.5 & 24.7 & 38.9 & 320 & 50000 & 1 \\
\hline $752-27$ & $\mathrm{H}$ & 75 & 290 & 204.7 & 528.8 & 843.9 & 25.8 & 49.4 & 350 & 2570 & 1 \\
\hline $754-09$ & $\mathrm{H}$ & 75 & 290 & 219.8 & 614.1 & 918.6 & 24.5 & 44.8 & 350 & 10000 & 1 \\
\hline $75-120$ & V & 75 & 290 & 209.9 & 595.6 & 854.7 & 32.7 & 35.4 & 350 & 10000 & 1 \\
\hline $753-12$ & $\mathrm{H}$ & 75 & 290 & 264.4 & 615.9 & 809.3 & 23.0 & 30.2 & 350 & 30000 & 1 \\
\hline $754-12$ & $\mathrm{H}$ & 75 & 290 & 227.2 & 613.8 & 839.4 & 24.2 & 31.2 & 350 & 30000 & 1 \\
\hline $75-270$ & V & 75 & 290 & 209.0 & 584.6 & 823.5 & 26.2 & 32.9 & 350 & 30000 & 1 \\
\hline $752-18$ & $\mathrm{H}$ & 75 & 290 & 203.2 & 591.1 & 799.6 & 26.4 & 41.0 & 400 & 2570 & 1 \\
\hline $752-24$ & $\mathrm{H}$ & 75 & 290 & 208.2 & 630.4 & 858.4 & 23.7 & 31.4 & 400 & 10000 & 1 \\
\hline $75-110$ & V & 75 & 290 & 206.7 & 593.1 & 884.1 & 25.3 & 42.4 & 400 & 10000 & 1 \\
\hline $751-06$ & $\mathrm{H}$ & 75 & 290 & 218.7 & 598.2 & 749.6 & 24.3 & 24.7 & 450 & 2570 & 1 \\
\hline $752-09$ & $\mathrm{H}$ & 75 & 290 & 197.7 & 606.2 & 888.1 & 25.5 & 33.0 & 450 & 2570 & 1 \\
\hline
\end{tabular}

a Designation $\mathrm{L}=$ longitudinal or axial direction, $\mathrm{C}=$ circumferential direction, $\mathrm{H}=$ horizontal direction in the plane of the slab, and $\mathrm{V}=$ vertical direction transverse to the plane of the slab.

b Heats I, P2, P1, KRB, and 205 are commercial and Heats 69, 68, 73, 74, and 75 are experimental.

c 1: Argonne National Laboratory

2: Materials Engineering Associates 

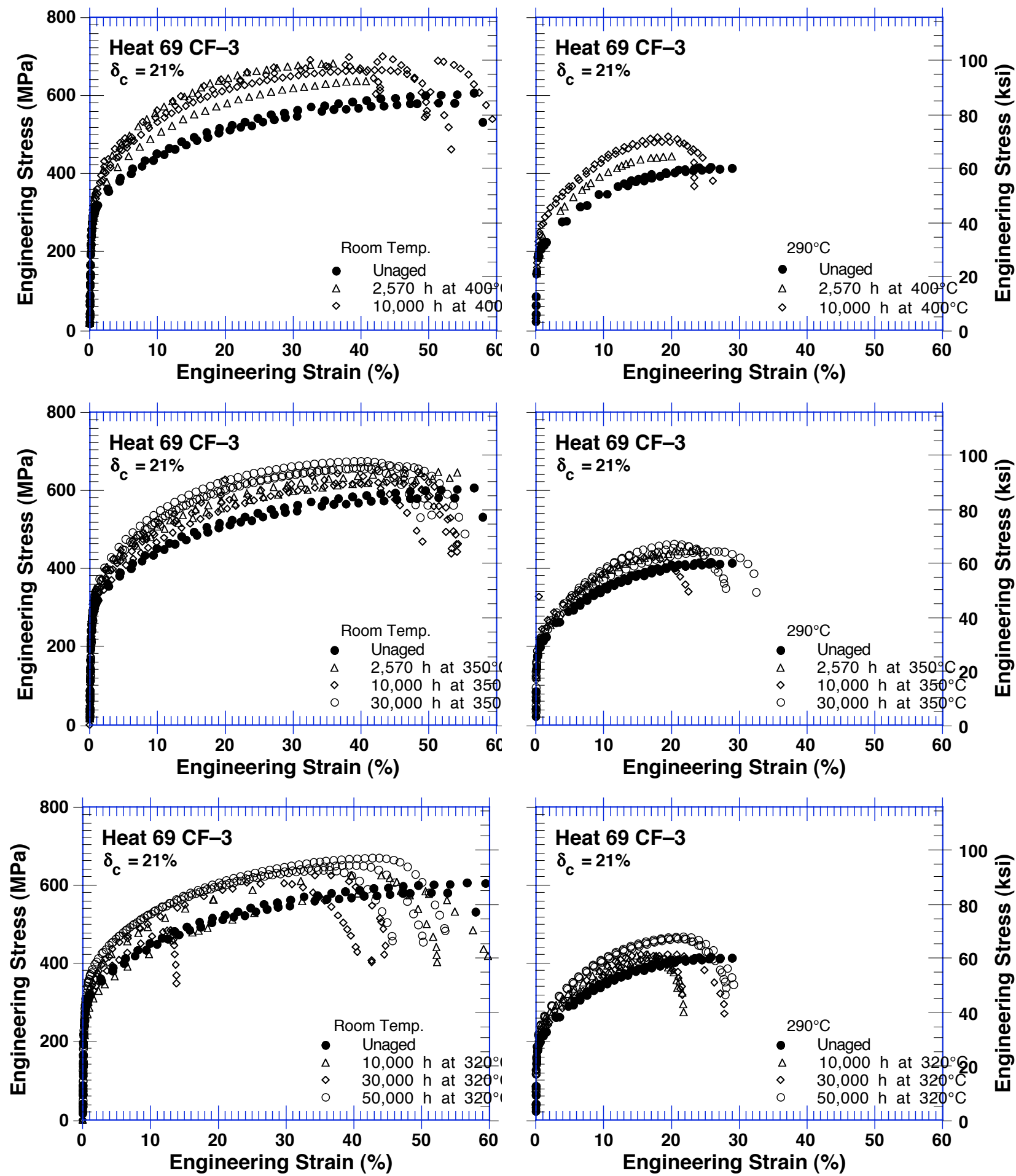

Figure 9. Effect of thermal aging on engineering stress vs. strain curves for Heat 69 

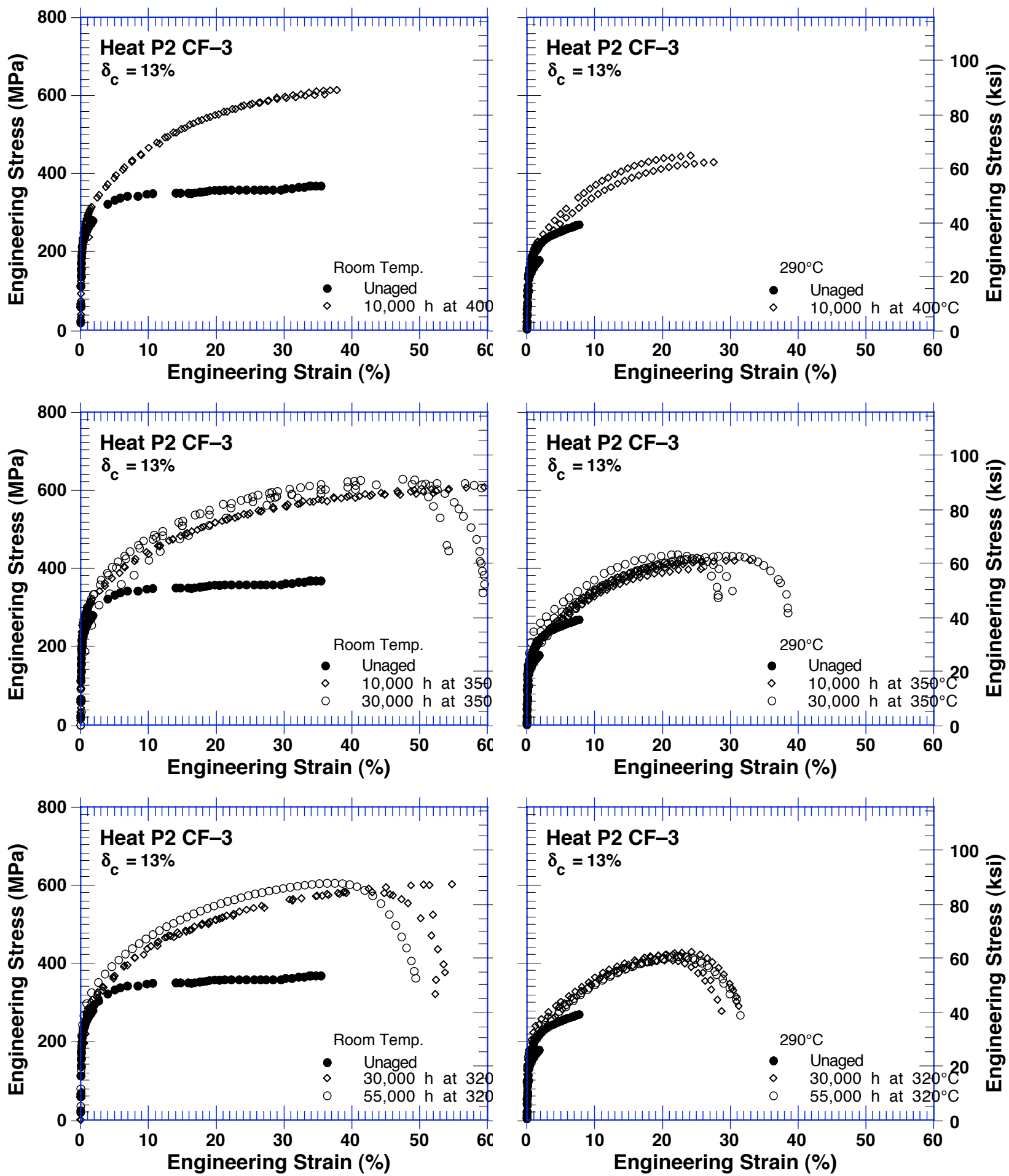

Figure 10. Effect of thermal aging on engineering stress vs. strain curves for Heat P2 

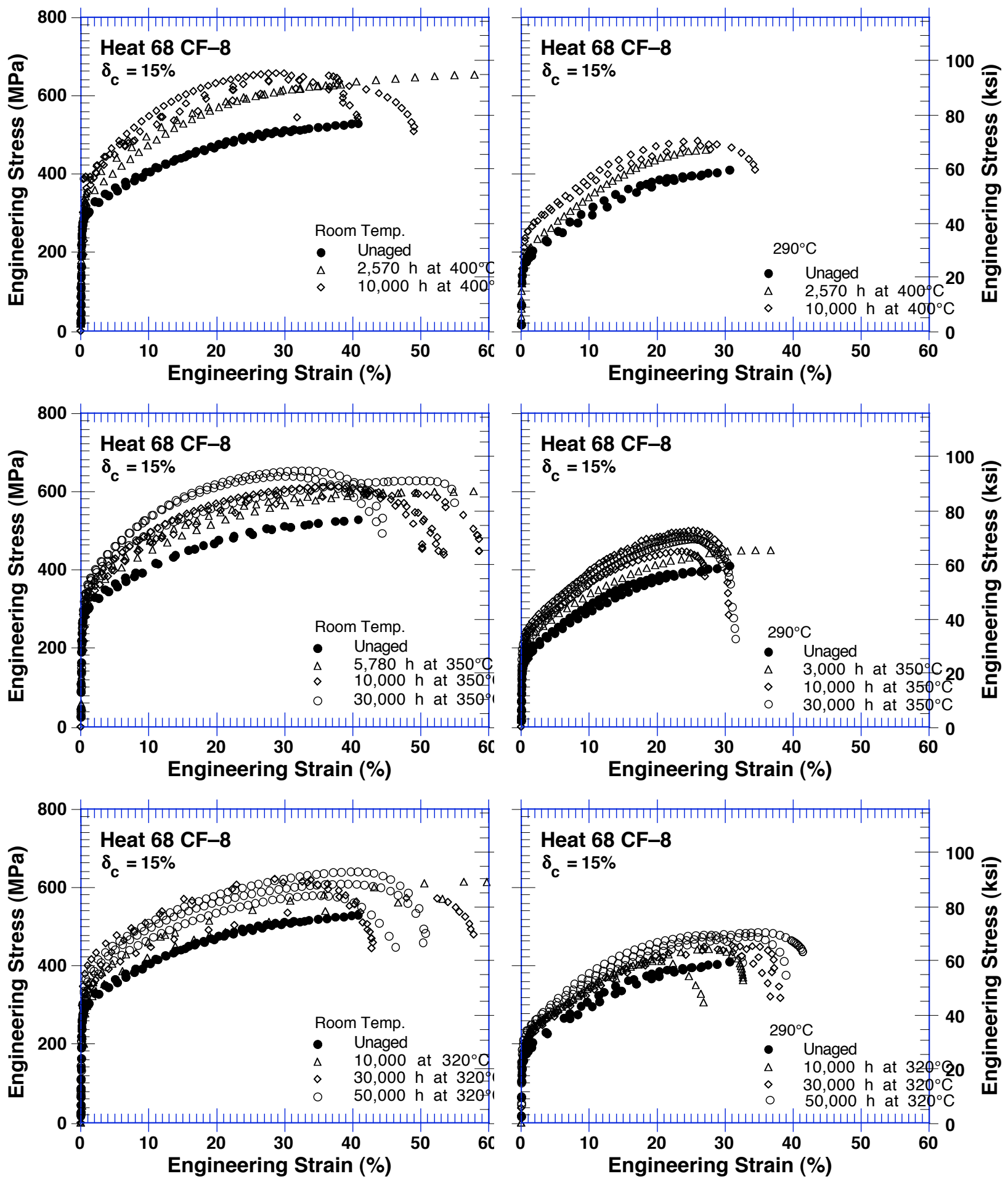

Figure 11. Effect of thermal aging on engineering stress vs. strain curves for Heat 68 

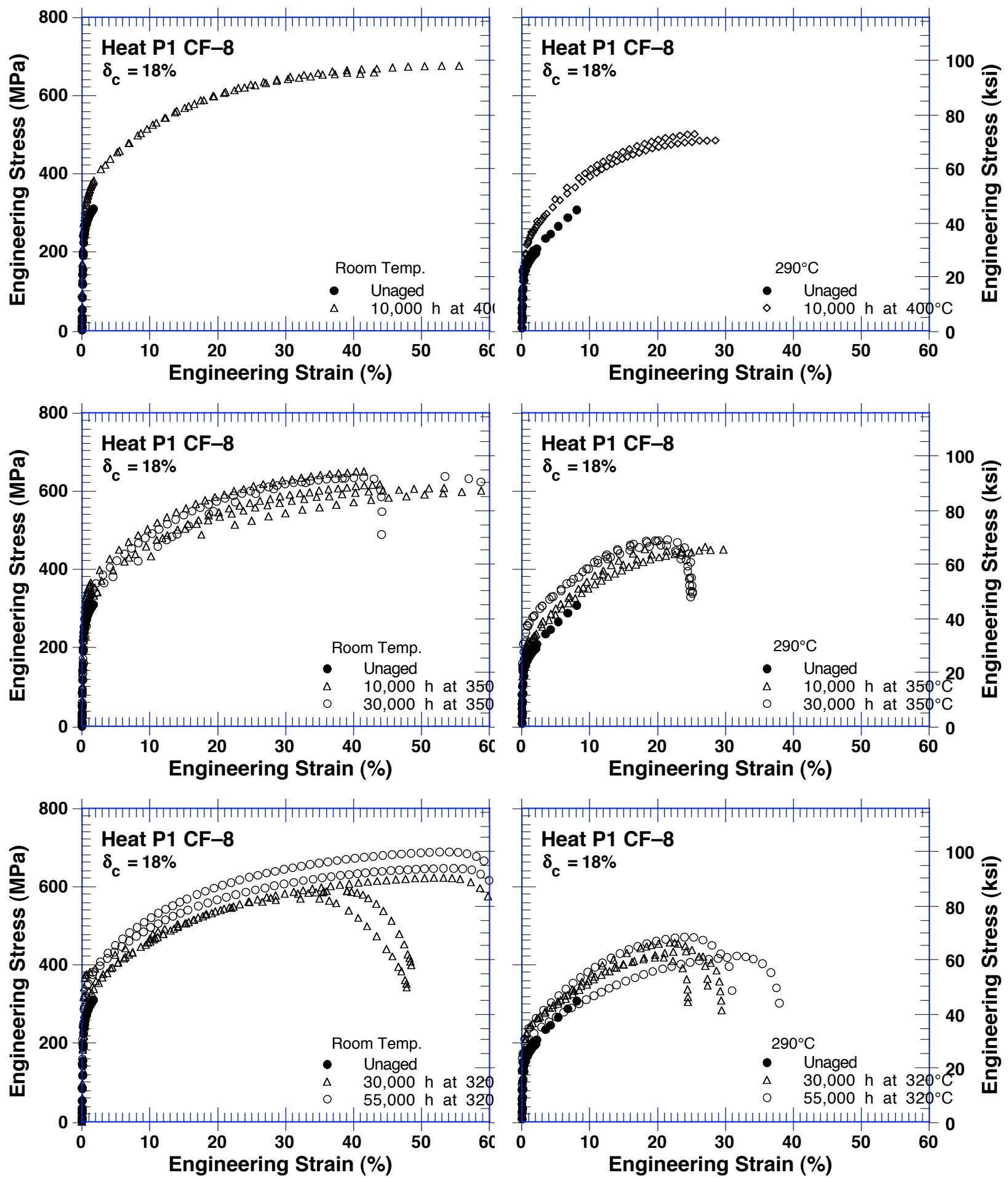

Figure 12. Effect of thermal aging on engineering stress vs. strain curves for Heat P1 

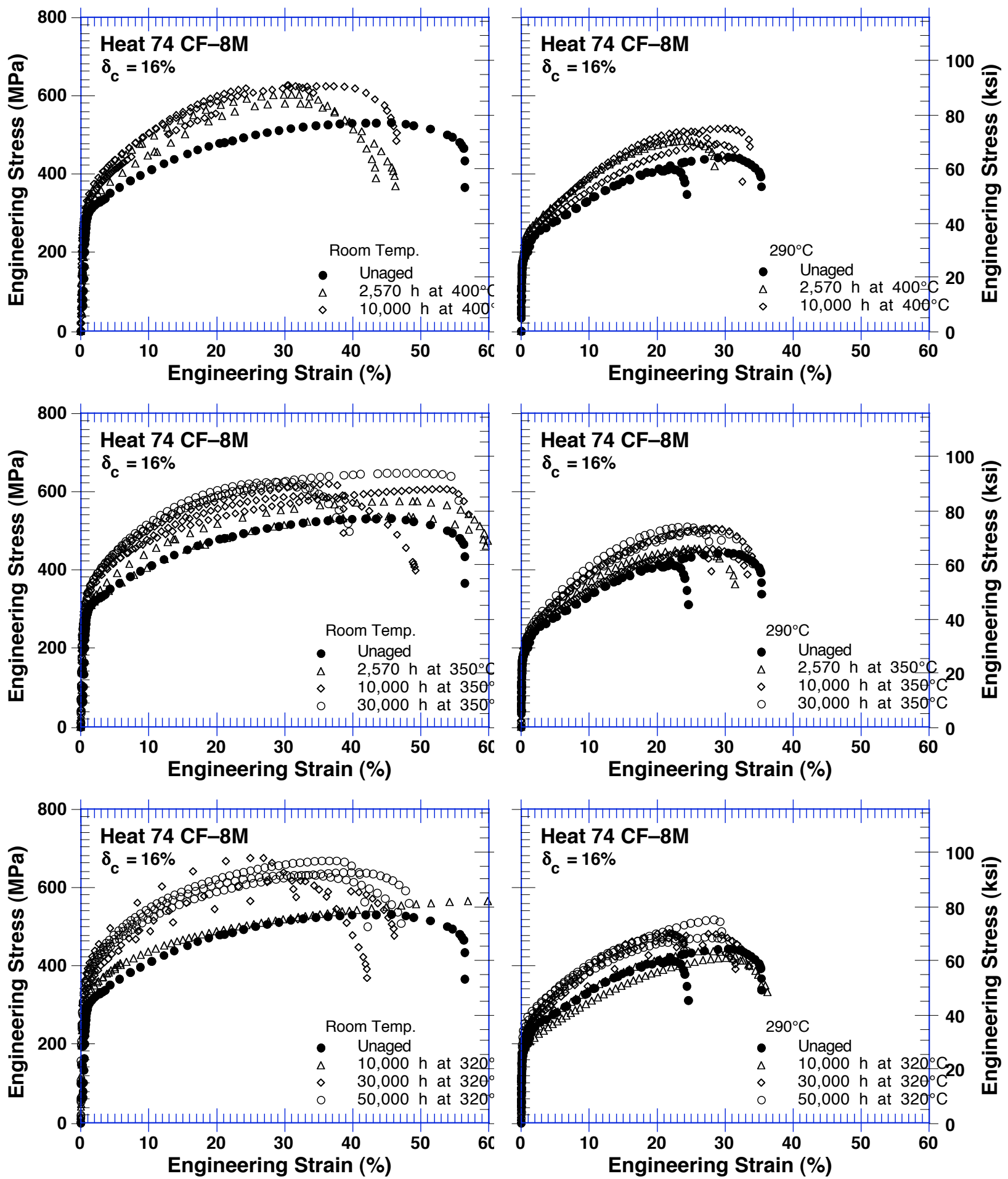

Figure 13. Effect of thermal aging on engineering stress vs. strain curves for Heat 74 

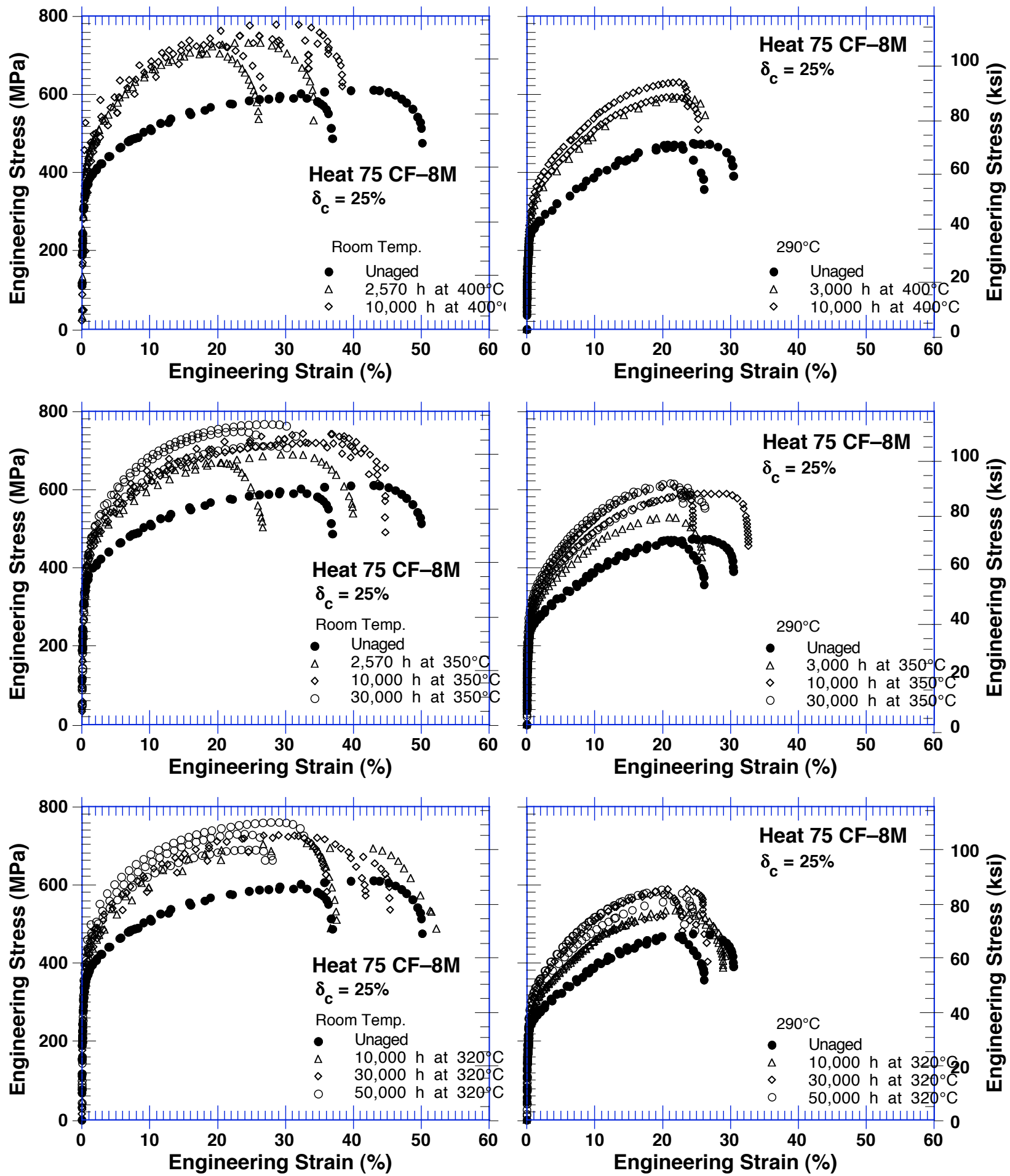

Figure 14. Effect of thermal aging on engineering stress vs. strain curves for Heat 75 

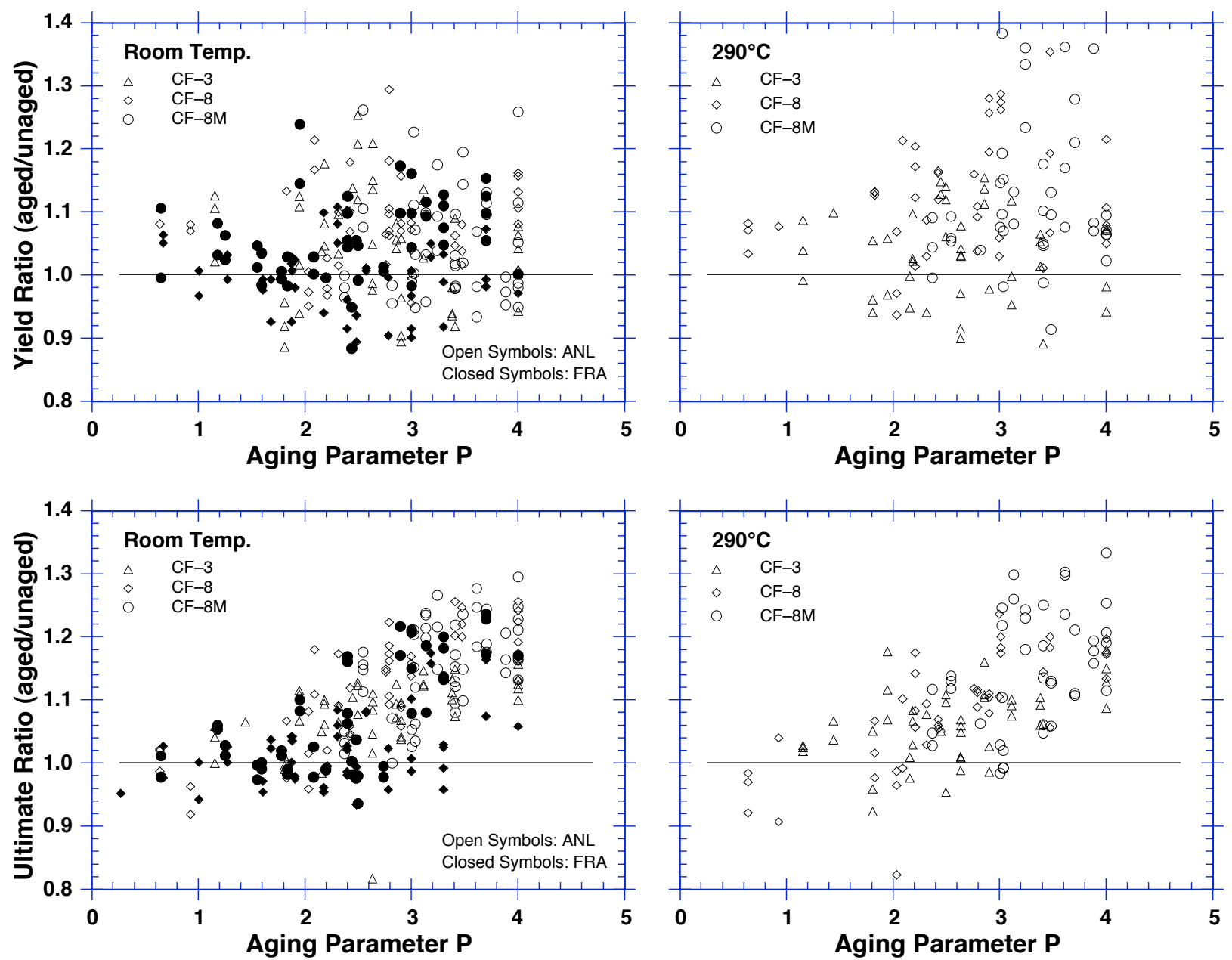

Figure 15. Ratio of yield and ultimate stress of aged and unaged cast stainless steel as a function of the aging parameter $P$

temperatures, thermal aging led to an increase in yield and ultimate stress and a slight decrease in ductility. For all heats, the increase in ultimate stress is substantially greater than the increase in yield stress. The Mo-bearing CF-8M steels are the most sensitive to thermal aging and Mo-free low-C CF-3 steels the least sensitive. Some heats show no change in yield stress. Furthermore, specimens aged for short times at high temperatures, e.g., $\approx 3,000 \mathrm{~h}$ at $400\left(752^{\circ} \mathrm{F}\right)$, often show a decrease in yield stress.

The ratios of the yield and ultimate stress of aged and unaged cast stainless steels at room temperature and $290^{\circ} \mathrm{C}\left(554^{\circ} \mathrm{F}\right)$ are plotted as a function of the aging parameter $\mathrm{P}$ in Fig. 15 . The results from tensile tests conducted at FRA ${ }^{15}$ are also shown in the figure. The aging parameter $P$ is determined from the equation

$$
P=\log _{10}[t]-\frac{1000 Q}{19.143}\left[\frac{1}{T_{S}+273}-\frac{1}{673}\right],
$$

where $Q$ is the activation energy and $t$ and $T_{S}$ are the time and temperature of aging, respectively. Equation 1 considers aging at $400^{\circ} \mathrm{C}$ as the baseline aging behavior for the material and 
parameter $\mathrm{P}$ is the log of the aging time at $400^{\circ} \mathrm{C}$. Activation energies for the various heats of cast stainless steel were obtained from room-temperature Charpy-impact data for materials that were aged up to $30,000 \mathrm{~h}$ at $290-400^{\circ} \mathrm{C} .5,6$

The data show considerable scatter, particularly for the yield stress. However, for the various heats of cast stainless steel, increases in yield and ultimate stress are observed for $\mathrm{P}$ values of $1.5-2.5$, which correspond to aging times of $30-300 \mathrm{~h}$ at $400^{\circ} \mathrm{C}$. The increase in ultimate stress is substantially greater than the increase in yield stress. The changes in yield and ultimate stress range from -10 to $20 \%$ and from -5 to $25 \%$, respectively. These results are consistent with the Charpy data, i.e., for a specific heat, an increase in tensile stress corresponds to a decrease in impact energy.

Photomicrographs of the fracture surface of unaged and aged Heats $\mathrm{P} 1,68$, and 75 , tested at room temperature and $290^{\circ} \mathrm{C}$, are shown in Figs. 16-18. In most cases, the fracture mode was predominantly a dimpled ductile failure, particularly for the unaged materials. The aged materials showed some cleavage and, in addition, the high-C steels, e.g., Heats 68 and P1, exhibit phase boundary separation.

Tensile properties of the cast materials can also be obtained from Charpy-impact data. For a Charpy specimen, the yield stress is estimated from the expression

$$
\sigma_{y}=C P_{y} B / W b^{2},
$$

taken from Ref. 28 , where Py is the yield load, $W$ is the specimen width, $B$ is the specimen thickness, $\mathrm{b}$ is the uncracked ligament, and $\mathrm{C}$ is a constant. The yield load was obtained from load-time traces of the Charpy tests. Deviation from linearity in the load-time traces occurred at $125-150 \mu$ s for the various heats. The load at $200 \mu$ s was estimated to represent $0.2 \%$ yield stress. Ultimate stress was also obtained from impact data by means of Eq. 2 and the maximum load. The constant $C$ was determined by comparing tensile and Charpy-impact data. The best values of the constant were 1.50 and 2.36 for yield stress and ultimate stress, respectively. The estimated values of tensile stress are based on the assumption that strain rate effects are approximately the same for all heats and aging conditions. The estimated values of tensile stress are not accurate at temperatures corresponding to lower-shelf and transition regions. The estimated values of yield and ultimate stress for Heats $68,69,74$, and 75 , along with the values obtained from actual tensile tests, are shown in Fig. 19. The estimated tensile properties are in good agreement with the measured values.

\subsection{Engineering Stress-Strain Behavior}

Thermal aging increases the strain-hardening rate of all grades of cast stainless steel. The increase is greater at room temperature than at $290^{\circ} \mathrm{C}$, and CF-8M steels show a larger increase than do Mo-free grades of steel (Figs. 9-14). The influence of thermal aging on tensile stress-strain behavior of cast stainless steels was evaluated by fitting the engineering stress $(\sigma)$ vs. strain $(\varepsilon)$ data with the RO equation

$$
\frac{\varepsilon}{\varepsilon_{o}}=\frac{\sigma}{\sigma_{o}}+\alpha\left(\frac{\sigma}{\sigma_{o}}\right)^{n}
$$


$25^{\circ} \mathrm{C}$

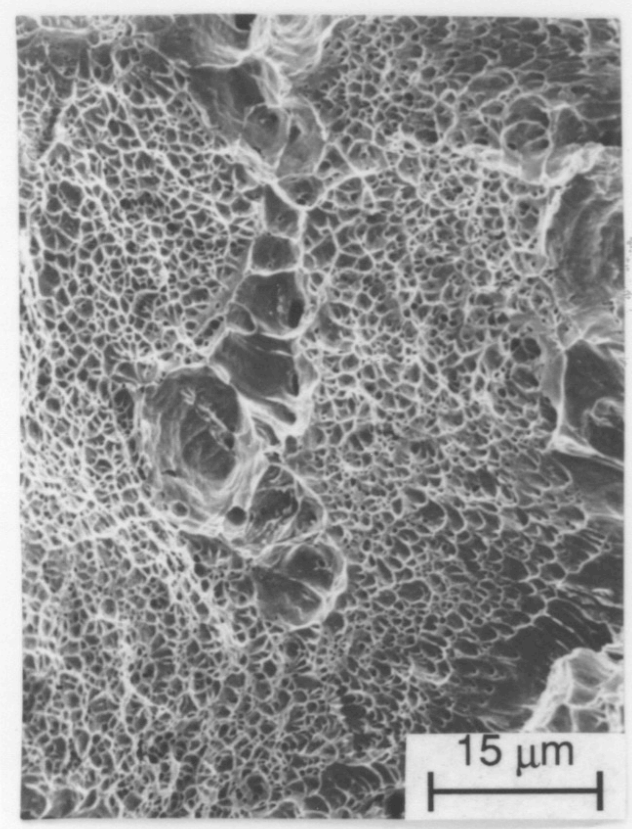

$290^{\circ} \mathrm{C}$

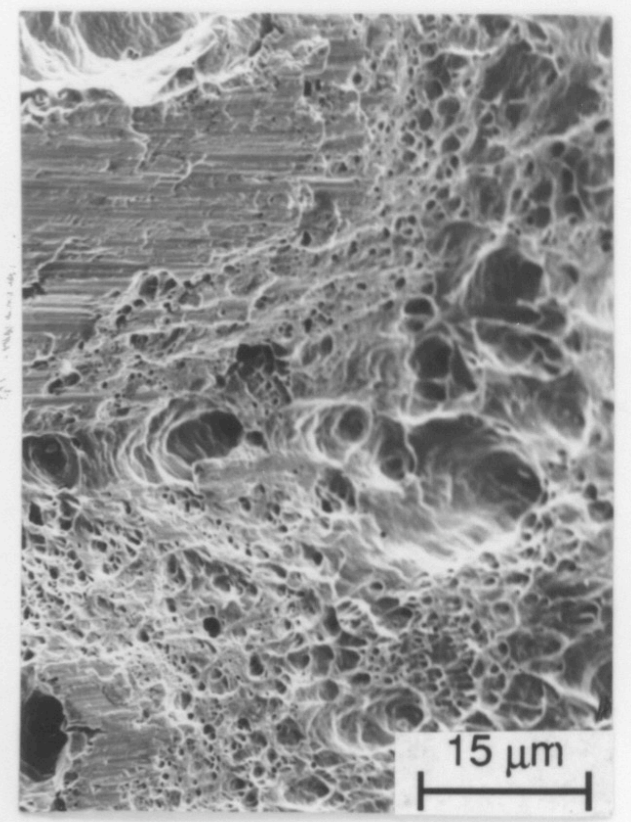

\section{Unaged}
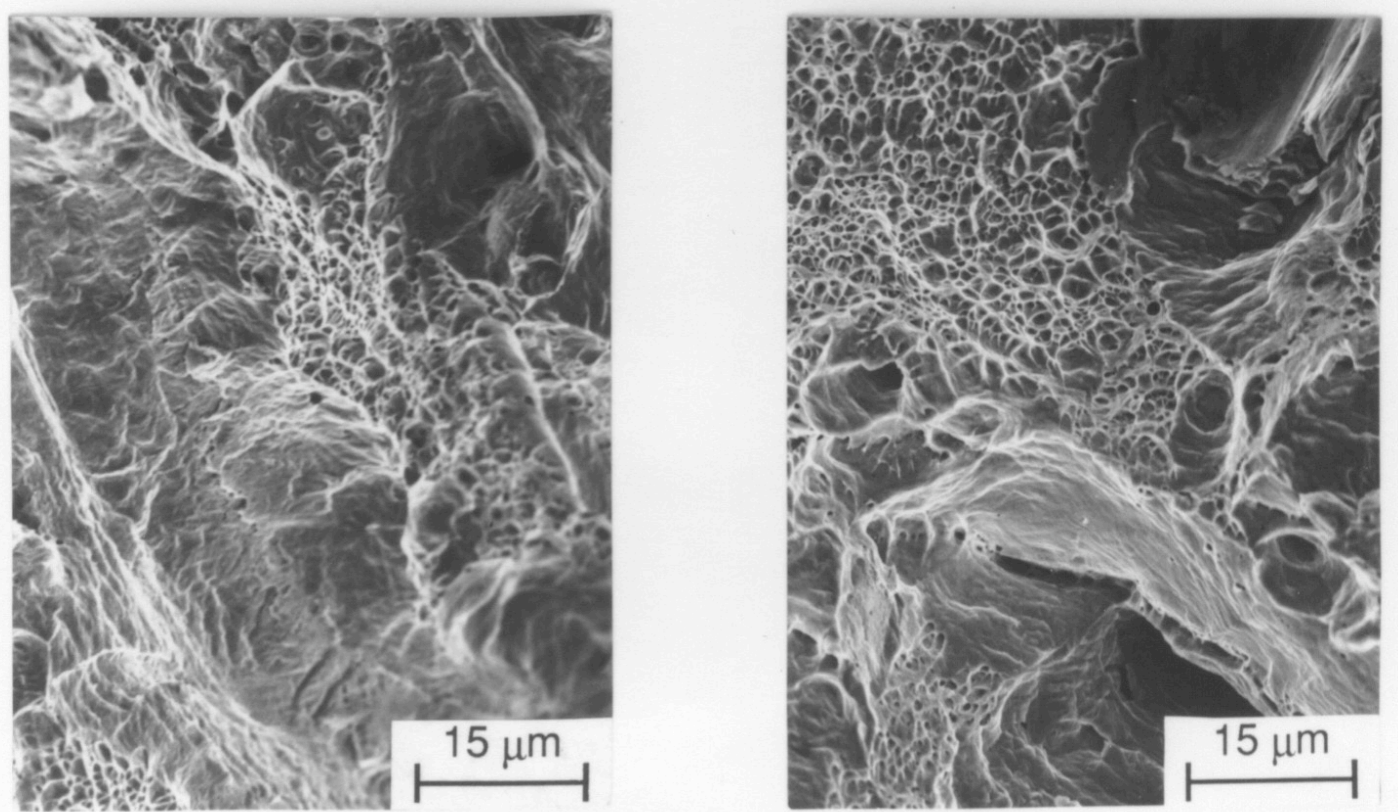

\section{Aged $10,000 \mathrm{~h}$ at $400^{\circ} \mathrm{C}$}

Figure 16. Fracture surface of tensile specimens of unaged and aged Heat $P 1$ tested at room temperature and $290^{\circ} \mathrm{C}$ 

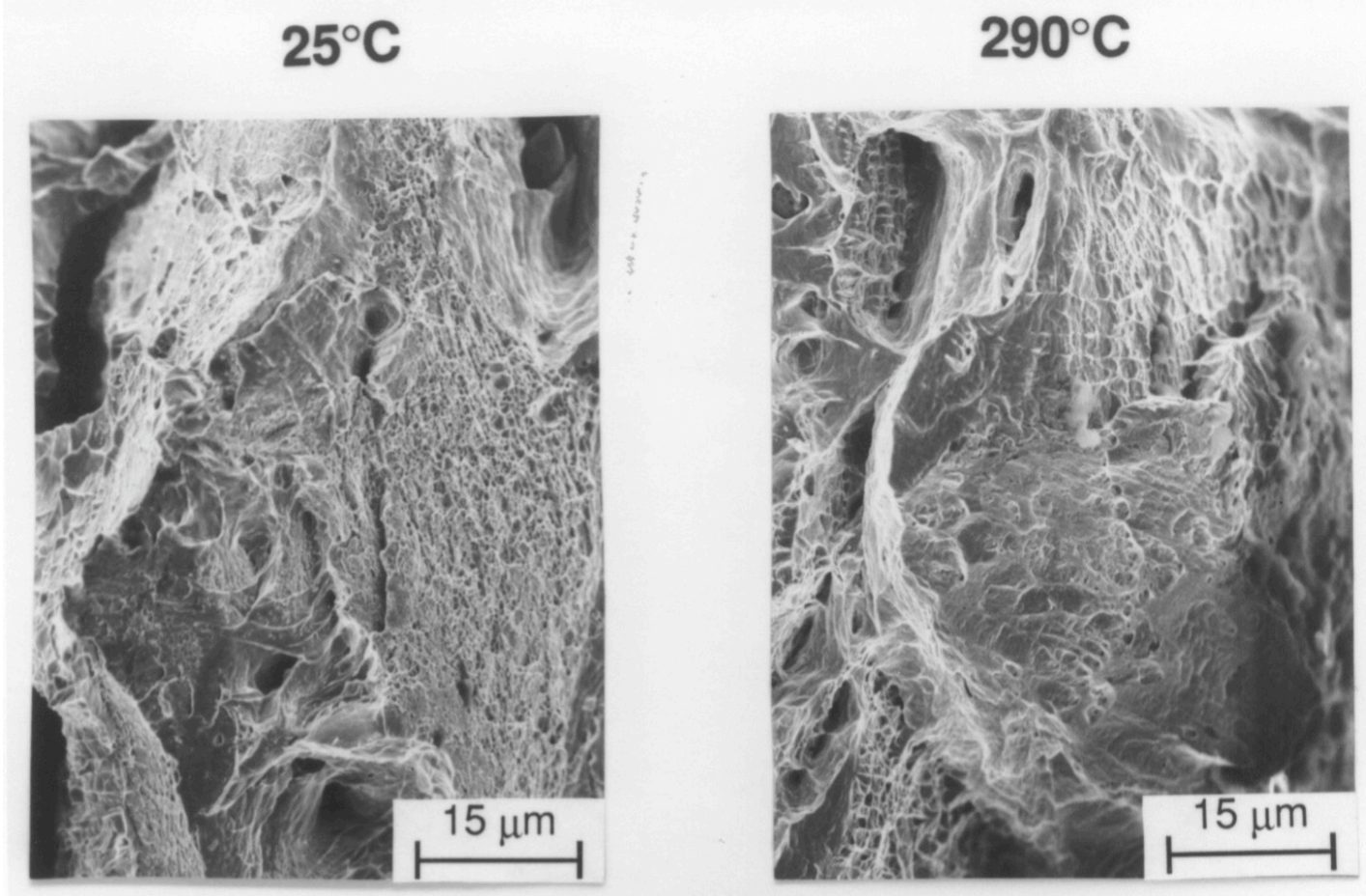

\section{Unaged}
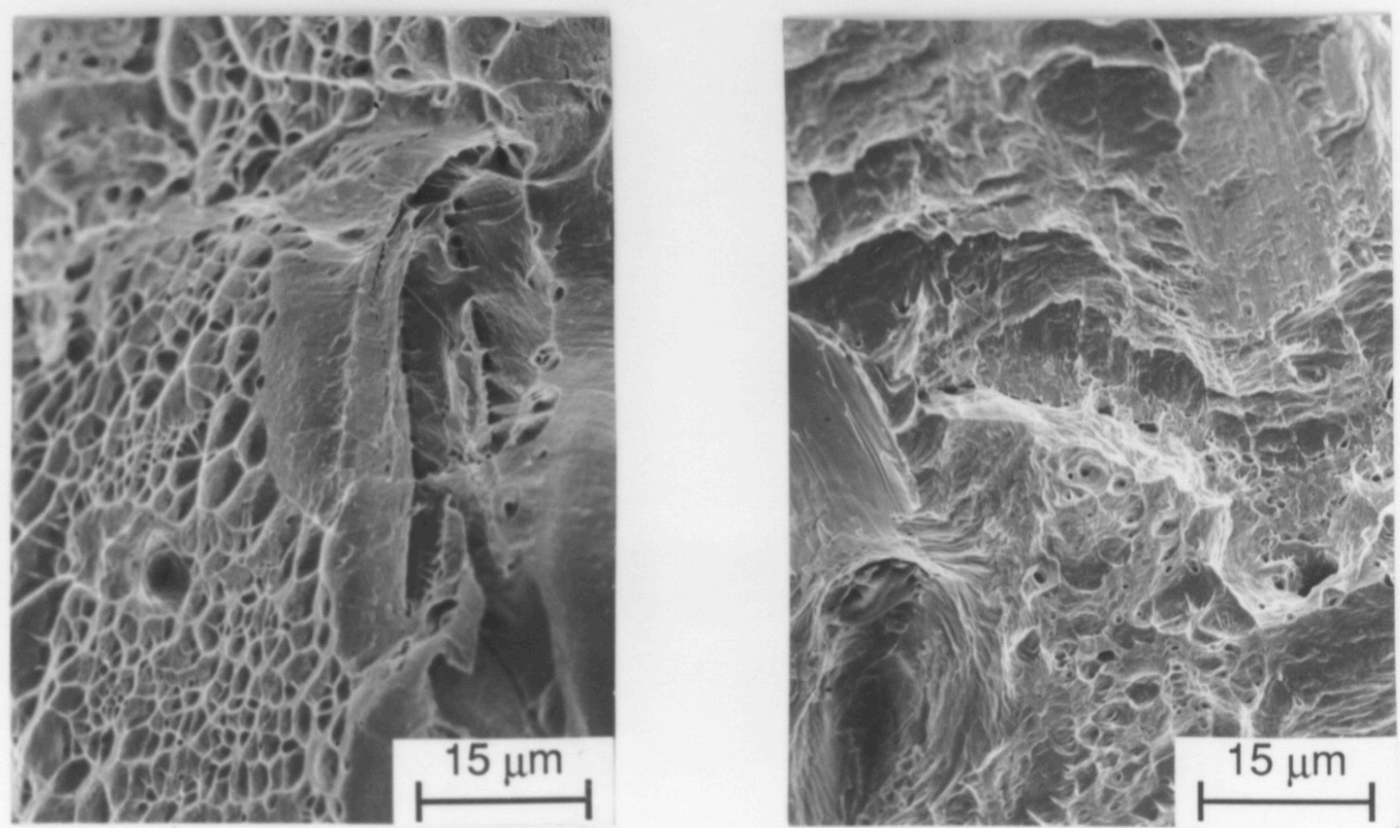

Aged $10,000 \mathrm{~h}$ at $400^{\circ} \mathrm{C}$

Figure 17. Fracture surface of tensile specimens of unaged and aged Heat 68 tested at room temperature and $290^{\circ} \mathrm{C}$ 

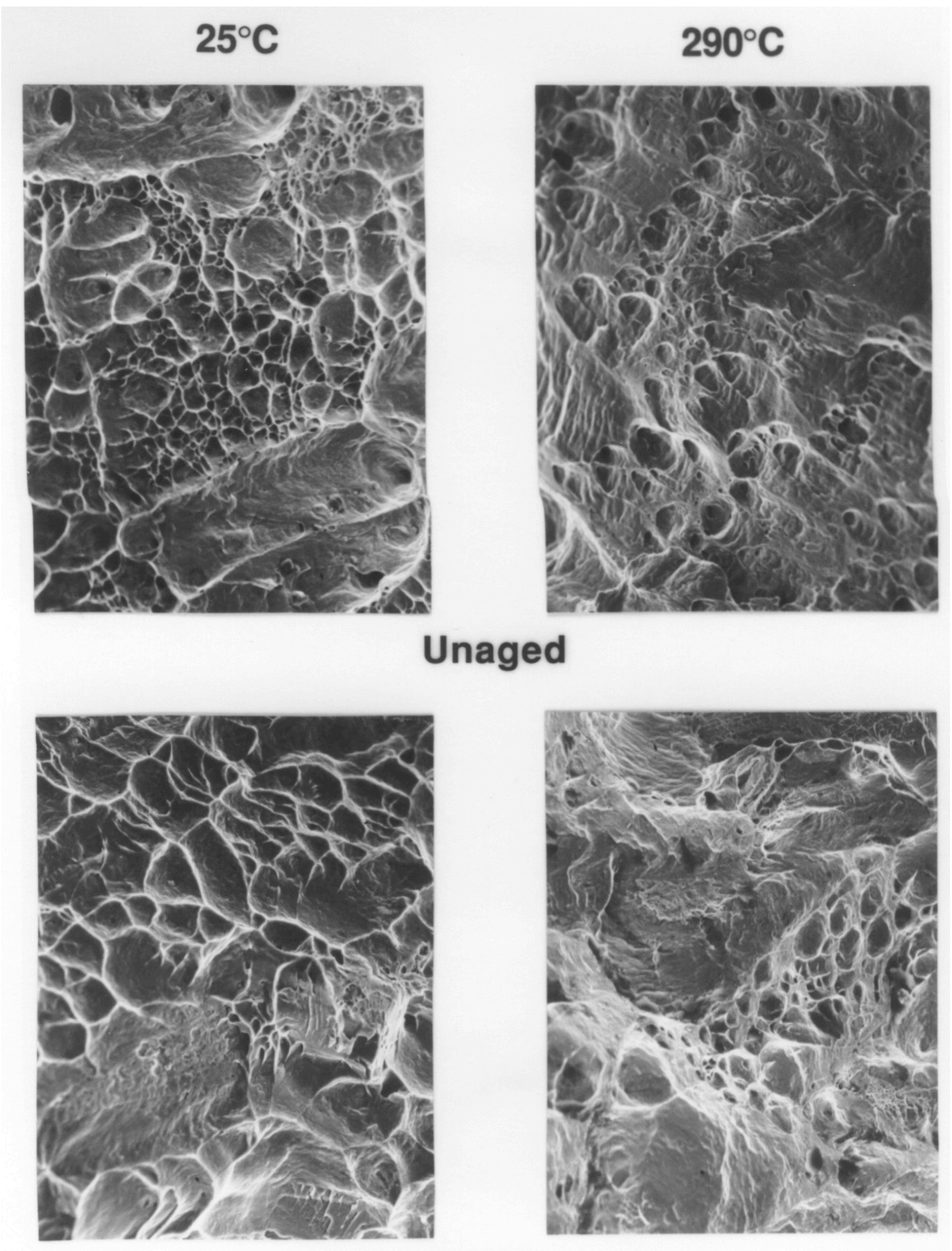

\section{Aged $10,000 \mathrm{~h}$ at $400^{\circ} \mathrm{C}$}

Figure 18. Fracture surface of tensile specimens of unaged and aged Heat 75 tested at room temperature and $290^{\circ} \mathrm{C}$ 

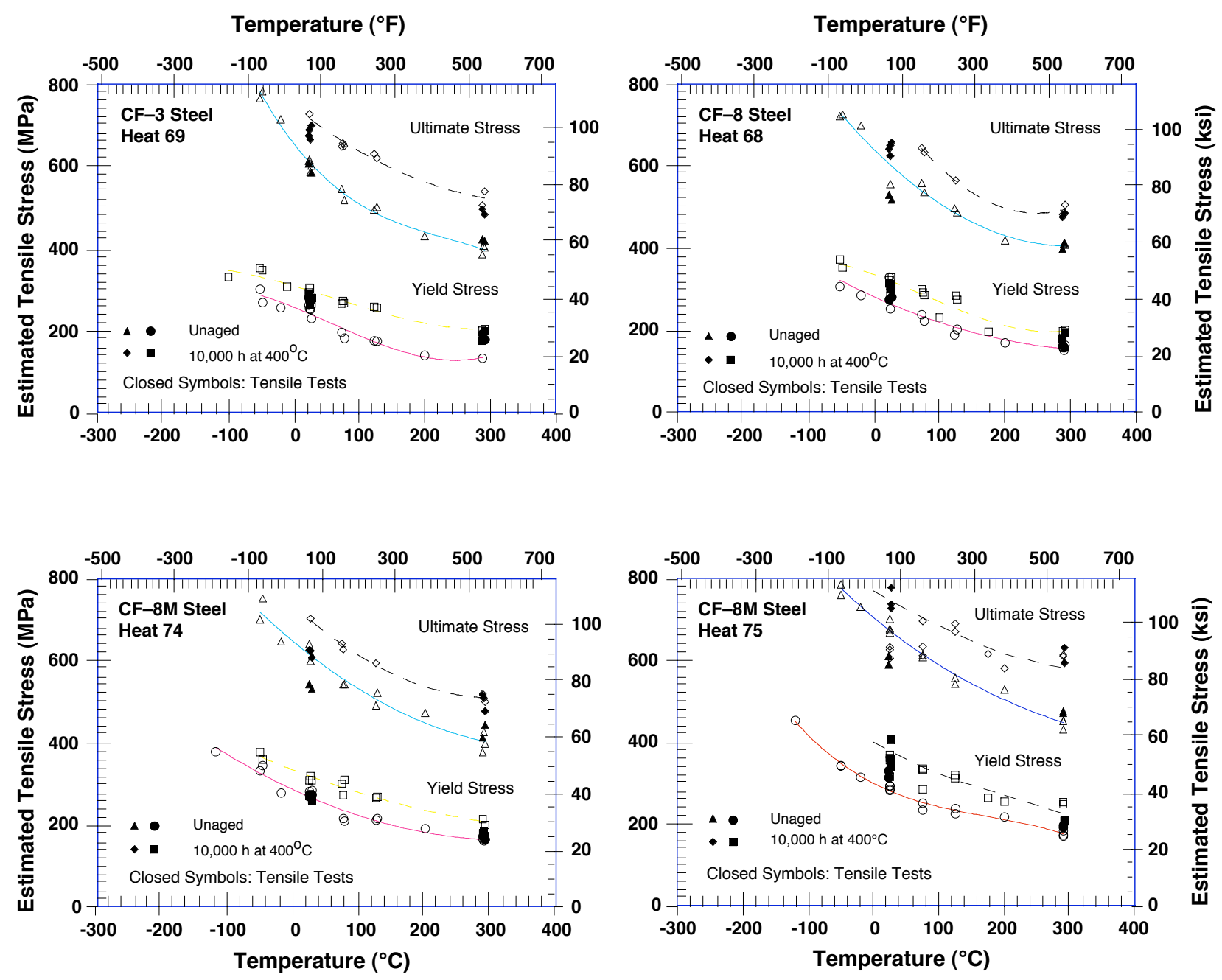

Figure 19. Values of yield and ultimate stress estimated from Charpy-impact data and those obtained from tensile tests for Heats 69, 68, 74, and 75

where $\sigma_{0}$ is an arbitary reference stress, often assumed to be equal to flow or yield stress; the reference strain $\varepsilon_{0}=\sigma_{0} / E ; \alpha$ and $n$ are $R O$ parameters; and $E$ is elastic modulus. The RO equation can be rearranged to the form

$$
\frac{E \varepsilon-\sigma}{\sigma_{o}}=\alpha\left(\frac{\sigma}{\sigma_{o}}\right)^{n}
$$

which is more convenient for fitting stress vs. strain data; $\alpha$ can be determined at $\sigma / \sigma_{0}=1$ and $\mathrm{n}$ can be obtained from the slope of the log-log plot of Eq. 4. For each test, the RO parameters were obtained from the best-fit of the tensile data to Eq. 4, with flow stress $\sigma_{f}$ as the reference stress

$$
\frac{E \varepsilon-\sigma}{\sigma_{f}}=\alpha_{1}\left(\frac{\sigma}{\sigma_{f}}\right)^{n_{1}}
$$


and also with yield stress $\sigma_{\mathrm{y}}$ as the reference stress

$$
\frac{E \varepsilon-\sigma}{\sigma_{y}}=\alpha_{2}\left(\frac{\sigma}{\sigma_{y}}\right)^{n_{2}} .
$$

The ROFIT computer program developed by Battelle* was used for the analyses. For each reference stress, three different sets of values of the RO parameters were obtained from the best fit of the engineering stress-vs.-strain data for $<5 \%$ strain, $<15 \%$ strain, and the entire strain range of the test. Typical examples of the ROFIT analyses with $\sigma_{f}$ as the reference stress are shown in Fig. 20. The best-fit values of the constant for all the tensile tests are given in Tables 4 and 5. As expected, the exponents $n_{1}$ and $n_{2}$ are the same from the two analyses, where $\sigma_{f}$ or $\sigma_{y}$ are used as the reference stress. The parameters $\alpha_{1}$ and $\alpha_{2}$ follow the relationship

$$
\alpha_{2}=\alpha_{1}\left(\frac{\sigma_{y}}{\sigma_{f}}\right)^{n_{1}-1}
$$

and can be determined from one another. The results indicate that the exponent $n_{1}$ or $n_{2}$ does not change with thermal aging and differs for the three grades of steel. The parameters $\alpha_{1}$ and $\alpha_{2}$ decrease with thermal aging.

\section{Estimation of Tensile Properties}

\subsection{Flow Stress}

Tensile flow stress of aged cast stainless steels can be estimated from the correlation between the ratio of the tensile flow stress of aged and unaged cast stainless steels and a normalized aging parameter. Correlations have been developed for estimating the tensile flow stress of aged cast stainless steels from the kinetics of thermal embrittlement. 6 The ratio of the tensile flow stress of aged and unaged cast stainless steels at room temperature and $290^{\circ} \mathrm{C}\left(554^{\circ} \mathrm{F}\right)$ is plotted as a function of a normalized aging parameter in Fig. 21. Flow stress is characterized as the mean of the $0.2 \%$ yield stress and ultimate stress, and the aging parameter is normalized with respect to a $\theta$ value of 2.9. The aging parameter $P$ was determined from Eq. 1 and experimental values of activation energy. At both temperatures, the flow stress ratio increased with thermal aging; the increase in flow stress of CF-3 steels was the smallest and that of CF$8 \mathrm{M}$ steels the largest. The correlations (shown as solid lines in Fig. 21) were obtained by subtracting one times the standard deviation for the fit to the data from the best-fit curve. The tensile-flow-stress ratio $R_{f}=\left(\sigma_{f}\right.$ aged $\left./ \sigma_{f_{\text {unaged }}}\right)$, is given by

$$
R_{f}=a_{1}+b_{1} P \quad\left(1.00 \leq R_{f} \leq c_{1}\right) .
$$

Values of the constants $a_{1}, b_{1}$, and $c_{1}$ for various grades of steel and test temperatures are given in Table 6 . Equation 8 is valid for $R_{f}$ values between 1 and $c_{1}$, service temperatures

* ROFIT (Version 1.0) computer program for IBM PC developed by Battelle Columbus for the U.S. Nuclear Regulatory Commission. Contact N. D. Ghadiali. 

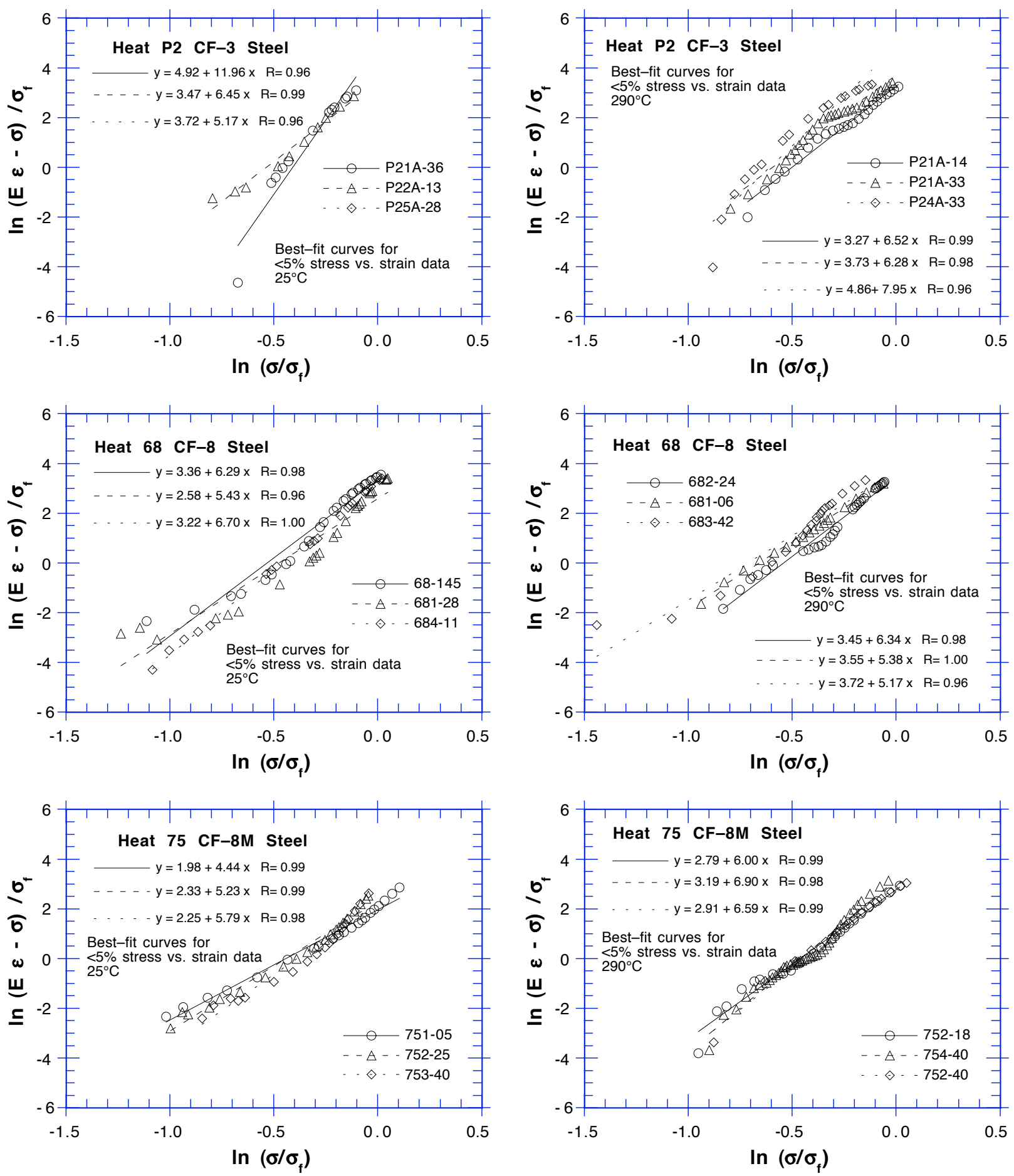

Figure 20. Examples of ROFIT analysis of tensile stress-vs.-strain curves at room temperature and $290^{\circ} \mathrm{C}$ for cast stainless steels using flow stress as the reference stress 

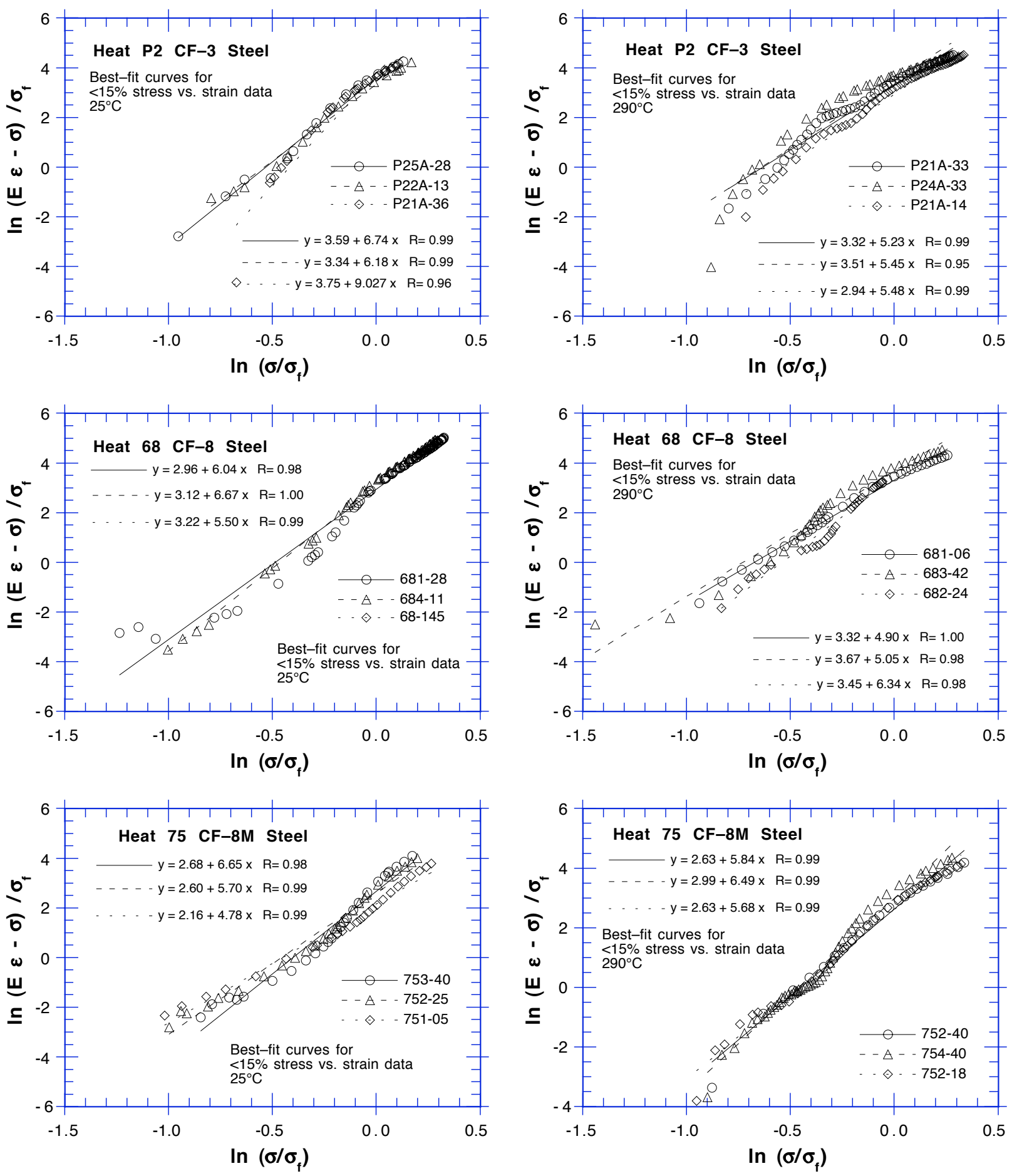

Figure 20. (Contd.) 

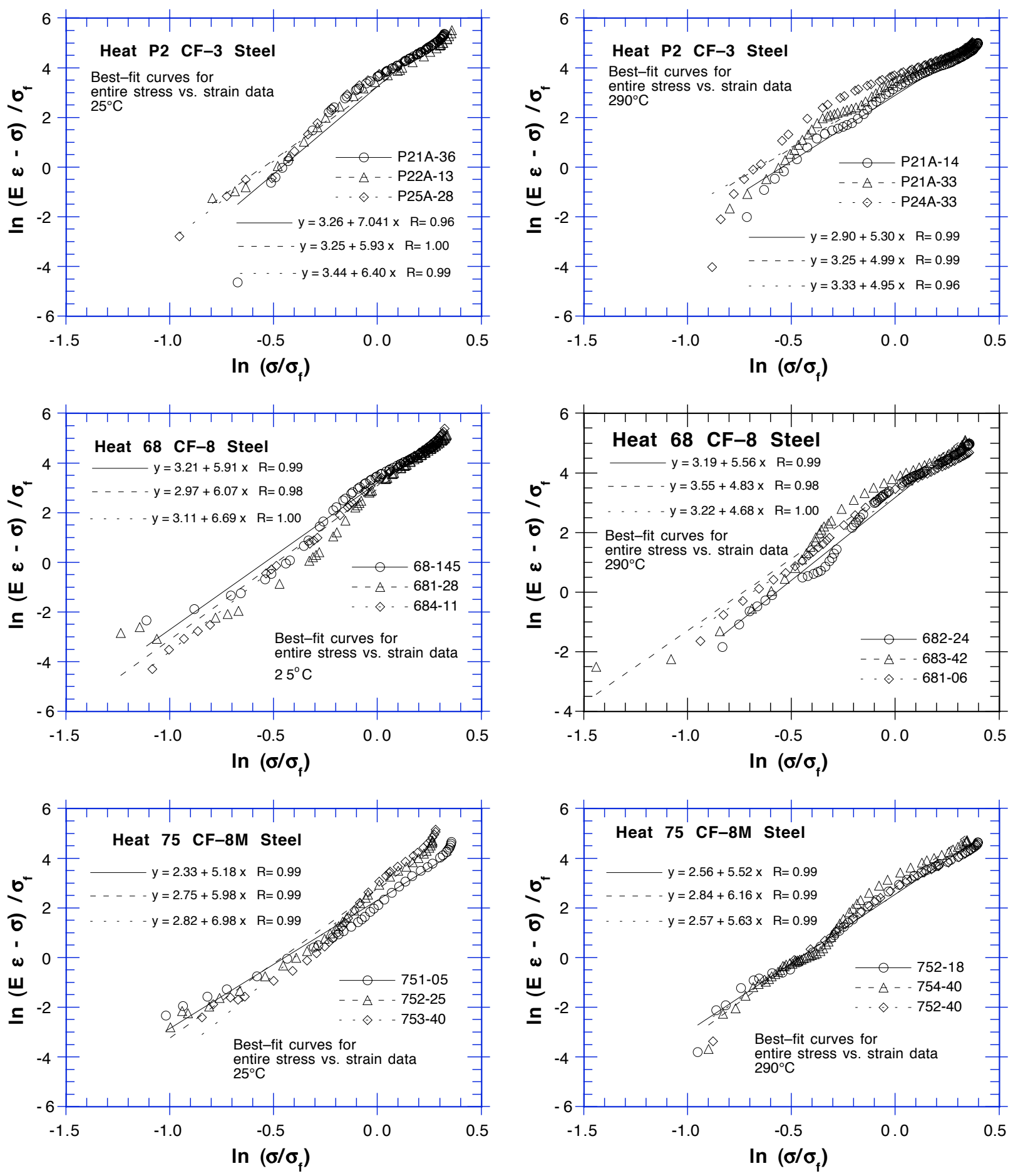

Figure 20. (Contd.) 
Table 4. Best-fit values of the Ramberg-Osgood constants for unaged and aged cast stainless steels at room temperature

\begin{tabular}{|c|c|c|c|c|c|c|c|c|c|c|c|c|}
\hline \multirow{3}{*}{$\begin{array}{c}\text { Specimen } \\
\text { Id }\end{array}$} & \multirow{3}{*}{$\begin{array}{l}\text { Aging } \\
\text { Temp. } \\
\left({ }^{\circ} \mathrm{C}\right)\end{array}$} & \multirow{3}{*}{$\begin{array}{c}\text { Aging } \\
\text { Time } \\
(\mathrm{h})\end{array}$} & \multirow{3}{*}{$\begin{array}{l}\text { Flow } \\
\text { Stress } \\
(\mathrm{MPa})\end{array}$} & \multirow{3}{*}{$\begin{array}{l}\text { Yield } \\
\text { Stress } \\
\text { (MPa) }\end{array}$} & \multicolumn{4}{|c|}{ Stress-Strain Data $<5 \%$ Strain } & \multicolumn{4}{|c|}{ Stress-Strain Data <15\% Strain } \\
\hline & & & & & \multicolumn{2}{|c|}{$\begin{array}{c}\text { Flow Stress } \\
\text { Reference }\end{array}$} & \multicolumn{2}{|c|}{$\begin{array}{l}\text { Yield Stress } \\
\text { Reference }\end{array}$} & \multicolumn{2}{|c|}{$\begin{array}{c}\text { Flow Stress } \\
\text { Reference }\end{array}$} & \multicolumn{2}{|c|}{$\begin{array}{c}\text { Yield Stress } \\
\text { Reference }\end{array}$} \\
\hline & & & & & $\alpha_{1}$ & $\mathrm{n}_{1}$ & $\alpha_{2}$ & $\mathrm{n}_{2}$ & $\alpha_{1}$ & $\mathrm{n}_{1}$ & $\alpha_{2}$ & $\mathrm{n}_{2}$ \\
\hline & - & aged & - & - & - & - & - & - & - & - & - & - \\
\hline & - & & - & - & - & - & - & - & 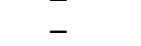 & - & - & - \\
\hline & - & Unaged & - & - & - & - & - & - & - & - & - & - \\
\hline & - & aged & _ & - & - & _- & - & - & - & _- & - & _- \\
\hline & & laged & - & - & - & - & - & - & - & - & - & - \\
\hline & 20 & 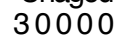 & - & - & - & - & - & - & - & - & - & - \\
\hline & 0 & & - & 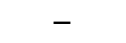 & - & - & - & - & - & - & - & - \\
\hline & 0 & 000 & - & - & - & - & - & - & - & - & - & - \\
\hline & 0 & & - & - & - & - & - & - & - & - & - & - \\
\hline & & & - & - & - & - & - & - & - & - & - & - \\
\hline & 0 & 100 & - & - & - & - & - & - & - & - & - & - \\
\hline $12 v$ & 50 & 10000 & - & 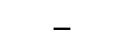 & - & - & - & & 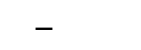 & - & - & - \\
\hline $\mathrm{P} 21 \mathrm{~T}-01$ & & Unaged & 33.9 & 16.3 & 10 & 444 & 0 & 9 & 59 & & 50 & .457 \\
\hline & & U & & & & & 4.3 & & & 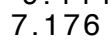 & 4.585 & 167 \\
\hline & & $\mathrm{U}$ & 3.9 & & & & & & & & & \\
\hline & & U & 3.9 & 7 & 5.760 & 9.724 & 1.487 & 9.574 & 15.760 & 9.724 & 1.487 & 9.57 \\
\hline & 90 & & & & & 7.114 & 1.668 & & & 6.437 & & 6.402 \\
\hline & 290 & & & & & 4.613 & 3.7 & & & 5.341 & & .307 \\
\hline & 290 & & & & & & 10 & & & & & 799 \\
\hline & 290 & 58 & & & 1 & 9.3 & 1.3 & 9.3 & & & 1.897 & 7.274 \\
\hline & 320 & 300 & & & & 6.7 & 1.312 & & & & & 6.200 \\
\hline & 320 & & & & & & 3.9 & & & & & 5.396 \\
\hline & 320 & & 0.6 & & & 7.072 & 1.079 & 7.0 & & & & 6.486 \\
\hline & 320 & & & & & 7.061 & 1.6 & 7.0 & & & 1.956 & 5.977 \\
\hline & 350 & & & & & 7.512 & 1.725 & 7.5 & & 6.682 & 1.863 & 6.661 \\
\hline & & & & & & & - & . & & . & - & - \\
\hline & 350 & & & & 4 & 5.133 & 3.614 & 5.2 & 34 & 5.571 & & 5.609 \\
\hline & & & & & & & & & & & & \\
\hline & & & & & & & & & & & & 6.167 \\
\hline & 350 & & & & 9 & 6.3 & 1.2 & 6.3 & & 48 & & 6.148 \\
\hline & 400 & & & & & & 2.8 & 4.6 & & & & 4.842 \\
\hline & 400 & & 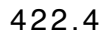 & & 1 & 4. & 3.007 & 4.5 & 1 & 4.936 & 3.059 & 4012 \\
\hline & - & & & & - & & & - & & & & - \\
\hline & & & & & & & & - & & - & & - \\
\hline & 290 & & & & .5 & 728 & 4 & & 3. & 6.1 & & \\
\hline & 290 & & & & & & 2 & & & & 2.574 & .920 \\
\hline & & & & & & & & & & & & \\
\hline & & & & & & & & & & & & 5.224 \\
\hline & 320 & & & & & & & & 44 & & & 6.334 \\
\hline & 320 & & & & & & 2.5 & & 15. & 37 & 2.5 & 5.82 \\
\hline & & & & & & & & & & & & \\
\hline & & & & & & & & & & & & \\
\hline & & & & & & & & & & & & \\
\hline & & & & & & & & & & & & 6.421 \\
\hline & & & & & & & & & & & & \\
\hline & & & & & & & & & & & & \\
\hline & & & & & & & 1.4 & & 23. & & 1.4 & 7. \\
\hline & & & & & & & & & & & & \\
\hline & & & & & & & & & & & & \\
\hline & & & & & & & & & & & & \\
\hline & & & & & & & & & & & & \\
\hline & & & & & & & 2.6 & & 14. & & & \\
\hline & & & & & & & & & & & & \\
\hline & 350 & & & & 0 & & 0.0 & & & & 0.011 & \\
\hline & 40 & & & & & & 2.5 & 4.3 & & & 2.745 & \\
\hline & & 2010 & & & & & & & & & 1.769 & \\
\hline & & & & & & & & & 22. & & 3.412 & \\
\hline $6 s$ & 400 & & $\ldots$ & & 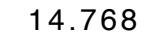 & 3025 & 8 & 3.906 & 18.461 & 4.490 & 2.867 & 10 \\
\hline
\end{tabular}


Table 4. (Contd.)

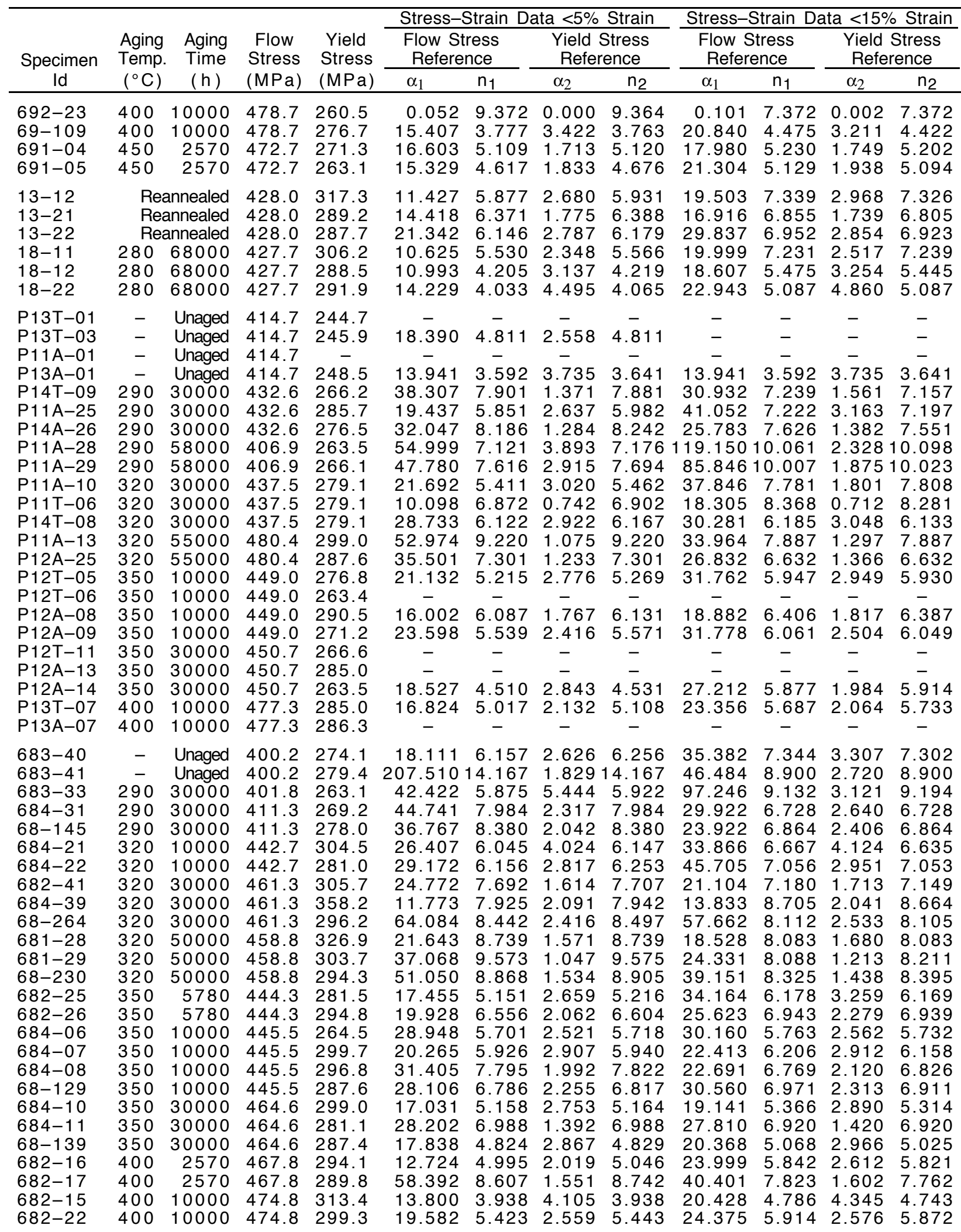


Table 4. (Contd.)

\begin{tabular}{|c|c|c|c|c|c|c|c|c|c|c|c|c|}
\hline \multirow{3}{*}{$\begin{array}{c}\text { Specimen } \\
\text { Id }\end{array}$} & \multirow{3}{*}{$\begin{array}{l}\text { Aging } \\
\text { Temp. } \\
\left({ }^{\circ} \mathrm{C}\right) \\
\end{array}$} & \multirow{3}{*}{$\begin{array}{c}\text { Aging } \\
\text { Time } \\
(\mathrm{h})\end{array}$} & \multirow{3}{*}{$\begin{array}{c}\text { Flow } \\
\text { Stress } \\
\text { (MPa) }\end{array}$} & \multirow{3}{*}{$\begin{array}{c}\text { Yield } \\
\text { Stress } \\
\text { (MPa) }\end{array}$} & \multicolumn{4}{|c|}{ Stress-Strain Data $<5 \%$ Strain } & \multicolumn{4}{|c|}{ Stress-Strain Data $<15 \%$ Strain } \\
\hline & & & & & \multicolumn{2}{|c|}{$\begin{array}{c}\text { Flow Stress } \\
\text { Reference }\end{array}$} & \multicolumn{2}{|c|}{$\begin{array}{c}\text { Yield Stress } \\
\text { Reference } \\
\end{array}$} & \multicolumn{2}{|c|}{$\begin{array}{c}\text { Flow Stress } \\
\text { Reference }\end{array}$} & \multicolumn{2}{|c|}{$\begin{array}{c}\text { Yield Stress } \\
\text { Reference } \\
\end{array}$} \\
\hline & & & & & $\alpha_{1}$ & 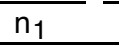 & $\alpha_{2}$ & $\mathrm{n}_{2}$ & 0 & $n_{1}$ & $\alpha_{2}$ & nz \\
\hline & 0 & $\begin{array}{l}00 \\
00 \\
70 \\
70\end{array}$ & & & $\begin{array}{l}22.124 \\
11.958 \\
12.073 \\
23.584\end{array}$ & & $\begin{array}{l}980 \\
041 \\
340 \\
151\end{array}$ & 8.147 & & & $\begin{array}{l}.077 \\
.982 \\
.990 \\
.285\end{array}$ & $\begin{array}{l}7.150 \\
7.718 \\
5.705 \\
6.855\end{array}$ \\
\hline & $\begin{array}{l}20 \\
50 \\
50 \\
50 \\
50 \\
50 \\
50 \\
00\end{array}$ & $\begin{array}{l}70 \\
0 \\
0 \\
0 \\
0 \\
0 \\
0 \\
0 \\
0 \\
0 \\
0 \\
0\end{array}$ & & & $\begin{array}{r}42.1 \\
82.0 \\
78.6 \\
31.3 \\
39.0 \\
37.0 \\
92.6 \\
77.5 \\
45.4 \\
30.8 \\
79.1 \\
59.2 \\
141.4 \\
26.1 \\
39.0\end{array}$ & $\begin{array}{l}.265 \\
.949 \\
.642 \\
.508 \\
.668 \\
.082 \\
.636 \\
.376 \\
.914 \\
.952 \\
.551 \\
.745 \\
.429 \\
.653 \\
.8511 \\
.884\end{array}$ & $\begin{array}{r}6.497 \\
6.744 \\
8.583 \\
6.307 \\
5.909 \\
6.108 \\
6.482 \\
3.370 \\
5.223 \\
6.315 \\
6.243 \\
2.961 \\
4.664 \\
3.769 \\
12.319 \\
5.495\end{array}$ & $\begin{array}{l}5.740 \\
6.600 \\
4.741 \\
5.162 \\
4.662\end{array}$ & $\begin{array}{r}53 \\
51 \\
50 \\
161 \\
160 \\
58 \\
53 \\
138\end{array}$ & $\begin{array}{l}5.9 \\
5.6 \\
7.0 \\
10.3 \\
5.4 \\
5.5 \\
5.7 \\
9.4 \\
8.1 \\
5.3 \\
5.4 \\
9.9 \\
7.3 \\
9.0 \\
3.7 \\
5.4\end{array}$ & $\begin{array}{l}7.280 \\
7.556 \\
7.003 \\
3.163 \\
6.962 \\
6.481 \\
5.093 \\
1.954 \\
3.088 \\
6.786 \\
7.143 \\
1.775 \\
2.776 \\
2.801 \\
6.259 \\
5.781\end{array}$ & $\begin{array}{l}5.950 \\
5.687 \\
7.213 \\
0.418 \\
5.489 \\
5.556 \\
5.805 \\
9.440 \\
8.212 \\
5.313 \\
5.445 \\
9.899 \\
7.397 \\
9.124 \\
3.812 \\
5.447\end{array}$ \\
\hline & & & & & & & & & & & & \\
\hline 1 & $\begin{array}{l}- \\
-\end{array}$ & & & & $\begin{array}{l}7 \\
9 \\
3 \\
2 \\
9 \\
9 \\
4 \\
4 \\
7 \\
2 \\
3 \\
4 \\
3 \\
3 \\
3 \\
\end{array}$ & & $\begin{array}{l}0 \\
0 \\
9 \\
6 \\
4 \\
7 \\
3 \\
1 \\
6 \\
8 \\
4 \\
3 \\
3 \\
2 \\
8 \\
5 \\
0 \\
1 \\
4\end{array}$ & $\begin{array}{l}5 . \\
5 . \\
3 . \\
4 . \\
4 . \\
4 .\end{array}$ & 1 & $\begin{array}{l}2 \\
0 \\
9 \\
6 \\
1 \\
1 \\
4 \\
3 \\
0 \\
1 \\
9 \\
8 \\
5 \\
7 \\
4\end{array}$ & $\begin{array}{l}7 \\
2 \\
9 \\
2 \\
7 \\
8 \\
8 \\
7 \\
5 \\
4 \\
3 \\
3 \\
3 \\
8 \\
0 \\
0 \\
8 \\
8 \\
9 \\
0 \\
8 \\
5 \\
1 \\
4 \\
0 \\
8 \\
4\end{array}$ & 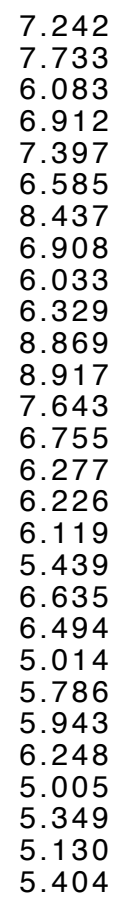 \\
\hline $\begin{array}{l}753-41 \\
753-30 \\
754-28 \\
75-135 \\
754-21 \\
754-25\end{array}$ & $\begin{array}{l}290 \\
290\end{array}$ & 10000 & 1 & & $\begin{array}{r}13.029 \\
20.617 \\
8.964 \\
9.692 \\
16.380 \\
10.041\end{array}$ & $\begin{array}{l}2 \\
4 \\
6\end{array}$ & $\begin{array}{r}3 \\
74 \\
57 \\
57 \\
72 \\
70\end{array}$ & 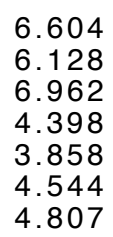 & $\begin{array}{l}4 \\
1 \\
8\end{array}$ & $\begin{array}{l}6.940 \\
6.823 \\
5.985 \\
4.842 \\
5.353 \\
6.161\end{array}$ & $\begin{array}{l}2.624 \\
2.005 \\
1.979 \\
2.565 \\
3.588 \\
3.776\end{array}$ & $\begin{array}{l}6.823 \\
6.026 \\
4.877 \\
5.313 \\
6.136\end{array}$ \\
\hline
\end{tabular}


Table 4. (Contd.)

\begin{tabular}{|c|c|c|c|c|c|c|c|c|c|c|c|c|}
\hline \multirow{3}{*}{$\begin{array}{l}\text { Specimen } \\
\text { Id }\end{array}$} & \multirow{3}{*}{$\begin{array}{l}\text { Aging } \\
\text { Temp. } \\
\left({ }^{\circ} \mathrm{C}\right)\end{array}$} & \multirow{3}{*}{$\begin{array}{c}\text { Aging } \\
\text { Time } \\
(\mathrm{h})\end{array}$} & \multirow{3}{*}{$\begin{array}{l}\text { Flow } \\
\text { Stress } \\
\text { (MPa) }\end{array}$} & \multirow{3}{*}{$\begin{array}{c}\text { Yield } \\
\text { Stress } \\
\text { (MPa } \\
\text { ) }\end{array}$} & \multicolumn{4}{|c|}{ Stress-Strain Data $<5 \%$ Strain } & \multicolumn{4}{|c|}{ Stress-Strain Data $<15 \%$ Strain } \\
\hline & & & & & \multicolumn{2}{|c|}{$\begin{array}{c}\text { Flow Stress } \\
\text { Reference }\end{array}$} & \multicolumn{2}{|c|}{$\begin{array}{c}\text { Yield Stress } \\
\text { Reference }\end{array}$} & \multicolumn{2}{|c|}{$\begin{array}{c}\text { Flow Stress } \\
\text { Reference }\end{array}$} & \multicolumn{2}{|c|}{$\begin{array}{c}\text { Yield Stress } \\
\text { Reference }\end{array}$} \\
\hline & & & & & $\alpha_{1}$ & $\mathrm{n}_{1}$ & $\alpha_{2}$ & $\mathrm{n}_{2}$ & $\alpha_{1}$ & $\mathrm{n}_{1}$ & $\alpha_{2}$ & $\mathrm{n}_{2}$ \\
\hline & & & & & & & & & & & & 0.595 \\
\hline & & & & & & & & & & & & \\
\hline & & & & & & & & & & & & \\
\hline & & & & & 10. & & 26 & & 26 & & & 515 \\
\hline & 0 & & & & 11. & & 322 & & 40 & & & - \\
\hline & & & & & & & 977 & & & & & 6.811 \\
\hline & & & & & 13. & 6.5 & 50 & 0 & 776 & & & 6.639 \\
\hline & & & & & & & 1.680 & & 60 & & & 6.130 \\
\hline & & & & & & 4.126 & 2.404 & & 558 & & & 4.893 \\
\hline & & & & & 11.133 & 4.345 & 2.713 & 4.349 & 96 & 5. & 15 & 5.088 \\
\hline & & & & & 15 & 5.636 & 1.157 & & 361 & 5. & 56 & 5.761 \\
\hline & & & & & 10 & 2.9 & 4.750 & 2. & 13 & & & 3.953 \\
\hline & & & & & & 4.609 & 1.275 & & 9.648 & & & 5.005 \\
\hline & & & & & & & 2.147 & & 10.552 & & & 4.737 \\
\hline & & & & & 1 & & & & & & & \\
\hline & & & & & 12.5 & & & & & & & \\
\hline & & & & & 9. & & 1.723 & & & & & 87 \\
\hline & & & & & 7. & 3. & 3.893 & & 86 & & & 90 \\
\hline & & & & & 3. & 3.1 & 1.473 & & 7.472 & 5. & & 5.128 \\
\hline & 400 & 00 & 7.6 & 3.9 & 11.999 & 5.662 & 1.178 & & 12.404 & & & 5.745 \\
\hline & 400 & 10000 & 7.6 & 359.0 & 16.151 & 3.879 & 4.547 & 3.879 & 19.749 & 4.616 & 4.018 & 4.616 \\
\hline & 450 & & & & 8.651 & 4.794 & 1.233 & 4.763 & 10.631 & 5.384 & 1.134 & 5.331 \\
\hline & 450 & 2570 & 523.5 & 314.7 & 7.661 & 4.949 & 1.027 & 4.949 & 8.863 & 5.447 & 0.922 & 5.447 \\
\hline
\end{tabular}


Table 5. Best-fit values of the Ramberg-Osgood constants for unaged and aged cast stainless steels at $290^{\circ} \mathrm{C}$

\begin{tabular}{|c|c|c|c|c|c|c|c|c|c|c|c|c|}
\hline \multirow{3}{*}{$\begin{array}{c}\text { Specimen } \\
\text { Id }\end{array}$} & \multirow{3}{*}{$\begin{array}{c}\text { Aging } \\
\text { Temp. } \\
\left({ }^{\circ} \mathrm{C}\right)\end{array}$} & \multirow{3}{*}{$\begin{array}{l}\text { Aging } \\
\text { Time } \\
(\mathrm{h})\end{array}$} & \multirow{3}{*}{$\begin{array}{c}\text { Flow } \\
\text { Stress } \\
\text { (MPa) }\end{array}$} & \multirow{3}{*}{$\begin{array}{c}\text { Yield } \\
\text { Stress } \\
\text { (MPa) }\end{array}$} & \multicolumn{4}{|c|}{ Stress-Strain Data $<5 \%$ Strain } & \multicolumn{4}{|c|}{ Stress-Strain Data $<15 \%$ Strain } \\
\hline & & & & & \multicolumn{2}{|c|}{$\begin{array}{l}\text { Flow Stress } \\
\text { Reference } \\
\end{array}$} & \multicolumn{2}{|c|}{$\begin{array}{c}\text { Yield Stress } \\
\text { Reference }\end{array}$} & \multicolumn{2}{|c|}{$\begin{array}{c}\text { Flow Stress } \\
\text { Reference } \\
\end{array}$} & \multicolumn{2}{|c|}{$\begin{array}{c}\text { Yield Stress } \\
\text { Reference } \\
\end{array}$} \\
\hline & & & & & $\alpha_{1}$ & 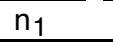 & $\alpha_{2}$ & $\mathrm{n}_{2}$ & $\alpha_{1}$ & $\mathrm{n}_{1}$ & $\alpha_{2}$ & $\mathrm{n}_{2}$ \\
\hline $\begin{array}{l}2 \\
4 \\
5 \\
0\end{array}$ & $\begin{array}{c}- \\
- \\
- \\
320 \\
320 \\
320 \\
350 \\
350 \\
350\end{array}$ & $\begin{array}{l}\text { naged } \\
\text { naged } \\
\text { naged } \\
000 \\
000 \\
000 \\
000 \\
000 \\
000\end{array}$ & $\begin{array}{l}- \\
- \\
- \\
- \\
- \\
- \\
- \\
- \\
-\end{array}$ & $\begin{array}{l}- \\
- \\
- \\
- \\
- \\
- \\
- \\
- \\
-\end{array}$ & $\begin{array}{l}- \\
- \\
- \\
- \\
- \\
- \\
- \\
- \\
-\end{array}$ & $\begin{array}{l}- \\
- \\
- \\
- \\
- \\
- \\
- \\
- \\
-\end{array}$ & $\begin{array}{l}- \\
- \\
- \\
- \\
- \\
- \\
- \\
- \\
-\end{array}$ & $\begin{array}{l}- \\
- \\
- \\
- \\
- \\
- \\
- \\
- \\
-\end{array}$ & $\begin{array}{l}- \\
- \\
- \\
- \\
- \\
- \\
- \\
- \\
-\end{array}$ & $\begin{array}{l}- \\
- \\
- \\
- \\
- \\
- \\
- \\
- \\
-\end{array}$ & $\begin{array}{l}- \\
- \\
- \\
- \\
- \\
- \\
- \\
- \\
-\end{array}$ & $\begin{array}{l}- \\
- \\
- \\
- \\
- \\
- \\
- \\
- \\
-\end{array}$ \\
\hline $\begin{array}{l}\text { P23T-0 } \\
\text { P22A-0 } \\
\text { P23A-0 } \\
\text { P21A-3 } \\
\text { P21A-3 } \\
\text { P24T-1 } \\
\text { P21A-3 } \\
\text { P22T-1 } \\
\text { P21A-1 } \\
\text { P21A-1 } \\
\text { P21A-1 } \\
\text { P25T-1 } \\
\text { P21A-1 } \\
\text { P21T-0 } \\
\text { P22T-0 } \\
\text { P23A-1 } \\
\text { P23A-2 } \\
\text { P22T-1 } \\
\text { P24A-3 } \\
\text { P24A-3 } \\
\text { P24T-0 } \\
\text { P24A-0 }\end{array}$ & $\begin{array}{l}- \\
- \\
- \\
- \\
290 \\
290 \\
290 \\
290 \\
290 \\
320 \\
320 \\
320 \\
320 \\
320 \\
350 \\
350 \\
350 \\
350 \\
350 \\
350 \\
350 \\
400 \\
400\end{array}$ & $\begin{array}{l}\text { Un } \\
\text { Un } \\
\text { Un } \\
30 \\
30 \\
30 \\
58 \\
58 \\
30 \\
30 \\
30 \\
55 \\
55 \\
10 \\
10 \\
10 \\
10 \\
30 \\
30 \\
30 \\
10 \\
10\end{array}$ & $\begin{array}{l}0.3 \\
0.3 \\
0.3 \\
3.8 \\
3.8 \\
8.2 \\
8.2 \\
8.2 \\
3.7 \\
3.7 \\
5.2 \\
5.2 \\
5.2 \\
5.2 \\
2.1 \\
2.1 \\
2.1 \\
6.2 \\
6.2\end{array}$ & $\begin{array}{l}16 \\
15 \\
14 \\
16 \\
17 \\
15 \\
16 \\
15 \\
15 \\
16 \\
15 \\
15 \\
16 \\
15 \\
16 \\
14 \\
14 \\
16 \\
14\end{array}$ & $\begin{array}{r}30.130 \\
9.867 \\
28.481 \\
- \\
133.790 \\
40.329 \\
593.540 \\
114.150 \\
14.069 \\
25.048 \\
62.046 \\
32.615 \\
100.920 \\
62.313 \\
74.163 \\
45.695 \\
30.569 \\
37.588 \\
21.989 \\
42.270 \\
129.200 \\
34.062 \\
58.077\end{array}$ & $\begin{array}{c}3.599 \\
2.779 \\
2.892 \\
- \\
8.911 \\
6.136 \\
9.612 \\
8.300 \\
3.600 \\
6.266 \\
7.180 \\
5.864 \\
9.045 \\
7.568 \\
6.743 \\
4.933 \\
2.874 \\
4.893 \\
6.231 \\
6.000 \\
7.900 \\
5.237 \\
5.333\end{array}$ & $\begin{array}{c}7.750 \\
3.629 \\
7.894 \\
- \\
1.745 \\
1.901 \\
2.439 \\
1.630 \\
3.860 \\
0.867 \\
1.864 \\
1.451 \\
1.283 \\
1.589 \\
2.271 \\
4.095 \\
10.639 \\
3.464 \\
1.165 \\
1.160 \\
1.210 \\
2.550 \\
2.742\end{array}$ & $\begin{array}{c}3.602 \\
2.833 \\
2.928 \\
- \\
8.902 \\
6.160 \\
9.593 \\
8.300 \\
3.600 \\
6.336 \\
7.194 \\
5.895 \\
9.057 \\
7.590 \\
6.737 \\
4.955 \\
2.927 \\
4.905 \\
6.258 \\
6.000 \\
7.900 \\
5.237 \\
5.335\end{array}$ & $\begin{array}{r}40.384 \\
9.867 \\
28.481 \\
- \\
62.867 \\
27.515 \\
19.110 \\
35.770 \\
25.090 \\
19.474 \\
40.888 \\
23.481 \\
55.847 \\
44.166 \\
32.202 \\
35.083 \\
30.569 \\
29.679 \\
17.282 \\
28.880 \\
33.430 \\
24.125 \\
34.889\end{array}$ & $\begin{array}{l}4.108 \\
2.779 \\
2.892 \\
- \\
6.959 \\
4.876 \\
6.359 \\
5.800 \\
4.000 \\
5.115 \\
6.076 \\
4.847 \\
7.457 \\
6.695 \\
4.799 \\
4.366 \\
2.8741 \\
4.349 \\
5.227 \\
5.100 \\
5.400 \\
4.335 \\
4.173\end{array}$ & $\begin{array}{r}8.083 \\
3.629 \\
7.894 \\
- \\
2.255 \\
2.619 \\
3.760 \\
2.202 \\
5.470 \\
1.290 \\
2.211 \\
1.871 \\
1.572 \\
1.782 \\
3.100 \\
4.567 \\
10.639 \\
3.930 \\
1.529 \\
1.550 \\
1.680 \\
2.995 \\
3.602\end{array}$ & $\begin{array}{c}4.076 \\
2.833 \\
2.928 \\
- \\
7.108 \\
4.966 \\
6.417 \\
5.800 \\
4.000 \\
5.262 \\
6.174 \\
4.956 \\
7.652 \\
6.786 \\
4.860 \\
4.311 \\
2.927 \\
4.290 \\
5.313 \\
5.100 \\
5.400 \\
4.392 \\
4.228\end{array}$ \\
\hline $\begin{array}{l}-40 \\
-32 \\
-33 \\
236 \\
-26 \\
-27 \\
-42 \\
-39 \\
246 \\
-28 \\
-29 \\
130 \\
-27 \\
-09 \\
120\end{array}$ & $\begin{array}{r}- \\
- \\
290 \\
290 \\
290 \\
320 \\
320 \\
320 \\
320 \\
320 \\
320 \\
320 \\
320 \\
350 \\
350 \\
350\end{array}$ & $\begin{array}{l}\text { ed } \\
0 \\
0 \\
0 \\
0 \\
0 \\
0 \\
0 \\
0 \\
0 \\
0 \\
0 \\
0 \\
0 \\
0 \\
0\end{array}$ & $\begin{array}{l}4 \\
8 \\
8 \\
8 \\
2 \\
0\end{array}$ & $\begin{array}{l}5 \\
2 \\
5 \\
1\end{array}$ & $\begin{array}{l}64.420 \\
36.631 \\
67.208 \\
57.898 \\
28.885 \\
23.782 \\
17.580 \\
22.021 \\
31.424 \\
31.758 \\
39.539 \\
28.152 \\
26.381 \\
28.175 \\
27.831 \\
16.671\end{array}$ & $\begin{array}{l}5.468 \\
7.773 \\
7.371 \\
6.811 \\
6.627 \\
6.625 \\
6.693 \\
6.043 \\
6.747 \\
8.602 \\
7.328 \\
6.390 \\
5.109 \\
6.155 \\
5.960\end{array}$ & $\begin{array}{l}2.108 \\
1.869 \\
2.609 \\
1.177 \\
1.192 \\
1.235 \\
1.830 \\
1.121 \\
1.019 \\
1.495 \\
1.998 \\
2.513 \\
1.047 \\
1.444\end{array}$ & $\begin{array}{l}8 \\
3 \\
9 \\
6 \\
1 \\
4 \\
9 \\
3 \\
4 \\
3 \\
0 \\
4 \\
5 \\
5\end{array}$ & $\begin{array}{l}31.210 \\
46.935 \\
41.700 \\
29.038 \\
18.479 \\
14.487 \\
15.351 \\
21.323 \\
21.168 \\
19.246 \\
18.314 \\
19.024 \\
22.891 \\
23.302 \\
14.764\end{array}$ & & $\begin{array}{l}3.738 \\
2.302 \\
2.115 \\
1.499 \\
1.464 \\
1.453 \\
1.590 \\
2.224 \\
1.580 \\
1.681 \\
1.975 \\
2.354 \\
2.716 \\
1.237 \\
1.697\end{array}$ & $\begin{array}{l}4.998 \\
6.868 \\
6.575 \\
8.056 \\
5.722 \\
5.730 \\
5.476 \\
5.004 \\
5.467 \\
6.069 \\
5.795 \\
5.359 \\
4.622 \\
5.618 \\
5.343\end{array}$ \\
\hline $\begin{array}{r}3-14 \\
3-15 \\
-270 \\
-18 \\
-24 \\
-110\end{array}$ & 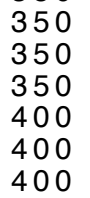 & $\begin{array}{r}30 \\
30 \\
2 \\
10 \\
10\end{array}$ & $\begin{array}{l}3 \\
3 \\
3 \\
4 \\
6\end{array}$ & $\begin{array}{l}4 \\
8 \\
6 \\
9 \\
9 \\
3\end{array}$ & $\begin{array}{l}87.807 \\
18.280 \\
20.517 \\
15.209 \\
16.846\end{array}$ & $\begin{array}{c}-\overline{6} 18 \\
4.700 \\
5.282 \\
5.798 \\
5.161\end{array}$ & $\begin{array}{c}-\overline{1} \\
1.147 \\
2.720 \\
1.433 \\
0.644 \\
1.828\end{array}$ & $\begin{array}{l}9 . \overline{6} \\
4.7 \\
5.3 \\
5.7 \\
5.1\end{array}$ & $\begin{array}{l}19.7 \\
17.2 \\
13.2 \\
15.8\end{array}$ & $\begin{array}{l}64 \\
00 \\
04 \\
24 \\
94\end{array}$ & $\begin{array}{l}2.344 \\
2.830 \\
1.667 \\
0.772 \\
2.008\end{array}$ & $\begin{array}{l}5.856 \\
4.800 \\
4.759 \\
5.289 \\
4.865\end{array}$ \\
\hline $\begin{array}{l}691-06 \\
692-09\end{array}$ & $\begin{array}{l}450 \\
450\end{array}$ & $\begin{array}{l}2570 \\
2570\end{array}$ & & & $16 . \overline{7} 53$ & $\overline{4} 888$ & $3 . \overline{5} 52$ & $3 . \overline{498}$ & $20 . \overline{5} 67$ & $3 . \overline{8} 57$ & $3 . \overline{5} 80$ & $3 . \overline{8} 21$ \\
\hline & & & & & $\begin{array}{l}23.371 \\
43.387\end{array}$ & $\begin{array}{l}6.542 \\
7.274\end{array}$ & $\begin{array}{l}1.730 \\
1.472\end{array}$ & $\begin{array}{l}6.606 \\
7.304\end{array}$ & $\begin{array}{l}22.522 \\
35.599\end{array}$ & $\begin{array}{l}6.420 \\
6.830\end{array}$ & $\begin{array}{l}1.791 \\
1.515\end{array}$ & $\begin{array}{l}6.398 \\
6.841\end{array}$ \\
\hline
\end{tabular}


Table 5. (Contd.)

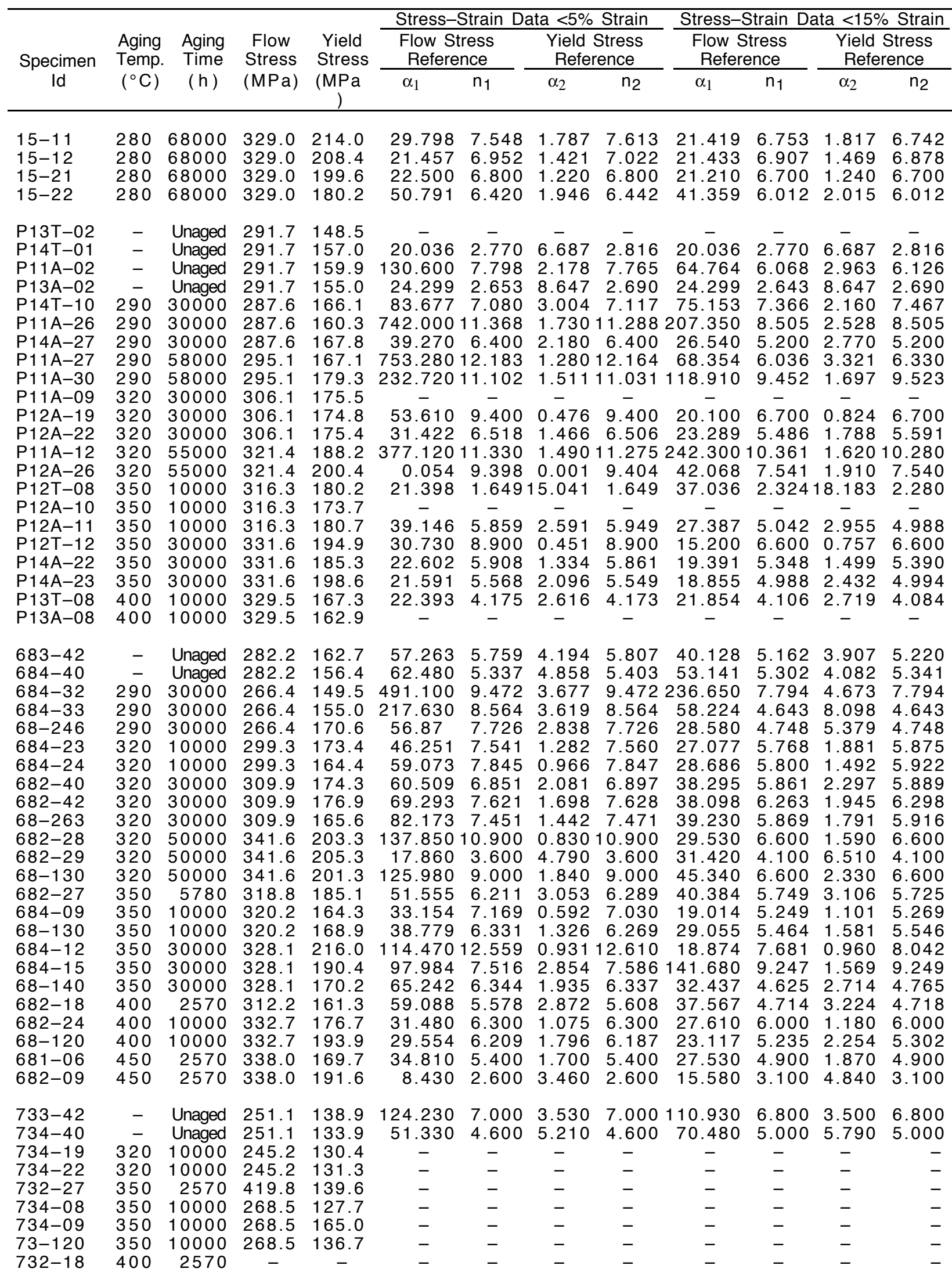


Table 5. (Contd.)

\begin{tabular}{|c|c|c|c|c|c|c|c|c|c|c|c|c|}
\hline \multirow{3}{*}{$\begin{array}{l}\text { Specimen } \\
\text { Id }\end{array}$} & \multirow{3}{*}{$\begin{array}{l}\text { Aging } \\
\text { Temp. } \\
\left({ }^{\circ} \mathrm{C}\right)\end{array}$} & \multirow{3}{*}{$\begin{array}{l}\text { Aging } \\
\text { Time } \\
(\mathrm{h})\end{array}$} & \multirow{3}{*}{$\begin{array}{c}\text { Flow } \\
\text { Stress } \\
\text { (MPa) }\end{array}$} & \multirow{3}{*}{$\begin{array}{c}\text { Yield } \\
\text { Stress } \\
\text { (MPa) }\end{array}$} & \multicolumn{4}{|c|}{ Stress-Strain Data $<5 \%$ Strain } & \multicolumn{4}{|c|}{ Stress-Strain Data $<15 \%$ Strain } \\
\hline & & & & & \multicolumn{2}{|c|}{$\begin{array}{c}\text { Flow Stress } \\
\text { Reference }\end{array}$} & \multicolumn{2}{|c|}{$\begin{array}{c}\text { Yield Stress } \\
\text { Reference } \\
\end{array}$} & \multicolumn{2}{|c|}{$\begin{array}{c}\text { Flow Stress } \\
\text { Reference }\end{array}$} & \multicolumn{2}{|c|}{$\begin{array}{c}\text { Yield Stress } \\
\text { Reference }\end{array}$} \\
\hline & & & & & $\alpha_{1}$ & 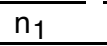 & $\alpha_{2}$ & $\mathrm{n}_{2}$ & $\alpha_{1}$ & 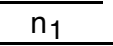 & 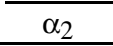 & $\mathrm{n}_{2}$ \\
\hline 3 & 0 & $\begin{array}{r}10000 \\
10000 \\
10000 \\
2570 \\
2570\end{array}$ & $\begin{array}{l}.9 \\
.9 \\
.9 \\
.9 \\
.9\end{array}$ & $\begin{array}{l}38.5 \\
43.0 \\
39.4 \\
44.1 \\
36.2\end{array}$ & $\begin{array}{l}34.550 \\
31.760 \\
6.310 \\
4.410 \\
32.370\end{array}$ & $\begin{array}{l}.356 \\
.357 \\
.000 \\
.497 \\
.535\end{array}$ & $\begin{array}{l}1.740 \\
1.888 \\
2.044 \\
2.607 \\
2.001\end{array}$ & $\begin{array}{l}9.282 \\
8.318 \\
7.000 \\
6.549 \\
7.566\end{array}$ & $\begin{array}{l}52 . \\
80 .\end{array}$ & $\begin{array}{l}7.213 \\
7.114 \\
6.300 \\
5.311 \\
5.958\end{array}$ & $\begin{array}{l}626 \\
.282 \\
440 \\
829 \\
.157\end{array}$ & $\begin{array}{l}.225 \\
.136 \\
.300 \\
.327 \\
.965\end{array}$ \\
\hline & & & & $\begin{array}{l}.4 \\
.3 \\
.2\end{array}$ & $\begin{array}{c}18.003 \\
21.084 \\
-\end{array}$ & $\begin{array}{l}151 \\
.212 \\
-\end{array}$ & $\begin{array}{l}286 \\
.381 \\
-\end{array}$ & $\begin{array}{l}155 \\
212 \\
-\end{array}$ & 5. & $\begin{array}{l}4.713 \\
4.646 \\
-\end{array}$ & $\begin{array}{l}.419 \\
.555 \\
-\end{array}$ & $\begin{array}{l}.763 \\
.712 \\
-\end{array}$ \\
\hline $\begin{array}{l}0 \\
2 \\
5 \\
6 \\
6 \\
6\end{array}$ & 0 & $\begin{array}{l}d \\
d \\
0 \\
0 \\
0 \\
0 \\
0 \\
0 \\
0 \\
0 \\
0 \\
0 \\
0 \\
0 \\
0 \\
0 \\
0 \\
0 \\
0 \\
0 \\
0 \\
0 \\
0 \\
0\end{array}$ & & $\begin{array}{l}2 \\
9 \\
7\end{array}$ & $\begin{array}{r}86.188 \\
60.021 \\
90.681 \\
230.811 \\
75.777 \\
88.686 \\
228.170 \\
75.219 \\
26.614 \\
34.808 \\
37.451 \\
40.274 \\
36.297 \\
42.692 \\
85.841 \\
42.453 \\
- \\
40.940 \\
58.921 \\
65.448 \\
16.677 \\
46.860 \\
52.170 \\
67.830 \\
18.336 \\
19.035\end{array}$ & $\begin{array}{l}9.360 \\
7.722 \\
8.199 \\
8.266 \\
7.258 \\
8.381 \\
9.397 \\
7.633 \\
5.765 \\
5.726 \\
5.691 \\
7.600 \\
8.371 \\
7.360 \\
7.577 \\
7.166 \\
- \\
5.910 \\
6.192 \\
6.614 \\
5.443 \\
6.366 \\
6.733 \\
6.166 \\
6.049 \\
5.811\end{array}$ & $\begin{array}{l}0.861 \\
1.113 \\
7.462 \\
2.746 \\
2.842 \\
2.744 \\
2.586 \\
0.530 \\
2.110 \\
2.431 \\
2.850 \\
1.547\end{array}$ & $\begin{array}{l}9.291 \\
7.722 \\
8.199 \\
8.266 \\
7.258 \\
8.381 \\
9.397 \\
7.633 \\
5.765 \\
5.721 \\
5.700 \\
7.594 \\
8.349 \\
7.321 \\
7.577 \\
7.166 \\
- \\
5.910 \\
6.192 \\
6.600 \\
5.459 \\
6.377 \\
6.746 \\
6.166 \\
6.089 \\
5.795\end{array}$ & $\begin{array}{l}0 \\
3 \\
7 \\
6 \\
6 \\
4 \\
3 \\
6\end{array}$ & $\begin{array}{l}7.111 \\
6.220 \\
4.883 \\
4.985 \\
5.001 \\
5.100 \\
5.424 \\
5.823 \\
5.072 \\
4.989 \\
5.317 \\
5.886 \\
6.896 \\
6.086 \\
5.450 \\
4.967 \\
- \\
4.790 \\
4.759 \\
4.747 \\
5.959 \\
4.658 \\
6.243 \\
5.005\end{array}$ & $\begin{array}{l}.023 \\
.390 \\
.511 \\
.511 \\
.041 \\
.311 \\
.588 \\
.025 \\
.343 \\
.715 \\
.085 \\
.265 \\
.267\end{array}$ & $\begin{array}{l}.154 \\
.220 \\
4.883 \\
.985 \\
.001 \\
.100 \\
.424 \\
.823 \\
.115 \\
.025 \\
.258 \\
.744 \\
.755 \\
5.109 \\
.450 \\
4.967 \\
- \\
4.790 \\
4.759 \\
4.856 \\
5.977 \\
4.777 \\
5.311 \\
5.005 \\
5.279 \\
5.252\end{array}$ \\
\hline $\begin{array}{l}12 \\
12 \\
70 \\
18 \\
24 \\
10 \\
06 \\
09\end{array}$ & 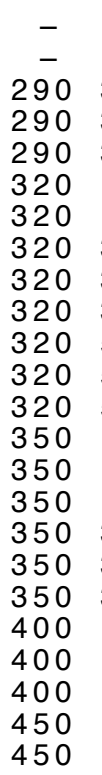 & $\begin{array}{r}\text { Unaged } \\
30000 \\
30000 \\
30000 \\
100000 \\
10000 \\
30000 \\
30000 \\
300000 \\
500000 \\
50000 \\
50000 \\
25700 \\
10000 \\
10000 \\
30000 \\
30000 \\
30000 \\
2570 \\
10000 \\
10000 \\
2570 \\
2570\end{array}$ & $\begin{array}{l}9 \\
.9 \\
5 \\
5 \\
.0 \\
.0 \\
0 \\
0 \\
.0 \\
.0 \\
8 \\
.9 \\
.9 \\
1 \\
1 \\
.1 \\
.2 \\
6\end{array}$ & $\begin{array}{l}4 \\
2 \\
0 \\
2 \\
2 \\
7\end{array}$ & $\begin{array}{l}17.526 \\
24.102 \\
17.324 \\
10.689 \\
15.317 \\
10.515 \\
13.264\end{array}$ & $\begin{array}{l}4 \\
4 \\
8 \\
8 \\
6 \\
4 \\
5 \\
1 \\
4 \\
5\end{array}$ & $\begin{array}{l}0.77 \\
0.99 \\
1.01 \\
2.43 \\
1.61 \\
1.03 \\
1.55 \\
1.35 \\
0.67 \\
0.85 \\
-\end{array}$ & $\begin{array}{l}5.072 \\
4.891 \\
6.207 \\
5.189 \\
5.415 \\
5.045 \\
4.891\end{array}$ & $\begin{array}{l}14.2 \\
11.4 \\
12.9\end{array}$ & $\begin{array}{l}4.645 \\
4.573 \\
5.555 \\
5.073 \\
5.113 \\
5.293 \\
4.829\end{array}$ & $\begin{array}{l}1.604 \\
1.739 \\
0.624 \\
0.694 \\
0.841 \\
0.798 \\
0.789\end{array}$ & $\begin{array}{l}5.024 \\
5.097 \\
5.716 \\
5.744 \\
5.525 \\
6.021 \\
5.098 \\
- \\
5.711 \\
4.645 \\
4.573 \\
5.601 \\
5.033\end{array}$ \\
\hline
\end{tabular}



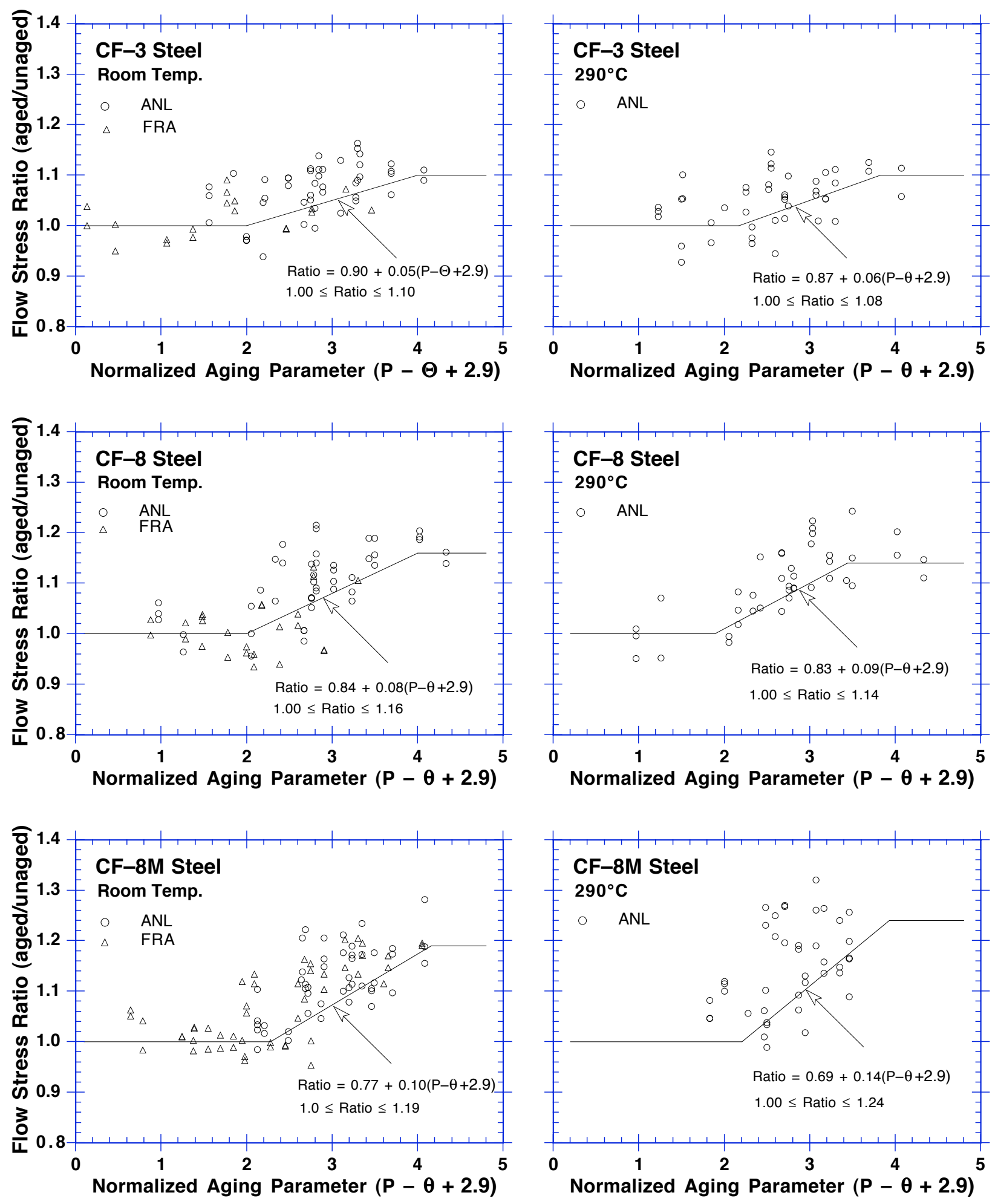

Figure 21. Flow stress ratio of aged cast stainless steels at $R T$ and $290^{\circ} \mathrm{C}$ as a function of the normalized aging parameter. The solid lines represent correlations obtained by subtracting $\sigma$ from the best fit curve. 
Table 6. Values of constants in Eq. 8 for estimating tensile flow stress of aged cast stainless steels

\begin{tabular}{|c|c|c|c|c|c|c|}
\hline \multirow[b]{2}{*}{ Grade } & \multicolumn{3}{|c|}{ Room Temp. } & \multicolumn{3}{|c|}{$290^{\circ} \mathrm{C}$} \\
\hline & $a_{1}$ & $b_{1}$ & $c_{1}$ & $a_{1}$ & $b_{1}$ & $c_{1}$ \\
\hline CF-3 & 0.90 & 0.05 & 1.10 & 0.87 & 0.06 & 1.08 \\
\hline CF-8 & 0.84 & 0.08 & 1.16 & 0.83 & 0.09 & 1.14 \\
\hline CF-8M & 0.77 & 0.10 & 1.19 & 0.69 & 0.14 & 1.24 \\
\hline
\end{tabular}

between 280 and $330^{\circ} \mathrm{C}\left(536\right.$ and $626^{\circ} \mathrm{F}$ ), and ferrite content $>7 \%$ for $\mathrm{CF}-8 \mathrm{M}$ steel and $>10 \%$ for CF-3 and CF-8 steels. Thermal aging has little or no effect on the tensile strength of cast stainless steels with low ferrite content. The available data base is inadequate for estimating the tensile properties at service temperatures $<280^{\circ} \mathrm{C}\left(<536^{\circ} \mathrm{F}\right)$.

The flow stress for a specific composition of cast stainless steel, as a function of time and temperature of aging, is estimated from Eqs. 8 and 1. The activation energy $Q$ in Eq. 1 is determined from the composition of the steel6,7 expressed as

$$
Q=10\left[74.52-7.20 \theta-3.46 S i-1.78 C r-4.35 I_{1} M n+\left(148-125 I_{1}\right) N-61 I_{2} C\right] \text {, }
$$

where the indicators $I_{1} I_{2}$ are 0 and 1 , respectively, for CF-3 or CF-8 steels and assume the values of 1 and 0 , respectively, for CF-8M steels, and $\theta$ is a constant. Parametric studies show that the aging response at reactor temperatures is relatively insensitive to the values of $\theta .5,6$ A $\theta$ value of 2.9 (i.e., mean value of the experimental data) is used to estimate thermal aging effects at $280-330^{\circ} \mathrm{C}\left(536-626^{\circ} \mathrm{F}\right)$, a value of 3.3 is used at temperatures $<280^{\circ} \mathrm{C}\left(<536^{\circ} \mathrm{F}\right)$, and 2.5 at $330-360^{\circ} \mathrm{C}\left(626-680^{\circ} \mathrm{F}\right)$. Tensile flow stress of aged material can be determined from $R_{f}$ and the initial tensile flow stress. The minimum value of 1 and maximum value of $c_{1}$ for the tensile-flow-stress ratio $R_{f}$ is assumed, respectively, when the ratio calculated from Eq. 8 is $<1$ or $>\mathrm{c}_{1}$.

Experimental and estimated tensile flow stress at $290^{\circ} \mathrm{C}\left(554^{\circ} \mathrm{F}\right)$ and at $\mathrm{RT}$ for various heats of aged cast stainless steel are shown in Fig. 22. For each heat, the aging parameter and activation energy were obtained from Eqs. 1 and 9 and a $\theta$ value of 2.9. Tensile flow stress was then estimated from Eq. 8 and the initial flow stress of the materials. The estimated values are either accurate or conservative for all materials and aging conditions.

The data on tensile properties of cast stainless steels indicate that the increase in yield stress due to thermal aging is much lower than the increase in ultimate stress. The tensile-yield-stress ratio $R_{y}=\left(\sigma_{\text {yaged }} / \sigma_{\text {yunaged }}\right)$, is given by

$$
R_{y}=a_{2}+b_{2} P \quad\left(1.00 \leq R_{y} \leq c_{2}\right) .
$$

Values of the constants $a_{2}, b_{2}$, and $c_{2}$ for different grades of steel and test temperatures are given in Table 7. Equation 10 is valid for $R_{y}$ values between 1 and $c_{2}$, service temperatures between 280 and $330^{\circ} \mathrm{C}\left(536\right.$ and $626^{\circ} \mathrm{F}$ ), and ferrite content $>7 \%$ for $\mathrm{CF}-8 \mathrm{M}$ steel and $>10 \%$ for CF-3 and CF-8 steels. 

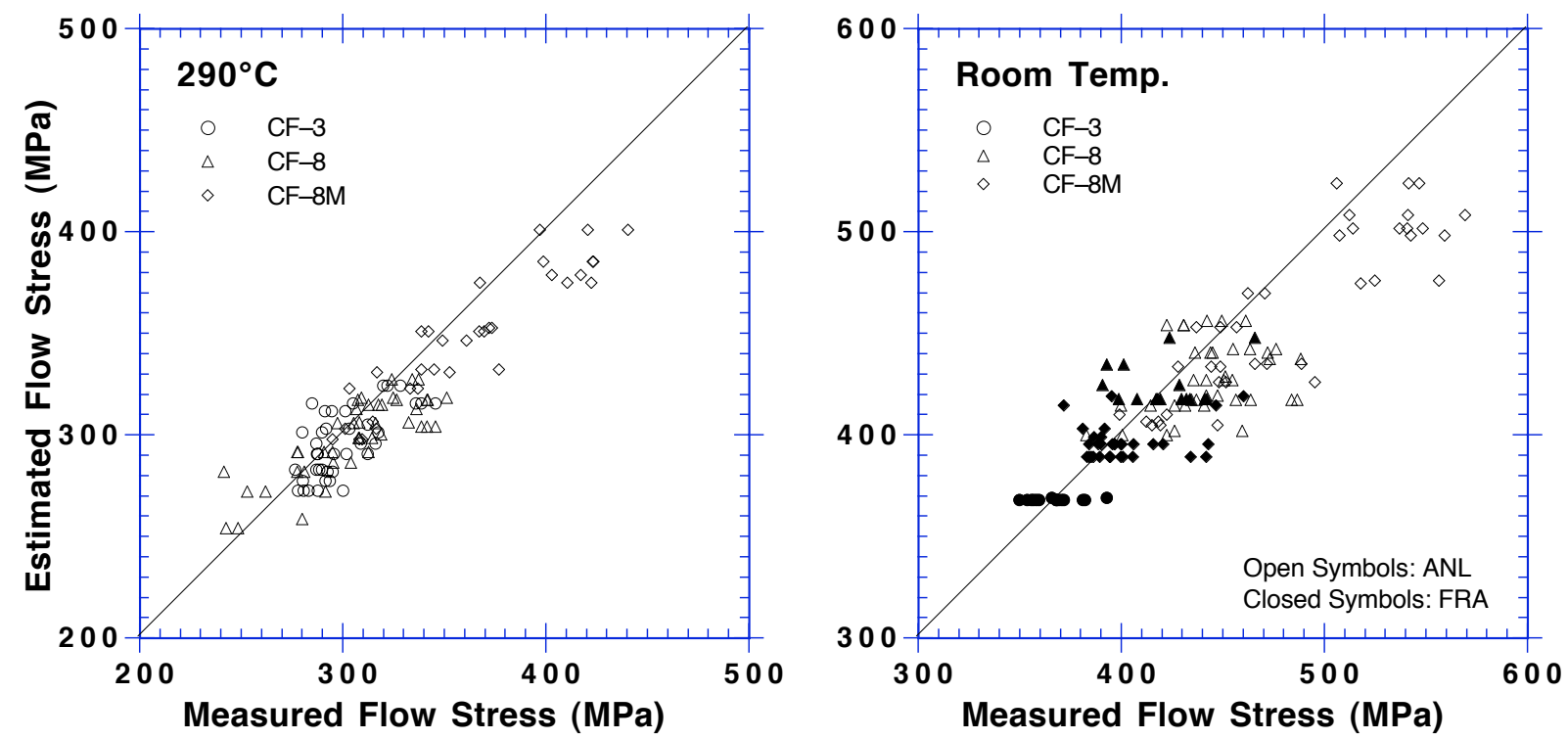

Figure 22. Experimental and estimated flow stress of aged cast stainless steel at $290^{\circ} \mathrm{C}$ and $R T$

Table 7. Values of constants in Eq. 10 for estimating tensile yield stress of aged cast stainless steels

\begin{tabular}{lcccccc}
\hline & \multicolumn{3}{c}{ Room Temp. } & & \multicolumn{3}{c}{$290^{\circ} \mathrm{C}$} \\
\cline { 2 - 3 } \cline { 6 - 7 } Grade & $\mathrm{a}_{2}$ & $\mathrm{~b}_{2}$ & $\mathrm{c}_{2}$ & $\mathrm{a}_{2}$ & $\mathrm{~b}_{2}$ & $\mathrm{c}_{2}$ \\
\hline & & & & & & \\
CF-3 & 0.873 & 0.048 & 1.07 & 0.844 & 0.058 & 1.05 \\
CF-8 & 0.798 & 0.076 & 1.10 & 0.788 & 0.086 & 1.09 \\
CF-8M & 0.708 & 0.092 & 1.10 & 0.635 & 0.129 & 1.14 \\
\hline
\end{tabular}

Experimental and estimated tensile yield stress at $290^{\circ} \mathrm{C}\left(554^{\circ} \mathrm{F}\right)$ and at $\mathrm{RT}$ for various heats of aged cast stainless steel are shown in Fig. 23. The aging parameter and activation energy were obtained from Eqs. 1 and 9 and a $\theta$ value of 2.9. Tensile yield stress was then estimated from Eq. 10 and the initial yield stress of the materials. The estimated values are conservative for most materials and aging conditions.

\subsection{Ramberg-Osgood Parameters}

The engineering stress vs. strain behavior of aged cast stainless steel can also be obtained from the estimated flow stress. For all grades of cast stainless steel, the RO parameter $n_{1}$ does not change with thermal aging. The parameter $\alpha_{1}$ decreases with aging and shows good correlation with the flow stress $\sigma_{f}$ of the material, Fig. 24. The RO parameter is correlated to flow stress $\sigma_{f}$ by the expression

$$
\alpha_{1}=a_{3}+b_{3} \sigma_{f} .
$$

The RO parameter $n_{1}$ and values of the constants $a_{3}$ and $b_{3}$ for different grades of steel, test temperatures, and range of engineering strain are given in Table 8. Examples of engineering 

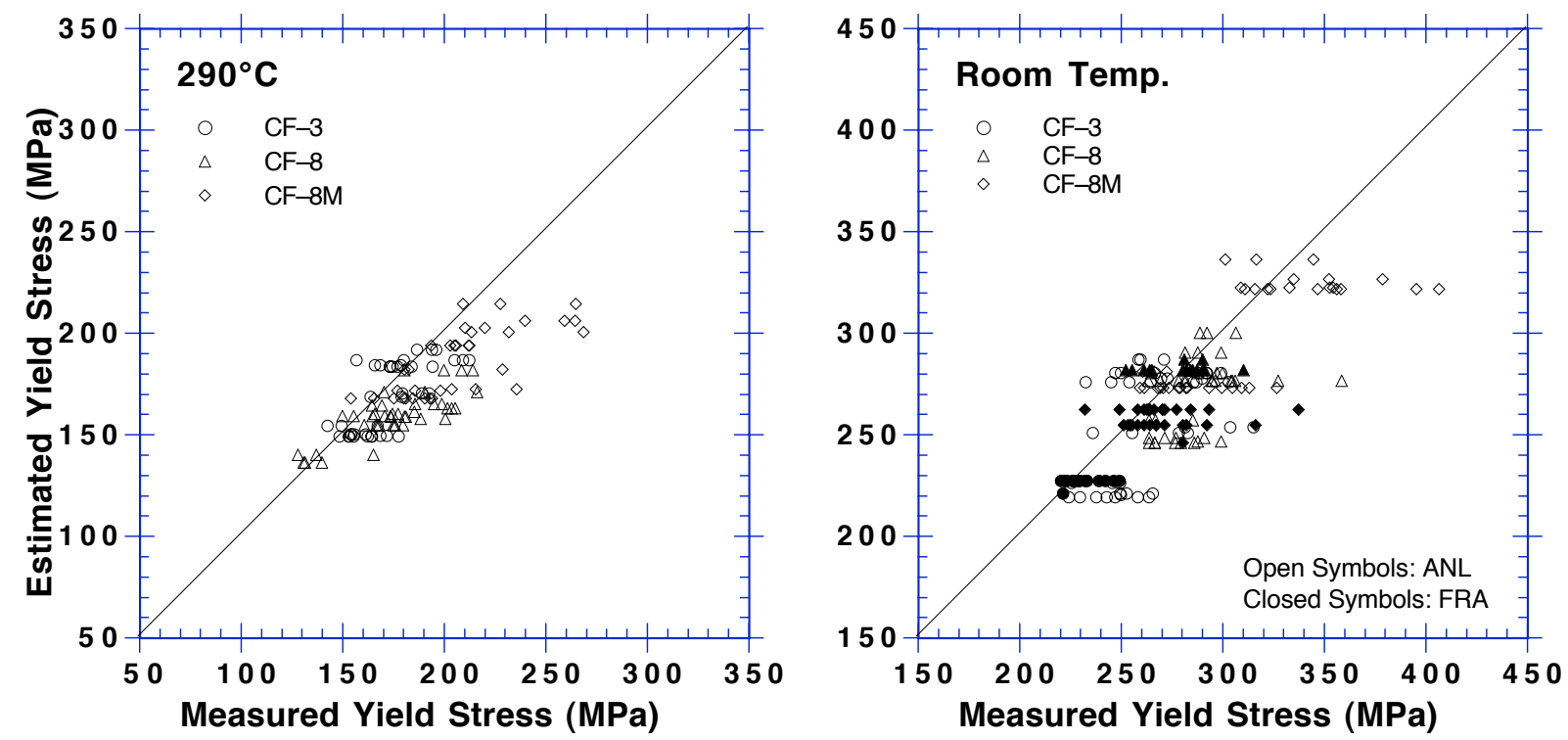

Figure 23. Experimental and estimated yield stress of aged cast stainless steel at $290^{\circ} \mathrm{C}$ and $R T$

Table 8. Values of Ramberg/Osgood parameter $n_{1}$ and the constants in Eq. 11 for estimating parameter $\alpha_{1}$ for aged cast stainless steels

\begin{tabular}{|c|c|c|c|c|c|c|c|}
\hline \multirow{2}{*}{$\begin{array}{c}\text { Strain } \\
\%\end{array}$} & \multirow[b]{2}{*}{ Grade } & \multicolumn{3}{|c|}{ Room Temp. } & \multicolumn{3}{|c|}{$290^{\circ} \mathrm{C}$} \\
\hline & & $a_{3}$ & $b_{3}$ & $\mathrm{n}_{1}$ & $a_{3}$ & $b_{3}$ & $\mathrm{n}_{1}$ \\
\hline \multirow[t]{3}{*}{$<5$} & $\mathrm{CF}-3$ & 143.9 & -0.267 & 6.1 & 102.1 & -0.235 & 6.2 \\
\hline & CF-8 & 157.9 & -0.300 & 6.4 & 153.3 & -0.373 & 7.1 \\
\hline & CF-8M & 50.9 & -0.072 & 5.6 & 145.9 & -0.314 & 6.6 \\
\hline \multirow{3}{*}{$<15$} & CF-3 & 104.5 & -0.176 & 6.0 & 91.3 & -0.221 & 5.3 \\
\hline & CF-8 & 149.0 & -0.271 & 6.9 & 137.5 & -0.336 & 5.9 \\
\hline & CF-8M & 65.7 & -0.097 & 6.1 & 103.3 & -0.217 & 5.4 \\
\hline \multirow[t]{3}{*}{$>15$} & CF-3 & 76.2 & -0.116 & 6.0 & 95.5 & -0.233 & 5.1 \\
\hline & CF-8 & 122.0 & -0.216 & 6.2 & 124.2 & -0.303 & 5.5 \\
\hline & CF-8M & 44.6 & -0.056 & 5.8 & 81.4 & -0.162 & 5.4 \\
\hline
\end{tabular}

stress-vs.-strain curves determined from estimated flow stress and Eq. 11 and those observed experimentally for several heats of aged CF-3, CF-8, and CF-8M steels, are shown in Fig. 25. The estimated curves are essentially conservative.

If required, the engineering stress-vs.-strain curve can also be obtained from Eq. 6, i.e., when yield stress is used as the reference stress. The RO parameter $\alpha_{2}$ is determined from estimated values of $\alpha_{1}, \sigma_{\mathrm{f}}, \sigma_{\mathrm{y}}$, and Eq. 7. The parameter $\mathrm{n}_{2}$ is same as $n_{1}$. 

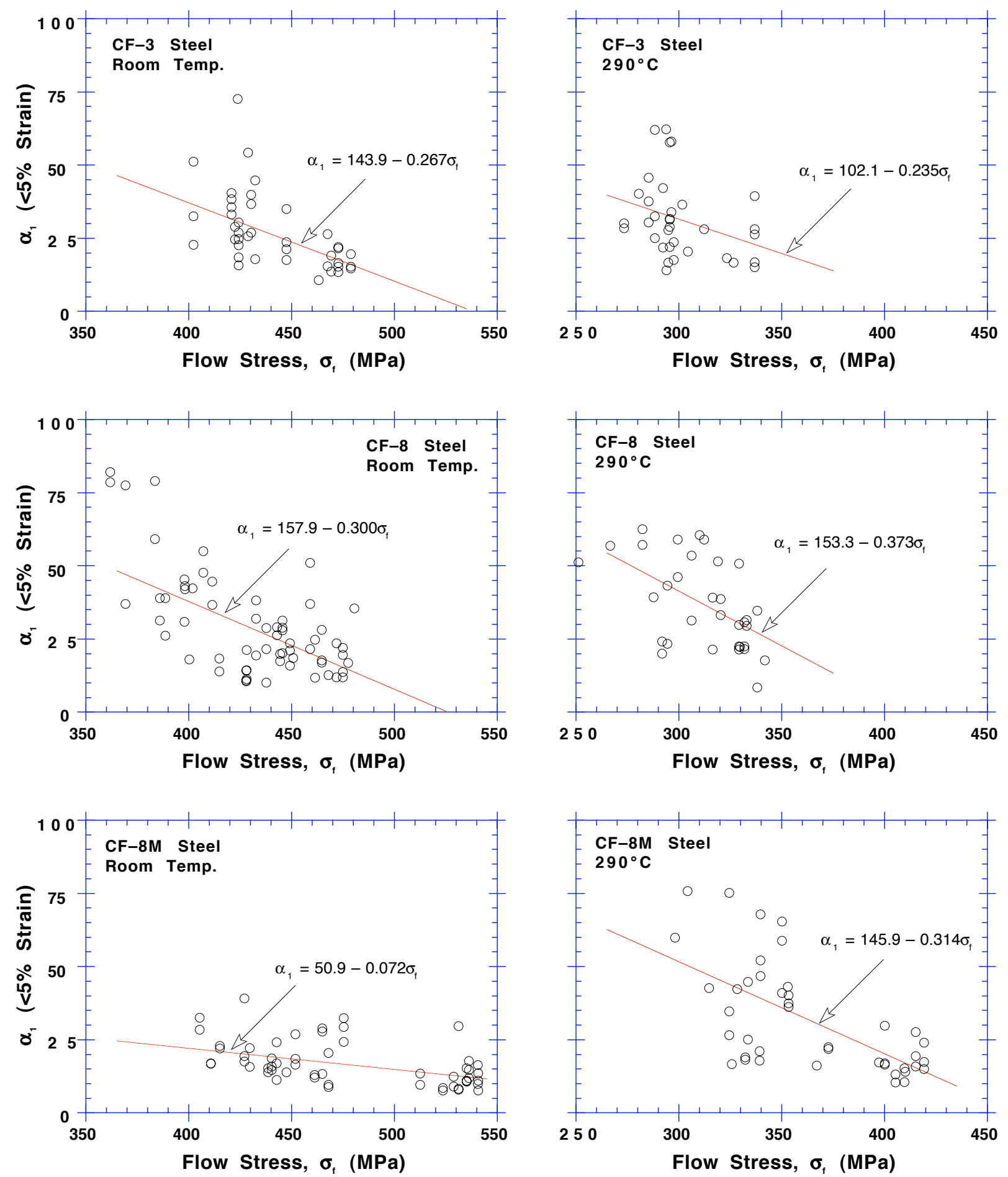

Figure 24. Correlation between the Ramberg/Osgood parameter $\alpha_{1}$ and flow stress at RT and $290^{\circ} \mathrm{C}$ for cast stainless steels 

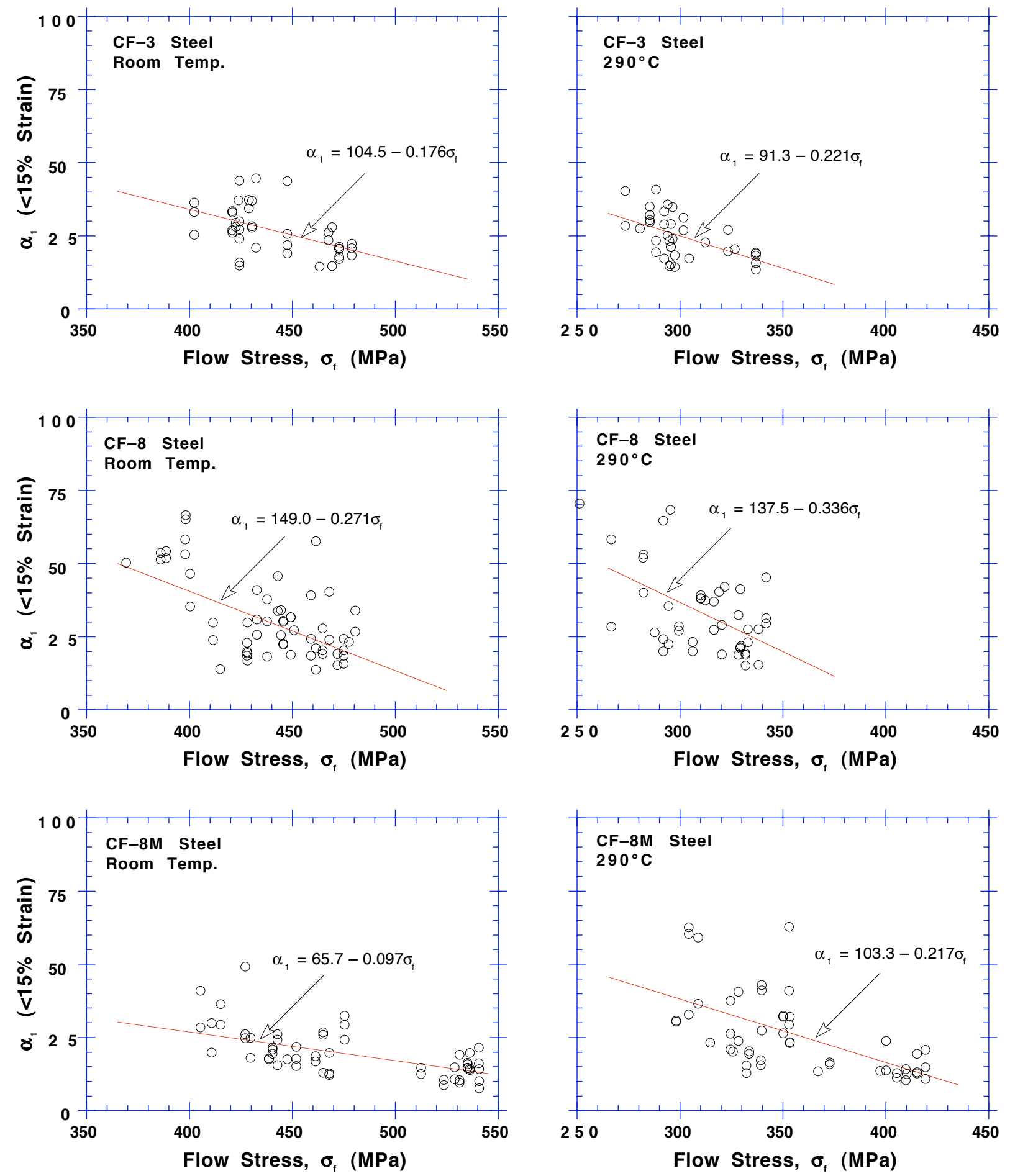

Figure 24. (Contd.) 

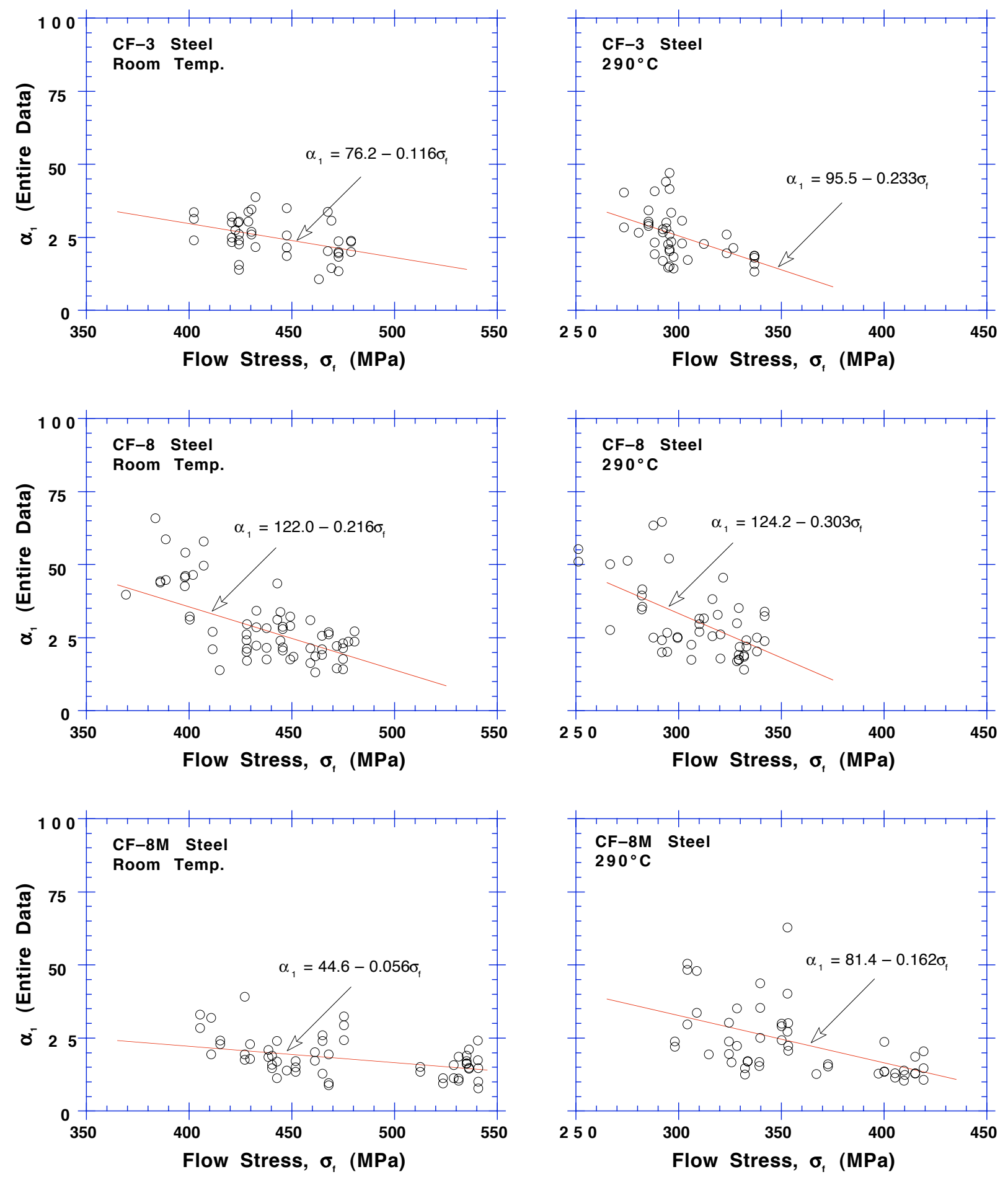

Figure 24. (Contd.) 

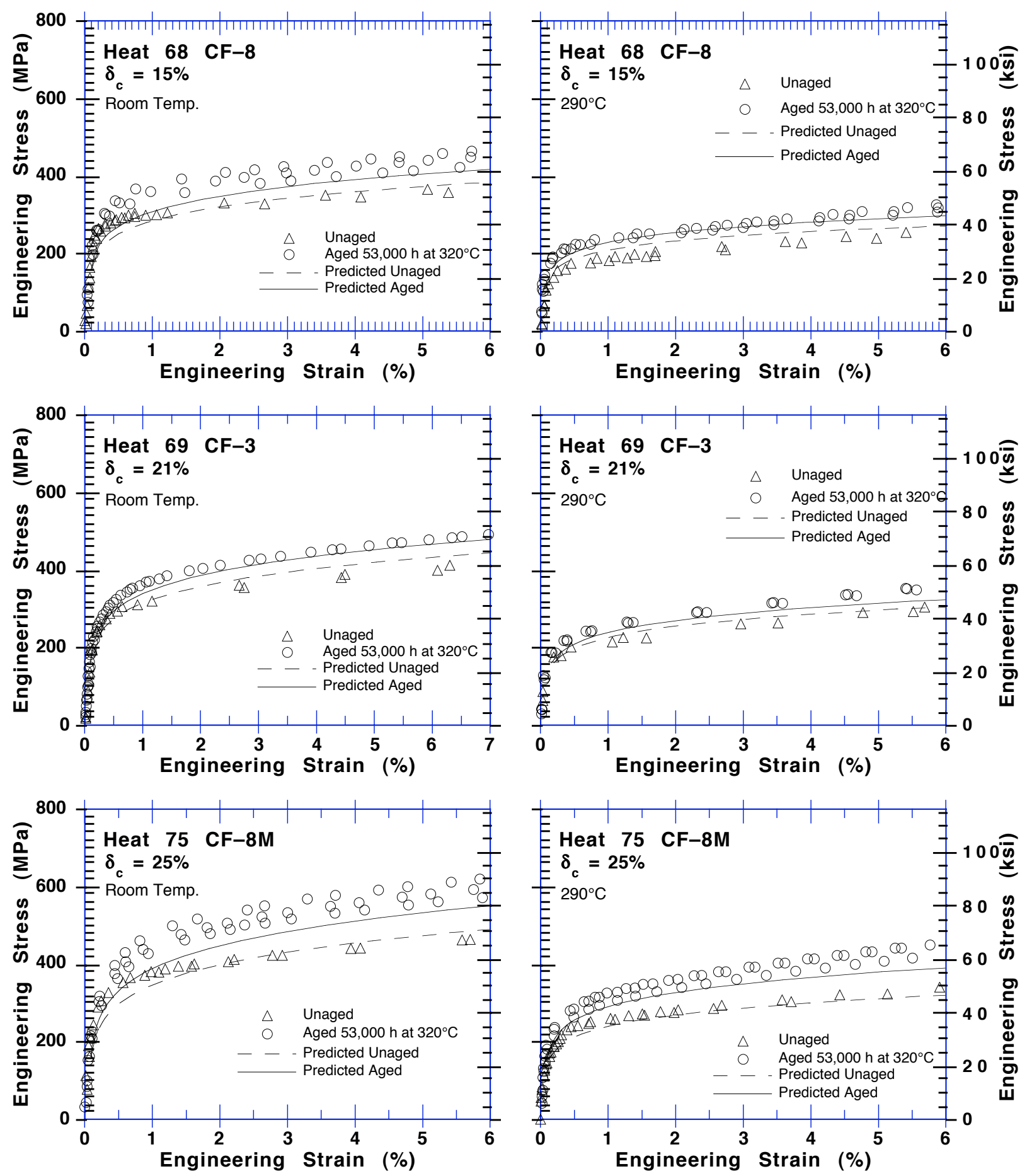

Figure 25. Engineering stress-vs.-strain curve at RT and $290^{\circ} \mathrm{C}$, estimated from the chemical composition and initial tensile strength and determined experimentally for thermally aged $\mathrm{CF}-3, \mathrm{CF}-8$, and $\mathrm{CF}-8 \mathrm{M}$ steels 

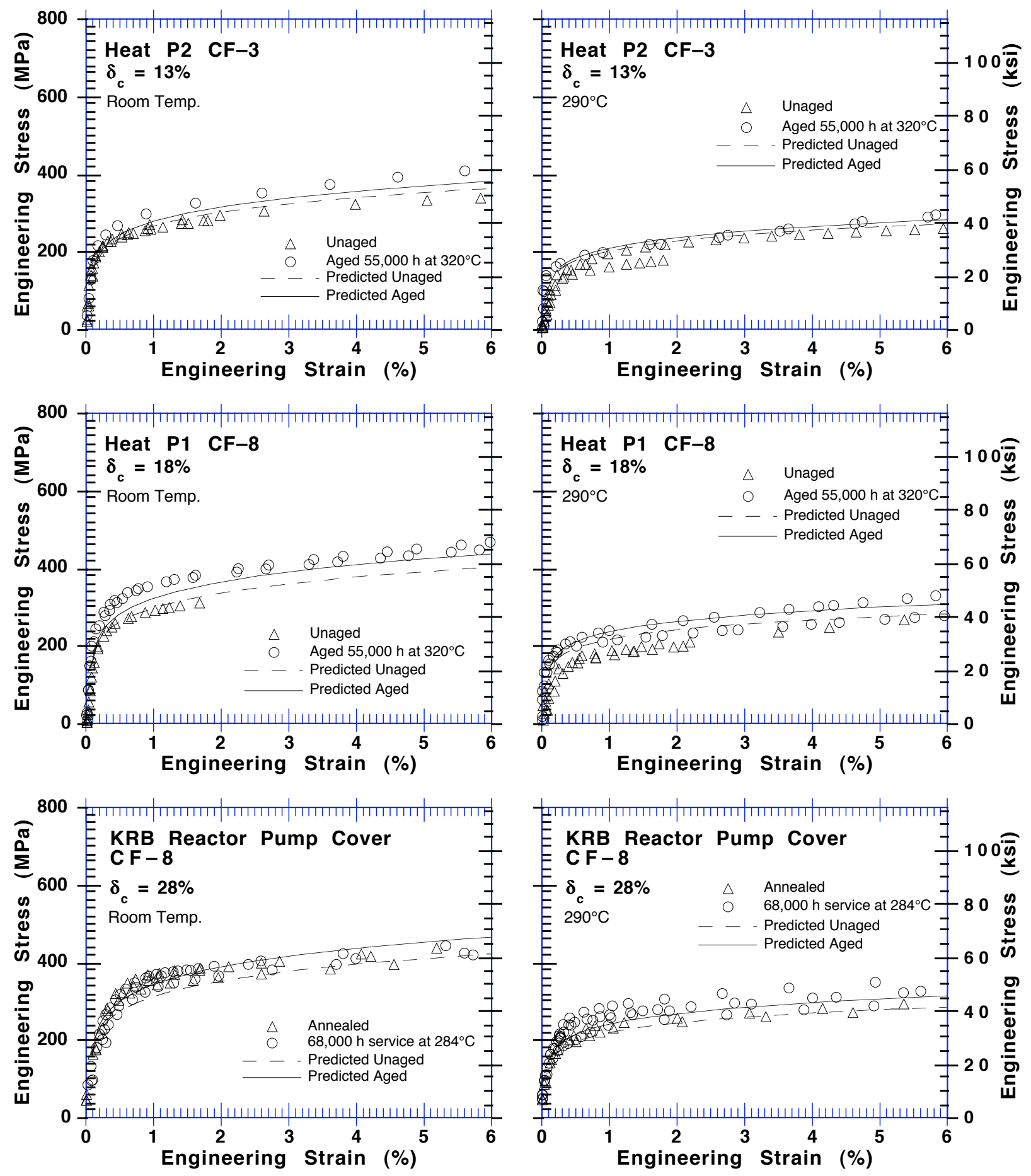

Figure 25. (Contd.) 


\section{Conclusions}

The effect of thermal aging on tensile properties of cast stainless steels has been evaluated. Tensile data for several experimental and commercial heats of cast stainless steels are presented. The materials were thermally aged up to $58,000 \mathrm{~h}$ at temperatures between 290 and $450^{\circ} \mathrm{C}\left(554\right.$ and $\left.842^{\circ} \mathrm{F}\right)$. Aging at these temperatures increases the tensile strength of these steels. The high-C Mo-bearing CF-8M steels are the most and the low-C Mo-free CF-3 steels least susceptible to thermal aging.

A procedure and correlations are presented for predicting the change in tensile flow and yield stress and engineering stress-vs.-strain curve (represented by the Ramberg/Osgood equation) of cast stainless steel components that is due to thermal aging during service in LWRs at $280-330^{\circ} \mathrm{C}\left(535-625^{\circ} \mathrm{C}\right)$. The tensile properties of aged cast stainless steel are estimated from information that is readily available from certified material test records for the component, i.e., chemical composition and the initial tensile strength of unaged material. The correlations described in this report may be used to assess thermal embrittlement of cast stainless steel components. The estimated tensile properties may be used as input to a structural analysis code, such as leak-before-break analysis of nuclear power plant piping, or for performing fitness-for-service evaluations of safety-related components in support of plant life extension and license renewal.

\section{Acknowledgments}

This work was supported by the Office of the Nuclear Regulatory Research in the U.S. Nuclear Regulatory Commission. The author are grateful to T. M. Galvin, A. Sather, and W. F. Burke for their contributions to the experimental effort. The authors also thank W. J. Shack and T. F. Kassner for their helpful discussions. This work was sponsored by the Office of Nuclear Regulatory Research, U.S. Nuclear Regulatory Commission, under FIN A2243; Project Manager: Dr. J. Muscara.

\section{References}

1. O. K. Chopra and H. M. Chung, "Aging Degradation of Cast Stainless Steels: Effects on Mechanical Properties," in Environmental Degradation of Materials in Nuclear Power Systems-Water Reactors, G. J. Theus and J. R. Weeks, eds., The Metallurgical Society, Warrendale, PA, pp. 737-748 (1988).

2. O. K. Chopra and H. M. Chung, "Effect of Low-Temperature Aging on the Mechanical Properties of Cast Stainless Steels," in Properties of Stainless Stee/s in Elevated-Temperature Service, M. Prager, ed., MPC Vol. 26, PVP Vol. 132, ASME, New York, pp. 79-105 (1988).

3. O. K. Chopra, "Thermal Aging of Cast Stainless Steels: Mechanisms and Predictions," in Fatigue, Degradation, and Fracture - 1990, W. H. Bamford, C. Becht, S. Bhandari, J. D. Gilman, L. A. James, and M. Prager, eds., MPC Vol. 30, PVP Vol. 195, ASME, New York, pp. 193-214 (1990). 
4. O. K. Chopra and A. Sather, Initial Assessment of the Mechanisms and Significance of Low-Temperature Embrittlement of Cast Stainless Steels in LWR Systems, NUREG/CR-5385, ANL-89/17 (Aug. 1990).

5. O. K. Chopra, Estimation of Fracture Toughness of Cast Stainless Steels during Thermal Aging in LWR Systems, NUREG/CR-4513, ANL-90/42 (June 1991).

6. O. K. Chopra, "Thermal Aging of Cast Stainless Steels in LWR Systems: Estimation of Mechanical Properties," in Nuclear Plant Systems/Components Aging Management and Life Extension, I. T. Kisisel, J. Sinnappan, R. W. Carlson, and W. H. Lake, eds., PVP Vol. 228, ASME, New York, pp. 79-92 (1992).

7. O. K. Chopra, "Prediction of Aging Degradation of Cast Stainless Steel Components in LWR Systems," in Proceedings of the Aging Research Information Conference, NUREG/CP-0122, Vol. 2, pp. 324-340 (1992).

8. H. M. Chung and O. K. Chopra, "Kinetics and Mechanism of Thermal Aging Embrittlement of Duplex Stainless Steels," in Environmental Degradation of Materials in Nuclear Power Systems-Water Reactors, G. J. Theus and J. R. Weeks, eds., The Metallurgical Society, Warrendale, PA, pp. 359-370 (1988).

9. H. M. Chung and O. K. Chopra, "Long-Term Aging Embrittlement of Cast Austenitic Stainless Steels - Mechanism and Kinetics," in Properties of Stainless Steels in Elevated-Temperature Service, M. Prager, ed., MPC Vol. 26, PVP Vol. 132, ASME, New York, pp. 17-34 (1988).

10. H. M. Chung and T. R. Leax, "Embrittlement of Laboratory- and Reactor-Aged CF3, CF8, and CF8M Duplex Stainless Steels," Mater. Sci. Technol., 6, 249-262 (1990).

11. A. Trautwein and W. Gysel, "Influence of Long Time Aging of CF-8 and CF-8M Cast Steel at Temperatures Between 300 and $500^{\circ} \mathrm{C}$ on the Impact Toughness and the Structure Properties," in Spectrum, Technische Mitteilungen aus dem+GF+Konzern, No. 5 (May 1981); also in Stainless Steel Castings, V. G. Behal and A. S. Melilli, eds., STP 756, ASTM, Philadelphia, PA, pp. 165-189 (1982).

12. E. I. Landerman and W. H. Bamford, "Fracture Toughness and Fatigue Characteristics of Centrifugally Cast Type 316 Stainless Steel Pipe after Simulated Thermal Service Conditions," in Ductility and Toughness Considerations in Elevated-Temperature Service, MPC 8, ASME, New York, pp. 99-127 (1978).

13. S. Bonnet, J. Bourgoin, J. Champredonde, D. Guttmann, and M. Guttmann, "Relationship between Evolution of Mechanical Properties of Various Cast Duplex Stainless Steels and Metallurgical and Aging Parameters: An Outline of Current EDF Programmes," Mater. Sci. Technol., 6, 221-229 (1990).

14. P. H. Pumphrey and K. N. Akhurst, "Aging Kinetics of CF3 Cast Stainless Steel in Temperature Range $300-400^{\circ} \mathrm{C}, "$ Mater. Sci. Technol., 6, 211-219 (1990).

15. G. Slama, P. Petrequin, and T. Mager, "Effect of Aging on Mechanical Properties of Austenitic Stainless Steel Castings and Welds," presented at SMIRT Post-Conference Seminar 6, Assuring Structural Integrity of Steel Reactor Pressure Boundary Components, Aug. 29-30, 1983, Monterey, CA. 
16. P. McConnell and J. W. Sheckherd, Fracture Toughness Characterization of Thermally Embrittled Cast Duplex Stainless Steel, Report NP-5439, Electric Power Research Institute, Palo Alto, CA (September 1987).

17. P. Auger, F. Danoix, A. Menand, S. Bonnet, J. Bourgoin, and M. Guttmann, "Atom Probe and Transmission Electron Microscopy Study of Aging of Cast Duplex Stainless Steels," Mater. Sci. Technol., 6, 301-313 (1990).

18. M. Vrinat, P. Cozar, and Y. Meyzaud, "Precipitated Phases in the Ferrite of Aged Cast Duplex Stainless Steels," Scripta Metall., 2 0, 1101-1106 (1986).

19. P. Joly, R. Cozar, and A. Pineau, "Effect of Crystallographic Orientation of Austenite on the Formation of Cleavage Cracks in Ferrite in an Aged Duplex Stainless Steel," Scripta Metall., 24 , 2235-2240 (1990).

20. J. M. Sassen, M. G. Hetherington, T. J. Godfrey, and G. D. W. Smith, "Kinetics of Spinodal Reaction in the Ferrite Phase of a Duplex Stainless Steel," in Properties of Stainless Steels in Elevated Temperature Service, M. Prager, ed., MPC Vol. 26, PVP Vol. 132, ASME, New York, pp. 65-78 (1988).

21. J. E. Brown, A. Cerezo, T. J. Godfrey, M. G. Hetherington, and G. D. W. Smith, "Quantitative Atom Probe Analysis of Spinodal Reaction in Ferrite Phase of Duplex Stainless Steel," Mater. Sci. Technol., 6, 293-300 (1990).

22. O. K. Chopra and L. Y. Bush, Long-Term Embrittlement of Cast Duplex Stainless Steels in LWR Systems: Semiannual Report October 1989-March 1990, NUREG/CR-4744, Vol. 5, No. 1, ANL-91/7 (July 1991).

23. A. W. Bowen and G. M. Leak, "Diffusion in BCC Iron Base Alloys," Metall. Trans., 1, 2767 (1970).

24. L. S. Aubrey, P. F. Wieser, W. J. Pollard, and E. A. Schoefer, "Ferrite Measurement and Control in Cast Duplex Stainless Steel," in Stainless Steel Castings, V. G. Behal and A. S. Melilli, eds., STP 756, ASTM, Philadelphia, PA, pp. 126-164 (1982).

25. A. L. Hiser, Compilation of Tensile and J-R Curve Data from Thermally-Aged Stainless Steel, MEA-2239, Vols. I-III, Materials Engineering Associates, Inc., Lanham, MD (October 1987).

26. A. L. Hiser, Tensile and $\mathcal{J}-R$ Curve Characterization of Thermally Aged Cast Stainless Steels, NUREG/CR-5024, MEA-2229, Materials Engineering Associates, Inc., Lanham, MD (September 1988).

27. O. K. Chopra,Long-Term Embrittlement of Cast Stainless Steels in LWR Systems: Semiannual Report October 1990-March 1991, NUREG/CR-4744, Vol. 6, No. 1, ANL-91/22 (August 1992).

28. W. L. Server, "Impact Three-Point Bend Testing for Notched and Precracked Specimens," J. Test. Eval. 6, 29 (1978). 


\section{Appendix}




\section{Introduction}

Tensile tests were conducted according to ASTM Specification E 8 and E 21 in an Instron tensile test machine with a maximum loading capacity of $90 \mathrm{kN}$ (20 kips). Cylindrical specimens with a diameter of $5.08 \mathrm{~mm}(0.2 \mathrm{in}$.) and a gauge length of $20.3 \mathrm{~mm}(0.8 \mathrm{in}$.) were used for all the tests. An axial extensometer, with an initial gauge length of $20.3 \mathrm{~mm}$ (0.8 in.), was used for continuous measurement of strain during room-temperature tests. An IBM computer was used to digitize load, crosshead movement, and axial displacement data, and to store the data on floppy disks. Analog traces of load-vs.-crosshead displacement and load-vs.-extensometer displacement were also obtained for each test.

The tests at $290^{\circ} \mathrm{C}\left(\approx 550^{\circ} \mathrm{F}\right)$ were conducted in a forced-air recirculating furnace. Thermocouples were mounted above and below the specimen gauge length to monitor and control the temperature within $\pm 2^{\circ} \mathrm{C}$. For the tests on samples aged for $<10,000 \mathrm{~h}$, an axial extensometer was not used for the elevated-temperature tests. Total strain in the specimen gauge length was determined from correlations developed from the room-temperature tests. The total elongation was determined from the crosshead displacement multiplied by 0.64 . Elevatedtemperature tests on the long-term-aged samples were conducted with a clip gauge mounted on the specimen grips. Total strain in the specimen gauge length was determined from correlations developed from room-temperature tests conducted with clip gauge attached to the specimen grips and the extensometer mounted on the specimen gauge length.

The engineering stress vs. strain data for ten commercial and experimental heats aged up to $58,000 \mathrm{~h}$ at temperatures between 290 and $450^{\circ} \mathrm{C}$ are presented in this appendix; results from the tests at Materials Engineering Associates (MEA) are also included. 
Table 1. Test data for specimen /1V-01

\begin{tabular}{|c|c|c|c|c|c|}
\hline $\begin{array}{l}\text { Test Number } \\
\text { Aging Temp. } \\
\text { Yield Stress }\end{array}$ & $\begin{array}{l}: M \\
: \cup 1 \\
: 26\end{array}$ & est & $\begin{array}{l}\text { Tes } \\
\text { Agi } \\
\text { Ulti }\end{array}$ & $\begin{array}{l}\text { np. } \\
\text { ne } \\
\text { Stress }\end{array}$ & $\begin{array}{l}25^{\circ} \mathrm{C} \\
- \\
598.8 \mathrm{MPa}\end{array}$ \\
\hline Engr. & & Engr. & & Engr. & \\
\hline $\begin{array}{l}\text { Stress } \\
\text { (MPa) }\end{array}$ & $\begin{array}{l}\text { Engr. } \\
\text { Strain }\end{array}$ & $\begin{array}{l}\text { Stress } \\
(\mathrm{MPa})\end{array}$ & $\begin{array}{l}\text { Engr. } \\
\text { Strain }\end{array}$ & $\begin{array}{l}\text { Stress } \\
(\mathrm{MPa})\end{array}$ & $\begin{array}{l}\text { Engr. } \\
\text { Strain }\end{array}$ \\
\hline 23.72 & 0.0001 & 298.27 & 0.0101 & 530.28 & 0.2536 \\
\hline 24.20 & 0.0001 & 304.13 & 0.0116 & 539.31 & 0.2763 \\
\hline 51.30 & 0.0002 & 306.47 & 0.0130 & 546.48 & 0.2990 \\
\hline 87.22 & 0.0002 & 312.06 & 0.0144 & 553.99 & 0.3222 \\
\hline 122.52 & 0.0002 & 314.68 & 0.0159 & 560.41 & 0.3454 \\
\hline 160.44 & 0.0002 & 353.15 & 0.0365 & 566.82 & 0.3691 \\
\hline 194.02 & 0.0003 & 384.38 & 0.0579 & 572.54 & 0.3930 \\
\hline 222.29 & 0.0007 & 410.44 & 0.0792 & 575.99 & 0.4172 \\
\hline 242.63 & 0.0015 & 433.82 & 0.1006 & 581.64 & 0.4418 \\
\hline 255.86 & 0.0024 & 452.09 & 0.1221 & 585.64 & 0.4668 \\
\hline 268.07 & 0.0035 & 469.46 & 0.1436 & 588.54 & 0.4922 \\
\hline 277.93 & 0.0047 & 485.12 & 0.1652 & 592.12 & 0.5182 \\
\hline 283.51 & 0.0060 & 497.25 & 0.1870 & 593.91 & 0.5451 \\
\hline 289.37 & 0.0073 & 509.52 & 0.2090 & 596.12 & 0.5730 \\
\hline 296.34 & 0.0088 & 520.76 & 0.2311 & 597.29 & 0.6027 \\
\hline
\end{tabular}

Table 2. Test data for specimen /1V-02

\begin{tabular}{|c|c|c|c|c|c|}
\hline $\begin{array}{l}\text { Test Number } \\
\text { Aging Temp. } \\
\text { Yield Stress }\end{array}$ & $\begin{array}{l}: M \\
: U \\
: 24\end{array}$ & & $\begin{array}{l}\text { Tes } \\
\text { Agi } \\
\text { Ulti }\end{array}$ & $\begin{array}{l}\text { np. } \\
\text { ne } \\
\text { Stress }\end{array}$ & $\begin{array}{l}25^{\circ} \mathrm{C} \\
- \\
583.4 \mathrm{MPa}\end{array}$ \\
\hline Engr. & & Engr. & & Engr. & \\
\hline $\begin{array}{l}\text { Stress } \\
(\mathrm{MPa})\end{array}$ & $\begin{array}{l}\text { Engr. } \\
\text { Strain }\end{array}$ & $\begin{array}{l}\text { Stress } \\
(\mathrm{MPa})\end{array}$ & $\begin{array}{l}\text { Engr. } \\
\text { Strain }\end{array}$ & $\begin{array}{l}\text { Stress } \\
(\mathrm{MPa})\end{array}$ & $\begin{array}{l}\text { Engr. } \\
\text { Strain }\end{array}$ \\
\hline 22.75 & 0.0000 & 302.20 & 0.0146 & 525.79 & 0.2703 \\
\hline 69.09 & 0.0004 & 305.44 & 0.0160 & 534.34 & 0.2921 \\
\hline 105.42 & 0.0006 & 309.44 & 0.0175 & 541.38 & 0.3142 \\
\hline 145.82 & 0.0009 & 311.44 & 0.0189 & 546.75 & 0.3362 \\
\hline 179.06 & 0.0013 & 349.01 & 0.0390 & 554.55 & 0.3589 \\
\hline 207.67 & 0.0019 & 377.56 & 0.0599 & 558.41 & 0.3816 \\
\hline 228.22 & 0.0027 & 403.48 & 0.0808 & 562.41 & 0.4049 \\
\hline 245.52 & 0.0037 & 423.48 & 0.1015 & 566.34 & 0.4286 \\
\hline 258.21 & 0.0049 & 442.02 & 0.1225 & 570.13 & 0.4526 \\
\hline 269.31 & 0.0062 & 459.12 & 0.1434 & 572.95 & 0.4771 \\
\hline 276.41 & 0.0075 & 472.84 & 0.1642 & 576.06 & 0.5021 \\
\hline 282.41 & 0.0088 & 486.22 & 0.1852 & 578.19 & 0.5278 \\
\hline 288.27 & 0.0102 & 496.63 & 0.2062 & 579.99 & 0.5544 \\
\hline 293.10 & 0.0117 & 507.73 & 0.2273 & 581.09 & 0.5820 \\
\hline 297.23 & 0.0131 & 517.59 & 0.2488 & 582.19 & 0.6116 \\
\hline
\end{tabular}


Table 3. Test data for specimen 12V-01

\begin{tabular}{|c|c|c|c|c|c|}
\hline $\begin{array}{l}\text { Test Number } \\
\text { Aging Temp. } \\
\text { Y }\end{array}$ & $\begin{array}{l}: \mathrm{Mt} \\
: \mathrm{Ur}\end{array}$ & $\begin{array}{l}\text { est } \\
\text { d } \\
\text { MPa }\end{array}$ & $\begin{array}{l}\text { Tes } \\
\text { Agir } \\
\text { Ultir }\end{array}$ & $\begin{array}{l}\text { n. } \\
\text { ne } \\
\text { Stress }\end{array}$ & $\begin{array}{l}25^{\circ} \mathrm{C} \\
- \\
579.9 \mathrm{MPa}\end{array}$ \\
\hline Engr. & & Engr. & & Engr. & \\
\hline $\begin{array}{l}\text { Stress } \\
(\mathrm{MPa})\end{array}$ & $\begin{array}{l}\text { Engr. } \\
\text { Strain }\end{array}$ & $\begin{array}{l}\text { Stress } \\
(\mathrm{MPa})\end{array}$ & $\begin{array}{l}\text { Engr. } \\
\text { Strain }\end{array}$ & $\begin{array}{l}\text { Stress } \\
(\mathrm{MPa})\end{array}$ & $\begin{array}{l}\text { Engr. } \\
\text { Strain }\end{array}$ \\
\hline 22.75 & 0.0001 & 303.92 & 0.0151 & 519.66 & 0.2644 \\
\hline 78.26 & 0.0004 & 307.99 & 0.0166 & 527.52 & 0.2855 \\
\hline 117.07 & 0.0006 & 309.92 & 0.0180 & 534.76 & 0.3068 \\
\hline 158.23 & 0.0009 & 313.50 & 0.0195 & 540.62 & 0.3283 \\
\hline 192.36 & 0.0012 & 347.91 & 0.0396 & 544.41 & 0.3501 \\
\hline 220.15 & 0.0018 & 375.21 & 0.0604 & 550.82 & 0.3723 \\
\hline 239.80 & 0.0027 & 399.00 & 0.0808 & 557.37 & 0.3946 \\
\hline 254.42 & 0.0037 & 419.13 & 0.1011 & 560.47 & 0.4175 \\
\hline 266.07 & 0.0050 & 436.85 & 0.1214 & 563.99 & 0.4406 \\
\hline 274.20 & 0.0063 & 452.50 & 0.1417 & 567.99 & 0.4643 \\
\hline 282.20 & 0.0077 & 466.84 & 0.1620 & 570.47 & 0.4882 \\
\hline 287.72 & 0.0091 & 479.88 & 0.1822 & 573.64 & 0.5129 \\
\hline 292.54 & 0.0106 & 490.63 & 0.2026 & 575.30 & 0.5384 \\
\hline 297.58 & 0.0121 & 502.01 & 0.2230 & 577.64 & 0.5650 \\
\hline 300.75 & 0.0136 & 510.69 & 0.2436 & 579.09 & 0.5934 \\
\hline
\end{tabular}

Table 4. Test data for specimen 12V-02

\begin{tabular}{|c|c|c|c|c|c|}
\hline $\begin{array}{l}\text { Test Number } \\
\text { Aging Temp. } \\
\text { Yield Stress }\end{array}$ & $\begin{array}{l}: M \\
: U \\
: 25\end{array}$ & & $\begin{array}{l}\text { Tes } \\
\text { Agi } \\
\text { Ulti }\end{array}$ & $\begin{array}{l}\text { np. } \\
\text { ne } \\
\text { Stress }\end{array}$ & $\begin{array}{l}25^{\circ} \mathrm{C} \\
- \\
578.1 \mathrm{MPa}\end{array}$ \\
\hline Engr. & & Engr. & & Engr. & \\
\hline $\begin{array}{l}\text { Stress } \\
(\mathrm{MPa})\end{array}$ & $\begin{array}{l}\text { Engr. } \\
\text { Strain }\end{array}$ & $\begin{array}{l}\text { Stress } \\
(\mathrm{MPa})\end{array}$ & $\begin{array}{l}\text { Engr. } \\
\text { Strain }\end{array}$ & $\begin{array}{l}\text { Stress } \\
(\mathrm{MPa})\end{array}$ & $\begin{array}{l}\text { Engr. } \\
\text { Strain }\end{array}$ \\
\hline 21.65 & 0.0001 & 307.71 & 0.0150 & 519.73 & 0.2586 \\
\hline 65.84 & 0.0004 & 311.30 & 0.0165 & 526.76 & 0.2789 \\
\hline 103.63 & 0.0006 & 313.71 & 0.0181 & 534.69 & 0.2992 \\
\hline 142.51 & 0.0008 & 316.88 & 0.0196 & 539.86 & 0.3199 \\
\hline 178.64 & 0.0010 & 350.12 & 0.0394 & 546.27 & 0.3409 \\
\hline 212.08 & 0.0015 & 377.14 & 0.0600 & 551.10 & 0.3620 \\
\hline 236.83 & 0.0022 & 401.48 & 0.0802 & 555.92 & 0.3835 \\
\hline 254.76 & 0.0033 & 421.27 & 0.1003 & 560.75 & 0.4054 \\
\hline 267.52 & 0.0044 & 438.02 & 0.1203 & 563.51 & 0.4276 \\
\hline 276.96 & 0.0058 & 452.99 & 0.1400 & 567.37 & 0.4502 \\
\hline 284.06 & 0.0073 & 465.40 & 0.1597 & 568.96 & 0.4732 \\
\hline 289.30 & 0.0088 & 481.53 & 0.1793 & 572.33 & 0.4969 \\
\hline 294.96 & 0.0104 & 492.35 & 0.1990 & 573.85 & 0.5212 \\
\hline 299.65 & 0.0119 & 501.11 & 0.2188 & 574.75 & 0.5463 \\
\hline 301.09 & 0.0134 & 511.66 & 0.2387 & 575.09 & 0.5735 \\
\hline
\end{tabular}


Table 5. Test data for specimen 13C-01

\begin{tabular}{|c|c|c|c|c|c|}
\hline $\begin{array}{l}\text { Test Number } \\
\text { Aging Temp. } \\
\text { Yield Stress }\end{array}$ & $\begin{array}{l}: M \\
: U 1 \\
: 23\end{array}$ & & $\begin{array}{l}\text { Tes } \\
\text { Agir } \\
\text { Ulti }\end{array}$ & $\begin{array}{l}\text { np. } \\
\text { ne } \\
\text { Stress }\end{array}$ & $\begin{array}{l}25^{\circ} \mathrm{C} \\
- \\
517.6 \mathrm{MPa}\end{array}$ \\
\hline Engr. & & Engr. & & Engr. & \\
\hline $\begin{array}{l}\text { Stress } \\
(\mathrm{MPa})\end{array}$ & $\begin{array}{l}\text { Engr. } \\
\text { Strain }\end{array}$ & $\begin{array}{l}\text { Stress } \\
(\mathrm{MPa})\end{array}$ & $\begin{array}{l}\text { Engr. } \\
\text { Strain }\end{array}$ & $\begin{array}{l}\text { Stress } \\
(\mathrm{MPa})\end{array}$ & $\begin{array}{l}\text { Engr. } \\
\text { Strain }\end{array}$ \\
\hline 21.79 & 0.0001 & 292.20 & 0.0149 & 462.57 & 0.2234 \\
\hline 70.46 & 0.0004 & 294.68 & 0.0164 & 468.64 & 0.2410 \\
\hline 109.97 & 0.0006 & 298.41 & 0.0180 & 475.60 & 0.2589 \\
\hline 148.44 & 0.0008 & 300.89 & 0.0195 & 480.22 & 0.2768 \\
\hline 182.71 & 0.0012 & 327.23 & 0.0356 & 486.56 & 0.2951 \\
\hline 209.53 & 0.0018 & 347.01 & 0.0525 & 491.73 & 0.3135 \\
\hline 229.11 & 0.0026 & 365.49 & 0.0692 & 495.87 & 0.3323 \\
\hline 244.42 & 0.0037 & 381.56 & 0.0860 & 499.39 & 0.3513 \\
\hline 254.97 & 0.0049 & 395.35 & 0.1028 & 503.52 & 0.3707 \\
\hline 262.35 & 0.0061 & 408.79 & 0.1197 & 506.49 & 0.3903 \\
\hline 268.90 & 0.0075 & 419.89 & 0.1368 & 509.11 & 0.4105 \\
\hline 274.14 & 0.0089 & 429.82 & 0.1540 & 511.94 & 0.4314 \\
\hline 279.24 & 0.0104 & 438.64 & 0.1711 & 514.21 & 0.4528 \\
\hline 283.65 & 0.0119 & 448.09 & 0.1884 & 516.35 & 0.4752 \\
\hline 288.13 & 0.0134 & 454.92 & 0.2058 & 516.49 & 0.4987 \\
\hline
\end{tabular}

Table 6. Test data for specimen $12 \mathrm{~V}-23$

\begin{tabular}{|c|c|c|c|c|c|}
\hline \multirow{2}{*}{$\begin{array}{l}\text { Test Number } \\
\text { Aging Temp. } \\
\text { Yield Stress } \\
\text { Engr. } \\
\text { Stress } \\
\text { (MPa) }\end{array}$} & \multicolumn{2}{|c|}{$\begin{array}{l}: 119 \\
: 320^{\circ} \mathrm{C} \\
: 235.8 \mathrm{MPa}\end{array}$} & \multicolumn{2}{|c|}{$\begin{array}{l}\text { Test Temp. } \\
\text { Aging Time } \\
\text { Ultimate Stress }\end{array}$} & $\begin{array}{l}25^{\circ} \mathrm{C} \\
30,000 \mathrm{~h} \\
637.3 \mathrm{MPa}\end{array}$ \\
\hline & \multicolumn{2}{|l|}{ Engr. } & \multicolumn{2}{|l|}{ Engr. } \\
\hline & $\begin{array}{l}\text { Engr. } \\
\text { Strain }\end{array}$ & $\begin{array}{l}\text { Stress } \\
\text { (MPa) }\end{array}$ & $\begin{array}{l}\text { Engr. } \\
\text { Strain }\end{array}$ & $\begin{array}{l}\text { Stress } \\
(\mathrm{MPa})\end{array}$ & $\begin{array}{l}\text { Engr. } \\
\text { Strain }\end{array}$ \\
\hline 0.00 & 0.00 & 384.59 & 0.0324 & 603.57 & 0.3157 \\
\hline 43.6040 & 0.00 & 386.62 & 0.0330 & 605.86 & 0.3371 \\
\hline 190.25 & 0.0015 & 436.18 & 0.0642 & 611.71 & 0.3616 \\
\hline 274.48 & 0.0042 & 457.78 & 0.0815 & 615.35 & 0.3771 \\
\hline 296.47 & 0.0056 & 479.29 & 0.0983 & 619.50 & 0.3984 \\
\hline 303.00 & 0.0063 & 486.13 & 0.1062 & 631.01 & 0.4890 \\
\hline 333.23 & 0.0116 & 534.59 & 0.1614 & 634.39 & 0.5164 \\
\hline 360.34 & 0.0208 & 574.76 & 0.2402 & 637.33 & 0.5749 \\
\hline
\end{tabular}


Table 7. Test data for specimen 13C-14

\begin{tabular}{|c|c|c|c|c|c|}
\hline $\begin{array}{l}\text { Test Number } \\
\text { Aging Temp. } \\
\text { Yield Stress }\end{array}$ & \multicolumn{2}{|c|}{$\begin{array}{l}: 121 \\
: 320^{\circ} \mathrm{C} \\
: 278.2 \mathrm{MPa}\end{array}$} & \multicolumn{2}{|c|}{$\begin{array}{l}\text { Test Temp. } \\
\text { Aging Time } \\
\text { Ultimate Stress }\end{array}$} & $\begin{array}{l}25^{\circ} \mathrm{C} \\
30,000 \mathrm{~h} \\
633.9 \mathrm{MPa}\end{array}$ \\
\hline \multirow{2}{*}{$\begin{array}{l}\text { Engr. } \\
\text { Stress } \\
\text { (MPa) }\end{array}$} & \multicolumn{2}{|r|}{ Engr. } & \multicolumn{3}{|c|}{ Engr. } \\
\hline & $\begin{array}{l}\text { Engr. } \\
\text { Strain }\end{array}$ & $\begin{array}{l}\text { Stress } \\
(\mathrm{MPa})\end{array}$ & $\begin{array}{l}\text { Engr. } \\
\text { Strain }\end{array}$ & $\begin{array}{l}\text { Stress } \\
(\mathrm{MPa})\end{array}$ & $\begin{array}{l}\text { Engr. } \\
\text { Strain }\end{array}$ \\
\hline 1.04 & 0.0000 & 430.45 & 0.0536 & 590.35 & 0.2479 \\
\hline 68.95 & 0.0005 & 451.69 & 0.0731 & 606.17 & 0.2892 \\
\hline 196.77 & 0.0028 & 474.35 & 0.0867 & 606.87 & 0.2891 \\
\hline 283.55 & 0.0054 & 482.80 & 0.0930 & 613.18 & 0.3155 \\
\hline 311.96 & 0.0082 & 492.80 & 0.0997 & 623.97 & 0.3807 \\
\hline 324.49 & 0.0099 & 526.96 & 0.1350 & 624.47 & 0.3806 \\
\hline 356.89 & 0.0172 & 562.69 & 0.1863 & 631.93 & 0.4557 \\
\hline 378.50 & 0.0253 & 564.45 & 0.1916 & 633.91 & 0.5043 \\
\hline 414.69 & 0.0429 & & & & \\
\hline
\end{tabular}

Table 8. Test data for specimen $13 \mathrm{~V}-38$

\begin{tabular}{|c|c|c|c|c|c|}
\hline $\begin{array}{l}\text { Test Number } \\
\text { Aging Temp. } \\
\text { Yield Stress }\end{array}$ & $\begin{array}{l}: 2 \\
: 32 \\
: 25\end{array}$ & & $\begin{array}{l}\text { Tes } \\
\text { Agi } \\
\text { Ulti }\end{array}$ & $\begin{array}{l}\text { np. } \\
\text { ne } \\
\text { Stress }\end{array}$ & $\begin{array}{l}25^{\circ} \mathrm{C} \\
30,000 \mathrm{~h} \\
609.6 \mathrm{MPa}\end{array}$ \\
\hline Engr. & & Engr. & & Engr. & \\
\hline $\begin{array}{l}\text { Stress } \\
(\mathrm{MPa})\end{array}$ & $\begin{array}{l}\text { Engr. } \\
\text { Strain }\end{array}$ & $\begin{array}{l}\text { Stress } \\
\text { (MPa) }\end{array}$ & $\begin{array}{l}\text { Engr. } \\
\text { Strain }\end{array}$ & $\begin{array}{l}\text { Stress } \\
(\mathrm{MPa})\end{array}$ & $\begin{array}{l}\text { Engr. } \\
\text { Strain }\end{array}$ \\
\hline 15.31 & 0.0001 & 343.18 & 0.0197 & 512.07 & 0.1530 \\
\hline 58.64 & 0.0005 & 358.13 & 0.0259 & 519.27 & 0.1633 \\
\hline 91.12 & 0.0008 & 370.99 & 0.0320 & 532.16 & 0.1839 \\
\hline 127.95 & 0.0010 & 382.74 & 0.0381 & 544.08 & 0.2045 \\
\hline 152.36 & 0.0013 & 393.50 & 0.0443 & 556.49 & 0.2295 \\
\hline 176.22 & 0.0015 & 403.34 & 0.0504 & 567.12 & 0.2547 \\
\hline 188.00 & 0.0017 & 412.73 & 0.0565 & 577.22 & 0.2819 \\
\hline 199.29 & 0.0019 & 421.62 & 0.0626 & 587.01 & 0.3138 \\
\hline 219.44 & 0.0024 & 429.86 & 0.0687 & 594.55 & 0.3440 \\
\hline 242.09 & 0.0032 & 441.64 & 0.0780 & 601.63 & 0.3814 \\
\hline 259.00 & 0.0043 & 451.14 & 0.0861 & 606.43 & 0.4150 \\
\hline 272.01 & 0.0053 & 460.27 & 0.0943 & 608.93 & 0.4501 \\
\hline 285.98 & 0.0067 & 468.83 & 0.1025 & 607.43 & 0.4928 \\
\hline 297.47 & 0.0082 & 476.91 & 0.1107 & 588.07 & 0.5213 \\
\hline 310.53 & 0.0105 & 487.33 & 0.1222 & 554.04 & 0.5400 \\
\hline 321.27 & 0.0129 & 496.22 & 0.1325 & 508.75 & 0.5549 \\
\hline 332.68 & 0.0161 & 504.19 & 0.1428 & 451.10 & 0.5656 \\
\hline
\end{tabular}


Table 9. Test data for specimen 13V-39

\begin{tabular}{|c|c|c|c|c|c|}
\hline $\begin{array}{l}\text { Test Number } \\
\text { Aging Temp. } \\
\text { Yield Stress }\end{array}$ & \multicolumn{2}{|c|}{$\begin{array}{l}: 120 \\
: 320^{\circ} \mathrm{C} \\
: 282.5 \mathrm{MPa}\end{array}$} & \multicolumn{2}{|c|}{$\begin{array}{l}\text { Test Temp. } \\
\text { Aging Time } \\
\text { Ultimate Stress }\end{array}$} & $\begin{array}{l}: 25^{\circ} \mathrm{C} \\
: 30,000 \mathrm{~h} \\
: 452.7 \mathrm{MPa}\end{array}$ \\
\hline Engr. & & Engr. & & Engr. & \\
\hline $\begin{array}{l}\text { Stress } \\
\text { (MPa) }\end{array}$ & $\begin{array}{l}\text { Engr. } \\
\text { Strain }\end{array}$ & $\begin{array}{l}\text { Stress } \\
\text { (MPa) }\end{array}$ & $\begin{array}{l}\text { Engr. } \\
\text { Strain }\end{array}$ & $\begin{array}{l}\text { Stress } \\
\text { (MPa) }\end{array}$ & $\begin{array}{l}\text { Engr. } \\
\text { Strain }\end{array}$ \\
\hline 0.44 & 0.0000 & 36462 & 0.0269 & & \\
\hline 58.55 & 0.0009 & 389.67 & 0.0413 & & \\
\hline 210.64 & 0.0036 & 409.75 & 0.0553 & & \\
\hline 276.76 & 0.0058 & 430.67 & 0.0701 & & \\
\hline 309.50 & 0.0089 & 443.50 & 0.0803 & & \\
\hline 323.52 & 0.0115 & 450.68 & 0.0874 & & \\
\hline 344.97 & 0.0180 & & & & \\
\hline
\end{tabular}

Table 10. Test data for specimen /1 V-26

\begin{tabular}{|c|c|c|c|c|c|}
\hline $\begin{array}{l}\text { Test Number } \\
\text { Aging Temp. } \\
\text { Yield Stress }\end{array}$ & $\begin{array}{l}: M \\
: 35 \\
: 28\end{array}$ & & $\begin{array}{l}\text { Tes } \\
\text { Agi } \\
\text { Ulti }\end{array}$ & $\begin{array}{l}\text { np. } \\
\text { ne } \\
\text { Stress }\end{array}$ & $\begin{array}{l}25^{\circ} \mathrm{C} \\
10,000 \mathrm{~h} \\
615.4 \mathrm{MPa}\end{array}$ \\
\hline Engr. & & Engr. & & Engr. & \\
\hline $\begin{array}{l}\text { Stress } \\
(\mathrm{MPa})\end{array}$ & $\begin{array}{l}\text { Engr. } \\
\text { Strain }\end{array}$ & $\begin{array}{l}\text { Stress } \\
(\mathrm{MPa})\end{array}$ & $\begin{array}{l}\text { Engr. } \\
\text { Strain }\end{array}$ & $\begin{array}{l}\text { Stress } \\
(\mathrm{MPa})\end{array}$ & $\begin{array}{l}\text { Engr. } \\
\text { Strain }\end{array}$ \\
\hline 22.34 & 0.0001 & 347.91 & 0.0144 & 557.72 & 0.2101 \\
\hline 31.65 & 0.0002 & 352.25 & 0.0159 & 565.99 & 0.2270 \\
\hline 64.74 & 0.0004 & 356.46 & 0.0174 & 573.44 & 0.2439 \\
\hline 103.42 & 0.0006 & 359.98 & 0.0188 & 579.44 & 0.2612 \\
\hline 143.27 & 0.0009 & 391.35 & 0.0337 & 585.30 & 0.2787 \\
\hline 184.43 & 0.0012 & 417.68 & 0.0495 & 590.05 & 0.2964 \\
\hline 222.36 & 0.0016 & 440.92 & 0.0652 & 594.95 & 0.3145 \\
\hline 252.42 & 0.0022 & 460.09 & 0.0809 & 598.67 & 0.3329 \\
\hline 274.20 & 0.0032 & 477.32 & 0.0968 & 603.08 & 0.3518 \\
\hline 290.48 & 0.0044 & 492.70 & 0.1126 & 605.91 & 0.3710 \\
\hline 303.23 & 0.0057 & 505.73 & 0.1286 & 609.43 & 0.3908 \\
\hline 314.47 & 0.0071 & 518.76 & 0.1447 & 611.56 & 0.4114 \\
\hline 323.09 & 0.0085 & 529.10 & 0.1609 & 612.74 & 0.4330 \\
\hline 330.53 & 0.0099 & 540.76 & 0.1771 & 613.22 & 0.4560 \\
\hline 336.46 & 0.0114 & 549.58 & 0.1936 & 613.22 & 0.4813 \\
\hline 341.98 & 0.0128 & & & & \\
\hline
\end{tabular}


Table 11. Test data for specimen $11 \mathrm{~V}-27$

\begin{tabular}{|c|c|c|c|c|c|}
\hline $\begin{array}{l}\text { Test Number } \\
\text { Aging Temp. } \\
\text { Yield Stress }\end{array}$ & $\begin{array}{l}: M \\
: 35 \\
: 30\end{array}$ & & $\begin{array}{l}\text { Tes } \\
\text { Agi } \\
\text { Ulti }\end{array}$ & $\begin{array}{l}\text { np. } \\
\text { ne } \\
\text { Stress }\end{array}$ & $\begin{array}{l}25^{\circ} \mathrm{C} \\
10,000 \mathrm{~h} \\
644.7 \mathrm{MPa}\end{array}$ \\
\hline Engr. & & Engr. & & Engr. & \\
\hline $\begin{array}{l}\text { Stress } \\
(\mathrm{MPa})\end{array}$ & $\begin{array}{l}\text { Engr. } \\
\text { Strain }\end{array}$ & $\begin{array}{l}\text { Stress } \\
(\mathrm{MPa})\end{array}$ & $\begin{array}{l}\text { Engr. } \\
\text { Strain }\end{array}$ & $\begin{array}{l}\text { Stress } \\
(\mathrm{MPa})\end{array}$ & $\begin{array}{l}\text { Engr. } \\
\text { Strain }\end{array}$ \\
\hline 22.82 & 0.0001 & 374.25 & 0.0113 & 593.22 & 0.2240 \\
\hline 33.72 & 0.0002 & 379.76 & 0.0126 & 600.40 & 0.2404 \\
\hline 65.98 & 0.0003 & 384.11 & 0.0139 & 605.91 & 0.2568 \\
\hline 103.90 & 0.0005 & 390.73 & 0.0152 & 611.84 & 0.2735 \\
\hline 143.41 & 0.0007 & 421.34 & 0.0303 & 616.18 & 0.2903 \\
\hline 184.02 & 0.0009 & 448.30 & 0.0466 & 619.63 & 0.3075 \\
\hline 222.98 & 0.0013 & 471.33 & 0.0628 & 623.98 & 0.3248 \\
\hline 257.31 & 0.0018 & 489.67 & 0.0789 & 627.01 & 0.3422 \\
\hline 283.24 & 0.0025 & 508.01 & 0.0950 & 630.32 & 0.3599 \\
\hline 303.44 & 0.0034 & 523.38 & 0.1110 & 633.35 & 0.3779 \\
\hline 320.68 & 0.0044 & 536.21 & 0.1270 & 634.94 & 0.3962 \\
\hline 333.64 & 0.0054 & 548.48 & 0.1431 & 637.97 & 0.4149 \\
\hline 344.60 & 0.0066 & 559.16 & 0.1591 & 639.28 & 0.4337 \\
\hline 353.49 & 0.0077 & 569.44 & 0.1753 & 641.76 & 0.4529 \\
\hline 361.63 & 0.0089 & 579.78 & 0.1916 & 642.18 & 0.4723 \\
\hline 367.01 & 0.0101 & 587.64 & 0.2078 & 643.83 & 0.4919 \\
\hline
\end{tabular}

Table 12. Test data for specimen I2V-19

\begin{tabular}{|c|c|c|c|c|c|}
\hline $\begin{array}{l}\text { Test Number } \\
\text { Aging Temp. } \\
\text { Yield Stress }\end{array}$ & \multicolumn{2}{|c|}{$\begin{array}{l}: \text { MEA Test } \\
: 350^{\circ} \mathrm{C} \\
: 314.7 \mathrm{MPa}\end{array}$} & \multicolumn{2}{|c|}{$\begin{array}{l}\text { Test Temp. } \\
\text { Aging Time } \\
\text { Ultimate Stress }\end{array}$} & $\begin{array}{l}25^{\circ} \mathrm{C} \\
10,000 \mathrm{~h} \\
642.0 \mathrm{MPa}\end{array}$ \\
\hline Engr. & & Engr. & & Engr. & \\
\hline $\begin{array}{l}\text { Stress } \\
\text { (MPa) }\end{array}$ & $\begin{array}{l}\text { Engr. } \\
\text { Strain }\end{array}$ & $\begin{array}{l}\text { Stress } \\
(\mathrm{MPa})\end{array}$ & $\begin{array}{l}\text { Engr. } \\
\text { Strain }\end{array}$ & $\begin{array}{l}\text { Stress } \\
(\mathrm{MPa})\end{array}$ & $\begin{array}{l}\text { Engr. } \\
\text { Strain }\end{array}$ \\
\hline 23.79 & 0.0001 & 360.04 & 0.0100 & 592.47 & 0.2152 \\
\hline 36.75 & 0.0002 & 365.49 & 0.0114 & 600.67 & 0.2344 \\
\hline 71.08 & 0.0004 & 372.94 & 0.0129 & 605.50 & 0.2538 \\
\hline 107.08 & 0.0004 & 376.38 & 0.0144 & 612.74 & 0.2736 \\
\hline 144.24 & 0.0006 & 380.59 & 0.0159 & 617.56 & 0.2939 \\
\hline 182.99 & 0.0008 & 418.10 & 0.0331 & 620.60 & 0.3144 \\
\hline 220.63 & 0.0010 & 445.61 & 0.0510 & 627.56 & 0.3352 \\
\hline 250.69 & 0.0013 & 470.91 & 0.0689 & 629.22 & 0.3567 \\
\hline 278.55 & 0.0019 & 491.04 & 0.0867 & 632.59 & 0.3786 \\
\hline 297.92 & 0.0028 & 509.80 & 0.1045 & 634.80 & 0.4009 \\
\hline 314.75 & 0.0038 & 541.58 & 0.1226 & 637.97 & 0.4240 \\
\hline 326.47 & 0.0049 & 554.48 & 0.1408 & 638.59 & 0.4480 \\
\hline 338.39 & 0.0060 & 564.54 & 0.1591 & 639.97 & 0.4728 \\
\hline 347.01 & 0.0073 & 575.30 & 0.1775 & 640.18 & 0.4988 \\
\hline 355.42 & 0.0086 & 583.64 & 0.1962 & 640.32 & 0.5265 \\
\hline
\end{tabular}


Table 13. Test data for specimen I2V-03

\begin{tabular}{|c|c|c|c|c|c|}
\hline $\begin{array}{l}\text { Test Number } \\
\text { Aging Temp. } \\
\text { Yield Stress }\end{array}$ & $\begin{array}{l}: M \\
: U \\
: 16\end{array}$ & & $\begin{array}{l}\text { Tes } \\
\text { Agi } \\
\text { Ulti }\end{array}$ & $\begin{array}{l}\text { np. } \\
\text { ne } \\
\text { Stress }\end{array}$ & $\begin{array}{l}290^{\circ} \mathrm{C} \\
- \\
409.2 \mathrm{MPa}\end{array}$ \\
\hline Engr. & & Engr. & & Engr. & \\
\hline $\begin{array}{l}\text { Stress } \\
(\mathrm{MPa})\end{array}$ & $\begin{array}{l}\text { Engr. } \\
\text { Strain }\end{array}$ & $\begin{array}{l}\text { Stress } \\
(\mathrm{MPa})\end{array}$ & $\begin{array}{l}\text { Engr. } \\
\text { Strain }\end{array}$ & $\begin{array}{l}\text { Stress } \\
(\mathrm{MPa})\end{array}$ & $\begin{array}{l}\text { Engr. } \\
\text { Strain }\end{array}$ \\
\hline 35.99 & 0.0001 & 203.12 & 0.0108 & 358.94 & 0.1225 \\
\hline 35.44 & 0.0001 & 205.67 & 0.0119 & 365.28 & 0.1303 \\
\hline 36.40 & 0.0001 & 208.36 & 0.0130 & 369.63 & 0.1372 \\
\hline 39.44 & 0.0001 & 225.11 & 0.0202 & 374.18 & 0.1472 \\
\hline 59.29 & 0.0002 & 240.97 & 0.0283 & 381.00 & 0.1534 \\
\hline 85.15 & 0.0004 & 255.24 & 0.0362 & 381.62 & 0.1643 \\
\hline 108.32 & 0.0007 & 268.14 & 0.0440 & 386.93 & 0.1751 \\
\hline 128.10 & 0.0011 & 279.58 & 0.0518 & 391.76 & 0.1779 \\
\hline 145.76 & 0.0016 & 292.06 & 0.0596 & 393.83 & 0.1911 \\
\hline 160.17 & 0.0023 & 303.71 & 0.6750 & 398.03 & 0.1995 \\
\hline 170.44 & 0.0031 & 312.26 & 0.0752 & 397.90 & 0.2061 \\
\hline 177.68 & 0.0040 & 320.54 & 0.0830 & 401.55 & 0.2118 \\
\hline 184.16 & 0.0051 & 331.43 & 0.0909 & 403.89 & 0.2261 \\
\hline 189.67 & 0.0062 & 339.43 & 0.0988 & 405.76 & 0.2345 \\
\hline 194.16 & 0.0073 & 346.67 & 0.1066 & 406.10 & 0.2451 \\
\hline 196.85 & 0.0084 & 353.15 & 0.1145 & 407.76 & 0.2464 \\
\hline 202.15 & 0.0096 & & & & \\
\hline
\end{tabular}

Table 14. Test data for specimen 12V-06

\begin{tabular}{|c|c|c|c|c|c|}
\hline \multirow{3}{*}{$\begin{array}{l}\text { Engr. } \\
\text { Stress } \\
\text { (MPa) }\end{array}$} & \multicolumn{2}{|c|}{$\begin{array}{l}: \text { MEA Test } \\
: \text { Unaged } \\
: 178.5 \mathrm{MPa}\end{array}$} & \multicolumn{2}{|c|}{$\begin{array}{l}\text { Test Temp. } \\
\text { Aging Time } \\
\text { Ultimate Stress }\end{array}$} & $\begin{array}{l}290^{\circ} \mathrm{C} \\
- \\
402.4 \mathrm{MPa}\end{array}$ \\
\hline & & Engr. & & Engr. & \\
\hline & $\begin{array}{l}\text { Engr. } \\
\text { Strain }\end{array}$ & $\begin{array}{l}\text { Stress } \\
\text { (MPa) }\end{array}$ & $\begin{array}{l}\text { Engr. } \\
\text { Strain }\end{array}$ & $\begin{array}{l}\text { Stress } \\
(\mathrm{MPa})\end{array}$ & $\begin{array}{l}\text { Engr. } \\
\text { Strain }\end{array}$ \\
\hline 21.44 & 0.0002 & 200.43 & 0.0101 & 332.19 & 0.0994 \\
\hline 21.93 & 0.0002 & 202.71 & 0.0113 & 341.01 & 0.1090 \\
\hline 42.20 & 0.0003 & 205.26 & 0.0125 & 349.22 & 0.1187 \\
\hline 69.43 & 0.0005 & 207.26 & 0.0137 & 355.84 & 0.1289 \\
\hline 97.49 & 0.0007 & 210.57 & 0.0148 & 362.32 & 0.1386 \\
\hline 128.38 & 0.0009 & 210.98 & 0.0158 & 370.59 & 0.1459 \\
\hline 156.51 & 0.0011 & 228.91 & 0.0243 & 373.42 & 0.1578 \\
\hline 168.37 & 0.0019 & 246.90 & 0.0339 & 378.25 & 0.1660 \\
\hline 175.33 & 0.0029 & 262.76 & 0.0435 & 382.04 & 0.1801 \\
\hline 182.64 & 0.0041 & 277.10 & 0.0527 & 383.76 & 0.1921 \\
\hline 185.74 & 0.0053 & 291.79 & 0.0620 & 391.42 & 0.2000 \\
\hline 189.81 & 0.0064 & 302.75 & 0.0713 & 395.21 & 0.2108 \\
\hline 193.95 & 0.0077 & 313.23 & 0.0808 & 396.79 & 0.2269 \\
\hline 197.05 & 0.0089 & 324.12 & 0.0900 & 401.62 & 0.2296 \\
\hline
\end{tabular}


Table 15. Test data for specimen 13C-02

\begin{tabular}{|c|c|c|c|c|c|}
\hline \multirow{3}{*}{$\begin{array}{l}\text { Engr. } \\
\text { Stress } \\
\text { (MPa) }\end{array}$} & \multicolumn{2}{|c|}{$\begin{array}{l}: \text { MEA Test } \\
: \text { Unaged } \\
: 158.6 \mathrm{MPa}\end{array}$} & \multicolumn{2}{|c|}{$\begin{array}{l}\text { Test Temp. } \\
\text { Aging Time } \\
\text { Ultimate Stress }\end{array}$} & $\begin{array}{l}290^{\circ} \mathrm{C} \\
- \\
387.3 \mathrm{MPa}\end{array}$ \\
\hline & & Engr. & & Engr. & \\
\hline & $\begin{array}{l}\text { Engr. } \\
\text { Strain }\end{array}$ & $\begin{array}{l}\text { Stress } \\
\text { (MPa) }\end{array}$ & $\begin{array}{l}\text { Engr. } \\
\text { Strain }\end{array}$ & $\begin{array}{l}\text { Stress } \\
(\mathrm{MPa})\end{array}$ & $\begin{array}{l}\text { Engr. } \\
\text { Strain }\end{array}$ \\
\hline 35.16 & 0.0003 & 192.85 & 0.0113 & 324.88 & 0.0940 \\
\hline 36.06 & 0.0003 & 195.88 & 0.0123 & 333.02 & 0.1021 \\
\hline 57.23 & 0.0003 & 198.71 & 0.0135 & 340.39 & 0.1101 \\
\hline 84.32 & 0.0005 & 201.40 & 0.0146 & 346.19 & 0.1185 \\
\hline 109.70 & 0.0008 & 202.64 & 0.0156 & 353.08 & 0.1266 \\
\hline 128.38 & 0.0013 & 218.49 & 0.0228 & 358.25 & 0.1353 \\
\hline 145.27 & 0.0018 & 233.87 & 0.0308 & 363.28 & 0.1441 \\
\hline 155.27 & 0.0026 & 248.69 & 0.0385 & 365.01 & 0.1539 \\
\hline 163.54 & 0.0035 & 262.35 & 0.0464 & 371.49 & 0.1614 \\
\hline 169.68 & 0.0045 & 274.69 & 0.0542 & 377.76 & 0.1728 \\
\hline 176.16 & 0.0056 & 285.24 & 0.0621 & 377.49 & 0.1832 \\
\hline 180.78 & 0.0068 & 297.78 & 0.0701 & 383.97 & 0.1869 \\
\hline 183.40 & 0.0079 & 307.85 & 0.0781 & 384.38 & 0.2002 \\
\hline 187.40 & 0.0090 & 315.02 & 0.0861 & 386.73 & 0.2136 \\
\hline 190.36 & 0.0102 & & & & \\
\hline
\end{tabular}

Table 16. Test data for specimen 12V-24

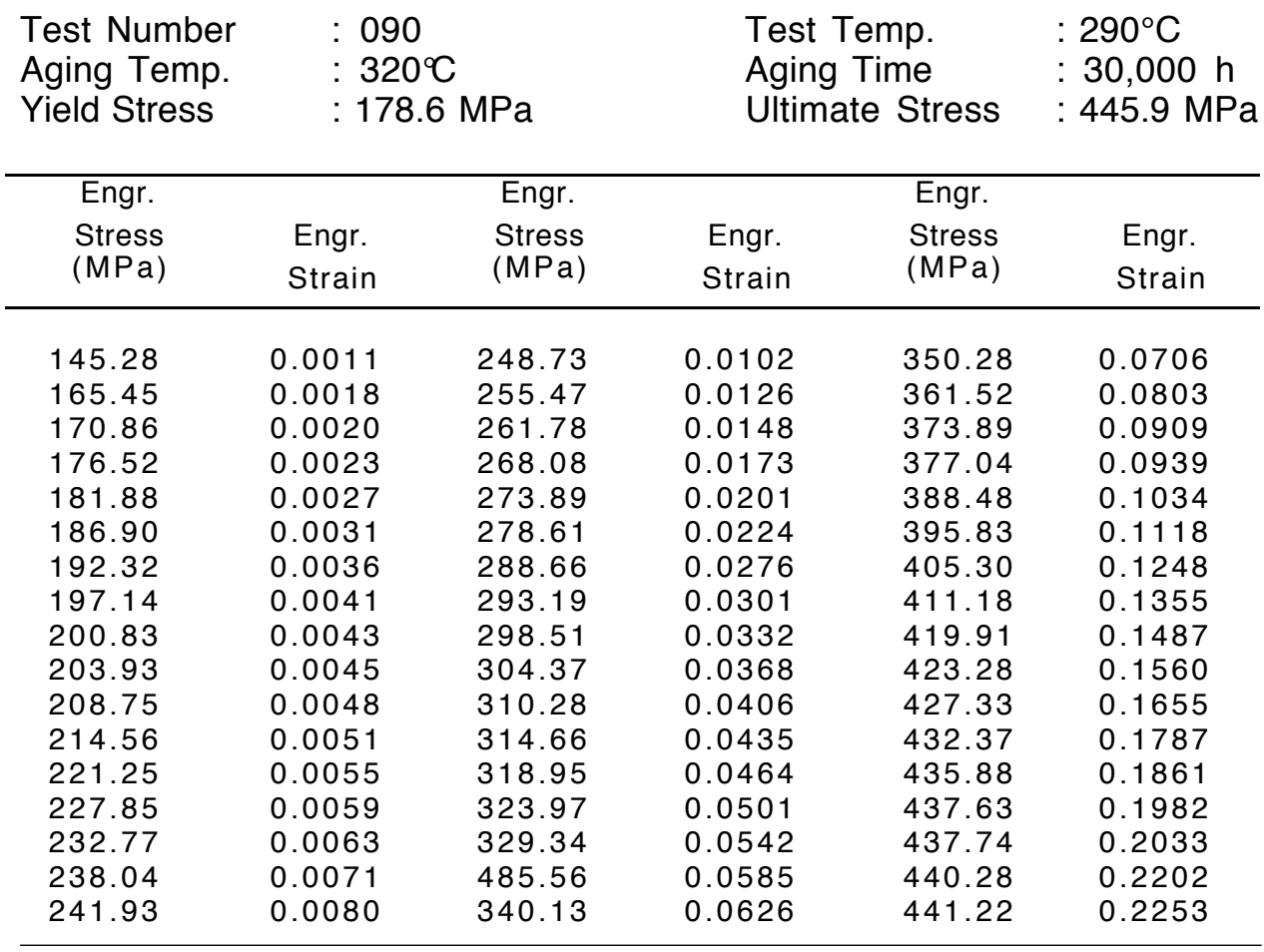


Table 17. Test data for specimen 13C-15

$\begin{array}{llll}\text { Test Number } & : 089 & \text { Test Temp. } & : 290^{\circ} \mathrm{C} \\ \text { Aging Temp. } & : 320^{\circ} \mathrm{C} & \text { Aging Time } & : 30,000 \mathrm{~h} \\ \text { Yield Stress } & : 163.6 \mathrm{MPa} & \text { Ultimate Stress } & : 427.2 \mathrm{MPa}\end{array}$

\begin{tabular}{cccccc}
\hline $\begin{array}{c}\text { Engr. } \\
\text { Stress } \\
(\mathrm{MPa})\end{array}$ & $\begin{array}{c}\text { Engr. } \\
\text { Strain }\end{array}$ & $\begin{array}{c}\text { Engr. } \\
\text { Stress } \\
(\mathrm{MPa})\end{array}$ & $\begin{array}{c}\text { Engr. } \\
\text { Strain }\end{array}$ & $\begin{array}{c}\text { Engr. } \\
\text { Stress } \\
(\mathrm{MPa})\end{array}$ & $\begin{array}{c}\text { Engr. } \\
\text { Strain }\end{array}$ \\
\hline & & & & & \\
135.46 & 0.0010 & 290.05 & 0.0352 & 389.27 & 0.1184 \\
140.82 & 0.0012 & 293.13 & 0.0374 & 393.63 & 0.1243 \\
166.39 & 0.0022 & 296.36 & 0.0392 & 398.03 & 0.1307 \\
184.42 & 0.0035 & 299.43 & 0.0416 & 403.25 & 0.1394 \\
193.07 & 0.0044 & 309.75 & 0.0487 & 407.55 & 0.1466 \\
200.97 & 0.0054 & 315.76 & 0.0531 & 409.39 & 0.1497 \\
226.12 & 0.0074 & 323.77 & 0.0594 & 410.97 & 0.1528 \\
233.47 & 0.0081 & 485.56 & 0.0717 & 419.81 & 0.1723 \\
241.11 & 0.0099 & 346.25 & 0.0783 & 420.88 & 0.1743 \\
244.69 & 0.0112 & 348.99 & 0.0805 & 423.02 & 0.1809 \\
247.82 & 0.0125 & 355.71 & 0.0863 & 424.34 & 0.1860 \\
250.90 & 0.0138 & 362.14 & 0.0916 & 425.25 & 0.1888 \\
254.62 & 0.0156 & 364.72 & 0.0942 & 425.76 & 0.1916 \\
257.80 & 0.0170 & 367.02 & 0.0962 & 425.83 & 0.1943 \\
261.08 & 0.0185 & 369.00 & 0.0985 & 426.28 & 0.1977 \\
284.03 & 0.0315 & 386.36 & 0.1142 & 427.03 & 0.2017 \\
286.92 & 0.0333 & 387.86 & 0.1162 & 427.24 & 0.2045 \\
\hline
\end{tabular}

Table 18. Test data for specimen $13 \mathrm{~V}-40$

\begin{tabular}{|c|c|c|c|c|c|}
\hline $\begin{array}{l}\text { Test Number } \\
\text { Aging Temp. } \\
\text { Y }\end{array}$ & $\begin{array}{l}: 0 \\
: 32 \\
-\end{array}$ & & $\begin{array}{l}\text { Tes } \\
\text { Agi } \\
\text { Ulti }\end{array}$ & $\begin{array}{l}\text { np. } \\
\text { me } \\
\text { Stress }\end{array}$ & $\begin{array}{l}: 290^{\circ} \mathrm{C} \\
: 30,000 \mathrm{~h} \\
: 470.5 \mathrm{MPa}\end{array}$ \\
\hline Engr. & & Engr. & & Engr. & \\
\hline $\begin{array}{l}\text { Stress } \\
(\mathrm{MPa})\end{array}$ & $\begin{array}{l}\text { Engr. } \\
\text { Strain }\end{array}$ & $\begin{array}{l}\text { Stress } \\
(\mathrm{MPa})\end{array}$ & $\begin{array}{l}\text { Engr. } \\
\text { Strain }\end{array}$ & $\begin{array}{l}\text { Stress } \\
(\mathrm{MPa})\end{array}$ & $\begin{array}{l}\text { Engr. } \\
\text { Strain }\end{array}$ \\
\hline 212.71 & 0.0017 & 380.70 & 0.0127 & 437.61 & 0.0570 \\
\hline 224.61 & 0.0019 & 384.77 & 0.0143 & 442.37 & 0.0625 \\
\hline 235.42 & 0.0020 & 388.73 & 0.0160 & 445.21 & 0.0676 \\
\hline 246.68 & 0.0022 & 392.05 & 0.0174 & 448.56 & 0.0720 \\
\hline 258.83 & 0.0024 & 394.94 & 0.0189 & 452.66 & 0.0794 \\
\hline 271.47 & 0.0026 & 485.56 & 0.0205 & 454.44 & 0.0826 \\
\hline 288.43 & 0.0029 & 402.90 & 0.0238 & 453.98 & 0.0874 \\
\hline 298.12 & 0.0032 & 408.90 & 0.0280 & 454.42 & 0.0916 \\
\hline 308.54 & 0.0035 & 411.34 & 0.0299 & 456.78 & 0.0958 \\
\hline 318.12 & 0.0038 & 417.04 & 0.0347 & 457.92 & 0.0982 \\
\hline 329.87 & 0.0043 & 420.85 & 0.0378 & 459.79 & 0.1004 \\
\hline 334.79 & 0.0047 & 422.94 & 0.0396 & 461.50 & 0.1053 \\
\hline 341.12 & 0.0052 & 424.50 & 0.0416 & 462.96 & 0.1100 \\
\hline 346.43 & 0.0056 & 426.73 & 0.0440 & 463.79 & 0.1144 \\
\hline 355.36 & 0.0068 & 429.70 & 0.0472 & 464.36 & 0.1164 \\
\hline 361.00 & 0.0078 & 432.42 & 0.0507 & 465.60 & 0.1235 \\
\hline 367.97 & 0.0090 & 434.51 & 0.0529 & & \\
\hline
\end{tabular}


Table 19. Test data for specimen /1 V-28

\begin{tabular}{|c|c|c|c|c|c|}
\hline $\begin{array}{l}\text { Test Number } \\
\text { Aging Temp. } \\
\text { Yield Stress }\end{array}$ & $\begin{array}{l}: M \\
: 35 \\
: 15\end{array}$ & & $\begin{array}{l}\text { Tes } \\
\text { Agi } \\
\text { Ulti }\end{array}$ & $\begin{array}{l}\text { np. } \\
\text { ne } \\
\text { Stress }\end{array}$ & $\begin{array}{l}290^{\circ} \mathrm{C} \\
10,000 \mathrm{~h} \\
381.1 \mathrm{MPa}\end{array}$ \\
\hline Engr. & & Engr. & & Engr. & \\
\hline $\begin{array}{l}\text { Stress } \\
(\mathrm{MPa})\end{array}$ & $\begin{array}{l}\text { Engr. } \\
\text { Strain }\end{array}$ & $\begin{array}{l}\text { Stress } \\
(\mathrm{MPa})\end{array}$ & $\begin{array}{l}\text { Engr. } \\
\text { Strain }\end{array}$ & $\begin{array}{l}\text { Stress } \\
(\mathrm{MPa})\end{array}$ & $\begin{array}{l}\text { Engr. } \\
\text { Strain }\end{array}$ \\
\hline 22.82 & 0.0002 & 225.39 & 0.0091 & 329.78 & 0.0585 \\
\hline 28.06 & 0.0002 & 229.39 & 0.0101 & 333.22 & 0.0618 \\
\hline 46.54 & 0.0003 & 233.11 & 0.0110 & 339.02 & 0.0652 \\
\hline 66.60 & 0.0004 & 240.76 & 0.0134 & 342.60 & 0.0686 \\
\hline 95.22 & 0.0006 & 251.31 & 0.0170 & 346.81 & 0.0719 \\
\hline 122.11 & 0.0007 & 259.31 & 0.0205 & 351.56 & 0.0753 \\
\hline 146.24 & 0.0009 & 268.27 & 0.0240 & 356.39 & 0.0784 \\
\hline 160.23 & 0.0012 & 274.96 & 0.0275 & 360.18 & 0.0816 \\
\hline 173.33 & 0.0018 & 282.89 & 0.0310 & 362.32 & 0.0846 \\
\hline 183.88 & 0.0024 & 288.96 & 0.0345 & 364.39 & 0.0876 \\
\hline 191.61 & 0.0032 & 294.27 & 0.0379 & 368.59 & 0.0903 \\
\hline 198.57 & 0.0040 & 301.99 & 0.0414 & 371.83 & 0.0932 \\
\hline 204.36 & 0.0048 & 309.09 & 0.0448 & 373.35 & 0.0959 \\
\hline 209.74 & 0.0056 & 314.26 & 0.0482 & 374.73 & 0.0984 \\
\hline 215.19 & 0.0065 & 318.54 & 0.0516 & 377.90 & 0.1007 \\
\hline 218.43 & 0.0073 & 324.81 & 0.0551 & 380.66 & 0.1021 \\
\hline 222.36 & 0.0082 & & & & \\
\hline
\end{tabular}

Table 20. Test data for specimen /1 V-29

\begin{tabular}{|c|c|c|c|c|c|}
\hline $\begin{array}{l}\text { Test Number } \\
\text { Aging Temp. } \\
\text { Yield Stress }\end{array}$ & $\begin{array}{l}: M \\
: 35 \\
: 18\end{array}$ & & $\begin{array}{l}\text { Tes } \\
\text { Agi } \\
\text { Ulti }\end{array}$ & $\begin{array}{l}\text { np. } \\
\text { ne } \\
\text { Stress }\end{array}$ & $\begin{array}{l}290^{\circ} \mathrm{C} \\
10,000 \mathrm{~h} \\
442.8 \mathrm{MPa}\end{array}$ \\
\hline Engr. & & Engr. & & Engr. & \\
\hline $\begin{array}{l}\text { Stress } \\
\text { (MPa) }\end{array}$ & $\begin{array}{l}\text { Engr. } \\
\text { Strain }\end{array}$ & $\begin{array}{l}\text { Stress } \\
\text { (MPa) }\end{array}$ & $\begin{array}{l}\text { Engr. } \\
\text { Strain }\end{array}$ & $\begin{array}{l}\text { Stress } \\
(\mathrm{MPa})\end{array}$ & $\begin{array}{l}\text { Engr. } \\
\text { Strain }\end{array}$ \\
\hline 22.55 & 0.0001 & 211.46 & 0.0057 & 375.21 & 0.0873 \\
\hline 21.17 & 0.0001 & 214.77 & 0.0065 & 384.66 & 0.0953 \\
\hline 21.79 & 0.0001 & 219.80 & 0.0074 & 391.62 & 0.1035 \\
\hline 38.13 & 0.0002 & 224.15 & 0.0082 & 398.52 & 0.1117 \\
\hline 55.71 & 0.0003 & 226.84 & 0.0090 & 405.27 & 0.1201 \\
\hline 78.19 & 0.0004 & 246.00 & 0.0150 & 409.55 & 0.1286 \\
\hline 102.80 & 0.0006 & 265.93 & 0.0219 & 414.86 & 0.1372 \\
\hline 126.45 & 0.0008 & 281.10 & 0.0290 & 421.41 & 0.1458 \\
\hline 148.38 & 0.0011 & 296.47 & 0.0362 & 424.72 & 0.1547 \\
\hline 164.30 & 0.0015 & 311.09 & 0.0434 & 429.89 & 0.1639 \\
\hline 175.40 & 0.0021 & 324.26 & 0.0506 & 432.58 & 0.1731 \\
\hline 185.40 & 0.0027 & 334.95 & 0.0578 & 434.99 & 0.1829 \\
\hline 192.71 & 0.0034 & 344.88 & 0.0650 & 436.92 & 0.1925 \\
\hline 199.26 & 0.0041 & 357.36 & 0.0724 & 438.92 & 0.2046 \\
\hline 205.74 & 0.0049 & 366.53 & 0.0798 & 442.02 & 0.2140 \\
\hline
\end{tabular}


Table 21. Test data for specimen I2V-20

\begin{tabular}{|c|c|c|c|c|c|}
\hline $\begin{array}{l}\text { Test Number } \\
\text { Aging Temp. } \\
\text { Yield Stress }\end{array}$ & $\begin{array}{l}: M \\
: 35 \\
: 17\end{array}$ & & $\begin{array}{l}\text { Tes } \\
\text { Agi } \\
\text { Ulti }\end{array}$ & $\begin{array}{l}\text { np. } \\
\text { ne } \\
\text { Stress }\end{array}$ & $\begin{array}{l}290^{\circ} \mathrm{C} \\
10,000 \mathrm{~h} \\
437.8 \mathrm{MPa}\end{array}$ \\
\hline Engr. & & Engr. & & Engr. & \\
\hline $\begin{array}{l}\text { Stress } \\
(\mathrm{MPa})\end{array}$ & $\begin{array}{l}\text { Engr. } \\
\text { Strain }\end{array}$ & $\begin{array}{l}\text { Stress } \\
(\mathrm{MPa})\end{array}$ & $\begin{array}{l}\text { Engr. } \\
\text { Strain }\end{array}$ & $\begin{array}{l}\text { Stress } \\
(\mathrm{MPa})\end{array}$ & $\begin{array}{l}\text { Engr. } \\
\text { Strain }\end{array}$ \\
\hline 24.06 & 0.0001 & 215.74 & 0.0090 & 383.28 & 0.1135 \\
\hline 24.41 & 0.0001 & 221.11 & 0.0100 & 390.66 & 0.1231 \\
\hline 38.20 & 0.0002 & 222.29 & 0.0111 & 397.55 & 0.1331 \\
\hline 56.88 & 0.0004 & 226.42 & 0.0123 & 402.17 & 0.1432 \\
\hline 81.01 & 0.0005 & 229.11 & 0.0135 & 408.79 & 0.1534 \\
\hline 106.73 & 0.0008 & 252.42 & 0.0218 & 413.75 & 0.1636 \\
\hline 129.48 & 0.0010 & 270.62 & 0.0309 & 417.55 & 0.1743 \\
\hline 148.51 & 0.0015 & 288.61 & 0.0398 & 423.27 & 0.1854 \\
\hline 163.34 & 0.0021 & 305.44 & 0.0487 & 424.99 & 0.1966 \\
\hline 174.37 & 0.0029 & 319.85 & 0.0576 & 429.54 & 0.2086 \\
\hline 183.61 & 0.0037 & 333.29 & 0.0667 & 430.85 & 0.2210 \\
\hline 192.36 & 0.0047 & 344.32 & 0.0758 & 432.23 & 0.2336 \\
\hline 199.88 & 0.0057 & 354.74 & 0.0852 & 435.89 & 0.2483 \\
\hline 204.15 & 0.0067 & 365.28 & 0.0945 & 436.30 & 0.2624 \\
\hline 209.74 & 0.0078 & 373.42 & 0.1039 & 436.78 & 0.2795 \\
\hline
\end{tabular}

Table 22. Test data for specimen P21T-01

\begin{tabular}{|c|c|c|c|c|c|}
\hline $\begin{array}{l}\text { Test Number } \\
\text { Aging Temp. } \\
\text { Yield Stress }\end{array}$ & $\begin{array}{l}M \\
\text { Ur } \\
21\end{array}$ & & $\begin{array}{l}\text { Tes } \\
\text { Agi } \\
\text { Ulti }\end{array}$ & $\begin{array}{l}\text { np. } \\
\text { ne } \\
\text { Stress }\end{array}$ & $\begin{array}{l}25^{\circ} \mathrm{C} \\
- \\
538.3 \mathrm{MPa}\end{array}$ \\
\hline Engr. & & Engr. & & Engr. & \\
\hline $\begin{array}{l}\text { Stress } \\
(\mathrm{MPa})\end{array}$ & $\begin{array}{l}\text { Engr. } \\
\text { Strain }\end{array}$ & $\begin{array}{l}\text { Stress } \\
(\mathrm{MPa})\end{array}$ & $\begin{array}{l}\text { Engr. } \\
\text { Strain }\end{array}$ & $\begin{array}{l}\text { Stress } \\
(\mathrm{MPa})\end{array}$ & $\begin{array}{l}\text { Engr. } \\
\text { Strain }\end{array}$ \\
\hline 75.77 & 0.0002 & 225.73 & 0.0031 & 263.10 & 0.0101 \\
\hline 95.56 & 0.0003 & 232.84 & 0.0038 & 267.31 & 0.0110 \\
\hline 112.94 & 0.0004 & 239.59 & 0.0047 & 267.93 & 0.0120 \\
\hline 133.55 & 0.0005 & 245.38 & 0.0055 & 271.93 & 0.0130 \\
\hline 154.30 & 0.0007 & 248.90 & 0.0063 & 272.62 & 0.0141 \\
\hline 171.47 & 0.0010 & 253.38 & 0.0073 & 275.38 & 0.0152 \\
\hline 189.81 & 0.0014 & 257.59 & 0.0082 & 279.31 & 0.0164 \\
\hline 204.64 & 0.0018 & 259.66 & 0.0091 & 280.13 & 0.0174 \\
\hline 215.53 & 0.0025 & & & & \\
\hline
\end{tabular}


Table 23. Test data for specimen P23T-01

\begin{tabular}{|c|c|c|c|c|c|}
\hline \multirow{3}{*}{$\begin{array}{l}\text { Engr. } \\
\text { Stress } \\
\text { (MPa) }\end{array}$} & \multicolumn{2}{|c|}{$\begin{array}{l}: \text { MEA Test } \\
: \text { Unaged } \\
: 238.1 \mathrm{MPa}\end{array}$} & \multicolumn{2}{|c|}{$\begin{array}{l}\text { Test Temp. } \\
\text { Aging Time } \\
\text { Ultimate Stress }\end{array}$} & $\begin{array}{l}25^{\circ} \mathrm{C} \\
- \\
556.8 \mathrm{MPa}\end{array}$ \\
\hline & & Engr. & & Engr. & \\
\hline & $\begin{array}{l}\text { Engr. } \\
\text { Strain }\end{array}$ & $\begin{array}{l}\text { Stress } \\
(\mathrm{MPa})\end{array}$ & $\begin{array}{l}\text { Engr. } \\
\text { Strain }\end{array}$ & $\begin{array}{l}\text { Stress } \\
(\mathrm{MPa})\end{array}$ & $\begin{array}{l}\text { Engr. } \\
\text { Strain }\end{array}$ \\
\hline 0.00 & 0.0000 & 342.95 & 0.0845 & 358.18 & 0.2337 \\
\hline 59.50 & 0.0004 & 347.50 & 0.0975 & 358.18 & 0.2444 \\
\hline 136.45 & 0.0010 & 349.08 & 0.1064 & 358.18 & 0.2560 \\
\hline 212.36 & 0.0024 & 350.46 & 0.1406 & 358.18 & 0.2653 \\
\hline 226.56 & 0.0032 & 350.46 & 0.1474 & 358.18 & 0.2736 \\
\hline 246.14 & 0.0052 & 350.46 & 0.1587 & 358.18 & 0.2837 \\
\hline 256.35 & 0.0064 & 349.08 & 0.1633 & 358.18 & 0.2953 \\
\hline 268.90 & 0.0094 & 350.94 & 0.1683 & 361.97 & 0.3024 \\
\hline 282.34 & 0.0142 & 352.87 & 0.1755 & 361.97 & 0.3118 \\
\hline 294.41 & 0.0198 & 353.15 & 0.1798 & 365.22 & 0.3214 \\
\hline 304.06 & 0.0263 & 354.46 & 0.1848 & 365.22 & 0.3297 \\
\hline 313.44 & 0.0330 & 356.32 & 0.1901 & 368.66 & 0.3373 \\
\hline 321.99 & 0.0398 & 357.70 & 0.1973 & 368.66 & 0.3398 \\
\hline 332.19 & 0.0504 & 356.87 & 0.2048 & 368.66 & 0.3472 \\
\hline 337.84 & 0.0584 & 358.18 & 0.2124 & 368.66 & 0.3547 \\
\hline 342.95 & 0.0687 & 358.18 & 0.2225 & & \\
\hline
\end{tabular}

Table 24. Test data for specimen P22A-01

Test Number : MEA Test

Aging Temp. : Unaged

Yield Stress $\quad: 206.4 \mathrm{MPa}$
Test Temp. $\quad: 25^{\circ} \mathrm{C}$

Aging Time :-

Ultimate Stress $\quad: 561.7 \mathrm{MPa}$

Experimental Data Not Available.

Table 25. Test data for specimen P23A-01

\begin{tabular}{|c|c|c|c|c|c|}
\hline \multirow{3}{*}{$\begin{array}{l}\text { Test Number } \\
\text { Aging Temp. } \\
\text { Yield Stress } \\
\text { Engr. } \\
\text { Stress } \\
\text { (MPa) }\end{array}$} & \multicolumn{2}{|c|}{$\begin{array}{l}: \text { MEA Test } \\
: \text { Unaged } \\
: 216.7 \mathrm{MPa}\end{array}$} & \multicolumn{2}{|c|}{$\begin{array}{l}\text { Test Temp. } \\
\text { Aging Time } \\
\text { Ultimate Stress }\end{array}$} & $\begin{array}{l}25^{\circ} \mathrm{C} \\
- \\
536.9 \mathrm{MPa}\end{array}$ \\
\hline & \multirow[b]{2}{*}{$\begin{array}{l}\text { Engr. } \\
\text { Strain }\end{array}$} & \multirow{2}{*}{$\begin{array}{l}\text { Engr. } \\
\text { Stress } \\
\text { (MPa) }\end{array}$} & \multicolumn{3}{|c|}{ Engr. } \\
\hline & & & $\begin{array}{l}\text { Engr. } \\
\text { Strain }\end{array}$ & $\begin{array}{l}\text { Stress } \\
(\mathrm{MPa})\end{array}$ & $\begin{array}{l}\text { Engr. } \\
\text { Strain }\end{array}$ \\
\hline 33.72 & 0.0000 & 210.84 & 0.0025 & 260.28 & 0.0105 \\
\hline 49.92 & 0.0002 & 218.70 & 0.0031 & 262.97 & 0.0113 \\
\hline 66.47 & 0.0003 & 225.94 & 0.0037 & 265.17 & 0.0123 \\
\hline 97.77 & 0.0004 & 232.56 & 0.0045 & 268.28 & 0.0132 \\
\hline 118.04 & 0.0005 & 237.73 & 0.0053 & 270.62 & 0.0141 \\
\hline 139.14 & 0.0007 & 243.45 & 0.0061 & 272.83 & 0.0151 \\
\hline 156.44 & 0.0009 & 246.83 & 0.0070 & 275.31 & 0.0160 \\
\hline 171.06 & 0.0011 & 250.56 & 0.0078 & 277.10 & 0.0170 \\
\hline 185.47 & 0.0014 & 254.42 & 0.0087 & 280.00 & 0.0180 \\
\hline 199.53 & 0.0019 & 257.24 & 0.0096 & & \\
\hline
\end{tabular}


Table 26. Test data for specimen P22T-16

\begin{tabular}{|c|c|c|c|c|c|}
\hline $\begin{array}{l}\text { Test Number } \\
\text { Aging Temp. } \\
\text { Yield Stress }\end{array}$ & \multicolumn{2}{|c|}{$\begin{array}{l}: 116 \\
: 290^{\circ} \mathrm{C} \\
: 247.0 \mathrm{MPa}\end{array}$} & \multicolumn{2}{|c|}{$\begin{array}{l}\text { Test Temp. } \\
\text { Aging Time } \\
\text { Ultimate Stress }\end{array}$} & $\begin{array}{l}25^{\circ} \mathrm{C} \\
30,000 \mathrm{~h} \\
580.1 \mathrm{MPa}\end{array}$ \\
\hline \multirow{2}{*}{$\begin{array}{l}\text { Engr. } \\
\text { Stress } \\
(\mathrm{MPa})\end{array}$} & \multicolumn{2}{|r|}{ Engr. } & \multicolumn{3}{|c|}{ Engr. } \\
\hline & $\begin{array}{l}\text { Engr. } \\
\text { Strain }\end{array}$ & $\begin{array}{l}\text { Stress } \\
(\mathrm{MPa})\end{array}$ & $\begin{array}{l}\text { Engr. } \\
\text { Strain }\end{array}$ & $\begin{array}{l}\text { Stress } \\
(\mathrm{MPa})\end{array}$ & $\begin{array}{l}\text { Engr. } \\
\text { Strain }\end{array}$ \\
\hline 43.91 & 0.0000 & 342.66 & 0.0248 & 481.02 & 0.1430 \\
\hline 189.97 & 0.0015 & 363.69 & 0.0376 & 485.81 & 0.1553 \\
\hline 201.17 & 0.0018 & 388.73 & 0.0551 & 508.05 & 0.1923 \\
\hline 223.14 & 0.0021 & 421.22 & 0.0830 & 526.44 & 0.2290 \\
\hline 261.09 & 0.0032 & 448.33 & 0.1060 & 541.88 & 0.2665 \\
\hline 275.55 & 0.0042 & 468.51 & 0.1272 & 562.32 & 0.3484 \\
\hline 303.36 & 0.0088 & 475.56 & 0.1355 & 576.12 & 0.4433 \\
\hline 323.48 & 0.0172 & 477.51 & 0.1399 & 580.05 & 0.5090 \\
\hline
\end{tabular}

Table 27. Test data for specimen P21A-31

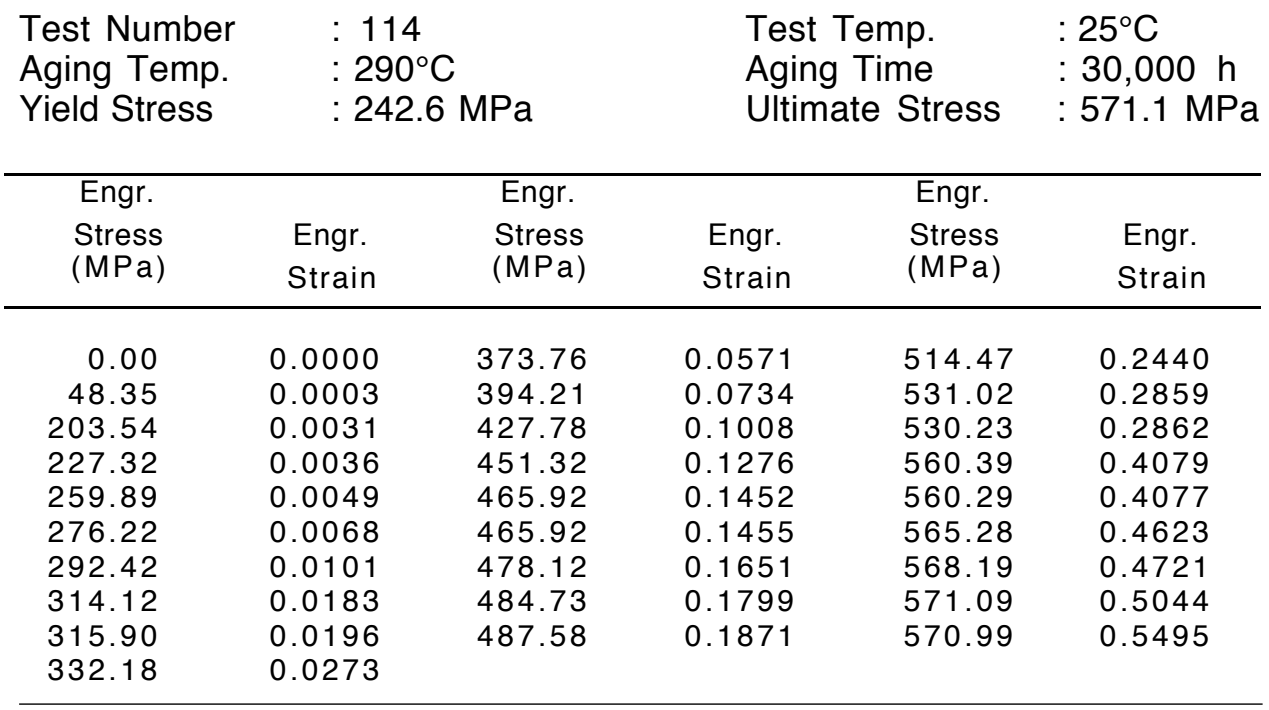


Table 28. Test data for specimen P25A-28

\begin{tabular}{|c|c|c|c|c|c|}
\hline $\begin{array}{l}\text { Test Number } \\
\text { Aging Temp. } \\
\text { Yield Stress }\end{array}$ & $\begin{array}{l}: 1 \\
: 25 \\
: 22\end{array}$ & $\mathrm{MPa}$ & $\begin{array}{l}\text { Tes } \\
\text { Agi } \\
\text { Ulti }\end{array}$ & $\begin{array}{l}\text { np. } \\
\text { me } \\
\text { Stress }\end{array}$ & $\begin{array}{l}25^{\circ} \mathrm{C} \\
30,000 \mathrm{~h} \\
548.3 \mathrm{MPa}\end{array}$ \\
\hline Engr. & & Engr. & & Engr. & \\
\hline $\begin{array}{l}\text { Stress } \\
(\mathrm{MPa})\end{array}$ & $\begin{array}{l}\text { Engr. } \\
\text { Strain }\end{array}$ & $\begin{array}{l}\text { Stress } \\
(\mathrm{MPa})\end{array}$ & $\begin{array}{l}\text { Engr. } \\
\text { Strain }\end{array}$ & $\begin{array}{l}\text { Stress } \\
(\mathrm{MPa})\end{array}$ & $\begin{array}{l}\text { Engr. } \\
\text { Strain }\end{array}$ \\
\hline 0.00 & 0.0000 & 321.89 & 0.0234 & 476.75 & 0.1723 \\
\hline 86.41 & 0.0002 & 347.19 & 0.0401 & 483.95 & 0.1925 \\
\hline 155.03 & 0.0009 & 368.69 & 0.0567 & 504.89 & 0.2369 \\
\hline 194.51 & 0.0016 & 383.86 & 0.0691 & 511.41 & 0.2558 \\
\hline 213.11 & 0.0023 & 405.18 & 0.0880 & 512.94 & 0.2598 \\
\hline 241.77 & 0.0025 & 419.23 & 0.0991 & 529.77 & 0.3109 \\
\hline 261.95 & 0.0042 & 436.03 & 0.1167 & 536.79 & 0.3411 \\
\hline 269.65 & 0.0052 & 447.42 & 0.1308 & 543.63 & 0.3742 \\
\hline 287.33 & 0.0090 & 457.87 & 0.1450 & 548.27 & 0.4148 \\
\hline 301.36 & 0.0135 & 458.27 & 0.1448 & & \\
\hline
\end{tabular}

Table 29. Test data for specimen P21A-36

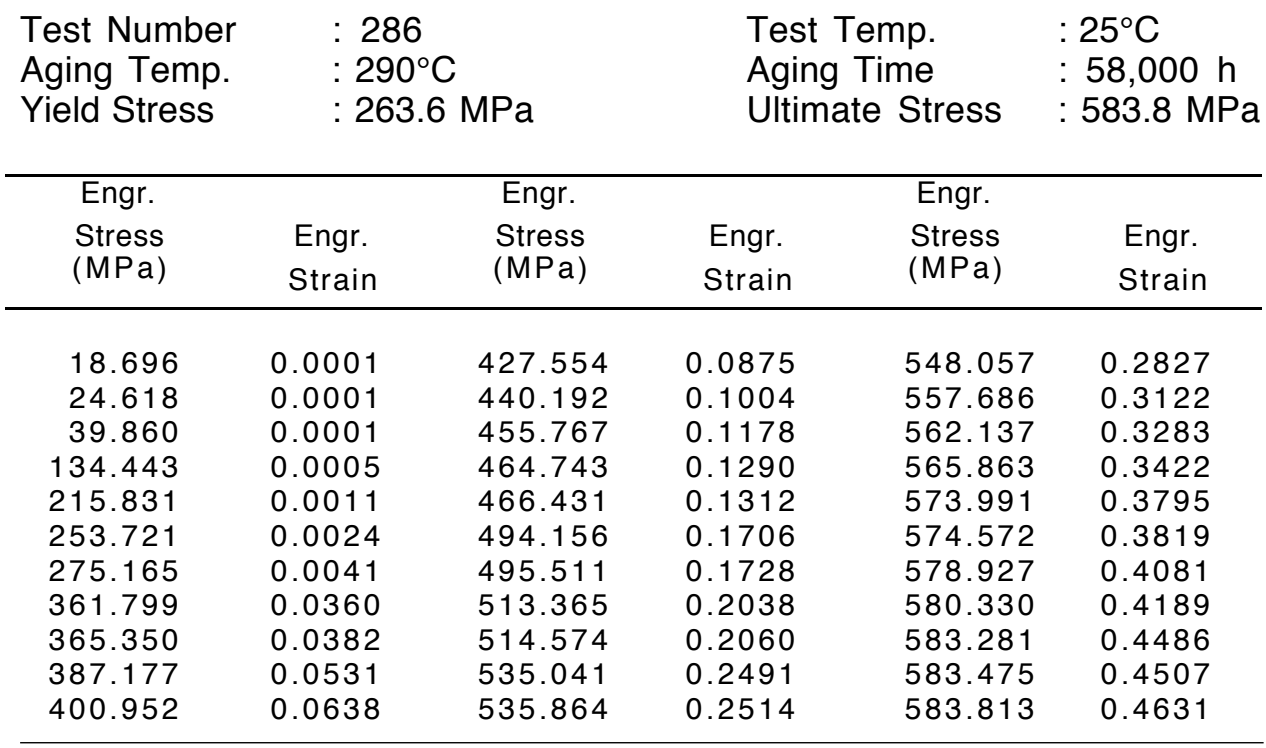


Table 30. Test data for specimen P24T-14

\begin{tabular}{|c|c|c|c|c|c|}
\hline $\begin{array}{l}\text { Test Number } \\
\text { Aging Temp. } \\
\text { Yield Stress }\end{array}$ & \multicolumn{2}{|c|}{$\begin{array}{l}: 118 \\
: 320^{\circ} \mathrm{C} \\
: 229.4 \mathrm{MPa}\end{array}$} & \multicolumn{2}{|c|}{$\begin{array}{l}\text { Test Temp. } \\
\text { Aging Time } \\
\text { Ultimate Stress }\end{array}$} & $\begin{array}{l}25^{\circ} \mathrm{C} \\
30,000 \mathrm{~h} \\
600.0 \mathrm{MPa}\end{array}$ \\
\hline \multirow{2}{*}{$\begin{array}{l}\text { Engr. } \\
\text { Stress } \\
\text { (MPa) }\end{array}$} & \multicolumn{2}{|r|}{ Engr. } & \multicolumn{3}{|c|}{ Engr. } \\
\hline & $\begin{array}{l}\text { Engr. } \\
\text { Strain }\end{array}$ & $\begin{array}{l}\text { Stress } \\
(\mathrm{MPa})\end{array}$ & $\begin{array}{l}\text { Engr. } \\
\text { Strain }\end{array}$ & $\begin{array}{l}\text { Stress } \\
(\mathrm{MPa})\end{array}$ & $\begin{array}{l}\text { Engr. } \\
\text { Strain }\end{array}$ \\
\hline 1.08 & 0.0000 & 368.40 & 0.0424 & 521.97 & 0.2166 \\
\hline 72.59 & 0.0001 & 395.11 & 0.0601 & 542.22 & 0.2682 \\
\hline 207.27 & 0.0022 & 421.93 & 0.0814 & 560.21 & 0.3171 \\
\hline 241.78 & 0.0027 & 444.92 & 0.1003 & 571.16 & 0.3615 \\
\hline 272.19 & 0.0048 & 467.12 & 0.1241 & 579.92 & 0.4024 \\
\hline 285.75 & 0.0067 & 474.79 & 0.1323 & 594.04 & 0.5004 \\
\hline 307.05 & 0.0117 & 486.37 & 0.1514 & 599.32 & 0.5632 \\
\hline 326.49 & 0.0178 & 500.36 & 0.1771 & 600.04 & 0.6237 \\
\hline 340.41 & 0.0239 & & & & \\
\hline
\end{tabular}

Table 31. Test data for specimen P25T-10

\begin{tabular}{|c|c|c|c|c|c|}
\hline $\begin{array}{l}\text { Test Number } \\
\text { Aging Temp. } \\
Y\end{array}$ & $\begin{array}{l}: 10 \\
: 32\end{array}$ & & $\begin{array}{l}\text { Tes } \\
\text { Agir } \\
\text { Ultir }\end{array}$ & $\begin{array}{l}\text { np. } \\
\text { ne } \\
\text { Stress }\end{array}$ & $\begin{array}{l}25^{\circ} \mathrm{C} \\
30,000 \mathrm{~h} \\
581.8 \mathrm{MPa}\end{array}$ \\
\hline Engr. & & Engr. & & Engr. & \\
\hline $\begin{array}{l}\text { Stress } \\
(\mathrm{MPa})\end{array}$ & $\begin{array}{l}\text { Engr. } \\
\text { Strain }\end{array}$ & $\begin{array}{l}\text { Stress } \\
\text { (MPa) }\end{array}$ & $\begin{array}{l}\text { Engr. } \\
\text { Strain }\end{array}$ & $\begin{array}{l}\text { Stress } \\
(\mathrm{MPa})\end{array}$ & $\begin{array}{l}\text { Engr. } \\
\text { Strain }\end{array}$ \\
\hline 0.00 & 0.0000 & 414.63 & 0.0790 & 534.40 & 0.2365 \\
\hline 64.28 & 0.0003 & 443.49 & 0.0996 & 537.07 & 0.2424 \\
\hline 164.98 & 0.0022 & 452.36 & 0.1085 & 540.08 & 0.2500 \\
\hline 198.69 & 0.0024 & 466.92 & 0.1227 & 541.90 & 0.2527 \\
\hline 213.37 & 0.0029 & 469.64 & 0.1279 & 547.20 & 0.2668 \\
\hline 219.84 & 0.0030 & 478.91 & 0.1372 & 566.01 & 0.3318 \\
\hline 249.13 & 0.0040 & 484.19 & 0.1494 & 567.78 & 0.3355 \\
\hline 251.89 & 0.0043 & 493.73 & 0.1618 & 572.87 & 0.3572 \\
\hline 275.62 & 0.0069 & 499.40 & 0.1732 & 572.56 & 0.3660 \\
\hline 287.20 & 0.0091 & 501.58 & 0.1740 & 574.42 & 0.3763 \\
\hline 300.95 & 0.0124 & 505.86 & 0.1857 & 574.32 & 0.3757 \\
\hline 320.76 & 0.0189 & 511.37 & 0.1946 & 576.85 & 0.3951 \\
\hline 339.21 & 0.0279 & 517.49 & 0.2045 & 579.98 & 0.4058 \\
\hline 366.24 & 0.0431 & 526.82 & 0.2223 & 581.80 & 0.4462 \\
\hline 394.74 & 0.0629 & 531.63 & 0.2313 & & \\
\hline
\end{tabular}


Table 32. Test data for specimen P22A-36

\begin{tabular}{|c|c|c|c|c|c|}
\hline $\begin{array}{l}\text { Test Number } \\
\text { Aging Temp. } \\
\text { Yield Stress }\end{array}$ & $\begin{array}{l}: 1 \\
: 32 \\
: 23\end{array}$ & & $\begin{array}{l}\text { Tes } \\
\text { Agi } \\
\text { Ulti }\end{array}$ & $\begin{array}{l}\text { np. } \\
\text { me } \\
\text { Stress }\end{array}$ & $\begin{array}{l}25^{\circ} \mathrm{C} \\
30,000 \mathrm{~h} \\
603.0 \mathrm{MPa}\end{array}$ \\
\hline Engr. & & Engr. & & Engr. & \\
\hline $\begin{array}{l}\text { Stress } \\
(\mathrm{MPa})\end{array}$ & $\begin{array}{l}\text { Engr. } \\
\text { Strain }\end{array}$ & $\begin{array}{l}\text { Stress } \\
(\mathrm{MPa})\end{array}$ & $\begin{array}{l}\text { Engr. } \\
\text { Strain }\end{array}$ & $\begin{array}{l}\text { Stress } \\
(\mathrm{MPa})\end{array}$ & $\begin{array}{l}\text { Engr. } \\
\text { Strain }\end{array}$ \\
\hline 0.00 & 0.0000 & 437.15 & 0.0830 & 521.86 & 0.1973 \\
\hline 67.86 & 0.0001 & 455.92 & 0.0989 & 541.57 & 0.2361 \\
\hline 195.99 & 0.0015 & 465.90 & 0.1055 & 563.14 & 0.2942 \\
\hline 220.01 & 0.0020 & 471.02 & 0.1109 & 563.62 & 0.2993 \\
\hline 260.93 & 0.0031 & 481.35 & 0.1347 & 577.37 & 0.3565 \\
\hline 283.12 & 0.0050 & 484.70 & 0.1395 & 582.02 & 0.3841 \\
\hline 295.07 & 0.0065 & 497.55 & 0.1568 & 583.56 & 0.3885 \\
\hline 312.40 & 0.0099 & 504.35 & 0.1669 & 590.65 & 0.4283 \\
\hline 330.98 & 0.0152 & 510.57 & 0.1782 & 601.19 & 0.5273 \\
\hline 361.40 & 0.0298 & 516.28 & 0.1882 & 602.66 & 0.5777 \\
\hline 391.84 & 0.0483 & 521.81 & 0.1946 & 602.98 & 0.6590 \\
\hline 414.44 & 0.0649 & & & & \\
\hline
\end{tabular}

Table 33. Test data for specimen P21A-19

\begin{tabular}{|c|c|c|c|c|c|}
\hline $\begin{array}{l}\text { Test Number } \\
\text { Aging Temp. } \\
\text { Yield Stress }\end{array}$ & $\begin{array}{l}: 2 \\
: 32 \\
: 24\end{array}$ & & $\begin{array}{l}\text { Tes } \\
\text { Agi } \\
\text { Ulti }\end{array}$ & $\begin{array}{l}\text { np. } \\
\text { ne } \\
\text { Stress }\end{array}$ & $\begin{array}{l}25^{\circ} \mathrm{C} \\
55,000 \mathrm{~h} \\
605.2 \mathrm{MPa}\end{array}$ \\
\hline Engr. & & Engr. & & Engr. & \\
\hline $\begin{array}{l}\text { Stress } \\
\text { (MPa) }\end{array}$ & $\begin{array}{l}\text { Engr. } \\
\text { Strain }\end{array}$ & $\begin{array}{l}\text { Stress } \\
(\mathrm{MPa})\end{array}$ & $\begin{array}{l}\text { Engr. } \\
\text { Strain }\end{array}$ & $\begin{array}{l}\text { Stress } \\
(\mathrm{MPa})\end{array}$ & $\begin{array}{l}\text { Engr. } \\
\text { Strain }\end{array}$ \\
\hline 15.30 & 0.0001 & 351.39 & 0.0260 & 523.10 & 0.1626 \\
\hline 19.83 & 0.0001 & 372.83 & 0.0360 & 524.69 & 0.1646 \\
\hline 57.42 & 0.0003 & 376.63 & 0.0380 & 530.32 & 0.1726 \\
\hline 68.12 & 0.0003 & 398.34 & 0.0500 & 538.61 & 0.1846 \\
\hline 127.65 & 0.0006 & 401.60 & 0.0520 & 546.12 & 0.1966 \\
\hline 140.24 & 0.0007 & 404.98 & 0.0540 & 556.47 & 0.2146 \\
\hline 152.69 & 0.0008 & 420.58 & 0.0640 & 563.74 & 0.2287 \\
\hline 207.14 & 0.0015 & 426.26 & 0.0679 & 564.75 & 0.2307 \\
\hline 223.63 & 0.0020 & 443.08 & 0.0802 & 571.49 & 0.2450 \\
\hline 259.10 & 0.0039 & 445.53 & 0.0822 & 577.56 & 0.2593 \\
\hline 266.89 & 0.0046 & 462.18 & 0.0963 & 581.56 & 0.2695 \\
\hline 273.90 & 0.0053 & 473.10 & 0.1064 & 582.28 & 0.2715 \\
\hline 308.66 & 0.0113 & 487.57 & 0.1207 & 594.80 & 0.3112 \\
\hline 314.87 & 0.0129 & 500.52 & 0.1347 & 599.37 & 0.3304 \\
\hline
\end{tabular}


Table 34. Test data for specimen P22T-04

\begin{tabular}{|c|c|c|c|c|c|}
\hline \multirow{3}{*}{$\begin{array}{l}\text { Engr. } \\
\text { Stress } \\
\text { (MPa) }\end{array}$} & \multicolumn{2}{|c|}{$\begin{array}{l}: \text { MEA Test } \\
: 350^{\circ} \mathrm{C} \\
: 252.3 \mathrm{MPa}\end{array}$} & \multicolumn{2}{|c|}{$\begin{array}{l}\text { Test Temp. } \\
\text { Aging Time } \\
\text { Ultimate Stress }\end{array}$} & \multirow{2}{*}{$\begin{array}{l}25^{\circ} \mathrm{C} \\
10,000 \mathrm{~h} \\
601.8 \mathrm{MPa} \\
\end{array}$} \\
\hline & & Engr. & & Engr. & \\
\hline & $\begin{array}{l}\text { Engr. } \\
\text { Strain }\end{array}$ & $\begin{array}{l}\text { Stress } \\
\text { (MPa) }\end{array}$ & $\begin{array}{l}\text { Engr. } \\
\text { Strain }\end{array}$ & $\begin{array}{l}\text { Stress } \\
(\mathrm{MPa})\end{array}$ & $\begin{array}{l}\text { Engr. } \\
\text { Strain }\end{array}$ \\
\hline 59.78 & 0.0003 & 314.81 & 0.0148 & 546.62 & 0.2670 \\
\hline 91.70 & 0.0004 & 320.54 & 0.0163 & 552.41 & 0.2844 \\
\hline 125.00 & 0.0006 & 362.39 & 0.0358 & 559.72 & 0.3022 \\
\hline 160.85 & 0.0008 & 404.72 & 0.0641 & 564.40 & 0.3201 \\
\hline 196.16 & 0.0011 & 424.99 & 0.0808 & 570.20 & 0.3379 \\
\hline 217.81 & 0.0017 & 441.82 & 0.0974 & 575.16 & 0.3561 \\
\hline 239.45 & 0.0024 & 457.81 & 0.1139 & 579.16 & 0.3743 \\
\hline 254.35 & 0.0033 & 470.64 & 0.1306 & 583.37 & 0.3926 \\
\hline 268.28 & 0.0044 & 482.98 & 0.1474 & 584.61 & 0.4112 \\
\hline 277.24 & 0.0056 & 494.84 & 0.1641 & 588.33 & 0.4299 \\
\hline 285.86 & 0.0068 & 506.70 & 0.1811 & 589.98 & 0.4487 \\
\hline 290.41 & 0.0081 & 517.52 & 0.1982 & 592.81 & 0.4679 \\
\hline 298.41 & 0.0094 & 524.62 & 0.2154 & 596.67 & 0.4868 \\
\hline 302.13 & 0.0107 & 533.38 & 0.2325 & 596.05 & 0.5056 \\
\hline 306.82 & 0.0121 & 541.10 & 0.2496 & 597.36 & 0.5240 \\
\hline 314.88 & 0.0134 & & & & \\
\hline
\end{tabular}

Table 35. Test data for specimen P23A-14

\begin{tabular}{|c|c|c|c|c|c|}
\hline $\begin{array}{l}\text { Test Number } \\
\text { Aging Temp. } \\
\text { Yield Stress }\end{array}$ & $\begin{array}{l}: M \\
: 35 \\
: 24\end{array}$ & & $\begin{array}{l}\text { Tes } \\
\text { Agi } \\
\text { Ulti }\end{array}$ & $\begin{array}{l}\text { np. } \\
\text { ne } \\
\text { Stress }\end{array}$ & $\begin{array}{l}25^{\circ} \mathrm{C} \\
10,000 \mathrm{~h} \\
594.3 \mathrm{MPa}\end{array}$ \\
\hline Engr. & & Engr. & & Engr. & \\
\hline $\begin{array}{l}\text { Stress } \\
(\mathrm{MPa})\end{array}$ & $\begin{array}{l}\text { Engr. } \\
\text { Strain }\end{array}$ & $\begin{array}{l}\text { Stress } \\
(\mathrm{MPa})\end{array}$ & $\begin{array}{l}\text { Engr. } \\
\text { Strain }\end{array}$ & $\begin{array}{l}\text { Stress } \\
(\mathrm{MPa})\end{array}$ & $\begin{array}{l}\text { Engr. } \\
\text { Strain }\end{array}$ \\
\hline 22.82 & 0.0002 & 288.48 & 0.0086 & 537.79 & 0.2465 \\
\hline 43.37 & 0.0003 & 294.27 & 0.0096 & 545.58 & 0.2655 \\
\hline 66.26 & 0.0004 & 297.51 & 0.0107 & 552.96 & 0.2845 \\
\hline 93.22 & 0.0005 & 302.68 & 0.0119 & 557.85 & 0.3039 \\
\hline 121.28 & 0.0006 & 342.53 & 0.0278 & 565.85 & 0.3237 \\
\hline 149.00 & 0.0008 & 374.73 & 0.0461 & 570.82 & 0.3436 \\
\hline 175.54 & 0.0010 & 402.93 & 0.0655 & 571.92 & 0.3637 \\
\hline 199.81 & 0.0014 & 437.27 & 0.1004 & 579.85 & 0.3844 \\
\hline 220.63 & 0.0019 & 458.85 & 0.1176 & 580.61 & 0.4054 \\
\hline 238.08 & 0.0027 & 474.22 & 0.1356 & 585.02 & 0.4267 \\
\hline 250.28 & 0.0035 & 486.49 & 0.1538 & 588.54 & 0.4487 \\
\hline 259.24 & 0.0044 & 498.63 & 0.1720 & 588.61 & 0.4714 \\
\hline 270.14 & 0.0054 & 510.56 & 0.1904 & 590.74 & 0.4951 \\
\hline 275.03 & 0.0064 & 521.38 & 0.2090 & 592.40 & 0.5204 \\
\hline 285.10 & 0.0075 & 529.79 & 0.2277 & & \\
\hline
\end{tabular}


Table 36. Test data for specimen P23A-36

\begin{tabular}{|c|c|c|c|c|c|}
\hline $\begin{array}{l}\text { Test Number } \\
\text { Aging Temp. } \\
\text { Yield Stress }\end{array}$ & $\begin{array}{l}: M \\
: 35 \\
: 26\end{array}$ & & $\begin{array}{l}\text { Tes } \\
\text { Agi } \\
\text { Ulti }\end{array}$ & $\begin{array}{l}\text { np. } \\
\text { ne } \\
\text { Stress }\end{array}$ & $\begin{array}{l}25^{\circ} \mathrm{C} \\
10,000 \mathrm{~h} \\
608.5 \mathrm{MPa}\end{array}$ \\
\hline Engr. & & Engr. & & Engr. & \\
\hline $\begin{array}{l}\text { Stress } \\
(\mathrm{MPa})\end{array}$ & $\begin{array}{l}\text { Engr. } \\
\text { Strain }\end{array}$ & $\begin{array}{l}\text { Stress } \\
(\mathrm{MPa})\end{array}$ & $\begin{array}{l}\text { Engr. } \\
\text { Strain }\end{array}$ & $\begin{array}{l}\text { Stress } \\
(\mathrm{MPa})\end{array}$ & $\begin{array}{l}\text { Engr. } \\
\text { Strain }\end{array}$ \\
\hline 3.17 & 0.0002 & 298.20 & 0.0106 & 548.34 & 0.2622 \\
\hline 33.58 & 0.0002 & 301.30 & 0.0119 & 556.82 & 0.2838 \\
\hline 61.98 & 0.0004 & 306.13 & 0.0131 & 564.13 & 0.3057 \\
\hline 94.32 & 0.0005 & 308.13 & 0.0144 & 571.44 & 0.3279 \\
\hline 130.66 & 0.0008 & 311.92 & 0.0157 & 576.61 & 0.3503 \\
\hline 166.03 & 0.0010 & 355.42 & 0.0359 & 581.64 & 0.3732 \\
\hline 203.81 & 0.0012 & 388.17 & 0.0576 & 585.92 & 0.3961 \\
\hline 237.66 & 0.0015 & 418.30 & 0.0798 & 590.19 & 0.4196 \\
\hline 254.76 & 0.0024 & 455.95 & 0.1164 & 593.50 & 0.4435 \\
\hline 262.21 & 0.0034 & 474.29 & 0.1365 & 597.57 & 0.4676 \\
\hline 271.24 & 0.0045 & 490.22 & 0.1572 & 600.12 & 0.4922 \\
\hline 279.10 & 0.0057 & 504.35 & 0.1778 & 602.19 & 0.5174 \\
\hline 282.82 & 0.0069 & 517.18 & 0.1986 & 603.91 & 0.5429 \\
\hline 289.44 & 0.0081 & 528.48 & 0.2196 & 606.33 & 0.5689 \\
\hline 292.96 & 0.0093 & 539.17 & 0.2409 & 607.98 & 0.5955 \\
\hline
\end{tabular}

Table 37. Test data for specimen P22T-11

\begin{tabular}{|c|c|c|c|c|c|}
\hline $\begin{array}{l}\text { Test Number } \\
\text { Aging Temp. } \\
\text { Yield Stress }\end{array}$ & $\begin{array}{l}: 1 \\
: 35 \\
: 24\end{array}$ & & $\begin{array}{l}\text { Tes } \\
\text { Agir } \\
\text { Ulti }\end{array}$ & $\begin{array}{l}\text { np. } \\
\text { ne } \\
\text { Stress }\end{array}$ & $\begin{array}{l}25^{\circ} \mathrm{C} \\
30,000 \mathrm{~h} \\
628.4 \mathrm{MPa}\end{array}$ \\
\hline Engr. & & Engr. & & Engr. & \\
\hline $\begin{array}{l}\text { Stress } \\
(\mathrm{MPa})\end{array}$ & $\begin{array}{l}\text { Engr. } \\
\text { Strain }\end{array}$ & $\begin{array}{l}\text { Stress } \\
(\mathrm{MPa})\end{array}$ & $\begin{array}{l}\text { Engr. } \\
\text { Strain }\end{array}$ & $\begin{array}{l}\text { Stress } \\
(\mathrm{MPa})\end{array}$ & $\begin{array}{l}\text { Engr. } \\
\text { Strain }\end{array}$ \\
\hline 0.00 & 0.0000 & 437.70 & 0.0736 & 578.79 & 0.2605 \\
\hline 63.87 & 0.0003 & 459.69 & 0.0898 & 582.98 & 0.2690 \\
\hline 203.91 & 0.0025 & 471.05 & 0.0973 & 587.96 & 0.2787 \\
\hline 222.66 & 0.0028 & 484.95 & 0.1108 & 589.58 & 0.2852 \\
\hline 229.32 & 0.0029 & 497.35 & 0.1235 & 592.32 & 0.2982 \\
\hline 249.74 & 0.0036 & 518.00 & 0.1476 & 594.90 & 0.3027 \\
\hline 286.31 & 0.0062 & 521.30 & 0.1541 & 597.52 & 0.3136 \\
\hline 300.38 & 0.0080 & 527.36 & 0.1624 & 603.41 & 0.3340 \\
\hline 333.68 & 0.0158 & 536.88 & 0.1732 & 607.02 & 0.3542 \\
\hline 370.93 & 0.0297 & 540.59 & 0.1816 & 610.92 & 0.3707 \\
\hline 377.32 & 0.0333 & 549.45 & 0.1968 & 614.92 & 0.3913 \\
\hline 388.17 & 0.0403 & 563.77 & 0.2188 & 621.68 & 0.4432 \\
\hline 403.38 & 0.0502 & 564.52 & 0.2301 & 623.50 & 0.4576 \\
\hline 417.59 & 0.0575 & 569.11 & 0.2389 & 625.01 & 0.4709 \\
\hline 432.23 & 0.0689 & 573.91 & 0.2496 & 628.40 & 0.5554 \\
\hline
\end{tabular}


Table 38. Test data for specimen P22A-13

\begin{tabular}{|c|c|c|c|c|c|}
\hline $\begin{array}{l}\text { Test Number } \\
\text { Aging Temp. } \\
\text { Yield Stress }\end{array}$ & \multicolumn{2}{|c|}{$\begin{array}{l}: 112 \\
: 350^{\circ} \mathrm{C} \\
: 245.5 \mathrm{MPa}\end{array}$} & \multicolumn{2}{|c|}{$\begin{array}{l}\text { Test Temp. } \\
\text { Aging Time } \\
\text { Ultimate Stress }\end{array}$} & $\begin{array}{l}: 25^{\circ} \mathrm{C} \\
: 30,000 \mathrm{~h} \\
: 615.4 \mathrm{MPa}\end{array}$ \\
\hline Engr. & & Engr. & & Engr. & \\
\hline $\begin{array}{l}\text { Stress } \\
(\mathrm{MPa})\end{array}$ & $\begin{array}{l}\text { Engr. } \\
\text { Strain }\end{array}$ & $\begin{array}{l}\text { Stress } \\
(\mathrm{MPa})\end{array}$ & $\begin{array}{l}\text { Engr. } \\
\text { Strain }\end{array}$ & $\begin{array}{l}\text { Stress } \\
(\mathrm{MPa})\end{array}$ & $\begin{array}{l}\text { Engr. } \\
\text { Strain }\end{array}$ \\
\hline 0.00 & 0.0000 & 382.98 & 0.0400 & 570.86 & 0.2623 \\
\hline 92.46 & 0.0003 & 402.17 & 0.0535 & 580.24 & 0.2911 \\
\hline 194.32 & 0.0016 & 424.97 & 0.0691 & 581.92 & 0.2985 \\
\hline 216.44 & 0.0019 & 451.94 & 0.0896 & 583.79 & 0.3018 \\
\hline 228.12 & 0.0021 & 474.33 & 0.1065 & 589.03 & 0.3270 \\
\hline 266.02 & 0.0036 & 479.54 & 0.1115 & 590.51 & 0.3272 \\
\hline 281.00 & 0.0048 & 484.08 & 0.1211 & 601.41 & 0.3777 \\
\hline 301.46 & 0.0076 & 509.21 & 0.1497 & 602.22 & 0.3855 \\
\hline 321.99 & 0.0124 & 509.31 & 0.1496 & 607.76 & 0.4254 \\
\hline 335.17 & 0.0174 & 537.42 & 0.1913 & 614.32 & 0.4766 \\
\hline 358.57 & 0.0266 & 554.68 & 0.2264 & 615.39 & 0.5406 \\
\hline
\end{tabular}

Table 39. Test data for specimen P24A-32

\begin{tabular}{|c|c|c|c|c|c|}
\hline $\begin{array}{l}\text { Test Number } \\
\text { Aging Temp. } \\
\text { Yield Stress }\end{array}$ & & $\mathrm{Pa}$ & $\begin{array}{l}\text { Tes } \\
\text { Agi } \\
\text { Ulti }\end{array}$ & $\begin{array}{l}\text { mp. } \\
\text { me } \\
\text { Stress }\end{array}$ & $\begin{array}{l}25^{\circ} \mathrm{C} \\
30,000 \mathrm{~h} \\
617.2 \mathrm{MPa}\end{array}$ \\
\hline Engr. & & Engr. & & Engr. & \\
\hline $\begin{array}{l}\text { Stress } \\
\text { (MPa) }\end{array}$ & $\begin{array}{l}\text { Engr. } \\
\text { Strain }\end{array}$ & $\begin{array}{l}\text { Stress } \\
\text { (MPa) }\end{array}$ & $\begin{array}{l}\text { Engr. } \\
\text { Strain }\end{array}$ & $\begin{array}{l}\text { Stress } \\
(\mathrm{MPa})\end{array}$ & $\begin{array}{l}\text { Engr. } \\
\text { Strain }\end{array}$ \\
\hline $\begin{array}{r}0.00 \\
187.93 \\
204.63 \\
216.16 \\
254.69 \\
285.18 \\
306.94 \\
336.38\end{array}$ & $\begin{array}{l}0.0000 \\
0.0013 \\
0.0014 \\
0.0017 \\
0.0030 \\
0.0065 \\
0.0101 \\
0.0197\end{array}$ & $\begin{array}{l}359.05 \\
382.89 \\
421.03 \\
444.60 \\
476.19 \\
484.47 \\
510.06\end{array}$ & $\begin{array}{l}0.0305 \\
0.0445 \\
0.0707 \\
0.0878 \\
0.1127 \\
0.1257 \\
0.1561\end{array}$ & $\begin{array}{l}528.87 \\
561.70 \\
580.29 \\
591.20 \\
602.95 \\
612.92 \\
617.20\end{array}$ & $\begin{array}{l}0.1818 \\
0.2462 \\
0.2982 \\
0.3395 \\
0.3998 \\
0.4005 \\
0.5470\end{array}$ \\
\hline
\end{tabular}


Table 40. Test data for specimen P24T-05

\begin{tabular}{|c|c|c|c|c|c|}
\hline \multirow{3}{*}{$\begin{array}{l}\text { Engr. } \\
\text { Stress } \\
\text { (MPa) }\end{array}$} & \multicolumn{2}{|c|}{$\begin{array}{l}: \text { MEA Test } \\
: 400^{\circ} \mathrm{C} \\
: 233.5 \mathrm{MPa}\end{array}$} & \multicolumn{2}{|c|}{$\begin{array}{l}\text { Test Temp. } \\
\text { Aging Time } \\
\text { Ultimate Stress }\end{array}$} & \multirow[t]{2}{*}{$\begin{array}{l}25^{\circ} \mathrm{C} \\
10,000 \mathrm{~h} \\
603.1 \mathrm{MPa}\end{array}$} \\
\hline & & Engr. & & Engr. & \\
\hline & $\begin{array}{l}\text { Engr. } \\
\text { Strain }\end{array}$ & $\begin{array}{l}\text { Stress } \\
(\mathrm{MPa})\end{array}$ & $\begin{array}{l}\text { Engr. } \\
\text { Strain }\end{array}$ & $\begin{array}{l}\text { Stress } \\
(\mathrm{MPa})\end{array}$ & $\begin{array}{l}\text { Engr. } \\
\text { Strain }\end{array}$ \\
\hline 20.13 & 0.0000 & 301.65 & 0.0126 & 534.27 & 0.1735 \\
\hline 40.20 & 0.0003 & 306.54 & 0.0138 & 542.62 & 0.1860 \\
\hline 74.95 & 0.0005 & 311.02 & 0.0149 & 549.72 & 0.1985 \\
\hline 112.38 & 0.0007 & 314.61 & 0.0162 & 558.41 & 0.2112 \\
\hline 147.27 & 0.0010 & 345.36 & 0.0276 & 565.23 & 0.2240 \\
\hline 176.64 & 0.0014 & 373.01 & 0.0399 & 571.64 & 0.2369 \\
\hline 201.53 & 0.0020 & 395.90 & 0.0521 & 575.99 & 0.2497 \\
\hline 220.91 & 0.0028 & 415.34 & 0.0643 & 580.68 & 0.2629 \\
\hline 237.25 & 0.0037 & 433.40 & 0.0763 & 585.09 & 0.2763 \\
\hline 250.28 & 0.0047 & 449.81 & 0.0884 & 590.60 & 0.2898 \\
\hline 261.17 & 0.0057 & 466.09 & 0.1004 & 592.88 & 0.3034 \\
\hline 269.86 & 0.0068 & 479.32 & 0.1124 & 595.57 & 0.3171 \\
\hline 277.51 & 0.0079 & 492.42 & 0.1246 & 599.43 & 0.3312 \\
\hline 284.34 & 0.0090 & 504.63 & 0.1368 & 600.46 & 0.3455 \\
\hline 292.13 & 0.0102 & 513.80 & 0.1489 & 601.77 & 0.3599 \\
\hline 296.96 & 0.0114 & 525.38 & 0.1613 & & \\
\hline
\end{tabular}

Table 41. Test data for specimen P24A-04

\begin{tabular}{|c|c|c|c|c|c|}
\hline $\begin{array}{l}\text { Test Number } \\
\text { Aging Temp. } \\
\text { Yield Stress }\end{array}$ & $\begin{array}{l}: M \\
: 4 C \\
: 23\end{array}$ & & $\begin{array}{l}\text { Tes } \\
\text { Agi } \\
\text { Ulti }\end{array}$ & $\begin{array}{l}\text { np. } \\
\text { ne } \\
\text { Stress }\end{array}$ & $\begin{array}{l}25^{\circ} \mathrm{C} \\
10,000 \mathrm{~h} \\
616.8 \mathrm{MPa}\end{array}$ \\
\hline Engr. & & Engr. & & Engr. & \\
\hline $\begin{array}{l}\text { Stress } \\
\text { (MPa) }\end{array}$ & $\begin{array}{l}\text { Engr. } \\
\text { Strain }\end{array}$ & $\begin{array}{l}\text { Stress } \\
\text { (MPa) }\end{array}$ & $\begin{array}{l}\text { Engr. } \\
\text { Strain }\end{array}$ & $\begin{array}{l}\text { Stress } \\
(\mathrm{MPa})\end{array}$ & $\begin{array}{l}\text { Engr. } \\
\text { Strain }\end{array}$ \\
\hline 23.72 & 0.0002 & 284.41 & 0.0091 & 528.07 & 0.1661 \\
\hline 33.44 & 0.0002 & 287.86 & 0.0101 & 536.89 & 0.1786 \\
\hline 62.74 & 0.0004 & 294.96 & 0.0111 & 544.69 & 0.1912 \\
\hline 93.01 & 0.0005 & 237.66 & 0.0122 & 551.30 & 0.2036 \\
\hline 124.45 & 0.0007 & 303.51 & 0.0133 & 558.27 & 0.2160 \\
\hline 150.58 & 0.0009 & 339.15 & 0.0247 & 565.16 & 0.2283 \\
\hline 177.47 & 0.0012 & 365.70 & 0.0374 & 573.78 & 0.2406 \\
\hline 197.88 & 0.0016 & 388.31 & 0.0498 & 578.54 & 0.2530 \\
\hline 218.15 & 0.0023 & 410.17 & 0.0625 & 582.19 & 0.2652 \\
\hline 232.35 & 0.0031 & 430.09 & 0.0757 & 586.95 & 0.2776 \\
\hline 246.21 & 0.0041 & 447.61 & 0.0892 & 594.26 & 0.2894 \\
\hline 255.31 & 0.0050 & 476.77 & 0.1165 & 596.60 & 0.3015 \\
\hline 263.66 & 0.0060 & 493.87 & 0.1285 & 600.74 & 0.3135 \\
\hline 272.96 & 0.0070 & 504.35 & 0.1410 & 602.33 & 0.3252 \\
\hline 276.82 & 0.0080 & 515.73 & 0.1535 & 606.46 & 0.3367 \\
\hline
\end{tabular}


Table 42. Test data for specimen P21T-02

\begin{tabular}{|c|c|c|c|c|c|}
\hline $\begin{array}{l}\text { Test Number } \\
\text { Aging Temp. } \\
\text { Y }\end{array}$ & $\begin{array}{l}: M \\
: U r\end{array}$ & $\begin{array}{l}\text { est } \\
\mathrm{dPa}\end{array}$ & $\begin{array}{l}\text { Tes } \\
\text { Agi } \\
\text { Ulti }\end{array}$ & $\begin{array}{l}\text { np. } \\
\text { ne } \\
\text { Stress }\end{array}$ & $\begin{array}{l}290^{\circ} \mathrm{C} \\
- \\
387.1 \mathrm{MPa}\end{array}$ \\
\hline Engr. & & Engr. & & Engr. & \\
\hline $\begin{array}{l}\text { Stress } \\
(\mathrm{MPa})\end{array}$ & $\begin{array}{l}\text { Engr. } \\
\text { Strain }\end{array}$ & $\begin{array}{l}\text { Stress } \\
(\mathrm{MPa})\end{array}$ & $\begin{array}{l}\text { Engr. } \\
\text { Strain }\end{array}$ & $\begin{array}{l}\text { Stress } \\
(\mathrm{MPa})\end{array}$ & $\begin{array}{l}\text { Engr. } \\
\text { Strain }\end{array}$ \\
\hline 14.07 & 0.0000 & 181.95 & 0.0074 & 247.11 & 0.0423 \\
\hline 25.86 & 0.0005 & 194.16 & 0.0097 & 250.56 & 0.0465 \\
\hline 36.68 & 0.0007 & 204.02 & 0.0125 & 253.52 & 0.0509 \\
\hline 67.84 & 0.0012 & 211.53 & 0.0153 & 256.14 & 0.0551 \\
\hline 83.63 & 0.0016 & 217.60 & 0.0182 & 259.59 & 0.0594 \\
\hline 100.66 & 0.0020 & 224.56 & 0.0217 & 261.73 & 0.0639 \\
\hline 117.90 & 0.0026 & 230.97 & 0.0257 & 264.28 & 0.0682 \\
\hline 135.00 & 0.0032 & 235.32 & 0.0299 & 267.52 & 0.0726 \\
\hline 152.37 & 0.0043 & 240.01 & 0.0340 & 269.45 & 0.0772 \\
\hline 167.20 & 0.0056 & 243.52 & 0.0381 & & \\
\hline
\end{tabular}

Table 43. Test data for specimen P23T-02

\begin{tabular}{|c|c|c|c|c|c|}
\hline $\begin{array}{l}\text { Test Number } \\
\text { Aging Temp. } \\
\text { Yield Stress }\end{array}$ & $\begin{array}{l}: \mathrm{ME} \\
: \mathrm{Ur} \\
: 15\end{array}$ & $\begin{array}{l}\text { est } \\
\mathrm{d} \\
\mathrm{MPa}\end{array}$ & $\begin{array}{l}\text { Tes } \\
\text { Agi } \\
\text { Ulti }\end{array}$ & $\begin{array}{l}\text { np. } \\
\text { me } \\
\text { Stress }\end{array}$ & $\begin{array}{l}290^{\circ} \mathrm{C} \\
- \\
405.0 \mathrm{MPa}\end{array}$ \\
\hline Engr. & & Engr. & & Engr. & \\
\hline $\begin{array}{l}\text { Stress } \\
\text { (MPa) }\end{array}$ & $\begin{array}{l}\text { Engr. } \\
\text { Strain }\end{array}$ & $\begin{array}{l}\text { Stress } \\
\text { (MPa) }\end{array}$ & $\begin{array}{l}\text { Engr. } \\
\text { Strain }\end{array}$ & $\begin{array}{l}\text { Stress } \\
(\mathrm{MPa})\end{array}$ & $\begin{array}{l}\text { Engr. } \\
\text { Strain }\end{array}$ \\
\hline 4.48 & 0.0001 & 53.71 & 0.0005 & 140.17 & 0.0022 \\
\hline 10.20 & 0.0001 & 70.40 & 0.0008 & 148.51 & 0.0029 \\
\hline 14.89 & 0.0002 & 86.80 & 0.0009 & 154.58 & 0.0037 \\
\hline 20.55 & 0.0003 & 101.70 & 0.0011 & 159.13 & 0.0045 \\
\hline 29.99 & 0.0003 & 117.69 & 0.0013 & 163.61 & 0.0056 \\
\hline 41.71 & 0.0004 & 130.59 & 0.0017 & 166.99 & 0.0064 \\
\hline
\end{tabular}

Table 44. Test data for specimen P22A-02

\begin{tabular}{|c|c|c|c|c|c|}
\hline \multirow[b]{2}{*}{ Engr. } & \multicolumn{2}{|c|}{$\begin{array}{l}: \text { MEA Test } \\
: \text { Unaged } \\
: 137.9 \mathrm{MPa}\end{array}$} & \multicolumn{2}{|c|}{$\begin{array}{l}\text { Test Temp. } \\
\text { Aging Time } \\
\text { Ultimate Stress }\end{array}$} & $\begin{array}{l}290^{\circ} \mathrm{C} \\
- \\
406.9 \mathrm{MPa}\end{array}$ \\
\hline & & Engr. & & Engr. & \\
\hline $\begin{array}{l}\text { Stress } \\
(\mathrm{MPa})\end{array}$ & $\begin{array}{l}\text { Engr. } \\
\text { Strain }\end{array}$ & $\begin{array}{l}\text { Stress } \\
\text { (MPa) }\end{array}$ & $\begin{array}{l}\text { Engr. } \\
\text { Strain }\end{array}$ & $\begin{array}{l}\text { Stress } \\
(\mathrm{MPa})\end{array}$ & $\begin{array}{l}\text { Engr. } \\
\text { Strain }\end{array}$ \\
\hline 10.69 & 0.0002 & 61.78 & 0.0009 & 154.37 & 0.0079 \\
\hline 12.41 & 0.0002 & 74.12 & 0.0011 & 156.58 & 0.0089 \\
\hline 14.27 & 0.0002 & 89.01 & 0.0013 & 159.82 & 0.0099 \\
\hline 15.38 & 0.0002 & 102.32 & 0.0015 & 161.61 & 0.0107 \\
\hline 16.27 & 0.0002 & 113.63 & 0.0019 & 164.16 & 0.0117 \\
\hline 17.37 & 0.0003 & 123.07 & 0.0024 & 166.92 & 0.0125 \\
\hline 18.48 & 0.0003 & 131.07 & 0.0030 & 168.09 & 0.0134 \\
\hline 19.72 & 0.0003 & 136.31 & 0.0037 & 170.37 & 0.0144 \\
\hline 21.03 & 0.0003 & 141.00 & 0.0045 & 172.78 & 0.0152 \\
\hline 25.65 & 0.0004 & 144.86 & 0.0053 & 174.44 & 0.0161 \\
\hline 31.65 & 0.0004 & 148.51 & 0.0062 & 176.37 & 0.0171 \\
\hline
\end{tabular}


Table 45. Test data for specimen P23A-02

$\begin{array}{llll}\text { Test Number } & : \text { MEA Test } & \text { Test Temp. } & : 290^{\circ} \mathrm{C} \\ \text { Aging Temp. } & : \text { Unaged } & \text { Aging Time } & :- \\ \text { Yield Stress } & : 144.0 \mathrm{MPa} & \text { Ultimate Stress } & : 385.1 \mathrm{MPa}\end{array}$

Experimental Data Not Available.

Table 46. Test data for specimen P21A-32

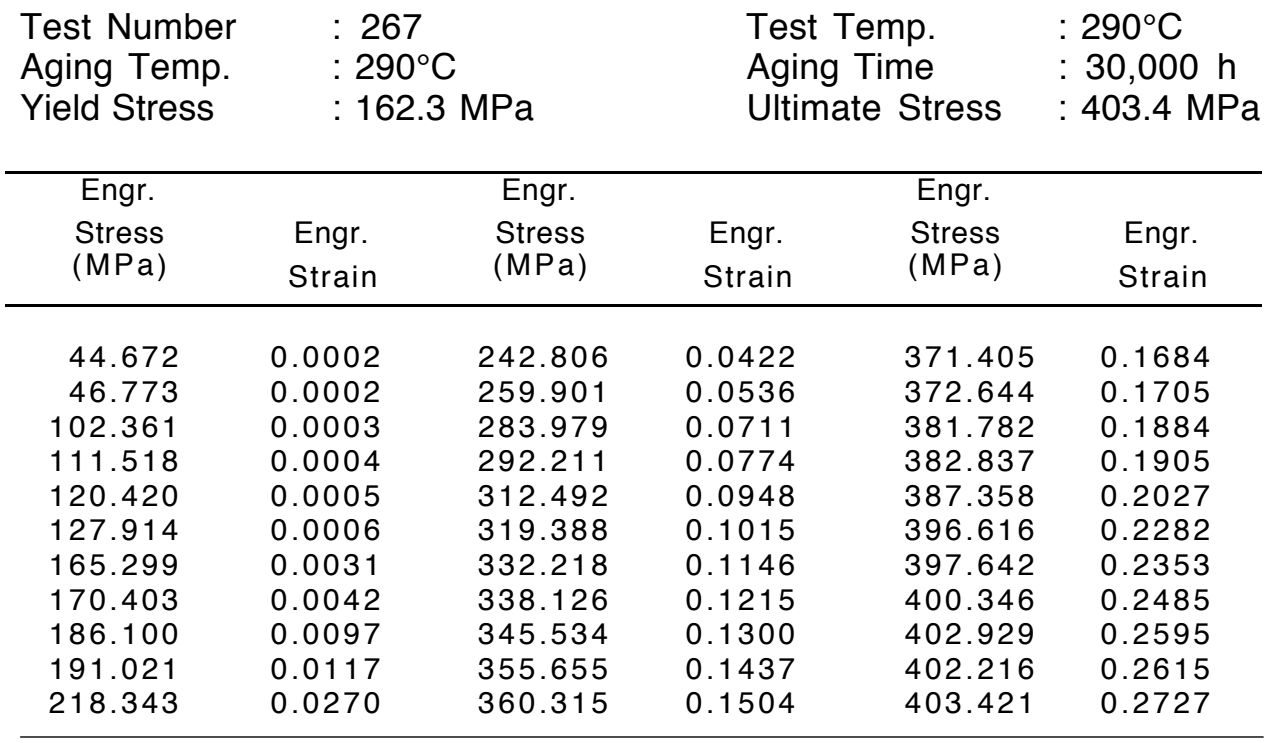

Table 47. Test data for specimen P21A-33

\begin{tabular}{|c|c|c|c|c|c|}
\hline $\begin{array}{l}\text { Test Number } \\
\text { Aging Temp. } \\
\text { Yield Stress }\end{array}$ & $\begin{array}{l}: 0 \\
: 25 \\
: 15\end{array}$ & & $\begin{array}{l}\text { Tes } \\
\text { Agi } \\
\text { Ulti }\end{array}$ & $\begin{array}{l}\text { np. } \\
\text { me } \\
\text { Stress }\end{array}$ & $\begin{array}{l}290^{\circ} \mathrm{C} \\
30,000 \mathrm{~h} \\
405.9 \mathrm{MPa}\end{array}$ \\
\hline Engr. & & Engr. & & Engr. & \\
\hline $\begin{array}{l}\text { Stress } \\
(\mathrm{MPa})\end{array}$ & $\begin{array}{l}\text { Engr. } \\
\text { Strain }\end{array}$ & $\begin{array}{l}\text { Stress } \\
(\mathrm{MPa})\end{array}$ & $\begin{array}{l}\text { Engr. } \\
\text { Strain }\end{array}$ & $\begin{array}{l}\text { Stress } \\
(\mathrm{MPa})\end{array}$ & $\begin{array}{l}\text { Engr. } \\
\text { Strain }\end{array}$ \\
\hline 126.22 & 0.0010 & 231.75 & 0.0186 & 351.63 & 0.1175 \\
\hline 159.63 & 0.0024 & 237.87 & 0.0205 & 354.16 & 0.1213 \\
\hline 164.81 & 0.0030 & 242.83 & 0.0232 & 362.63 & 0.1327 \\
\hline 168.80 & 0.0036 & 250.06 & 0.0283 & 365.60 & 0.1367 \\
\hline 172.02 & 0.0041 & 254.96 & 0.0320 & 368.00 & 0.1402 \\
\hline 179.21 & 0.0057 & 260.16 & 0.0359 & 374.97 & 0.1516 \\
\hline 184.07 & 0.0069 & 266.55 & 0.0410 & 381.91 & 0.1652 \\
\hline 187.40 & 0.0082 & 275.01 & 0.0476 & 384.64 & 0.1709 \\
\hline 192.53 & 0.0102 & 286.24 & 0.0572 & 388.73 & 0.1803 \\
\hline 197.56 & 0.0123 & 297.42 & 0.0666 & 393.29 & 0.1924 \\
\hline 202.25 & 0.0132 & 307.75 & 0.0757 & 396.45 & 0.2015 \\
\hline 204.73 & 0.0139 & 318.13 & 0.0850 & 399.57 & 0.2143 \\
\hline 208.74 & 0.0145 & 328.51 & 0.0948 & 401.57 & 0.2232 \\
\hline 212.31 & 0.0151 & 336.87 & 0.1013 & 403.44 & 0.2319 \\
\hline 217.96 & 0.0161 & 340.72 & 0.1052 & 405.35 & 0.2407 \\
\hline 226.73 & 0.0177 & 345.13 & 0.1101 & 405.91 & 0.2449 \\
\hline
\end{tabular}


Table 48. Test data for specimen P24T-16

$\begin{array}{llll}\text { Test Number } & : \text { MEA Test } & \text { Test Temp. } & : 290^{\circ} \mathrm{C} \\ \text { Aging Temp. } & : 290^{\circ} \mathrm{C} & \text { Aging Time } & : 30,000 \mathrm{~h} \\ \text { Yield Stress } & : 148.2 \mathrm{MPa} & \text { Ultimate Stress } & : 407.0 \mathrm{MPa}\end{array}$

Experimental Data Not Available.

Table 49. Test data for specimen P21A-37

\begin{tabular}{|c|c|c|c|c|c|}
\hline $\begin{array}{l}\text { Test Number } \\
\text { Aging Temp. } \\
\text { Yield Stress }\end{array}$ & $\begin{array}{l}: 3 \\
: 25 \\
: 16\end{array}$ & & $\begin{array}{l}\text { Tes } \\
\text { Agi } \\
\text { Ulti }\end{array}$ & $\begin{array}{l}\text { np. } \\
\text { ne } \\
\text { Stress }\end{array}$ & $\begin{array}{l}290^{\circ} \mathrm{C} \\
58,000 \mathrm{~h} \\
410.7 \mathrm{MPa}\end{array}$ \\
\hline Engr. & & Engr. & & Engr. & \\
\hline $\begin{array}{l}\text { Stress } \\
\text { (MPa) }\end{array}$ & $\begin{array}{l}\text { Engr. } \\
\text { Strain }\end{array}$ & $\begin{array}{l}\text { Stress } \\
(\mathrm{MPa})\end{array}$ & $\begin{array}{l}\text { Engr. } \\
\text { Strain }\end{array}$ & $\begin{array}{l}\text { Stress } \\
(\mathrm{MPa})\end{array}$ & $\begin{array}{l}\text { Engr. } \\
\text { Strain }\end{array}$ \\
\hline 24.800 & 0.000 & 190.360 & 0.009 & 360.330 & 0.134 \\
\hline 59.530 & 0.000 & 196.230 & 0.011 & 390.370 & 0.183 \\
\hline 70.650 & 0.000 & 235.510 & 0.031 & 394.480 & 0.192 \\
\hline 81.700 & 0.000 & 246.880 & 0.038 & 396.880 & 0.199 \\
\hline 112.760 & 0.001 & 261.030 & 0.047 & 400.100 & 0.208 \\
\hline 121.120 & 0.001 & 267.710 & 0.051 & 400.920 & 0.211 \\
\hline 128.690 & 0.001 & 280.710 & 0.060 & 409.240 & 0.243 \\
\hline 135.470 & 0.001 & 298.400 & 0.073 & 409.740 & 0.246 \\
\hline 154.940 & 0.002 & 333.400 & 0.104 & 409.940 & 0.248 \\
\hline 161.630 & 0.003 & 335.600 & 0.106 & 410.590 & 0.257 \\
\hline 167.120 & 0.003 & 351.350 & 0.123 & 410.590 & 0.259 \\
\hline
\end{tabular}

Table 50. Test data for specimen P22T-17

\begin{tabular}{|c|c|c|c|c|c|}
\hline $\begin{array}{l}\text { Test Number } \\
\text { Aging Temp. } \\
\text { Y }\end{array}$ & & & $\begin{array}{l}\text { Tes } \\
\text { Agi } \\
\text { Ulti }\end{array}$ & $\begin{array}{l}\text { np. } \\
\text { ne } \\
\text { Stress }\end{array}$ & $\begin{array}{l}290^{\circ} \mathrm{C} \\
58,000 \mathrm{~h} \\
422.7 \mathrm{MPa}\end{array}$ \\
\hline Engr. & & Engr. & & Engr. & \\
\hline $\begin{array}{l}\text { Stress } \\
(\mathrm{MPa})\end{array}$ & $\begin{array}{l}\text { Engr. } \\
\text { Strain }\end{array}$ & $\begin{array}{l}\text { Stress } \\
(\mathrm{MPa})\end{array}$ & $\begin{array}{l}\text { Engr. } \\
\text { Strain }\end{array}$ & $\begin{array}{l}\text { Stress } \\
(\mathrm{MPa})\end{array}$ & $\begin{array}{l}\text { Engr. } \\
\text { Strain }\end{array}$ \\
\hline 16.930 & 0.000 & 252.800 & 0.035 & 406.590 & 0.200 \\
\hline 20.250 & 0.000 & 256.530 & 0.037 & 409.090 & 0.207 \\
\hline 34.980 & 0.000 & 260.240 & 0.039 & 411.600 & 0.216 \\
\hline 46.610 & 0.000 & 305.830 & 0.070 & 414.150 & 0.225 \\
\hline 58.520 & 0.000 & 319.660 & 0.081 & 414.850 & 0.227 \\
\hline 92.410 & 0.000 & 322.270 & 0.083 & 416.820 & 0.239 \\
\hline 103.720 & 0.000 & 324.820 & 0.085 & 417.460 & 0.241 \\
\hline 151.770 & 0.001 & 374.050 & 0.137 & 418.280 & 0.245 \\
\hline 177.440 & 0.003 & 384.420 & 0.153 & 418.920 & 0.252 \\
\hline 195.040 & 0.006 & 398.700 & 0.179 & 419.580 & 0.254 \\
\hline 200.220 & 0.008 & 399.640 & 0.182 & 420.160 & 0.256 \\
\hline
\end{tabular}


Table 51. Test data for specimen P21A-14

\begin{tabular}{|c|c|c|c|c|c|}
\hline $\begin{array}{l}\text { Test Number } \\
\text { Aging Temp. } \\
\text { Yield Stress }\end{array}$ & $\begin{array}{l}: 07 \\
: 32 \\
: 15\end{array}$ & & $\begin{array}{l}\text { Tes } \\
\text { Agir } \\
\text { Ulti }\end{array}$ & $\begin{array}{l}\text { np. } \\
\text { me } \\
\text { Stress }\end{array}$ & $\begin{array}{l}: 290^{\circ} \mathrm{C} \\
30,000 \mathrm{~h} \\
: 428.9 \mathrm{MPa}\end{array}$ \\
\hline Engr. & & Engr. & & Engr. & \\
\hline $\begin{array}{l}\text { Stress } \\
(\mathrm{MPa})\end{array}$ & $\begin{array}{l}\text { Engr. } \\
\text { Strain }\end{array}$ & $\begin{array}{l}\text { Stress } \\
\text { (MPa) }\end{array}$ & $\begin{array}{l}\text { Engr. } \\
\text { Strain }\end{array}$ & $\begin{array}{l}\text { Stress } \\
(\mathrm{MPa})\end{array}$ & $\begin{array}{l}\text { Engr. } \\
\text { Strain }\end{array}$ \\
\hline 0.00 & 0.0000 & 240.59 & 0.0126 & 339.96 & 0.0811 \\
\hline 126.47 & 0.0007 & 247.15 & 0.0151 & 351.42 & 0.0912 \\
\hline 140.94 & 0.0010 & 251.64 & 0.0176 & 364.20 & 0.1004 \\
\hline 153.12 & 0.0015 & 257.87 & 0.0214 & 376.09 & 0.1122 \\
\hline 161.24 & 0.0019 & 262.91 & 0.0242 & 390.12 & 0.1303 \\
\hline 168.47 & 0.0023 & 268.07 & 0.0276 & 397.02 & 0.1402 \\
\hline 179.44 & 0.0032 & 275.15 & 0.0318 & 404.68 & 0.1528 \\
\hline 188.51 & 0.0046 & 280.09 & 0.0349 & 409.96 & 0.1637 \\
\hline 197.32 & 0.0062 & 285.05 & 0.0386 & 414.54 & 0.1735 \\
\hline 205.07 & 0.0073 & 291.29 & 0.0429 & 418.00 & 0.1823 \\
\hline 212.49 & 0.0082 & 297.23 & 0.0470 & 419.93 & 0.1911 \\
\hline 218.37 & 0.0089 & 302.06 & 0.0503 & 422.02 & 0.2012 \\
\hline 223.76 & 0.0094 & 316.65 & 0.0616 & 424.43 & 0.2107 \\
\hline 228.26 & 0.0100 & 323.63 & 0.0675 & 426.22 & 0.2229 \\
\hline 233.74 & 0.0108 & 332.60 & 0.0748 & 428.89 & 0.2429 \\
\hline
\end{tabular}

Table 52. Test data for specimen P21A-15

\begin{tabular}{|c|c|c|c|c|c|}
\hline $\begin{array}{l}\text { Test Number } \\
\text { Aging Temp. } \\
\text { Yield Stress }\end{array}$ & \multicolumn{2}{|c|}{$\begin{array}{l}: 268 \\
: 320^{\circ} \mathrm{C} \\
: 163.9 \mathrm{MPa}\end{array}$} & \multicolumn{2}{|c|}{$\begin{array}{l}\text { Test Temp. } \\
\text { Aging Time } \\
\text { Ultimate Stress }\end{array}$} & $\begin{array}{l}290^{\circ} \mathrm{C} \\
30,000 \mathrm{~h} \\
422.7 \mathrm{MPa}\end{array}$ \\
\hline \multicolumn{2}{|l|}{ Engr. } & \multicolumn{2}{|l|}{ Engr. } & \multicolumn{2}{|l|}{ Engr. } \\
\hline $\begin{array}{l}\text { Stress } \\
(\mathrm{MPa})\end{array}$ & $\begin{array}{l}\text { Engr. } \\
\text { Strain }\end{array}$ & $\begin{array}{l}\text { Stress } \\
\text { (MPa) }\end{array}$ & $\begin{array}{l}\text { Engr. } \\
\text { Strain }\end{array}$ & $\begin{array}{l}\text { Stress } \\
(\mathrm{MPa})\end{array}$ & $\begin{array}{l}\text { Engr. } \\
\text { Strain }\end{array}$ \\
\hline 41.587 & 0.0002 & 181.122 & 0.0053 & 352.778 & 0.1018 \\
\hline 57.834 & 0.0002 & 202.641 & 0.0119 & 368.889 & 0.1170 \\
\hline 67.757 & 0.0002 & 207.981 & 0.0142 & 371.000 & 0.1192 \\
\hline 87.450 & 0.0002 & 212.072 & 0.0162 & 390.322 & 0.1413 \\
\hline 97.436 & 0.0003 & 221.866 & 0.0205 & 398.230 & 0.1522 \\
\hline 106.786 & 0.0003 & 267.526 & 0.0440 & 403.850 & 0.1608 \\
\hline 115.687 & 0.0004 & 274.437 & 0.0481 & 410.038 & 0.1718 \\
\hline 123.186 & 0.0006 & 282.514 & 0.0526 & 415.142 & 0.1827 \\
\hline 130.199 & 0.0006 & 292.529 & 0.0586 & 419.248 & 0.1936 \\
\hline 169.039 & 0.0033 & 302.756 & 0.0651 & 420.728 & 0.1997 \\
\hline 175.526 & 0.0042 & 318.588 & 0.0756 & 421.191 & 0.2021 \\
\hline
\end{tabular}


Table 53. Test data for specimen P21A-16

\begin{tabular}{|c|c|c|c|c|c|}
\hline $\begin{array}{l}\text { Test Number } \\
\text { Aging Temp. } \\
\text { Yield Stress }\end{array}$ & $\begin{array}{l}: 0 \\
: 32 \\
: 15\end{array}$ & & $\begin{array}{l}\text { Tes } \\
\text { Agir } \\
\text { Ulti }\end{array}$ & $\begin{array}{l}\text { np. } \\
\text { ne } \\
\text { Stress }\end{array}$ & $\begin{array}{l}290^{\circ} \mathrm{C} \\
30,000 \mathrm{~h} \\
407.7 \mathrm{MPa}\end{array}$ \\
\hline Engr. & & Engr. & & Engr. & \\
\hline $\begin{array}{l}\text { Stress } \\
(\mathrm{MPa})\end{array}$ & $\begin{array}{l}\text { Engr. } \\
\text { Strain }\end{array}$ & $\begin{array}{l}\text { Stress } \\
(\mathrm{MPa})\end{array}$ & $\begin{array}{l}\text { Engr. } \\
\text { Strain }\end{array}$ & $\begin{array}{l}\text { Stress } \\
(\mathrm{MPa})\end{array}$ & $\begin{array}{l}\text { Engr. } \\
\text { Strain }\end{array}$ \\
\hline 117.51 & 0.0008 & 276.83 & 0.0394 & 369.41 & 0.1158 \\
\hline 129.27 & 0.0011 & 288.36 & 0.0476 & 372.84 & 0.1193 \\
\hline 139.60 & 0.0014 & 297.98 & 0.0550 & 378.80 & 0.1272 \\
\hline 151.74 & 0.0020 & 305.49 & 0.0606 & 381.74 & 0.1318 \\
\hline 162.95 & 0.0028 & 312.11 & 0.0658 & 385.80 & 0.1367 \\
\hline 175.68 & 0.0043 & 317.36 & 0.0701 & 388.53 & 0.1428 \\
\hline 186.25 & 0.0062 & 323.09 & 0.0746 & 392.00 & 0.1497 \\
\hline 195.49 & 0.0086 & 329.66 & 0.0803 & 393.84 & 0.1562 \\
\hline 202.07 & 0.0098 & 334.76 & 0.0849 & 396.52 & 0.1628 \\
\hline 207.07 & 0.0106 & 340.40 & 0.0894 & 399.06 & 0.1698 \\
\hline 218.81 & 0.0121 & 346.73 & 0.0946 & 401.88 & 0.1771 \\
\hline 230.35 & 0.0137 & 351.53 & 0.0993 & 403.67 & 0.1841 \\
\hline 243.26 & 0.0183 & 357.51 & 0.1028 & 405.27 & 0.1916 \\
\hline 252.14 & 0.0232 & 362.26 & 0.1077 & 407.10 & 0.2028 \\
\hline 264.26 & 0.0310 & 365.54 & 0.1113 & 407.71 & 0.2095 \\
\hline
\end{tabular}

Table 54. Test data for specimen P25T-12

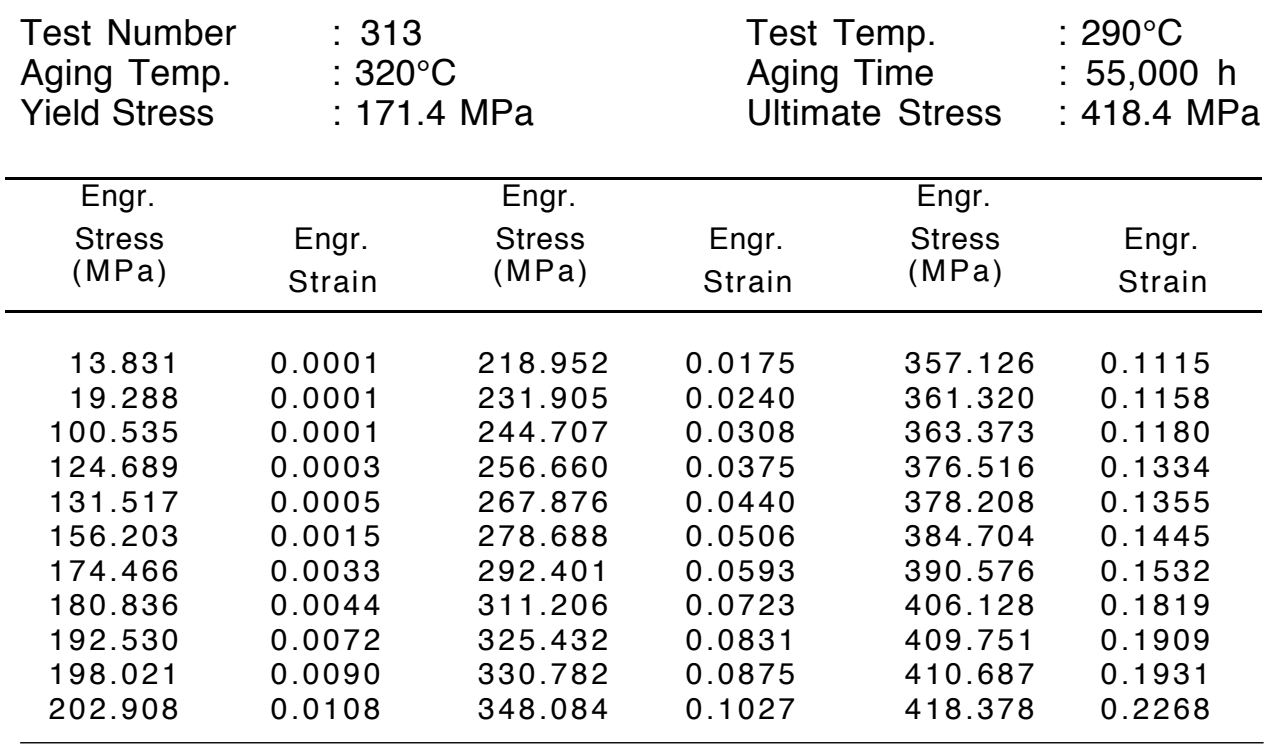


Table 55. Test data for specimen P21A-18

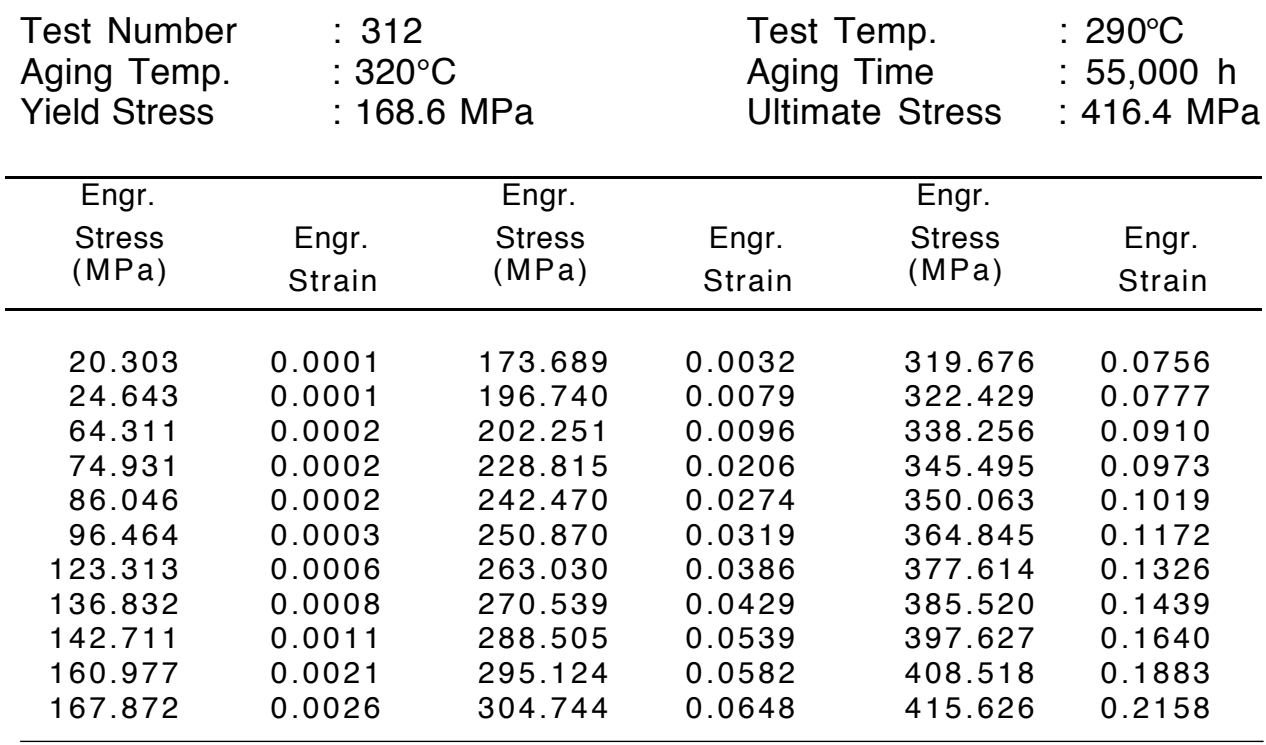

Table 56. Test data for specimen P21T-08

\begin{tabular}{|c|c|c|c|c|c|}
\hline $\begin{array}{l}\text { Test Number } \\
\text { Aging Temp. } \\
\text { Yield Stress }\end{array}$ & $\begin{array}{l}: \mathrm{ME} \\
: 35 \\
: 15\end{array}$ & $\mathrm{st}$ & $\begin{array}{l}\text { Tes } \\
\text { Agi } \\
\text { Ulti }\end{array}$ & $\begin{array}{l}\text { np. } \\
\text { ne } \\
\text { Stress }\end{array}$ & $\begin{array}{l}290^{\circ} \mathrm{C} \\
10,000 \mathrm{~h} \\
423.6 \mathrm{MPa}\end{array}$ \\
\hline Engr. & & Engr. & & Engr. & \\
\hline $\begin{array}{l}\text { Stress } \\
(\mathrm{MPa})\end{array}$ & $\begin{array}{l}\text { Engr. } \\
\text { Strain }\end{array}$ & $\begin{array}{l}\text { Stress } \\
(\mathrm{MPa})\end{array}$ & $\begin{array}{l}\text { Engr. } \\
\text { Strain }\end{array}$ & $\begin{array}{l}\text { Stress } \\
(\mathrm{MPa})\end{array}$ & $\begin{array}{l}\text { Engr. } \\
\text { Strain }\end{array}$ \\
\hline 37.65 & 0.0002 & 202.84 & 0.0139 & 359.91 & 0.1300 \\
\hline 60.05 & 0.0003 & 206.57 & 0.0155 & 371.56 & 0.1418 \\
\hline 85.01 & 0.0005 & 209.88 & 0.0170 & 379.90 & 0.1537 \\
\hline 112.80 & 0.0008 & 212.77 & 0.0184 & 385.14 & 0.1657 \\
\hline 132.66 & 0.0014 & 233.04 & 0.0288 & 393.00 & 0.1780 \\
\hline 150.31 & 0.0024 & 252.97 & 0.0402 & 397.34 & 0.1906 \\
\hline 162.79 & 0.0036 & 270.96 & 0.0513 & 402.24 & 0.2035 \\
\hline 171.82 & 0.0049 & 288.41 & 0.0624 & 407.55 & 0.2168 \\
\hline 179.47 & 0.0064 & 303.78 & 0.0735 & 411.13 & 0.2305 \\
\hline 185.47 & 0.0079 & 316.68 & 0.0847 & 414.86 & 0.2448 \\
\hline 190.16 & 0.0094 & 330.26 & 0.0958 & 417.48 & 0.2593 \\
\hline 195.19 & 0.0109 & 341.64 & 0.1071 & 417.75 & 0.2756 \\
\hline 198.84 & 0.0125 & 352.39 & 0.1184 & 420.37 & 0.2938 \\
\hline
\end{tabular}


Table 57. Test data for specimen P22T-05

\begin{tabular}{|c|c|c|c|c|c|}
\hline $\begin{array}{l}\text { Test Number } \\
\text { Aging Temp. } \\
\text { Yield Stress }\end{array}$ & $\begin{array}{l}: M \\
: 35 \\
: 15\end{array}$ & & $\begin{array}{l}\text { Tes } \\
\text { Agi } \\
\text { Ulti }\end{array}$ & $\begin{array}{l}\text { mp. } \\
\text { me } \\
\text { Stress }\end{array}$ & $\begin{array}{l}290^{\circ} \mathrm{C} \\
10,000 \mathrm{~h} \\
399.0 \mathrm{MPa}\end{array}$ \\
\hline Engr. & & Engr. & & Engr. & \\
\hline $\begin{array}{l}\text { Stress } \\
(\mathrm{MPa})\end{array}$ & $\begin{array}{l}\text { Engr. } \\
\text { Strain }\end{array}$ & $\begin{array}{l}\text { Stress } \\
(\mathrm{MPa})\end{array}$ & $\begin{array}{l}\text { Engr. } \\
\text { Strain }\end{array}$ & $\begin{array}{l}\text { Stress } \\
(\mathrm{MPa})\end{array}$ & $\begin{array}{l}\text { Engr. } \\
\text { Strain }\end{array}$ \\
\hline 22.55 & 0.0002 & 195.60 & 0.0136 & 338.12 & 0.1086 \\
\hline 26.75 & 0.0002 & 200.36 & 0.0151 & 345.15 & 0.1180 \\
\hline 50.06 & 0.0003 & 202.91 & 0.0164 & 353.84 & 0.1275 \\
\hline 80.46 & 0.0005 & 206.08 & 0.0178 & 360.60 & 0.1370 \\
\hline 113.97 & 0.0008 & 208.50 & 0.0191 & 366.46 & 0.1468 \\
\hline 135.48 & 0.0016 & 224.91 & 0.0271 & 372.87 & 0.1567 \\
\hline 150.17 & 0.0026 & 241.73 & 0.0362 & 377.35 & 0.1668 \\
\hline 161.13 & 0.0038 & 256.90 & 0.0452 & 381.14 & 0.1773 \\
\hline 168.58 & 0.0051 & 271.52 & 0.0542 & 386.73 & 0.1881 \\
\hline 173.82 & 0.0065 & 283.86 & 0.0632 & 388.59 & 0.1993 \\
\hline 180.64 & 0.0079 & 296.20 & 0.0723 & 391.83 & 0.2110 \\
\hline 184.37 & 0.0094 & 307.78 & 0.0813 & 396.10 & 0.2236 \\
\hline 189.47 & 0.0108 & 318.12 & 0.0904 & 397.48 & 0.2380 \\
\hline 193.33 & 0.0122 & 328.33 & 0.0995 & 398.79 & 0.2563 \\
\hline
\end{tabular}

Table 58. Test data for specimen P23A-15

\begin{tabular}{|c|c|c|c|c|c|}
\hline $\begin{array}{l}\text { Test Number } \\
\text { Aging Temp. } \\
\text { Yield Stress }\end{array}$ & $\begin{array}{l}: M \\
: 35 \\
: 16\end{array}$ & st & $\begin{array}{l}\text { Tes } \\
\text { Agi } \\
\text { Ulti }\end{array}$ & $\begin{array}{l}\text { np. } \\
\text { me } \\
\text { Stress }\end{array}$ & $\begin{array}{l}290^{\circ} \mathrm{C} \\
10,000 \mathrm{~h} \\
415.4 \mathrm{MPa}\end{array}$ \\
\hline Engr. & & Engr. & & Engr. & \\
\hline $\begin{array}{l}\text { Stress } \\
(\mathrm{MPa})\end{array}$ & $\begin{array}{l}\text { Engr. } \\
\text { Strain }\end{array}$ & $\begin{array}{l}\text { Stress } \\
(\mathrm{MPa})\end{array}$ & $\begin{array}{l}\text { Engr. } \\
\text { Strain }\end{array}$ & $\begin{array}{l}\text { Stress } \\
(\mathrm{MPa})\end{array}$ & $\begin{array}{l}\text { Engr. } \\
\text { Strain }\end{array}$ \\
\hline 10.69 & 0.0002 & 61.78 & 0.0009 & 154.37 & 0.0079 \\
\hline 12.41 & 0.0002 & 74.12 & 0.0011 & 156.58 & 0.0089 \\
\hline 14.27 & 0.0002 & 89.01 & 0.0013 & 159.82 & 0.0099 \\
\hline 15.38 & 0.0002 & 102.32 & 0.0015 & 161.61 & 0.0107 \\
\hline 16.27 & 0.0002 & 113.63 & 0.0019 & 164.16 & 0.0117 \\
\hline 17.37 & 0.0003 & 123.07 & 0.0024 & 166.92 & 0.0125 \\
\hline 18.48 & 0.0003 & 131.07 & 0.0030 & 168.09 & 0.0134 \\
\hline 19.72 & 0.0003 & 136.31 & 0.0037 & 170.37 & 0.0144 \\
\hline 21.03 & 0.0003 & 141.00 & 0.0045 & 172.78 & 0.0152 \\
\hline 25.65 & 0.0004 & 144.86 & 0.0053 & 174.44 & 0.0161 \\
\hline 31.65 & 0.0004 & 148.51 & 0.0062 & 176.37 & 0.0171 \\
\hline 39.85 & 0.0006 & 151.55 & 0.0071 & 178.51 & 0.0179 \\
\hline 49.64 & 0.0007 & & & & \\
\hline
\end{tabular}


Table 59. Test data for specimen P23A-27

\begin{tabular}{|c|c|c|c|c|c|}
\hline $\begin{array}{l}\text { Test Number } \\
\text { Aging Temp. } \\
\text { Yield Stress }\end{array}$ & \multicolumn{2}{|c|}{$\begin{array}{l}: \text { MEA Test } \\
: 350^{\circ} \mathrm{C} \\
: 154.1 \mathrm{MPa}\end{array}$} & \multicolumn{2}{|c|}{$\begin{array}{l}\text { Test Temp. } \\
\text { Aging Time } \\
\text { Ultimate Stress }\end{array}$} & $\begin{array}{l}290^{\circ} \mathrm{C} \\
10,000 \mathrm{~h} \\
419.4 \mathrm{MPa}\end{array}$ \\
\hline \multirow{2}{*}{$\begin{array}{l}\text { Engr. } \\
\text { Stress } \\
\text { (MPa) }\end{array}$} & \multicolumn{2}{|r|}{ Engr. } & \multicolumn{3}{|c|}{ Engr. } \\
\hline & $\begin{array}{l}\text { Engr. } \\
\text { Strain }\end{array}$ & $\begin{array}{l}\text { Stress } \\
(\mathrm{MPa})\end{array}$ & $\begin{array}{l}\text { Engr. } \\
\text { Strain }\end{array}$ & $\begin{array}{l}\text { Stress } \\
(\mathrm{MPa})\end{array}$ & $\begin{array}{l}\text { Engr. } \\
\text { Strain }\end{array}$ \\
\hline 21.99 & 0.0002 & 205.19 & 0.0138 & 359.35 & 0.1180 \\
\hline 31.44 & 0.0002 & 207.60 & 0.0151 & 366.94 & 0.1275 \\
\hline 63.57 & 0.0004 & 211.94 & 0.0164 & 374.11 & 0.1371 \\
\hline 98.73 & 0.0006 & 215.05 & 0.0177 & 380.73 & 0.1469 \\
\hline 121.69 & 0.0012 & 217.94 & 0.0190 & 387.76 & 0.1568 \\
\hline 141.07 & 0.0020 & 234.15 & 0.0271 & 392.52 & 0.1668 \\
\hline 154.03 & 0.0029 & 251.18 & 0.0361 & 396.72 & 0.1770 \\
\hline 164.99 & 0.0039 & 266.07 & 0.0450 & 402.93 & 0.1875 \\
\hline 172.51 & 0.0050 & 281.58 & 0.0542 & 406.38 & 0.1983 \\
\hline 178.85 & 0.0062 & 296.13 & 0.0632 & 409.34 & 0.2094 \\
\hline 185.47 & 0.0074 & 308.40 & 0.0721 & 413.34 & 0.2204 \\
\hline 188.78 & 0.0086 & 319.64 & 0.0813 & 415.68 & 0.2333 \\
\hline 194.98 & 0.0099 & 330.53 & 0.0902 & 417.62 & 0.2471 \\
\hline 197.40 & 0.0112 & 340.74 & 0.0993 & 418.24 & 0.2568 \\
\hline 200.91 & 0.0125 & 350.67 & 0.1086 & & \\
\hline
\end{tabular}

Table 60. Test data for specimen P22T-12

\begin{tabular}{|c|c|c|c|c|c|}
\hline $\begin{array}{l}\text { Test Number } \\
\text { Aging Temp. } \\
\text { Yield Stress }\end{array}$ & $\begin{array}{l}: 0 \\
: 35 \\
: 16\end{array}$ & & $\begin{array}{l}\text { Tes } \\
\text { Agir } \\
\text { Ulti }\end{array}$ & $\begin{array}{l}\mathrm{np} . \\
\text { me } \\
\text { Stress }\end{array}$ & $\begin{array}{l}290^{\circ} \mathrm{C} \\
30,000 \mathrm{~h} \\
436.2 \mathrm{MPa}\end{array}$ \\
\hline Engr. & & Engr. & & Engr. & \\
\hline $\begin{array}{l}\text { Stress } \\
\text { (MPa) }\end{array}$ & $\begin{array}{l}\text { Engr. } \\
\text { Strain }\end{array}$ & $\begin{array}{l}\text { Stress } \\
\text { (MPa) }\end{array}$ & $\begin{array}{l}\text { Engr. } \\
\text { Strain }\end{array}$ & $\begin{array}{l}\text { Stress } \\
(\mathrm{MPa})\end{array}$ & $\begin{array}{l}\text { Engr. } \\
\text { Strain }\end{array}$ \\
\hline 130.91 & 0.0009 & 268.41 & 0.0233 & 395.75 & 0.1236 \\
\hline 153.22 & 0.0015 & 277.06 & 0.0282 & 399.34 & 0.1286 \\
\hline 163.93 & 0.0019 & 285.77 & 0.0337 & 403.47 & 0.1335 \\
\hline 174.58 & 0.0026 & 296.03 & 0.0405 & 407.24 & 0.1393 \\
\hline 183.67 & 0.0034 & 304.54 & 0.0461 & 411.61 & 0.1450 \\
\hline 195.59 & 0.0050 & 315.14 & 0.0538 & 414.86 & 0.1495 \\
\hline 203.53 & 0.0058 & 320.76 & 0.0580 & 418.07 & 0.1533 \\
\hline 211.42 & 0.0063 & 325.59 & 0.0620 & 421.65 & 0.1645 \\
\hline 218.28 & 0.0069 & 333.05 & 0.0683 & 424.69 & 0.1706 \\
\hline 224.89 & 0.0074 & 341.35 & 0.0749 & 428.54 & 0.1836 \\
\hline 230.92 & 0.0080 & 350.28 & 0.0819 & 430.45 & 0.1904 \\
\hline 239.05 & 0.0098 & 357.50 & 0.0881 & 432.49 & 0.1978 \\
\hline 244.67 & 0.0121 & 365.29 & 0.0947 & 434.25 & 0.2047 \\
\hline 253.04 & 0.0154 & 376.10 & 0.1022 & 435.40 & 0.2131 \\
\hline 261.70 & 0.0198 & 387.44 & 0.1140 & 436.23 & 0.2183 \\
\hline
\end{tabular}


Table 61. Test data for specimen P24A-30

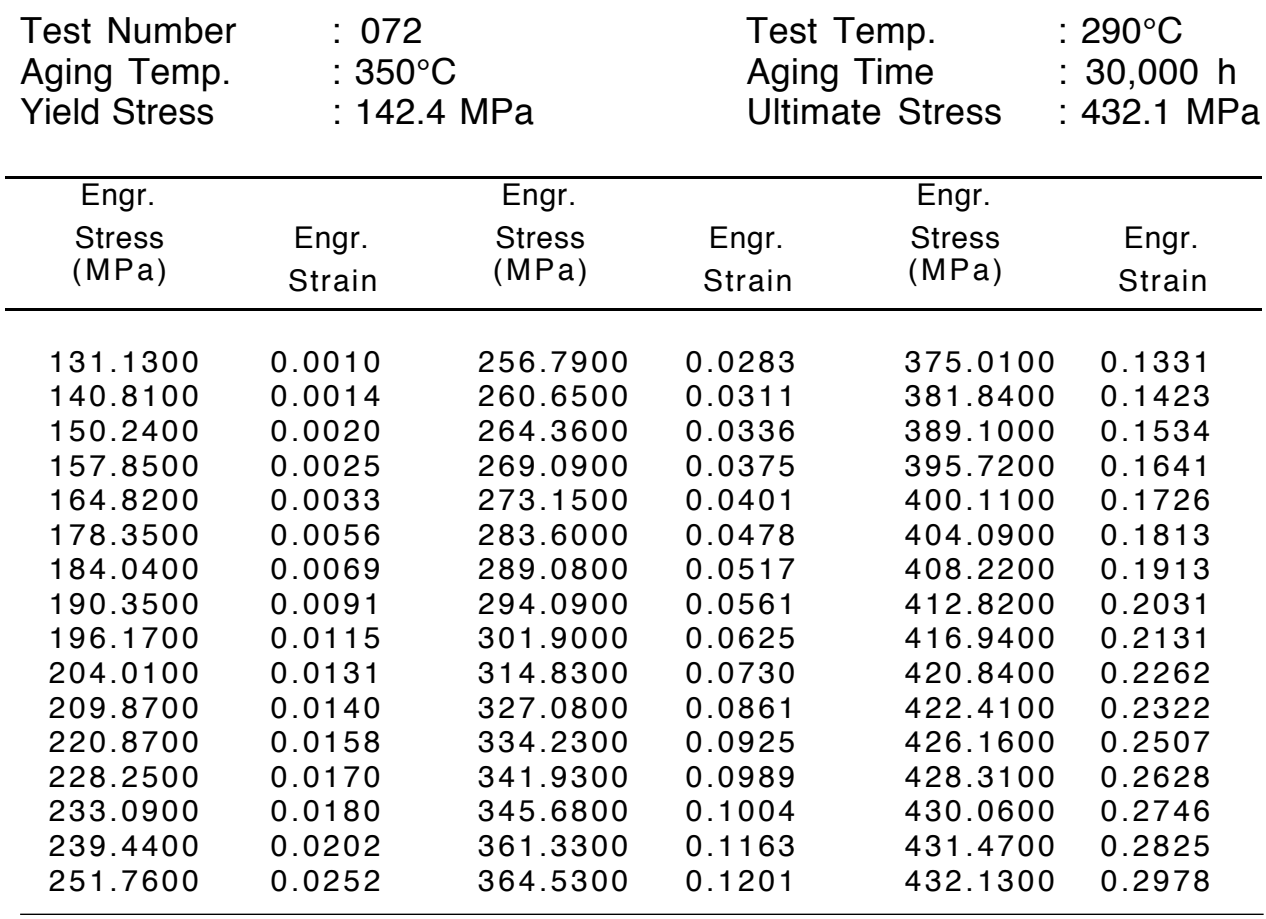

Table 62. Test data for specimen P24A-33

\begin{tabular}{|c|c|c|c|c|c|}
\hline $\begin{array}{l}\text { Test Number } \\
\text { Aging Temp. } \\
\text { Yield Stress }\end{array}$ & $\begin{array}{l}: 2 \\
: 35 \\
: 12\end{array}$ & & $\begin{array}{l}\text { Tes } \\
\text { Agi } \\
\text { Ulti }\end{array}$ & $\begin{array}{l}\text { np. } \\
\text { ne } \\
\text { Stress }\end{array}$ & $\begin{array}{l}290^{\circ} \mathrm{C} \\
30,000 \mathrm{~h} \\
425.7 \mathrm{MPa}\end{array}$ \\
\hline Engr. & & Engr. & & Engr. & \\
\hline $\begin{array}{l}\text { Stress } \\
(\mathrm{MPa})\end{array}$ & $\begin{array}{l}\text { Engr. } \\
\text { Strain }\end{array}$ & $\begin{array}{l}\text { Stress } \\
(\mathrm{MPa})\end{array}$ & $\begin{array}{l}\text { Engr. } \\
\text { Strain }\end{array}$ & $\begin{array}{l}\text { Stress } \\
(\mathrm{MPa})\end{array}$ & $\begin{array}{l}\text { Engr. } \\
\text { Strain }\end{array}$ \\
\hline 40.469 & 0.0002 & 174.335 & 0.0071 & 377.208 & 0.1414 \\
\hline 62.385 & 0.0002 & 191.185 & 0.0125 & 387.253 & 0.1546 \\
\hline 72.151 & 0.0002 & 224.120 & 0.0276 & 391.765 & 0.1609 \\
\hline 99.523 & 0.0004 & 228.372 & 0.0300 & 403.010 & 0.1784 \\
\hline 107.616 & 0.0005 & 255.185 & 0.0450 & 404.316 & 0.1805 \\
\hline 114.890 & 0.0005 & 274.007 & 0.0561 & 411.705 & 0.1958 \\
\hline 120.765 & 0.0007 & 316.520 & 0.0848 & 415.330 & 0.2043 \\
\hline 125.966 & 0.0009 & 324.415 & 0.0910 & 415.990 & 0.2064 \\
\hline 134.208 & 0.0013 & 345.233 & 0.1085 & 422.545 & 0.2264 \\
\hline 141.149 & 0.0018 & 347.565 & 0.1106 & 424.256 & 0.2331 \\
\hline 169.298 & 0.0057 & 370.991 & 0.1344 & 425.780 & 0.2460 \\
\hline
\end{tabular}


Table 63. Test data for specimen P24T-06

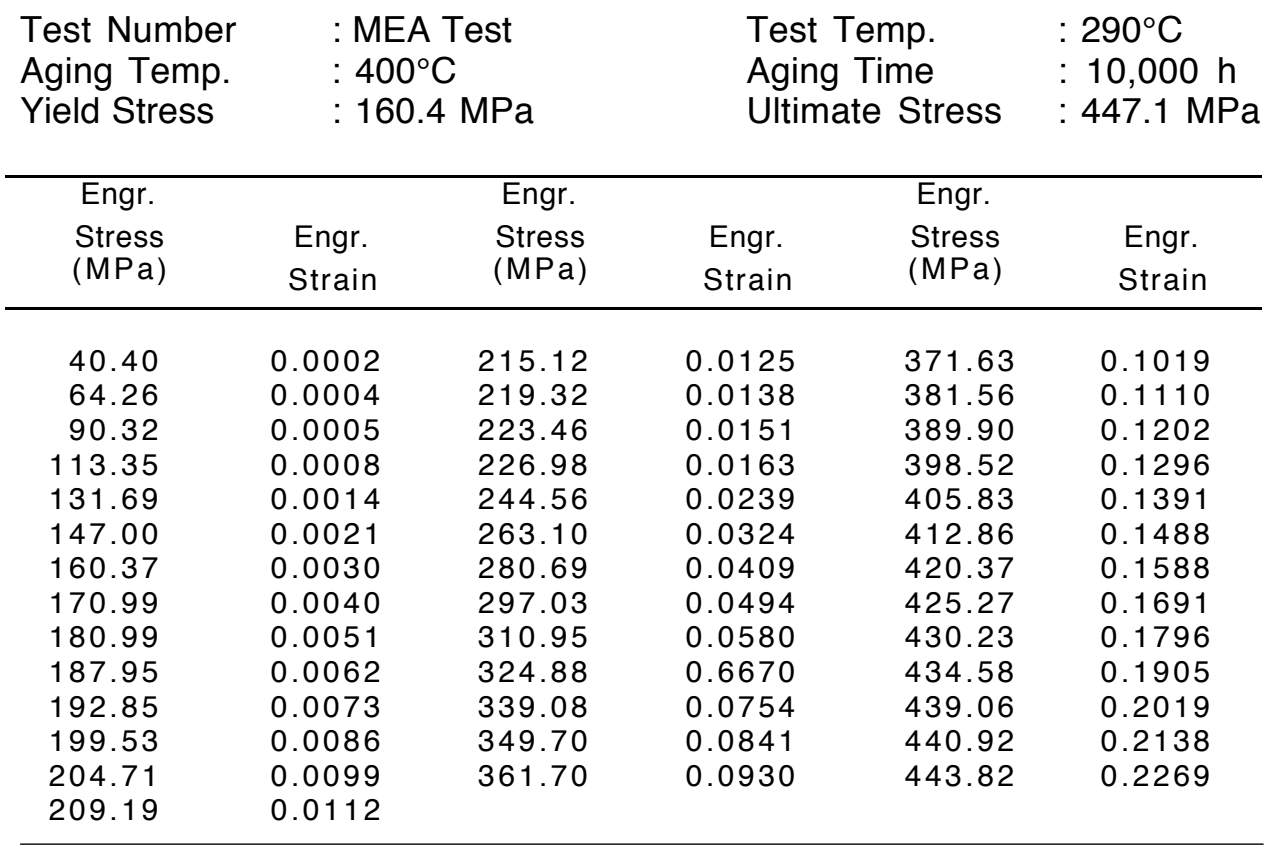

Table 64. Test data for specimen P24A-05

\begin{tabular}{|c|c|c|c|c|c|}
\hline $\begin{array}{l}\text { Test Number } \\
\text { Aging Temp. } \\
\text { Y }\end{array}$ & $\begin{array}{l}: M \\
: 4 C \\
: 14\end{array}$ & $\begin{array}{l}\text { est } \\
\mathrm{MPa}\end{array}$ & $\begin{array}{l}\text { Tes } \\
\text { Agi } \\
\text { Ulti }\end{array}$ & $\begin{array}{l}\text { np. } \\
\text { ne } \\
\text { Stress }\end{array}$ & $\begin{array}{l}290^{\circ} \mathrm{C} \\
10,000 \mathrm{~h} \\
430.6 \mathrm{MPa}\end{array}$ \\
\hline Engr. & & Engr. & & Engr. & \\
\hline $\begin{array}{l}\text { Stress } \\
(\mathrm{MPa})\end{array}$ & $\begin{array}{l}\text { Engr. } \\
\text { Strain }\end{array}$ & $\begin{array}{l}\text { Stress } \\
(\mathrm{MPa})\end{array}$ & $\begin{array}{l}\text { Engr. } \\
\text { Strain }\end{array}$ & $\begin{array}{l}\text { Stress } \\
(\mathrm{MPa})\end{array}$ & $\begin{array}{l}\text { Engr. } \\
\text { Strain }\end{array}$ \\
\hline 56.54 & 0.0003 & 207.05 & 0.0169 & 365.42 & 0.1241 \\
\hline 87.77 & 0.0006 & 212.70 & 0.0184 & 374.52 & 0.1340 \\
\hline 115.07 & 0.0011 & 215.19 & 0.0198 & 381.62 & 0.1442 \\
\hline 133.07 & 0.0020 & 218.22 & 0.0212 & 389.14 & 0.1546 \\
\hline 146.58 & 0.0030 & 236.83 & 0.0300 & 395.07 & 0.1650 \\
\hline 157.48 & 0.0041 & 255.45 & 0.0395 & 401.69 & 0.1757 \\
\hline 166.30 & 0.0053 & 271.58 & 0.0489 & 406.79 & 0.1868 \\
\hline 173.47 & 0.0066 & 287.24 & 0.0581 & 411.69 & 0.1980 \\
\hline 180.37 & 0.0080 & 299.16 & 0.0673 & 416.37 & 0.2096 \\
\hline 186.02 & 0.0095 & 312.95 & 0.0767 & 420.37 & 0.2218 \\
\hline 190.09 & 0.0109 & 325.02 & 0.0860 & 423.34 & 0.2342 \\
\hline 195.60 & 0.0124 & 336.46 & 0.0953 & 425.41 & 0.2470 \\
\hline 200.15 & 0.0139 & 347.50 & 0.1048 & 427.75 & 0.2608 \\
\hline 202.91 & 0.0154 & 357.42 & 0.1144 & 428.92 & 0.2753 \\
\hline
\end{tabular}


Table 65. Test data for specimen 693-40

\begin{tabular}{|c|c|c|c|c|c|}
\hline $\begin{array}{l}\text { Test Number } \\
\text { Aging Temp. } \\
\text { Yield Stress }\end{array}$ & $\begin{array}{l}: \mathrm{M} \\
: \mathrm{Ur} \\
: 27\end{array}$ & $\begin{array}{l}\text { est } \\
\mathrm{d} \\
\mathrm{MPa}\end{array}$ & $\begin{array}{l}\text { Tes } \\
\text { Agi } \\
\text { Ulti }\end{array}$ & $\begin{array}{l}\text { np. } \\
\text { ne } \\
\text { Stress }\end{array}$ & $\begin{array}{l}25^{\circ} \mathrm{C} \\
- \\
606.0 \mathrm{MPa}\end{array}$ \\
\hline Engr. & & Engr. & & Engr. & \\
\hline $\begin{array}{l}\text { Stress } \\
(\mathrm{MPa})\end{array}$ & $\begin{array}{l}\text { Engr. } \\
\text { Strain }\end{array}$ & $\begin{array}{l}\text { Stress } \\
(\mathrm{MPa})\end{array}$ & $\begin{array}{l}\text { Engr. } \\
\text { Strain }\end{array}$ & $\begin{array}{l}\text { Stress } \\
(\mathrm{MPa})\end{array}$ & $\begin{array}{l}\text { Engr. } \\
\text { Strain }\end{array}$ \\
\hline 16.62 & 0.0001 & 360.73 & 0.0266 & 562.20 & 0.3066 \\
\hline 34.27 & 0.0002 & 389.00 & 0.0449 & 570.20 & 0.3265 \\
\hline 73.77 & 0.0004 & 412.44 & 0.0630 & 573.02 & 0.3465 \\
\hline 118.45 & 0.0007 & 433.40 & 0.0811 & 578.88 & 0.3669 \\
\hline 143.34 & 0.0008 & 450.64 & 0.0991 & 583.23 & 0.3876 \\
\hline 195.81 & 0.0012 & 464.64 & 0.1172 & 586.61 & 0.4084 \\
\hline 219.46 & 0.0015 & 481.32 & 0.1356 & 590.95 & 0.4294 \\
\hline 239.87 & 0.0020 & 492.84 & 0.1539 & 592.88 & 0.4509 \\
\hline 258.35 & 0.0026 & 505.25 & 0.1725 & 597.02 & 0.4728 \\
\hline 274.20 & 0.0034 & 515.11 & 0.1912 & 599.50 & 0.4952 \\
\hline 288.27 & 0.0043 & 523.45 & 0.2099 & 600.88 & 0.5182 \\
\hline 296.89 & 0.0053 & 532.07 & 0.2289 & 601.98 & 0.5421 \\
\hline 305.85 & 0.0064 & 541.79 & 0.2480 & 605.98 & 0.5671 \\
\hline 312.13 & 0.0075 & 551.17 & 0.2675 & 604.26 & 0.5947 \\
\hline 316.95 & 0.0088 & 555.37 & 0.2869 & 580.26 & 0.6277 \\
\hline
\end{tabular}

Table 66. Test data for specimen 693-41

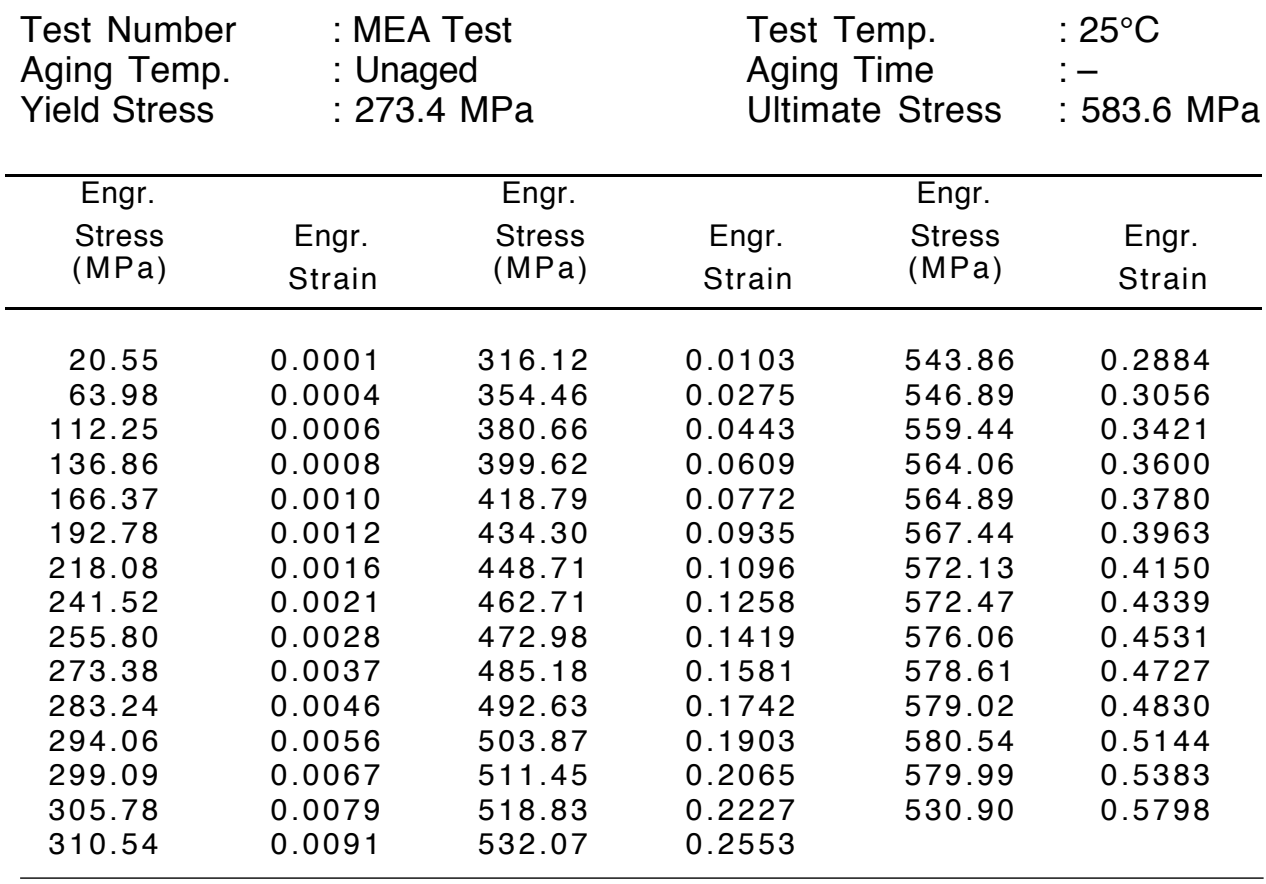


Table 67. Test data for specimen 694-30

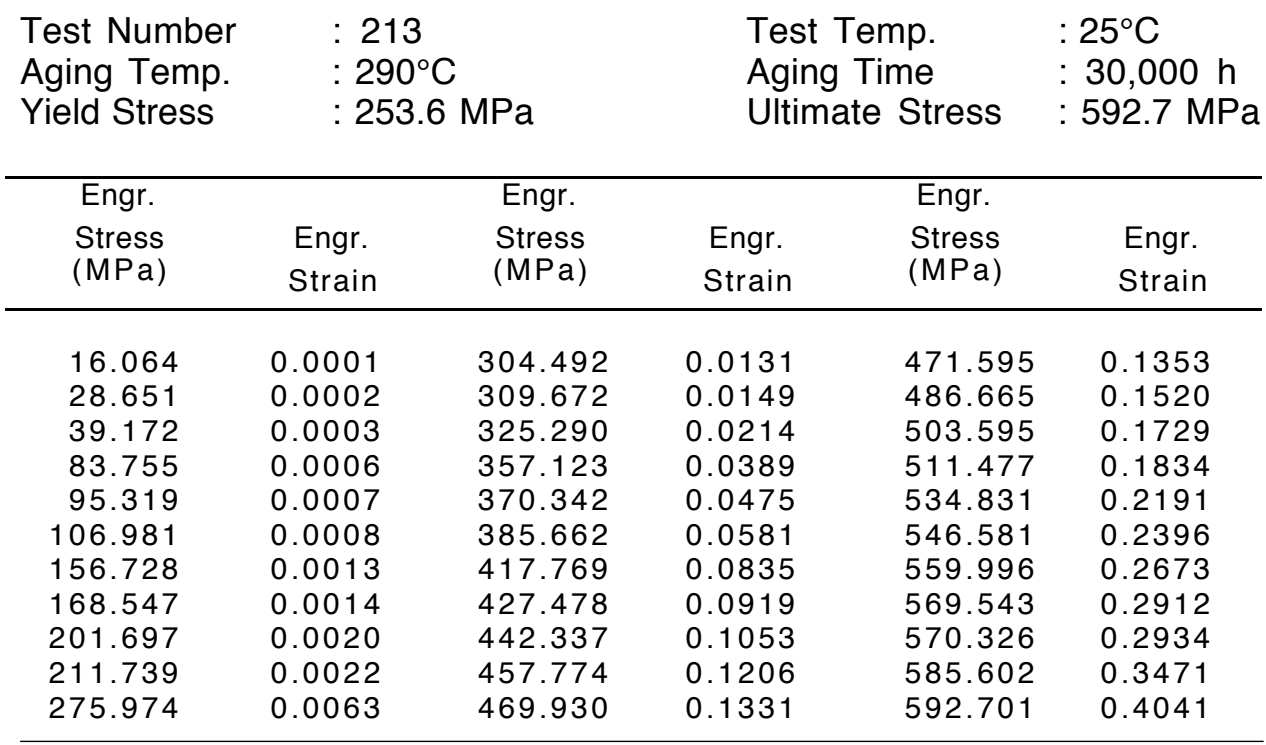

Table 68. Test data for specimen 694-31

\begin{tabular}{|c|c|c|c|c|c|}
\hline $\begin{array}{l}\text { Test Number } \\
\text { Aging Temp. } \\
\text { Yield Stress }\end{array}$ & $\begin{array}{l}: 2 \\
: 25 \\
: 26\end{array}$ & $\mathrm{MPa}$ & $\begin{array}{l}\text { Tes } \\
\text { Agi } \\
\text { Ulti }\end{array}$ & $\begin{array}{l}\text { np. } \\
\text { me } \\
\text { Stress }\end{array}$ & $\begin{array}{l}25^{\circ} \mathrm{C} \\
30,000 \mathrm{~h} \\
588.9 \mathrm{MPa}\end{array}$ \\
\hline Engr. & & Engr. & & Engr. & \\
\hline $\begin{array}{l}\text { Stress } \\
(\mathrm{MPa})\end{array}$ & $\begin{array}{l}\text { Engr. } \\
\text { Strain }\end{array}$ & $\begin{array}{l}\text { Stress } \\
(\mathrm{MPa})\end{array}$ & $\begin{array}{l}\text { Engr. } \\
\text { Strain }\end{array}$ & $\begin{array}{l}\text { Stress } \\
(\mathrm{MPa})\end{array}$ & $\begin{array}{l}\text { Engr. } \\
\text { Strain }\end{array}$ \\
\hline 16.244 & 0.0001 & 353.863 & 0.0233 & 490.937 & 0.1322 \\
\hline 30.299 & 0.0002 & 371.853 & 0.0323 & 508.542 & 0.1564 \\
\hline 75.004 & 0.0004 & 376.140 & 0.0346 & 514.150 & 0.1651 \\
\hline 86.479 & 0.0005 & 387.844 & 0.0413 & 529.756 & 0.1913 \\
\hline 134.548 & 0.0008 & 391.394 & 0.0436 & 537.120 & 0.2055 \\
\hline 146.345 & 0.0009 & 405.317 & 0.0527 & 538.046 & 0.2075 \\
\hline 202.168 & 0.0015 & 424.102 & 0.0663 & 541.167 & 0.2142 \\
\hline 266.385 & 0.0037 & 443.453 & 0.0823 & 557.456 & 0.2551 \\
\hline 288.237 & 0.0056 & 453.475 & 0.0915 & 561.650 & 0.2671 \\
\hline 293.641 & 0.0062 & 467.432 & 0.1053 & 574.865 & 0.3158 \\
\hline 316.566 & 0.0103 & 477.244 & 0.1161 & 586.472 & 0.3843 \\
\hline
\end{tabular}


Table 69. Test data for specimen 69-135

\begin{tabular}{|c|c|c|c|c|c|}
\hline $\begin{array}{l}\text { Test Number } \\
\text { Aging Temp. } \\
\text { Yield Stress }\end{array}$ & $\begin{array}{l}: 2 \\
: 25 \\
: 24\end{array}$ & & $\begin{array}{l}\text { Tes } \\
\text { Agi } \\
\text { Ulti }\end{array}$ & $\begin{array}{l}\text { np. } \\
\text { ne } \\
\text { Stress }\end{array}$ & $\begin{array}{l}25^{\circ} \mathrm{C} \\
30,000 \mathrm{~h} \\
600.6 \mathrm{MPa}\end{array}$ \\
\hline Engr. & & Engr. & & Engr. & \\
\hline $\begin{array}{l}\text { Stress } \\
\text { (MPa) }\end{array}$ & $\begin{array}{l}\text { Engr. } \\
\text { Strain }\end{array}$ & $\begin{array}{l}\text { Stress } \\
(\mathrm{MPa})\end{array}$ & $\begin{array}{l}\text { Engr. } \\
\text { Strain }\end{array}$ & $\begin{array}{l}\text { Stress } \\
(\mathrm{MPa})\end{array}$ & $\begin{array}{l}\text { Engr. } \\
\text { Strain }\end{array}$ \\
\hline 14.941 & 0.0001 & 373.098 & 0.0399 & 537.746 & 0.2300 \\
\hline 28.372 & 0.0002 & 395.391 & 0.0539 & 559.708 & 0.2875 \\
\hline 39.164 & 0.0003 & 401.251 & 0.0579 & 560.534 & 0.2896 \\
\hline 82.127 & 0.0006 & 427.169 & 0.0780 & 561.166 & 0.2916 \\
\hline 93.196 & 0.0007 & 443.763 & 0.0931 & 564.956 & 0.3041 \\
\hline 162.694 & 0.0014 & 463.539 & 0.1132 & 567.920 & 0.3145 \\
\hline 201.857 & 0.0022 & 488.281 & 0.1438 & 578.707 & 0.3584 \\
\hline 244.583 & 0.0039 & 506.308 & 0.1703 & 581.234 & 0.3689 \\
\hline 250.010 & 0.0042 & 514.908 & 0.1846 & 590.514 & 0.4220 \\
\hline 286.788 & 0.0077 & 515.880 & 0.1867 & 599.746 & 0.5053 \\
\hline 310.806 & 0.0127 & 522.828 & 0.1990 & 600.670 & 0.5351 \\
\hline
\end{tabular}

Table 70. Test data for specimen 694-21

\begin{tabular}{|c|c|c|c|c|c|}
\hline $\begin{array}{l}\text { Test Number } \\
\text { Aging Temp. } \\
\text { Yield Stress }\end{array}$ & $\begin{array}{l}: 1 \\
: 32 \\
: 2 \varepsilon\end{array}$ & & $\begin{array}{l}\text { Tes } \\
\text { Agi } \\
\text { Ulti }\end{array}$ & $\begin{array}{l}\text { mp. } \\
\text { me } \\
\text { Stress }\end{array}$ & $\begin{array}{l}25^{\circ} \mathrm{C} \\
10,000 \mathrm{~h} \\
624.6 \mathrm{MPa}\end{array}$ \\
\hline Engr. & & Engr. & & Engr. & \\
\hline $\begin{array}{l}\text { Stress } \\
(\mathrm{MPa})\end{array}$ & $\begin{array}{l}\text { Engr. } \\
\text { Strain }\end{array}$ & $\begin{array}{l}\text { Stress } \\
(\mathrm{MPa})\end{array}$ & $\begin{array}{l}\text { Engr. } \\
\text { Strain }\end{array}$ & $\begin{array}{l}\text { Stress } \\
(\mathrm{MPa})\end{array}$ & $\begin{array}{l}\text { Engr. } \\
\text { Strain }\end{array}$ \\
\hline 0.00 & 0.0000 & 337.70 & 0.0100 & 516.64 & 0.1202 \\
\hline 25.29 & 0.0001 & 355.85 & 0.0139 & 540.48 & 0.1493 \\
\hline 25.78 & 0.0001 & 375.32 & 0.0230 & 563.50 & 0.1909 \\
\hline 202.11 & 0.0028 & 401.68 & 0.0314 & 562.73 & 0.1925 \\
\hline 220.67 & 0.0031 & 423.52 & 0.0439 & 587.68 & 0.2523 \\
\hline 220.47 & 0.0032 & 456.54 & 0.0688 & 609.91 & 0.3300 \\
\hline 278.46 & 0.0047 & 478.25 & 0.0847 & 624.63 & 0.4169 \\
\hline 314.64 & 0.0073 & 492.58 & 0.0976 & & \\
\hline
\end{tabular}


Table 71. Test data for specimen 694-25

\begin{tabular}{|c|c|c|c|c|c|}
\hline $\begin{array}{l}\text { Test Number } \\
\text { Aging Temp. } \\
\text { Yield Stress }\end{array}$ & \multicolumn{2}{|c|}{$\begin{array}{l}: 138 \\
: 320^{\circ} \mathrm{C} \\
: 232.1 \mathrm{MPa}\end{array}$} & \multicolumn{2}{|c|}{$\begin{array}{l}\text { Test Temp. } \\
\text { Aging Time } \\
\text { Ultimate Stress }\end{array}$} & $\begin{array}{l}25^{\circ} \mathrm{C} \\
10,000 \mathrm{~h} \\
585.4 \mathrm{MPa}\end{array}$ \\
\hline \multirow{2}{*}{$\begin{array}{l}\text { Engr. } \\
\text { Stress } \\
\text { (MPa) }\end{array}$} & \multicolumn{2}{|r|}{ Engr. } & \multicolumn{3}{|c|}{ Engr. } \\
\hline & $\begin{array}{l}\text { Engr. } \\
\text { Strain }\end{array}$ & $\begin{array}{l}\text { Stress } \\
\text { (MPa) }\end{array}$ & $\begin{array}{l}\text { Engr. } \\
\text { Strain }\end{array}$ & $\begin{array}{l}\text { Stress } \\
(\mathrm{MPa})\end{array}$ & $\begin{array}{l}\text { Engr. } \\
\text { Strain }\end{array}$ \\
\hline 0.00 & 0.0000 & 345.97 & 0.0272 & 489.97 & 0.1759 \\
\hline 63.76 & 0.0002 & 367.11 & 0.0422 & 511.55 & 0.2187 \\
\hline 196.90 & 0.0020 & 391.60 & 0.0609 & 528.74 & 0.2605 \\
\hline 216.59 & 0.0026 & 423.07 & 0.0910 & 559.66 & 0.3535 \\
\hline 270.79 & 0.0047 & 458.08 & 0.1248 & 578.29 & 0.4746 \\
\hline 286.44 & 0.0066 & 478.44 & 0.1529 & 578.29 & 0.4747 \\
\hline 310.53 & 0.0112 & 483.92 & 0.1637 & 585.42 & 0.5537 \\
\hline 329.69 & 0.0183 & & & & \\
\hline
\end{tabular}

Table 72. Test data for specimen 692-40

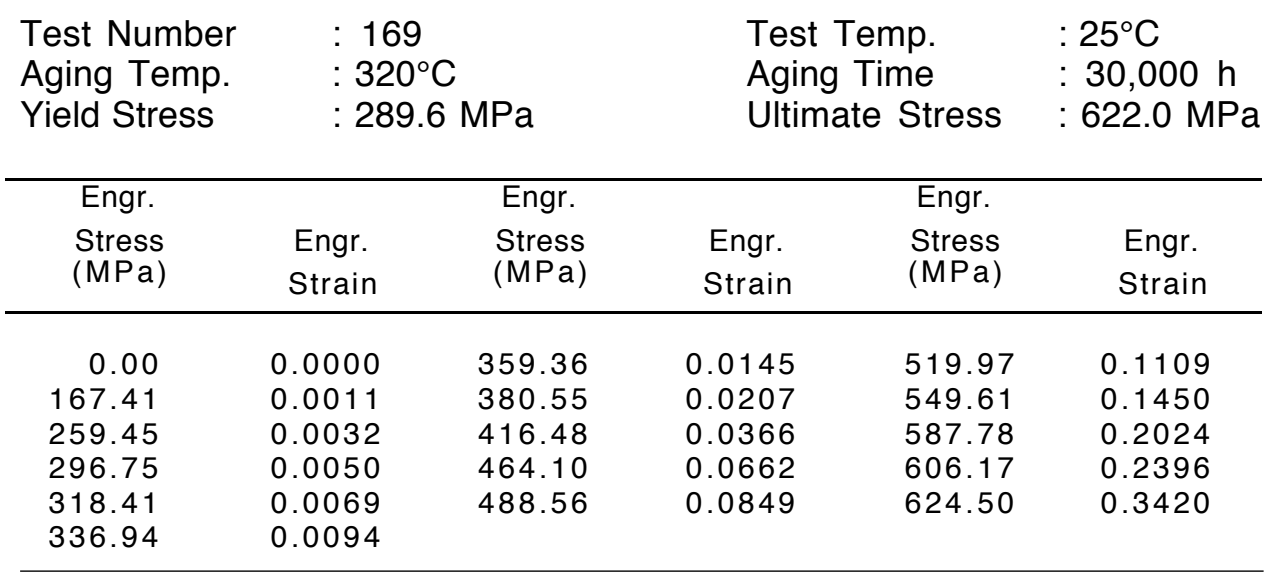

Table 73. Test data for specimen 692-41

\begin{tabular}{|c|c|c|c|c|c|}
\hline $\begin{array}{l}\text { Test Number } \\
\text { Aging Temp. } \\
\text { Yield Stress }\end{array}$ & \multicolumn{2}{|c|}{$\begin{array}{l}: 158 \\
: 320^{\circ} \mathrm{C} \\
: 269.4 \mathrm{MPa}\end{array}$} & \multicolumn{2}{|c|}{$\begin{array}{l}\text { Test Temp. } \\
\text { Aging Time } \\
\text { Ultimate Stress }\end{array}$} & $\begin{array}{l}: 25^{\circ} \mathrm{C} \\
: 30,000 \mathrm{~h} \\
: 604.4 \mathrm{MPa}\end{array}$ \\
\hline Engr. & & Engr. & & Engr. & \\
\hline $\begin{array}{l}\text { Stress } \\
(\mathrm{MPa})\end{array}$ & $\begin{array}{l}\text { Engr. } \\
\text { Strain }\end{array}$ & $\begin{array}{l}\text { Stress } \\
(\mathrm{MPa})\end{array}$ & $\begin{array}{l}\text { Engr. } \\
\text { Strain }\end{array}$ & $\begin{array}{l}\text { Stress } \\
(\mathrm{MPa})\end{array}$ & $\begin{array}{l}\text { Engr. } \\
\text { Strain }\end{array}$ \\
\hline 0.00 & 0.0000 & 360.37 & 0.0128 & 490.75 & 0.0851 \\
\hline 216.85 & 0.0011 & 375.03 & 0.0172 & 500.69 & 0.0948 \\
\hline 249.30 & 0.0017 & 395.81 & 0.0250 & 542.63 & 0.1332 \\
\hline 281.66 & 0.0029 & 410.58 & 0.0325 & 571.23 & 0.1713 \\
\hline 302.70 & 0.0058 & 436.92 & 0.0470 & 591.06 & 0.2121 \\
\hline 312.62 & 0.0049 & 454.19 & 0.0585 & 603.15 & 0.2525 \\
\hline 323.14 & 0.0060 & 470.45 & 0.0702 & 604.40 & 0.2857 \\
\hline 342.94 & 0.0091 & & & & \\
\hline
\end{tabular}


Table 74. Test data for specimen 69-245

\begin{tabular}{|c|c|c|c|c|c|}
\hline $\begin{array}{l}\text { Test Number } \\
\text { Aging Temp. } \\
\text { Yield Stress }\end{array}$ & \multicolumn{2}{|c|}{$\begin{array}{l}: 160 \\
: 320^{\circ} \mathrm{C} \\
: 272.5 \mathrm{MPa}\end{array}$} & \multicolumn{2}{|c|}{$\begin{array}{l}\text { Test Temp. } \\
\text { Aging Time } \\
\text { Ultimate Stress }\end{array}$} & $\begin{array}{l}25^{\circ} \mathrm{C} \\
30,000 \mathrm{~h} \\
485.8 \mathrm{MPa}\end{array}$ \\
\hline \multicolumn{2}{|l|}{ Engr. } & Engr. & \multicolumn{3}{|c|}{ Engr. } \\
\hline $\begin{array}{l}\text { Stress } \\
\text { (MPa) }\end{array}$ & $\begin{array}{l}\text { Engr. } \\
\text { Strain }\end{array}$ & $\begin{array}{l}\text { Stress } \\
\text { (MPa) }\end{array}$ & $\begin{array}{l}\text { Engr. } \\
\text { Strain }\end{array}$ & $\begin{array}{l}\text { Stress } \\
(\mathrm{MPa})\end{array}$ & $\begin{array}{l}\text { Engr. } \\
\text { Strain }\end{array}$ \\
\hline 0.00 & 0.0000 & 341.82 & 0.0144 & 450.74 & 0.0793 \\
\hline 124.00 & 0.0006 & 360.03 & 0.0205 & 469.20 & 0.0961 \\
\hline 250.85 & 0.0030 & 381.06 & 0.0299 & 480.46 & 0.1091 \\
\hline 292.97 & 0.0059 & 394.62 & 0.0379 & 483.88 & 0.1156 \\
\hline 314.66 & 0.0086 & 409.01 & 0.0475 & 485.82 & 0.1185 \\
\hline 330.39 & 0.0113 & 426.91 & 0.0601 & & \\
\hline
\end{tabular}

Table 75. Test data for specimen 691-28

\begin{tabular}{|c|c|c|c|c|c|}
\hline $\begin{array}{l}\text { Test Number } \\
\text { Aging Temp. } \\
\text { Yield Stress }\end{array}$ & \multicolumn{2}{|c|}{$\begin{array}{l}: 290 \\
: 320^{\circ} \mathrm{C} \\
: 291.6 \mathrm{MPa}\end{array}$} & \multicolumn{2}{|c|}{$\begin{array}{l}\text { Test Temp. } \\
\text { Aging Time } \\
\text { Ultimate Stress }\end{array}$} & \multirow[t]{2}{*}{$\begin{array}{l}25^{\circ} \mathrm{C} \\
50,000 \mathrm{~h} \\
637.9 \mathrm{MPa}\end{array}$} \\
\hline Engr. & & Engr. & & Engr. & \\
\hline $\begin{array}{l}\text { Stress } \\
(\mathrm{MPa})\end{array}$ & $\begin{array}{l}\text { Engr. } \\
\text { Strain }\end{array}$ & $\begin{array}{l}\text { Stress } \\
\text { (MPa) }\end{array}$ & $\begin{array}{l}\text { Engr. } \\
\text { Strain }\end{array}$ & $\begin{array}{l}\text { Stress } \\
(\mathrm{MPa})\end{array}$ & $\begin{array}{l}\text { Engr. } \\
\text { Strain }\end{array}$ \\
\hline 20.217 & 0.0001 & 285.141 & 0.0031 & 513.943 & 0.0884 \\
\hline 49.211 & 0.0002 & 291.618 & 0.0034 & 529.028 & 0.1031 \\
\hline 59.313 & 0.0003 & 319.590 & 0.0049 & 531.148 & 0.1053 \\
\hline 103.425 & 0.0005 & 327.870 & 0.0055 & 549.125 & 0.1258 \\
\hline 114.924 & 0.0005 & 371.732 & 0.0111 & 559.101 & 0.1388 \\
\hline 126.683 & 0.0006 & 438.943 & 0.0350 & 560.643 & 0.1410 \\
\hline 197.707 & 0.0011 & 442.567 & 0.0370 & 572.643 & 0.1583 \\
\hline 208.454 & 0.0012 & 469.498 & 0.0532 & 603.488 & 0.2156 \\
\hline 218.725 & 0.0013 & 472.467 & 0.0553 & 610.958 & 0.2338 \\
\hline 263.449 & 0.0023 & 489.364 & 0.0676 & 623.247 & 0.2704 \\
\hline 271.030 & 0.0026 & 492.111 & 0.0697 & 635.055 & 0.3245 \\
\hline
\end{tabular}


Table 76. Test data for specimen 691-29

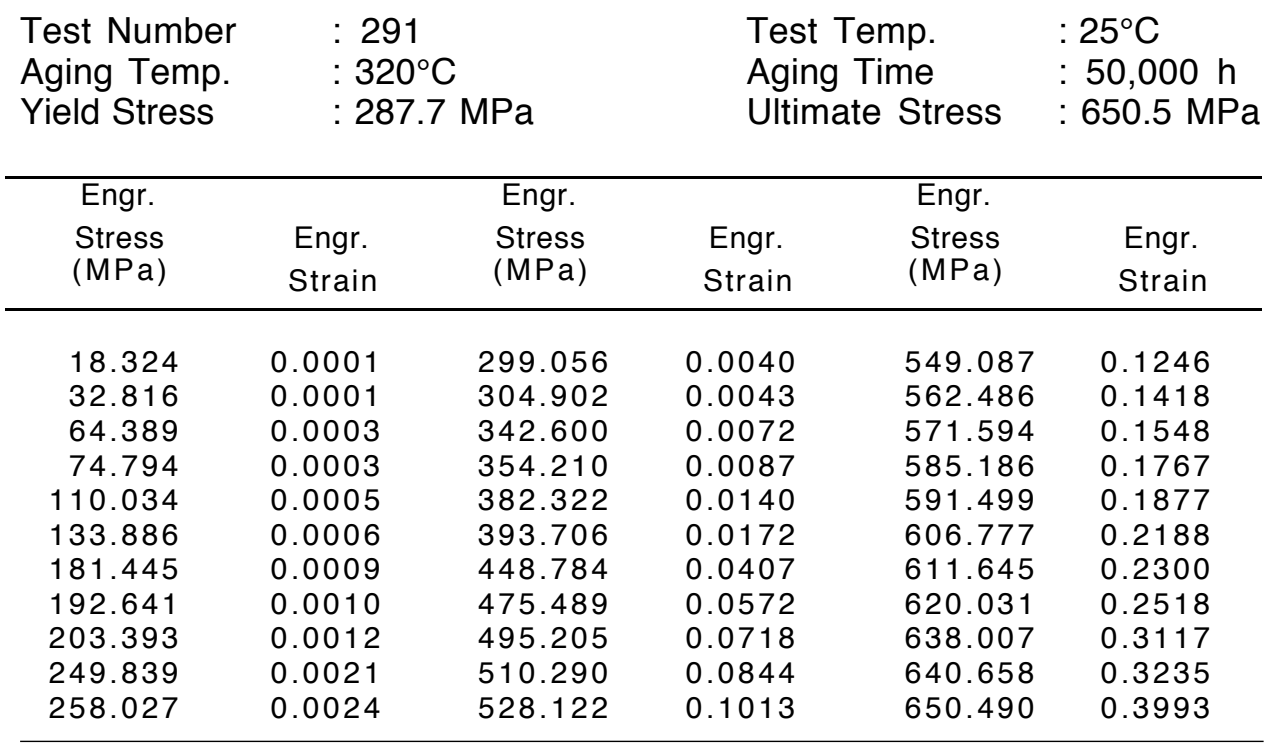

Table 77. Test data for specimen 69-230

\begin{tabular}{|c|c|c|c|c|c|}
\hline $\begin{array}{l}\text { Test Number } \\
\text { Aging Temp. } \\
\text { Yield Stress }\end{array}$ & $\begin{array}{l}: 2 \\
: 32 \\
: 25\end{array}$ & & $\begin{array}{l}\text { Tes } \\
\text { Agi } \\
\text { Ulti }\end{array}$ & $\begin{array}{l}\mathrm{mp.} \\
\text { me } \\
\text { Stress }\end{array}$ & $\begin{array}{l}25^{\circ} \mathrm{C} \\
50,000 \mathrm{~h} \\
669.4 \mathrm{MPa}\end{array}$ \\
\hline Engr. & & Engr. & & Engr. & \\
\hline $\begin{array}{l}\text { Stress } \\
\text { (MPa) }\end{array}$ & $\begin{array}{l}\text { Engr. } \\
\text { Strain }\end{array}$ & $\begin{array}{l}\text { Stress } \\
(\mathrm{MPa})\end{array}$ & $\begin{array}{l}\text { Engr. } \\
\text { Strain }\end{array}$ & $\begin{array}{l}\text { Stress } \\
(\mathrm{MPa})\end{array}$ & $\begin{array}{l}\text { Engr. } \\
\text { Strain }\end{array}$ \\
\hline 20.574 & 0.0001 & 401.408 & 0.0192 & 550.954 & 0.1236 \\
\hline 67.134 & 0.0004 & 407.220 & 0.0213 & 567.002 & 0.1424 \\
\hline 78.055 & 0.0004 & 431.264 & 0.0317 & 578.039 & 0.1570 \\
\hline 89.323 & 0.0005 & 435.510 & 0.0338 & 595.389 & 0.1823 \\
\hline 174.631 & 0.0011 & 447.375 & 0.0401 & 601.702 & 0.1929 \\
\hline 186.713 & 0.0012 & 461.207 & 0.0484 & 615.293 & 0.2182 \\
\hline 230.179 & 0.0018 & 471.063 & 0.0547 & 623.245 & 0.2351 \\
\hline 295.588 & 0.0037 & 486.085 & 0.0651 & 633.992 & 0.2607 \\
\hline 301.988 & 0.0040 & 505.073 & 0.0797 & 649.511 & 0.3080 \\
\hline 390.983 & 0.0158 & 526.230 & 0.0984 & 652.450 & 0.3190 \\
\hline 396.420 & 0.0175 & 537.074 & 0.1088 & 669.415 & 0.4275 \\
\hline
\end{tabular}


Table 78. Test data for specimen 692-25

\begin{tabular}{|c|c|c|c|c|c|}
\hline $\begin{array}{l}\text { Test Number } \\
\text { Aging Temp. } \\
\text { Yield Stress }\end{array}$ & $\begin{array}{l}: M \\
: 35 \\
: 28\end{array}$ & & $\begin{array}{l}\text { Tes } \\
\text { Agi } \\
\text { Ulti }\end{array}$ & $\begin{array}{l}\text { np. } \\
\text { ne } \\
\text { Stress }\end{array}$ & $\begin{array}{l}25^{\circ} \mathrm{C} \\
2,570 \mathrm{~h} \\
633.4 \mathrm{MPa}\end{array}$ \\
\hline Engr. & & Engr. & & Engr. & \\
\hline $\begin{array}{l}\text { Stress } \\
\text { (MPa) }\end{array}$ & $\begin{array}{l}\text { Engr. } \\
\text { Strain }\end{array}$ & $\begin{array}{l}\text { Stress } \\
(\mathrm{MPa})\end{array}$ & $\begin{array}{l}\text { Engr. } \\
\text { Strain }\end{array}$ & $\begin{array}{l}\text { Stress } \\
(\mathrm{MPa})\end{array}$ & $\begin{array}{l}\text { Engr. } \\
\text { Strain }\end{array}$ \\
\hline 17.03 & 0.0002 & 315.99 & 0.0060 & 575.44 & 0.2356 \\
\hline 30.96 & 0.0003 & 326.54 & 0.0069 & 582.26 & 0.2567 \\
\hline 48.61 & 0.0003 & 332.19 & 0.0078 & 588.74 & 0.2782 \\
\hline 69.15 & 0.0004 & 337.15 & 0.0088 & 597.09 & 0.2997 \\
\hline 93.77 & 0.0006 & 397.28 & 0.0281 & 600.95 & 0.3215 \\
\hline 116.52 & 0.0007 & 430.58 & 0.0488 & 606.33 & 0.3434 \\
\hline 143.89 & 0.0009 & 457.81 & 0.0694 & 611.70 & 0.3655 \\
\hline 168.58 & 0.0011 & 479.94 & 0.0899 & 615.43 & 0.3878 \\
\hline 195.19 & 0.0014 & 501.25 & 0.1105 & 617.98 & 0.4106 \\
\hline 222.70 & 0.0018 & 516.14 & 0.1311 & 622.18 & 0.4338 \\
\hline 243.38 & 0.0023 & 529.10 & 0.1518 & 624.67 & 0.4572 \\
\hline 262.97 & 0.0029 & 543.65 & 0.1725 & 625.91 & 0.4813 \\
\hline 279.79 & 0.0035 & 553.92 & 0.1934 & 629.35 & 0.5058 \\
\hline 292.96 & 0.0043 & 565.85 & 0.2145 & 631.01 & 0.5310 \\
\hline 305.92 & 0.0051 & & & & \\
\hline
\end{tabular}

Table 79. Test data for specimen 692-26

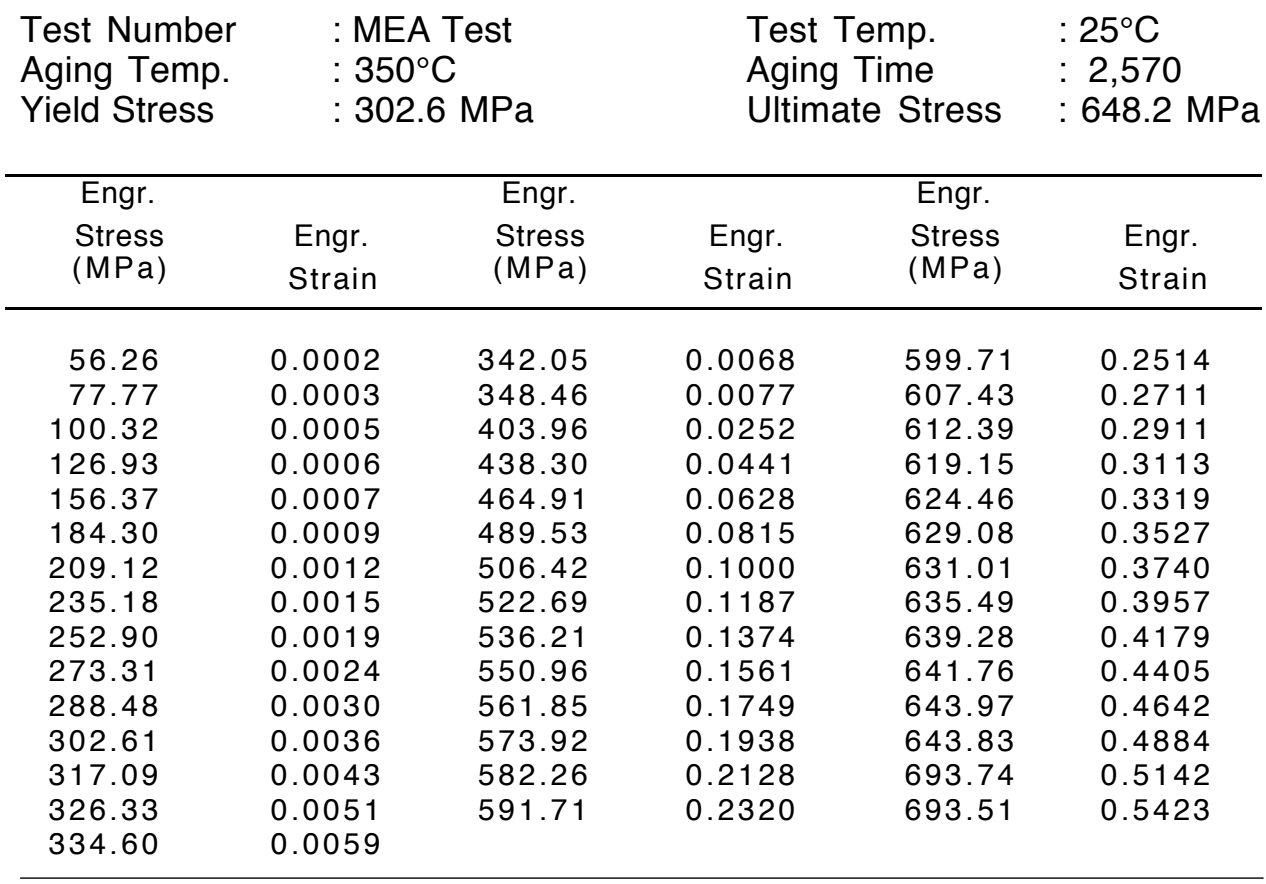


Table 80. Test data for specimen 694-06

\begin{tabular}{|c|c|c|c|c|c|}
\hline $\begin{array}{l}\text { Test Number } \\
\text { Aging Temp. } \\
\text { Yield Stress }\end{array}$ & $\begin{array}{l}: 1 \\
: 35 \\
: 25\end{array}$ & & $\begin{array}{l}\text { Tes } \\
\text { Agi } \\
\text { Ulti }\end{array}$ & $\begin{array}{l}\text { np. } \\
\text { me } \\
\text { Stress }\end{array}$ & $\begin{array}{l}: 25^{\circ} \mathrm{C} \\
10,000 \mathrm{~h} \\
: 652.0 \mathrm{MPa}\end{array}$ \\
\hline Engr. & & Engr. & & Engr. & \\
\hline $\begin{array}{l}\text { Stress } \\
(\mathrm{MPa})\end{array}$ & $\begin{array}{l}\text { Engr. } \\
\text { Strain }\end{array}$ & $\begin{array}{l}\text { Stress } \\
(\mathrm{MPa})\end{array}$ & $\begin{array}{l}\text { Engr. } \\
\text { Strain }\end{array}$ & $\begin{array}{l}\text { Stress } \\
(\mathrm{MPa})\end{array}$ & $\begin{array}{l}\text { Engr. } \\
\text { Strain }\end{array}$ \\
\hline 0.00 & 0.0000 & 424.24 & 0.0347 & 586.25 & 0.1890 \\
\hline 52.67 & 0.0002 & 446.83 & 0.0477 & 598.94 & 0.2068 \\
\hline 205.14 & 0.0024 & 472.38 & 0.0649 & 613.91 & 0.2894 \\
\hline 300.54 & 0.0049 & 479.82 & 0.0705 & 627.40 & 0.2075 \\
\hline 333.72 & 0.0076 & 490.91 & 0.0808 & 635.87 & 0.3284 \\
\hline 352.83 & 0.0102 & 516.46 & 0.0999 & 643.32 & 0.3695 \\
\hline 374.54 & 0.0151 & 539.41 & 0.1211 & 651.98 & 0.4355 \\
\hline 397.47 & 0.0223 & 567.83 & 0.1593 & 652.00 & 0.4794 \\
\hline 397.98 & 0.0225 & & & & \\
\hline
\end{tabular}

Table 81. Test data for specimen 694-07

\begin{tabular}{|c|c|c|c|c|c|}
\hline $\begin{array}{l}\text { Test Number } \\
\text { Aging Temp. } \\
\text { Yield Stress }\end{array}$ & $\begin{array}{l}: 1 \\
: 35 \\
: 26\end{array}$ & & $\begin{array}{l}\text { Tes } \\
\text { Agi } \\
\text { Ulti }\end{array}$ & $\begin{array}{l}\text { np. } \\
\text { ne } \\
\text { Stress }\end{array}$ & $\begin{array}{l}25^{\circ} \mathrm{C} \\
10,000 \mathrm{~h} \\
635.5 \mathrm{MPa}\end{array}$ \\
\hline Engr. & & Engr. & & Engr. & \\
\hline $\begin{array}{l}\text { Stress } \\
(\mathrm{MPa})\end{array}$ & $\begin{array}{l}\text { Engr. } \\
\text { Strain }\end{array}$ & $\begin{array}{l}\text { Stress } \\
\text { (MPa) }\end{array}$ & $\begin{array}{l}\text { Engr. } \\
\text { Strain }\end{array}$ & $\begin{array}{l}\text { Stress } \\
(\mathrm{MPa})\end{array}$ & $\begin{array}{l}\text { Engr. } \\
\text { Strain }\end{array}$ \\
\hline 0.00 & 0.0000 & 351.64 & 0.0125 & 522.00 & 0.1168 \\
\hline 48.83 & 0.0000 & 375.73 & 0.0192 & 521.61 & 0.1170 \\
\hline 190.46 & 0.0014 & 394.91 & 0.0266 & 546.67 & 0.1430 \\
\hline 205.90 & 0.0019 & 413.76 & 0.0361 & 575.73 & 0.1892 \\
\hline 219.80 & 0.0021 & 432.19 & 0.0471 & 600.01 & 0.2344 \\
\hline 224.58 & 0.0021 & 457.56 & 0.0635 & 616.14 & 0.2782 \\
\hline 282.30 & 0.0038 & 479.87 & 0.0794 & 625.95 & 0.3272 \\
\hline 311.61 & 0.0063 & 490.93 & 0.0897 & 635.48 & 0.3858 \\
\hline 336.66 & 0.0096 & & & & \\
\hline
\end{tabular}


Table 82. Test data for specimen 694-08

\begin{tabular}{|c|c|c|c|c|c|}
\hline $\begin{array}{l}\text { Test Number } \\
\text { Aging Temp. } \\
\text { Yield Stress }\end{array}$ & $\begin{array}{l}: 2 \\
: 35 \\
: 24\end{array}$ & & $\begin{array}{l}\text { Tes } \\
\text { Agi } \\
\text { Ulti }\end{array}$ & $\begin{array}{l}\text { np. } \\
\text { ne } \\
\text { Stress }\end{array}$ & $\begin{array}{l}25^{\circ} \mathrm{C} \\
10,000 \mathrm{~h} \\
619.9 \mathrm{MPa}\end{array}$ \\
\hline Engr. & & Engr. & & Engr. & \\
\hline $\begin{array}{l}\text { Stress } \\
\text { (MPa) }\end{array}$ & $\begin{array}{l}\text { Engr. } \\
\text { Strain }\end{array}$ & $\begin{array}{l}\text { Stress } \\
(\mathrm{MPa})\end{array}$ & $\begin{array}{l}\text { Engr. } \\
\text { Strain }\end{array}$ & $\begin{array}{l}\text { Stress } \\
(\mathrm{MPa})\end{array}$ & $\begin{array}{l}\text { Engr. } \\
\text { Strain }\end{array}$ \\
\hline 29.804 & 0.0001 & 251.978 & 0.0037 & 489.372 & 0.1201 \\
\hline 57.140 & 0.0003 & 257.323 & 0.0040 & 505.084 & 0.1375 \\
\hline 69.410 & 0.0003 & 290.110 & 0.0073 & 529.567 & 0.1677 \\
\hline 114.844 & 0.0006 & 295.546 & 0.0081 & 540.362 & 0.1829 \\
\hline 126.319 & 0.0007 & 300.549 & 0.0090 & 580.171 & 0.2557 \\
\hline 139.722 & 0.0008 & 323.287 & 0.0140 & 584.943 & 0.2668 \\
\hline 151.033 & 0.0009 & 356.050 & 0.0254 & 600.606 & 0.3117 \\
\hline 193.474 & 0.0015 & 383.049 & 0.0383 & 609.329 & 0.3458 \\
\hline 202.824 & 0.0016 & 387.059 & 0.0405 & 615.161 & 0.3782 \\
\hline 211.624 & 0.0019 & 428.034 & 0.0664 & 615.547 & 0.3805 \\
\hline 246.493 & 0.0033 & 450.503 & 0.0840 & 619.932 & 0.4381 \\
\hline
\end{tabular}

Table 83. Test data for specimen 69-119

\begin{tabular}{|c|c|c|c|c|c|}
\hline $\begin{array}{l}\text { Test Number } \\
\text { Aging Temp. } \\
\text { Yield Stress }\end{array}$ & & $\mathrm{MPa}$ & $\begin{array}{l}\text { Tes } \\
\text { Agi } \\
\text { Ulti }\end{array}$ & $\begin{array}{l}\text { np. } \\
\text { me } \\
\text { Stress }\end{array}$ & $\begin{array}{l}25^{\circ} \mathrm{C} \\
10,000 \mathrm{~h} \\
617.3 \mathrm{MPa}\end{array}$ \\
\hline Engr. & & Engr. & & Engr. & \\
\hline $\begin{array}{l}\text { Stress } \\
(\mathrm{MPa})\end{array}$ & $\begin{array}{l}\text { Engr. } \\
\text { Strain }\end{array}$ & $\begin{array}{l}\text { Stress } \\
\text { (MPa) }\end{array}$ & $\begin{array}{l}\text { Engr. } \\
\text { Strain }\end{array}$ & $\begin{array}{l}\text { Stress } \\
(\mathrm{MPa})\end{array}$ & $\begin{array}{l}\text { Engr. } \\
\text { Strain }\end{array}$ \\
\hline 0.15 & 0.0000 & 371.71 & 0.0220 & 517.93 & 0.1238 \\
\hline 61.59 & 0.0002 & 398.15 & 0.0329 & 540.92 & 0.1492 \\
\hline 198.49 & 0.0023 & 418.93 & 0.0460 & 567.66 & 0.1938 \\
\hline 212.81 & 0.0025 & 443.63 & 0.0641 & 584.92 & 0.2359 \\
\hline 288.30 & 0.0051 & 468.44 & 0.0800 & 602.70 & 0.3007 \\
\hline 318.24 & 0.0090 & 474.73 & 0.0861 & 605.60 & 0.2987 \\
\hline 338.72 & 0.0117 & 475.41 & 0.0858 & 617.30 & 0.3745 \\
\hline 358.66 & 0.0183 & 487.14 & 0.0970 & & \\
\hline
\end{tabular}


Table 84. Test data for specimen 693-12

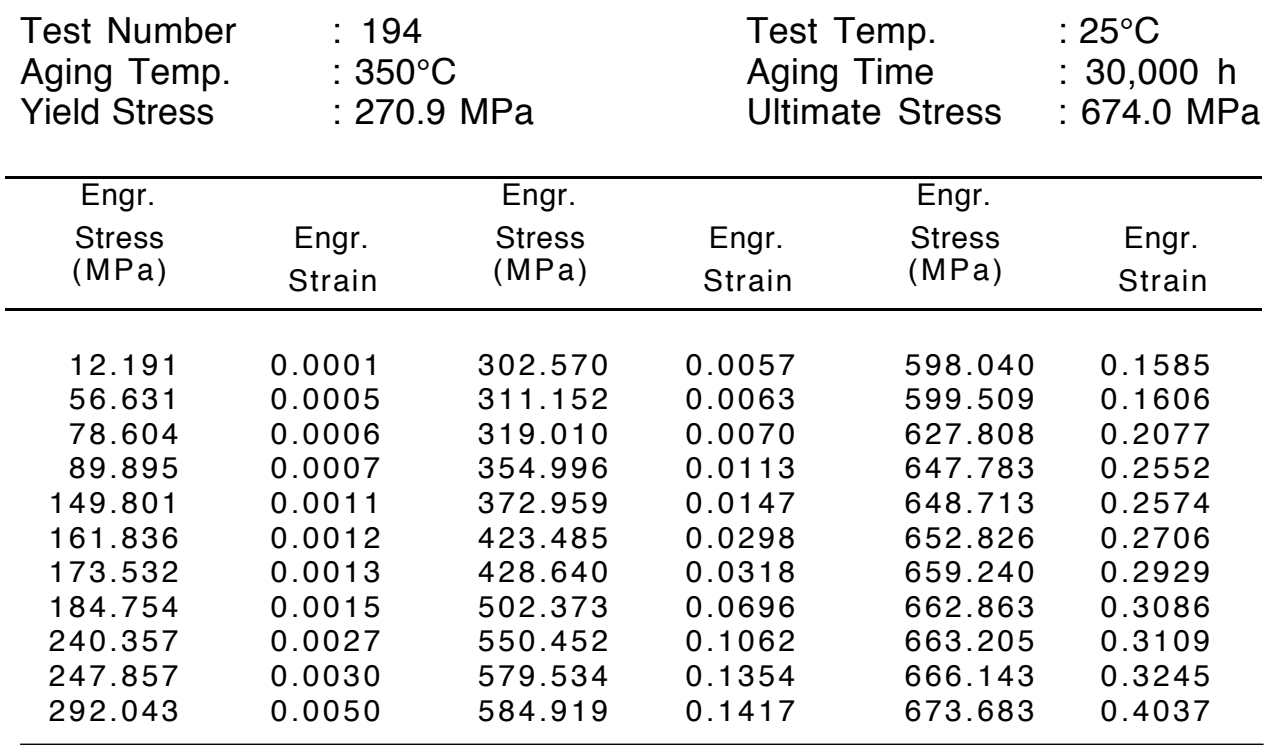

Table 85. Test data for specimen 693-13

\begin{tabular}{|c|c|c|c|c|c|}
\hline $\begin{array}{l}\text { Test Number } \\
\text { Aging Temp. } \\
\text { Yield Stress }\end{array}$ & $\begin{array}{l}: 1 \\
: 35 \\
: 25\end{array}$ & & $\begin{array}{l}\text { Tes } \\
\text { Agi } \\
\text { Ulti }\end{array}$ & $\begin{array}{l}\text { np. } \\
\text { ne } \\
\text { Stress }\end{array}$ & $\begin{array}{l}25^{\circ} \mathrm{C} \\
30,000 \mathrm{~h} \\
655.1 \mathrm{MPa}\end{array}$ \\
\hline Engr. & & Engr. & & Engr. & \\
\hline $\begin{array}{l}\text { Stress } \\
\text { (MPa) }\end{array}$ & $\begin{array}{l}\text { Engr. } \\
\text { Strain }\end{array}$ & $\begin{array}{l}\text { Stress } \\
(\mathrm{MPa})\end{array}$ & $\begin{array}{l}\text { Engr. } \\
\text { Strain }\end{array}$ & $\begin{array}{l}\text { Stress } \\
(\mathrm{MPa})\end{array}$ & $\begin{array}{l}\text { Engr. } \\
\text { Strain }\end{array}$ \\
\hline 16.518 & 0.0001 & 306.978 & 0.0074 & 557.880 & 0.1354 \\
\hline 29.428 & 0.0002 & 341.770 & 0.0124 & 583.972 & 0.1679 \\
\hline 39.967 & 0.0003 & 352.485 & 0.0145 & 593.580 & 0.1824 \\
\hline 82.031 & 0.0006 & 358.620 & 0.0160 & 603.382 & 0.1991 \\
\hline 92.775 & 0.0007 & 392.335 & 0.0267 & 615.038 & 0.2223 \\
\hline 103.705 & 0.0008 & 413.189 & 0.0350 & 622.500 & 0.2394 \\
\hline 183.741 & 0.0016 & 451.464 & 0.0536 & 629.231 & 0.2582 \\
\hline 193.934 & 0.0017 & 482.282 & 0.0718 & 630.157 & 0.2604 \\
\hline 242.138 & 0.0030 & 501.307 & 0.0850 & 641.667 & 0.2995 \\
\hline 284.841 & 0.0054 & 523.790 & 0.1026 & 642.155 & 0.3017 \\
\hline 292.874 & 0.0060 & 545.883 & 0.1229 & 649.860 & 0.3381 \\
\hline
\end{tabular}


Table 86. Test data for specimen 69-130

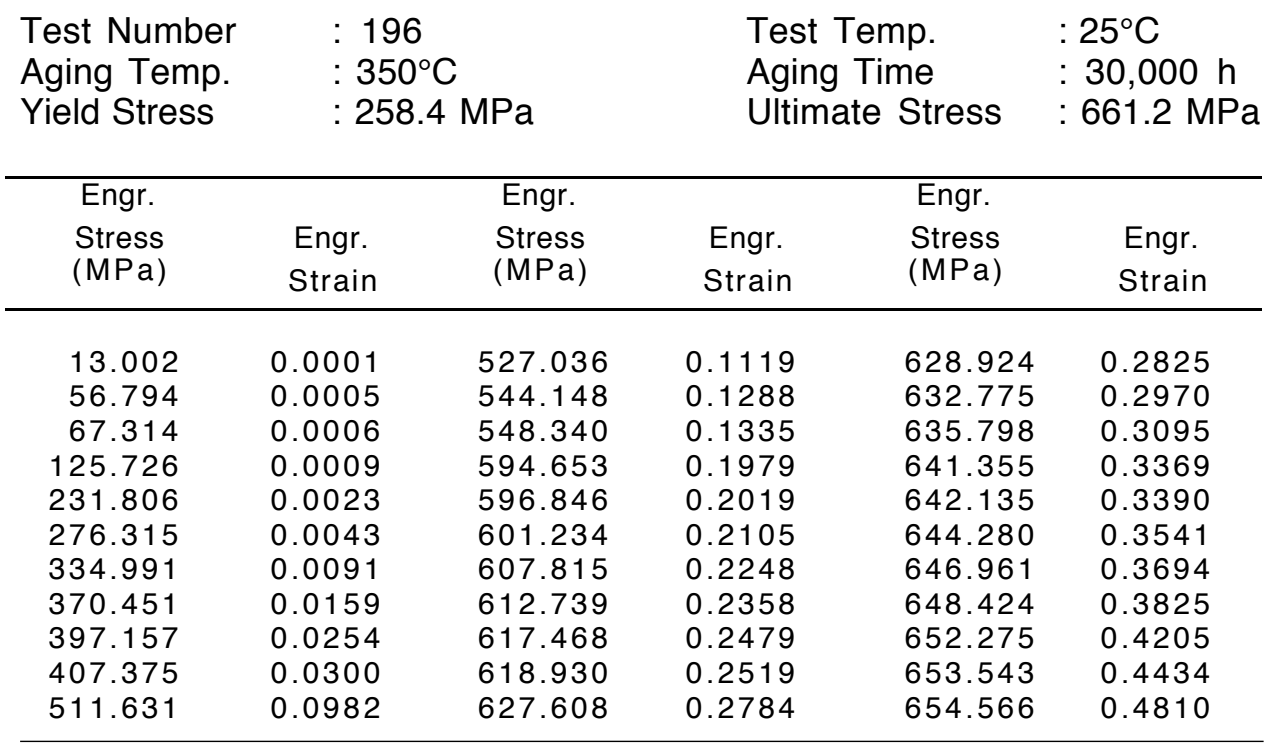

Table 87. Test data for specimen 692-16

\begin{tabular}{|c|c|c|c|c|c|}
\hline $\begin{array}{l}\text { Test Number } \\
\text { Aging Temp. } \\
\text { Yield Stress }\end{array}$ & $\begin{array}{l}: M \\
: 4 C \\
: 25\end{array}$ & & $\begin{array}{l}\text { Tes } \\
\text { Agi } \\
\text { Ulti }\end{array}$ & $\begin{array}{l}\text { np. } \\
\text { ne } \\
\text { Stress }\end{array}$ & $\begin{array}{l}25^{\circ} \mathrm{C} \\
2,570 \mathrm{~h} \\
638.8 \mathrm{MPa}\end{array}$ \\
\hline Engr. & & Engr. & & Engr. & \\
\hline $\begin{array}{l}\text { Stress } \\
(\mathrm{MPa})\end{array}$ & $\begin{array}{l}\text { Engr. } \\
\text { Strain }\end{array}$ & $\begin{array}{l}\text { Stress } \\
(\mathrm{MPa})\end{array}$ & $\begin{array}{l}\text { Engr. } \\
\text { Strain }\end{array}$ & $\begin{array}{l}\text { Stress } \\
(\mathrm{MPa})\end{array}$ & $\begin{array}{l}\text { Engr. } \\
\text { Strain }\end{array}$ \\
\hline 5.35 & 0.0003 & 308.82 & 0.0080 & 579.50 & 0.2027 \\
\hline 57.02 & 0.0004 & 318.06 & 0.0091 & 589.16 & 0.2191 \\
\hline 78.12 & 0.0005 & 323.50 & 0.0101 & 595.98 & 0.2352 \\
\hline 102.04 & 0.0006 & 378.80 & 0.0250 & 601.84 & 0.2514 \\
\hline 123.90 & 0.0007 & 416.99 & 0.0412 & 609.01 & 0.2676 \\
\hline 148.65 & 0.0009 & 444.57 & 0.0573 & 614.05 & 0.2839 \\
\hline 174.09 & 0.0011 & 468.36 & 0.0734 & 618.46 & 0.3000 \\
\hline 198.84 & 0.0015 & 487.87 & 0.0893 & 621.91 & 0.3161 \\
\hline 218.70 & 0.0020 & 507.11 & 0.1054 & 624.39 & 0.3320 \\
\hline 237.52 & 0.0027 & 521.86 & 0.1215 & 629.15 & 0.3478 \\
\hline 253.59 & 0.0035 & 538.69 & 0.1377 & 632.04 & 0.3634 \\
\hline 268.21 & 0.0043 & 551.51 & 0.1540 & 635.15 & 0.3787 \\
\hline 279.93 & 0.0052 & 560.61 & 0.1702 & 635.83 & 0.3935 \\
\hline 291.37 & 0.0061 & 571.30 & 0.1865 & 636.52 & 0.4076 \\
\hline 300.20 & 0.0070 & & & & \\
\hline
\end{tabular}


Table 88. Test data for specimen 692-17

\begin{tabular}{|c|c|c|c|c|c|}
\hline $\begin{array}{l}\text { Test Number } \\
\text { Aging Temp. } \\
\text { Yield Stress }\end{array}$ & $\begin{array}{l}: M \\
: 40 \\
: 30\end{array}$ & & $\begin{array}{l}\text { Tes } \\
\text { Agi } \\
\text { Ulti }\end{array}$ & $\begin{array}{l}\text { np. } \\
\text { ne } \\
\text { Stress }\end{array}$ & $\begin{array}{l}25^{\circ} \mathrm{C} \\
2,570 \\
683.2 \mathrm{MPa}\end{array}$ \\
\hline Engr. & & Engr. & & Engr. & \\
\hline $\begin{array}{l}\text { Stress } \\
(\mathrm{MPa})\end{array}$ & $\begin{array}{l}\text { Engr. } \\
\text { Strain }\end{array}$ & $\begin{array}{l}\text { Stress } \\
(\mathrm{MPa})\end{array}$ & $\begin{array}{l}\text { Engr. } \\
\text { Strain }\end{array}$ & $\begin{array}{l}\text { Stress } \\
(\mathrm{MPa})\end{array}$ & $\begin{array}{l}\text { Engr. } \\
\text { Strain }\end{array}$ \\
\hline 37.16 & 0.0002 & 338.33 & 0.0061 & 619.98 & 0.1643 \\
\hline 57.09 & 0.0003 & 347.08 & 0.0070 & 630.73 & 0.1774 \\
\hline 77.36 & 0.0004 & 356.18 & 0.0080 & 637.28 & 0.1907 \\
\hline 98.80 & 0.0005 & 409.76 & 0.0198 & 643.56 & 0.2042 \\
\hline 124.24 & 0.0006 & 446.57 & 0.0330 & 650.45 & 0.2178 \\
\hline 147.75 & 0.0007 & 474.22 & 0.0459 & 658.38 & 0.2318 \\
\hline 174.71 & 0.0009 & 497.53 & 0.0586 & 661.48 & 0.2462 \\
\hline 201.88 & 0.0011 & 520.07 & 0.0714 & 665.83 & 0.2608 \\
\hline 226.35 & 0.0014 & 538.83 & 0.0840 & 670.86 & 0.2761 \\
\hline 249.93 & 0.0017 & 554.82 & 0.0967 & 673.62 & 0.2915 \\
\hline 267.38 & 0.0022 & 571.30 & 0.1093 & 676.38 & 0.3074 \\
\hline 285.93 & 0.0028 & 584.88 & 0.1221 & 677.89 & 0.3253 \\
\hline 300.89 & 0.0036 & 597.22 & 0.1397 & 680.72 & 0.3420 \\
\hline 315.57 & 0.0043 & 607.08 & 0.1517 & 681.41 & 0.3592 \\
\hline 325.98 & 0.0051 & & & & \\
\hline
\end{tabular}

Table 89. Test data for specimen 692-15

\begin{tabular}{|c|c|c|c|c|c|}
\hline $\begin{array}{l}\text { Test Number } \\
\text { Aging Temp. } \\
\text { Yield Stress }\end{array}$ & $\begin{array}{l}: 1 \\
: 4 c \\
: 2 \varepsilon\end{array}$ & & $\begin{array}{l}\text { Tes } \\
\text { Agi } \\
\text { Ulti }\end{array}$ & $\begin{array}{l}\text { np. } \\
\text { ne } \\
\text { Stress }\end{array}$ & $\begin{array}{l}25^{\circ} \mathrm{C} \\
10,000 \mathrm{~h} \\
673.9 \mathrm{MPa}\end{array}$ \\
\hline \multicolumn{2}{|l|}{ Engr. } & \multicolumn{2}{|l|}{ Engr. } & \multicolumn{2}{|l|}{ Engr. } \\
\hline $\begin{array}{l}\text { Stress } \\
(\mathrm{MPa})\end{array}$ & $\begin{array}{l}\text { Engr. } \\
\text { Strain }\end{array}$ & $\begin{array}{l}\text { Stress } \\
(\mathrm{MPa})\end{array}$ & $\begin{array}{l}\text { Engr. } \\
\text { Strain }\end{array}$ & $\begin{array}{l}\text { Stress } \\
(\mathrm{MPa})\end{array}$ & $\begin{array}{l}\text { Engr. } \\
\text { Strain }\end{array}$ \\
\hline 0.00 & 0.0000 & 402.17 & 0.0234 & 588.75 & 0.1456 \\
\hline 48.36 & 0.0003 & 422.54 & 0.0303 & 614.28 & 0.1803 \\
\hline 204.28 & 0.0028 & 440.75 & 0.0392 & 637.49 & 0.2198 \\
\hline 220.26 & 0.0030 & 459.56 & 0.0486 & 639.37 & 0.2202 \\
\hline 280.31 & 0.0050 & 474.81 & 0.0582 & 656.16 & 0.2726 \\
\hline 310.80 & 0.0067 & 484.83 & 0.0662 & 669.09 & 0.3230 \\
\hline 336.12 & 0.0089 & 522.63 & 0.0900 & 673.06 & 0.3595 \\
\hline 356.52 & 0.0123 & 554.65 & 0.1125 & 673.92 & 0.3920 \\
\hline 380.47 & 0.0170 & & & & \\
\hline
\end{tabular}


Table 90. Test data for specimen 692-22

\begin{tabular}{|c|c|c|c|c|c|}
\hline $\begin{array}{l}\text { Test Number } \\
\text { Aging Temp. } \\
\text { Yield Stress }\end{array}$ & \multicolumn{2}{|c|}{$\begin{array}{l}: 133 \\
: 400^{\circ} \mathrm{C} \\
: 278.6 \mathrm{MPa}\end{array}$} & \multicolumn{2}{|c|}{$\begin{array}{l}\text { Test Temp. } \\
\text { Aging Time } \\
\text { Ultimate Stress }\end{array}$} & $\begin{array}{l}25^{\circ} \mathrm{C} \\
10,000 \mathrm{~h} \\
699.1 \mathrm{MPa}\end{array}$ \\
\hline \multirow{2}{*}{$\begin{array}{l}\text { Engr. } \\
\text { Stress } \\
(\mathrm{MPa})\end{array}$} & \multicolumn{2}{|r|}{ Engr. } & \multicolumn{3}{|c|}{ Engr. } \\
\hline & $\begin{array}{l}\text { Engr. } \\
\text { Strain }\end{array}$ & $\begin{array}{l}\text { Stress } \\
\text { (MPa) }\end{array}$ & $\begin{array}{l}\text { Engr. } \\
\text { Strain }\end{array}$ & $\begin{array}{l}\text { Stress } \\
(\mathrm{MPa})\end{array}$ & $\begin{array}{l}\text { Engr. } \\
\text { Strain }\end{array}$ \\
\hline 0.00 & 0.0000 & 436.88 & 0.0271 & 623.56 & 0.1674 \\
\hline 38.38 & 0.0003 & 463.13 & 0.0381 & 640.99 & 0.1934 \\
\hline 208.64 & 0.0027 & 478.68 & 0.0460 & 659.25 & 0.2328 \\
\hline 221.35 & 0.0030 & 490.47 & 0.0543 & 671.26 & 0.2732 \\
\hline 307.54 & 0.0057 & 532.36 & 0.0824 & 690.36 & 0.3315 \\
\hline 360.78 & 0.0101 & 561.09 & 0.0998 & 697.69 & 0.3936 \\
\hline 395.49 & 0.0154 & 585.36 & 0.1218 & 699.13 & 0.4474 \\
\hline 417.65 & 0.0208 & 597.45 & 0.1338 & & \\
\hline
\end{tabular}

Table 91. Test data for specimen 692-23

\begin{tabular}{|c|c|c|c|c|c|}
\hline $\begin{array}{l}\text { Test Number } \\
\text { Aging Temp. } \\
\text { Yield Stress }\end{array}$ & $\begin{array}{l}: 2 \\
: 40 \\
: 26\end{array}$ & & $\begin{array}{l}\text { Tes } \\
\text { Agi } \\
\text { Ulti }\end{array}$ & $\begin{array}{l}\mathrm{np} . \\
\text { me } \\
\text { Stress }\end{array}$ & $\begin{array}{l}25^{\circ} \mathrm{C} \\
10,000 \mathrm{~h} \\
664.7 \mathrm{MPa}\end{array}$ \\
\hline \multicolumn{2}{|l|}{ Engr. } & \multicolumn{2}{|l|}{ Engr. } & \multicolumn{2}{|l|}{ Engr. } \\
\hline $\begin{array}{l}\text { Stress } \\
(\mathrm{MPa})\end{array}$ & $\begin{array}{l}\text { Engr. } \\
\text { Strain }\end{array}$ & $\begin{array}{l}\text { Stress } \\
\text { (MPa) }\end{array}$ & $\begin{array}{l}\text { Engr. } \\
\text { Strain }\end{array}$ & $\begin{array}{l}\text { Stress } \\
(\mathrm{MPa})\end{array}$ & $\begin{array}{l}\text { Engr. } \\
\text { Strain }\end{array}$ \\
\hline 33.299 & 0.0002 & 338.892 & 0.0101 & 586.626 & 0.1587 \\
\hline 49.029 & 0.0003 & 361.067 & 0.0140 & 589.529 & 0.1629 \\
\hline 74.030 & 0.0004 & 393.989 & 0.0228 & 599.642 & 0.1780 \\
\hline 85.676 & 0.0005 & 454.954 & 0.0488 & 611.060 & 0.1975 \\
\hline 147.160 & 0.0009 & 458.695 & 0.0508 & 618.851 & 0.2128 \\
\hline 159.290 & 0.0010 & 486.807 & 0.0673 & 627.753 & 0.2327 \\
\hline 171.333 & 0.0011 & 516.370 & 0.0877 & 633.076 & 0.2460 \\
\hline 245.169 & 0.0028 & 536.450 & 0.1042 & 642.172 & 0.2731 \\
\hline 252.088 & 0.0031 & 543.418 & 0.1104 & 657.656 & 0.3407 \\
\hline 277.017 & 0.0044 & 564.078 & 0.1313 & 661.188 & 0.3663 \\
\hline 287.836 & 0.0051 & 578.449 & 0.1481 & 664.043 & 0.4000 \\
\hline
\end{tabular}


Table 92. Test data for specimen 69-109

\begin{tabular}{|c|c|c|c|c|c|}
\hline $\begin{array}{l}\text { Test Number } \\
\text { Aging Temp. } \\
\text { Yield Stress }\end{array}$ & \multicolumn{2}{|c|}{$\begin{array}{l}: 135 \\
: 400^{\circ} \mathrm{C} \\
: 276.7 \mathrm{MPa}\end{array}$} & \multicolumn{2}{|c|}{$\begin{array}{l}\text { Test Temp. } \\
\text { Aging Time } \\
\text { Ultimate Stress }\end{array}$} & $\begin{array}{l}25^{\circ} \mathrm{C} \\
10,000 \mathrm{~h} \\
688.0 \mathrm{MPa}\end{array}$ \\
\hline \multicolumn{2}{|l|}{ Engr. } & \multicolumn{2}{|l|}{ Engr. } & \multicolumn{2}{|l|}{ Engr. } \\
\hline $\begin{array}{l}\text { Stress } \\
(\mathrm{MPa})\end{array}$ & $\begin{array}{l}\text { Engr. } \\
\text { Strain }\end{array}$ & $\begin{array}{l}\text { Stress } \\
(\mathrm{MPa})\end{array}$ & $\begin{array}{l}\text { Engr. } \\
\text { Strain }\end{array}$ & $\begin{array}{l}\text { Stress } \\
(\mathrm{MPa})\end{array}$ & $\begin{array}{l}\text { Engr. } \\
\text { Strain }\end{array}$ \\
\hline 0.00 & 0.0000 & 431.75 & 0.0220 & 627.12 & 0.2021 \\
\hline 43.64 & 0.0003 & 461.67 & 0.0470 & 651.41 & 0.2642 \\
\hline 203.61 & 0.0031 & 479.14 & 0.0574 & 665.36 & 0.3196 \\
\hline 305.80 & 0.0065 & 492.48 & 0.0667 & 674.17 & 0.3623 \\
\hline 344.05 & 0.0100 & 542.45 & 0.0988 & 678.09 & 0.3952 \\
\hline 377.06 & 0.0149 & 573.46 & 0.1271 & 685.21 & 0.4499 \\
\hline 405.86 & 0.0221 & 601.41 & 0.1597 & 688.02 & 0.5581 \\
\hline
\end{tabular}

Table 93. Test data for specimen 691-04

\begin{tabular}{|c|c|c|c|c|c|}
\hline $\begin{array}{l}\text { Test Number } \\
\text { Aging Temp. } \\
\text { Yield Stress }\end{array}$ & \multicolumn{2}{|c|}{$\begin{array}{l}: \text { MEA Test } \\
: 450^{\circ} \mathrm{C} \\
: 271.3 \mathrm{MPa}\end{array}$} & \multicolumn{2}{|c|}{$\begin{array}{l}\text { Test Temp. } \\
\text { Aging Time } \\
\text { Ultimate Stress }\end{array}$} & \multirow[t]{2}{*}{$\begin{array}{l}25^{\circ} \mathrm{C} \\
2,570 \mathrm{~h} \\
691.9 \mathrm{MPa}\end{array}$} \\
\hline Engr. & & Engr. & & Engr. & \\
\hline $\begin{array}{l}\text { Stress } \\
(\mathrm{MPa})\end{array}$ & $\begin{array}{l}\text { Engr. } \\
\text { Strain }\end{array}$ & $\begin{array}{l}\text { Stress } \\
(\mathrm{MPa})\end{array}$ & $\begin{array}{l}\text { Engr. } \\
\text { Strain }\end{array}$ & $\begin{array}{l}\text { Stress } \\
(\mathrm{MPa})\end{array}$ & $\begin{array}{l}\text { Engr. } \\
\text { Strain }\end{array}$ \\
\hline 37.58 & 0.0000 & 325.78 & 0.0068 & 606.46 & 0.1622 \\
\hline 54.19 & 0.0003 & 387.69 & 0.0158 & 615.01 & 0.1738 \\
\hline 73.43 & 0.0004 & 424.23 & 0.0267 & 622.60 & 0.1854 \\
\hline 94.80 & 0.0005 & 452.99 & 0.0378 & 629.22 & 0.1970 \\
\hline 118.73 & 0.0006 & 475.26 & 0.0489 & 632.39 & 0.2085 \\
\hline 143.20 & 0.0007 & 494.56 & 0.0601 & 641.35 & 0.2203 \\
\hline 169.47 & 0.0010 & 514.14 & 0.0712 & 646.73 & 0.2322 \\
\hline 192.98 & 0.0012 & 530.69 & 0.0824 & 649.56 & 0.2442 \\
\hline 213.46 & 0.0016 & 199.67 & 0.0728 & 656.59 & 0.2559 \\
\hline 233.87 & 0.0021 & 543.38 & 0.0936 & 658.59 & 0.2669 \\
\hline 251.66 & 0.0026 & 558.75 & 0.1049 & 663.48 & 0.2779 \\
\hline 266.55 & 0.0032 & 571.30 & 0.1162 & 666.65 & 0.2894 \\
\hline 282.06 & 0.0039 & 579.78 & 0.1278 & 97.19 & 0.3008 \\
\hline 294.13 & 0.0045 & 589.57 & 0.1392 & 97.74 & 0.3109 \\
\hline 305.78 & 0.0053 & 599.02 & 0.1507 & 97.83 & 0.3197 \\
\hline 316.47 & 0.0060 & & & & \\
\hline
\end{tabular}


Table 94. Test data for specimen 691-05

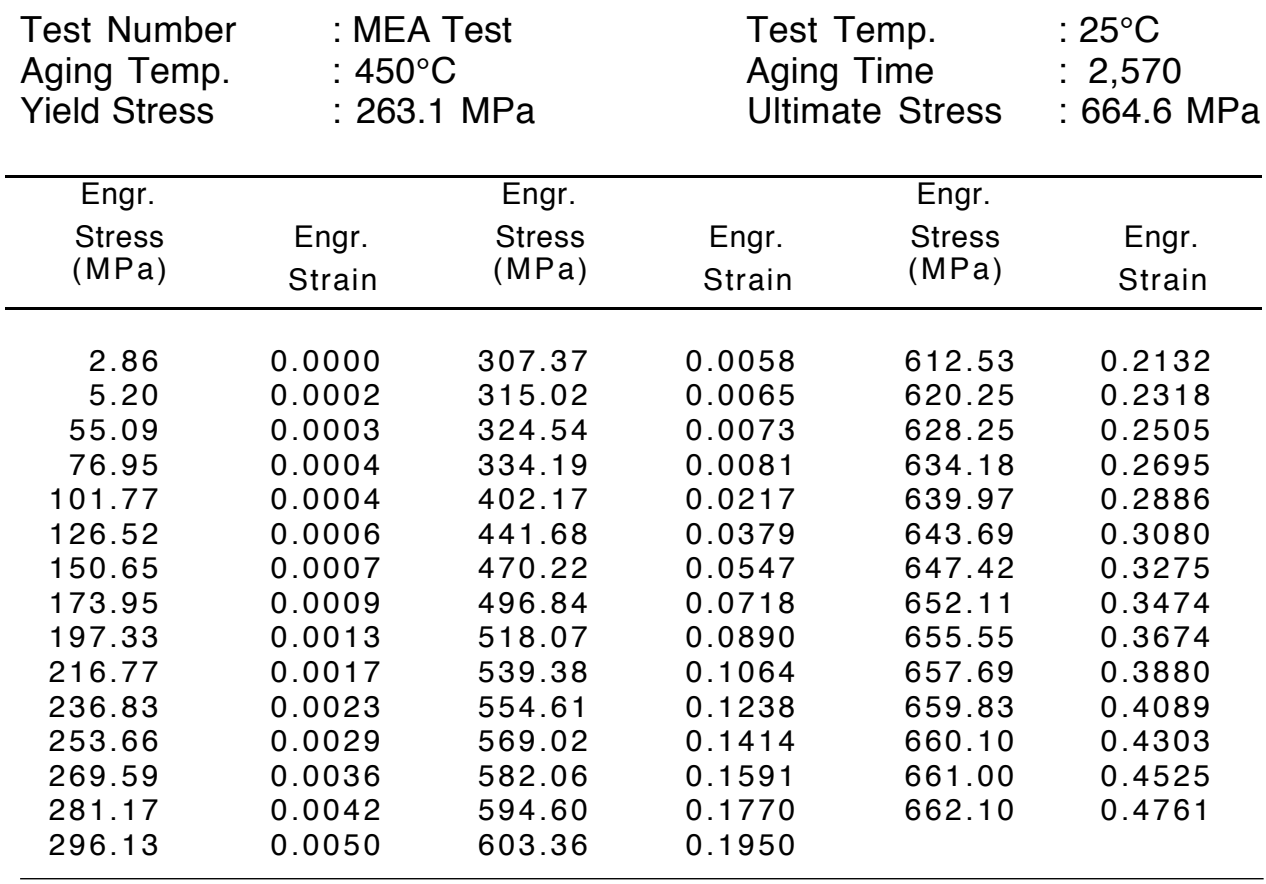

Table 95. Test data for specimen 693-42

\begin{tabular}{|c|c|c|c|c|c|}
\hline $\begin{array}{l}\text { Test Number } \\
\text { Aging Temp. } \\
\text { Yield Stress }\end{array}$ & \multicolumn{2}{|c|}{$\begin{array}{l}: \text { MEA Test } \\
: \text { Unaged } \\
: 190.8 \mathrm{MPa}\end{array}$} & \multicolumn{2}{|c|}{$\begin{array}{l}\text { Test Temp. } \\
\text { Aging Time } \\
\text { Ultimate Stress }\end{array}$} & $\begin{array}{l}290^{\circ} \mathrm{C} \\
- \\
420.9 \mathrm{MPa}\end{array}$ \\
\hline \multirow{2}{*}{$\begin{array}{l}\text { Engr. } \\
\text { Stress } \\
\text { (MPa) }\end{array}$} & \multicolumn{2}{|r|}{ Engr. } & \multicolumn{3}{|c|}{ Engr. } \\
\hline & $\begin{array}{l}\text { Engr. } \\
\text { Strain }\end{array}$ & $\begin{array}{l}\text { Stress } \\
(\mathrm{MPa})\end{array}$ & $\begin{array}{l}\text { Engr. } \\
\text { Strain }\end{array}$ & $\begin{array}{l}\text { Stress } \\
(\mathrm{MPa})\end{array}$ & $\begin{array}{l}\text { Engr. } \\
\text { Strain }\end{array}$ \\
\hline 38.40 & 0.0002 & 221.11 & 0.0099 & 367.35 & 0.1210 \\
\hline 62.81 & 0.0003 & 223.25 & 0.0111 & 374.66 & 0.1298 \\
\hline 88.25 & 0.0004 & 225.67 & 0.0122 & 382.25 & 0.1407 \\
\hline 116.38 & 0.0006 & 244.35 & 0.0204 & 386.04 & 0.1510 \\
\hline 143.76 & 0.0009 & 261.66 & 0.0296 & 391.14 & 0.1606 \\
\hline 162.03 & 0.0013 & 277.17 & 0.0386 & 397.69 & 0.1704 \\
\hline 175.33 & 0.0019 & 290.34 & 0.0477 & 399.96 & 0.1792 \\
\hline 186.50 & 0.0026 & 304.27 & 0.0568 & 402.38 & 0.1917 \\
\hline 193.74 & 0.0035 & 315.50 & 0.0658 & 409.00 & 0.1998 \\
\hline 201.60 & 0.0045 & 327.29 & 0.0748 & 409.76 & 0.2107 \\
\hline 206.22 & 0.0055 & 337.84 & 0.0837 & 410.17 & 0.2202 \\
\hline 209.95 & 0.0066 & 346.88 & 0.0928 & 412.10 & 0.2340 \\
\hline 213.19 & 0.0076 & 352.87 & 0.1024 & 415.00 & 0.2410 \\
\hline 217.87 & 0.0088 & 360.66 & 0.1113 & 416.31 & 0.2581 \\
\hline
\end{tabular}


Table 96. Test data for specimen 694-40

\begin{tabular}{|c|c|c|c|c|c|}
\hline $\begin{array}{l}\text { Test Number } \\
\text { Aging Temp. } \\
\text { Yield Stress }\end{array}$ & $\begin{array}{l}: M \\
: U r \\
: 17\end{array}$ & & $\begin{array}{l}\text { Tes } \\
\text { Agi } \\
\text { Ulti }\end{array}$ & $\begin{array}{l}\text { mp. } \\
\text { me } \\
\text { Stress }\end{array}$ & $\begin{array}{l}290^{\circ} \mathrm{C} \\
- \\
417.0 \mathrm{MPa}\end{array}$ \\
\hline Engr. & & Engr. & & Engr. & \\
\hline $\begin{array}{l}\text { Stress } \\
(\mathrm{MPa})\end{array}$ & $\begin{array}{l}\text { Engr. } \\
\text { Strain }\end{array}$ & $\begin{array}{l}\text { Stress } \\
(\mathrm{MPa})\end{array}$ & $\begin{array}{l}\text { Engr. } \\
\text { Strain }\end{array}$ & $\begin{array}{l}\text { Stress } \\
(\mathrm{MPa})\end{array}$ & $\begin{array}{l}\text { Engr. } \\
\text { Strain }\end{array}$ \\
\hline 5.84 & 0.0002 & 217.12 & 0.0120 & 364.80 & 0.1259 \\
\hline 62.05 & 0.0003 & 217.46 & 0.0134 & 369.70 & 0.1357 \\
\hline 86.12 & 0.0003 & 223.11 & 0.0143 & 380.18 & 0.1476 \\
\hline 110.25 & 0.0006 & 224.91 & 0.0156 & 382.38 & 0.1598 \\
\hline 130.59 & 0.0009 & 242.97 & 0.0248 & 388.52 & 0.1707 \\
\hline 148.72 & 0.0014 & 262.83 & 0.0351 & 393.48 & 0.1830 \\
\hline 166.03 & 0.0021 & 278.69 & 0.0451 & 399.62 & 0.1924 \\
\hline 178.92 & 0.0030 & 293.30 & 0.0551 & 402.03 & 0.2047 \\
\hline 188.50 & 0.0040 & 306.13 & 0.0652 & 405.20 & 0.2206 \\
\hline 195.74 & 0.0051 & 318.88 & 0.0753 & 407.48 & 0.2323 \\
\hline 198.71 & 0.0065 & 330.60 & 0.0853 & 409.20 & 0.2450 \\
\hline 206.43 & 0.0078 & 338.12 & 0.0950 & 411.55 & 0.2557 \\
\hline 206.77 & 0.0093 & 347.43 & 0.1052 & 411.13 & 0.2715 \\
\hline 214.22 & 0.0106 & 356.46 & 0.1155 & 413.62 & 0.2897 \\
\hline
\end{tabular}

Table 97. Test data for specimen 694-32

\begin{tabular}{|c|c|c|c|c|c|}
\hline $\begin{array}{l}\text { Test Number } \\
\text { Aging Temp. } \\
\text { Yield Stress }\end{array}$ & $\begin{array}{l}: 25 \\
: 29 \\
: 17\end{array}$ & $\mathrm{~Pa}$ & $\begin{array}{l}\text { Tes } \\
\text { Agi } \\
\text { Ulti }\end{array}$ & $\begin{array}{l}\text { np. } \\
\text { ne } \\
\text { Stress }\end{array}$ & $\begin{array}{l}290^{\circ} \mathrm{C} \\
30,000 \mathrm{~h} \\
401.9 \mathrm{MPa}\end{array}$ \\
\hline Engr. & & Engr. & & Engr. & \\
\hline $\begin{array}{l}\text { Stress } \\
(\mathrm{MPa})\end{array}$ & $\begin{array}{l}\text { Engr. } \\
\text { Strain }\end{array}$ & $\begin{array}{l}\text { Stress } \\
(\mathrm{MPa})\end{array}$ & $\begin{array}{l}\text { Engr. } \\
\text { Strain }\end{array}$ & $\begin{array}{l}\text { Stress } \\
(\mathrm{MPa})\end{array}$ & $\begin{array}{l}\text { Engr. } \\
\text { Strain }\end{array}$ \\
\hline $\begin{array}{r}92.1209 \\
101.7914 \\
112.1934 \\
131.3247 \\
147.5982 \\
154.0403 \\
159.5851 \\
167.7292 \\
174.1713 \\
190.5912\end{array}$ & $\begin{array}{l}0.0003 \\
0.0004 \\
0.0005 \\
0.0006 \\
0.0008 \\
0.0012 \\
0.0014 \\
0.0019 \\
0.0024 \\
0.0054\end{array}$ & $\begin{array}{l}196.3262 \\
201.4223 \\
207.0842 \\
243.0889 \\
255.0904 \\
258.7821 \\
266.2142 \\
289.8515 \\
305.3058 \\
313.5523 \\
326.3536\end{array}$ & $\begin{array}{l}0.0070 \\
0.0085 \\
0.0108 \\
0.0287 \\
0.0352 \\
0.0373 \\
0.0419 \\
0.0572 \\
0.0684 \\
0.0748 \\
0.0858\end{array}$ & $\begin{array}{l}335.6876 \\
365.2161 \\
368.0202 \\
379.9242 \\
378.3051 \\
388.0293 \\
391.2138 \\
397.4559 \\
395.7735 \\
400.2844 \\
401.8791\end{array}$ & $\begin{array}{l}0.0945 \\
0.1293 \\
0.1315 \\
0.1488 \\
0.1510 \\
0.1683 \\
0.1726 \\
0.1860 \\
0.1931 \\
0.2048 \\
0.2168\end{array}$ \\
\hline
\end{tabular}


Table 98. Test data for specimen 694-33

\begin{tabular}{|c|c|c|c|c|c|}
\hline $\begin{array}{l}\text { Test Number } \\
\text { Aging Temp. } \\
\text { Yield Stress }\end{array}$ & $\begin{array}{l}: 2 \\
: 25 \\
: 17\end{array}$ & & $\begin{array}{l}\text { Tes } \\
\text { Agi } \\
\text { Ulti }\end{array}$ & $\begin{array}{l}\text { np. } \\
\text { ne } \\
\text { Stress }\end{array}$ & $\begin{array}{l}290^{\circ} \mathrm{C} \\
30,000 \mathrm{~h} \\
386.7 \mathrm{MPa}\end{array}$ \\
\hline Engr. & & Engr. & & Engr. & \\
\hline $\begin{array}{l}\text { Stress } \\
\text { (MPa) }\end{array}$ & $\begin{array}{l}\text { Engr. } \\
\text { Strain }\end{array}$ & $\begin{array}{l}\text { Stress } \\
(\mathrm{MPa})\end{array}$ & $\begin{array}{l}\text { Engr. } \\
\text { Strain }\end{array}$ & $\begin{array}{l}\text { Stress } \\
(\mathrm{MPa})\end{array}$ & $\begin{array}{l}\text { Engr. } \\
\text { Strain }\end{array}$ \\
\hline 40.677 & 0.0002 & 183.059 & 0.0043 & 311.235 & 0.0687 \\
\hline 77.338 & 0.0001 & 188.797 & 0.0053 & 314.099 & 0.0708 \\
\hline 86.362 & 0.0002 & 193.883 & 0.0065 & 327.536 & 0.0815 \\
\hline 105.077 & 0.0003 & 199.723 & 0.0080 & 346.024 & 0.0987 \\
\hline 114.923 & 0.0005 & 205.021 & 0.0097 & 355.618 & 0.1093 \\
\hline 139.343 & 0.0007 & 210.938 & 0.0119 & 366.011 & 0.1221 \\
\hline 145.909 & 0.0011 & 221.791 & 0.0164 & 370.124 & 0.1287 \\
\hline 151.739 & 0.0012 & 231.511 & 0.0210 & 375.045 & 0.1351 \\
\hline 156.810 & 0.0015 & 264.427 & 0.0384 & 377.498 & 0.1415 \\
\hline 164.711 & 0.0020 & 279.195 & 0.0470 & 385.428 & 0.1618 \\
\hline 176.450 & 0.0031 & 296.023 & 0.0579 & 386.580 & 0.1705 \\
\hline
\end{tabular}

Table 99. Test data for specimen 69-236

\begin{tabular}{|c|c|c|c|c|c|}
\hline $\begin{array}{l}\text { Test Number } \\
\text { Aging Temp. } \\
\text { Yield Stress }\end{array}$ & $\begin{array}{l}: 25 \\
: 29 \\
: 19\end{array}$ & & $\begin{array}{l}\text { Tes } \\
\text { Agi } \\
\text { Ulti }\end{array}$ & $\begin{array}{l}\text { n. } \\
\text { ne } \\
\text { Stress }\end{array}$ & $\begin{array}{l}290^{\circ} \mathrm{C} \\
30,000 \mathrm{~h} \\
440.5 \mathrm{MPa}\end{array}$ \\
\hline Engr. & & Engr. & & Engr. & \\
\hline $\begin{array}{l}\text { Stress } \\
(\mathrm{MPa})\end{array}$ & $\begin{array}{l}\text { Engr. } \\
\text { Strain }\end{array}$ & $\begin{array}{l}\text { Stress } \\
(\mathrm{MPa})\end{array}$ & $\begin{array}{l}\text { Engr. } \\
\text { Strain }\end{array}$ & $\begin{array}{l}\text { Stress } \\
(\mathrm{MPa})\end{array}$ & $\begin{array}{l}\text { Engr. } \\
\text { Strain }\end{array}$ \\
\hline 29.931 & 0.0002 & 260.447 & 0.0256 & 402.820 & 0.1400 \\
\hline 128.317 & 0.0004 & 278.262 & 0.0348 & 416.634 & 0.1681 \\
\hline 153.637 & 0.0006 & 293.774 & 0.0434 & 422.237 & 0.1748 \\
\hline 160.551 & 0.0008 & 314.841 & 0.0562 & 427.859 & 0.1856 \\
\hline 177.960 & 0.0015 & 327.460 & 0.0649 & 427.540 & 0.1966 \\
\hline 185.697 & 0.0019 & 336.252 & 0.0711 & 428.628 & 0.1989 \\
\hline 191.963 & 0.0026 & 354.730 & 0.0862 & 430.196 & 0.2013 \\
\hline 208.602 & 0.0053 & 368.481 & 0.0989 & 435.668 & 0.2148 \\
\hline 214.752 & 0.0069 & 379.193 & 0.1098 & 438.387 & 0.2264 \\
\hline 220.210 & 0.0085 & 393.975 & 0.1292 & 437.134 & 0.2355 \\
\hline 236.327 & 0.0145 & 395.983 & 0.1313 & 437.841 & 0.2444 \\
\hline
\end{tabular}


Table 100. Test data for specimen 694-26

\begin{tabular}{|c|c|c|c|c|c|}
\hline \multirow{3}{*}{$\begin{array}{l}\text { Engr. } \\
\text { Stress } \\
\text { (MPa) }\end{array}$} & \multicolumn{2}{|c|}{$\begin{array}{l}: 093 \\
: 320^{\circ} \mathrm{C} \\
: 174.4 \mathrm{MPa}\end{array}$} & \multicolumn{2}{|c|}{$\begin{array}{l}\text { Test Temp. } \\
\text { Aging Time } \\
\text { Ultimate Stress }\end{array}$} & \multirow{2}{*}{$\begin{array}{l}290^{\circ} \mathrm{C} \\
10,000 \mathrm{~h} \\
408.7 \mathrm{MPa} \\
\end{array}$} \\
\hline & & Engr. & & Engr. & \\
\hline & $\begin{array}{l}\text { Engr. } \\
\text { Strain }\end{array}$ & $\begin{array}{l}\text { Stress } \\
\text { (MPa) }\end{array}$ & $\begin{array}{l}\text { Engr. } \\
\text { Strain }\end{array}$ & $\begin{array}{l}\text { Stress } \\
(\mathrm{MPa})\end{array}$ & $\begin{array}{l}\text { Engr. } \\
\text { Strain }\end{array}$ \\
\hline 138.84 & 0.0010 & 265.70 & 0.0209 & 356.35 & 0.0886 \\
\hline 160.95 & 0.0016 & 269.89 & 0.0232 & 359.43 & 0.0914 \\
\hline 167.45 & 0.0019 & 274.99 & 0.0261 & 368.11 & 0.0972 \\
\hline 173.71 & 0.0021 & 278.60 & 0.0284 & 370.90 & 0.1000 \\
\hline 179.57 & 0.0025 & 283.15 & 0.0313 & 378.15 & 0.1079 \\
\hline 185.44 & 0.0030 & 291.95 & 0.0367 & 380.44 & 0.1108 \\
\hline 197.02 & 0.0044 & 299.03 & 0.0418 & 388.90 & 0.1219 \\
\hline 207.49 & 0.0053 & 303.34 & 0.0449 & 394.34 & 0.1299 \\
\hline 217.36 & 0.0060 & 307.25 & 0.0478 & 396.33 & 0.1330 \\
\hline 227.69 & 0.0070 & 312.20 & 0.0518 & 399.83 & 0.1394 \\
\hline 236.43 & 0.0083 & 323.14 & 0.0601 & 401.68 & 0.1426 \\
\hline 242.41 & 0.0101 & 332.16 & 0.0677 & 404.39 & 0.1490 \\
\hline 248.98 & 0.0127 & 335.03 & 0.0702 & 405.75 & 0.1522 \\
\hline 252.15 & 0.0140 & 344.10 & 0.0777 & 406.52 & 0.1557 \\
\hline 257.29 & 0.0163 & 346.68 & 0.0800 & 407.38 & 0.1584 \\
\hline 260.90 & 0.0183 & 353.77 & 0.0861 & 408.66 & 0.1643 \\
\hline
\end{tabular}

Table 101. Test data for specimen 694-27

\begin{tabular}{|c|c|c|c|c|c|}
\hline $\begin{array}{l}\text { Test Number } \\
\text { Aging Temp. } \\
\text { Yield Stress }\end{array}$ & $\begin{array}{l}: 0 \\
: 32 \\
: 18\end{array}$ & & $\begin{array}{l}\text { Tes } \\
\text { Agi } \\
\text { Ulti }\end{array}$ & $\begin{array}{l}\text { np. } \\
\text { me } \\
\text { Stress }\end{array}$ & $\begin{array}{l}290^{\circ} \mathrm{C} \\
10,000 \mathrm{~h} \\
422.8 \mathrm{MPa}\end{array}$ \\
\hline Engr. & & Engr. & & Engr. & \\
\hline $\begin{array}{l}\text { Stress } \\
\text { (MPa) }\end{array}$ & $\begin{array}{l}\text { Engr. } \\
\text { Strain }\end{array}$ & $\begin{array}{l}\text { Stress } \\
(\mathrm{MPa})\end{array}$ & $\begin{array}{l}\text { Engr. } \\
\text { Strain }\end{array}$ & $\begin{array}{l}\text { Stress } \\
(\mathrm{MPa})\end{array}$ & $\begin{array}{l}\text { Engr. } \\
\text { Strain }\end{array}$ \\
\hline 146.2500 & 0.0011 & 272.5800 & 0.0190 & 382.9700 & 0.0966 \\
\hline 165.8300 & 0.0016 & 276.2900 & 0.0207 & 391.2300 & 0.1026 \\
\hline 192.3000 & 0.0030 & 289.1900 & 0.0275 & 398.8000 & 0.1111 \\
\hline 204.5200 & 0.0039 & 293.6400 & 0.0300 & 406.0500 & 0.1209 \\
\hline 217.7800 & 0.0045 & 303.1800 & 0.0355 & 410.6100 & 0.1286 \\
\hline 224.7300 & 0.0050 & 311.4900 & 0.0408 & 412.9100 & 0.1319 \\
\hline 235.6900 & 0.0060 & 464.0200 & 0.0456 & 416.2700 & 0.1375 \\
\hline 241.1200 & 0.0070 & 326.2900 & 0.0506 & 418.5200 & 0.1408 \\
\hline 246.4500 & 0.0087 & 334.9000 & 0.0571 & 419.8900 & 0.1488 \\
\hline 252.7700 & 0.0108 & 342.1100 & 0.0623 & 420.7900 & 0.1547 \\
\hline 257.1700 & 0.0124 & 348.4300 & 0.0671 & 421.5300 & 0.1583 \\
\hline 261.1700 & 0.0139 & 355.2700 & 0.0726 & 422.4500 & 0.1621 \\
\hline 265.0200 & 0.0155 & 365.4400 & 0.0809 & 422.8100 & 0.1716 \\
\hline 268.6800 & 0.0172 & 375.4200 & 0.0895 & & \\
\hline
\end{tabular}


Table 102. Test data for specimen 692-42

\begin{tabular}{|c|c|c|c|c|c|}
\hline $\begin{array}{l}\text { Test Number } \\
\text { Aging Temp. } \\
\text { Yield Stress }\end{array}$ & $\begin{array}{l}: 1 \\
: 32 \\
: 17\end{array}$ & & $\begin{array}{l}\text { Tes } \\
\text { Agi } \\
\text { Ulti }\end{array}$ & $\begin{array}{l}\text { np. } \\
\text { me } \\
\text { Stress }\end{array}$ & $\begin{array}{l}290^{\circ} \mathrm{C} \\
30,000 \mathrm{~h} \\
423.2 \mathrm{MPa}\end{array}$ \\
\hline Engr. & & Engr. & & Engr. & \\
\hline $\begin{array}{l}\text { Stress } \\
(\mathrm{MPa})\end{array}$ & $\begin{array}{l}\text { Engr. } \\
\text { Strain }\end{array}$ & $\begin{array}{l}\text { Stress } \\
(\mathrm{MPa})\end{array}$ & $\begin{array}{l}\text { Engr. } \\
\text { Strain }\end{array}$ & $\begin{array}{l}\text { Stress } \\
(\mathrm{MPa})\end{array}$ & $\begin{array}{l}\text { Engr. } \\
\text { Strain }\end{array}$ \\
\hline 0.00 & 0.0000 & 220.77 & 0.0069 & 328.14 & 0.0550 \\
\hline 32.82 & 0.0000 & 225.25 & 0.0079 & 335.92 & 0.0603 \\
\hline 87.60 & 0.0003 & 232.51 & 0.0096 & 343.22 & 0.0654 \\
\hline 122.11 & 0.0007 & 244.00 & 0.0129 & 356.66 & 0.0752 \\
\hline 147.65 & 0.0012 & 246.99 & 0.0140 & 365.52 & 0.0821 \\
\hline 166.30 & 0.0017 & 251.47 & 0.0153 & 373.47 & 0.0883 \\
\hline 174.93 & 0.0020 & 255.50 & 0.0169 & 380.38 & 0.0937 \\
\hline 178.85 & 0.0022 & 259.44 & 0.0185 & 383.34 & 0.0965 \\
\hline 187.88 & 0.0027 & 264.02 & 0.0202 & 389.76 & 0.1002 \\
\hline 194.33 & 0.0033 & 279.22 & 0.0272 & 398.90 & 0.1098 \\
\hline 198.16 & 0.0037 & 289.34 & 0.0326 & 406.36 & 0.1197 \\
\hline 202.33 & 0.0041 & 297.11 & 0.0367 & 412.27 & 0.1287 \\
\hline 208.24 & 0.0049 & 310.43 & 0.0442 & 416.28 & 0.1365 \\
\hline 213.17 & 0.0056 & 319.00 & 0.0493 & 420.05 & 0.1444 \\
\hline 215.60 & 0.0060 & 321.95 & 0.0514 & 423.15 & 0.1633 \\
\hline
\end{tabular}

Table 103. Test data for specimen 694-39

\begin{tabular}{|c|c|c|c|c|c|}
\hline $\begin{array}{l}\text { Test Number } \\
\text { Aging Temp. } \\
\text { Yield Stress }\end{array}$ & $\begin{array}{l}: 1 \\
: 32 \\
: 16\end{array}$ & & $\begin{array}{l}\text { Tes } \\
\text { Agir } \\
\text { Ulti }\end{array}$ & $\begin{array}{l}\text { np. } \\
\text { ne } \\
\text { Stress }\end{array}$ & $\begin{array}{l}290^{\circ} \mathrm{C} \\
30,000 \mathrm{~h} \\
413.8 \mathrm{MPa}\end{array}$ \\
\hline Engr. & & Engr. & & Engr. & \\
\hline $\begin{array}{l}\text { Stress } \\
(\mathrm{MPa})\end{array}$ & $\begin{array}{l}\text { Engr. } \\
\text { Strain }\end{array}$ & $\begin{array}{l}\text { Stress } \\
(\mathrm{MPa})\end{array}$ & $\begin{array}{l}\text { Engr. } \\
\text { Strain }\end{array}$ & $\begin{array}{l}\text { Stress } \\
(\mathrm{MPa})\end{array}$ & $\begin{array}{l}\text { Engr. } \\
\text { Strain }\end{array}$ \\
\hline 0.00 & 0.0000 & 221.61 & 0.0109 & 342.46 & 0.0788 \\
\hline 38.24 & 0.0000 & 225.99 & 0.0124 & 356.38 & 0.0897 \\
\hline 77.18 & 0.0003 & 230.03 & 0.0137 & 362.54 & 0.0945 \\
\hline 118.92 & 0.0009 & 233.81 & 0.0153 & 370.79 & 0.0999 \\
\hline 142.34 & 0.0014 & 242.89 & 0.0185 & 377.89 & 0.1069 \\
\hline 154.72 & 0.0018 & 246.67 & 0.0203 & 386.27 & 0.1162 \\
\hline 166.44 & 0.0024 & 255.09 & 0.0238 & 391.19 & 0.1222 \\
\hline 177.42 & 0.0031 & 263.06 & 0.0277 & 399.65 & 0.1348 \\
\hline 183.21 & 0.0037 & 267.79 & 0.0302 & 403.16 & 0.1411 \\
\hline 188.10 & 0.0043 & 275.61 & 0.0344 & 406.77 & 0.1481 \\
\hline 197.17 & 0.0055 & 281.19 & 0.0374 & 409.33 & 0.1544 \\
\hline 202.51 & 0.0065 & 286.87 & 0.0411 & 411.89 & 0.1614 \\
\hline 207.90 & 0.0076 & 301.45 & 0.0499 & 413.02 & 0.1666 \\
\hline 212.68 & 0.0085 & 311.21 & 0.0560 & 413.54 & 0.1712 \\
\hline 217.02 & 0.0096 & 328.38 & 0.0683 & 413.84 & 0.1796 \\
\hline
\end{tabular}


Table 104. Test data for specimen 69-246

\begin{tabular}{|c|c|c|c|c|c|}
\hline $\begin{array}{l}\text { Test Number } \\
\text { Aging Temp. } \\
\text { Yield Stress }\end{array}$ & $\begin{array}{l}: 1 \\
: 32 \\
: 16\end{array}$ & & $\begin{array}{l}\text { Tes } \\
\text { Agi } \\
\text { Ulti }\end{array}$ & $\begin{array}{l}\text { np. } \\
\text { ne } \\
\text { Stress }\end{array}$ & $\begin{array}{l}290^{\circ} \mathrm{C} \\
30,000 \mathrm{~h} \\
423.0 \mathrm{MPa}\end{array}$ \\
\hline Engr. & & Engr. & & Engr. & \\
\hline $\begin{array}{l}\text { Stress } \\
(\mathrm{MPa})\end{array}$ & $\begin{array}{l}\text { Engr. } \\
\text { Strain }\end{array}$ & $\begin{array}{l}\text { Stress } \\
(\mathrm{MPa})\end{array}$ & $\begin{array}{l}\text { Engr. } \\
\text { Strain }\end{array}$ & $\begin{array}{l}\text { Stress } \\
(\mathrm{MPa})\end{array}$ & $\begin{array}{l}\text { Engr. } \\
\text { Strain }\end{array}$ \\
\hline 0.00 & 0.0000 & 226.65 & 0.0109 & 333.06 & 0.0719 \\
\hline 38.39 & 0.0000 & 233.55 & 0.0131 & 336.77 & 0.0751 \\
\hline 88.32 & 0.0003 & 241.50 & 0.0160 & 342.78 & 0.0801 \\
\hline 125.36 & 0.0008 & 251.71 & 0.0205 & 353.04 & 0.0882 \\
\hline 151.10 & 0.0014 & 263.56 & 0.0261 & 366.00 & 0.0976 \\
\hline 164.85 & 0.0020 & 273.17 & 0.0310 & 380.33 & 0.1127 \\
\hline 175.80 & 0.0025 & 281.62 & 0.0363 & 388.44 & 0.1227 \\
\hline 184.49 & 0.0031 & 285.82 & 0.0389 & 397.96 & 0.1349 \\
\hline 190.74 & 0.0036 & 291.13 & 0.0422 & 402.18 & 0.1416 \\
\hline 198.19 & 0.0045 & 300.58 & 0.0482 & 408.70 & 0.1540 \\
\hline 203.84 & 0.0054 & 304.64 & 0.0512 & 412.71 & 0.1631 \\
\hline 210.14 & 0.0065 & 307.84 & 0.0535 & 418.09 & 0.1773 \\
\hline 212.44 & 0.0070 & 312.19 & 0.0568 & 419.24 & 0.1818 \\
\hline 216.34 & 0.0080 & 318.80 & 0.0616 & 422.96 & 0.1947 \\
\hline 220.29 & 0.0091 & 322.65 & 0.0645 & 423.02 & 0.1987 \\
\hline
\end{tabular}

Table 105. Test data for specimen 692-28

\begin{tabular}{|c|c|c|c|c|c|}
\hline $\begin{array}{l}\text { Test Number } \\
\text { Aging Temp. } \\
\text { Yield Stress }\end{array}$ & \multicolumn{2}{|c|}{$\begin{array}{ll}: & 322 \\
: & 320^{\circ} \mathrm{C} \\
: & 209.1 \mathrm{MPa}\end{array}$} & \multicolumn{2}{|c|}{$\begin{array}{l}\text { Test Temp. } \\
\text { Aging Time } \\
\text { Ultimate Stress }\end{array}$} & $\begin{array}{l}290^{\circ} \mathrm{C} \\
55,000 \mathrm{~h} \\
462.5 \mathrm{MPa}\end{array}$ \\
\hline Engr. & & Engr. & & Engr. & \\
\hline $\begin{array}{l}\text { Stress } \\
(\mathrm{MPa})\end{array}$ & $\begin{array}{l}\text { Engr. } \\
\text { Strain }\end{array}$ & $\begin{array}{l}\text { Stress } \\
(\mathrm{MPa})\end{array}$ & $\begin{array}{l}\text { Engr. } \\
\text { Strain }\end{array}$ & $\begin{array}{l}\text { Stress } \\
(\mathrm{MPa})\end{array}$ & $\begin{array}{l}\text { Engr. } \\
\text { Strain }\end{array}$ \\
\hline 40.830 & 0.0001 & 231.050 & 0.0052 & 404.400 & 0.0993 \\
\hline 107.640 & 0.0002 & 248.300 & 0.0089 & 422.080 & 0.1191 \\
\hline 118.020 & 0.0003 & 253.150 & 0.0102 & 430.380 & 0.1302 \\
\hline 127.670 & 0.0004 & 274.850 & 0.0178 & 437.620 & 0.1412 \\
\hline 153.380 & 0.0006 & 300.340 & 0.0291 & 442.680 & 0.1501 \\
\hline 161.060 & 0.0007 & 318.020 & 0.0380 & 448.180 & 0.1612 \\
\hline 168.070 & 0.0009 & 337.670 & 0.0490 & 452.890 & 0.1724 \\
\hline 187.220 & 0.0016 & 348.550 & 0.0556 & 453.660 & 0.1746 \\
\hline 197.770 & 0.0020 & 368.160 & 0.0687 & 456.090 & 0.1812 \\
\hline 220.280 & 0.0038 & 382.460 & 0.0795 & 459.530 & 0.1923 \\
\hline 225.950 & 0.0044 & 387.760 & 0.0839 & 462.410 & 0.2119 \\
\hline
\end{tabular}


Table 106. Test data for specimen 692-29

\begin{tabular}{|c|c|c|c|c|c|}
\hline $\begin{array}{l}\text { Test Number } \\
\text { Aging Temp. } \\
\text { Yield Stress }\end{array}$ & $\begin{array}{l}: 3 \\
: 32 \\
: 21\end{array}$ & & $\begin{array}{l}\text { Tes } \\
\text { Agi } \\
\text { Ulti }\end{array}$ & $\begin{array}{l}\text { np. } \\
\text { ne } \\
\text { Stress }\end{array}$ & $\begin{array}{l}290^{\circ} \mathrm{C} \\
55,000 \mathrm{~h} \\
464.4 \mathrm{MPa}\end{array}$ \\
\hline Engr. & & Engr. & & Engr. & \\
\hline $\begin{array}{l}\text { Stress } \\
\text { (MPa) }\end{array}$ & $\begin{array}{l}\text { Engr. } \\
\text { Strain }\end{array}$ & $\begin{array}{l}\text { Stress } \\
(\mathrm{MPa})\end{array}$ & $\begin{array}{l}\text { Engr. } \\
\text { Strain }\end{array}$ & $\begin{array}{l}\text { Stress } \\
(\mathrm{MPa})\end{array}$ & $\begin{array}{l}\text { Engr. } \\
\text { Strain }\end{array}$ \\
\hline 15.50 & 0.0001 & 313.79 & 0.0358 & 436.19 & 0.1390 \\
\hline 52.59 & 0.0002 & 330.08 & 0.0447 & 441.32 & 0.1479 \\
\hline 107.64 & 0.0002 & 348.55 & 0.0556 & 442.68 & 0.1501 \\
\hline 153.38 & 0.0006 & 355.38 & 0.0598 & 447.10 & 0.1590 \\
\hline 187.22 & 0.0016 & 368.16 & 0.0687 & 449.08 & 0.1634 \\
\hline 197.77 & 0.0020 & 374.18 & 0.0730 & 452.89 & 0.1724 \\
\hline 220.28 & 0.0038 & 382.46 & 0.0795 & 453.66 & 0.1746 \\
\hline 225.95 & 0.0044 & 387.76 & 0.0839 & 456.09 & 0.1812 \\
\hline 231.05 & 0.0052 & 395.23 & 0.0905 & 456.81 & 0.1835 \\
\hline 248.30 & 0.0089 & 404.40 & 0.0993 & 459.07 & 0.1901 \\
\hline 253.15 & 0.0102 & 412.71 & 0.1081 & 459.53 & 0.1923 \\
\hline 269.03 & 0.0155 & 414.79 & 0.1102 & 461.46 & 0.2002 \\
\hline 274.85 & 0.0178 & 422.08 & 0.1191 & 461.52 & 0.2026 \\
\hline 290.71 & 0.0246 & 423.95 & 0.1212 & 462.28 & 0.2096 \\
\hline 300.34 & 0.0291 & 430.38 & 0.1302 & 462.41 & 0.2119 \\
\hline
\end{tabular}

Table 107. Test data for specimen 69-130

\begin{tabular}{|c|c|c|c|c|c|}
\hline $\begin{array}{l}\text { Test Number } \\
\text { Aging Temp. } \\
\text { Yield Stress }\end{array}$ & $\begin{array}{l}: 3 \\
: 32 \\
: 20\end{array}$ & & $\begin{array}{l}\text { Tes } \\
\text { Agi } \\
\text { Ulti }\end{array}$ & $\begin{array}{l}\text { np. } \\
\text { ne } \\
\text { Stress }\end{array}$ & $\begin{array}{l}290^{\circ} \mathrm{C} \\
55,000 \mathrm{~h} \\
468.0 \mathrm{MPa}\end{array}$ \\
\hline \multicolumn{2}{|l|}{ Engr. } & \multicolumn{2}{|l|}{ Engr. } & \multicolumn{2}{|l|}{ Engr. } \\
\hline $\begin{array}{l}\text { Stress } \\
(\mathrm{MPa})\end{array}$ & $\begin{array}{l}\text { Engr. } \\
\text { Strain }\end{array}$ & $\begin{array}{l}\text { Stress } \\
\text { (MPa) }\end{array}$ & $\begin{array}{l}\text { Engr. } \\
\text { Strain }\end{array}$ & $\begin{array}{l}\text { Stress } \\
(\mathrm{MPa})\end{array}$ & $\begin{array}{l}\text { Engr. } \\
\text { Strain }\end{array}$ \\
\hline 14.9900 & 0.0001 & 297.9800 & 0.0256 & 442.2700 & 0.1422 \\
\hline 51.6000 & 0.0002 & 316.5400 & 0.0345 & 444.7000 & 0.1465 \\
\hline 82.8700 & 0.0003 & 329.1100 & 0.0410 & 448.3000 & 0.1534 \\
\hline 137.9900 & 0.0007 & 348.1400 & 0.0519 & 450.4400 & 0.1577 \\
\hline 164.9200 & 0.0011 & 361.8000 & 0.0606 & 451.5000 & 0.1599 \\
\hline 193.2100 & 0.0019 & 368.2400 & 0.0650 & 453.4500 & 0.1644 \\
\hline 199.1900 & 0.0021 & 380.1900 & 0.0738 & 456.9800 & 0.1734 \\
\hline 204.5900 & 0.0025 & 391.1000 & 0.0825 & 457.7800 & 0.1757 \\
\hline 224.7700 & 0.0042 & 396.1400 & 0.0870 & 460.0000 & 0.1824 \\
\hline 236.0400 & 0.0056 & 398.6300 & 0.0891 & 461.3400 & 0.1870 \\
\hline 242.8700 & 0.0067 & 405.5600 & 0.0956 & 462.8300 & 0.1937 \\
\hline 260.3900 & 0.0111 & 414.1800 & 0.1044 & 463.0500 & 0.1957 \\
\hline 266.0500 & 0.0127 & 427.2600 & 0.1198 & 464.1600 & 0.2028 \\
\hline 271.3400 & 0.0146 & 433.7100 & 0.1288 & 464.3300 & 0.2052 \\
\hline 287.6800 & 0.0210 & 436.8000 & 0.1332 & & \\
\hline
\end{tabular}


Table 108. Test data for specimen 692-27

\begin{tabular}{|c|c|c|c|c|c|}
\hline $\begin{array}{l}\text { Test Number } \\
\text { Aging Temp. } \\
\text { Yield Stress }\end{array}$ & $\begin{array}{l}: \mathrm{M} \\
: 35 \\
: 17\end{array}$ & & $\begin{array}{l}\text { Tes } \\
\text { Agi } \\
\text { Ulti }\end{array}$ & $\begin{array}{l}\text { np. } \\
\text { ne } \\
\text { Stress }\end{array}$ & $\begin{array}{l}290^{\circ} \mathrm{C} \\
2,570 \mathrm{~h} \\
451.3 \mathrm{MPa}\end{array}$ \\
\hline Engr. & & Engr. & & Engr. & \\
\hline $\begin{array}{l}\text { Stress } \\
(\mathrm{MPa})\end{array}$ & $\begin{array}{l}\text { Engr. } \\
\text { Strain }\end{array}$ & $\begin{array}{l}\text { Stress } \\
(\mathrm{MPa})\end{array}$ & $\begin{array}{l}\text { Engr. } \\
\text { Strain }\end{array}$ & $\begin{array}{l}\text { Stress } \\
(\mathrm{MPa})\end{array}$ & $\begin{array}{l}\text { Engr. } \\
\text { Strain }\end{array}$ \\
\hline 30.96 & 0.0002 & 218.91 & 0.0095 & 396.24 & 0.1123 \\
\hline 53.09 & 0.0003 & 224.15 & 0.0107 & 402.79 & 0.1220 \\
\hline 78.81 & 0.0005 & 229.60 & 0.0118 & 411.62 & 0.1316 \\
\hline 100.80 & 0.0006 & 230.77 & 0.0129 & 418.44 & 0.1416 \\
\hline 122.04 & 0.0010 & 254.55 & 0.0213 & 420.92 & 0.1519 \\
\hline 139.62 & 0.0015 & 276.89 & 0.0305 & 426.30 & 0.1607 \\
\hline 154.79 & 0.0021 & 295.79 & 0.0395 & 433.68 & 0.1711 \\
\hline 168.65 & 0.0027 & 312.68 & 0.0485 & 435.96 & 0.1819 \\
\hline 180.44 & 0.0035 & 328.95 & 0.0574 & 437.47 & 0.1949 \\
\hline 191.47 & 0.0044 & 341.84 & 0.0663 & 443.26 & 0.2042 \\
\hline 198.29 & 0.0054 & 354.46 & 0.0754 & 444.16 & 0.2147 \\
\hline 204.36 & 0.0064 & 366.53 & 0.0844 & 445.40 & 0.2278 \\
\hline 209.81 & 0.0074 & 376.66 & 0.0936 & 448.23 & 0.2386 \\
\hline 214.98 & 0.0084 & 386.52 & 0.1029 & & \\
\hline
\end{tabular}

Table 109. Test data for specimen 694-09

\begin{tabular}{|c|c|c|c|c|c|}
\hline $\begin{array}{l}\text { Test Number } \\
\text { Aging Temp. } \\
\text { Yield Stress }\end{array}$ & $\begin{array}{l}: 08 \\
: 35 \\
: 15\end{array}$ & & $\begin{array}{l}\text { Tes } \\
\text { Agi } \\
\text { Ulti }\end{array}$ & $\begin{array}{l}\text { np. } \\
\text { ne } \\
\text { Stress }\end{array}$ & $\begin{array}{l}290^{\circ} \mathrm{C} \\
10,000 \mathrm{~h} \\
413.1 \mathrm{MPa}\end{array}$ \\
\hline Engr. & & Engr. & & Engr. & \\
\hline $\begin{array}{l}\text { Stress } \\
(\mathrm{MPa})\end{array}$ & $\begin{array}{l}\text { Engr. } \\
\text { Strain }\end{array}$ & $\begin{array}{l}\text { Stress } \\
(\mathrm{MPa})\end{array}$ & $\begin{array}{l}\text { Engr. } \\
\text { Strain }\end{array}$ & $\begin{array}{l}\text { Stress } \\
(\mathrm{MPa})\end{array}$ & $\begin{array}{l}\text { Engr. } \\
\text { Strain }\end{array}$ \\
\hline 123.82 & 0.0008 & 285.16 & 0.0396 & 384.24 & 0.1428 \\
\hline 135.09 & 0.0011 & 288.91 & 0.0425 & 388.55 & 0.1516 \\
\hline 145.72 & 0.0014 & 292.03 & 0.0451 & 392.78 & 0.1621 \\
\hline 179.65 & 0.0034 & 297.24 & 0.0497 & 396.40 & 0.1704 \\
\hline 184.07 & 0.0039 & 318.71 & 0.0680 & 399.99 & 0.1807 \\
\hline 187.95 & 0.0044 & 322.01 & 0.0713 & 402.35 & 0.1886 \\
\hline 197.82 & 0.0059 & 328.13 & 0.0044 & 404.54 & 0.1971 \\
\hline 209.48 & 0.0070 & 331.83 & 0.0064 & 405.67 & 0.2006 \\
\hline 250.46 & 0.0161 & 334.79 & 0.0835 & 410.36 & 0.2219 \\
\hline 254.22 & 0.0183 & 342.03 & 0.0902 & 410.65 & 0.2254 \\
\hline 257.59 & 0.0205 & 351.67 & 0.0984 & 411.35 & 0.2291 \\
\hline 261.58 & 0.0233 & 354.18 & 0.1012 & 411.80 & 0.2324 \\
\hline 264.56 & 0.0251 & 362.98 & 0.1120 & 412.14 & 0.2359 \\
\hline 271.69 & 0.0300 & 368.77 & 0.1199 & 413.13 & 0.2504 \\
\hline 278.33 & 0.0344 & 370.36 & 0.1223 & & \\
\hline
\end{tabular}


Table 110. Test data for specimen 69-120

\begin{tabular}{|c|c|c|c|c|c|}
\hline $\begin{array}{l}\text { Test Number } \\
\text { Aging Temp. } \\
\text { Yield Stress }\end{array}$ & $\begin{array}{l}: 0 \\
: 35 \\
: 17\end{array}$ & $\mathrm{MPa}$ & $\begin{array}{l}\text { Tes } \\
\text { Agir } \\
\text { Ulti }\end{array}$ & $\begin{array}{l}\text { np. } \\
\text { ne } \\
\text { Stress }\end{array}$ & $\begin{array}{l}290^{\circ} \mathrm{C} \\
10,000 \mathrm{~h} \\
429.8 \mathrm{MPa}\end{array}$ \\
\hline Engr. & & Engr. & & Engr. & \\
\hline $\begin{array}{l}\text { Stress } \\
(\mathrm{MPa})\end{array}$ & $\begin{array}{l}\text { Engr. } \\
\text { Strain }\end{array}$ & $\begin{array}{l}\text { Stress } \\
(\mathrm{MPa})\end{array}$ & $\begin{array}{l}\text { Engr. } \\
\text { Strain }\end{array}$ & $\begin{array}{l}\text { Stress } \\
(\mathrm{MPa})\end{array}$ & $\begin{array}{l}\text { Engr. } \\
\text { Strain }\end{array}$ \\
\hline 136.20 & 0.0012 & 280.83 & 0.0234 & 384.06 & 0.0967 \\
\hline 155.69 & 0.0017 & 288.57 & 0.0269 & 386.73 & 0.0978 \\
\hline 163.40 & 0.0020 & 292.45 & 0.0293 & 388.24 & 0.0997 \\
\hline 169.57 & 0.0023 & 300.51 & 0.0341 & 394.84 & 0.1057 \\
\hline 181.02 & 0.0028 & 321.25 & 0.0472 & 400.41 & 0.1116 \\
\hline 190.90 & 0.0035 & 327.47 & 0.0516 & 404.27 & 0.1186 \\
\hline 200.80 & 0.0042 & 332.17 & 0.0563 & 406.32 & 0.1205 \\
\hline 213.12 & 0.0047 & 336.71 & 0.0598 & 417.53 & 0.1375 \\
\hline 223.46 & 0.0051 & 341.63 & 0.0630 & 418.61 & 0.1406 \\
\hline 233.17 & 0.0058 & 347.16 & 0.0666 & 423.94 & 0.1475 \\
\hline 237.09 & 0.0064 & 352.21 & 0.0711 & 424.93 & 0.1505 \\
\hline 241.80 & 0.0075 & 357.79 & 0.0753 & 426.86 & 0.1582 \\
\hline 245.58 & 0.0089 & 359.94 & 0.0773 & 427.76 & 0.1623 \\
\hline 268.51 & 0.0176 & 374.46 & 0.0886 & 428.79 & 0.1649 \\
\hline 272.59 & 0.0200 & 379.16 & 0.0925 & 429.62 & 0.1680 \\
\hline
\end{tabular}

Table 111. Test data for specimen 693-14

\begin{tabular}{|c|c|c|c|c|c|}
\hline $\begin{array}{l}\text { Test Number } \\
\text { Aging Temp. } \\
\text { Yield Stress }\end{array}$ & $\begin{array}{l}: 2 \\
: 35 \\
: 18\end{array}$ & & $\begin{array}{l}\text { Tes } \\
\text { Agi } \\
\text { Ulti }\end{array}$ & $\begin{array}{l}\text { np. } \\
\text { ne } \\
\text { Stress }\end{array}$ & $\begin{array}{l}290^{\circ} \mathrm{C} \\
30,000 \mathrm{~h} \\
457.6 \mathrm{MPa}\end{array}$ \\
\hline \multicolumn{2}{|l|}{ Engr. } & \multicolumn{2}{|l|}{ Engr. } & \multicolumn{2}{|l|}{ Engr. } \\
\hline $\begin{array}{l}\text { Stress } \\
(\mathrm{MPa})\end{array}$ & $\begin{array}{l}\text { Engr. } \\
\text { Strain }\end{array}$ & $\begin{array}{l}\text { Stress } \\
(\mathrm{MPa})\end{array}$ & $\begin{array}{l}\text { Engr. } \\
\text { Strain }\end{array}$ & $\begin{array}{l}\text { Stress } \\
(\mathrm{MPa})\end{array}$ & $\begin{array}{l}\text { Engr. } \\
\text { Strain }\end{array}$ \\
\hline 41.930 & 0.0002 & 222.230 & 0.0082 & 340.870 & 0.0606 \\
\hline 44.020 & 0.0002 & 227.610 & 0.0095 & 358.140 & 0.0714 \\
\hline 72.230 & 0.0002 & 232.580 & 0.0110 & 367.790 & 0.0779 \\
\hline 82.970 & 0.0002 & 254.330 & 0.0181 & 370.810 & 0.0800 \\
\hline 104.380 & 0.0003 & 265.620 & 0.0227 & 395.940 & 0.1000 \\
\hline 115.300 & 0.0004 & 270.950 & 0.0249 & 413.610 & 0.1151 \\
\hline 150.160 & 0.0008 & 295.360 & 0.0360 & 423.500 & 0.1263 \\
\hline 156.440 & 0.0011 & 299.860 & 0.0382 & 434.790 & 0.1416 \\
\hline 162.130 & 0.0014 & 321.040 & 0.0492 & 441.260 & 0.1783 \\
\hline 187.280 & 0.0029 & 324.970 & 0.0513 & 445.890 & 0.1869 \\
\hline 199.740 & 0.0044 & 328.880 & 0.0536 & 456.860 & 0.2177 \\
\hline
\end{tabular}


Table 112. Test data for specimen 693-15

\begin{tabular}{|c|c|c|c|c|c|}
\hline \multirow{2}{*}{$\begin{array}{l}\text { Test Number } \\
\text { Aging Temp. } \\
\text { Yield Stress } \\
\text { Engr. }\end{array}$} & \multicolumn{2}{|c|}{$\begin{array}{l}: 245 \\
: 350^{\circ} \mathrm{C} \\
: 195.8 \mathrm{MPa}\end{array}$} & \multicolumn{2}{|c|}{$\begin{array}{l}\text { Test Temp. } \\
\text { Aging Time } \\
\text { Ultimate Stress }\end{array}$} & $\begin{array}{l}290^{\circ} \mathrm{C} \\
30,000 \mathrm{~h} \\
444.0 \mathrm{MPa}\end{array}$ \\
\hline & & Engr. & & Engr. & \\
\hline $\begin{array}{l}\text { Stress } \\
(\mathrm{MPa})\end{array}$ & $\begin{array}{l}\text { Engr. } \\
\text { Strain }\end{array}$ & $\begin{array}{l}\text { Stress } \\
(\mathrm{MPa})\end{array}$ & $\begin{array}{l}\text { Engr. } \\
\text { Strain }\end{array}$ & $\begin{array}{l}\text { Stress } \\
(\mathrm{MPa})\end{array}$ & $\begin{array}{l}\text { Engr. } \\
\text { Strain }\end{array}$ \\
\hline 82.970 & 0.0002 & 199.740 & 0.0044 & 336.470 & 0.0582 \\
\hline 93.520 & 0.0003 & 209.870 & 0.0059 & 340.870 & 0.0606 \\
\hline 115.300 & 0.0004 & 216.440 & 0.0071 & 358.140 & 0.0714 \\
\hline 125.980 & 0.0005 & 222.230 & 0.0082 & 370.810 & 0.0800 \\
\hline 143.140 & 0.0007 & 232.580 & 0.0110 & 395.940 & 0.1000 \\
\hline 150.160 & 0.0008 & 238.500 & 0.0129 & 425.300 & 0.1285 \\
\hline 162.130 & 0.0014 & 249.310 & 0.0163 & 427.020 & 0.1307 \\
\hline 167.380 & 0.0016 & 265.620 & 0.0227 & 434.790 & 0.1416 \\
\hline 180.180 & 0.0025 & 270.950 & 0.0249 & 439.950 & 0.1761 \\
\hline 187.280 & 0.0029 & 299.860 & 0.0382 & 448.150 & 0.1914 \\
\hline 193.920 & 0.0036 & 321.040 & 0.0492 & 451.500 & 0.2004 \\
\hline & & & & 456.860 & 0.2177 \\
\hline
\end{tabular}

Table 113. Test data for specimen 69-270

\begin{tabular}{|c|c|c|c|c|c|}
\hline $\begin{array}{l}\text { Test Number } \\
\text { Aging Temp. } \\
\text { Yield Stress }\end{array}$ & $\begin{array}{l}: 2 \\
: 35 \\
: 15\end{array}$ & $\mathrm{PPa}$ & $\begin{array}{l}\text { Tes } \\
\text { Agi } \\
\text { Ulti }\end{array}$ & $\begin{array}{l}\text { np. } \\
\text { ne } \\
\text { Stress }\end{array}$ & $\begin{array}{l}290^{\circ} \mathrm{C} \\
30,000 \mathrm{~h} \\
462.4 \mathrm{MPa}\end{array}$ \\
\hline Engr. & & Engr. & & Engr. & \\
\hline $\begin{array}{l}\text { Stress } \\
(\mathrm{MPa})\end{array}$ & $\begin{array}{l}\text { Engr. } \\
\text { Strain }\end{array}$ & $\begin{array}{l}\text { Stress } \\
(\mathrm{MPa})\end{array}$ & $\begin{array}{l}\text { Engr. } \\
\text { Strain }\end{array}$ & $\begin{array}{l}\text { Stress } \\
(\mathrm{MPa})\end{array}$ & $\begin{array}{l}\text { Engr. } \\
\text { Strain }\end{array}$ \\
\hline 82.606 & 0.0001 & 228.049 & 0.0085 & 395.334 & 0.0963 \\
\hline 95.725 & 0.0002 & 234.184 & 0.0103 & 413.701 & 0.1135 \\
\hline 108.530 & 0.0002 & 277.671 & 0.0264 & 429.263 & 0.1313 \\
\hline 143.211 & 0.0003 & 306.192 & 0.0395 & 437.264 & 0.1421 \\
\hline 149.390 & 0.0005 & 310.405 & 0.0415 & 440.550 & 0.1470 \\
\hline 160.181 & 0.0006 & 327.972 & 0.0507 & 444.849 & 0.1536 \\
\hline 176.370 & 0.0014 & 335.471 & 0.0551 & 452.378 & 0.1682 \\
\hline 183.372 & 0.0019 & 346.937 & 0.0616 & 453.404 & 0.1704 \\
\hline 200.800 & 0.0036 & 356.335 & 0.0677 & 456.180 & 0.1769 \\
\hline 205.624 & 0.0043 & 367.072 & 0.0747 & 461.617 & 0.1955 \\
\hline 211.986 & 0.0052 & 387.093 & 0.0896 & 462.441 & 0.2042 \\
\hline
\end{tabular}


Table 114. Test data for specimen 692-18

\begin{tabular}{|c|c|c|c|c|c|}
\hline $\begin{array}{l}\text { Test Number } \\
\text { Aging Temp. } \\
\text { Yield Stress }\end{array}$ & $\begin{array}{l}: M \\
: 4 C \\
: 16\end{array}$ & & $\begin{array}{l}\text { Tes } \\
\text { Agi } \\
\text { Ulti }\end{array}$ & $\begin{array}{l}\text { np. } \\
\text { ne } \\
\text { Stress }\end{array}$ & $\begin{array}{l}290^{\circ} \mathrm{C} \\
2,570 \mathrm{~h} \\
444.8 \mathrm{MPa}\end{array}$ \\
\hline Engr. & & Engr. & & Engr. & \\
\hline $\begin{array}{l}\text { Stress } \\
(\mathrm{MPa})\end{array}$ & $\begin{array}{l}\text { Engr. } \\
\text { Strain }\end{array}$ & $\begin{array}{l}\text { Stress } \\
(\mathrm{MPa})\end{array}$ & $\begin{array}{l}\text { Engr. } \\
\text { Strain }\end{array}$ & $\begin{array}{l}\text { Stress } \\
(\mathrm{MPa})\end{array}$ & $\begin{array}{l}\text { Engr. } \\
\text { Strain }\end{array}$ \\
\hline 86.25 & 0.0002 & 233.32 & 0.0099 & 384.31 & 0.0873 \\
\hline 108.04 & 0.0005 & 237.94 & 0.0108 & 391.21 & 0.0936 \\
\hline 127.83 & 0.0009 & 257.24 & 0.0164 & 397.21 & 0.1000 \\
\hline 145.27 & 0.0015 & 274.69 & 0.0230 & 403.89 & 0.1066 \\
\hline 160.58 & 0.0022 & 290.75 & 0.0295 & 410.65 & 0.1131 \\
\hline 173.06 & 0.0029 & 304.75 & 0.0361 & 414.03 & 0.1197 \\
\hline 184.30 & 0.0037 & 316.47 & 0.0425 & 420.17 & 0.1265 \\
\hline 194.23 & 0.0045 & 328.74 & 0.0489 & 424.72 & 0.1332 \\
\hline 203.26 & 0.0053 & 340.19 & 0.0554 & 429.06 & 0.1402 \\
\hline 210.36 & 0.0062 & 349.15 & 0.0618 & 430.72 & 0.1472 \\
\hline 216.84 & 0.0071 & 357.84 & 0.0682 & 435.75 & 0.1544 \\
\hline 223.39 & 0.0080 & 367.77 & 0.0746 & 437.54 & 0.1618 \\
\hline 228.42 & 0.0089 & 376.38 & 0.0809 & & \\
\hline
\end{tabular}

Table 115. Test data for specimen 692-24

\begin{tabular}{|c|c|c|c|c|c|}
\hline $\begin{array}{l}\text { Test Number } \\
\text { Aging Temp. } \\
\text { Yield Stress }\end{array}$ & $\begin{array}{l}: 0 \\
: 40 \\
: 17\end{array}$ & $\mathrm{~Pa}$ & $\begin{array}{l}\text { Tes } \\
\text { Agi } \\
\text { Ulti }\end{array}$ & $\begin{array}{l}\text { mp. } \\
\text { me } \\
\text { Stress }\end{array}$ & $\begin{array}{l}290^{\circ} \mathrm{C} \\
10,000 \mathrm{~h} \\
494.5 \mathrm{MPa}\end{array}$ \\
\hline Engr. & & Engr. & & Engr. & \\
\hline $\begin{array}{l}\text { Stress } \\
\text { (MPa) }\end{array}$ & $\begin{array}{l}\text { Engr. } \\
\text { Strain }\end{array}$ & $\begin{array}{l}\text { Stress } \\
(\mathrm{MPa})\end{array}$ & $\begin{array}{l}\text { Engr. } \\
\text { Strain }\end{array}$ & $\begin{array}{l}\text { Stress } \\
(\mathrm{MPa})\end{array}$ & $\begin{array}{l}\text { Engr. } \\
\text { Strain }\end{array}$ \\
\hline 140.73 & 0.0007 & 317.37 & 0.0229 & 461.09 & 0.1205 \\
\hline 156.38 & 0.0012 & 322.53 & 0.0249 & 466.65 & 0.1281 \\
\hline 172.08 & 0.0017 & 327.39 & 0.0268 & 468.26 & 0.1305 \\
\hline 207.80 & 0.0034 & 334.90 & 0.0301 & 474.50 & 0.1394 \\
\hline 226.34 & 0.0038 & 342.76 & 0.0344 & 475.86 & 0.1426 \\
\hline 238.59 & 0.0044 & 349.63 & 0.0383 & 486.49 & 0.1584 \\
\hline 245.57 & 0.0050 & 367.64 & 0.0486 & 486.86 & 0.1622 \\
\hline 255.51 & 0.0061 & 375.24 & 0.0531 & 489.64 & 0.1719 \\
\hline 264.06 & 0.0075 & 392.99 & 0.0648 & 491.96 & 0.1770 \\
\hline 268.78 & 0.0082 & 403.24 & 0.0715 & 492.83 & 0.1794 \\
\hline 299.05 & 0.0163 & 416.33 & 0.0811 & 493.41 & 0.1818 \\
\hline 303.87 & 0.0180 & 439.77 & 0.0983 & 493.88 & 0.1844 \\
\hline 308.58 & 0.0195 & 448.48 & 0.1067 & 494.54 & 0.1875 \\
\hline 313.34 & 0.0212 & 456.50 & 0.1149 & 494.22 & 0.1914 \\
\hline
\end{tabular}


Table 116. Test data for specimen 69-110

\begin{tabular}{|c|c|c|c|c|c|}
\hline $\begin{array}{l}\text { Test Number } \\
\text { Aging Temp. } \\
\text { Yield Stress }\end{array}$ & $\begin{array}{l}: 0 \\
: 40 \\
: 15\end{array}$ & & $\begin{array}{l}\text { Tes } \\
\text { Agi } \\
\text { Ulti }\end{array}$ & $\begin{array}{l}\text { np. } \\
\text { ne } \\
\text { Stress }\end{array}$ & $\begin{array}{l}290^{\circ} \mathrm{C} \\
10,000 \mathrm{~h} \\
481.6 \mathrm{MPa}\end{array}$ \\
\hline Engr. & & Engr. & & Engr. & \\
\hline $\begin{array}{l}\text { Stress } \\
\text { (MPa) }\end{array}$ & $\begin{array}{l}\text { Engr. } \\
\text { Strain }\end{array}$ & $\begin{array}{l}\text { Stress } \\
\text { (MPa) }\end{array}$ & $\begin{array}{l}\text { Engr. } \\
\text { Strain }\end{array}$ & $\begin{array}{l}\text { Stress } \\
(\mathrm{MPa})\end{array}$ & $\begin{array}{l}\text { Engr. } \\
\text { Strain }\end{array}$ \\
\hline 160.02 & 0.0019 & 289.49 & 0.0156 & 435.97 & 0.1017 \\
\hline 167.34 & 0.0022 & 296.70 & 0.0180 & 446.92 & 0.1135 \\
\hline 180.56 & 0.0028 & 302.91 & 0.0200 & 452.79 & 0.1209 \\
\hline 198.25 & 0.0037 & 317.92 & 0.0264 & 460.29 & 0.1310 \\
\hline 211.86 & 0.0042 & 329.33 & 0.0318 & 463.60 & 0.1362 \\
\hline 225.14 & 0.0045 & 341.63 & 0.0386 & 466.27 & 0.1410 \\
\hline 243.42 & 0.0056 & 352.95 & 0.0445 & 470.77 & 0.1497 \\
\hline 248.87 & 0.0061 & 357.77 & 0.0473 & 473.15 & 0.1542 \\
\hline 256.00 & 0.0074 & 362.99 & 0.0506 & 475.87 & 0.1620 \\
\hline 260.72 & 0.0085 & 378.40 & 0.0606 & 478.16 & 0.1701 \\
\hline 266.72 & 0.0097 & 394.94 & 0.0716 & 480.01 & 0.1788 \\
\hline 272.41 & 0.0110 & 407.10 & 0.0808 & 481.00 & 0.1823 \\
\hline 277.15 & 0.0122 & 416.71 & 0.0875 & 481.56 & 0.1897 \\
\hline 282.46 & 0.0135 & 426.51 & 0.0956 & 481.39 & 0.1945 \\
\hline
\end{tabular}

Table 117. Test data for specimen 691-06

\begin{tabular}{|c|c|c|c|c|c|}
\hline $\begin{array}{l}\text { Test Number } \\
\text { Aging Temp. } \\
\text { Yield Stress }\end{array}$ & $\begin{array}{l}: M \\
: 45 \\
: 17\end{array}$ & & $\begin{array}{l}\text { Tes } \\
\text { Agi } \\
\text { Ulti }\end{array}$ & $\begin{array}{l}\mathrm{np} . \\
\text { me } \\
\text { Stress }\end{array}$ & $\begin{array}{l}290^{\circ} \mathrm{C} \\
2,570 \mathrm{~h} \\
479.7 \mathrm{MPa}\end{array}$ \\
\hline Engr. & & Engr. & & Engr. & \\
\hline $\begin{array}{l}\text { Stress } \\
(\mathrm{MPa})\end{array}$ & $\begin{array}{l}\text { Engr. } \\
\text { Strain }\end{array}$ & $\begin{array}{l}\text { Stress } \\
(\mathrm{MPa})\end{array}$ & $\begin{array}{l}\text { Engr. } \\
\text { Strain }\end{array}$ & $\begin{array}{l}\text { Stress } \\
(\mathrm{MPa})\end{array}$ & $\begin{array}{l}\text { Engr. } \\
\text { Strain }\end{array}$ \\
\hline 44.40 & 0.0003 & 244.83 & 0.0095 & 418.30 & 0.0858 \\
\hline 69.36 & 0.0003 & 250.49 & 0.0106 & 425.89 & 0.0928 \\
\hline 97.15 & 0.0004 & 255.73 & 0.0115 & 432.71 & 0.0997 \\
\hline 121.28 & 0.0008 & 260.83 & 0.0125 & 441.06 & 0.1066 \\
\hline 140.31 & 0.0012 & 283.24 & 0.0181 & 446.37 & 0.1138 \\
\hline 157.48 & 0.0019 & 306.27 & 0.0248 & 452.71 & 0.1208 \\
\hline 170.09 & 0.0025 & 324.19 & 0.3160 & 457.40 & 0.1280 \\
\hline 182.78 & 0.0033 & 339.50 & 0.3830 & 461.26 & 0.1353 \\
\hline 195.67 & 0.0041 & 353.98 & 0.0451 & 466.43 & 0.1425 \\
\hline 206.22 & 0.0049 & 366.73 & 0.0518 & 468.43 & 0.1500 \\
\hline 216.22 & 0.0059 & 378.38 & 0.0586 & 472.36 & 0.1577 \\
\hline 223.60 & 0.0067 & 389.76 & 0.0655 & 474.22 & 0.1655 \\
\hline 231.11 & 0.0077 & 399.69 & 0.0723 & 476.77 & 0.1739 \\
\hline 238.08 & 0.0086 & 409.76 & 0.0790 & & \\
\hline
\end{tabular}


Table 118. Test data for specimen 692-09

\begin{tabular}{|c|c|c|c|c|c|}
\hline $\begin{array}{l}\text { Test Number } \\
\text { Aging Temp. } \\
\text { Yield Stress }\end{array}$ & $\begin{array}{l}: M \\
: 45 \\
: 17\end{array}$ & & $\begin{array}{l}\text { Tes } \\
\text { Agi } \\
\text { Ulti }\end{array}$ & $\begin{array}{l}\text { mp. } \\
\text { me } \\
\text { Stress }\end{array}$ & $\begin{array}{l}290^{\circ} \mathrm{C} \\
2,570 \mathrm{~h} \\
477.1 \mathrm{MPa}\end{array}$ \\
\hline Engr. & & Engr. & & Engr. & \\
\hline $\begin{array}{l}\text { Stress } \\
(\mathrm{MPa})\end{array}$ & $\begin{array}{l}\text { Engr. } \\
\text { Strain }\end{array}$ & $\begin{array}{l}\text { Stress } \\
(\mathrm{MPa})\end{array}$ & $\begin{array}{l}\text { Engr. } \\
\text { Strain }\end{array}$ & $\begin{array}{l}\text { Stress } \\
(\mathrm{MPa})\end{array}$ & $\begin{array}{l}\text { Engr. } \\
\text { Strain }\end{array}$ \\
\hline 2.92 & 0.0002 & 218.70 & 0.0074 & 408.65 & 0.0914 \\
\hline 23.44 & 0.0002 & 226.42 & 0.0084 & 418.58 & 0.0998 \\
\hline 40.40 & 0.0004 & 232.01 & 0.0094 & 426.23 & 0.1086 \\
\hline 59.50 & 0.0005 & 238.35 & 0.0105 & 433.40 & 0.1176 \\
\hline 81.29 & 0.0007 & 243.80 & 0.0115 & 440.85 & 0.1268 \\
\hline 104.04 & 0.0009 & 271.86 & 0.0180 & 447.88 & 0.1361 \\
\hline 120.24 & 0.0014 & 296.68 & 0.0261 & 451.81 & 0.1457 \\
\hline 141.07 & 0.0019 & 317.92 & 0.0340 & 455.95 & 0.1553 \\
\hline 156.30 & 0.0025 & 334.67 & 0.0421 & 460.23 & 0.1653 \\
\hline 169.34 & 0.0032 & 349.84 & 0.0500 & 464.43 & 0.1755 \\
\hline 180.57 & 0.0040 & 363.97 & 0.0580 & 466.78 & 0.1861 \\
\hline 192.85 & 0.0047 & 376.73 & 0.0661 & 471.12 & 0.1971 \\
\hline 202.02 & 0.0056 & 387.55 & 0.0744 & 474.36 & 0.2090 \\
\hline 210.36 & 0.0065 & 398.52 & 0.0828 & 475.05 & 0.2219 \\
\hline
\end{tabular}

Table 119. Test data for specimen 18-11

\begin{tabular}{|c|c|c|c|c|c|}
\hline $\begin{array}{l}\text { Test Number } \\
\text { Aging Temp. } \\
\text { Yield Stress }\end{array}$ & $\begin{array}{l}: 0 \\
: 28 \\
: 30\end{array}$ & & $\begin{array}{l}\text { Tes } \\
\text { Agi } \\
\text { Ulti }\end{array}$ & $\begin{array}{l}\text { np. } \\
\text { me } \\
\text { Stress }\end{array}$ & $\begin{array}{l}25^{\circ} \mathrm{C} \\
68,000 \mathrm{~h} \\
554.9 \mathrm{MPa}\end{array}$ \\
\hline Engr. & & Engr. & & Engr. & \\
\hline $\begin{array}{l}\text { Stress } \\
(\mathrm{MPa})\end{array}$ & $\begin{array}{l}\text { Engr. } \\
\text { Strain }\end{array}$ & $\begin{array}{l}\text { Stress } \\
(\mathrm{MPa})\end{array}$ & $\begin{array}{l}\text { Engr. } \\
\text { Strain }\end{array}$ & $\begin{array}{l}\text { Stress } \\
(\mathrm{MPa})\end{array}$ & $\begin{array}{l}\text { Engr. } \\
\text { Strain }\end{array}$ \\
\hline 48.09 & 0.0000 & 287.83 & 0.0037 & 424.09 & 0.0559 \\
\hline 84.24 & 0.0002 & 293.75 & 0.0040 & 442.61 & 0.0788 \\
\hline 107.98 & 0.0005 & 303.24 & 0.0044 & 457.71 & 0.0977 \\
\hline 131.91 & 0.0007 & 307.15 & 0.0046 & 464.98 & 0.1083 \\
\hline 155.85 & 0.0011 & 312.18 & 0.0048 & 469.05 & 0.1153 \\
\hline 179.66 & 0.0015 & 317.03 & 0.0050 & 473.27 & 0.1224 \\
\hline 203.60 & 0.0019 & 321.96 & 0.0052 & 482.07 & 0.1402 \\
\hline 215.66 & 0.0019 & 326.61 & 0.0055 & 499.86 & 0.1778 \\
\hline 227.53 & 0.0021 & 336.68 & 0.0062 & 515.08 & 0.2170 \\
\hline 233.61 & 0.0021 & 348.23 & 0.0071 & 525.78 & 0.2579 \\
\hline 239.97 & 0.0022 & 357.04 & 0.0082 & 531.32 & 0.2791 \\
\hline 249.70 & 0.0024 & 363.74 & 0.0093 & 532.42 & 0.3007 \\
\hline 256.30 & 0.0026 & 373.20 & 0.0117 & 546.59 & 0.3914 \\
\hline 265.98 & 0.0029 & 378.00 & 0.0135 & 553.42 & 0.4691 \\
\hline 275.32 & 0.0032 & 383.90 & 0.0165 & 553.66 & 0.4791 \\
\hline 278.40 & 0.0033 & 394.22 & 0.0240 & 554.08 & 0.4840 \\
\hline 283.24 & 0.0035 & 410.58 & 0.0399 & 554.87 & 0.4891 \\
\hline
\end{tabular}


Table 120. Test data for specimen 18-12

\begin{tabular}{|c|c|c|c|c|c|}
\hline $\begin{array}{l}\text { Test Number } \\
\text { Aging Temp. } \\
\text { Yield Stress }\end{array}$ & $\begin{array}{l}: 0 \\
: 2 \varepsilon \\
: 2 \varepsilon\end{array}$ & & $\begin{array}{l}\text { Tes } \\
\text { Agi } \\
\text { Ulti }\end{array}$ & $\begin{array}{l}\text { np. } \\
\text { me } \\
\text { Stress }\end{array}$ & $\begin{array}{l}25^{\circ} \mathrm{C} \\
68,000 \mathrm{~h} \\
555.9 \mathrm{MPa}\end{array}$ \\
\hline Engr. & & Engr. & & Engr. & \\
\hline $\begin{array}{l}\text { Stress } \\
(\mathrm{MPa})\end{array}$ & $\begin{array}{l}\text { Engr. } \\
\text { Strain }\end{array}$ & $\begin{array}{l}\text { Stress } \\
(\mathrm{MPa})\end{array}$ & $\begin{array}{l}\text { Engr. } \\
\text { Strain }\end{array}$ & $\begin{array}{l}\text { Stress } \\
(\mathrm{MPa})\end{array}$ & $\begin{array}{l}\text { Engr. } \\
\text { Strain }\end{array}$ \\
\hline 14.90 & 0.0000 & 336.25 & 0.0074 & 485.06 & 0.0940 \\
\hline 32.56 & 0.0001 & 341.27 & 0.0080 & 494.87 & 0.1032 \\
\hline 54.54 & 0.0003 & 350.19 & 0.0090 & 505.27 & 0.1157 \\
\hline 95.04 & 0.0008 & 359.80 & 0.0099 & 518.78 & 0.1330 \\
\hline 148.66 & 0.0017 & 365.26 & 0.0112 & 528.90 & 0.1481 \\
\hline 201.65 & 0.0024 & 379.81 & 0.0154 & 535.04 & 0.1613 \\
\hline 210.18 & 0.0025 & 389.69 & 0.0190 & 540.25 & 0.1711 \\
\hline 227.65 & 0.0027 & 404.24 & 0.0258 & 547.31 & 0.1880 \\
\hline 237.14 & 0.0033 & 422.76 & 0.0380 & 551.00 & 0.2022 \\
\hline 238.48 & 0.0033 & 442.28 & 0.0532 & 554.99 & 0.2214 \\
\hline 250.50 & 0.0034 & 460.52 & 0.0701 & 556.14 & 0.2421 \\
\hline 287.81 & 0.0048 & 479.33 & 0.0888 & 556.32 & 0.2550 \\
\hline 312.16 & 0.0059 & & & & \\
\hline
\end{tabular}

Table 121. Test data for specimen 18-22

\begin{tabular}{|c|c|c|c|c|c|}
\hline $\begin{array}{l}\text { Test Number } \\
\text { Aging Temp. } \\
\text { Yield Stress }\end{array}$ & $\begin{array}{l}: 0 \\
: 28 \\
: 28\end{array}$ & $\mathrm{MPa}$ & $\begin{array}{l}\text { Tes } \\
\text { Agi } \\
\text { Ulti }\end{array}$ & $\begin{array}{l}\text { np. } \\
\text { ne } \\
\text { Stress }\end{array}$ & $\begin{array}{l}25^{\circ} \mathrm{C} \\
68,000 \mathrm{~h} \\
568.9 \mathrm{MPa}\end{array}$ \\
\hline Engr. & & Engr. & & Engr. & \\
\hline $\begin{array}{l}\text { Stress } \\
(\mathrm{MPa})\end{array}$ & $\begin{array}{l}\text { Engr. } \\
\text { Strain }\end{array}$ & $\begin{array}{l}\text { Stress } \\
(\mathrm{MPa})\end{array}$ & $\begin{array}{l}\text { Engr. } \\
\text { Strain }\end{array}$ & $\begin{array}{l}\text { Stress } \\
(\mathrm{MPa})\end{array}$ & $\begin{array}{l}\text { Engr. } \\
\text { Strain }\end{array}$ \\
\hline 23.90 & 0.0000 & 313.52 & 0.0075 & 419.35 & 0.0572 \\
\hline 48.59 & 0.0003 & 323.34 & 0.0087 & 457.49 & 0.0934 \\
\hline 97.26 & 0.0009 & 332.66 & 0.0099 & 481.22 & 0.1144 \\
\hline 145.01 & 0.0018 & 337.20 & 0.0107 & 484.37 & 0.1155 \\
\hline 193.33 & 0.0030 & 347.14 & 0.0129 & 512.14 & 0.1563 \\
\hline 241.06 & 0.0036 & 356.58 & 0.0161 & 537.27 & 0.2026 \\
\hline 265.32 & 0.0046 & 365.48 & 0.0196 & 552.11 & 0.2431 \\
\hline 289.62 & 0.0059 & 380.58 & 0.0276 & 558.67 & 0.2798 \\
\hline 304.09 & 0.0068 & 394.97 & 0.0370 & 568.94 & 0.3292 \\
\hline
\end{tabular}


Table 122. Test data for specimen 13-12

\begin{tabular}{|c|c|c|c|c|c|}
\hline $\begin{array}{l}\text { Test Number } \\
\text { Aging Temp. } \\
\text { Yield Stress }\end{array}$ & $\begin{array}{l}: 0 \\
: \mathrm{R} \\
: 31\end{array}$ & $\begin{array}{l}\text { ealed } \\
\mathrm{MPa}\end{array}$ & $\begin{array}{l}\text { Tes } \\
\text { Agi } \\
\text { Ulti }\end{array}$ & $\begin{array}{l}\text { np. } \\
\text { ne } \\
\text { Stress }\end{array}$ & $\begin{array}{l}25^{\circ} \mathrm{C} \\
- \\
567.4 \mathrm{MPa}\end{array}$ \\
\hline Engr. & & Engr. & & Engr. & \\
\hline $\begin{array}{l}\text { Stress } \\
(\mathrm{MPa})\end{array}$ & $\begin{array}{l}\text { Engr. } \\
\text { Strain }\end{array}$ & $\begin{array}{l}\text { Stress } \\
(\mathrm{MPa})\end{array}$ & $\begin{array}{l}\text { Engr. } \\
\text { Strain }\end{array}$ & $\begin{array}{l}\text { Stress } \\
(\mathrm{MPa})\end{array}$ & $\begin{array}{l}\text { Engr. } \\
\text { Strain }\end{array}$ \\
\hline 59.50 & 0.0001 & 352.87 & 0.0067 & 496.24 & 0.1460 \\
\hline 99.30 & 0.0003 & 357.20 & 0.0073 & 510.41 & 0.1747 \\
\hline 133.00 & 0.0006 & 361.96 & 0.0080 & 522.24 & 0.2028 \\
\hline 163.07 & 0.0010 & 367.66 & 0.0091 & 529.11 & 0.2228 \\
\hline 193.54 & 0.0016 & 372.86 & 0.0108 & 536.15 & 0.2462 \\
\hline 201.94 & 0.0018 & 378.36 & 0.0130 & 542.22 & 0.2717 \\
\hline 215.15 & 0.0020 & 385.83 & 0.0168 & 547.41 & 0.2928 \\
\hline 229.30 & 0.0021 & 398.00 & 0.0259 & 552.36 & 0.3249 \\
\hline 237.36 & 0.0022 & 415.43 & 0.0421 & 558.66 & 0.3637 \\
\hline 280.74 & 0.0031 & 434.03 & 0.0634 & 562.70 & 0.4037 \\
\hline 300.55 & 0.0036 & 449.93 & 0.0811 & 566.12 & 0.4461 \\
\hline 319.41 & 0.0043 & 464.70 & 0.0982 & 567.34 & 0.4761 \\
\hline 332.93 & 0.0050 & 474.03 & 0.1080 & 567.40 & 0.4995 \\
\hline 346.78 & 0.0061 & 478.62 & 0.1167 & & \\
\hline
\end{tabular}

Table 123. Test data for specimen 13-21

\begin{tabular}{|c|c|c|c|c|c|}
\hline \multirow{3}{*}{$\begin{array}{l}\text { Test Number } \\
\text { Aging Temp. } \\
\text { Yield Stress } \\
\text { Engr. } \\
\text { Stress } \\
\text { (MPa) }\end{array}$} & \multicolumn{2}{|c|}{$\begin{array}{l}: 026 \\
: \text { Reannealed } \\
: 289.2 \mathrm{MPa}\end{array}$} & \multicolumn{2}{|c|}{$\begin{array}{l}\text { Test Temp. } \\
\text { Aging Time } \\
\text { Ultimate Stress }\end{array}$} & $\begin{array}{l}25^{\circ} \mathrm{C} \\
- \\
575.2 \mathrm{MPa}\end{array}$ \\
\hline & & Engr. & & Engr. & \\
\hline & $\begin{array}{l}\text { Engr. } \\
\text { Strain }\end{array}$ & $\begin{array}{l}\text { Stress } \\
\text { (MPa) }\end{array}$ & $\begin{array}{l}\text { Engr. } \\
\text { Strain }\end{array}$ & $\begin{array}{l}\text { Stress } \\
(\mathrm{MPa})\end{array}$ & $\begin{array}{l}\text { Engr. } \\
\text { Strain }\end{array}$ \\
\hline 43.57 & 0.0000 & 347.01 & 0.0081 & 488.50 & 0.0968 \\
\hline 85.02 & 0.0002 & 353.99 & 0.0092 & 505.80 & 0.1120 \\
\hline 130.85 & 0.0006 & 360.46 & 0.0107 & 523.90 & 0.1343 \\
\hline 179.40 & 0.0014 & 368.83 & 0.0131 & 543.60 & 0.1667 \\
\hline 232.59 & 0.0022 & 379.05 & 0.0167 & 555.16 & 0.1962 \\
\hline 262.35 & 0.0028 & 388.82 & 0.0210 & 564.90 & 0.2333 \\
\hline 276.49 & 0.0032 & 403.16 & 0.0286 & 571.38 & 0.2689 \\
\hline 297.17 & 0.0040 & 421.66 & 0.0407 & 573.98 & 0.2871 \\
\hline 310.87 & 0.0046 & 436.44 & 0.0518 & 573.92 & 0.2947 \\
\hline 322.33 & 0.0053 & 453.33 & 0.0654 & 574.89 & 0.3157 \\
\hline 331.48 & 0.0062 & 470.21 & 0.0788 & 575.17 & 0.3364 \\
\hline 339.10 & 0.0070 & 483.57 & 0.0905 & 575.22 & 0.3509 \\
\hline
\end{tabular}


Table 124. Test data for specimen 13-22

\begin{tabular}{|c|c|c|c|c|c|}
\hline \multirow{3}{*}{$\begin{array}{l}\text { Test Number } \\
\text { Aging Temp. } \\
\text { Yield Stress } \\
\text { Engr. } \\
\text { Stress } \\
\text { (MPa) }\end{array}$} & \multicolumn{2}{|c|}{$\begin{array}{l}: 027 \\
: \text { Reannealed } \\
: 287.7 \mathrm{MPa}\end{array}$} & \multicolumn{2}{|c|}{$\begin{array}{l}\text { Test Temp. } \\
\text { Aging Time } \\
\text { Ultimate Stress }\end{array}$} & $\begin{array}{l}25^{\circ} \mathrm{C} \\
- \\
531.2 \mathrm{MPa}\end{array}$ \\
\hline & \multirow[b]{2}{*}{$\begin{array}{l}\text { Engr. } \\
\text { Strain }\end{array}$} & \multirow{2}{*}{$\begin{array}{l}\text { Engr. } \\
\text { Stress } \\
\text { (MPa) }\end{array}$} & \multicolumn{3}{|c|}{ Engr. } \\
\hline & & & $\begin{array}{l}\text { Engr. } \\
\text { Strain }\end{array}$ & $\begin{array}{l}\text { Stress } \\
(\mathrm{MPa})\end{array}$ & $\begin{array}{l}\text { Engr. } \\
\text { Strain }\end{array}$ \\
\hline 44.21 & 0.0000 & 331.47 & 0.0081 & 442.35 & 0.0957 \\
\hline 85.37 & 0.0003 & 336.80 & 0.0093 & 455.83 & 0.1097 \\
\hline 131.85 & 0.0008 & 341.69 & 0.0105 & 468.19 & 0.1252 \\
\hline 174.19 & 0.0014 & 346.72 & 0.0124 & 473.23 & 0.1269 \\
\hline 196.69 & 0.0019 & 354.13 & 0.0158 & 477.17 & 0.1347 \\
\hline 214.31 & 0.0022 & 360.84 & 0.0195 & 478.12 & 0.1390 \\
\hline 230.61 & 0.0024 & 370.52 & 0.0259 & 492.78 & 0.1656 \\
\hline 260.12 & 0.0031 & 383.34 & 0.0361 & 504.02 & 0.1894 \\
\hline 290.13 & 0.0042 & 394.21 & 0.0455 & 516.50 & 0.2247 \\
\hline 302.55 & 0.0048 & 413.05 & 0.0640 & 526.42 & 0.2619 \\
\hline 312.26 & 0.0056 & 429.39 & 0.0806 & 530.27 & 0.2926 \\
\hline 320.50 & 0.0064 & 434.61 & 0.0856 & 531.25 & 0.3066 \\
\hline 327.75 & 0.0074 & 436.25 & 0.0876 & & \\
\hline
\end{tabular}

Table 125. Test data for specimen 15-11

\begin{tabular}{|c|c|c|c|c|c|}
\hline $\begin{array}{l}\text { Test Number } \\
\text { Aging Temp. } \\
\text { Yield Stress }\end{array}$ & $\begin{array}{l}: 0 \\
: 28 \\
: 21\end{array}$ & $\mathrm{MPa}$ & $\begin{array}{l}\text { Tes } \\
\text { Agir } \\
\text { Ulti }\end{array}$ & $\begin{array}{l}\text { mp. } \\
\text { me } \\
\text { Stress }\end{array}$ & $\begin{array}{l}290^{\circ} \mathrm{C} \\
68,000 \mathrm{~h} \\
469.0 \mathrm{MPa}\end{array}$ \\
\hline Engr. & & Engr. & & Engr. & \\
\hline $\begin{array}{l}\text { Stress } \\
(\mathrm{MPa})\end{array}$ & $\begin{array}{l}\text { Engr. } \\
\text { Strain }\end{array}$ & $\begin{array}{l}\text { Stress } \\
\text { (MPa) }\end{array}$ & $\begin{array}{l}\text { Engr. } \\
\text { Strain }\end{array}$ & $\begin{array}{l}\text { Stress } \\
(\mathrm{MPa})\end{array}$ & $\begin{array}{l}\text { Engr. } \\
\text { Strain }\end{array}$ \\
\hline 48.99 & 0.0000 & 278.02 & 0.0076 & 438.50 & 0.1433 \\
\hline 83.20 & 0.0002 & 281.34 & 0.0085 & 443.15 & 0.1514 \\
\hline 122.05 & 0.0006 & 284.82 & 0.0095 & 452.28 & 0.1714 \\
\hline 162.68 & 0.0013 & 287.99 & 0.0104 & 460.92 & 0.1953 \\
\hline 189.28 & 0.0019 & 294.38 & 0.0127 & 466.31 & 0.2175 \\
\hline 195.54 & 0.0021 & 319.77 & 0.0266 & 468.95 & 0.2414 \\
\hline 201.11 & 0.0024 & 333.17 & 0.0365 & 468.76 & 0.2577 \\
\hline 213.49 & 0.0026 & 348.54 & 0.0493 & 460.47 & 0.2747 \\
\hline 220.74 & 0.0028 & 365.08 & 0.0638 & 434.38 & 0.2978 \\
\hline 235.41 & 0.0030 & 380.97 & 0.0782 & 387.87 & 0.3198 \\
\hline 245.10 & 0.0034 & 390.25 & 0.0864 & 308.74 & 0.3294 \\
\hline 252.35 & 0.0039 & 402.23 & 0.0982 & 269.78 & 0.3309 \\
\hline 260.15 & 0.0045 & 410.60 & 0.1048 & 213.57 & 0.3327 \\
\hline 265.27 & 0.0052 & 420.39 & 0.1163 & 166.50 & 0.3340 \\
\hline 271.57 & 0.0062 & 429.58 & 0.1286 & 156.46 & 0.3341 \\
\hline
\end{tabular}


Table 126. Test data for specimen 15-12

\begin{tabular}{|c|c|c|c|c|c|}
\hline $\begin{array}{l}\text { Test Number } \\
\text { Aging Temp. } \\
\text { Yield Stress }\end{array}$ & $\begin{array}{l}: 0 \\
: 2 \varepsilon \\
: 20\end{array}$ & & $\begin{array}{l}\text { Tes } \\
\text { Agi } \\
\text { Ulti }\end{array}$ & $\begin{array}{l}\text { mp. } \\
\text { me } \\
\text { Stress }\end{array}$ & $\begin{array}{l}290^{\circ} \mathrm{C} \\
68,000 \mathrm{~h} \\
444.5 \mathrm{MPa}\end{array}$ \\
\hline Engr. & & Engr. & & Engr. & \\
\hline $\begin{array}{l}\text { Stress } \\
\text { (MPa) }\end{array}$ & $\begin{array}{l}\text { Engr. } \\
\text { Strain }\end{array}$ & $\begin{array}{l}\text { Stress } \\
\text { (MPa) }\end{array}$ & $\begin{array}{l}\text { Engr. } \\
\text { Strain }\end{array}$ & $\begin{array}{l}\text { Stress } \\
(\mathrm{MPa})\end{array}$ & $\begin{array}{l}\text { Engr. } \\
\text { Strain }\end{array}$ \\
\hline 46.39 & 0.0000 & 243.65 & 0.0045 & 355.26 & 0.0853 \\
\hline 83.04 & 0.0003 & 247.23 & 0.0052 & 367.60 & 0.0977 \\
\hline 119.69 & 0.0007 & 251.20 & 0.0060 & 374.04 & 0.1017 \\
\hline 140.89 & 0.0010 & 255.94 & 0.0073 & 384.35 & 0.1144 \\
\hline 158.51 & 0.0014 & 260.29 & 0.0086 & 392.07 & 0.1237 \\
\hline 177.59 & 0.0019 & 264.10 & 0.0100 & 407.55 & 0.1429 \\
\hline 186.93 & 0.0022 & 267.86 & 0.0116 & 413.00 & 0.1531 \\
\hline 200.26 & 0.0026 & 270.93 & 0.0131 & 423.69 & 0.1712 \\
\hline 208.37 & 0.0028 & 274.64 & 0.0149 & 429.49 & 0.1877 \\
\hline 214.65 & 0.0029 & 278.49 & 0.0171 & 432.18 & 0.1915 \\
\hline 219.32 & 0.0031 & 285.89 & 0.0220 & 435.42 & 0.1987 \\
\hline 228.30 & 0.0033 & 294.90 & 0.0285 & 437.28 & 0.2026 \\
\hline 235.71 & 0.0036 & 308.51 & 0.0400 & 441.72 & 0.2144 \\
\hline 238.60 & 0.0039 & 325.75 & 0.0560 & 440.08 & 0.2189 \\
\hline 240.86 & 0.0042 & 342.68 & 0.0724 & 444.39 & 0.2366 \\
\hline
\end{tabular}

Table 127. Test data for specimen 15-21

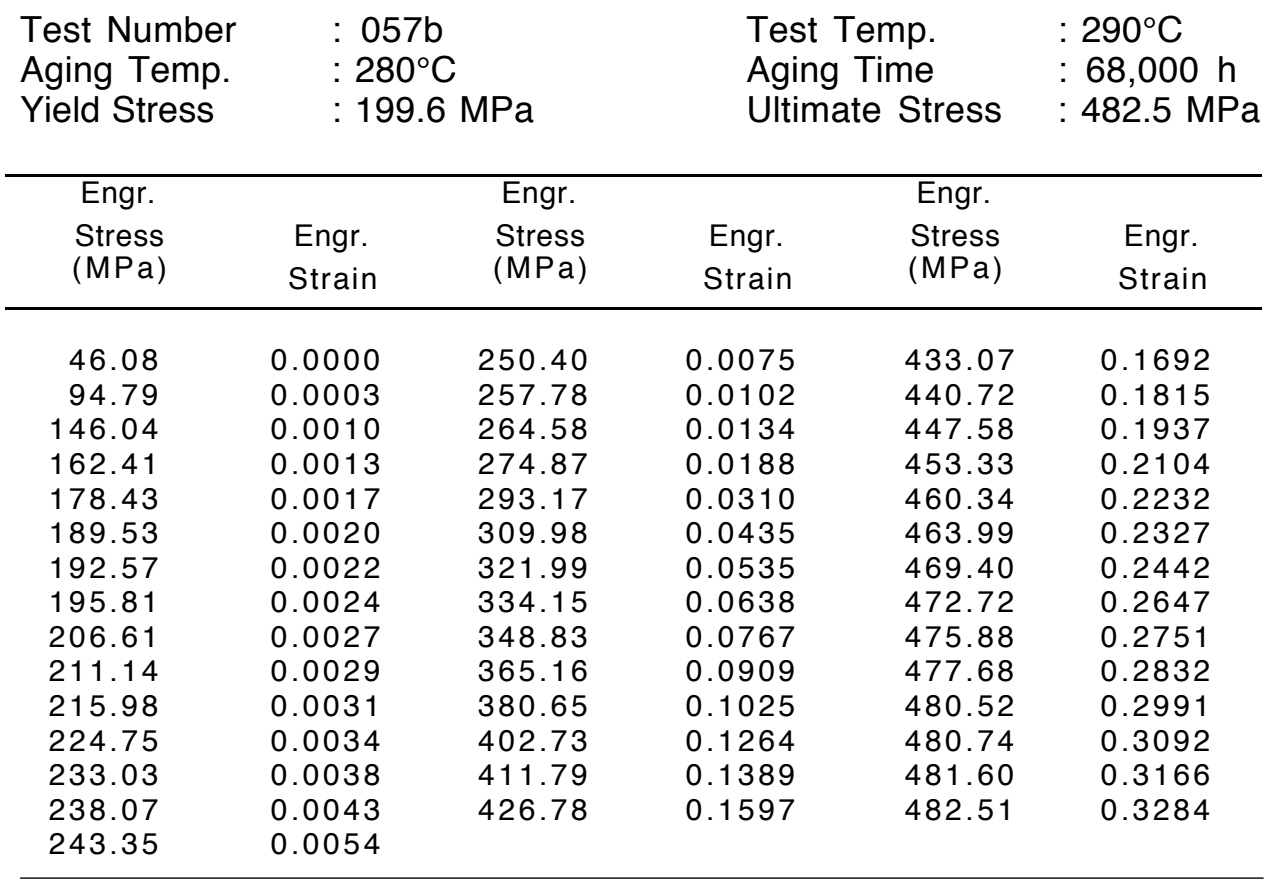


Table 128. Test data for specimen 15-22

\begin{tabular}{|c|c|c|c|c|c|}
\hline $\begin{array}{l}\text { Test Number } \\
\text { Aging Temp. } \\
\text { Yield Stress }\end{array}$ & $\begin{array}{l}: 0 \\
: 2 \varepsilon \\
: 1 \varepsilon\end{array}$ & $\mathrm{MPa}$ & $\begin{array}{l}\text { Tes } \\
\text { Agir } \\
\text { Ulti }\end{array}$ & $\begin{array}{l}\text { np. } \\
\text { ne } \\
\text { Stress }\end{array}$ & $\begin{array}{l}290^{\circ} \mathrm{C} \\
68,000 \mathrm{~h} \\
434.0 \mathrm{MPa}\end{array}$ \\
\hline Engr. & & Engr. & & Engr. & \\
\hline $\begin{array}{l}\text { Stress } \\
(\mathrm{MPa})\end{array}$ & $\begin{array}{l}\text { Engr. } \\
\text { Strain }\end{array}$ & $\begin{array}{l}\text { Stress } \\
(\mathrm{MPa})\end{array}$ & $\begin{array}{l}\text { Engr. } \\
\text { Strain }\end{array}$ & $\begin{array}{l}\text { Stress } \\
(\mathrm{MPa})\end{array}$ & $\begin{array}{l}\text { Engr. } \\
\text { Strain }\end{array}$ \\
\hline 34.92 & 0.0000 & 204.38 & 0.0055 & 364.37 & 0.1249 \\
\hline 81.09 & 0.0003 & 209.24 & 0.0059 & 370.79 & 0.1328 \\
\hline 120.94 & 0.0009 & 214.55 & 0.0065 & 375.87 & 0.1406 \\
\hline 144.27 & 0.0014 & 220.45 & 0.0072 & 381.69 & 0.1491 \\
\hline 159.54 & 0.0019 & 227.00 & 0.0080 & 391.10 & 0.1642 \\
\hline 165.71 & 0.0021 & 237.95 & 0.0098 & 399.87 & 0.1797 \\
\hline 172.82 & 0.0024 & 252.42 & 0.0181 & 401.75 & 0.1814 \\
\hline 178.83 & 0.0027 & 264.31 & 0.0273 & 409.03 & 0.1954 \\
\hline 184.30 & 0.0032 & 277.23 & 0.0387 & 411.64 & 0.2005 \\
\hline 186.79 & 0.0034 & 288.27 & 0.0491 & 419.25 & 0.2181 \\
\hline 190.91 & 0.0038 & 302.62 & 0.0627 & 422.72 & 0.2286 \\
\hline 194.69 & 0.0043 & 314.15 & 0.0741 & 426.14 & 0.2408 \\
\hline 196.38 & 0.0045 & 326.02 & 0.0859 & 428.19 & 0.2480 \\
\hline 199.56 & 0.0050 & 330.52 & 0.0903 & 431.89 & 0.2636 \\
\hline 201.99 & 0.0053 & 347.48 & 0.1053 & 434.03 & 0.2816 \\
\hline
\end{tabular}

Table 129. Test data for specimen 16-21

\begin{tabular}{|c|c|c|c|c|c|}
\hline \multirow[t]{3}{*}{$\begin{array}{l}\text { Test Number } \\
\text { Aging Temp. } \\
\text { Yield Stress }\end{array}$} & \multicolumn{2}{|c|}{$\begin{array}{l}: 060 \\
: \text { Reannealed } \\
: 184.0 \mathrm{MPa}\end{array}$} & \multicolumn{2}{|c|}{$\begin{array}{l}\text { Test Temp. } \\
\text { Aging Time } \\
\text { Ultimate Stress }\end{array}$} & \multirow[t]{2}{*}{$\begin{array}{l}290^{\circ} \mathrm{C} \\
- \\
405.0 \mathrm{MPa}\end{array}$} \\
\hline & & Engr. & & Engr. & \\
\hline & $\begin{array}{l}\text { Engr. } \\
\text { Strain }\end{array}$ & $\begin{array}{l}\text { Stress } \\
(\mathrm{MPa})\end{array}$ & $\begin{array}{l}\text { Engr. } \\
\text { Strain }\end{array}$ & $\begin{array}{l}\text { Stress } \\
(\mathrm{MPa})\end{array}$ & $\begin{array}{l}\text { Engr. } \\
\text { Strain }\end{array}$ \\
\hline 73.83 & 0.0002 & 263.54 & 0.0096 & 427.35 & 0.0939 \\
\hline 105.52 & 0.0006 & 274.92 & 0.0124 & 446.96 & 0.1061 \\
\hline 127.98 & 0.0009 & 285.57 & 0.0155 & 462.80 & 0.1199 \\
\hline 141.76 & 0.0012 & 294.26 & 0.0185 & 473.64 & 0.1309 \\
\hline 156.10 & 0.0016 & 301.80 & 0.0212 & 482.97 & 0.1419 \\
\hline 167.01 & 0.0020 & 317.07 & 0.0277 & 483.46 & 0.1437 \\
\hline 176.70 & 0.0024 & 343.30 & 0.0410 & 485.46 & 0.1484 \\
\hline 191.30 & 0.0033 & 363.02 & 0.0523 & 494.14 & 0.1610 \\
\hline 202.85 & 0.0039 & 379.90 & 0.0624 & 500.51 & 0.1719 \\
\hline 226.10 & 0.0048 & 395.39 & 0.0723 & 504.16 & 0.1812 \\
\hline 240.81 & 0.0057 & 414.01 & 0.0844 & 505.94 & 0.1900 \\
\hline 251.80 & 0.0072 & 432.47 & 0.0972 & & \\
\hline
\end{tabular}


Table 130. Test data for specimen 17-21

\begin{tabular}{|c|c|c|c|c|c|}
\hline $\begin{array}{l}\text { Test Number } \\
\text { Aging Temp. } \\
\text { Yield Stress }\end{array}$ & $\begin{array}{l}: 0 \\
: \operatorname{Re} \\
: 17\end{array}$ & $\begin{array}{l}\text { aled } \\
\text { MPa }\end{array}$ & $\begin{array}{l}\text { Tes } \\
\text { Agi } \\
\text { Ulti }\end{array}$ & $\begin{array}{l}\text { np. } \\
\text { ne } \\
\text { Stress }\end{array}$ & $\begin{array}{l}290^{\circ} \mathrm{C} \\
- \\
416.2 \mathrm{MPa}\end{array}$ \\
\hline Engr. & & Engr. & & Engr. & \\
\hline $\begin{array}{l}\text { Stress } \\
(\mathrm{MPa})\end{array}$ & $\begin{array}{l}\text { Engr. } \\
\text { Strain }\end{array}$ & $\begin{array}{l}\text { Stress } \\
(\mathrm{MPa})\end{array}$ & $\begin{array}{l}\text { Engr. } \\
\text { Strain }\end{array}$ & $\begin{array}{l}\text { Stress } \\
(\mathrm{MPa})\end{array}$ & $\begin{array}{l}\text { Engr. } \\
\text { Strain }\end{array}$ \\
\hline 47.67 & 0.0000 & 206.10 & 0.0063 & 371.77 & 0.1798 \\
\hline 85.55 & 0.0003 & 208.03 & 0.0065 & 379.86 & 0.1942 \\
\hline 114.31 & 0.0007 & 210.94 & 0.0070 & 386.78 & 0.2092 \\
\hline 140.22 & 0.0012 & 220.28 & 0.0086 & 390.72 & 0.2216 \\
\hline 156.57 & 0.0017 & 230.71 & 0.0105 & 393.02 & 0.2293 \\
\hline 164.89 & 0.0020 & 246.73 & 0.0207 & 395.16 & 0.2356 \\
\hline 173.91 & 0.0024 & 259.45 & 0.0331 & 400.01 & 0.2503 \\
\hline 180.02 & 0.0028 & 270.48 & 0.0460 & 403.95 & 0.2639 \\
\hline 185.68 & 0.0033 & 286.56 & 0.0643 & 406.29 & 0.2704 \\
\hline 189.43 & 0.0039 & 301.82 & 0.0830 & 410.18 & 0.2900 \\
\hline 193.47 & 0.0045 & 316.97 & 0.0985 & 412.48 & 0.3016 \\
\hline 195.79 & 0.0050 & 330.80 & 0.1155 & 414.70 & 0.3139 \\
\hline 198.36 & 0.0056 & 340.41 & 0.1284 & 416.19 & 0.3330 \\
\hline 201.41 & 0.0059 & 348.69 & 0.1405 & & \\
\hline 203.83 & 0.0061 & 358.81 & 0.1550 & & \\
\hline
\end{tabular}

Table 131. Test data for specimen P13T-01

$\begin{array}{llll}\text { Test Number } & : \text { MEA Test } & \text { Test Temp. } & : 25^{\circ} \mathrm{C} \\ \text { Aging Temp. } & : \text { Unaged } & \text { Aging Time } & :- \\ \text { Yield Stress } & : 244.7 \mathrm{MPa} & \text { Ultimate Stress } & : 584.5 \mathrm{MPa}\end{array}$

Experimental Data Not Available.

Table 132. Test data for specimen P13T-03

\begin{tabular}{|c|c|c|c|c|c|}
\hline $\begin{array}{l}\text { Test Number } \\
\text { Aging Temp. } \\
\text { Yield Stress }\end{array}$ & $\begin{array}{l}: M \\
: U r \\
: 24\end{array}$ & $\begin{array}{l}\text { est } \\
\mathrm{dPa}\end{array}$ & $\begin{array}{l}\text { Tes } \\
\text { Agi } \\
\text { Ulti }\end{array}$ & $\begin{array}{l}\text { np. } \\
\text { ne } \\
\text { Stress }\end{array}$ & $\begin{array}{l}25^{\circ} \mathrm{C} \\
- \\
579.9 \mathrm{MPa}\end{array}$ \\
\hline Engr. & & Engr. & & Engr. & \\
\hline $\begin{array}{l}\text { Stress } \\
(\mathrm{MPa})\end{array}$ & $\begin{array}{l}\text { Engr. } \\
\text { Strain }\end{array}$ & $\begin{array}{l}\text { Stress } \\
(\mathrm{MPa})\end{array}$ & $\begin{array}{l}\text { Engr. } \\
\text { Strain }\end{array}$ & $\begin{array}{l}\text { Stress } \\
(\mathrm{MPa})\end{array}$ & $\begin{array}{l}\text { Engr. } \\
\text { Strain }\end{array}$ \\
\hline 10.20 & 0.0002 & 136.31 & 0.0010 & 263.38 & 0.0053 \\
\hline 18.68 & 0.0003 & 156.17 & 0.0012 & 270.48 & 0.0062 \\
\hline 28.96 & 0.0003 & 175.54 & 0.0014 & 275.72 & 0.0070 \\
\hline 37.78 & 0.0003 & 192.16 & 0.0017 & 279.72 & 0.0078 \\
\hline 54.26 & 0.0004 & 210.08 & 0.0021 & 285.65 & 0.0087 \\
\hline 68.95 & 0.0005 & 224.70 & 0.0026 & 288.20 & 0.0095 \\
\hline 84.25 & 0.0006 & 236.15 & 0.0031 & 291.72 & 0.0104 \\
\hline 100.11 & 0.0007 & 247.80 & 0.0038 & 296.68 & 0.0113 \\
\hline 118.59 & 0.0008 & 256.76 & 0.0045 & 298.06 & 0.0123 \\
\hline
\end{tabular}


Table 133. Test data for specimen P11A-01

$\begin{array}{llll}\text { Test Number } & : \text { MEA Test } & \text { Test Temp. } & : 25^{\circ} \mathrm{C} \\ \text { Aging Temp. } & : \text { Unaged } & \text { Aging Time } & :- \\ \mathrm{Y} & -\mathrm{MPa} & \text { Ultimate Stress } & : 584.9 \mathrm{MPa}\end{array}$

Experimental Data Not Available.

Table 134. Test data for specimen P13A-01

\begin{tabular}{|c|c|c|c|c|c|}
\hline $\begin{array}{l}\text { Test Number } \\
\text { Aging Temp. } \\
\text { Yield Stress }\end{array}$ & $\begin{array}{l}: M \\
: U r \\
: 24\end{array}$ & $\begin{array}{l}\text { est } \\
\mathrm{dPa}\end{array}$ & $\begin{array}{l}\text { Tes } \\
\text { Agir } \\
\text { Ulti }\end{array}$ & $\begin{array}{l}\text { np. } \\
\text { ne } \\
\text { Stress }\end{array}$ & $\begin{array}{l}25^{\circ} \mathrm{C} \\
- \\
584.5 \mathrm{MPa}\end{array}$ \\
\hline Engr. & & Engr. & & Engr. & \\
\hline $\begin{array}{l}\text { Stress } \\
(\mathrm{MPa})\end{array}$ & $\begin{array}{l}\text { Engr. } \\
\text { Strain }\end{array}$ & $\begin{array}{l}\text { Stress } \\
(\mathrm{MPa})\end{array}$ & $\begin{array}{l}\text { Engr. } \\
\text { Strain }\end{array}$ & $\begin{array}{l}\text { Stress } \\
(\mathrm{MPa})\end{array}$ & $\begin{array}{l}\text { Engr. } \\
\text { Strain }\end{array}$ \\
\hline 17.93 & 0.0002 & 125.42 & 0.0008 & 275.79 & 0.0067 \\
\hline 20.96 & 0.0002 & 143.20 & 0.0009 & 280.62 & 0.0076 \\
\hline 23.44 & 0.0002 & 162.65 & 0.0011 & 285.79 & 0.0084 \\
\hline 26.89 & 0.0002 & 181.81 & 0.0014 & 288.20 & 0.0093 \\
\hline 29.30 & 0.0002 & 197.95 & 0.0017 & 292.06 & 0.0102 \\
\hline 30.89 & 0.0002 & 214.36 & 0.0021 & 295.92 & 0.0111 \\
\hline 33.09 & 0.0003 & 228.63 & 0.0025 & 297.16 & 0.0120 \\
\hline 34.89 & 0.0003 & 239.11 & 0.0030 & 300.96 & 0.0130 \\
\hline 53.57 & 0.0004 & 249.93 & 0.0037 & 303.30 & 0.0139 \\
\hline 70.95 & 0.0005 & 257.45 & 0.0043 & 305.51 & 0.0149 \\
\hline 86.32 & 0.0005 & 264.55 & 0.0051 & 309.44 & 0.0159 \\
\hline 104.39 & 0.0006 & 271.52 & 0.0059 & 310.40 & 0.0168 \\
\hline
\end{tabular}

Table 135. Test data for specimen P14T-09

\begin{tabular}{|c|c|c|c|c|c|}
\hline $\begin{array}{l}\text { Test Number } \\
\text { Aging Temp. } \\
\text { Yield Stress }\end{array}$ & $\begin{array}{l}: 1 \\
: 25 \\
: 26\end{array}$ & $\mathrm{MPa}$ & $\begin{array}{l}\text { Tes } \\
\text { Agi } \\
\text { Ulti }\end{array}$ & $\begin{array}{l}\text { np. } \\
\text { ne } \\
\text { Stress }\end{array}$ & $\begin{array}{l}25^{\circ} \mathrm{C} \\
30,000 \mathrm{~h} \\
596.2 \mathrm{MPa}\end{array}$ \\
\hline Engr. & & Engr. & & Engr. & \\
\hline $\begin{array}{l}\text { Stress } \\
\text { (MPa) }\end{array}$ & $\begin{array}{l}\text { Engr. } \\
\text { Strain }\end{array}$ & $\begin{array}{l}\text { Stress } \\
(\mathrm{MPa})\end{array}$ & $\begin{array}{l}\text { Engr. } \\
\text { Strain }\end{array}$ & $\begin{array}{l}\text { Stress } \\
(\mathrm{MPa})\end{array}$ & $\begin{array}{l}\text { Engr. } \\
\text { Strain }\end{array}$ \\
\hline 0.00 & 0.0000 & 398.64 & 0.0486 & 545.00 & 0.2716 \\
\hline 77.21 & 0.0002 & 422.46 & 0.0680 & 559.25 & 0.3307 \\
\hline 212.73 & 0.0018 & 442.43 & 0.0858 & 561.89 & 0.3462 \\
\hline 258.67 & 0.0025 & 465.90 & 0.1064 & 566.23 & 0.3725 \\
\hline 291.99 & 0.0042 & 478.01 & 0.1209 & 577.95 & 0.4433 \\
\hline 311.40 & 0.0062 & 481.09 & 0.1354 & 591.37 & 0.5609 \\
\hline 326.33 & 0.0093 & 481.39 & 0.1361 & 590.97 & 0.5612 \\
\hline 354.39 & 0.0201 & 507.72 & 0.1791 & 595.58 & 0.6312 \\
\hline 354.49 & 0.0204 & 529.83 & 0.2271 & 596.15 & 0.6717 \\
\hline 380.79 & 0.0348 & 537.08 & 0.2433 & & \\
\hline
\end{tabular}


Table 136. Test data for specimen P11A-25

\begin{tabular}{|c|c|c|c|c|c|}
\hline $\begin{array}{l}\text { Test Number } \\
\text { Aging Temp. } \\
\text { Yield Stress }\end{array}$ & $\begin{array}{l}: 10 \\
: 29 \\
: 28\end{array}$ & & $\begin{array}{l}\text { Tes } \\
\text { Agi } \\
\text { Ulti }\end{array}$ & $\begin{array}{l}\text { np. } \\
\text { ne } \\
\text { Stress }\end{array}$ & $\begin{array}{l}25^{\circ} \mathrm{C} \\
30,000 \mathrm{~h} \\
595.2 \mathrm{MPa}\end{array}$ \\
\hline Engr. & & Engr. & & Engr. & \\
\hline $\begin{array}{l}\text { Stress } \\
\text { (MPa) }\end{array}$ & $\begin{array}{l}\text { Engr. } \\
\text { Strain }\end{array}$ & $\begin{array}{l}\text { Stress } \\
(\mathrm{MPa})\end{array}$ & $\begin{array}{l}\text { Engr. } \\
\text { Strain }\end{array}$ & $\begin{array}{l}\text { Stress } \\
(\mathrm{MPa})\end{array}$ & $\begin{array}{l}\text { Engr. } \\
\text { Strain }\end{array}$ \\
\hline 0.00 & 0.0000 & 327.99 & 0.0134 & 485.84 & 0.1723 \\
\hline 68.60 & 0.0002 & 330.65 & 0.0149 & 503.01 & 0.2040 \\
\hline 147.31 & 0.0010 & 391.70 & 0.0575 & 524.41 & 0.2531 \\
\hline 181.90 & 0.0016 & 395.97 & 0.0605 & 546.10 & 0.3130 \\
\hline 205.98 & 0.0015 & 410.10 & 0.0741 & 557.67 & 0.3579 \\
\hline 222.63 & 0.0017 & 420.72 & 0.0850 & 564.19 & 0.3855 \\
\hline 258.83 & 0.0025 & 431.76 & 0.0957 & 575.74 & 0.4433 \\
\hline 283.36 & 0.0036 & 445.71 & 0.1083 & 582.95 & 0.4831 \\
\hline 299.93 & 0.0050 & 445.67 & 0.1083 & 590.96 & 0.5406 \\
\hline 307.02 & 0.0061 & 467.17 & 0.1347 & 595.22 & 0.6055 \\
\hline 311.57 & 0.0060 & 483.61 & 0.1593 & & \\
\hline
\end{tabular}

Table 137. Test data for specimen P14A-26

\begin{tabular}{|c|c|c|c|c|c|}
\hline $\begin{array}{l}\text { Test Number } \\
\text { Aging Temp. } \\
\text { Yield Stress }\end{array}$ & \multicolumn{2}{|c|}{$\begin{array}{l}: 104 \\
: 290^{\circ} \mathrm{C} \\
: 276.5 \mathrm{MPa}\end{array}$} & \multicolumn{2}{|c|}{$\begin{array}{l}\text { Test Temp. } \\
\text { Aging Time } \\
\text { Ultimate Stress }\end{array}$} & $\begin{array}{l}25^{\circ} \mathrm{C} \\
30,000 \mathrm{~h} \\
575.7 \mathrm{MPa}\end{array}$ \\
\hline Engr. & & Engr. & & Engr. & \\
\hline $\begin{array}{l}\text { Stress } \\
\text { (MPa) }\end{array}$ & $\begin{array}{l}\text { Engr. } \\
\text { Strain }\end{array}$ & $\begin{array}{l}\text { Stress } \\
\text { (MPa) }\end{array}$ & $\begin{array}{l}\text { Engr. } \\
\text { Strain }\end{array}$ & $\begin{array}{l}\text { Stress } \\
(\mathrm{MPa})\end{array}$ & $\begin{array}{l}\text { Engr. } \\
\text { Strain }\end{array}$ \\
\hline 73.86 & 0.0001 & 360.33 & 0.0196 & 494.94 & 0.1349 \\
\hline 202.47 & 0.0013 & 391.83 & 0.0374 & 512.42 & 0.1575 \\
\hline 219.88 & 0.0015 & 422.46 & 0.0598 & 537.40 & 0.2028 \\
\hline 230.30 & 0.0016 & 442.39 & 0.0783 & 553.09 & 0.2403 \\
\hline 269.71 & 0.0022 & 457.87 & 0.0911 & 564.42 & 0.2757 \\
\hline 296.90 & 0.0035 & 469.61 & 0.0986 & 569.81 & 0.3036 \\
\hline 314.51 & 0.0054 & 480.19 & 0.1109 & 573.78 & 0.3211 \\
\hline 330.05 & 0.0088 & 488.72 & 0.1263 & 575.68 & 0.3664 \\
\hline 345.39 & 0.0137 & & & & \\
\hline
\end{tabular}


Table 138. Test data for specimen P11A-28

\begin{tabular}{|c|c|c|c|c|c|}
\hline $\begin{array}{l}\text { Test Number } \\
\text { Aging Temp. } \\
\text { Yield Stress }\end{array}$ & $\begin{array}{l}: 2 \\
: 25 \\
: 26\end{array}$ & & $\begin{array}{l}\text { Tes } \\
\text { Agi } \\
\text { Ulti }\end{array}$ & $\begin{array}{l}\text { np. } \\
\text { ne } \\
\text { Stress }\end{array}$ & $\begin{array}{l}25^{\circ} \mathrm{C} \\
58,000 \mathrm{~h} \\
536.0 \mathrm{MPa}\end{array}$ \\
\hline Engr. & & Engr. & & Engr. & \\
\hline $\begin{array}{l}\text { Stress } \\
\text { (MPa) }\end{array}$ & $\begin{array}{l}\text { Engr. } \\
\text { Strain }\end{array}$ & $\begin{array}{l}\text { Stress } \\
(\mathrm{MPa})\end{array}$ & $\begin{array}{l}\text { Engr. } \\
\text { Strain }\end{array}$ & $\begin{array}{l}\text { Stress } \\
(\mathrm{MPa})\end{array}$ & $\begin{array}{l}\text { Engr. } \\
\text { Strain }\end{array}$ \\
\hline 18.772 & 0.0001 & 323.730 & 0.0296 & 492.111 & 0.2443 \\
\hline 41.505 & 0.0002 & 353.702 & 0.0567 & 498.424 & 0.2577 \\
\hline 53.992 & 0.0003 & 377.558 & 0.0811 & 510.328 & 0.2873 \\
\hline 111.117 & 0.0006 & 379.486 & 0.0833 & 514.377 & 0.2989 \\
\hline 135.575 & 0.0008 & 389.501 & 0.0944 & 515.051 & 0.3012 \\
\hline 197.871 & 0.0012 & 399.024 & 0.1055 & 518.666 & 0.3128 \\
\hline 209.283 & 0.0014 & 418.340 & 0.1289 & 522.281 & 0.3268 \\
\hline 219.264 & 0.0016 & 426.745 & 0.1400 & 526.040 & 0.3431 \\
\hline 256.470 & 0.0030 & 441.324 & 0.1597 & 532.642 & 0.3867 \\
\hline 266.986 & 0.0038 & 460.765 & 0.1885 & 534.233 & 0.4051 \\
\hline 297.088 & 0.0107 & 468.973 & 0.2017 & 535.968 & 0.4516 \\
\hline
\end{tabular}

Table 139. Test data for specimen P11A-29

\begin{tabular}{|c|c|c|c|c|c|}
\hline $\begin{array}{l}\text { Test Number } \\
\text { Aging Temp. } \\
\text { Yield Stress }\end{array}$ & \multicolumn{2}{|c|}{$\begin{array}{l}: 285 \\
: 290^{\circ} \mathrm{C} \\
: 266.1 \mathrm{MPa}\end{array}$} & \multicolumn{2}{|c|}{$\begin{array}{l}\text { Test Temp. } \\
\text { Aging Time } \\
\text { Ultimate Stress }\end{array}$} & $\begin{array}{l}25^{\circ} \mathrm{C} \\
58,000 \mathrm{~h} \\
562.0 \mathrm{MPa}\end{array}$ \\
\hline Engr. & & Engr. & & Engr. & \\
\hline $\begin{array}{l}\text { Stress } \\
(\mathrm{MPa})\end{array}$ & $\begin{array}{l}\text { Engr. } \\
\text { Strain }\end{array}$ & $\begin{array}{l}\text { Stress } \\
(\mathrm{MPa})\end{array}$ & $\begin{array}{l}\text { Engr. } \\
\text { Strain }\end{array}$ & $\begin{array}{l}\text { Stress } \\
(\mathrm{MPa})\end{array}$ & $\begin{array}{l}\text { Engr. } \\
\text { Strain }\end{array}$ \\
\hline 14.859 & 0.0001 & 292.610 & 0.0071 & 472.097 & 0.2052 \\
\hline 20.549 & 0.0001 & 318.089 & 0.0177 & 483.990 & 0.2298 \\
\hline 35.176 & 0.0002 & 350.081 & 0.0412 & 491.974 & 0.2477 \\
\hline 76.772 & 0.0004 & 367.359 & 0.0571 & 502.618 & 0.2745 \\
\hline 87.465 & 0.0004 & 384.826 & 0.0751 & 515.005 & 0.3103 \\
\hline 99.875 & 0.0005 & 394.609 & 0.0864 & 524.536 & 0.3420 \\
\hline 160.545 & 0.0008 & 411.273 & 0.1068 & 531.262 & 0.3672 \\
\hline 172.878 & 0.0009 & 427.903 & 0.1300 & 549.938 & 0.4652 \\
\hline 184.911 & 0.0010 & 439.883 & 0.1481 & 552.503 & 0.4836 \\
\hline 249.253 & 0.0025 & 460.214 & 0.1828 & 560.583 & 0.5582 \\
\hline 281.777 & 0.0050 & 465.207 & 0.1917 & 561.986 & 0.5795 \\
\hline
\end{tabular}


Table 140. Test data for specimen P11A-10

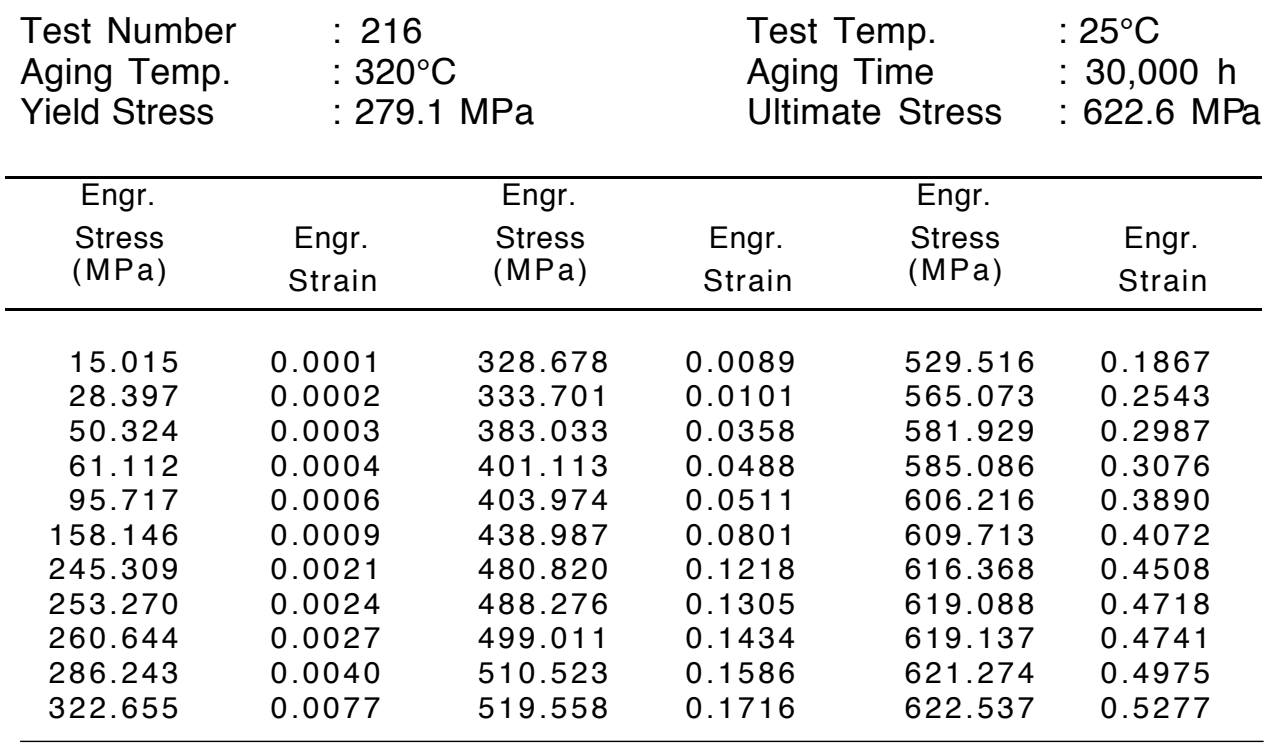

Table 141. Test data for specimen P11T-06

\begin{tabular}{|c|c|c|c|c|c|}
\hline $\begin{array}{l}\text { Test Number } \\
\text { Aging Temp. } \\
\text { Yield Stress }\end{array}$ & $\begin{array}{l}: 1 \\
: 32 \\
: 28\end{array}$ & & $\begin{array}{l}\text { Tes } \\
\text { Agi } \\
\text { Ulti }\end{array}$ & $\begin{array}{l}\mathrm{np} . \\
\text { me } \\
\text { Stress }\end{array}$ & $\begin{array}{l}25^{\circ} \mathrm{C} \\
30,000 \mathrm{~h} \\
569.3 \mathrm{MPa}\end{array}$ \\
\hline Engr. & & Engr. & & Engr. & \\
\hline $\begin{array}{l}\text { Stress } \\
(\mathrm{MPa})\end{array}$ & $\begin{array}{l}\text { Engr. } \\
\text { Strain }\end{array}$ & $\begin{array}{l}\text { Stress } \\
(\mathrm{MPa})\end{array}$ & $\begin{array}{l}\text { Engr. } \\
\text { Strain }\end{array}$ & $\begin{array}{l}\text { Stress } \\
(\mathrm{MPa})\end{array}$ & $\begin{array}{l}\text { Engr. } \\
\text { Strain }\end{array}$ \\
\hline 0.00 & 0.0000 & 342.55 & 0.0035 & 479.03 & 0.1020 \\
\hline 97.90 & 0.0002 & 366.58 & 0.0043 & 485.02 & 0.1088 \\
\hline 186.78 & 0.0011 & 374.51 & 0.0049 & 491.88 & 0.1191 \\
\hline 195.30 & 0.0013 & 374.45 & 0.0048 & 504.42 & 0.1376 \\
\hline 210.26 & 0.0016 & 376.06 & 0.0061 & 529.48 & 0.1813 \\
\hline 210.85 & 0.0017 & 381.40 & 0.0094 & 546.00 & 0.2204 \\
\hline 229.20 & 0.0017 & 390.14 & 0.0159 & 560.78 & 0.2703 \\
\hline 248.99 & 0.0020 & 425.73 & 0.0470 & 565.59 & 0.2916 \\
\hline 318.37 & 0.0029 & 442.01 & 0.0635 & 569.21 & 0.3316 \\
\hline 321.97 & 0.0031 & 468.24 & 0.0982 & 569.34 & 0.3603 \\
\hline
\end{tabular}


Table 142. Test data for specimen P14T-08

\begin{tabular}{|c|c|c|c|c|c|}
\hline $\begin{array}{l}\text { Test Number } \\
\text { Aging Temp. } \\
\text { Yield Stress }\end{array}$ & $\begin{array}{l}: 1 \\
: 32 \\
: 27\end{array}$ & & $\begin{array}{l}\text { Tes } \\
\text { Agir } \\
\text { Ulti }\end{array}$ & $\begin{array}{l}\text { np. } \\
\text { ne } \\
\text { Stress }\end{array}$ & $\begin{array}{l}25^{\circ} \mathrm{C} \\
30,000 \mathrm{~h} \\
595.9 \mathrm{MPa}\end{array}$ \\
\hline Engr. & & Engr. & & Engr. & \\
\hline $\begin{array}{l}\text { Stress } \\
(\mathrm{MPa})\end{array}$ & $\begin{array}{l}\text { Engr. } \\
\text { Strain }\end{array}$ & $\begin{array}{l}\text { Stress } \\
(\mathrm{MPa})\end{array}$ & $\begin{array}{l}\text { Engr. } \\
\text { Strain }\end{array}$ & $\begin{array}{l}\text { Stress } \\
(\mathrm{MPa})\end{array}$ & $\begin{array}{l}\text { Engr. } \\
\text { Strain }\end{array}$ \\
\hline 0.00 & 0.0000 & 379.65 & 0.0340 & 515.57 & 0.1577 \\
\hline 130.91 & 0.0008 & 393.44 & 0.0422 & 521.05 & 0.1650 \\
\hline 195.03 & 0.0019 & 404.80 & 0.0504 & 528.04 & 0.1762 \\
\hline 218.36 & 0.0024 & 416.36 & 0.0590 & 536.84 & 0.1915 \\
\hline 242.09 & 0.0027 & 426.20 & 0.0661 & 543.64 & 0.2056 \\
\hline 251.86 & 0.0030 & 431.24 & 0.0709 & 485.56 & 0.2200 \\
\hline 261.87 & 0.0033 & 451.27 & 0.0884 & 560.09 & 0.2446 \\
\hline 271.49 & 0.0037 & 460.07 & 0.0954 & 570.86 & 0.2784 \\
\hline 280.91 & 0.0043 & 465.27 & 0.0983 & 581.68 & 0.3217 \\
\hline 295.96 & 0.0055 & 471.26 & 0.1042 & 583.65 & 0.3378 \\
\hline 314.86 & 0.0079 & 478.93 & 0.1127 & 585.00 & 0.3490 \\
\hline 329.57 & 0.0111 & 492.34 & 0.1283 & 585.65 & 0.3596 \\
\hline 339.05 & 0.0140 & 498.90 & 0.1343 & 587.54 & 0.3614 \\
\hline 354.26 & 0.0203 & 506.39 & 0.1460 & 588.05 & 0.3651 \\
\hline 367.74 & 0.0262 & 509.78 & 0.1502 & 589.10 & 0.3950 \\
\hline
\end{tabular}

Table 143. Test data for specimen P11A-13

\begin{tabular}{|c|c|c|c|c|c|}
\hline $\begin{array}{l}\text { Test Number } \\
\text { Aging Temp. } \\
\text { Yield Stress }\end{array}$ & \multicolumn{2}{|c|}{$\begin{array}{ll}: & 281 \\
: & 320^{\circ} \mathrm{C} \\
: & 299.0 \mathrm{MPa}\end{array}$} & \multicolumn{2}{|c|}{$\begin{array}{l}\text { Test Temp. } \\
\text { Aging Time } \\
\text { Ultimate Stress }\end{array}$} & $\begin{array}{l}25^{\circ} \mathrm{C} \\
55,000 \mathrm{~h} \\
646.8 \mathrm{MPa}\end{array}$ \\
\hline Engr. & & Engr. & & Engr. & \\
\hline $\begin{array}{l}\text { Stress } \\
(\mathrm{MPa})\end{array}$ & $\begin{array}{l}\text { Engr. } \\
\text { Strain }\end{array}$ & $\begin{array}{l}\text { Stress } \\
(\mathrm{MPa})\end{array}$ & $\begin{array}{l}\text { Engr. } \\
\text { Strain }\end{array}$ & $\begin{array}{l}\text { Stress } \\
(\mathrm{MPa})\end{array}$ & $\begin{array}{l}\text { Engr. } \\
\text { Strain }\end{array}$ \\
\hline 22.902 & 0.0001 & 359.101 & 0.0102 & 614.679 & 0.3235 \\
\hline 25.967 & 0.0001 & 365.251 & 0.0119 & 626.149 & 0.3694 \\
\hline 64.721 & 0.0002 & 370.441 & 0.0136 & 626.631 & 0.3714 \\
\hline 75.416 & 0.0003 & 406.347 & 0.0308 & 629.764 & 0.3871 \\
\hline 173.574 & 0.0006 & 429.615 & 0.0456 & 632.222 & 0.3995 \\
\hline 186.428 & 0.0007 & 438.753 & 0.0519 & 632.752 & 0.4016 \\
\hline 198.896 & 0.0008 & 452.705 & 0.0623 & 638.632 & 0.4379 \\
\hline 211.070 & 0.0009 & 478.162 & 0.0840 & 640.994 & 0.4568 \\
\hline 278.813 & 0.0023 & 561.231 & 0.1897 & 641.524 & 0.4609 \\
\hline 286.481 & 0.0026 & 588.172 & 0.2458 & 646.006 & 0.5110 \\
\hline 300.163 & 0.0032 & 610.534 & 0.3092 & 646.777 & 0.5475 \\
\hline
\end{tabular}


Table 144. Test data for specimen P12A-25

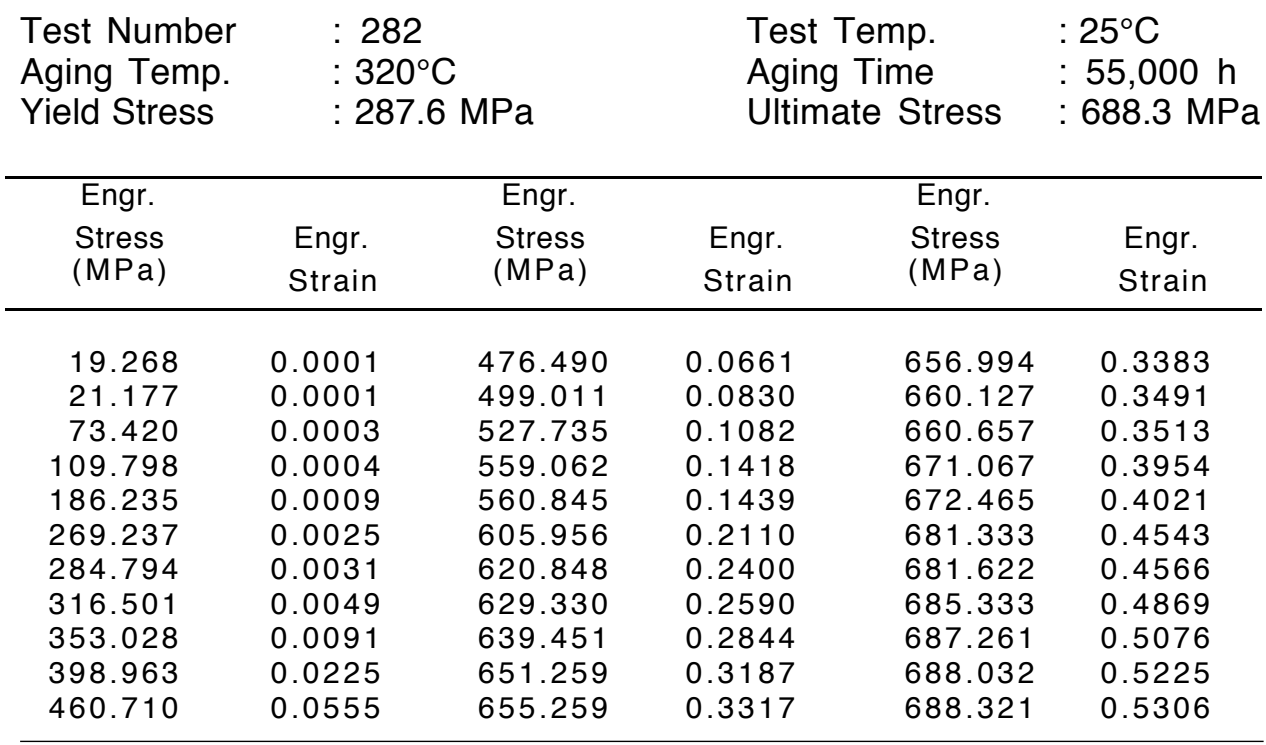

Table 145. Test data for specimen P12T-05

\begin{tabular}{|c|c|c|c|c|c|}
\hline $\begin{array}{l}\text { Test Number } \\
\text { Aging Temp. } \\
\text { Yield Stress }\end{array}$ & $\begin{array}{l}: M \\
: 35 \\
: 27\end{array}$ & & & $\begin{array}{l}\text { np. } \\
\text { ne } \\
\text { Stress }\end{array}$ & $\begin{array}{l}25^{\circ} \mathrm{C} \\
10,000 \mathrm{~h} \\
610.8 \mathrm{MPa}\end{array}$ \\
\hline Engr. & & Engr. & & Engr. & \\
\hline $\begin{array}{l}\text { Stress } \\
(\mathrm{MPa})\end{array}$ & $\begin{array}{l}\text { Engr. } \\
\text { Strain }\end{array}$ & $\begin{array}{l}\text { Stress } \\
(\mathrm{MPa})\end{array}$ & $\begin{array}{l}\text { Engr. } \\
\text { Strain }\end{array}$ & $\begin{array}{l}\text { Stress } \\
(\mathrm{MPa})\end{array}$ & $\begin{array}{l}\text { Engr. } \\
\text { Strain }\end{array}$ \\
\hline 49.6422 & 0.0003 & 346.8752 & 0.0156 & 549.5121 & 0.2378 \\
\hline 82.9439 & 0.0005 & 349.8400 & 0.0172 & 557.4411 & 0.2554 \\
\hline 121.3477 & 0.0007 & 354.0457 & 0.0188 & 563.5774 & 0.2731 \\
\hline 161.4062 & 0.0010 & 383.6932 & 0.0355 & 569.3000 & 0.2910 \\
\hline 197.6727 & 0.0013 & 409.3417 & 0.0526 & 574.4022 & 0.3091 \\
\hline 229.7333 & 0.0019 & 429.8881 & 0.0690 & 579.7801 & 0.3273 \\
\hline 255.6576 & 0.0027 & 448.2281 & 0.0853 & 585.1580 & 0.3457 \\
\hline 276.8245 & 0.0037 & 464.0861 & 0.1019 & 586.6748 & 0.3642 \\
\hline 294.5440 & 0.0049 & 480.0819 & 0.1184 & 592.1907 & 0.3832 \\
\hline 307.3683 & 0.0062 & 492.0788 & 0.1352 & 595.5002 & 0.4029 \\
\hline 316.8141 & 0.0077 & 503.7999 & 0.1520 & 598.0512 & 0.4225 \\
\hline 325.8462 & 0.0092 & 516.0036 & 0.1690 & 600.7401 & 0.4432 \\
\hline 330.6036 & 0.0107 & 525.1047 & 0.1860 & 602.7396 & 0.4670 \\
\hline 337.9810 & 0.0124 & 534.6194 & 0.2031 & 605.4976 & 0.4893 \\
\hline 341.9799 & 0.0140 & 542.3416 & 0.2204 & & \\
\hline
\end{tabular}


Table 146. Test data for specimen P12T-06

\begin{tabular}{|c|c|c|c|c|c|}
\hline \multirow{3}{*}{$\begin{array}{l}\text { Engr. } \\
\text { Stress } \\
\text { (MPa) }\end{array}$} & \multicolumn{2}{|c|}{$\begin{array}{l}: \text { MEA Test } \\
: 350^{\circ} \mathrm{C} \\
: 263.4 \mathrm{MPa}\end{array}$} & \multicolumn{2}{|c|}{$\begin{array}{l}\text { Test Temp. } \\
\text { Aging Time } \\
\text { Ultimate Stress }\end{array}$} & \multirow{2}{*}{$\begin{array}{l}25^{\circ} \mathrm{C} \\
10,000 \mathrm{~h} \\
608.7 \mathrm{MPa} \\
\end{array}$} \\
\hline & & Engr. & & Engr. & \\
\hline & $\begin{array}{l}\text { Engr. } \\
\text { Strain }\end{array}$ & $\begin{array}{l}\text { Stress } \\
\text { (MPa) }\end{array}$ & $\begin{array}{l}\text { Engr. } \\
\text { Strain }\end{array}$ & $\begin{array}{l}\text { Stress } \\
(\mathrm{MPa})\end{array}$ & $\begin{array}{l}\text { Engr. } \\
\text { Strain }\end{array}$ \\
\hline 2.42 & 0.0002 & 48.37 & 0.0174 & 552.27 & 0.3247 \\
\hline 4.53 & 0.0003 & 48.85 & 0.0192 & 558.20 & 0.3496 \\
\hline 9.51 & 0.0006 & 49.33 & 0.0210 & 564.82 & 0.3746 \\
\hline 14.84 & 0.0010 & 49.67 & 0.0228 & 570.89 & 0.4001 \\
\hline 21.36 & 0.0015 & 55.26 & 0.0502 & 577.64 & 0.4259 \\
\hline 27.03 & 0.0019 & 409.62 & 0.0764 & 582.47 & 0.4517 \\
\hline 31.95 & 0.0027 & 433.89 & 0.1016 & 586.67 & 0.4780 \\
\hline 35.57 & 0.0037 & 454.50 & 0.1265 & 591.23 & 0.5047 \\
\hline 38.61 & 0.0048 & 473.81 & 0.1512 & 593.78 & 0.5319 \\
\hline 40.95 & 0.0060 & 488.56 & 0.1759 & 597.71 & 0.5596 \\
\hline 42.94 & 0.0074 & 502.35 & 0.2007 & 600.88 & 0.5880 \\
\hline 44.43 & 0.0089 & 514.28 & 0.2253 & 602.95 & 0.6174 \\
\hline 45.41 & 0.0104 & 524.69 & 0.2501 & 605.43 & 0.6479 \\
\hline 46.52 & 0.0121 & 535.38 & 0.2750 & 607.01 & 0.6804 \\
\hline 47.03 & 0.0138 & 543.31 & 0.2997 & 608.74 & 0.7155 \\
\hline 47.88 & 0.0156 & & & & \\
\hline
\end{tabular}

Table 147. Test data for specimen P12A-08

\begin{tabular}{|c|c|c|c|c|c|}
\hline $\begin{array}{l}\text { Test Number } \\
\text { Aging Temp. } \\
\text { Yield Stress }\end{array}$ & $\begin{array}{l}: \mathrm{ML} \\
: 35 \\
: 29\end{array}$ & & $\begin{array}{l}\text { Test } \\
\text { Agin } \\
\text { Ultin }\end{array}$ & $\begin{array}{l}\text { mp. } \\
\text { me } \\
\text { Stress }\end{array}$ & $\begin{array}{l}: 25^{\circ} \mathrm{C} \\
: 10,000 \mathrm{~h} \\
: 652.9 \mathrm{MPa}\end{array}$ \\
\hline Engr. & & Engr. & & Engr. & \\
\hline $\begin{array}{l}\text { Stress } \\
(\mathrm{MPa})\end{array}$ & $\begin{array}{l}\text { Engr. } \\
\text { Strain }\end{array}$ & $\begin{array}{l}\text { Stress } \\
(\mathrm{MPa})\end{array}$ & $\begin{array}{l}\text { Engr. } \\
\text { Strain }\end{array}$ & $\begin{array}{l}\text { Stress } \\
(\mathrm{MPa})\end{array}$ & $\begin{array}{l}\text { Engr. } \\
\text { Strain }\end{array}$ \\
\hline 33.78 & 0.0002 & 357.56 & 0.0107 & 590.88 & 0.2098 \\
\hline 64.40 & 0.0003 & 361.70 & 0.0118 & 599.77 & 0.2241 \\
\hline 98.87 & 0.0005 & 366.53 & 0.0129 & 605.01 & 0.2383 \\
\hline 135.76 & 0.0007 & 398.38 & 0.0263 & 612.25 & 0.2525 \\
\hline 171.61 & 0.0009 & 427.06 & 0.0406 & 617.43 & 0.2667 \\
\hline 207.74 & 0.0012 & 449.88 & 0.0547 & 621.77 & 0.2810 \\
\hline 238.01 & 0.0018 & 469.60 & 0.0687 & 626.94 & 0.2952 \\
\hline 262.21 & 0.0024 & 487.18 & 0.0828 & 629.84 & 0.3093 \\
\hline 282.62 & 0.0031 & 503.11 & 0.0968 & 633.63 & 0.3232 \\
\hline 298.89 & 0.0039 & 519.11 & 0.1109 & 637.70 & 0.3372 \\
\hline 311.99 & 0.0047 & 533.38 & 0.1250 & 640.66 & 0.3509 \\
\hline 324.81 & 0.0055 & 545.44 & 0.1391 & 643.63 & 0.3647 \\
\hline 334.53 & 0.0065 & 555.72 & 0.1531 & 645.56 & 0.3781 \\
\hline 341.43 & 0.0075 & 567.37 & 0.1674 & 646.31 & 0.3911 \\
\hline 347.43 & 0.0085 & 576.13 & 0.1815 & 649.62 & 0.4038 \\
\hline 353.70 & 0.0096 & 584.47 & 0.1957 & 649.97 & 0.4154 \\
\hline
\end{tabular}


Table 148. Test data for specimen P12A-09

\begin{tabular}{|c|c|c|c|c|c|}
\hline $\begin{array}{l}\text { Test Number } \\
\text { Aging Temp. } \\
\text { Yield Stress }\end{array}$ & $\begin{array}{l}: M \\
: 35 \\
: 27\end{array}$ & & $\begin{array}{l}\text { Tes } \\
\text { Agir } \\
\text { Ulti }\end{array}$ & $\begin{array}{l}\text { np. } \\
\text { ne } \\
\text { Stress }\end{array}$ & $\begin{array}{l}25^{\circ} \mathrm{C} \\
10,000 \mathrm{~h} \\
618.0 \mathrm{MPa}\end{array}$ \\
\hline Engr. & & Engr. & & Engr. & \\
\hline $\begin{array}{l}\text { Stress } \\
(\mathrm{MPa})\end{array}$ & $\begin{array}{l}\text { Engr. } \\
\text { Strain }\end{array}$ & $\begin{array}{l}\text { Stress } \\
(\mathrm{MPa})\end{array}$ & $\begin{array}{l}\text { Engr. } \\
\text { Strain }\end{array}$ & $\begin{array}{l}\text { Stress } \\
(\mathrm{MPa})\end{array}$ & $\begin{array}{l}\text { Engr. } \\
\text { Strain }\end{array}$ \\
\hline 52.33 & 0.0003 & 337.71 & 0.0125 & 569.30 & 0.2471 \\
\hline 88.32 & 0.0005 & 341.77 & 0.0135 & 574.82 & 0.2621 \\
\hline 125.14 & 0.0007 & 370.87 & 0.0289 & 579.37 & 0.2771 \\
\hline 161.89 & 0.0010 & 397.69 & 0.0458 & 586.61 & 0.2918 \\
\hline 196.57 & 0.0013 & 419.68 & 0.0624 & 590.19 & 0.3064 \\
\hline 225.18 & 0.0018 & 439.47 & 0.0785 & 593.85 & 0.3209 \\
\hline 247.11 & 0.0025 & 458.71 & 0.0944 & 596.74 & 0.3352 \\
\hline 264.76 & 0.0033 & 474.91 & 0.1099 & 600.81 & 0.3494 \\
\hline 281.79 & 0.0042 & 487.46 & 0.1255 & 604.67 & 0.3632 \\
\hline 292.75 & 0.0052 & 501.59 & 0.1408 & 605.98 & 0.3770 \\
\hline 304.33 & 0.0063 & 515.73 & 0.1561 & 608.46 & 0.3905 \\
\hline 313.16 & 0.0074 & 525.86 & 0.1714 & 612.12 & 0.4034 \\
\hline 318.47 & 0.0084 & 537.79 & 0.1866 & 614.05 & 0.4159 \\
\hline 323.64 & 0.0093 & 546.13 & 0.2017 & 615.49 & 0.4278 \\
\hline 329.29 & 0.0103 & 555.37 & 0.2169 & 616.60 & 0.4387 \\
\hline
\end{tabular}

Table 149. Test data for specimen P12T-11

$\begin{array}{llll}\text { Test Number } & : 099 & \text { Test Temp. } & : 25^{\circ} \mathrm{C} \\ \text { Aging Temp. } & : 350^{\circ} \mathrm{C} & \text { Aging Time } & : 30,000 \mathrm{~h} \\ \text { Yield Stress } & : 266.6 \mathrm{MPa} & \text { Ultimate Stress } & : 617.0 \mathrm{MPa}\end{array}$

Experimental Data Not Available.

Table 150. Test data for specimen P12A-13

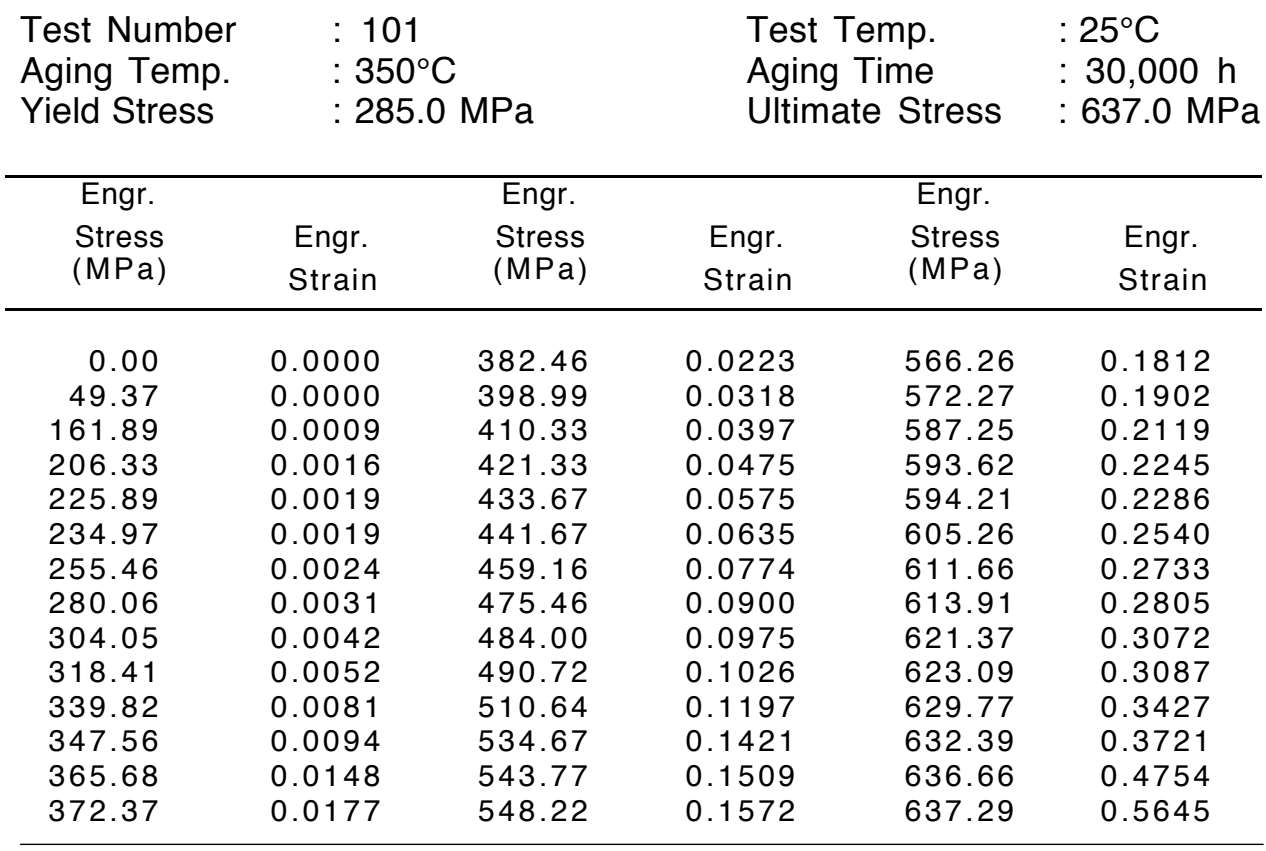


Table 151. Test data for specimen P12A-14

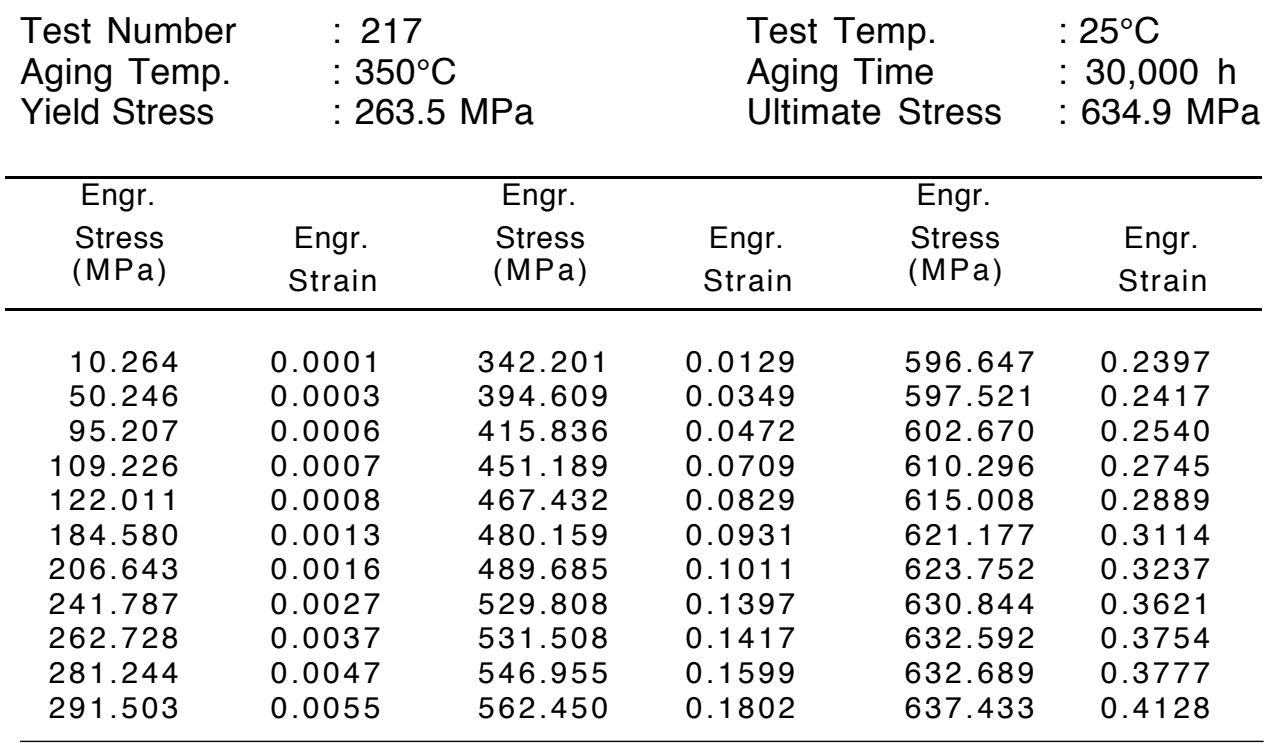

Table 152. Test data for specimen P13T-07

\begin{tabular}{|c|c|c|c|c|c|}
\hline $\begin{array}{l}\text { Test Number } \\
\text { Aging Temp. } \\
\text { Yield Stress }\end{array}$ & \multicolumn{2}{|c|}{$\begin{array}{l}: \text { MEA Test } \\
: 400^{\circ} \mathrm{C} \\
: 285.0 \mathrm{MPa}\end{array}$} & \multicolumn{2}{|c|}{$\begin{array}{l}\text { Test Temp. } \\
\text { Aging Time } \\
\text { Ultimate Stress }\end{array}$} & $\begin{array}{l}25^{\circ} \mathrm{C} \\
10,000 \mathrm{~h} \\
660.0 \mathrm{MPa}\end{array}$ \\
\hline \multirow{2}{*}{$\begin{array}{l}\text { Engr. } \\
\text { Stress } \\
(\mathrm{MPa})\end{array}$} & \multicolumn{2}{|r|}{ Engr. } & \multicolumn{3}{|c|}{ Engr. } \\
\hline & $\begin{array}{l}\text { Engr. } \\
\text { Strain }\end{array}$ & $\begin{array}{l}\text { Stress } \\
\text { (MPa) }\end{array}$ & $\begin{array}{l}\text { Engr. } \\
\text { Strain }\end{array}$ & $\begin{array}{l}\text { Stress } \\
(\mathrm{MPa})\end{array}$ & $\begin{array}{l}\text { Engr. } \\
\text { Strain }\end{array}$ \\
\hline 94.94 & 0.0004 & 375.01 & 0.0145 & 606.39 & 0.2093 \\
\hline 135.34 & 0.0005 & 378.52 & 0.0159 & 613.01 & 0.2243 \\
\hline 171.96 & 0.0006 & 412.31 & 0.0281 & 620.11 & 0.2396 \\
\hline 205.33 & 0.0010 & 438.23 & 0.0417 & 625.77 & 0.2551 \\
\hline 233.53 & 0.0015 & 458.85 & 0.0552 & 631.42 & 0.2708 \\
\hline 258.14 & 0.0022 & 478.36 & 0.0687 & 636.94 & 0.2868 \\
\hline 278.89 & 0.0031 & 498.22 & 0.0823 & 641.49 & 0.3033 \\
\hline 297.16 & 0.0042 & 514.35 & 0.0957 & 645.56 & 0.3200 \\
\hline 312.95 & 0.0053 & 530.48 & 0.1094 & 648.87 & 0.3370 \\
\hline 327.57 & 0.0064 & 542.76 & 0.1232 & 653.00 & 0.3544 \\
\hline 337.91 & 0.0077 & 556.61 & 0.1371 & 654.24 & 0.3724 \\
\hline 346.05 & 0.0089 & 568.61 & 0.1512 & 657.69 & 0.3907 \\
\hline 354.67 & 0.0103 & 578.19 & 0.1655 & 655.62 & 0.4099 \\
\hline 361.22 & 0.0117 & 587.98 & 0.1797 & 658.79 & 0.4301 \\
\hline 368.94 & 0.0131 & 597.64 & 0.1944 & & \\
\hline
\end{tabular}


Table 153. Test data for specimen P13A-07

\begin{tabular}{|c|c|c|c|c|c|}
\hline \multirow{3}{*}{$\begin{array}{l}\text { Engr. } \\
\text { Stress } \\
\text { (MPa) }\end{array}$} & \multicolumn{2}{|c|}{$\begin{array}{l}: \text { MEA Test } \\
: 400^{\circ} \mathrm{C} \\
: 286.3 \mathrm{MPa}\end{array}$} & \multicolumn{2}{|c|}{$\begin{array}{l}\text { Test Temp. } \\
\text { Aging Time } \\
\text { Ultimate Stress }\end{array}$} & \multirow[t]{2}{*}{$\begin{array}{l}25^{\circ} \mathrm{C} \\
10,000 \mathrm{~h} \\
677.7 \mathrm{MPa}\end{array}$} \\
\hline & & Engr. & & Engr. & \\
\hline & $\begin{array}{l}\text { Engr. } \\
\text { Strain }\end{array}$ & $\begin{array}{l}\text { Stress } \\
(\mathrm{MPa})\end{array}$ & $\begin{array}{l}\text { Engr. } \\
\text { Strain }\end{array}$ & $\begin{array}{l}\text { Stress } \\
(\mathrm{MPa})\end{array}$ & $\begin{array}{l}\text { Engr. } \\
\text { Strain }\end{array}$ \\
\hline 4.14 & 0.0002 & 372.59 & 0.0144 & 627.49 & 0.2488 \\
\hline 57.36 & 0.0003 & 379.00 & 0.0160 & 634.39 & 0.2679 \\
\hline 90.39 & 0.0004 & 382.93 & 0.0176 & 640.66 & 0.2873 \\
\hline 127.55 & 0.0006 & 422.79 & 0.0344 & 647.69 & 0.3070 \\
\hline 168.03 & 0.0008 & 454.99 & 0.0522 & 652.52 & 0.3272 \\
\hline 210.15 & 0.0010 & 478.36 & 0.0696 & 657.07 & 0.3477 \\
\hline 250.56 & 0.0014 & 503.80 & 0.0870 & 661.41 & 0.3686 \\
\hline 274.07 & 0.0024 & 524.97 & 0.1043 & 664.10 & 0.3901 \\
\hline 289.37 & 0.0035 & 542.76 & 0.1218 & 666.59 & 0.4119 \\
\hline 307.09 & 0.0046 & 560.20 & 0.1394 & 668.17 & 0.4344 \\
\hline 320.88 & 0.0058 & 572.82 & 0.1572 & 671.55 & 0.4573 \\
\hline 333.84 & 0.0071 & 587.30 & 0.1750 & 673.14 & 0.4807 \\
\hline 342.88 & 0.0084 & 598.88 & 0.1932 & 674.24 & 0.5049 \\
\hline 352.32 & 0.0099 & 609.36 & 0.2116 & 675.69 & 0.5298 \\
\hline 359.15 & 0.0113 & 619.22 & 0.2301 & 675.96 & 0.5555 \\
\hline 366.66 & 0.0128 & & & & \\
\hline
\end{tabular}

Table 154. Test data for specimen P13T-02

\begin{tabular}{|c|c|c|c|c|c|}
\hline $\begin{array}{l}\text { Test Number } \\
\text { Aging Temp. } \\
\text { Yield Stress }\end{array}$ & $\begin{array}{l}: M \\
: U r \\
: 14\end{array}$ & & $\begin{array}{l}\text { Tes } \\
\text { Agi } \\
\text { Ulti }\end{array}$ & $\begin{array}{l}\text { mp. } \\
\text { me } \\
\text { Stress }\end{array}$ & $\begin{array}{l}290^{\circ} \mathrm{C} \\
- \\
408.8 \mathrm{MPa}\end{array}$ \\
\hline Engr. & & Engr. & & Engr. & \\
\hline $\begin{array}{l}\text { Stress } \\
(\mathrm{MPa})\end{array}$ & $\begin{array}{l}\text { Engr. } \\
\text { Strain }\end{array}$ & $\begin{array}{l}\text { Stress } \\
(\mathrm{MPa})\end{array}$ & $\begin{array}{l}\text { Engr. } \\
\text { Strain }\end{array}$ & $\begin{array}{l}\text { Stress } \\
(\mathrm{MPa})\end{array}$ & $\begin{array}{l}\text { Engr. } \\
\text { Strain }\end{array}$ \\
\hline 19.44 & 0.0000 & 119.28 & 0.0014 & 179.26 & 0.0112 \\
\hline 22.06 & 0.0002 & 130.72 & 0.0019 & 181.06 & 0.0120 \\
\hline 25.72 & 0.0003 & 140.45 & 0.0025 & 183.81 & 0.0129 \\
\hline 29.65 & 0.0003 & 146.51 & 0.0030 & 185.47 & 0.0136 \\
\hline 35.23 & 0.0003 & 152.99 & 0.0038 & 186.50 & 0.0145 \\
\hline 39.85 & 0.0004 & 156.86 & 0.0045 & 188.57 & 0.0153 \\
\hline 40.82 & 0.0004 & 161.48 & 0.0054 & 189.88 & 0.0160 \\
\hline 45.78 & 0.0004 & 164.85 & 0.0061 & 191.95 & 0.0170 \\
\hline 51.92 & 0.0004 & 166.92 & 0.0070 & 192.92 & 0.0177 \\
\hline 57.09 & 0.0005 & 170.09 & 0.0079 & 195.47 & 0.0185 \\
\hline 62.12 & 0.0005 & 172.30 & 0.0087 & 196.36 & 0.0192 \\
\hline 67.50 & 0.0005 & 174.99 & 0.0096 & 198.29 & 0.0201 \\
\hline 83.77 & 0.0007 & 176.99 & 0.0103 & 199.12 & 0.0209 \\
\hline 102.11 & 0.0010 & & & & \\
\hline
\end{tabular}


Table 155. Test data for specimen P14T-01

\begin{tabular}{|c|c|c|c|c|c|}
\hline $\begin{array}{l}\text { Test Number } \\
\text { Aging Temp. } \\
\text { Yield Stress }\end{array}$ & $\begin{array}{l}: M \\
: U r \\
: 15\end{array}$ & $\begin{array}{l}\text { est } \\
\mathrm{d} \\
\mathrm{MPa}\end{array}$ & $\begin{array}{l}\text { Tes } \\
\text { Agi } \\
\text { Ulti }\end{array}$ & $\begin{array}{l}\text { mp. } \\
\text { me } \\
\text { Stress }\end{array}$ & $\begin{array}{l}290^{\circ} \mathrm{C} \\
- \\
437.5 \mathrm{MPa}\end{array}$ \\
\hline Engr. & & Engr. & & Engr. & \\
\hline $\begin{array}{l}\text { Stress } \\
(\mathrm{MPa})\end{array}$ & $\begin{array}{l}\text { Engr. } \\
\text { Strain }\end{array}$ & $\begin{array}{l}\text { Stress } \\
(\mathrm{MPa})\end{array}$ & $\begin{array}{l}\text { Engr. } \\
\text { Strain }\end{array}$ & $\begin{array}{l}\text { Stress } \\
(\mathrm{MPa})\end{array}$ & $\begin{array}{l}\text { Engr. } \\
\text { Strain }\end{array}$ \\
\hline 5.03 & 0.0000 & 121.69 & 0.0024 & 184.50 & 0.0096 \\
\hline 5.93 & 0.0002 & 135.41 & 0.0031 & 187.26 & 0.0103 \\
\hline 7.52 & 0.0002 & 146.44 & 0.0039 & 189.81 & 0.0112 \\
\hline 16.41 & 0.0003 & 154.72 & 0.0044 & 192.02 & 0.0121 \\
\hline 26.61 & 0.0005 & 162.10 & 0.0050 & 194.91 & 0.0129 \\
\hline 36.54 & 0.0006 & 167.40 & 0.0055 & 196.71 & 0.0138 \\
\hline 48.54 & 0.0008 & 171.75 & 0.0063 & 198.02 & 0.0147 \\
\hline 63.50 & 0.0010 & 175.75 & 0.0071 & 201.05 & 0.0156 \\
\hline 76.67 & 0.0012 & 178.92 & 0.0078 & 203.05 & 0.0166 \\
\hline 91.98 & 0.0015 & 181.95 & 0.0087 & 205.05 & 0.0173 \\
\hline 108.25 & 0.0019 & & & & \\
\hline
\end{tabular}

Table 156. Test data for specimen P11A-02

\begin{tabular}{|c|c|c|c|c|c|}
\hline $\begin{array}{l}\text { Test Number } \\
\text { Aging Temp. } \\
\text { Yield Stress }\end{array}$ & \multicolumn{2}{|c|}{$\begin{array}{l}: \text { MEA Test } \\
: \text { Unaged } \\
: 159.9 \mathrm{MPa}\end{array}$} & \multicolumn{2}{|c|}{$\begin{array}{l}\text { Test Temp. } \\
\text { Aging Time } \\
\text { Ultimate Stress }\end{array}$} & $\begin{array}{l}290^{\circ} \mathrm{C} \\
- \\
442.7 \mathrm{MPa}\end{array}$ \\
\hline Engr. & & Engr. & & Engr. & \\
\hline $\begin{array}{l}\text { Stress } \\
(\mathrm{MPa})\end{array}$ & $\begin{array}{l}\text { Engr. } \\
\text { Strain }\end{array}$ & $\begin{array}{l}\text { Stress } \\
(\mathrm{MPa})\end{array}$ & $\begin{array}{l}\text { Engr. } \\
\text { Strain }\end{array}$ & $\begin{array}{l}\text { Stress } \\
(\mathrm{MPa})\end{array}$ & $\begin{array}{l}\text { Engr. } \\
\text { Strain }\end{array}$ \\
\hline 39.99 & 0.0000 & 174.37 & 0.0059 & 235.32 & 0.0350 \\
\hline 45.16 & 0.0001 & 177.61 & 0.0071 & 239.32 & 0.0374 \\
\hline 50.68 & 0.0002 & 181.26 & 0.0087 & 243.45 & 0.0401 \\
\hline 55.36 & 0.0002 & 187.12 & 0.0108 & 247.04 & 0.0425 \\
\hline 60.88 & 0.0003 & 190.43 & 0.0124 & 251.18 & 0.0453 \\
\hline 70.26 & 0.0003 & 196.02 & 0.0151 & 257.52 & 0.0493 \\
\hline 81.36 & 0.0003 & 199.81 & 0.0172 & 266.76 & 0.0536 \\
\hline 117.42 & 0.0005 & 204.64 & 0.0192 & 277.86 & 0.0610 \\
\hline 137.76 & 0.0009 & 209.67 & 0.0219 & 288.82 & 0.0677 \\
\hline 152.72 & 0.0018 & 214.01 & 0.0249 & 298.96 & 0.0752 \\
\hline 163.61 & 0.0031 & 222.56 & 0.0283 & 308.40 & 0.0810 \\
\hline 169.06 & 0.0045 & 229.60 & 0.0319 & & \\
\hline
\end{tabular}


Table 157. Test data for specimen P13A-02

\begin{tabular}{|c|c|c|c|c|c|}
\hline $\begin{array}{l}\text { Test Number } \\
\text { Aging Temp. } \\
\text { Yield Stress }\end{array}$ & $\begin{array}{l}: M \\
: U r \\
: 15\end{array}$ & $\begin{array}{l}\text { est } \\
\text { MPa }\end{array}$ & $\begin{array}{l}\text { Tes } \\
\text { Agi } \\
\text { Ulti }\end{array}$ & $\begin{array}{l}\text { np. } \\
\text { ne } \\
\text { Stress }\end{array}$ & $\begin{array}{l}290^{\circ} \mathrm{C} \\
- \\
424.4 \mathrm{MPa}\end{array}$ \\
\hline Engr. & & Engr. & & Engr. & \\
\hline $\begin{array}{l}\text { Stress } \\
(\mathrm{MPa})\end{array}$ & $\begin{array}{l}\text { Engr. } \\
\text { Strain }\end{array}$ & $\begin{array}{l}\text { Stress } \\
(\mathrm{MPa})\end{array}$ & $\begin{array}{l}\text { Engr. } \\
\text { Strain }\end{array}$ & $\begin{array}{l}\text { Stress } \\
(\mathrm{MPa})\end{array}$ & $\begin{array}{l}\text { Engr. } \\
\text { Strain }\end{array}$ \\
\hline 4.55 & 0.0006 & 139.48 & 0.0037 & 179.33 & 0.0116 \\
\hline 9.03 & 0.0006 & 148.24 & 0.0045 & 181.88 & 0.0126 \\
\hline 19.44 & 0.0006 & 155.13 & 0.0053 & 184.43 & 0.0135 \\
\hline 33.58 & 0.0008 & 160.30 & 0.0061 & 186.37 & 0.0145 \\
\hline 50.26 & 0.0011 & 164.72 & 0.0071 & 188.30 & 0.0155 \\
\hline 65.50 & 0.0013 & 168.44 & 0.0080 & 190.50 & 0.0163 \\
\hline 83.36 & 0.0018 & 171.47 & 0.0089 & 192.36 & 0.0173 \\
\hline 99.84 & 0.0022 & 174.37 & 0.0098 & 194.43 & 0.0186 \\
\hline 113.76 & 0.0026 & 177.06 & 0.0107 & 196.43 & 0.0195 \\
\hline 128.31 & 0.0031 & & & & \\
\hline
\end{tabular}

Table 158. Test data for specimen P14T-10

\begin{tabular}{|c|c|c|c|c|c|}
\hline $\begin{array}{l}\text { Test Number } \\
\text { Aging Temp. } \\
\text { Yield Stress }\end{array}$ & $\begin{array}{l}: 2 \\
: 29 \\
: 16\end{array}$ & & $\begin{array}{l}\text { Tes } \\
\text { Agi } \\
\text { Ulti }\end{array}$ & $\begin{array}{l}\text { np. } \\
\text { ne } \\
\text { Stress }\end{array}$ & $\begin{array}{l}290^{\circ} \mathrm{C} \\
30,000 \mathrm{~h} \\
415.3 \mathrm{MPa}\end{array}$ \\
\hline Engr. & & Engr. & & Engr. & \\
\hline $\begin{array}{l}\text { Stress } \\
(\mathrm{MPa})\end{array}$ & $\begin{array}{l}\text { Engr. } \\
\text { Strain }\end{array}$ & $\begin{array}{l}\text { Stress } \\
(\mathrm{MPa})\end{array}$ & $\begin{array}{l}\text { Engr. } \\
\text { Strain }\end{array}$ & $\begin{array}{l}\text { Stress } \\
(\mathrm{MPa})\end{array}$ & $\begin{array}{l}\text { Engr. } \\
\text { Strain }\end{array}$ \\
\hline 49.686 & 0.0003 & 196.581 & 0.0124 & 356.289 & 0.1380 \\
\hline 51.137 & 0.0003 & 200.884 & 0.0146 & 358.380 & 0.1401 \\
\hline 64.981 & 0.0002 & 220.424 & 0.0261 & 376.128 & 0.1592 \\
\hline 73.645 & 0.0002 & 231.175 & 0.0328 & 383.481 & 0.1701 \\
\hline 79.114 & 0.0002 & 250.488 & 0.0455 & 387.668 & 0.1814 \\
\hline 110.354 & 0.0003 & 280.509 & 0.0671 & 397.185 & 0.1978 \\
\hline 126.569 & 0.0005 & 294.898 & 0.0782 & 397.773 & 0.2001 \\
\hline 145.661 & 0.0010 & 313.219 & 0.0934 & 402.525 & 0.2112 \\
\hline 160.219 & 0.0018 & 325.714 & 0.1050 & 406.577 & 0.2203 \\
\hline 176.655 & 0.0047 & 337.274 & 0.1163 & 410.779 & 0.2642 \\
\hline 181.604 & 0.0060 & 346.762 & 0.1270 & 415.188 & 0.2843 \\
\hline
\end{tabular}


Table 159. Test data for specimen P11A-26

\begin{tabular}{|c|c|c|c|c|c|}
\hline $\begin{array}{l}\text { Test Number } \\
\text { Aging Temp. } \\
\text { Yield Stress }\end{array}$ & $\begin{array}{l}: 2 \\
: 25 \\
: 16\end{array}$ & & $\begin{array}{l}\text { Tes } \\
\text { Agi } \\
\text { Ulti }\end{array}$ & $\begin{array}{l}\text { np. } \\
\text { ne } \\
\text { Stress }\end{array}$ & $\begin{array}{l}290^{\circ} \mathrm{C} \\
30,000 \mathrm{~h} \\
394.6 \mathrm{MPa}\end{array}$ \\
\hline Engr. & & Engr. & & Engr. & \\
\hline $\begin{array}{l}\text { Stress } \\
\text { (MPa) }\end{array}$ & $\begin{array}{l}\text { Engr. } \\
\text { Strain }\end{array}$ & $\begin{array}{l}\text { Stress } \\
(\mathrm{MPa})\end{array}$ & $\begin{array}{l}\text { Engr. } \\
\text { Strain }\end{array}$ & $\begin{array}{l}\text { Stress } \\
(\mathrm{MPa})\end{array}$ & $\begin{array}{l}\text { Engr. } \\
\text { Strain }\end{array}$ \\
\hline 42.339 & 0.0002 & 205.024 & 0.0281 & 325.205 & 0.1856 \\
\hline 46.026 & 0.0002 & 215.729 & 0.0372 & 336.590 & 0.2027 \\
\hline 100.931 & 0.0004 & 233.148 & 0.0528 & 347.127 & 0.2183 \\
\hline 110.489 & 0.0005 & 244.451 & 0.0633 & 360.859 & 0.2450 \\
\hline 133.822 & 0.0008 & 259.567 & 0.0782 & 365.983 & 0.2559 \\
\hline 139.770 & 0.0010 & 270.238 & 0.0891 & 369.260 & 0.2627 \\
\hline 157.315 & 0.0022 & 276.340 & 0.0955 & 381.426 & 0.2957 \\
\hline 162.839 & 0.0029 & 285.966 & 0.1060 & 383.735 & 0.3046 \\
\hline 177.574 & 0.0089 & 301.592 & 0.1241 & 384.747 & 0.3067 \\
\hline 181.396 & 0.0109 & 315.777 & 0.1397 & 390.256 & 0.3254 \\
\hline 191.523 & 0.0175 & 317.286 & 0.1419 & 390.473 & 0.3319 \\
\hline
\end{tabular}

Table 160. Test data for specimen P14A-27

\begin{tabular}{|c|c|c|c|c|c|}
\hline $\begin{array}{l}\text { Test Number } \\
\text { Aging Temp. } \\
\text { Yield Stress }\end{array}$ & $\begin{array}{l}: 06 \\
: 29 \\
: 16\end{array}$ & & $\begin{array}{l}\text { Tes } \\
\text { Agi } \\
\text { Ulti }\end{array}$ & $\begin{array}{l}\text { n. } \\
\text { ne } \\
\text { Stress }\end{array}$ & $\begin{array}{l}290^{\circ} \mathrm{C} \\
30,000 \mathrm{~h} \\
421.6 \mathrm{MPa}\end{array}$ \\
\hline Engr. & & Engr. & & Engr. & \\
\hline $\begin{array}{l}\text { Stress } \\
(\mathrm{MPa})\end{array}$ & $\begin{array}{l}\text { Engr. } \\
\text { Strain }\end{array}$ & $\begin{array}{l}\text { Stress } \\
\text { (MPa) }\end{array}$ & $\begin{array}{l}\text { Engr. } \\
\text { Strain }\end{array}$ & $\begin{array}{l}\text { Stress } \\
(\mathrm{MPa})\end{array}$ & $\begin{array}{l}\text { Engr. } \\
\text { Strain }\end{array}$ \\
\hline 134.280 & 0.0012 & 265.850 & 0.0374 & 376.520 & 0.1388 \\
\hline 146.280 & 0.0015 & 277.030 & 0.0468 & 381.110 & 0.1481 \\
\hline 161.390 & 0.0022 & 287.480 & 0.0538 & 383.420 & 0.1503 \\
\hline 169.850 & 0.0028 & 302.560 & 0.0676 & 393.740 & 0.1639 \\
\hline 181.710 & 0.0043 & 313.840 & 0.0770 & 401.590 & 0.1785 \\
\hline 194.170 & 0.0076 & 326.900 & 0.0858 & 403.420 & 0.1817 \\
\hline 197.230 & 0.0102 & 339.030 & 0.0983 & 408.260 & 0.1914 \\
\hline 204.710 & 0.0114 & 350.120 & 0.1068 & 412.750 & 0.2018 \\
\hline 212.740 & 0.0124 & 360.970 & 0.1193 & 417.410 & 0.2177 \\
\hline 239.090 & 0.0190 & 366.940 & 0.1261 & 420.670 & 0.2220 \\
\hline 243.280 & 0.0220 & 368.280 & 0.1283 & 421.580 & 0.2393 \\
\hline
\end{tabular}


Table 161. Test data for specimen P11A-27

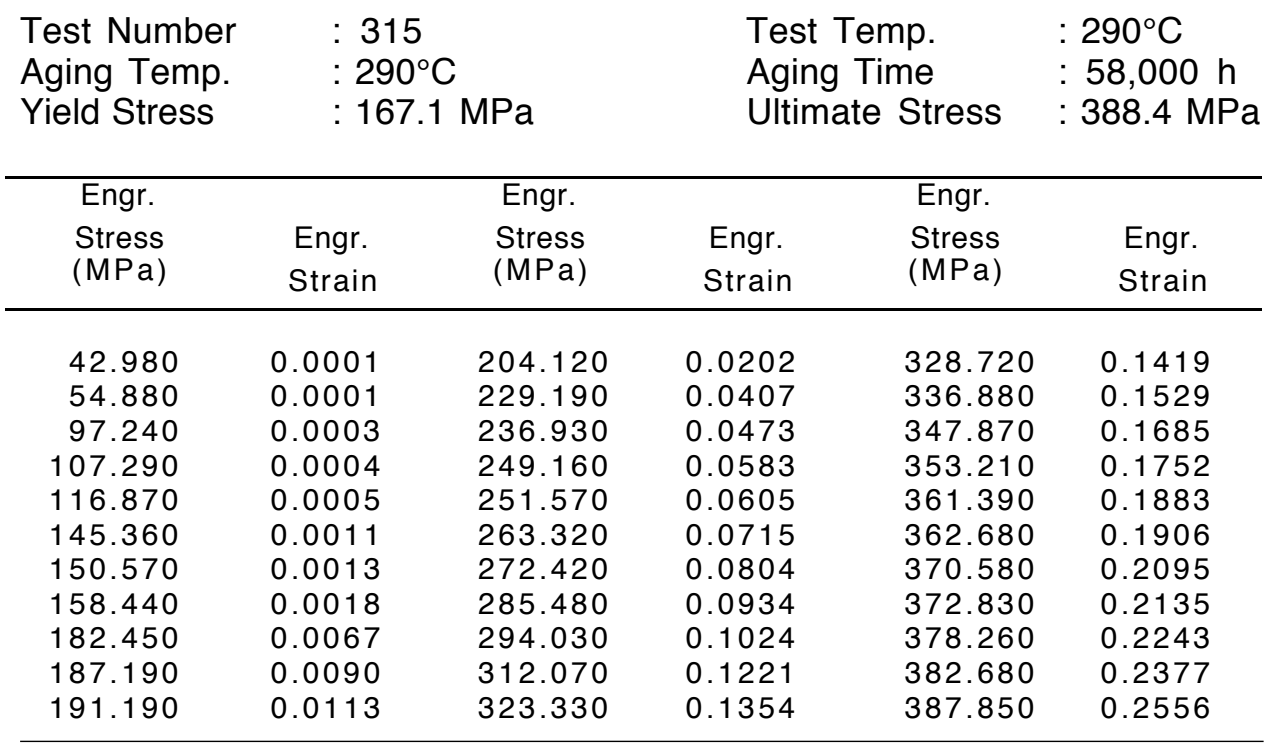

Table 162. Test data for specimen P11A-30

\begin{tabular}{|c|c|c|c|c|c|}
\hline $\begin{array}{l}\text { Test Number } \\
\text { Aging Temp. } \\
\text { Yield Stress }\end{array}$ & $\begin{array}{l}: 3 \\
: 25 \\
: 17\end{array}$ & $\mathrm{MPa}$ & $\begin{array}{l}\text { Tes } \\
\text { Agi } \\
\text { Ulti }\end{array}$ & $\begin{array}{l}\text { np. } \\
\text { ne } \\
\text { Stress }\end{array}$ & $\begin{array}{l}290^{\circ} \mathrm{C} \\
58,000 \mathrm{~h} \\
445.5 \mathrm{MPa}\end{array}$ \\
\hline Engr. & & Engr. & & Engr. & \\
\hline $\begin{array}{l}\text { Stress } \\
(\mathrm{MPa})\end{array}$ & $\begin{array}{l}\text { Engr. } \\
\text { Strain }\end{array}$ & $\begin{array}{l}\text { Stress } \\
(\mathrm{MPa})\end{array}$ & $\begin{array}{l}\text { Engr. } \\
\text { Strain }\end{array}$ & $\begin{array}{l}\text { Stress } \\
(\mathrm{MPa})\end{array}$ & $\begin{array}{l}\text { Engr. } \\
\text { Strain }\end{array}$ \\
\hline 14.878 & 0.0001 & 202.720 & 0.0102 & 370.226 & 0.1596 \\
\hline 20.989 & 0.0001 & 220.071 & 0.0210 & 376.627 & 0.1684 \\
\hline 48.486 & 0.0002 & 244.253 & 0.0389 & 383.251 & 0.1772 \\
\hline 60.940 & 0.0002 & 247.113 & 0.0410 & 393.064 & 0.1922 \\
\hline 107.906 & 0.0003 & 261.265 & 0.0523 & 397.907 & 0.2020 \\
\hline 118.106 & 0.0004 & 285.056 & 0.0718 & 424.204 & 0.2578 \\
\hline 127.299 & 0.0005 & 297.408 & 0.0828 & 426.516 & 0.2647 \\
\hline 135.616 & 0.0006 & 318.571 & 0.1026 & 431.229 & 0.2787 \\
\hline 176.196 & 0.0023 & 329.685 & 0.1135 & 436.938 & 0.2944 \\
\hline 192.908 & 0.0059 & 346.387 & 0.1310 & 440.770 & 0.3198 \\
\hline 198.148 & 0.0080 & 355.396 & 0.1421 & 444.927 & 0.3280 \\
\hline
\end{tabular}

Table 163. Test data for specimen P11A-09

$\begin{array}{llll}\text { Test Number } & : 073 & \text { Test Temp. } & : 290^{\circ} \mathrm{C} \\ \text { Aging Temp. } & : 320^{\circ} \mathrm{C} & \text { Aging Time } & : 30,000 \mathrm{~h} \\ \text { Yield Stress } & : 175.5 \mathrm{MPa} & \text { Ultimate Stress } & : 435.1 \mathrm{MPa}\end{array}$

Experimental Data Not Available. 
Table 164. Test data for specimen P12A-19

\begin{tabular}{|c|c|c|c|c|c|}
\hline $\begin{array}{l}\text { Test Number } \\
\text { Aging Temp. } \\
\text { Yield Stress }\end{array}$ & $\begin{array}{l}: 0 \\
: 32 \\
: 17\end{array}$ & & $\begin{array}{l}\text { Tes } \\
\text { Agir } \\
\text { Ulti }\end{array}$ & $\begin{array}{l}\text { np. } \\
\text { me } \\
\text { Stress }\end{array}$ & $\begin{array}{l}290^{\circ} \mathrm{C} \\
30,000 \mathrm{~h} \\
456.9 \mathrm{MPa}\end{array}$ \\
\hline Engr. & & Engr. & & Engr. & \\
\hline $\begin{array}{l}\text { Stress } \\
(\mathrm{MPa})\end{array}$ & $\begin{array}{l}\text { Engr. } \\
\text { Strain }\end{array}$ & $\begin{array}{l}\text { Stress } \\
(\mathrm{MPa})\end{array}$ & $\begin{array}{l}\text { Engr. } \\
\text { Strain }\end{array}$ & $\begin{array}{l}\text { Stress } \\
(\mathrm{MPa})\end{array}$ & $\begin{array}{l}\text { Engr. } \\
\text { Strain }\end{array}$ \\
\hline 131.38 & 0.0006 & 262.30 & 0.0224 & 378.76 & 0.1026 \\
\hline 168.14 & 0.0014 & 269.07 & 0.0268 & 390.99 & 0.1123 \\
\hline 180.81 & 0.0020 & 276.04 & 0.0311 & 398.61 & 0.1187 \\
\hline 190.28 & 0.0029 & 283.06 & 0.0357 & 404.47 & 0.1246 \\
\hline 197.47 & 0.0039 & 290.72 & 0.0404 & 408.04 & 0.1292 \\
\hline 202.65 & 0.0046 & 303.47 & 0.0495 & 413.26 & 0.1348 \\
\hline 205.37 & 0.0051 & 307.01 & 0.0532 & 425.04 & 0.1485 \\
\hline 211.97 & 0.0056 & 316.34 & 0.0608 & 430.30 & 0.1550 \\
\hline 220.18 & 0.0063 & 326.74 & 0.0668 & 435.21 & 0.1623 \\
\hline 228.18 & 0.0072 & 332.58 & 0.0705 & 438.72 & 0.1677 \\
\hline 236.77 & 0.0087 & 343.90 & 0.0795 & 442.50 & 0.1747 \\
\hline 241.73 & 0.0111 & 350.90 & 0.0850 & 447.03 & 0.1836 \\
\hline 245.57 & 0.0135 & 357.43 & 0.0888 & 450.03 & 0.1908 \\
\hline 250.99 & 0.0160 & 365.46 & 0.0937 & 453.65 & 0.2018 \\
\hline 255.46 & 0.0191 & 372.84 & 0.0975 & 456.90 & 0.2180 \\
\hline
\end{tabular}

Table 165. Test data for specimen P12A-22

\begin{tabular}{|c|c|c|c|c|c|}
\hline \multirow{3}{*}{$\begin{array}{l}\text { Engr. } \\
\text { Stress } \\
\text { (MPa) }\end{array}$} & \multicolumn{2}{|c|}{$\begin{array}{l}: 067 \\
: 320^{\circ} \mathrm{C} \\
: 175.4 \mathrm{MPa}\end{array}$} & \multicolumn{2}{|c|}{$\begin{array}{l}\text { Test Temp. } \\
\text { Aging Time } \\
\text { Ultimate Stress }\end{array}$} & \multirow[t]{2}{*}{$\begin{array}{l}290^{\circ} \mathrm{C} \\
30,000 \mathrm{~h} \\
418.7 \mathrm{MPa}\end{array}$} \\
\hline & & Engr. & & Engr. & \\
\hline & $\begin{array}{l}\text { Engr. } \\
\text { Strain }\end{array}$ & $\begin{array}{l}\text { Stress } \\
(\mathrm{MPa})\end{array}$ & $\begin{array}{l}\text { Engr. } \\
\text { Strain }\end{array}$ & $\begin{array}{l}\text { Stress } \\
(\mathrm{MPa})\end{array}$ & $\begin{array}{l}\text { Engr. } \\
\text { Strain }\end{array}$ \\
\hline 138.43 & 0.0011 & 289.17 & 0.0404 & 384.32 & 0.1234 \\
\hline 166.32 & 0.0019 & 297.39 & 0.0463 & 388.06 & 0.1280 \\
\hline 176.63 & 0.0025 & 304.19 & 0.0518 & 390.71 & 0.1316 \\
\hline 186.34 & 0.0033 & 310.83 & 0.0574 & 394.10 & 0.1367 \\
\hline 195.62 & 0.0045 & 316.83 & 0.0621 & 397.10 & 0.1411 \\
\hline 202.28 & 0.0052 & 323.18 & 0.0673 & 401.91 & 0.1495 \\
\hline 209.26 & 0.0059 & 328.93 & 0.0720 & 403.92 & 0.1535 \\
\hline 216.53 & 0.0065 & 334.40 & 0.0771 & 406.13 & 0.1583 \\
\hline 223.90 & 0.0072 & 340.70 & 0.0821 & 407.94 & 0.1627 \\
\hline 231.96 & 0.0082 & 346.22 & 0.0872 & 410.95 & 0.1687 \\
\hline 240.23 & 0.0102 & 352.72 & 0.0925 & 412.72 & 0.1736 \\
\hline 248.20 & 0.0138 & 358.78 & 0.0979 & 414.59 & 0.1791 \\
\hline 256.42 & 0.0183 & 363.96 & 0.1009 & 416.12 & 0.1842 \\
\hline 263.99 & 0.0229 & 370.68 & 0.1078 & 417.60 & 0.1905 \\
\hline 271.92 & 0.0278 & 376.35 & 0.1139 & 418.34 & 0.1961 \\
\hline 280.94 & 0.0344 & 380.58 & 0.1187 & 418.67 & 0.2057 \\
\hline
\end{tabular}


Table 166. Test data for specimen P12A-22

\begin{tabular}{|c|c|c|c|c|c|}
\hline $\begin{array}{l}\text { Test Number } \\
\text { Aging Temp. } \\
\text { Yield Stress }\end{array}$ & $\begin{array}{l}: 0 \\
: \text { Ur } \\
: 17\end{array}$ & & $\begin{array}{l}\text { Tes } \\
\text { Agi } \\
\text { Ulti }\end{array}$ & $\begin{array}{l}\text { np. } \\
\text { me } \\
\text { Stress }\end{array}$ & $\begin{array}{l}290^{\circ} \mathrm{C} \\
- \\
418 . .7 \mathrm{MPa}\end{array}$ \\
\hline Engr. & & Engr. & & Engr. & \\
\hline $\begin{array}{l}\text { Stress } \\
(\mathrm{MPa})\end{array}$ & $\begin{array}{l}\text { Engr. } \\
\text { Strain }\end{array}$ & $\begin{array}{l}\text { Stress } \\
(\mathrm{MPa})\end{array}$ & $\begin{array}{l}\text { Engr. } \\
\text { Strain }\end{array}$ & $\begin{array}{l}\text { Stress } \\
(\mathrm{MPa})\end{array}$ & $\begin{array}{l}\text { Engr. } \\
\text { Strain }\end{array}$ \\
\hline 138.430 & 0.0011 & 240.230 & 0.0102 & 380.580 & 0.1187 \\
\hline 154.150 & 0.0015 & 248.200 & 0.0138 & 384.320 & 0.1234 \\
\hline 166.320 & 0.0019 & 256.420 & 0.0183 & 388.060 & 0.1280 \\
\hline 176.630 & 0.0025 & 263.990 & 0.0229 & 390.710 & 0.1316 \\
\hline 186.340 & 0.0033 & 271.920 & 0.0278 & 399.600 & 0.1454 \\
\hline 195.620 & 0.0045 & 280.940 & 0.0344 & 401.910 & 0.1495 \\
\hline 202.280 & 0.0052 & 297.390 & 0.0463 & 410.950 & 0.1687 \\
\hline 209.260 & 0.0059 & 323.180 & 0.0673 & 412.720 & 0.1736 \\
\hline 216.530 & 0.0065 & 328.930 & 0.0720 & 414.590 & 0.1791 \\
\hline 223.900 & 0.0072 & 352.720 & 0.0925 & 416.120 & 0.1842 \\
\hline 231.960 & 0.0082 & 367.340 & 0.1040 & 418.670 & 0.2057 \\
\hline
\end{tabular}

Table 167. Test data for specimen P11A-12

\begin{tabular}{|c|c|c|c|c|c|}
\hline $\begin{array}{l}\text { Test Number } \\
\text { Aging Temp. } \\
\text { Yield Stress }\end{array}$ & $\begin{array}{l}: 3 \\
: \text { Ur } \\
: 18\end{array}$ & & $\begin{array}{l}\text { Tes } \\
\text { Agi } \\
\text { Ulti }\end{array}$ & $\begin{array}{l}\text { np. } \\
\text { ne } \\
\text { Stress }\end{array}$ & $\begin{array}{l}25^{\circ} \mathrm{C} \\
- \\
424.8 \mathrm{MPa}\end{array}$ \\
\hline Engr. & & Engr. & & Engr. & \\
\hline $\begin{array}{l}\text { Stress } \\
(\mathrm{MPa})\end{array}$ & $\begin{array}{l}\text { Engr. } \\
\text { Strain }\end{array}$ & $\begin{array}{l}\text { Stress } \\
\text { (MPa) }\end{array}$ & $\begin{array}{l}\text { Engr. } \\
\text { Strain }\end{array}$ & $\begin{array}{l}\text { Stress } \\
\text { (MPa) }\end{array}$ & $\begin{array}{l}\text { Engr. } \\
\text { Strain }\end{array}$ \\
\hline 11.551 & 0.0001 & 247.372 & 0.0335 & 359.065 & 0.1530 \\
\hline 17.176 & 0.0001 & 259.991 & 0.0440 & 372.345 & 0.1731 \\
\hline 123.234 & 0.0002 & 277.304 & 0.0595 & 377.431 & 0.1816 \\
\hline 131.103 & 0.0003 & 286.567 & 0.0685 & 389.453 & 0.2048 \\
\hline 151.510 & 0.0007 & 293.333 & 0.0751 & 392.761 & 0.2117 \\
\hline 175.865 & 0.0017 & 304.078 & 0.0860 & 397.788 & 0.2230 \\
\hline 182.490 & 0.0022 & 308.212 & 0.0905 & 402.369 & 0.2343 \\
\hline 187.882 & 0.0030 & 322.206 & 0.1061 & 413.527 & 0.2687 \\
\hline 210.120 & 0.0090 & 329.793 & 0.1148 & 417.995 & 0.2912 \\
\hline 222.346 & 0.0154 & 347.479 & 0.1371 & 418.821 & 0.2935 \\
\hline 235.500 & 0.0246 & 352.444 & 0.1437 & 424.466 & 0.3186 \\
\hline
\end{tabular}


Table 168. Test data for specimen P12T-08

\begin{tabular}{|c|c|c|c|c|c|}
\hline $\begin{array}{l}\text { Test Number } \\
\text { Aging Temp. } \\
\text { Yield Stress }\end{array}$ & $\begin{array}{l}: M \\
: 35 \\
: 18\end{array}$ & & $\begin{array}{l}\text { Tes } \\
\text { Agi } \\
\text { Ulti }\end{array}$ & $\begin{array}{l}\text { mp. } \\
\text { me } \\
\text { Stress }\end{array}$ & $\begin{array}{l}290^{\circ} \mathrm{C} \\
10,000 \mathrm{~h} \\
454.2 \mathrm{MPa}\end{array}$ \\
\hline Engr. & & Engr. & & Engr. & \\
\hline $\begin{array}{l}\text { Stress } \\
(\mathrm{MPa})\end{array}$ & $\begin{array}{l}\text { Engr. } \\
\text { Strain }\end{array}$ & $\begin{array}{l}\text { Stress } \\
(\mathrm{MPa})\end{array}$ & $\begin{array}{l}\text { Engr. } \\
\text { Strain }\end{array}$ & $\begin{array}{l}\text { Stress } \\
(\mathrm{MPa})\end{array}$ & $\begin{array}{l}\text { Engr. } \\
\text { Strain }\end{array}$ \\
\hline 3.19 & 0.0000 & 214.63 & 0.0086 & 375.49 & 0.1017 \\
\hline 4.50 & 0.0003 & 218.63 & 0.0097 & 383.56 & 0.1078 \\
\hline 6.80 & 0.0005 & 232.90 & 0.0151 & 391.07 & 0.1138 \\
\hline 9.48 & 0.0006 & 247.73 & 0.0218 & 397.00 & 0.1199 \\
\hline 12.72 & 0.0008 & 260.90 & 0.0285 & 404.10 & 0.1259 \\
\hline 16.01 & 0.0010 & 273.17 & 0.0351 & 411.34 & 0.1317 \\
\hline 18.54 & 0.0013 & 286.68 & 0.0428 & 416.24 & 0.1374 \\
\hline 20.88 & 0.0017 & 299.72 & 0.0506 & 422.03 & 0.1430 \\
\hline 22.77 & 0.0021 & 311.37 & 0.0575 & 427.06 & 0.1486 \\
\hline 24.28 & 0.0027 & 321.50 & 0.0642 & 431.82 & 0.1540 \\
\hline 25.76 & 0.0034 & 331.36 & 0.0706 & 436.85 & 0.1590 \\
\hline 26.99 & 0.0044 & 340.95 & 0.0769 & 440.58 & 0.1639 \\
\hline 28.23 & 0.0054 & 350.39 & 0.0832 & 444.16 & 0.1692 \\
\hline 29.46 & 0.0064 & 359.01 & 0.0894 & 446.44 & 0.1728 \\
\hline 210.57 & 0.0074 & 367.90 & 0.0956 & 450.23 & 0.1775 \\
\hline
\end{tabular}

Table 169. Test data for specimen P12A-10

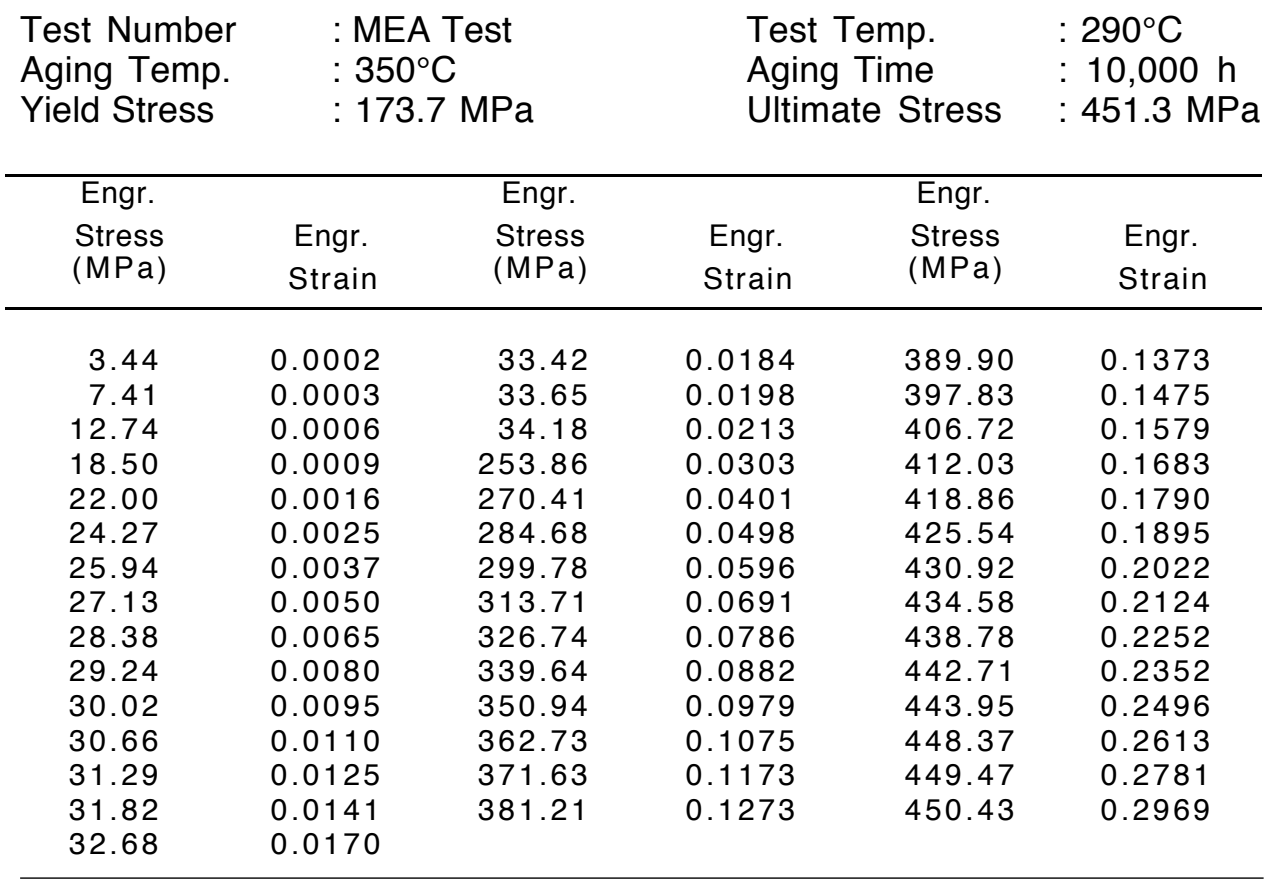


Table 170. Test data for specimen P12A-11

\begin{tabular}{|c|c|c|c|c|c|}
\hline $\begin{array}{l}\text { Test Number } \\
\text { Aging Temp. } \\
\text { Yield Stress }\end{array}$ & $\begin{array}{l}: M \\
: 35 \\
: 18\end{array}$ & & $\begin{array}{l}\text { Tes } \\
\text { Agi } \\
\text { Ulti }\end{array}$ & $\begin{array}{l}\text { mp. } \\
\text { me } \\
\text { Stress }\end{array}$ & $\begin{array}{l}290^{\circ} \mathrm{C} \\
10,000 \mathrm{~h} \\
457.7 \mathrm{MPa}\end{array}$ \\
\hline Engr. & & Engr. & & Engr. & \\
\hline $\begin{array}{l}\text { Stress } \\
(\mathrm{MPa})\end{array}$ & $\begin{array}{l}\text { Engr. } \\
\text { Strain }\end{array}$ & $\begin{array}{l}\text { Stress } \\
(\mathrm{MPa})\end{array}$ & $\begin{array}{l}\text { Engr. } \\
\text { Strain }\end{array}$ & $\begin{array}{l}\text { Stress } \\
(\mathrm{MPa})\end{array}$ & $\begin{array}{l}\text { Engr. } \\
\text { Strain }\end{array}$ \\
\hline 25.23 & 0.0002 & 208.29 & 0.0076 & 396.45 & 0.1291 \\
\hline 49.09 & 0.0002 & 212.70 & 0.0083 & 404.31 & 0.1391 \\
\hline 75.36 & 0.0003 & 227.73 & 0.0120 & 412.10 & 0.1491 \\
\hline 104.94 & 0.0005 & 249.11 & 0.0217 & 419.55 & 0.1594 \\
\hline 136.45 & 0.0006 & 267.24 & 0.0311 & 425.89 & 0.1698 \\
\hline 154.03 & 0.0011 & 284.20 & 0.0409 & 431.89 & 0.1805 \\
\hline 167.96 & 0.0016 & 300.06 & 0.0501 & 437.54 & 0.1914 \\
\hline 177.20 & 0.0022 & 313.78 & 0.0594 & 441.82 & 0.2009 \\
\hline 181.61 & 0.0030 & 327.50 & 0.0690 & 446.99 & 0.2133 \\
\hline 186.02 & 0.0038 & 342.95 & 0.0802 & 449.33 & 0.2265 \\
\hline 190.50 & 0.0045 & 355.01 & 0.0905 & 452.78 & 0.2358 \\
\hline 194.91 & 0.0053 & 367.56 & 0.1003 & 454.71 & 0.2503 \\
\hline 199.40 & 0.0060 & 376.59 & 0.1095 & 457.05 & 0.2700 \\
\hline 203.81 & 0.0068 & 387.14 & 0.1193 & & \\
\hline
\end{tabular}

Table 171. Test data for specimen P12T-12

\begin{tabular}{|c|c|c|c|c|c|}
\hline $\begin{array}{l}\text { Test Number } \\
\text { Aging Temp. } \\
\text { Yield Stress }\end{array}$ & $\begin{array}{l}: 06 \\
: 35 \\
: 19\end{array}$ & & $\begin{array}{l}\text { Tes } \\
\text { Agi } \\
\text { Ulti }\end{array}$ & $\begin{array}{l}\text { np. } \\
\text { ne } \\
\text { Stress }\end{array}$ & $\begin{array}{l}290^{\circ} \mathrm{C} \\
30,000 \mathrm{~h} \\
472.7 \mathrm{MPa}\end{array}$ \\
\hline Engr. & & Engr. & & Engr. & \\
\hline $\begin{array}{l}\text { Stress } \\
(\mathrm{MPa})\end{array}$ & $\begin{array}{l}\text { Engr. } \\
\text { Strain }\end{array}$ & $\begin{array}{l}\text { Stress } \\
(\mathrm{MPa})\end{array}$ & $\begin{array}{l}\text { Engr. } \\
\text { Strain }\end{array}$ & $\begin{array}{l}\text { Stress } \\
(\mathrm{MPa})\end{array}$ & $\begin{array}{l}\text { Engr. } \\
\text { Strain }\end{array}$ \\
\hline 0.00 & 0.0000 & 285.06 & 0.0185 & 418.11 & 0.1059 \\
\hline 160.70 & 0.0008 & 292.20 & 0.0220 & 428.42 & 0.1152 \\
\hline 177.07 & 0.0011 & 300.36 & 0.0263 & 433.66 & 0.1203 \\
\hline 193.08 & 0.0017 & 307.97 & 0.0307 & 442.72 & 0.1305 \\
\hline 203.09 & 0.0021 & 314.18 & 0.0343 & 447.23 & 0.1357 \\
\hline 210.49 & 0.0023 & 323.99 & 0.0404 & 451.05 & 0.1407 \\
\hline 218.79 & 0.0026 & 333.07 & 0.0464 & 455.25 & 0.1471 \\
\hline 227.36 & 0.0029 & 340.93 & 0.0516 & 459.15 & 0.1535 \\
\hline 236.13 & 0.0034 & 349.86 & 0.0578 & 462.72 & 0.1593 \\
\hline 243.53 & 0.0044 & 358.15 & 0.0636 & 465.26 & 0.1645 \\
\hline 251.71 & 0.0063 & 366.79 & 0.0696 & 468.03 & 0.1708 \\
\hline 257.38 & 0.0079 & 375.95 & 0.0760 & 470.67 & 0.1769 \\
\hline 264.33 & 0.0101 & 383.11 & 0.0814 & 472.16 & 0.1833 \\
\hline 271.72 & 0.0127 & 402.11 & 0.0953 & 472.69 & 0.1882 \\
\hline 278.71 & 0.0157 & & & & \\
\hline
\end{tabular}


Table 172. Test data for specimen P14A-22

\begin{tabular}{|c|c|c|c|c|c|}
\hline $\begin{array}{l}\text { Test Number } \\
\text { Aging Temp. } \\
\text { Yield Stress }\end{array}$ & $\begin{array}{l}: 07 \\
: 35 \\
: 18\end{array}$ & & $\begin{array}{l}\text { Tes } \\
\text { Agi } \\
\text { Ulti }\end{array}$ & $\begin{array}{l}\text { np. } \\
\text { me } \\
\text { Stress }\end{array}$ & $\begin{array}{l}290^{\circ} \mathrm{C} \\
30,000 \mathrm{~h} \\
462.3 \mathrm{MPa}\end{array}$ \\
\hline Engr. & & Engr. & & Engr. & \\
\hline $\begin{array}{l}\text { Stress } \\
(\mathrm{MPa})\end{array}$ & $\begin{array}{l}\text { Engr. } \\
\text { Strain }\end{array}$ & $\begin{array}{l}\text { Stress } \\
\text { (MPa) }\end{array}$ & $\begin{array}{l}\text { Engr. } \\
\text { Strain }\end{array}$ & $\begin{array}{l}\text { Stress } \\
(\mathrm{MPa})\end{array}$ & $\begin{array}{l}\text { Engr. } \\
\text { Strain }\end{array}$ \\
\hline 142.29 & 0.0011 & 322.47 & 0.0412 & 416.55 & 0.1124 \\
\hline 162.23 & 0.0017 & 331.01 & 0.0462 & 420.31 & 0.1164 \\
\hline 175.77 & 0.0022 & 334.55 & 0.0496 & 424.96 & 0.1213 \\
\hline 184.78 & 0.0027 & 340.61 & 0.0541 & 431.85 & 0.1305 \\
\hline 190.30 & 0.0030 & 346.03 & 0.0576 & 437.80 & 0.1393 \\
\hline 216.79 & 0.0042 & 353.29 & 0.0630 & 444.69 & 0.1490 \\
\hline 233.27 & 0.0049 & 359.55 & 0.0672 & 447.63 & 0.1541 \\
\hline 239.75 & 0.0057 & 366.71 & 0.0723 & 452.41 & 0.1637 \\
\hline 248.93 & 0.0073 & 375.77 & 0.0794 & 455.58 & 0.1686 \\
\hline 256.89 & 0.0089 & 380.67 & 0.0837 & 456.88 & 0.1728 \\
\hline 263.22 & 0.0108 & 386.30 & 0.0883 & 458.33 & 0.1776 \\
\hline 282.83 & 0.0181 & 393.80 & 0.0928 & 458.58 & 0.1816 \\
\hline 291.04 & 0.0212 & 401.42 & 0.0983 & 458.98 & 0.1878 \\
\hline 306.67 & 0.0297 & 406.60 & 0.1025 & 462.34 & 0.1927 \\
\hline 315.65 & 0.0350 & 411.50 & 0.1075 & & \\
\hline
\end{tabular}

Table 173. Test data for specimen P14A-23

\begin{tabular}{|c|c|c|c|c|c|}
\hline $\begin{array}{l}\text { Test Number } \\
\text { Aging Temp. } \\
\text { Yield Stress }\end{array}$ & $\begin{array}{l}: 0 \\
: 35 \\
: 15\end{array}$ & & $\begin{array}{l}\text { Tes } \\
\text { Agi } \\
\text { Ulti }\end{array}$ & $\begin{array}{l}\text { mp. } \\
\text { me } \\
\text { Stress }\end{array}$ & $\begin{array}{l}290^{\circ} \mathrm{C} \\
30,000 \mathrm{~h} \\
475.6 \mathrm{MPa}\end{array}$ \\
\hline Engr. & & Engr. & & Engr. & \\
\hline $\begin{array}{l}\text { Stress } \\
(\mathrm{MPa})\end{array}$ & $\begin{array}{l}\text { Engr. } \\
\text { Strain }\end{array}$ & $\begin{array}{l}\text { Stress } \\
(\mathrm{MPa})\end{array}$ & $\begin{array}{l}\text { Engr. } \\
\text { Strain }\end{array}$ & $\begin{array}{l}\text { Stress } \\
(\mathrm{MPa})\end{array}$ & $\begin{array}{l}\text { Engr. } \\
\text { Strain }\end{array}$ \\
\hline 0.24 & 0.0000 & 316.66 & 0.0340 & 447.88 & 0.1415 \\
\hline 148.36 & 0.0016 & 324.27 & 0.0398 & 452.09 & 0.1475 \\
\hline 161.13 & 0.0020 & 330.13 & 0.0437 & 454.13 & 0.1521 \\
\hline 185.08 & 0.0030 & 343.05 & 0.0524 & 459.21 & 0.1600 \\
\hline 204.59 & 0.0038 & 352.07 & 0.0583 & 462.25 & 0.1658 \\
\hline 215.64 & 0.0042 & 363.77 & 0.0665 & 466.48 & 0.1752 \\
\hline 229.77 & 0.0047 & 375.32 & 0.0748 & 468.66 & 0.1843 \\
\hline 244.59 & 0.0067 & 385.61 & 0.0825 & 468.90 & 0.1843 \\
\hline 255.32 & 0.0088 & 394.27 & 0.0894 & 471.80 & 0.1906 \\
\hline 265.90 & 0.0116 & 403.49 & 0.0969 & 473.42 & 0.1963 \\
\hline 272.58 & 0.0140 & 414.95 & 0.1041 & 474.30 & 0.2015 \\
\hline 281.96 & 0.0175 & 417.78 & 0.1069 & 475.25 & 0.2054 \\
\hline 292.48 & 0.0222 & 423.95 & 0.1128 & 475.29 & 0.2100 \\
\hline 300.49 & 0.0260 & 433.53 & 0.1236 & 475.57 & 0.2145 \\
\hline 307.67 & 0.0301 & 443.46 & 0.1351 & & \\
\hline
\end{tabular}


Table 174. Test data for specimen P13T-08

\begin{tabular}{|c|c|c|c|c|c|}
\hline $\begin{array}{l}\text { Test Number } \\
\text { Aging Temp. } \\
\text { Yield Stress }\end{array}$ & $\begin{array}{l}: M \\
: 40 \\
: 16\end{array}$ & & $\begin{array}{l}\text { Tes } \\
\text { Agi } \\
\text { Ulti }\end{array}$ & $\begin{array}{l}\text { mp. } \\
\text { me } \\
\text { Stress }\end{array}$ & $\begin{array}{l}290^{\circ} \mathrm{C} \\
10,000 \mathrm{~h} \\
502.2 \mathrm{MPa}\end{array}$ \\
\hline Engr. & & Engr. & & Engr. & \\
\hline $\begin{array}{l}\text { Stress } \\
(\mathrm{MPa})\end{array}$ & $\begin{array}{l}\text { Engr. } \\
\text { Strain }\end{array}$ & $\begin{array}{l}\text { Stress } \\
(\mathrm{MPa})\end{array}$ & $\begin{array}{l}\text { Engr. } \\
\text { Strain }\end{array}$ & $\begin{array}{l}\text { Stress } \\
(\mathrm{MPa})\end{array}$ & $\begin{array}{l}\text { Engr. } \\
\text { Strain }\end{array}$ \\
\hline 34.13 & 0.0002 & 247.25 & 0.0129 & 431.61 & 0.1201 \\
\hline 52.61 & 0.0004 & 251.59 & 0.0141 & 441.20 & 0.1294 \\
\hline 74.05 & 0.0005 & 256.00 & 0.0151 & 448.78 & 0.1388 \\
\hline 99.28 & 0.0007 & 279.17 & 0.0226 & 456.50 & 0.1487 \\
\hline 119.07 & 0.0012 & 300.68 & 0.0317 & 463.40 & 0.1586 \\
\hline 135.55 & 0.0017 & 318.47 & 0.0402 & 470.98 & 0.1697 \\
\hline 151.27 & 0.0023 & 335.29 & 0.0491 & 477.26 & 0.1807 \\
\hline 165.13 & 0.0031 & 350.46 & 0.0577 & 482.08 & 0.1909 \\
\hline 178.16 & 0.0040 & 364.53 & 0.0665 & 486.70 & 0.2012 \\
\hline 193.12 & 0.0056 & 377.42 & 0.0752 & 491.25 & 0.2116 \\
\hline 208.08 & 0.0072 & 389.83 & 0.0841 & 494.29 & 0.2223 \\
\hline 223.11 & 0.0089 & 401.48 & 0.0930 & 497.66 & 0.2331 \\
\hline 237.46 & 0.0105 & 412.44 & 0.1019 & 500.01 & 0.2441 \\
\hline 241.52 & 0.0116 & 422.44 & 0.1109 & 501.52 & 0.2545 \\
\hline
\end{tabular}

Table 175. Test data for specimen P13A-08

\begin{tabular}{|c|c|c|c|c|c|}
\hline $\begin{array}{l}\text { Test Number } \\
\text { Aging Temp. } \\
\text { Yield Stress }\end{array}$ & $\begin{array}{l}: M \\
: 40 \\
: 16\end{array}$ & $\begin{array}{l}\text { est } \\
\mathrm{MPa}\end{array}$ & $\begin{array}{l}\text { Tes } \\
\text { Agi } \\
\text { Ulti }\end{array}$ & $\begin{array}{l}\text { np. } \\
\text { ne } \\
\text { Stress }\end{array}$ & $\begin{array}{l}290^{\circ} \mathrm{C} \\
10,000 \mathrm{~h} \\
485.6 \mathrm{MPa}\end{array}$ \\
\hline Engr. & & Engr. & & Engr. & \\
\hline $\begin{array}{l}\text { Stress } \\
(\mathrm{MPa})\end{array}$ & $\begin{array}{l}\text { Engr. } \\
\text { Strain }\end{array}$ & $\begin{array}{l}\text { Stress } \\
(\mathrm{MPa})\end{array}$ & $\begin{array}{l}\text { Engr. } \\
\text { Strain }\end{array}$ & $\begin{array}{l}\text { Stress } \\
(\mathrm{MPa})\end{array}$ & $\begin{array}{l}\text { Engr. } \\
\text { Strain }\end{array}$ \\
\hline 19.93 & 0.0000 & 278.96 & 0.0272 & 438.09 & 0.1479 \\
\hline 91.98 & 0.0007 & 284.34 & 0.0297 & 443.88 & 0.1556 \\
\hline 125.97 & 0.0015 & 291.92 & 0.0330 & 448.92 & 0.1638 \\
\hline 150.86 & 0.0027 & 297.58 & 0.0362 & 454.50 & 0.1730 \\
\hline 173.20 & 0.0041 & 315.23 & 0.0457 & 460.16 & 0.1829 \\
\hline 197.60 & 0.0058 & 333.29 & 0.0564 & 464.71 & 0.1923 \\
\hline 219.46 & 0.0075 & 349.84 & 0.0673 & 468.43 & 0.2018 \\
\hline 227.80 & 0.0095 & 366.11 & 0.0785 & 471.81 & 0.2109 \\
\hline 236.77 & 0.0117 & 380.66 & 0.0894 & 474.64 & 0.2201 \\
\hline 243.73 & 0.0137 & 392.86 & 0.1005 & 477.46 & 0.2300 \\
\hline 250.62 & 0.0161 & 403.48 & 0.1102 & 479.88 & 0.2401 \\
\hline 256.69 & 0.0185 & 411.69 & 0.1177 & 481.25 & 0.2507 \\
\hline 263.52 & 0.0207 & 418.58 & 0.1251 & 484.43 & 0.2617 \\
\hline 269.45 & 0.0229 & 425.41 & 0.1329 & 484.29 & 0.2730 \\
\hline 274.00 & 0.0251 & 431.89 & 0.1401 & 485.39 & 0.2854 \\
\hline
\end{tabular}


Table 176. Test data for specimen 683-40

\begin{tabular}{|c|c|c|c|c|c|}
\hline $\begin{array}{l}\text { Test Number } \\
\text { Aging Temp. } \\
\text { Yield Stress }\end{array}$ & $\begin{array}{l}: M \\
: U 1 \\
: 27\end{array}$ & & $\begin{array}{l}\text { Tes } \\
\text { Agi } \\
\text { Ulti }\end{array}$ & $\begin{array}{l}\text { np. } \\
\text { ne } \\
\text { Stress }\end{array}$ & $\begin{array}{l}25^{\circ} \mathrm{C} \\
- \\
530.0 \mathrm{MPa}\end{array}$ \\
\hline $\begin{array}{l}\text { Engr. } \\
\text { Stress } \\
(\mathrm{MPa})\end{array}$ & $\begin{array}{l}\text { Engr. } \\
\text { Strain }\end{array}$ & $\begin{array}{l}\text { Engr. } \\
\text { Stress } \\
(\mathrm{MPa})\end{array}$ & $\begin{array}{l}\text { Engr. } \\
\text { Strain }\end{array}$ & $\begin{array}{l}\text { Engr. } \\
\text { Stress } \\
(\mathrm{MPa}) \\
\end{array}$ & $\begin{array}{l}\text { Engr. } \\
\text { Strain }\end{array}$ \\
\hline 26.89 & 0.0002 & 303.23 & 0.0074 & 481.12 & 0.2244 \\
\hline 66.60 & 0.0003 & 329.64 & 0.0206 & 486.84 & 0.2387 \\
\hline 88.67 & 0.0004 & 349.70 & 0.0356 & 490.22 & 0.2528 \\
\hline 111.90 & 0.0005 & 365.63 & 0.0507 & 496.49 & 0.2669 \\
\hline 139.89 & 0.0006 & 380.04 & 0.0658 & 503.11 & 0.2809 \\
\hline 163.96 & 0.0007 & 392.93 & 0.0807 & 504.56 & 0.2948 \\
\hline 191.33 & 0.0009 & 405.41 & 0.0954 & 508.70 & 0.3086 \\
\hline 217.12 & 0.0012 & 417.06 & 0.1099 & 511.80 & 0.3223 \\
\hline 238.28 & 0.0017 & 426.58 & 0.1244 & 515.87 & 0.3357 \\
\hline 257.11 & 0.0023 & 437.75 & 0.1389 & 518.83 & 0.3489 \\
\hline 270.07 & 0.0030 & 443.75 & 0.1532 & 520.21 & 0.3620 \\
\hline 279.03 & 0.0038 & 452.99 & 0.1675 & 523.73 & 0.3746 \\
\hline 286.27 & 0.0046 & 460.23 & 0.1818 & 524.48 & 0.3869 \\
\hline 292.96 & 0.0054 & 466.84 & 0.1961 & 527.93 & 0.3983 \\
\hline 298.75 & 0.0064 & 475.53 & 0.2104 & 528.55 & 0.4082 \\
\hline
\end{tabular}

Table 177. Test data for specimen 683-41

\begin{tabular}{|c|c|c|c|c|c|}
\hline $\begin{array}{l}\text { Test Number } \\
\text { Aging Temp. } \\
\text { Yield Stress }\end{array}$ & $\begin{array}{l}: M \\
: U r \\
: 27\end{array}$ & $\begin{array}{l}\text { est } \\
\mathrm{d} \\
\mathrm{MPa}\end{array}$ & $\begin{array}{l}\text { Tes } \\
\text { Agi } \\
\text { Ulti }\end{array}$ & $\begin{array}{l}\text { np. } \\
\text { ne } \\
\text { Stress }\end{array}$ & $\begin{array}{l}25^{\circ} \mathrm{C} \\
- \\
517.2 \mathrm{MPa}\end{array}$ \\
\hline $\begin{array}{l}\text { Engr. } \\
\text { Stress } \\
(\mathrm{MPa}) \\
\end{array}$ & $\begin{array}{l}\text { Engr. } \\
\text { Strain }\end{array}$ & $\begin{array}{l}\text { Engr. } \\
\text { Stress } \\
\text { (MPa) }\end{array}$ & $\begin{array}{l}\text { Engr. } \\
\text { Strain }\end{array}$ & $\begin{array}{l}\text { Engr. } \\
\text { Stress } \\
(\mathrm{MPa}) \\
\end{array}$ & $\begin{array}{l}\text { Engr. } \\
\text { Strain }\end{array}$ \\
\hline 45.37 & 0.0002 & 299.92 & 0.0106 & 457.61 & 0.1693 \\
\hline 65.43 & 0.0003 & 304.06 & 0.0122 & 462.16 & 0.1802 \\
\hline 86.67 & 0.0004 & 327.43 & 0.0266 & 469.67 & 0.1913 \\
\hline 109.49 & 0.0005 & 345.08 & 0.0408 & 475.81 & 0.2026 \\
\hline 136.59 & 0.0007 & 357.15 & 0.0538 & 482.91 & 0.2138 \\
\hline 163.82 & 0.0008 & 370.46 & 0.0664 & 486.01 & 0.2253 \\
\hline 192.16 & 0.0009 & 383.00 & 0.0785 & 493.60 & 0.2369 \\
\hline 219.60 & 0.0011 & 392.59 & 0.0903 & 496.35 & 0.2487 \\
\hline 246.63 & 0.0014 & 405.55 & 0.1020 & 501.87 & 0.2609 \\
\hline 264.41 & 0.0020 & 415.48 & 0.1136 & 505.66 & 0.2733 \\
\hline 276.48 & 0.0030 & 424.30 & 0.1249 & 508.83 & 0.2860 \\
\hline 285.17 & 0.0044 & 432.58 & 0.1362 & 511.66 & 0.2993 \\
\hline 291.03 & 0.0059 & 440.16 & 0.1473 & 512.97 & 0.3132 \\
\hline 295.03 & 0.0074 & 450.16 & 0.1583 & 514.07 & 0.3290 \\
\hline 298.61 & 0.0090 & & & & \\
\hline
\end{tabular}


Table 178. Test data for specimen 683-33

\begin{tabular}{|c|c|c|c|c|c|}
\hline $\begin{array}{l}\text { Test Number } \\
\text { Aging Temp. } \\
\text { Yield Stress }\end{array}$ & $\begin{array}{l}: 2 \\
: 26 \\
: 26\end{array}$ & & $\begin{array}{l}\text { Tes } \\
\text { Agi } \\
\text { Ulti }\end{array}$ & $\begin{array}{l}\mathrm{mp.} \\
\text { me } \\
\text { Stress }\end{array}$ & $\begin{array}{l}25^{\circ} \mathrm{C} \\
30,000 \mathrm{~h} \\
502.3 \mathrm{MPa}\end{array}$ \\
\hline $\begin{array}{l}\text { Engr. } \\
\text { Stress } \\
\text { (MPa) }\end{array}$ & $\begin{array}{l}\text { Engr. } \\
\text { Strain }\end{array}$ & $\begin{array}{l}\text { Engr. } \\
\text { Stress } \\
\text { (MPa) }\end{array}$ & $\begin{array}{l}\text { Engr. } \\
\text { Strain }\end{array}$ & $\begin{array}{l}\text { Engr. } \\
\text { Stress } \\
\text { (MPa) }\end{array}$ & $\begin{array}{l}\text { Engr. } \\
\text { Strain }\end{array}$ \\
\hline 15.277 & 0.0001 & 351.581 & 0.0596 & 443.121 & 0.1839 \\
\hline 59.946 & 0.0004 & 363.526 & 0.0726 & 466.461 & 0.2323 \\
\hline 70.662 & 0.0005 & 365.541 & 0.0747 & 467.452 & 0.2344 \\
\hline 167.215 & 0.0011 & 376.495 & 0.0875 & 471.522 & 0.2452 \\
\hline 179.868 & 0.0012 & 378.511 & 0.0896 & 472.586 & 0.2473 \\
\hline 240.869 & 0.0023 & 386.982 & 0.1002 & 479.712 & 0.2668 \\
\hline 247.815 & 0.0027 & 409.652 & 0.1302 & 484.443 & 0.2821 \\
\hline 316.452 & 0.0264 & 411.134 & 0.1323 & 490.510 & 0.3043 \\
\hline 319.288 & 0.0287 & 412.518 & 0.1344 & 492.891 & 0.3156 \\
\hline 334.245 & 0.0421 & 439.657 & 0.1776 & 501.585 & 0.3781 \\
\hline 336.620 & 0.0443 & 440.721 & 0.1797 & 502.266 & 0.4007 \\
\hline
\end{tabular}

Table 179. Test data for specimen 684-31

\begin{tabular}{|c|c|c|c|c|c|}
\hline $\begin{array}{l}\text { Test Number } \\
\text { Aging Temp. } \\
\text { Yield Stress }\end{array}$ & $\begin{array}{l}: 2 \\
: 2 c \\
: 26\end{array}$ & $\mathrm{MPa}$ & $\begin{array}{l}\text { Tes } \\
\text { Agi } \\
\text { Ulti }\end{array}$ & $\begin{array}{l}\text { mp. } \\
\text { me } \\
\text { Stress }\end{array}$ & $\begin{array}{l}25^{\circ} \mathrm{C} \\
30,000 \mathrm{~h} \\
531.5 \mathrm{MPa}\end{array}$ \\
\hline $\begin{array}{l}\text { Engr. } \\
\text { Stress } \\
(\mathrm{MPa}) \\
\end{array}$ & $\begin{array}{l}\text { Engr. } \\
\text { Strain }\end{array}$ & $\begin{array}{l}\text { Engr. } \\
\text { Stress } \\
(\mathrm{MPa}) \\
\end{array}$ & $\begin{array}{l}\text { Engr. } \\
\text { Strain }\end{array}$ & $\begin{array}{l}\text { Engr. } \\
\text { Stress } \\
(\mathrm{MPa}) \\
\end{array}$ & $\begin{array}{l}\text { Engr. } \\
\text { Strain }\end{array}$ \\
\hline 15.603 & 0.0001 & 360.080 & 0.0352 & 481.976 & 0.1438 \\
\hline 28.245 & 0.0002 & 394.218 & 0.0583 & 497.647 & 0.1677 \\
\hline 49.499 & 0.0004 & 397.019 & 0.0604 & 499.002 & 0.1699 \\
\hline 92.316 & 0.0007 & 405.249 & 0.0667 & 511.484 & 0.1944 \\
\hline 186.523 & 0.0014 & 425.612 & 0.0834 & 512.452 & 0.1966 \\
\hline 197.849 & 0.0016 & 435.013 & 0.0918 & 516.370 & 0.2057 \\
\hline 227.787 & 0.0022 & 437.819 & 0.0943 & 519.660 & 0.2149 \\
\hline 283.764 & 0.0054 & 455.309 & 0.1119 & 525.563 & 0.2349 \\
\hline 289.304 & 0.0062 & 463.055 & 0.1204 & 527.208 & 0.2420 \\
\hline 312.856 & 0.0119 & 468.522 & 0.1268 & 527.595 & 0.2444 \\
\hline 349.219 & 0.0289 & 470.375 & 0.1289 & 531.369 & 0.2795 \\
\hline
\end{tabular}


Table 180. Test data for specimen 68-145

\begin{tabular}{|c|c|c|c|c|c|}
\hline $\begin{array}{l}\text { Test Number } \\
\text { Aging Temp. } \\
\text { Yield Stress }\end{array}$ & $\begin{array}{l}: 2 \\
: 29 \\
: 27\end{array}$ & & $\begin{array}{l}\text { Tes } \\
\text { Agir } \\
\text { Ulti }\end{array}$ & $\begin{array}{l}\text { np. } \\
\text { ne } \\
\text { Stress }\end{array}$ & $\begin{array}{l}25^{\circ} \mathrm{C} \\
30,000 \mathrm{~h} \\
566.4 \mathrm{MPa}\end{array}$ \\
\hline $\begin{array}{l}\text { Engr. } \\
\text { Stress } \\
\text { (MPa) }\end{array}$ & $\begin{array}{l}\text { Engr. } \\
\text { Strain }\end{array}$ & $\begin{array}{l}\text { Engr. } \\
\text { Stress } \\
\text { (MPa) }\end{array}$ & $\begin{array}{l}\text { Engr. } \\
\text { Strain }\end{array}$ & $\begin{array}{l}\text { Engr. } \\
\text { Stress } \\
(\mathrm{MPa}) \\
\end{array}$ & $\begin{array}{l}\text { Engr. } \\
\text { Strain }\end{array}$ \\
\hline 14.767 & 0.0001 & 407.302 & 0.0596 & 511.435 & 0.1588 \\
\hline 27.509 & 0.0002 & 410.232 & 0.0616 & 517.459 & 0.1669 \\
\hline 138.852 & 0.0009 & 418.155 & 0.0676 & 530.809 & 0.1873 \\
\hline 218.478 & 0.0017 & 439.177 & 0.0842 & 532.014 & 0.1893 \\
\hline 245.500 & 0.0023 & 448.344 & 0.0922 & 535.628 & 0.1955 \\
\hline 253.019 & 0.0026 & 455.192 & 0.0981 & 547.436 & 0.2182 \\
\hline 319.555 & 0.0106 & 478.504 & 0.1208 & 550.183 & 0.2245 \\
\hline 325.097 & 0.0125 & 480.605 & 0.1228 & 551.677 & 0.2281 \\
\hline 341.088 & 0.0190 & 488.109 & 0.1307 & 560.737 & 0.2534 \\
\hline 371.687 & 0.0356 & 489.940 & 0.1327 & 562.328 & 0.2599 \\
\hline 384.323 & 0.0436 & 495.097 & 0.1387 & 566.376 & 0.2892 \\
\hline
\end{tabular}

Table 181. Test data for specimen 684-21

\begin{tabular}{|c|c|c|c|c|c|}
\hline $\begin{array}{l}\text { Test Number } \\
\text { Aging Temp. } \\
\text { Yield Stress }\end{array}$ & $\begin{array}{l}: 12 \\
: 32 \\
: 30\end{array}$ & $\mathrm{~Pa}$ & $\begin{array}{l}\text { Tes } \\
\text { Agi } \\
\text { Ulti }\end{array}$ & $\begin{array}{l}\text { np. } \\
\text { ne } \\
\text { Stress }\end{array}$ & $\begin{array}{l}25^{\circ} \mathrm{C} \\
10,000 \mathrm{~h} \\
614.1 \mathrm{MPa}\end{array}$ \\
\hline $\begin{array}{l}\text { Engr. } \\
\text { Stress } \\
\text { (MPa) }\end{array}$ & $\begin{array}{l}\text { Engr. } \\
\text { Strain }\end{array}$ & $\begin{array}{l}\text { Engr. } \\
\text { Stress } \\
(\mathrm{MPa}) \\
\end{array}$ & $\begin{array}{l}\text { Engr. } \\
\text { Strain }\end{array}$ & $\begin{array}{l}\text { Engr. } \\
\text { Stress } \\
(\mathrm{MPa})\end{array}$ & $\begin{array}{l}\text { Engr. } \\
\text { Strain }\end{array}$ \\
\hline $\begin{array}{r}0.20 \\
43.86 \\
201.61 \\
299.97 \\
323.03 \\
331.67 \\
342.81 \\
358.17\end{array}$ & $\begin{array}{l}0.0000 \\
0.0001 \\
0.0025 \\
0.0052 \\
0.0077 \\
0.0093 \\
0.0127 \\
0.0193\end{array}$ & $\begin{array}{l}383.48 \\
405.09 \\
428.57 \\
459.36 \\
473.99 \\
478.54 \\
484.63\end{array}$ & $\begin{array}{l}0.0356 \\
0.0528 \\
0.0739 \\
0.1004 \\
0.1162 \\
0.1220 \\
0.1342\end{array}$ & $\begin{array}{l}514.78 \\
542.52 \\
565.75 \\
581.42 \\
599.71 \\
609.92 \\
614.05\end{array}$ & $\begin{array}{l}0.1775 \\
0.2325 \\
0.2918 \\
0.3475 \\
0.4324 \\
0.5201 \\
0.5901\end{array}$ \\
\hline
\end{tabular}


Table 182. Test data for specimen 684-22

\begin{tabular}{|c|c|c|c|c|c|}
\hline $\begin{array}{l}\text { Test Number } \\
\text { Aging Temp. } \\
\text { Yield Stress }\end{array}$ & & & $\begin{array}{l}\text { Tes } \\
\text { Agi } \\
\text { Ulti }\end{array}$ & $\begin{array}{l}\text { np. } \\
\text { ne } \\
\text { Stress }\end{array}$ & $\begin{array}{l}25^{\circ} \mathrm{C} \\
10,000 \mathrm{~h} \\
571.2 \mathrm{MPa}\end{array}$ \\
\hline $\begin{array}{l}\text { Engr. } \\
\text { Stress } \\
\text { (MPa) }\end{array}$ & $\begin{array}{l}\text { Engr. } \\
\text { Strain }\end{array}$ & $\begin{array}{l}\text { Engr. } \\
\text { Stress } \\
\text { (MPa) }\end{array}$ & $\begin{array}{l}\text { Engr. } \\
\text { Strain }\end{array}$ & $\begin{array}{l}\text { Engr. } \\
\text { Stress } \\
(\mathrm{MPa}) \\
\end{array}$ & $\begin{array}{l}\text { Engr. } \\
\text { Strain }\end{array}$ \\
\hline 0.20 & 0.0000 & 322.48 & 0.0097 & 485.20 & 0.1852 \\
\hline 32.30 & 0.0000 & 334.82 & 0.0140 & 485.89 & 0.1852 \\
\hline 205.66 & 0.0020 & 353.54 & 0.0251 & 511.51 & 0.2517 \\
\hline 206.25 & 0.0020 & 386.11 & 0.0506 & 539.46 & 0.3421 \\
\hline 222.40 & 0.0022 & 420.65 & 0.0852 & 560.89 & 0.4697 \\
\hline 279.06 & 0.0038 & 450.14 & 0.1178 & 570.53 & 0.5504 \\
\hline 297.49 & 0.0050 & 466.83 & 0.1429 & 571.22 & 0.6435 \\
\hline 311.09 & 0.0069 & 478.09 & 0.1629 & & \\
\hline
\end{tabular}

Table 183. Test data for specimen 682-41

\begin{tabular}{|c|c|c|c|c|c|}
\hline $\begin{array}{l}\text { Test Number } \\
\text { Aging Temp. } \\
\text { Yield Stress }\end{array}$ & $\begin{array}{l}: 1 \\
: 32 \\
: 30\end{array}$ & $\mathrm{MPa}$ & $\begin{array}{l}\text { Tes } \\
\text { Agi } \\
\text { Ulti }\end{array}$ & $\begin{array}{l}\text { np. } \\
\text { ne } \\
\text { Stress }\end{array}$ & $\begin{array}{l}25^{\circ} \mathrm{C} \\
30,000 \mathrm{~h} \\
621.0 \mathrm{MPa}\end{array}$ \\
\hline $\begin{array}{l}\text { Engr. } \\
\text { Stress } \\
\text { (MPa) }\end{array}$ & $\begin{array}{l}\text { Engr. } \\
\text { Strain }\end{array}$ & $\begin{array}{l}\text { Engr. } \\
\text { Stress } \\
\text { (MPa) }\end{array}$ & $\begin{array}{l}\text { Engr. } \\
\text { Strain }\end{array}$ & $\begin{array}{l}\text { Engr. } \\
\text { Stress } \\
(\mathrm{MPa})\end{array}$ & $\begin{array}{l}\text { Engr. } \\
\text { Strain }\end{array}$ \\
\hline $\begin{array}{r}0.00 \\
232.61 \\
291.45 \\
306.61 \\
331.78 \\
354.04\end{array}$ & $\begin{array}{l}0.0000 \\
0.0017 \\
0.0031 \\
0.0036 \\
0.0054 \\
0.0084\end{array}$ & $\begin{array}{l}379.18 \\
401.78 \\
434.95 \\
464.96 \\
488.25\end{array}$ & $\begin{array}{l}0.0145 \\
0.0249 \\
0.0420 \\
0.0635 \\
0.0806\end{array}$ & $\begin{array}{l}509.61 \\
539.21 \\
570.81 \\
607.20 \\
621.00\end{array}$ & $\begin{array}{l}0.0980 \\
0.1225 \\
0.1592 \\
0.2236 \\
0.2783\end{array}$ \\
\hline
\end{tabular}

Table 184. Test data for specimen 684-39

\begin{tabular}{|c|c|c|c|c|c|}
\hline $\begin{array}{l}\text { Test Number } \\
\text { Aging Temp. } \\
\text { Yield Stress }\end{array}$ & $\begin{array}{l}: 16 \\
: 32 \\
: 35\end{array}$ & & $\begin{array}{l}\text { Tes } \\
\text { Agi } \\
\text { Ulti }\end{array}$ & $\begin{array}{l}\text { np. } \\
\text { ne } \\
\text { Stress }\end{array}$ & $\begin{array}{l}25^{\circ} \mathrm{C} \\
30,000 \mathrm{~h} \\
614.4 \mathrm{MPa}\end{array}$ \\
\hline $\begin{array}{l}\text { Engr. } \\
\text { Stress } \\
\text { (MPa) }\end{array}$ & $\begin{array}{l}\text { Engr. } \\
\text { Strain }\end{array}$ & $\begin{array}{l}\text { Engr. } \\
\text { Stress } \\
\text { (MPa) }\end{array}$ & $\begin{array}{l}\text { Engr. } \\
\text { Strain }\end{array}$ & $\begin{array}{l}\text { Engr. } \\
\text { Stress } \\
(\mathrm{MPa}) \\
\end{array}$ & $\begin{array}{l}\text { Engr. } \\
\text { Strain }\end{array}$ \\
\hline $\begin{array}{r}0.00 \\
69.76 \\
298.81 \\
347.64 \\
379.70 \\
400.70\end{array}$ & $\begin{array}{l}0.0000 \\
0.0001 \\
0.0030 \\
0.0044 \\
0.0067 \\
0.0092\end{array}$ & $\begin{array}{l}416.93 \\
434.07 \\
457.04 \\
477.88 \\
495.73\end{array}$ & $\begin{array}{l}0.0133 \\
0.0200 \\
0.0334 \\
0.0502 \\
0.0735\end{array}$ & $\begin{array}{l}532.08 \\
566.06 \\
595.24 \\
612.83 \\
614.44\end{array}$ & $\begin{array}{l}0.1137 \\
0.1636 \\
0.2291 \\
0.3099 \\
0.3387\end{array}$ \\
\hline
\end{tabular}


Table 185. Test data for specimen 68-264

\begin{tabular}{|c|c|c|c|c|c|}
\hline $\begin{array}{l}\text { Test Number } \\
\text { Aging Temp. } \\
\text { Yield Stress }\end{array}$ & $\begin{array}{l}: 1 \\
: 32 \\
: 2 c\end{array}$ & & $\begin{array}{l}\text { Tes } \\
\text { Agi } \\
\text { Ulti }\end{array}$ & $\begin{array}{l}\text { np. } \\
\text { ne } \\
\text { Stress }\end{array}$ & $\begin{array}{l}25^{\circ} \mathrm{C} \\
30,000 \mathrm{~h} \\
572.5 \mathrm{MPa}\end{array}$ \\
\hline $\begin{array}{l}\text { Engr. } \\
\text { Stress } \\
(\mathrm{MPa}) \\
\end{array}$ & $\begin{array}{l}\text { Engr. } \\
\text { Strain }\end{array}$ & $\begin{array}{l}\text { Engr. } \\
\text { Stress } \\
\text { (MPa) }\end{array}$ & $\begin{array}{l}\text { Engr. } \\
\text { Strain }\end{array}$ & $\begin{array}{l}\text { Engr. } \\
\text { Stress } \\
(\mathrm{MPa}) \\
\end{array}$ & $\begin{array}{l}\text { Engr. } \\
\text { Strain } \\
\end{array}$ \\
\hline $\begin{array}{r}0.00 \\
261.43 \\
291.16 \\
306.26 \\
315.94 \\
325.70 \\
336.88 \\
345.85\end{array}$ & $\begin{array}{l}0.0000 \\
0.0032 \\
0.0046 \\
0.0058 \\
0.0068 \\
0.0085 \\
0.0111 \\
0.0141\end{array}$ & $\begin{array}{l}355.60 \\
355.90 \\
363.44 \\
376.07 \\
391.55 \\
410.68 \\
432.03\end{array}$ & $\begin{array}{l}0.0183 \\
0.0183 \\
0.0228 \\
0.0319 \\
0.0442 \\
0.0621 \\
0.0853\end{array}$ & $\begin{array}{l}447.85 \\
467.97 \\
491.74 \\
503.84 \\
535.15 \\
563.65 \\
572.53\end{array}$ & $\begin{array}{l}0.1035 \\
0.1347 \\
0.1833 \\
0.2217 \\
0.3130 \\
0.4398 \\
0.5390\end{array}$ \\
\hline
\end{tabular}

Table 186. Test data for specimen 681-28

\begin{tabular}{|c|c|c|c|c|c|}
\hline $\begin{array}{l}\text { Test Number } \\
\text { Aging Temp. } \\
\text { Yield Stress }\end{array}$ & $\begin{array}{l}: 28 \\
: 32 \\
: 32\end{array}$ & & $\begin{array}{l}\text { Tes } \\
\text { Agir } \\
\text { Ulti }\end{array}$ & $\begin{array}{l}\text { np. } \\
\text { ne } \\
\text { Stress }\end{array}$ & $\begin{array}{l}25^{\circ} \mathrm{C} \\
50,000 \mathrm{~h} \\
640.3 \mathrm{MPa}\end{array}$ \\
\hline $\begin{array}{l}\text { Engr. } \\
\text { Stress } \\
(\mathrm{MPa})\end{array}$ & $\begin{array}{l}\text { Engr. } \\
\text { Strain }\end{array}$ & $\begin{array}{l}\text { Engr. } \\
\text { Stress } \\
(\mathrm{MPa})\end{array}$ & $\begin{array}{l}\text { Engr. } \\
\text { Strain }\end{array}$ & $\begin{array}{l}\text { Engr. } \\
\text { Stress } \\
(\mathrm{MPa}) \\
\end{array}$ & $\begin{array}{l}\text { Engr. } \\
\text { Strain }\end{array}$ \\
\hline 13.740 & 0.0001 & 440.890 & 0.0401 & 568.650 & 0.1798 \\
\hline 16.160 & 0.0000 & 446.950 & 0.0444 & 576.090 & 0.1926 \\
\hline 73.320 & 0.0005 & 476.220 & 0.0679 & 577.180 & 0.1947 \\
\hline 84.350 & 0.0005 & 478.690 & 0.0700 & 594.630 & 0.2288 \\
\hline 133.140 & 0.0008 & 481.040 & 0.0721 & 600.430 & 0.2418 \\
\hline 222.700 & 0.0014 & 512.890 & 0.1038 & 613.920 & 0.2763 \\
\hline 286.290 & 0.0024 & 522.170 & 0.1144 & 619.030 & 0.2915 \\
\hline 346.130 & 0.0052 & 523.880 & 0.1165 & 628.870 & 0.3265 \\
\hline 370.930 & 0.0084 & 538.910 & 0.1354 & 633.110 & 0.3442 \\
\hline 392.980 & 0.0143 & 548.160 & 0.1481 & 634.720 & 0.3532 \\
\hline 412.490 & 0.0229 & 567.410 & 0.1777 & 640.200 & 0.4019 \\
\hline
\end{tabular}


Table 187. Test data for specimen 681-29

\begin{tabular}{|c|c|c|c|c|c|}
\hline $\begin{array}{l}\text { Test Number } \\
\text { Aging Temp. } \\
\text { Yield Stress }\end{array}$ & $\begin{array}{l}: 2 \\
: 32 \\
: 30\end{array}$ & & $\begin{array}{l}\text { Tes } \\
\text { Agi } \\
\text { Ulti }\end{array}$ & $\begin{array}{l}\mathrm{mp.} \\
\text { me } \\
\text { Stress }\end{array}$ & $\begin{array}{l}25^{\circ} \mathrm{C} \\
50,000 \mathrm{~h} \\
608.4 \mathrm{MPa}\end{array}$ \\
\hline $\begin{array}{l}\text { Engr. } \\
\text { Stress } \\
\text { (MPa) }\end{array}$ & $\begin{array}{l}\text { Engr. } \\
\text { Strain }\end{array}$ & $\begin{array}{l}\text { Engr. } \\
\text { Stress } \\
\text { (MPa) }\end{array}$ & $\begin{array}{l}\text { Engr. } \\
\text { Strain }\end{array}$ & $\begin{array}{l}\text { Engr. } \\
\text { Stress } \\
\text { (MPa) }\end{array}$ & $\begin{array}{l}\text { Engr. } \\
\text { Strain }\end{array}$ \\
\hline 15.690 & 0.0001 & 440.540 & 0.0508 & 568.460 & 0.2131 \\
\hline 70.570 & 0.0002 & 456.880 & 0.0634 & 580.810 & 0.2443 \\
\hline 131.820 & 0.0005 & 482.840 & 0.0866 & 588.550 & 0.2679 \\
\hline 241.370 & 0.0014 & 491.090 & 0.0952 & 589.270 & 0.2703 \\
\hline 251.140 & 0.0016 & 501.010 & 0.1060 & 590.700 & 0.2751 \\
\hline 292.890 & 0.0028 & 523.550 & 0.1343 & 599.450 & 0.3102 \\
\hline 299.860 & 0.0030 & 525.140 & 0.1365 & 601.830 & 0.3226 \\
\hline 354.120 & 0.0085 & 534.260 & 0.1497 & 602.240 & 0.3247 \\
\hline 387.160 & 0.0193 & 547.790 & 0.1720 & 605.580 & 0.3470 \\
\hline 391.690 & 0.0214 & 550.470 & 0.1766 & 606.700 & 0.3572 \\
\hline 434.680 & 0.0465 & 555.190 & 0.1856 & 608.430 & 0.3845 \\
\hline
\end{tabular}

Table 188. Test data for specimen 68-230

\begin{tabular}{|c|c|c|c|c|c|}
\hline $\begin{array}{l}\text { Test Number } \\
\text { Aging Temp. } \\
\text { Yield Stress }\end{array}$ & & $\mathrm{Pa}$ & $\begin{array}{l}\text { Tes } \\
\text { Agi } \\
\text { Ulti }\end{array}$ & $\begin{array}{l}\mathrm{mp} . \\
\text { me } \\
\text { Stress }\end{array}$ & $\begin{array}{l}25^{\circ} \mathrm{C} \\
50,000 \mathrm{~h} \\
578.9 \mathrm{MPa}\end{array}$ \\
\hline $\begin{array}{l}\text { Engr. } \\
\text { Stress } \\
\text { (MPa) }\end{array}$ & $\begin{array}{l}\text { Engr. } \\
\text { Strain }\end{array}$ & $\begin{array}{l}\text { Engr. } \\
\text { Stress } \\
\text { (MPa) }\end{array}$ & $\begin{array}{l}\text { Engr. } \\
\text { Strain }\end{array}$ & $\begin{array}{l}\text { Engr. } \\
\text { Stress } \\
\text { (MPa) }\end{array}$ & $\begin{array}{l}\text { Engr. } \\
\text { Strain }\end{array}$ \\
\hline 17.980 & 0.0001 & 391.280 & 0.0327 & 521.840 & 0.1759 \\
\hline 23.600 & 0.0001 & 394.710 & 0.0350 & 524.540 & 0.1805 \\
\hline 119.640 & 0.0005 & 430.170 & 0.0623 & 541.250 & 0.2125 \\
\hline 170.980 & 0.0008 & 432.620 & 0.0646 & 542.450 & 0.2148 \\
\hline 183.760 & 0.0009 & 435.270 & 0.0669 & 552.460 & 0.2387 \\
\hline 196.100 & 0.0010 & 454.540 & 0.0862 & 554.310 & 0.2434 \\
\hline 296.600 & 0.0036 & 469.050 & 0.1023 & 561.180 & 0.2612 \\
\hline 302.200 & 0.0040 & 471.040 & 0.1046 & 567.420 & 0.2793 \\
\hline 327.610 & 0.0067 & 497.960 & 0.1395 & 571.500 & 0.2959 \\
\hline 334.490 & 0.0079 & 501.210 & 0.1440 & 575.950 & 0.3158 \\
\hline 376.020 & 0.0236 & 508.950 & 0.1554 & 578.850 & 0.3491 \\
\hline
\end{tabular}


Table 189. Test data for specimen 682-25

\begin{tabular}{|c|c|c|c|c|c|}
\hline $\begin{array}{l}\text { Test Number } \\
\text { Aging Temp. } \\
\text { Yield Stress }\end{array}$ & $\begin{array}{l}: M \\
: 35 \\
: 28\end{array}$ & & $\begin{array}{l}\text { Tes } \\
\text { Agi } \\
\text { Ulti }\end{array}$ & $\begin{array}{l}\text { mp. } \\
\text { me } \\
\text { Stress }\end{array}$ & $\begin{array}{l}25^{\circ} \mathrm{C} \\
5780 \mathrm{~h} \\
601.6 \mathrm{MPa}\end{array}$ \\
\hline $\begin{array}{l}\text { Engr. } \\
\text { Stress } \\
(\mathrm{MPa})\end{array}$ & $\begin{array}{l}\text { Engr. } \\
\text { Strain }\end{array}$ & $\begin{array}{l}\text { Engr. } \\
\text { Stress } \\
(\mathrm{MPa})\end{array}$ & $\begin{array}{l}\text { Engr. } \\
\text { Strain }\end{array}$ & $\begin{array}{l}\text { Engr. } \\
\text { Stress } \\
\text { (MPa) } \\
\end{array}$ & $\begin{array}{l}\text { Engr. } \\
\text { Strain }\end{array}$ \\
\hline 40.06 & 0.0002 & 324.67 & 0.0088 & 557.37 & 0.2780 \\
\hline 59.43 & 0.0004 & 331.43 & 0.0102 & 564.20 & 0.3001 \\
\hline 80.88 & 0.0005 & 335.50 & 0.0117 & 572.20 & 0.3227 \\
\hline 105.70 & 0.0006 & 376.94 & 0.0359 & 577.02 & 0.3452 \\
\hline 130.38 & 0.0008 & 406.03 & 0.0600 & 582.47 & 0.3684 \\
\hline 156.92 & 0.0010 & 431.40 & 0.0832 & 588.19 & 0.3922 \\
\hline 183.95 & 0.0011 & 451.40 & 0.1054 & 591.98 & 0.4165 \\
\hline 211.67 & 0.0014 & 469.40 & 0.1272 & 593.29 & 0.4412 \\
\hline 235.39 & 0.0019 & 487.53 & 0.1487 & 595.22 & 0.4669 \\
\hline 255.86 & 0.0025 & 501.80 & 0.1702 & 596.60 & 0.4932 \\
\hline 274.20 & 0.0033 & 515.38 & 0.1914 & 599.29 & 0.5203 \\
\hline 288.06 & 0.0042 & 527.93 & 0.2129 & 599.02 & 0.5484 \\
\hline 300.40 & 0.0051 & 538.76 & 0.2343 & 600.67 & 0.5775 \\
\hline 309.78 & 0.0062 & 548.41 & 0.2560 & 598.74 & 0.6072 \\
\hline 318.12 & 0.0075 & & & & \\
\hline
\end{tabular}

Table 190. Test data for specimen 682-26

\begin{tabular}{|c|c|c|c|c|c|}
\hline $\begin{array}{l}\text { Test Number } \\
\text { Aging Temp. } \\
\text { Yield Stress }\end{array}$ & $\begin{array}{l}: M \\
: 35 \\
: 25\end{array}$ & & $\begin{array}{l}\text { Tes } \\
\text { Agi } \\
\text { Ulti }\end{array}$ & $\begin{array}{l}\text { np. } \\
\text { ne } \\
\text { Stress }\end{array}$ & $\begin{array}{l}25^{\circ} \mathrm{C} \\
5780 \mathrm{~h} \\
599.4 \mathrm{MPa}\end{array}$ \\
\hline $\begin{array}{l}\text { Engr. } \\
\text { Stress } \\
(\mathrm{MPa}) \\
\end{array}$ & $\begin{array}{l}\text { Engr. } \\
\text { Strain }\end{array}$ & $\begin{array}{l}\text { Engr. } \\
\text { Stress } \\
(\mathrm{MPa})\end{array}$ & $\begin{array}{l}\text { Engr. } \\
\text { Strain }\end{array}$ & $\begin{array}{l}\text { Engr. } \\
\text { Stress } \\
(\mathrm{MPa}) \\
\end{array}$ & $\begin{array}{l}\text { Engr. } \\
\text { Strain }\end{array}$ \\
\hline 39.02 & 0.0002 & 321.43 & 0.0059 & 546.20 & 0.2054 \\
\hline 58.33 & 0.0003 & 330.19 & 0.0070 & 554.06 & 0.2226 \\
\hline 78.26 & 0.0004 & 336.60 & 0.0081 & 563.03 & 0.2402 \\
\hline 100.25 & 0.0005 & 342.26 & 0.0091 & 567.65 & 0.2580 \\
\hline 123.90 & 0.0007 & 385.07 & 0.0281 & 573.37 & 0.2762 \\
\hline 150.37 & 0.0008 & 411.55 & 0.0472 & 578.61 & 0.2947 \\
\hline 178.71 & 0.0009 & 434.09 & 0.0658 & 582.47 & 0.3139 \\
\hline 208.57 & 0.0011 & 454.64 & 0.0839 & 586.47 & 0.3334 \\
\hline 233.66 & 0.0014 & 474.22 & 0.1017 & 590.74 & 0.3538 \\
\hline 253.24 & 0.0019 & 488.98 & 0.1192 & 593.43 & 0.3747 \\
\hline 272.62 & 0.0025 & 504.42 & 0.1367 & 595.09 & 0.3967 \\
\hline 288.41 & 0.0033 & 515.04 & 0.1538 & 597.15 & 0.4199 \\
\hline 301.44 & 0.0041 & 525.59 & 0.1710 & 596.33 & 0.4447 \\
\hline 314.13 & 0.0049 & 538.20 & 0.1882 & 597.78 & 0.4715 \\
\hline
\end{tabular}


Table 191. Test data for specimen 684-06

\begin{tabular}{|c|c|c|c|c|c|}
\hline $\begin{array}{l}\text { Test Number } \\
\text { Aging Temp. } \\
\text { Yield Stress }\end{array}$ & $\begin{array}{l}: 1 \\
: 35 \\
: 26\end{array}$ & & $\begin{array}{l}\text { Tes } \\
\text { Agi } \\
\text { Ulti }\end{array}$ & $\begin{array}{l}\text { mp. } \\
\text { me } \\
\text { Stress }\end{array}$ & $\begin{array}{l}25^{\circ} \mathrm{C} \\
10,000 \mathrm{~h} \\
606.5 \mathrm{MPa}\end{array}$ \\
\hline $\begin{array}{l}\text { Engr. } \\
\text { Stress } \\
\text { (MPa) }\end{array}$ & $\begin{array}{l}\text { Engr. } \\
\text { Strain }\end{array}$ & $\begin{array}{l}\text { Engr. } \\
\text { Stress } \\
\text { (MPa) }\end{array}$ & $\begin{array}{l}\text { Engr. } \\
\text { Strain }\end{array}$ & $\begin{array}{l}\text { Engr. } \\
\text { Stress } \\
\text { (MPa) }\end{array}$ & $\begin{array}{l}\text { Engr. } \\
\text { Strain }\end{array}$ \\
\hline $\begin{array}{r}42.91 \\
194.98 \\
219.11 \\
255.25 \\
280.26 \\
280.51 \\
297.62 \\
313.10\end{array}$ & $\begin{array}{l}0.0002 \\
0.0024 \\
0.0030 \\
0.0039 \\
0.0050 \\
0.0049 \\
0.0062 \\
0.0081\end{array}$ & $\begin{array}{l}329.49 \\
348.32 \\
362.95 \\
383.50 \\
398.95 \\
422.46 \\
450.71 \\
470.92\end{array}$ & $\begin{array}{l}0.0112 \\
0.0163 \\
0.0227 \\
0.0335 \\
0.0434 \\
0.0602 \\
0.0811 \\
0.0973\end{array}$ & $\begin{array}{l}480.34 \\
485.57 \\
520.34 \\
551.99 \\
571.40 \\
588.76 \\
601.37 \\
606.46\end{array}$ & $\begin{array}{l}0.1036 \\
0.1118 \\
0.1500 \\
0.1962 \\
0.2367 \\
0.2865 \\
0.3393 \\
0.3925\end{array}$ \\
\hline
\end{tabular}

Table 192. Test data for specimen 684-07

\begin{tabular}{|c|c|c|c|c|c|}
\hline $\begin{array}{l}\text { Test Number } \\
\text { Aging Temp. } \\
\text { Yield Stress }\end{array}$ & $\begin{array}{l}: 1 \\
: 35 \\
: 25\end{array}$ & & $\begin{array}{l}\text { Tes } \\
\text { Agi } \\
\text { Ulti }\end{array}$ & $\begin{array}{l}\text { ne. } \\
\text { Stress }\end{array}$ & $\begin{array}{l}25^{\circ} \mathrm{C} \\
10,000 \mathrm{~h} \\
601.1 \mathrm{MPa}\end{array}$ \\
\hline $\begin{array}{l}\text { Engr. } \\
\text { Stress } \\
\text { (MPa) }\end{array}$ & $\begin{array}{l}\text { Engr. } \\
\text { Strain }\end{array}$ & $\begin{array}{l}\text { Engr. } \\
\text { Stress } \\
\text { (MPa) }\end{array}$ & $\begin{array}{l}\text { Engr. } \\
\text { Strain }\end{array}$ & $\begin{array}{l}\text { Engr. } \\
\text { Stress } \\
\text { (MPa) }\end{array}$ & $\begin{array}{l}\text { Engr. } \\
\text { Strain }\end{array}$ \\
\hline $\begin{array}{r}0.20 \\
44.00 \\
207.78 \\
282.94 \\
320.64 \\
341.11\end{array}$ & $\begin{array}{l}0.0000 \\
0.0001 \\
0.0024 \\
0.0040 \\
0.0062 \\
0.0084\end{array}$ & $\begin{array}{l}359.88 \\
379.74 \\
402.32 \\
426.63 \\
452.65 \\
475.82\end{array}$ & $\begin{array}{l}0.0124 \\
0.0188 \\
0.0293 \\
0.0448 \\
0.0638 \\
0.0818\end{array}$ & $\begin{array}{l}483.99 \\
525.48 \\
552.96 \\
581.43 \\
592.69 \\
601.06\end{array}$ & $\begin{array}{l}0.0926 \\
0.1305 \\
0.1719 \\
0.2267 \\
0.2652 \\
0.3150\end{array}$ \\
\hline
\end{tabular}


Table 193. Test data for specimen 684-08

\begin{tabular}{|c|c|c|c|c|c|}
\hline $\begin{array}{l}\text { Test Number } \\
\text { Aging Temp. } \\
\text { Yield Stress }\end{array}$ & $\begin{array}{l}: 2 \\
: 35 \\
: 25\end{array}$ & & $\begin{array}{l}\text { Tes } \\
\text { Agi } \\
\text { Ulti }\end{array}$ & $\begin{array}{l}\text { mp. } \\
\text { me } \\
\text { Stress }\end{array}$ & $\begin{array}{l}25^{\circ} \mathrm{C} \\
10,000 \mathrm{~h} \\
612.0 \mathrm{MPa}\end{array}$ \\
\hline $\begin{array}{l}\text { Engr. } \\
\text { Stress } \\
(\mathrm{MPa}) \\
\end{array}$ & $\begin{array}{l}\text { Engr. } \\
\text { Strain }\end{array}$ & $\begin{array}{l}\text { Engr. } \\
\text { Stress } \\
\text { (MPa) }\end{array}$ & $\begin{array}{l}\text { Engr. } \\
\text { Strain }\end{array}$ & $\begin{array}{l}\text { Engr. } \\
\text { Stress } \\
\text { (MPa) } \\
\end{array}$ & $\begin{array}{l}\text { Engr. } \\
\text { Strain }\end{array}$ \\
\hline 39.27 & 0.0000 & 425.98 & 0.0489 & 562.39 & 0.1853 \\
\hline 61.70 & 0.0001 & 444.80 & 0.0611 & 573.01 & 0.2054 \\
\hline 132.87 & 0.0006 & 465.05 & 0.0758 & 575.27 & 0.2099 \\
\hline 168.93 & 0.0009 & 470.32 & 0.0801 & 580.15 & 0.2208 \\
\hline 224.62 & 0.0016 & 482.95 & 0.0904 & 585.60 & 0.2340 \\
\hline 241.26 & 0.0020 & 494.42 & 0.1006 & 589.42 & 0.2452 \\
\hline 286.33 & 0.0027 & 498.72 & 0.1047 & 593.91 & 0.2585 \\
\hline 322.46 & 0.0064 & 509.40 & 0.1152 & 594.69 & 0.2607 \\
\hline 333.59 & 0.0084 & 513.34 & 0.1194 & 597.78 & 0.2717 \\
\hline 355.79 & 0.0143 & 524.74 & 0.1321 & 602.34 & 0.2898 \\
\hline 361.40 & 0.0163 & 533.63 & 0.1427 & 602.70 & 0.2921 \\
\hline 366.72 & 0.0183 & 536.90 & 0.1469 & 605.66 & 0.3057 \\
\hline 371.80 & 0.0203 & 544.67 & 0.1577 & 608.51 & 0.3242 \\
\hline 393.42 & 0.0304 & 547.74 & 0.1620 & 610.47 & 0.3403 \\
\hline 401.42 & 0.0345 & 554.71 & 0.1726 & 611.19 & 0.3495 \\
\hline 419.31 & 0.0448 & 557.45 & 0.1768 & 612.03 & 0.3657 \\
\hline
\end{tabular}

Table 194. Test data for specimen 68-129

\begin{tabular}{|c|c|c|c|c|c|}
\hline $\begin{array}{l}\text { Test Number } \\
\text { Aging Temp. } \\
\text { Yield Stress }\end{array}$ & $\begin{array}{l}: 1 \\
: 35 \\
: 28\end{array}$ & & $\begin{array}{l}\text { Tes } \\
\text { Agi } \\
\text { Ulti }\end{array}$ & $\begin{array}{l}\text { np. } \\
\text { me } \\
\text { Stress }\end{array}$ & $\begin{array}{l}25^{\circ} \mathrm{C} \\
10,000 \mathrm{~h} \\
595.8 \mathrm{MPa}\end{array}$ \\
\hline $\begin{array}{l}\text { Engr. } \\
\text { Stress } \\
\text { (MPa) }\end{array}$ & $\begin{array}{l}\text { Engr. } \\
\text { Strain }\end{array}$ & $\begin{array}{l}\text { Engr. } \\
\text { Stress } \\
(\mathrm{MPa}) \\
\end{array}$ & $\begin{array}{l}\text { Engr. } \\
\text { Strain }\end{array}$ & $\begin{array}{l}\text { Engr. } \\
\text { Stress } \\
\text { (MPa) } \\
\end{array}$ & $\begin{array}{l}\text { Engr. } \\
\text { Strain } \\
\end{array}$ \\
\hline $\begin{array}{r}43.56 \\
196.46 \\
223.72 \\
284.67 \\
319.09 \\
332.24 \\
345.83 \\
358.73\end{array}$ & $\begin{array}{l}0.0000 \\
0.0017 \\
0.0021 \\
0.0036 \\
0.0060 \\
0.0080 \\
0.0080 \\
0.0164\end{array}$ & $\begin{array}{l}383.46 \\
400.23 \\
424.12 \\
446.75 \\
468.20 \\
480.50 \\
489.89 \\
515.18\end{array}$ & $\begin{array}{l}0.0300 \\
0.0420 \\
0.0606 \\
0.0807 \\
0.0978 \\
0.1101 \\
0.1261 \\
0.1569\end{array}$ & $\begin{array}{l}545.42 \\
568.01 \\
585.78 \\
594.05 \\
594.45 \\
595.56 \\
595.46 \\
595.81\end{array}$ & $\begin{array}{l}0.2116 \\
0.2740 \\
0.3478 \\
0.4167 \\
0.4169 \\
0.4226 \\
0.4220 \\
0.5039\end{array}$ \\
\hline
\end{tabular}


Table 195. Test data for specimen 684-10

$\begin{array}{llll}\text { Test Number } & : 191 & \text { Test Temp. } & : 25^{\circ} \mathrm{C} \\ \text { Aging Temp. } & : 350^{\circ} \mathrm{C} & \text { Aging Time } & : 30,000 \mathrm{~h} \\ \text { Yield Stress } & : 299.0 \mathrm{MPa} & \text { Ultimate Stress } & : 652.8 \mathrm{MPa}\end{array}$

\begin{tabular}{rccccc}
\hline $\begin{array}{r}\text { Engr. } \\
\text { Stress } \\
(\mathrm{MPa})\end{array}$ & $\begin{array}{c}\text { Engr. } \\
\text { Strain }\end{array}$ & $\begin{array}{c}\text { Engr. } \\
\text { Stress } \\
(\mathrm{MPa})\end{array}$ & $\begin{array}{c}\text { Engr. } \\
\text { Strain }\end{array}$ & $\begin{array}{c}\text { Engr. } \\
\text { Stress } \\
(\mathrm{MPa})\end{array}$ & $\begin{array}{c}\text { Engr. } \\
\text { Strain }\end{array}$ \\
\hline & & & & & \\
9.42 & 0.0000 & 412.04 & 0.0253 & 581.43 & 0.1448 \\
34.71 & 0.0002 & 426.38 & 0.0319 & 596.07 & 0.1630 \\
99.40 & 0.0006 & 456.56 & 0.0478 & 601.13 & 0.1699 \\
136.72 & 0.0009 & 464.56 & 0.0524 & 604.28 & 0.1745 \\
234.34 & 0.0018 & 468.30 & 0.0547 & 615.26 & 0.1922 \\
244.35 & 0.0020 & 497.14 & 0.0731 & 619.10 & 0.1992 \\
253.55 & 0.0022 & 507.17 & 0.0799 & 621.48 & 0.2038 \\
269.99 & 0.0027 & 513.66 & 0.0844 & 629.89 & 0.2217 \\
277.38 & 0.0029 & 533.80 & 0.1000 & 633.66 & 0.2317 \\
332.95 & 0.0061 & 542.24 & 0.1068 & 639.49 & 0.2485 \\
346.27 & 0.0075 & 544.86 & 0.1090 & 640.42 & 0.2505 \\
351.98 & 0.0083 & 547.56 & 0.1113 & 642.60 & 0.2585 \\
356.97 & 0.0090 & 551.92 & 0.1155 & 643.09 & 0.2606 \\
363.36 & 0.0101 & 571.03 & 0.1335 & 647.38 & 0.2789 \\
368.95 & 0.0113 & 575.28 & 0.1380 & 647.45 & 0.2809 \\
406.56 & 0.0229 & 577.47 & 0.1403 & 652.75 & 0.3335 \\
\hline
\end{tabular}

Table 196. Test data for specimen 684-11

\begin{tabular}{|c|c|c|c|c|c|}
\hline $\begin{array}{l}\text { Test Number } \\
\text { Aging Temp. } \\
\text { Yield Stress }\end{array}$ & $\begin{array}{l}: 1 \\
35 \\
: 28\end{array}$ & & $\begin{array}{l}\text { Tes } \\
\text { Agi } \\
\text { Ulti }\end{array}$ & $\begin{array}{l}\text { np. } \\
\text { ne } \\
\text { Stress }\end{array}$ & $\begin{array}{l}25^{\circ} \mathrm{C} \\
30,000 \mathrm{~h} \\
628.2 \mathrm{MPa}\end{array}$ \\
\hline $\begin{array}{l}\text { Engr. } \\
\text { Stress } \\
(\mathrm{MPa})\end{array}$ & $\begin{array}{l}\text { Engr. } \\
\text { Strain }\end{array}$ & $\begin{array}{l}\text { Engr. } \\
\text { Stress } \\
(\mathrm{MPa})\end{array}$ & $\begin{array}{l}\text { Engr. } \\
\text { Strain }\end{array}$ & $\begin{array}{l}\text { Engr. } \\
\text { Stress } \\
(\mathrm{MPa}) \\
\end{array}$ & $\begin{array}{l}\text { Engr. } \\
\text { Strain } \\
\end{array}$ \\
\hline 9.95 & 0.0001 & 480.97 & 0.0878 & 587.05 & 0.2655 \\
\hline 38.08 & 0.0002 & 485.35 & 0.0921 & 589.00 & 0.2721 \\
\hline 59.45 & 0.0003 & 498.90 & 0.1071 & 595.44 & 0.2918 \\
\hline 153.78 & 0.0008 & 502.77 & 0.1113 & 597.82 & 0.3006 \\
\hline 202.98 & 0.0012 & 519.28 & 0.1323 & 601.95 & 0.3161 \\
\hline 265.79 & 0.0028 & 529.92 & 0.1473 & 602.88 & 0.3205 \\
\hline 279.07 & 0.0034 & 534.16 & 0.1538 & 608.88 & 0.3449 \\
\hline 327.92 & 0.0065 & 543.50 & 0.1691 & 609.68 & 0.3493 \\
\hline 340.30 & 0.0079 & 554.33 & 0.1886 & 615.00 & 0.3761 \\
\hline 379.05 & 0.0170 & 556.39 & 0.1930 & 615.87 & 0.3806 \\
\hline 393.50 & 0.0230 & 557.77 & 0.1951 & 616.30 & 0.3828 \\
\hline 401.83 & 0.0272 & 564.88 & 0.2104 & 619.10 & 0.4007 \\
\hline 425.91 & 0.0418 & 567.95 & 0.2169 & 621.06 & 0.4141 \\
\hline 429.22 & 0.0439 & 574.55 & 0.2326 & 621.50 & 0.4185 \\
\hline 439.73 & 0.0515 & 576.44 & 0.2370 & 624.75 & 0.4452 \\
\hline 458.10 & 0.0665 & 579.07 & 0.2436 & 628.10 & 0.4976 \\
\hline 462.98 & 0.0708 & 585.41 & 0.2610 & & \\
\hline
\end{tabular}


Table 197. Test data for specimen 68-139

$\begin{array}{llll}\text { Test Number } & : 193 & \text { Test Temp. } & : 25^{\circ} \mathrm{C} \\ \text { Aging Temp. } & : 350^{\circ} \mathrm{C} & \text { Aging Time } & : 30,000 \mathrm{~h} \\ \text { Yield Stress } & : 287.4 \mathrm{MPa} & \text { Ultimate Stress } & : 639.0 \mathrm{MPa}\end{array}$

\begin{tabular}{rccccc}
\hline $\begin{array}{c}\text { Engr. } \\
\text { Stress } \\
(\mathrm{MPa})\end{array}$ & $\begin{array}{c}\text { Engr. } \\
\text { Strain }\end{array}$ & $\begin{array}{c}\text { Engr. } \\
\text { Stress } \\
(\mathrm{MPa})\end{array}$ & $\begin{array}{c}\text { Engr. } \\
\text { Strain }\end{array}$ & $\begin{array}{c}\text { Engr. } \\
\text { Stress } \\
(\mathrm{MPa})\end{array}$ & $\begin{array}{c}\text { Engr. } \\
\text { Strain }\end{array}$ \\
\hline & & & & & \\
14.50 & 0.0001 & 430.18 & 0.0356 & 590.30 & 0.1636 \\
79.76 & 0.0006 & 434.81 & 0.0378 & 598.00 & 0.1749 \\
163.03 & 0.0011 & 460.58 & 0.0515 & 601.84 & 0.1813 \\
198.39 & 0.0014 & 468.51 & 0.0561 & 607.28 & 0.1913 \\
255.48 & 0.0025 & 490.56 & 0.0697 & 610.59 & 0.1974 \\
270.00 & 0.0030 & 500.67 & 0.0766 & 619.13 & 0.2155 \\
283.13 & 0.0036 & 519.54 & 0.0903 & 621.46 & 0.2216 \\
319.14 & 0.0058 & 539.11 & 0.1063 & 626.71 & 0.2354 \\
326.46 & 0.0064 & 544.20 & 0.1109 & 628.58 & 0.2415 \\
339.28 & 0.0078 & 557.24 & 0.1238 & 629.28 & 0.2435 \\
372.47 & 0.0138 & 561.93 & 0.1284 & 633.18 & 0.2579 \\
378.08 & 0.0153 & 563.95 & 0.1308 & 633.52 & 0.2599 \\
383.14 & 0.0168 & 565.68 & 0.1327 & 633.89 & 0.2620 \\
389.09 & 0.0187 & 575.40 & 0.1440 & 635.40 & 0.2684 \\
394.44 & 0.0205 & 578.95 & 0.1483 & 635.76 & 0.2704 \\
425.35 & 0.0333 & 587.76 & 0.1596 & 638.85 & 0.2963 \\
\hline
\end{tabular}

Table 198. Test data for specimen 682-16

\begin{tabular}{|c|c|c|c|c|c|}
\hline $\begin{array}{l}\text { Test Number } \\
\text { Aging Temp. } \\
\text { Yield Stress }\end{array}$ & $\begin{array}{l}: M \\
: 4 C \\
: 25\end{array}$ & $\begin{array}{l}\text { est } \\
\mathrm{MPa}\end{array}$ & $\begin{array}{l}\text { Tes } \\
\text { Agi } \\
\text { Ulti }\end{array}$ & $\begin{array}{l}\text { np. } \\
\text { ne } \\
\text { Stress }\end{array}$ & $\begin{array}{l}25^{\circ} \mathrm{C} \\
2,570 \mathrm{~h} \\
657.8 \mathrm{MPa}\end{array}$ \\
\hline $\begin{array}{l}\text { Engr. } \\
\text { Stress } \\
\text { (MPa) }\end{array}$ & $\begin{array}{l}\text { Engr. } \\
\text { Strain }\end{array}$ & $\begin{array}{l}\text { Engr. } \\
\text { Stress } \\
\text { (MPa) }\end{array}$ & $\begin{array}{l}\text { Engr. } \\
\text { Strain }\end{array}$ & $\begin{array}{l}\text { Engr. } \\
\text { Stress } \\
(\mathrm{MPa})\end{array}$ & $\begin{array}{l}\text { Engr. } \\
\text { Strain }\end{array}$ \\
\hline 19.10 & 0.0002 & 323.71 & 0.0054 & 601.98 & 0.2667 \\
\hline 21.24 & 0.0002 & 331.16 & 0.0063 & 611.36 & 0.2940 \\
\hline 38.61 & 0.0002 & 342.95 & 0.0072 & 617.01 & 0.3216 \\
\hline 57.09 & 0.0003 & 350.46 & 0.0081 & 624.87 & 0.3493 \\
\hline 79.36 & 0.0004 & 407.27 & 0.0300 & 631.35 & 0.3774 \\
\hline 105.08 & 0.0005 & 441.06 & 0.0529 & 635.42 & 0.4055 \\
\hline 130.66 & 0.0007 & 468.71 & 0.0749 & 639.08 & 0.4341 \\
\hline 158.72 & 0.0008 & 495.53 & 0.0966 & 643.14 & 0.4627 \\
\hline 191.54 & 0.0010 & 519.59 & 0.1185 & 646.04 & 0.4916 \\
\hline 219.18 & 0.0012 & 537.72 & 0.1408 & 649.28 & 0.5206 \\
\hline 242.01 & 0.0017 & 555.17 & 0.1640 & 651.76 & 0.5497 \\
\hline 261.66 & 0.0022 & 570.75 & 0.1881 & 652.73 & 0.5789 \\
\hline 280.34 & 0.0029 & 584.74 & 0.2136 & 653.97 & 0.6084 \\
\hline 295.65 & 0.0036 & 595.78 & 0.2397 & 654.86 & 0.6382 \\
\hline 310.82 & 0.0045 & & & & \\
\hline
\end{tabular}


Table 199. Test data for specimen 682-17

\begin{tabular}{cccccc}
$\begin{array}{c}\text { Test Number } \\
\text { Aging Temp. } \\
\text { Yield Stress }\end{array}$ & \multicolumn{2}{c}{$\begin{array}{c}\text { : MEA Test } \\
\text { : } 280^{\circ} \mathrm{C}\end{array}$} & \multicolumn{2}{c}{$\begin{array}{c}\text { Test Temp. } \\
\text { Aging Time } \\
\text { Ultimate Stress }\end{array}$} & $\begin{array}{l}: 25^{\circ} \mathrm{C} \\
: 2,570 \mathrm{~h} \\
: 629.5 \mathrm{MPa}\end{array}$ \\
\hline $\begin{array}{c}\text { Engr. } \\
\text { Stress }\end{array}$ & $\begin{array}{c}\text { Engr. } \\
\text { Strain }\end{array}$ & $\begin{array}{c}\text { Engr. } \\
\text { Stress } \\
\text { (MPa) }\end{array}$ & $\begin{array}{c}\text { Engr. } \\
\text { Strain }\end{array}$ & $\begin{array}{c}\text { Engr. } \\
\text { Stress } \\
(\mathrm{MPa})\end{array}$ & $\begin{array}{c}\text { Engr. } \\
\text { Strain }\end{array}$ \\
\hline 39.16 & 0.0002 & 322.88 & 0.0075 & 570.47 & 0.2032 \\
60.54 & 0.0002 & 326.81 & 0.0085 & 577.78 & 0.2165 \\
79.15 & 0.0003 & 358.87 & 0.0204 & 586.61 & 0.2300 \\
102.66 & 0.0004 & 381.21 & 0.0337 & 592.05 & 0.2434 \\
126.86 & 0.0005 & 401.07 & 0.0468 & 598.95 & 0.2570 \\
154.10 & 0.0006 & 420.79 & 0.0599 & 603.15 & 0.2705 \\
180.09 & 0.0007 & 438.51 & 0.0727 & 607.50 & 0.2839 \\
206.91 & 0.0009 & 455.95 & 0.0856 & 614.46 & 0.2973 \\
234.63 & 0.0012 & 472.70 & 0.0985 & 615.70 & 0.3105 \\
257.04 & 0.0016 & 487.39 & 0.1114 & 618.11 & 0.3233 \\
272.76 & 0.0022 & 502.97 & 0.1244 & 622.67 & 0.3361 \\
285.58 & 0.0029 & 516.90 & 0.1374 & 623.42 & 0.3482 \\
296.13 & 0.0037 & 528.07 & 0.1505 & 625.97 & 0.3602 \\
305.58 & 0.0046 & 540.20 & 0.1635 & 626.04 & 0.3717 \\
312.06 & 0.0055 & 551.44 & 0.1767 & 628.25 & 0.3824 \\
318.54 & 0.0065 & 561.51 & 0.1899 & & \\
\hline
\end{tabular}

Table 200. Test data for specimen 682-15

\begin{tabular}{|c|c|c|c|c|c|}
\hline $\begin{array}{l}\text { Test Number } \\
\text { Aging Temp. } \\
\text { Yield Stress }\end{array}$ & $\begin{array}{l}: 1 \\
: 40 \\
: 31\end{array}$ & & $\begin{array}{l}\text { Tes } \\
\text { Agi } \\
\text { Ulti }\end{array}$ & $\begin{array}{l}\text { mp. } \\
\text { me } \\
\text { Stress }\end{array}$ & $\begin{array}{l}: 25^{\circ} \mathrm{C} \\
: 10,000 \mathrm{~h} \\
: 641.0 \mathrm{MPa}\end{array}$ \\
\hline $\begin{array}{l}\text { Engr. } \\
\text { Stress } \\
(\mathrm{MPa}) \\
\end{array}$ & $\begin{array}{l}\text { Engr. } \\
\text { Strain }\end{array}$ & $\begin{array}{l}\text { Engr. } \\
\text { Stress } \\
(\mathrm{MPa}) \\
\end{array}$ & $\begin{array}{l}\text { Engr. } \\
\text { Strain }\end{array}$ & $\begin{array}{l}\text { Engr. } \\
\text { Stress } \\
(\mathrm{MPa}) \\
\end{array}$ & $\begin{array}{l}\text { Engr. } \\
\text { Strain }\end{array}$ \\
\hline $\begin{array}{r}48.61 \\
194.81 \\
229.86 \\
307.85 \\
354.53 \\
385.15 \\
404.35\end{array}$ & $\begin{array}{l}0.0003 \\
0.0025 \\
0.0032 \\
0.0052 \\
0.0084 \\
0.0131 \\
0.0184\end{array}$ & $\begin{array}{l}426.81 \\
450.59 \\
475.06 \\
479.75 \\
485.66 \\
508.43 \\
545.28\end{array}$ & $\begin{array}{l}0.0265 \\
0.0376 \\
0.0526 \\
0.0561 \\
0.0635 \\
0.0787 \\
0.1038\end{array}$ & $\begin{array}{l}545.48 \\
580.02 \\
600.75 \\
623.67 \\
629.45 \\
638.11 \\
640.98\end{array}$ & $\begin{array}{l}0.1063 \\
0.1369 \\
0.1625 \\
0.2002 \\
0.2148 \\
0.2498 \\
0.2785\end{array}$ \\
\hline
\end{tabular}


Table 201. Test data for specimen 682-22

\begin{tabular}{|c|c|c|c|c|c|}
\hline $\begin{array}{l}\text { Test Number } \\
\text { Aging Temp. } \\
\text { Yield Stress }\end{array}$ & $\begin{array}{l}: 1 \\
: 40 \\
: 25\end{array}$ & $\mathrm{~Pa}$ & $\begin{array}{l}\text { Tes } \\
\text { Agi } \\
\text { Ulti }\end{array}$ & $\begin{array}{l}\text { ne. } \\
\text { Stress }\end{array}$ & $\begin{array}{l}25^{\circ} \mathrm{C} \\
10,000 \mathrm{~h} \\
650.4 \mathrm{MPa}\end{array}$ \\
\hline $\begin{array}{l}\text { Engr. } \\
\text { Stress } \\
(\mathrm{MPa}) \\
\end{array}$ & $\begin{array}{l}\text { Engr. } \\
\text { Strain }\end{array}$ & $\begin{array}{l}\text { Engr. } \\
\text { Stress } \\
(\mathrm{MPa}) \\
\end{array}$ & $\begin{array}{l}\text { Engr. } \\
\text { Strain }\end{array}$ & $\begin{array}{l}\text { Engr. } \\
\text { Stress } \\
(\mathrm{MPa}) \\
\end{array}$ & $\begin{array}{l}\text { Engr. } \\
\text { Strain }\end{array}$ \\
\hline $\begin{array}{r}33.34 \\
204.82 \\
218.93 \\
285.41 \\
322.45 \\
348.30 \\
372.86\end{array}$ & $\begin{array}{l}0.0000 \\
0.0018 \\
0.0021 \\
0.0040 \\
0.0058 \\
0.0084 \\
0.0131\end{array}$ & $\begin{array}{l}390.58 \\
401.82 \\
422.99 \\
449.59 \\
470.96 \\
483.35 \\
526.76\end{array}$ & $\begin{array}{l}0.0178 \\
0.0220 \\
0.0333 \\
0.0501 \\
0.0666 \\
0.0783 \\
0.1082\end{array}$ & $\begin{array}{l}559.42 \\
591.94 \\
614.57 \\
614.27 \\
636.06 \\
647.26 \\
650.39\end{array}$ & $\begin{array}{l}0.1414 \\
0.1803 \\
0.2202 \\
0.2202 \\
0.2756 \\
0.3307 \\
0.3705\end{array}$ \\
\hline
\end{tabular}

Table 202. Test data for specimen 682-23

\begin{tabular}{|c|c|c|c|c|c|}
\hline $\begin{array}{l}\text { Test Number } \\
\text { Aging Temp. } \\
\text { Yield Stress }\end{array}$ & $\begin{array}{l}: 2 \\
: 4 c \\
: 30\end{array}$ & & $\begin{array}{l}\text { Tes } \\
\text { Agir } \\
\text { Ulti }\end{array}$ & $\begin{array}{l}\text { mp. } \\
\text { me } \\
\text { Stress }\end{array}$ & $\begin{array}{l}25^{\circ} \mathrm{C} \\
10,000 \mathrm{~h} \\
657.4 \mathrm{MPa}\end{array}$ \\
\hline $\begin{array}{l}\text { Engr. } \\
\text { Stress } \\
(\mathrm{MPa})\end{array}$ & $\begin{array}{l}\text { Engr. } \\
\text { Strain }\end{array}$ & $\begin{array}{l}\text { Engr. } \\
\text { Stress } \\
(\mathrm{MPa})\end{array}$ & $\begin{array}{l}\text { Engr. } \\
\text { Strain }\end{array}$ & $\begin{array}{l}\text { Engr. } \\
\text { Stress } \\
(\mathrm{MPa}) \\
\end{array}$ & $\begin{array}{l}\text { Engr. } \\
\text { Strain }\end{array}$ \\
\hline 17.77 & 0.0001 & 461.40 & 0.0430 & 601.42 & 0.1524 \\
\hline 90.70 & 0.0004 & 469.39 & 0.0475 & 608.18 & 0.1611 \\
\hline 189.83 & 0.0008 & 488.52 & 0.0585 & 617.09 & 0.1742 \\
\hline 256.81 & 0.0017 & 492.15 & 0.0608 & 624.19 & 0.1852 \\
\hline 265.69 & 0.0019 & 509.41 & 0.0718 & 630.09 & 0.1962 \\
\hline 273.92 & 0.0022 & 525.21 & 0.0828 & 631.33 & 0.1984 \\
\hline 289.49 & 0.0027 & 542.89 & 0.0959 & 635.60 & 0.2072 \\
\hline 321.44 & 0.0041 & 556.44 & 0.1068 & 640.22 & 0.2182 \\
\hline 340.09 & 0.0054 & 559.13 & 0.1090 & 641.05 & 0.2204 \\
\hline 347.25 & 0.0060 & 568.84 & 0.1177 & 645.29 & 0.2314 \\
\hline 381.41 & 0.0111 & 571.11 & 0.1199 & 647.48 & 0.2382 \\
\hline 387.43 & 0.0125 & 573.48 & 0.1221 & 650.64 & 0.2494 \\
\hline 392.67 & 0.0140 & 584.31 & 0.1329 & 652.99 & 0.2584 \\
\hline 398.48 & 0.0158 & 586.42 & 0.1351 & 654.91 & 0.2693 \\
\hline 425.12 & 0.0256 & 588.40 & 0.1372 & 655.36 & 0.2715 \\
\hline 434.90 & 0.0299 & 597.82 & 0.1481 & 656.58 & 0.2806 \\
\hline 444.28 & 0.0343 & 599.62 & 0.1502 & 657.39 & 0.2942 \\
\hline
\end{tabular}


Table 203. Test data for specimen 68-119

\begin{tabular}{|c|c|c|c|c|c|}
\hline $\begin{array}{l}\text { Test Number } \\
\text { Aging Temp. } \\
\text { Yield Stress }\end{array}$ & $\begin{array}{l}: 1 \\
: 40 \\
: 30\end{array}$ & & $\begin{array}{l}\text { Tes } \\
\text { Agi } \\
\text { Ulti }\end{array}$ & $\begin{array}{l}\text { mp. } \\
\text { me } \\
\text { Stress }\end{array}$ & $\begin{array}{l}25^{\circ} \mathrm{C} \\
10,000 \mathrm{~h} \\
624.3 \mathrm{MPa}\end{array}$ \\
\hline $\begin{array}{l}\text { Engr. } \\
\text { Stress } \\
\text { (MPa) }\end{array}$ & $\begin{array}{l}\text { Engr. } \\
\text { Strain }\end{array}$ & $\begin{array}{l}\text { Engr. } \\
\text { Stress } \\
(\mathrm{MPa})\end{array}$ & $\begin{array}{l}\text { Engr. } \\
\text { Strain }\end{array}$ & $\begin{array}{l}\text { Engr. } \\
\text { Stress } \\
\text { (MPa) }\end{array}$ & $\begin{array}{l}\text { Engr. } \\
\text { Strain }\end{array}$ \\
\hline $\begin{array}{r}0.00 \\
195.52 \\
240.17 \\
386.78 \\
389.99 \\
392.85 \\
393.10\end{array}$ & $\begin{array}{l}0.0000 \\
0.0016 \\
0.0021 \\
0.0049 \\
0.0052 \\
0.0064 \\
0.0066\end{array}$ & $\begin{array}{l}422.97 \\
443.47 \\
473.25 \\
479.42 \\
486.10 \\
536.68 \\
560.09\end{array}$ & $\begin{array}{l}0.0206 \\
0.0336 \\
0.0576 \\
0.0625 \\
0.0718 \\
0.1179 \\
0.1484\end{array}$ & $\begin{array}{l}587.81 \\
604.31 \\
617.67 \\
622.63 \\
624.26 \\
615.88\end{array}$ & $\begin{array}{l}0.1922 \\
0.2298 \\
0.2781 \\
0.3245 \\
0.3521 \\
0.3758\end{array}$ \\
\hline
\end{tabular}

Table 204. Test data for specimen 681-04

\begin{tabular}{|c|c|c|c|c|c|}
\hline $\begin{array}{l}\text { Test Number } \\
\text { Aging Temp. } \\
\text { Yield Stress }\end{array}$ & $\begin{array}{l}: M \\
: 45 \\
: 29\end{array}$ & & $\begin{array}{l}\text { Tes } \\
\text { Agi } \\
\text { Ulti }\end{array}$ & $\begin{array}{l}\mathrm{np} . \\
\text { ne } \\
\text { Stress }\end{array}$ & $\begin{array}{l}25^{\circ} \mathrm{C} \\
2,570 \mathrm{~h} \\
653.1 \mathrm{MPa}\end{array}$ \\
\hline $\begin{array}{l}\text { Engr. } \\
\text { Stress } \\
(\mathrm{MPa})\end{array}$ & $\begin{array}{l}\text { Engr. } \\
\text { Strain }\end{array}$ & $\begin{array}{l}\text { Engr. } \\
\text { Stress } \\
(\mathrm{MPa})\end{array}$ & $\begin{array}{l}\text { Engr. } \\
\text { Strain }\end{array}$ & $\begin{array}{l}\text { Engr. } \\
\text { Stress } \\
(\mathrm{MPa}) \\
\end{array}$ & $\begin{array}{l}\text { Engr. } \\
\text { Strain }\end{array}$ \\
\hline 18.00 & 0.0000 & 338.67 & 0.0064 & 593.22 & 0.1786 \\
\hline 45.57 & 0.0003 & 348.32 & 0.0072 & 599.09 & 0.1921 \\
\hline 65.85 & 0.0004 & 356.80 & 0.0079 & 606.88 & 0.2058 \\
\hline 86.53 & 0.0005 & 401.55 & 0.0172 & 612.94 & 0.2193 \\
\hline 110.52 & 0.0007 & 430.30 & 0.0287 & 617.98 & 0.2332 \\
\hline 137.41 & 0.0009 & 454.50 & 0.0405 & 623.22 & 0.2472 \\
\hline 163.75 & 0.0011 & 475.32 & 0.0526 & 626.94 & 0.2612 \\
\hline 190.50 & 0.0014 & 493.25 & 0.0646 & 632.46 & 0.2753 \\
\hline 214.84 & 0.0017 & 508.01 & 0.0768 & 634.80 & 0.2896 \\
\hline 237.25 & 0.0022 & 521.80 & 0.0889 & 638.32 & 0.3040 \\
\hline 256.62 & 0.0027 & 534.83 & 0.1012 & 641.28 & 0.3185 \\
\hline 274.14 & 0.0032 & 548.27 & 0.1137 & 642.45 & 0.3331 \\
\hline 291.30 & 0.0038 & 559.30 & 0.1263 & 645.14 & 0.3477 \\
\hline 306.61 & 0.0044 & 565.99 & 0.1392 & 646.66 & 0.3626 \\
\hline 317.16 & 0.0051 & 577.99 & 0.1521 & 648.87 & 0.3777 \\
\hline
\end{tabular}


Table 205. Test data for specimen 681-05

$\begin{array}{llll}\text { Test Number } & : \text { MEA Test } & \text { Test Temp. } & : 25^{\circ} \mathrm{C} \\ \text { Aging Temp. } & : 450^{\circ} \mathrm{C} & \text { Aging Time } & : 2,570 \mathrm{~h} \\ \text { Yield Stress } & : 309.4 \mathrm{MPa} & \text { Ultimate Stress } & : 632.9 \mathrm{MPa}\end{array}$

\begin{tabular}{rccccc}
\hline $\begin{array}{c}\text { Engr. } \\
\text { Stress } \\
(\mathrm{MPa})\end{array}$ & $\begin{array}{c}\text { Engr. } \\
\text { Strain }\end{array}$ & $\begin{array}{c}\text { Engr. } \\
\text { Stress } \\
(\mathrm{MPa})\end{array}$ & $\begin{array}{c}\text { Engr. } \\
\text { Strain }\end{array}$ & $\begin{array}{c}\text { Engr. } \\
\text { Stress } \\
(\mathrm{MPa})\end{array}$ & $\begin{array}{c}\text { Engr. } \\
\text { Strain }\end{array}$ \\
\hline 61.16 & 0.0003 & 351.08 & 0.0071 & 554.41 & 0.1045 \\
86.60 & 0.0004 & 380.66 & 0.0122 & 562.82 & 0.1108 \\
114.94 & 0.0005 & 405.41 & 0.0187 & 570.06 & 0.1172 \\
142.79 & 0.0007 & 422.30 & 0.0254 & 576.95 & 0.1236 \\
172.16 & 0.0008 & 437.89 & 0.0323 & 583.57 & 0.1299 \\
200.29 & 0.0010 & 453.47 & 0.0391 & 589.36 & 0.1363 \\
225.80 & 0.0012 & 466.64 & 0.0459 & 596.47 & 0.1428 \\
247.66 & 0.0015 & 477.19 & 0.0526 & 600.95 & 0.1493 \\
267.72 & 0.0020 & 489.11 & 0.0592 & 605.64 & 0.1559 \\
283.10 & 0.0024 & 501.39 & 0.0657 & 611.84 & 0.1626 \\
298.06 & 0.0029 & 510.07 & 0.0723 & 614.94 & 0.1692 \\
309.51 & 0.0035 & 520.35 & 0.0788 & 619.49 & 0.1759 \\
320.61 & 0.0042 & 530.41 & 0.0852 & 623.29 & 0.1827 \\
337.43 & 0.0056 & 537.93 & 0.0916 & 627.70 & 0.1895 \\
348.12 & 0.0064 & 547.86 & 0.0980 & 628.59 & 0.1964 \\
\hline
\end{tabular}

Table 206. Test data for specimen 683-42

\begin{tabular}{|c|c|c|c|c|c|}
\hline $\begin{array}{l}\text { Test Number } \\
\text { Aging Temp. } \\
\text { Yield Stress }\end{array}$ & $\begin{array}{l}: M \\
: U 1 \\
: 16\end{array}$ & $\begin{array}{l}\text { est } \\
\text { d } \\
\text { MPa }\end{array}$ & $\begin{array}{l}\text { Tes } \\
\text { Agi } \\
\text { Ulti }\end{array}$ & $\begin{array}{l}\text { np. } \\
\text { ne } \\
\text { Stress }\end{array}$ & $\begin{array}{l}290^{\circ} \mathrm{C} \\
- \\
397.3 \mathrm{MPa}\end{array}$ \\
\hline $\begin{array}{l}\text { Engr. } \\
\text { Stress } \\
(\mathrm{MPa}) \\
\end{array}$ & $\begin{array}{l}\text { Engr. } \\
\text { Strain }\end{array}$ & $\begin{array}{l}\text { Engr. } \\
\text { Stress } \\
\text { (MPa) }\end{array}$ & $\begin{array}{l}\text { Engr. } \\
\text { Strain }\end{array}$ & $\begin{array}{l}\text { Engr. } \\
\text { Stress } \\
(\mathrm{MPa})\end{array}$ & $\begin{array}{l}\text { Engr. } \\
\text { Strain }\end{array}$ \\
\hline 66.74 & 0.0005 & 204.43 & 0.0170 & 341.29 & 0.1304 \\
\hline 95.91 & 0.0007 & 206.91 & 0.0184 & 348.12 & 0.1387 \\
\hline 121.28 & 0.0011 & 217.87 & 0.0268 & 355.36 & 0.1485 \\
\hline 141.20 & 0.0017 & 230.77 & 0.0362 & 362.39 & 0.1574 \\
\hline 155.20 & 0.0025 & 243.04 & 0.0452 & 365.28 & 0.1656 \\
\hline 165.61 & 0.0034 & 254.21 & 0.0541 & 370.87 & 0.1751 \\
\hline 174.16 & 0.0045 & 266.96 & 0.0628 & 374.32 & 0.1846 \\
\hline 179.82 & 0.0057 & 277.72 & 0.0716 & 380.45 & 0.1919 \\
\hline 183.88 & 0.0069 & 288.75 & 0.0799 & 385.42 & 0.2031 \\
\hline 187.40 & 0.0083 & 297.03 & 0.0883 & 389.55 & 0.2146 \\
\hline 191.81 & 0.0097 & 307.51 & 0.0968 & 389.21 & 0.2235 \\
\hline 192.36 & 0.0110 & 316.33 & 0.1056 & 394.04 & 0.2372 \\
\hline 195.88 & 0.0125 & 324.67 & 0.1137 & 394.93 & 0.2498 \\
\hline 197.40 & 0.0139 & 332.81 & 0.1218 & 394.93 & 0.2639 \\
\hline 200.22 & 0.0154 & & & & \\
\hline
\end{tabular}


Table 207. Test data for specimen 684-40

\begin{tabular}{|c|c|c|c|c|c|}
\hline $\begin{array}{l}\text { Test Number } \\
\text { Aging Temp. } \\
\text { Yield Stress }\end{array}$ & $\begin{array}{l}: M \\
: U 1 \\
: 15\end{array}$ & & $\begin{array}{l}\text { Tes } \\
\text { Agi } \\
\text { Ulti }\end{array}$ & $\begin{array}{l}\text { np. } \\
\text { ne } \\
\text { Stress }\end{array}$ & $\begin{array}{l}290^{\circ} \mathrm{C} \\
- \\
412.3 \mathrm{MPa}\end{array}$ \\
\hline $\begin{array}{l}\text { Engr. } \\
\text { Stress } \\
(\mathrm{MPa})\end{array}$ & $\begin{array}{l}\text { Engr. } \\
\text { Strain }\end{array}$ & $\begin{array}{l}\text { Engr. } \\
\text { Stress } \\
(\mathrm{MPa})\end{array}$ & $\begin{array}{l}\text { Engr. } \\
\text { Strain }\end{array}$ & $\begin{array}{l}\text { Engr. } \\
\text { Stress } \\
(\mathrm{MPa}) \\
\end{array}$ & $\begin{array}{l}\text { Engr. } \\
\text { Strain }\end{array}$ \\
\hline 43.02 & 0.0003 & 190.57 & 0.0142 & 336.12 & 0.1472 \\
\hline 64.33 & 0.0005 & 192.29 & 0.0156 & 343.15 & 0.1580 \\
\hline 86.32 & 0.0006 & 193.81 & 0.0169 & 353.22 & 0.1695 \\
\hline 105.15 & 0.0008 & 210.01 & 0.0273 & 359.91 & 0.1806 \\
\hline 123.28 & 0.0013 & 226.77 & 0.0386 & 367.42 & 0.1917 \\
\hline 137.96 & 0.0019 & 238.63 & 0.0497 & 372.73 & 0.2029 \\
\hline 150.44 & 0.0027 & 250.97 & 0.0608 & 379.28 & 0.2128 \\
\hline 158.86 & 0.0037 & 264.83 & 0.0718 & 383.56 & 0.2248 \\
\hline 165.89 & 0.0048 & 275.58 & 0.0831 & 390.17 & 0.2380 \\
\hline 172.37 & 0.0060 & 287.51 & 0.0942 & 395.83 & 0.2510 \\
\hline 175.61 & 0.0074 & 296.27 & 0.1044 & 397.90 & 0.2655 \\
\hline 177.88 & 0.0087 & 308.68 & 0.1151 & 402.45 & 0.2749 \\
\hline 181.88 & 0.0101 & 316.68 & 0.1264 & 403.34 & 0.2882 \\
\hline 186.16 & 0.0115 & 328.26 & 0.1382 & 410.10 & 0.3068 \\
\hline 188.23 & 0.0128 & & & & \\
\hline
\end{tabular}

Table 208. Test data for specimen 684-32

\begin{tabular}{|c|c|c|c|c|c|}
\hline $\begin{array}{l}\text { Test Number } \\
\text { Aging Temp. } \\
\text { Yield Stress }\end{array}$ & $\begin{array}{l}: 25 \\
: 29 \\
: 14\end{array}$ & & $\begin{array}{l}\text { Tes } \\
\text { Agir } \\
\text { Ulti }\end{array}$ & $\begin{array}{l}\text { np. } \\
\text { ne } \\
\text { Stress }\end{array}$ & $\begin{array}{l}290^{\circ} \mathrm{C} \\
30,000 \mathrm{~h} \\
333.2 \mathrm{MPa}\end{array}$ \\
\hline $\begin{array}{l}\text { Engr. } \\
\text { Stress } \\
\text { (MPa) }\end{array}$ & $\begin{array}{l}\text { Engr. } \\
\text { Strain }\end{array}$ & $\begin{array}{l}\text { Engr. } \\
\text { Stress } \\
(\mathrm{MPa})\end{array}$ & $\begin{array}{l}\text { Engr. } \\
\text { Strain }\end{array}$ & $\begin{array}{l}\text { Engr. } \\
\text { Stress } \\
(\mathrm{MPa}) \\
\end{array}$ & $\begin{array}{l}\text { Engr. } \\
\text { Strain }\end{array}$ \\
\hline 38.035 & 0.0002 & 189.629 & 0.0321 & 272.081 & 0.1269 \\
\hline 40.376 & 0.0002 & 202.477 & 0.0452 & 281.043 & 0.1400 \\
\hline 55.969 & 0.0001 & 208.233 & 0.0515 & 286.391 & 0.1465 \\
\hline 65.557 & 0.0002 & 210.259 & 0.0535 & 288.174 & 0.1488 \\
\hline 105.987 & 0.0005 & 225.677 & 0.0709 & 299.143 & 0.1662 \\
\hline 115.819 & 0.0006 & 227.751 & 0.0731 & 302.679 & 0.1705 \\
\hline 123.596 & 0.0007 & 233.046 & 0.0794 & 314.764 & 0.1903 \\
\hline 147.480 & 0.0023 & 245.258 & 0.0938 & 318.349 & 0.2002 \\
\hline 152.702 & 0.0035 & 250.596 & 0.1005 & 325.917 & 0.2151 \\
\hline 164.710 & 0.0099 & 265.256 & 0.1182 & 326.063 & 0.2174 \\
\hline 180.375 & 0.0231 & 267.287 & 0.1204 & 333.170 & 0.2438 \\
\hline
\end{tabular}


Table 209. Test data for specimen 684-33

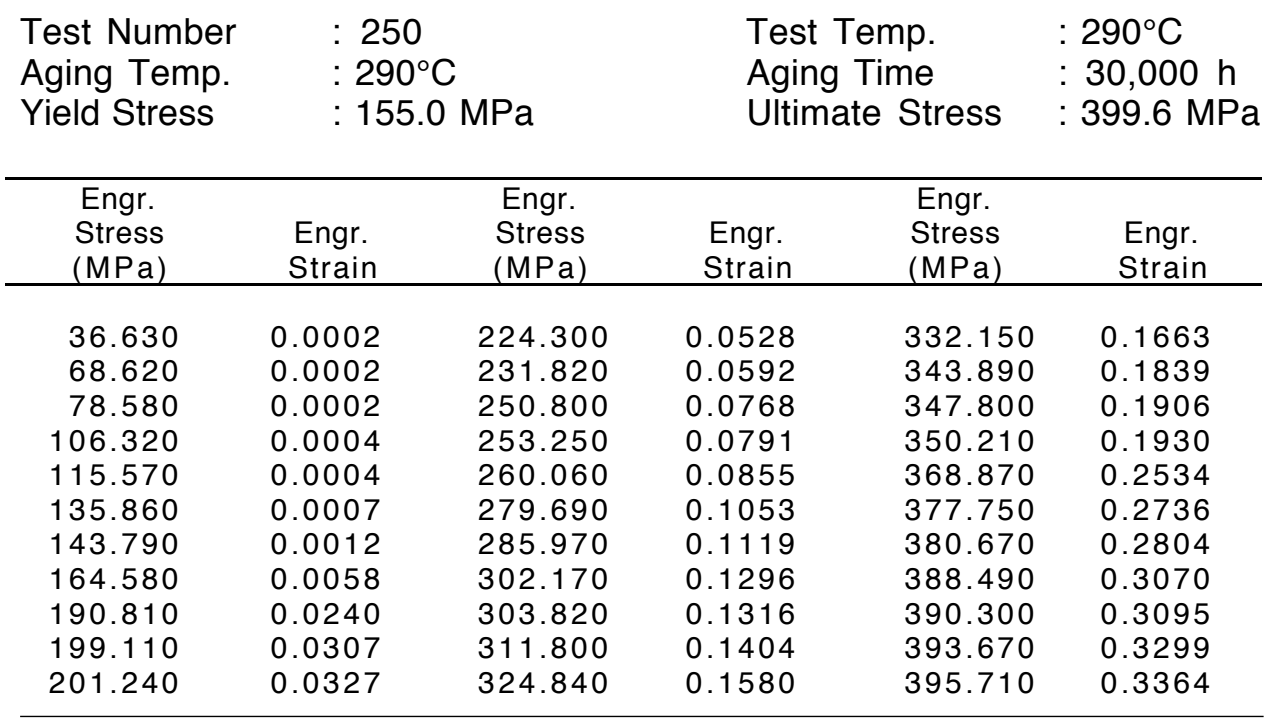

Table 210. Test data for specimen 68-246

\begin{tabular}{|c|c|c|c|c|c|}
\hline $\begin{array}{l}\text { Test Number } \\
\text { Aging Temp. } \\
\text { Yield Stress }\end{array}$ & $\begin{array}{l}: 2 \\
: 2 \\
: 17\end{array}$ & $\mathrm{MPa}$ & $\begin{array}{l}\text { Tes } \\
\text { Agi } \\
\text { Ulti }\end{array}$ & $\begin{array}{l}\text { np. } \\
\text { me } \\
\text { Stress }\end{array}$ & $\begin{array}{l}290^{\circ} \mathrm{C} \\
30,000 \mathrm{~h} \\
390.7 \mathrm{MPa}\end{array}$ \\
\hline $\begin{array}{l}\text { Engr. } \\
\text { Stress } \\
(\mathrm{MPa})\end{array}$ & $\begin{array}{l}\text { Engr. } \\
\text { Strain }\end{array}$ & $\begin{array}{l}\text { Engr. } \\
\text { Stress } \\
(\mathrm{MPa})\end{array}$ & $\begin{array}{l}\text { Engr. } \\
\text { Strain }\end{array}$ & $\begin{array}{l}\text { Engr. } \\
\text { Stress } \\
(\mathrm{MPa}) \\
\end{array}$ & $\begin{array}{l}\text { Engr. } \\
\text { Strain }\end{array}$ \\
\hline 41.940 & 0.0002 & 209.810 & 0.0183 & 326.070 & 0.1042 \\
\hline 64.890 & 0.0002 & 235.910 & 0.0344 & 342.730 & 0.1217 \\
\hline 75.350 & 0.0003 & 245.360 & 0.0407 & 348.040 & 0.1283 \\
\hline 117.050 & 0.0004 & 248.310 & 0.0430 & 363.210 & 0.1487 \\
\hline 126.570 & 0.0005 & 267.370 & 0.0561 & 364.310 & 0.1508 \\
\hline 134.690 & 0.0006 & 270.460 & 0.0583 & 369.340 & 0.1576 \\
\hline 161.000 & 0.0016 & 273.410 & 0.0605 & 376.300 & 0.1708 \\
\hline 166.350 & 0.0022 & 290.630 & 0.0734 & 380.330 & 0.1794 \\
\hline 172.140 & 0.0029 & 293.360 & 0.0755 & 387.400 & 0.1999 \\
\hline 201.510 & 0.0138 & 296.050 & 0.0777 & 388.030 & 0.2019 \\
\hline 205.820 & 0.0160 & 316.710 & 0.0954 & 390.650 & 0.2221 \\
\hline
\end{tabular}


Table 211. Test data for specimen 684-23

$\begin{array}{llll}\text { Test Number } & : 091 & \text { Test Temp. } & : 290^{\circ} \mathrm{C} \\ \text { Aging Temp. } & : 320^{\circ} \mathrm{C} & \text { Aging Time } & : 10,000 \mathrm{~h} \\ \text { Yield Stress } & : 173.4 \mathrm{MPa} & \text { Ultimate Stress } & : 416.6 \mathrm{MPa}\end{array}$

\begin{tabular}{cccccc}
\hline $\begin{array}{c}\text { Engr. } \\
\text { Stress } \\
(\mathrm{MPa})\end{array}$ & $\begin{array}{c}\text { Engr. } \\
\text { Strain }\end{array}$ & $\begin{array}{c}\text { Engr. } \\
\text { Stress } \\
(\mathrm{MPa})\end{array}$ & $\begin{array}{c}\text { Engr. } \\
\text { Strain }\end{array}$ & $\begin{array}{c}\text { Engr. } \\
\text { Stress } \\
(\mathrm{MPa})\end{array}$ & $\begin{array}{c}\text { Engr. } \\
\text { Strain }\end{array}$ \\
\hline & & & & & \\
137.03 & 0.0009 & 260.07 & 0.0280 & 374.87 & 0.1304 \\
166.79 & 0.0017 & 269.42 & 0.0354 & 380.58 & 0.1373 \\
175.09 & 0.0022 & 275.07 & 0.0400 & 383.43 & 0.1413 \\
186.21 & 0.0035 & 283.63 & 0.0476 & 390.10 & 0.1497 \\
191.45 & 0.0045 & 291.32 & 0.0545 & 393.67 & 0.1555 \\
195.90 & 0.0055 & 297.79 & 0.0619 & 395.70 & 0.1583 \\
199.81 & 0.0064 & 308.11 & 0.0690 & 398.82 & 0.1641 \\
206.73 & 0.0072 & 317.35 & 0.0771 & 400.26 & 0.1673 \\
213.91 & 0.0081 & 326.30 & 0.0847 & 402.70 & 0.1721 \\
222.01 & 0.0093 & 330.86 & 0.0889 & 405.86 & 0.1791 \\
227.13 & 0.0101 & 335.91 & 0.0934 & 407.25 & 0.1823 \\
234.78 & 0.0114 & 340.24 & 0.0972 & 409.32 & 0.1862 \\
238.58 & 0.0132 & 350.48 & 0.1044 & 410.74 & 0.1903 \\
242.49 & 0.0148 & 354.85 & 0.1088 & 412.18 & 0.1934 \\
246.97 & 0.0181 & 363.38 & 0.1176 & 412.36 & 0.1993 \\
251.74 & 0.0216 & 367.70 & 0.1221 & 412.41 & 0.2025 \\
255.64 & 0.0245 & 371.87 & 0.1266 & & \\
\hline
\end{tabular}

Table 212. Test data for specimen 684-24

\begin{tabular}{|c|c|c|c|c|c|}
\hline $\begin{array}{l}\text { Test Number } \\
\text { Aging Temp. } \\
\text { Yield Stress }\end{array}$ & $\begin{array}{l}: 0 \\
: 32 \\
: 16\end{array}$ & & $\begin{array}{l}\text { Tes } \\
\text { Agi } \\
\text { Ulti }\end{array}$ & $\begin{array}{l}\text { mp. } \\
\text { me } \\
\text { Stress }\end{array}$ & $\begin{array}{l}290^{\circ} \mathrm{C} \\
10,000 \mathrm{~h} \\
442.8 \mathrm{MPa}\end{array}$ \\
\hline $\begin{array}{l}\text { Engr. } \\
\text { Stress } \\
(\mathrm{MPa})\end{array}$ & $\begin{array}{l}\text { Engr. } \\
\text { Strain }\end{array}$ & $\begin{array}{l}\text { Engr. } \\
\text { Stress } \\
(\mathrm{MPa})\end{array}$ & $\begin{array}{l}\text { Engr. } \\
\text { Strain }\end{array}$ & $\begin{array}{l}\text { Engr. } \\
\text { Stress } \\
(\mathrm{MPa}) \\
\end{array}$ & $\begin{array}{l}\text { Engr. } \\
\text { Strain }\end{array}$ \\
\hline 131.09 & 0.0007 & 264.23 & 0.0338 & 395.13 & 0.1571 \\
\hline 161.38 & 0.0016 & 268.58 & 0.0370 & 399.64 & 0.1631 \\
\hline 170.77 & 0.0021 & 292.17 & 0.0571 & 406.01 & 0.1727 \\
\hline 177.76 & 0.0028 & 299.27 & 0.0630 & 408.98 & 0.1772 \\
\hline 184.73 & 0.0039 & 305.22 & 0.0682 & 410.77 & 0.1804 \\
\hline 188.47 & 0.0047 & 310.81 & 0.0730 & 414.03 & 0.1871 \\
\hline 191.92 & 0.0056 & 315.96 & 0.0778 & 423.79 & 0.2067 \\
\hline 195.08 & 0.0065 & 318.97 & 0.0805 & 425.05 & 0.2100 \\
\hline 198.78 & 0.0076 & 337.58 & 0.0967 & 429.03 & 0.2167 \\
\hline 201.57 & 0.0081 & 344.16 & 0.1001 & 430.50 & 0.2207 \\
\hline 207.31 & 0.0088 & 350.35 & 0.1059 & 431.77 & 0.2260 \\
\hline 236.70 & 0.0140 & 355.06 & 0.1103 & 432.94 & 0.2311 \\
\hline 241.45 & 0.0167 & 363.12 & 0.1186 & 435.81 & 0.2386 \\
\hline 246.41 & 0.0201 & 365.95 & 0.1218 & 437.16 & 0.2448 \\
\hline 251.57 & 0.0236 & 371.04 & 0.1273 & 439.01 & 0.2497 \\
\hline 256.17 & 0.0270 & 354.82 & 0.1306 & 439.80 & 0.2544 \\
\hline 260.33 & 0.0305 & 391.27 & 0.1513 & 442.04 & 0.2608 \\
\hline
\end{tabular}


Table 213. Test data for specimen 682-40

\begin{tabular}{|c|c|c|c|c|c|}
\hline $\begin{array}{l}\text { Test Number } \\
\text { Aging Temp. } \\
\text { Yield Stress }\end{array}$ & $\begin{array}{l}: 1 \\
: 32 \\
: 17\end{array}$ & & $\begin{array}{l}\text { Tes } \\
\text { Agi } \\
\text { Ulti }\end{array}$ & $\begin{array}{l}\text { mp. } \\
\text { me } \\
\text { Stress }\end{array}$ & $\begin{array}{l}290^{\circ} \mathrm{C} \\
30,000 \mathrm{~h} \\
440.8 \mathrm{MPa}\end{array}$ \\
\hline $\begin{array}{l}\text { Engr. } \\
\text { Stress } \\
(\mathrm{MPa}) \\
\end{array}$ & $\begin{array}{l}\text { Engr. } \\
\text { Strain }\end{array}$ & $\begin{array}{l}\text { Engr. } \\
\text { Stress } \\
(\mathrm{MPa}) \\
\end{array}$ & $\begin{array}{l}\text { Engr. } \\
\text { Strain }\end{array}$ & $\begin{array}{l}\text { Engr. } \\
\text { Stress } \\
\text { (MPa) }\end{array}$ & $\begin{array}{l}\text { Engr. } \\
\text { Strain }\end{array}$ \\
\hline 0.25 & 0.0000 & 218.20 & 0.0109 & 342.51 & 0.1025 \\
\hline 33.32 & 0.0001 & 220.60 & 0.0120 & 359.89 & 0.1192 \\
\hline 97.41 & 0.0007 & 224.95 & 0.0139 & 376.72 & 0.1375 \\
\hline 144.41 & 0.0015 & 229.50 & 0.0160 & 384.74 & 0.1472 \\
\hline 157.94 & 0.0018 & 233.25 & 0.0182 & 392.24 & 0.1570 \\
\hline 162.85 & 0.0021 & 238.24 & 0.0210 & 398.80 & 0.1659 \\
\hline 174.78 & 0.0026 & 247.78 & 0.0270 & 409.88 & 0.1835 \\
\hline 183.14 & 0.0033 & 253.92 & 0.0312 & 419.01 & 0.2008 \\
\hline 190.60 & 0.0041 & 260.91 & 0.0365 & 422.18 & 0.2068 \\
\hline 196.45 & 0.0049 & 271.49 & 0.0448 & 423.13 & 0.2086 \\
\hline 201.35 & 0.0058 & 280.12 & 0.0523 & 431.34 & 0.2305 \\
\hline 205.71 & 0.0069 & 292.09 & 0.0622 & 434.92 & 0.2482 \\
\hline 210.26 & 0.0080 & 305.66 & 0.0731 & 439.19 & 0.2666 \\
\hline 213.01 & 0.0091 & 317.23 & 0.0832 & 440.78 & 0.2804 \\
\hline 215.71 & 0.0099 & 329.89 & 0.0936 & 440.83 & 0.2903 \\
\hline
\end{tabular}

Table 214. Test data for specimen 682-42

\begin{tabular}{|c|c|c|c|c|c|}
\hline $\begin{array}{l}\text { Test Number } \\
\text { Aging Temp. } \\
\text { Yield Stress }\end{array}$ & $\begin{array}{l}: 1 \\
: 32 \\
: 17\end{array}$ & & $\begin{array}{l}\text { Tes } \\
\text { Agi } \\
\text { Ulti }\end{array}$ & $\begin{array}{l}\text { mp. } \\
\text { me } \\
\text { Stress }\end{array}$ & $\begin{array}{l}290^{\circ} \mathrm{C} \\
30,000 \mathrm{~h} \\
451.6 \mathrm{MPa}\end{array}$ \\
\hline $\begin{array}{l}\text { Engr. } \\
\text { Stress } \\
(\mathrm{MPa}) \\
\end{array}$ & $\begin{array}{l}\text { Engr. } \\
\text { Strain }\end{array}$ & $\begin{array}{l}\text { Engr. } \\
\text { Stress } \\
(\mathrm{MPa})\end{array}$ & $\begin{array}{l}\text { Engr. } \\
\text { Strain }\end{array}$ & $\begin{array}{l}\text { Engr. } \\
\text { Stress } \\
(\mathrm{MPa}) \\
\end{array}$ & $\begin{array}{l}\text { Engr. } \\
\text { Strain }\end{array}$ \\
\hline 0.64 & 0.0000 & 226.99 & 0.0137 & 376.65 & 0.1316 \\
\hline 30.11 & 0.0000 & 236.62 & 0.0188 & 383.92 & 0.1396 \\
\hline 95.68 & 0.0005 & 244.12 & 0.0235 & 393.44 & 0.1529 \\
\hline 134.65 & 0.0010 & 249.34 & 0.0268 & 401.17 & 0.1631 \\
\hline 161.74 & 0.0017 & 255.40 & 0.0306 & 409.59 & 0.1740 \\
\hline 167.78 & 0.0020 & 261.86 & 0.0360 & 413.59 & 0.1799 \\
\hline 178.19 & 0.0025 & 269.51 & 0.0415 & 417.63 & 0.1861 \\
\hline 186.21 & 0.0031 & 279.95 & 0.0497 & 422.07 & 0.1929 \\
\hline 189.13 & 0.0034 & 285.02 & 0.0538 & 429.12 & 0.2062 \\
\hline 194.24 & 0.0040 & 297.14 & 0.0634 & 433.29 & 0.2175 \\
\hline 198.55 & 0.0047 & 306.34 & 0.0708 & 437.63 & 0.2318 \\
\hline 206.44 & 0.0062 & 314.30 & 0.0777 & 442.07 & 0.2430 \\
\hline 209.77 & 0.0071 & 326.74 & 0.0880 & 447.30 & 0.2585 \\
\hline 214.33 & 0.0085 & 338.22 & 0.0975 & 448.61 & 0.2688 \\
\hline 217.60 & 0.0097 & 360.01 & 0.1145 & 450.08 & 0.2851 \\
\hline 221.43 & 0.0112 & 369.13 & 0.1240 & 451.61 & 0.3043 \\
\hline
\end{tabular}


Table 215. Test data for specimen 68-263

\begin{tabular}{|c|c|c|c|c|c|}
\hline $\begin{array}{l}\text { Test Number } \\
\text { Aging Temp. } \\
\text { Yield Stress }\end{array}$ & $\begin{array}{l}: 1 \\
: 32 \\
: 16\end{array}$ & & $\begin{array}{l}\text { Tes } \\
\text { Agi } \\
\text { Ulti }\end{array}$ & $\begin{array}{l}\text { mp. } \\
\text { me } \\
\text { Stress }\end{array}$ & $\begin{array}{l}290^{\circ} \mathrm{C} \\
30,000 \mathrm{~h} \\
450.3 \mathrm{MPa}\end{array}$ \\
\hline $\begin{array}{l}\text { Engr. } \\
\text { Stress } \\
\text { (MPa) }\end{array}$ & $\begin{array}{l}\text { Engr. } \\
\text { Strain }\end{array}$ & $\begin{array}{l}\text { Engr. } \\
\text { Stress } \\
(\mathrm{MPa})\end{array}$ & $\begin{array}{l}\text { Engr. } \\
\text { Strain }\end{array}$ & $\begin{array}{l}\text { Engr. } \\
\text { Stress } \\
\text { (MPa) } \\
\end{array}$ & $\begin{array}{l}\text { Engr. } \\
\text { Strain }\end{array}$ \\
\hline 0.00 & 0.0000 & 242.20 & 0.0266 & 376.97 & 0.1490 \\
\hline 80.05 & 0.0003 & 248.82 & 0.0312 & 382.37 & 0.1557 \\
\hline 129.13 & 0.0010 & 260.45 & 0.0401 & 388.36 & 0.1647 \\
\hline 157.15 & 0.0019 & 271.23 & 0.0491 & 392.52 & 0.1710 \\
\hline 164.98 & 0.0022 & 288.38 & 0.0639 & 400.29 & 0.1824 \\
\hline 175.45 & 0.0027 & 298.41 & 0.0723 & 406.13 & 0.1928 \\
\hline 180.09 & 0.0031 & 306.85 & 0.0799 & 413.00 & 0.2067 \\
\hline 188.86 & 0.0039 & 316.09 & 0.0880 & 413.88 & 0.2070 \\
\hline 192.50 & 0.0045 & 324.38 & 0.0952 & 417.98 & 0.2174 \\
\hline 195.84 & 0.0051 & 332.62 & 0.1006 & 422.37 & 0.2266 \\
\hline 199.02 & 0.0058 & 339.82 & 0.1078 & 427.99 & 0.2419 \\
\hline 205.20 & 0.0072 & 345.52 & 0.1134 & 438.53 & 0.2735 \\
\hline 215.00 & 0.0109 & 349.69 & 0.1175 & 444.23 & 0.2983 \\
\hline 220.87 & 0.0135 & 355.10 & 0.1231 & 449.22 & 0.3216 \\
\hline 223.96 & 0.0151 & 359.91 & 0.1288 & 450.01 & 0.3315 \\
\hline 228.83 & 0.0178 & 366.31 & 0.1352 & 450.32 & 0.3464 \\
\hline 233.71 & 0.0207 & 371.27 & 0.1413 & & \\
\hline
\end{tabular}

Table 216. Test data for specimen 682-28

\begin{tabular}{|c|c|c|c|c|c|}
\hline $\begin{array}{l}\text { Test Number } \\
\text { Aging Temp. } \\
\text { Yield Stress }\end{array}$ & $\begin{array}{l}: 3 \\
: 32 \\
: 20\end{array}$ & & $\begin{array}{l}\text { Tes } \\
\text { Agi } \\
\text { Ulti }\end{array}$ & $\begin{array}{l}\text { np. } \\
\text { ne } \\
\text { Stress }\end{array}$ & $\begin{array}{l}290^{\circ} \mathrm{C} \\
50,000 \mathrm{~h} \\
479.0 \mathrm{MPa}\end{array}$ \\
\hline $\begin{array}{l}\text { Engr. } \\
\text { Stress } \\
\text { (MPa) }\end{array}$ & $\begin{array}{l}\text { Engr. } \\
\text { Strain }\end{array}$ & $\begin{array}{l}\text { Engr. } \\
\text { Stress } \\
\text { (MPa) }\end{array}$ & $\begin{array}{l}\text { Engr. } \\
\text { Strain }\end{array}$ & $\begin{array}{l}\text { Engr. } \\
\text { Stress } \\
(\mathrm{MPa})\end{array}$ & $\begin{array}{l}\text { Engr. } \\
\text { Strain }\end{array}$ \\
\hline 95.970 & 0.0002 & 311.280 & 0.0498 & 422.850 & 0.1422 \\
\hline 118.450 & 0.0002 & 318.200 & 0.0543 & 428.320 & 0.1489 \\
\hline 155.240 & 0.0005 & 338.060 & 0.0673 & 431.790 & 0.1533 \\
\hline 162.540 & 0.0007 & 341.270 & 0.0695 & 442.830 & 0.1689 \\
\hline 204.750 & 0.0029 & 359.350 & 0.0826 & 455.890 & 0.1911 \\
\hline 210.160 & 0.0037 & 378.570 & 0.0979 & 457.050 & 0.1933 \\
\hline 228.290 & 0.0075 & 381.060 & 0.1001 & 464.650 & 0.2116 \\
\hline 264.380 & 0.0231 & 390.950 & 0.1090 & 466.090 & 0.2139 \\
\hline 268.810 & 0.0253 & 404.820 & 0.1223 & 466.860 & 0.2159 \\
\hline 281.090 & 0.0320 & 407.000 & 0.1245 & 473.630 & 0.2382 \\
\hline 300.340 & 0.0432 & 409.010 & 0.1268 & 478.960 & 0.2689 \\
\hline
\end{tabular}


Table 217. Test data for specimen 682-29

\begin{tabular}{|c|c|c|c|c|c|}
\hline $\begin{array}{l}\text { Test Number } \\
\text { Aging Temp. } \\
\text { Yield Stress }\end{array}$ & $\begin{array}{l}: 3 \\
: 32 \\
: 20\end{array}$ & & $\begin{array}{l}\text { Tes } \\
\text { Agi } \\
\text { Ulti }\end{array}$ & $\begin{array}{l}\text { np. } \\
\text { ne } \\
\text { Stress }\end{array}$ & $\begin{array}{l}290^{\circ} \mathrm{C} \\
50,000 \mathrm{~h} \\
485.6 \mathrm{MPa}\end{array}$ \\
\hline $\begin{array}{l}\text { Engr. } \\
\text { Stress } \\
\text { (MPa) }\end{array}$ & $\begin{array}{l}\text { Engr. } \\
\text { Strain }\end{array}$ & $\begin{array}{l}\text { Engr. } \\
\text { Stress } \\
\text { (MPa) }\end{array}$ & $\begin{array}{l}\text { Engr. } \\
\text { Strain }\end{array}$ & $\begin{array}{l}\text { Engr. } \\
\text { Stress } \\
(\mathrm{MPa})\end{array}$ & $\begin{array}{l}\text { Engr. } \\
\text { Strain }\end{array}$ \\
\hline 18.870 & 0.0001 & 301.950 & 0.0480 & 418.900 & 0.1578 \\
\hline 33.340 & 0.0002 & 305.100 & 0.0501 & 428.130 & 0.1711 \\
\hline 68.280 & 0.0003 & 319.730 & 0.0611 & 443.810 & 0.1979 \\
\hline 78.920 & 0.0003 & 338.970 & 0.0765 & 449.360 & 0.2072 \\
\hline 90.140 & 0.0003 & 351.940 & 0.0874 & 459.460 & 0.2311 \\
\hline 133.150 & 0.0005 & 354.440 & 0.0896 & 463.780 & 0.2405 \\
\hline 142.130 & 0.0006 & 375.810 & 0.1094 & 472.960 & 0.2706 \\
\hline 196.750 & 0.0022 & 377.910 & 0.1115 & 476.600 & 0.2818 \\
\hline 239.890 & 0.0098 & 388.780 & 0.1225 & 480.990 & 0.3041 \\
\hline 258.450 & 0.0189 & 398.960 & 0.1335 & 482.860 & 0.3129 \\
\hline 262.340 & 0.0212 & 417.320 & 0.1557 & 485.580 & 0.3506 \\
\hline
\end{tabular}

Table 218. Test data for specimen 681-30

\begin{tabular}{|c|c|c|c|c|c|}
\hline $\begin{array}{l}\text { Test Number } \\
\text { Aging Temp. } \\
\text { Yield Stress }\end{array}$ & $\begin{array}{l}3 \\
32 \\
20\end{array}$ & $\mathrm{MPa}$ & $\begin{array}{l}\text { Tes } \\
\text { Agi } \\
\text { Ulti }\end{array}$ & $\begin{array}{l}\text { np. } \\
\text { me } \\
\text { Stress }\end{array}$ & $\begin{array}{l}290^{\circ} \mathrm{C} \\
50,000 \mathrm{~h} \\
475.2 \mathrm{MPa}\end{array}$ \\
\hline $\begin{array}{l}\text { Engr. } \\
\text { Stress } \\
(\mathrm{MPa})\end{array}$ & $\begin{array}{l}\text { Engr. } \\
\text { Strain }\end{array}$ & $\begin{array}{l}\text { Engr. } \\
\text { Stress } \\
(\mathrm{MPa})\end{array}$ & $\begin{array}{l}\text { Engr. } \\
\text { Strain }\end{array}$ & $\begin{array}{l}\text { Engr. } \\
\text { Stress } \\
(\mathrm{MPa}) \\
\end{array}$ & $\begin{array}{l}\text { Engr. } \\
\text { Strain }\end{array}$ \\
\hline 112.840 & 0.0001 & 329.750 & 0.0780 & 430.620 & 0.1979 \\
\hline 119.940 & 0.0002 & 342.440 & 0.0891 & 443.920 & 0.2229 \\
\hline 149.250 & 0.0007 & 344.740 & 0.0912 & 445.330 & 0.2274 \\
\hline 168.720 & 0.0013 & 371.180 & 0.1176 & 457.120 & 0.2528 \\
\hline 180.220 & 0.0017 & 373.300 & 0.1198 & 455.730 & 0.2548 \\
\hline 244.130 & 0.0161 & 383.200 & 0.1308 & 464.740 & 0.2758 \\
\hline 248.410 & 0.0184 & 396.060 & 0.1461 & 463.810 & 0.2778 \\
\hline 252.380 & 0.0208 & 403.040 & 0.1550 & 466.760 & 0.2865 \\
\hline 292.290 & 0.0476 & 404.700 & 0.1572 & 470.830 & 0.3026 \\
\hline 295.130 & 0.0499 & 422.660 & 0.1835 & 471.970 & 0.3050 \\
\hline 306.500 & 0.0587 & 429.590 & 0.1956 & 475.140 & 0.3337 \\
\hline
\end{tabular}


Table 219. Test data for specimen 682-27

$\begin{array}{llll}\text { Test Number } & : \text { MEA Test } & \text { Test Temp. } & : 290^{\circ} \mathrm{C} \\ \text { Aging Temp. } & : 350^{\circ} \mathrm{C} & \text { Aging Time } & : 5,780 \mathrm{~h} \\ \text { Yield Stress } & : 185.1 \mathrm{MPa} & \text { Ultimate Stress } & : 452.4 \mathrm{MPa}\end{array}$

\begin{tabular}{cccccc}
\hline $\begin{array}{c}\text { Engr. } \\
\text { Stress } \\
(\mathrm{MPa})\end{array}$ & $\begin{array}{c}\text { Engr. } \\
\text { Strain }\end{array}$ & $\begin{array}{c}\text { Engr. } \\
\text { Stress } \\
(\mathrm{MPa})\end{array}$ & $\begin{array}{c}\text { Engr. } \\
\text { Strain }\end{array}$ & $\begin{array}{c}\text { Engr. } \\
\text { Stress } \\
(\mathrm{MPa})\end{array}$ & $\begin{array}{c}\text { Engr. } \\
\text { Strain }\end{array}$ \\
\hline 36.34 & 0.0002 & 213.39 & 0.0086 & 389.42 & 0.1610 \\
57.23 & 0.0004 & 216.98 & 0.0096 & 396.38 & 0.1726 \\
77.64 & 0.0005 & 220.84 & 0.0107 & 404.72 & 0.1848 \\
98.32 & 0.0006 & 241.11 & 0.0213 & 411.96 & 0.1970 \\
117.35 & 0.0007 & 257.45 & 0.0329 & 416.37 & 0.2091 \\
136.24 & 0.0011 & 273.10 & 0.0443 & 423.61 & 0.2200 \\
150.31 & 0.0016 & 289.79 & 0.0558 & 427.34 & 0.2358 \\
162.99 & 0.0021 & 301.78 & 0.0675 & 430.85 & 0.2474 \\
174.51 & 0.0026 & 316.54 & 0.0789 & 436.23 & 0.2601 \\
185.12 & 0.0032 & 327.50 & 0.0906 & 442.23 & 0.2769 \\
190.23 & 0.0039 & 340.60 & 0.1021 & 444.09 & 0.2891 \\
198.57 & 0.0047 & 349.36 & 0.1137 & 447.06 & 0.3037 \\
203.53 & 0.0057 & 360.39 & 0.1254 & 449.19 & 0.3238 \\
207.39 & 0.0066 & 370.11 & 0.1373 & 450.23 & 0.3447 \\
209.88 & 0.0076 & 378.87 & 0.1489 & 450.37 & 0.3672 \\
\hline
\end{tabular}

Table 220. Test data for specimen 684-09

\begin{tabular}{|c|c|c|c|c|c|}
\hline $\begin{array}{l}\text { Test Number } \\
\text { Aging Temp. } \\
\text { Yield Stress }\end{array}$ & $\begin{array}{l}: 0 \\
: 35 \\
: 16\end{array}$ & & $\begin{array}{l}\text { Tes } \\
\text { Agi } \\
\text { Ulti }\end{array}$ & $\begin{array}{l}\text { np. } \\
\text { ne } \\
\text { Stress }\end{array}$ & $\begin{array}{l}290^{\circ} \mathrm{C} \\
10,000 \mathrm{~h} \\
500.3 \mathrm{MPa}\end{array}$ \\
\hline $\begin{array}{l}\text { Engr. } \\
\text { Stress } \\
\text { (MPa) }\end{array}$ & $\begin{array}{l}\text { Engr. } \\
\text { Strain }\end{array}$ & $\begin{array}{l}\text { Engr. } \\
\text { Stress } \\
\text { (MPa) }\end{array}$ & $\begin{array}{l}\text { Engr. } \\
\text { Strain }\end{array}$ & $\begin{array}{l}\text { Engr. } \\
\text { Stress } \\
(\mathrm{MPa}) \\
\end{array}$ & $\begin{array}{l}\text { Engr. } \\
\text { Strain }\end{array}$ \\
\hline 112.43 & 0.0004 & 319.88 & 0.0463 & 451.18 & 0.1521 \\
\hline 136.35 & 0.0007 & 330.15 & 0.0531 & 458.54 & 0.1622 \\
\hline 153.62 & 0.0012 & 338.70 & 0.0596 & 462.12 & 0.1676 \\
\hline 188.85 & 0.0024 & 345.67 & 0.0645 & 465.31 & 0.1717 \\
\hline 199.52 & 0.0031 & 369.51 & 0.0813 & 474.65 & 0.1766 \\
\hline 216.98 & 0.0037 & 377.37 & 0.0873 & 477.80 & 0.1805 \\
\hline 234.00 & 0.0047 & 385.47 & 0.0929 & 479.90 & 0.1882 \\
\hline 240.14 & 0.0055 & 392.40 & 0.0977 & 482.34 & 0.1959 \\
\hline 244.67 & 0.0067 & 399.62 & 0.1010 & 487.25 & 0.2127 \\
\hline 248.80 & 0.0082 & 407.33 & 0.1074 & 492.37 & 0.2187 \\
\hline 254.35 & 0.0102 & 416.37 & 0.1147 & 494.34 & 0.2215 \\
\hline 283.26 & 0.0237 & 433.21 & 0.1314 & 494.83 & 0.2277 \\
\hline 293.73 & 0.0297 & 438.61 & 0.1371 & 495.71 & 0.2366 \\
\hline 305.03 & 0.0367 & 442.98 & 0.1420 & 497.57 & 0.2426 \\
\hline 315.10 & 0.0433 & 447.21 & 0.1471 & 500.32 & 0.2567 \\
\hline
\end{tabular}


Table 221. Test data for specimen 68-130

$\begin{array}{llll}\text { Test Number } & : 083 & \text { Test Temp. } & : 290^{\circ} \mathrm{C} \\ \text { Aging Temp. } & : 350^{\circ} \mathrm{C} & \text { Aging Time } & : 10,000 \mathrm{~h} \\ \text { Yield Stress } & : 168.9 \mathrm{MPa} & \text { Ultimate Stress } & : 447.2 \mathrm{MPa}\end{array}$

\begin{tabular}{cccccc}
\hline $\begin{array}{c}\text { Engr. } \\
\text { Stress } \\
(\mathrm{MPa})\end{array}$ & $\begin{array}{c}\text { Engr. } \\
\text { Strain }\end{array}$ & $\begin{array}{c}\text { Engr. } \\
\text { Stress } \\
(\mathrm{MPa})\end{array}$ & $\begin{array}{c}\text { Engr. } \\
\text { Strain }\end{array}$ & $\begin{array}{c}\text { Engr. } \\
\text { Stress } \\
(\mathrm{MPa})\end{array}$ & $\begin{array}{c}\text { Engr. } \\
\text { Strain }\end{array}$ \\
\hline & & & & & \\
122.1500 & 0.0008 & 266.7200 & 0.0244 & 395.3500 & 0.1314 \\
138.2200 & 0.0012 & 273.6900 & 0.0296 & 401.0800 & 0.1385 \\
155.0300 & 0.0018 & 280.2500 & 0.0342 & 404.1500 & 0.1428 \\
160.7000 & 0.0020 & 288.5200 & 0.0407 & 406.9700 & 0.1467 \\
166.4700 & 0.0023 & 293.2500 & 0.0446 & 410.0800 & 0.1509 \\
177.2300 & 0.0028 & 301.2700 & 0.0509 & 415.3200 & 0.1584 \\
180.9800 & 0.0031 & 308.6000 & 0.0572 & 418.6000 & 0.1637 \\
195.8000 & 0.0044 & 313.6800 & 0.0614 & 426.7800 & 0.1772 \\
203.5800 & 0.0052 & 322.4600 & 0.0688 & 428.7200 & 0.1812 \\
212.2400 & 0.0058 & 327.7500 & 0.0732 & 432.8700 & 0.1894 \\
221.2100 & 0.0066 & 334.3400 & 0.0790 & 436.2800 & 0.1970 \\
230.5300 & 0.0075 & 340.1700 & 0.0837 & 439.6400 & 0.2044 \\
234.2100 & 0.0080 & 364.9600 & 0.1007 & 442.6200 & 0.2129 \\
237.4900 & 0.0088 & 372.2600 & 0.1067 & 443.5500 & 0.2161 \\
240.8200 & 0.0101 & 377.6200 & 0.1124 & 446.0800 & 0.2243 \\
254.1300 & 0.0164 & 385.2900 & 0.1207 & 446.8500 & 0.2301 \\
260.4000 & 0.0204 & 392.7400 & 0.1281 & 447.1400 & 0.2333 \\
\hline
\end{tabular}

Table 222. Test data for specimen 684-12

\begin{tabular}{|c|c|c|c|c|c|}
\hline $\begin{array}{l}\text { Test Number } \\
\text { Aging Temp. } \\
\text { Yield Stress }\end{array}$ & $\begin{array}{l}: 2 \\
: 35 \\
: 21\end{array}$ & & $\begin{array}{l}\text { Tes } \\
\text { Agir } \\
\text { Ulti }\end{array}$ & $\begin{array}{l}\text { mp. } \\
\text { me } \\
\text { Stress }\end{array}$ & $\begin{array}{l}290^{\circ} \mathrm{C} \\
30,000 \mathrm{~h} \\
485.7 \mathrm{MPa}\end{array}$ \\
\hline $\begin{array}{l}\text { Engr. } \\
\text { Stress } \\
(\mathrm{MPa})\end{array}$ & $\begin{array}{l}\text { Engr. } \\
\text { Strain }\end{array}$ & $\begin{array}{l}\text { Engr. } \\
\text { Stress } \\
\text { (MPa) }\end{array}$ & $\begin{array}{l}\text { Engr. } \\
\text { Strain }\end{array}$ & $\begin{array}{l}\text { Engr. } \\
\text { Stress } \\
\text { (MPa) }\end{array}$ & $\begin{array}{l}\text { Engr. } \\
\text { Strain }\end{array}$ \\
\hline 64.060 & 0.0004 & 227.050 & 0.0060 & 386.180 & 0.0998 \\
\hline 93.730 & 0.0005 & 259.280 & 0.0181 & 389.000 & 0.1024 \\
\hline 103.610 & 0.0006 & 272.530 & 0.0251 & 409.010 & 0.1188 \\
\hline 125.630 & 0.0007 & 277.420 & 0.0276 & 421.990 & 0.1310 \\
\hline 132.320 & 0.0007 & 301.910 & 0.0421 & 442.810 & 0.1500 \\
\hline 138.680 & 0.0008 & 306.330 & 0.0447 & 443.850 & 0.1536 \\
\hline 163.920 & 0.0009 & 318.480 & 0.0521 & 460.970 & 0.1797 \\
\hline 172.010 & 0.0010 & 347.650 & 0.0713 & 462.490 & 0.1817 \\
\hline 179.520 & 0.0011 & 357.420 & 0.0783 & 472.180 & 0.1991 \\
\hline 203.880 & 0.0021 & 360.570 & 0.0806 & 477.380 & 0.2102 \\
\hline 221.270 & 0.0046 & 383.260 & 0.0975 & 485.710 & 0.2388 \\
\hline
\end{tabular}


Table 223. Test data for specimen 684-15

\begin{tabular}{|c|c|c|c|c|c|}
\hline $\begin{array}{l}\text { Test Number } \\
\text { Aging Temp. } \\
\text { Yield Stress }\end{array}$ & $\begin{array}{l}: 26 \\
: 35 \\
: 19\end{array}$ & & $\begin{array}{l}\text { Tes } \\
\text { Agir } \\
\text { Ulti }\end{array}$ & $\begin{array}{l}\text { np. } \\
\text { ne } \\
\text { Stress }\end{array}$ & $\begin{array}{l}290^{\circ} \mathrm{C} \\
30,000 \mathrm{~h} \\
427.3 \mathrm{MPa}\end{array}$ \\
\hline $\begin{array}{l}\text { Engr. } \\
\text { Stress } \\
(\mathrm{MPa})\end{array}$ & $\begin{array}{l}\text { Engr. } \\
\text { Strain } \\
\end{array}$ & $\begin{array}{l}\text { Engr. } \\
\text { Stress } \\
(\mathrm{MPa})\end{array}$ & $\begin{array}{l}\text { Engr. } \\
\text { Strain }\end{array}$ & $\begin{array}{l}\text { Engr. } \\
\text { Stress } \\
(\mathrm{MPa}) \\
\end{array}$ & $\begin{array}{l}\text { Engr. } \\
\text { Strain }\end{array}$ \\
\hline 28.682 & 0.0002 & 242.204 & 0.0235 & 354.865 & 0.1120 \\
\hline 35.020 & 0.0002 & 245.934 & 0.0258 & 369.129 & 0.1270 \\
\hline 61.873 & 0.0003 & 266.270 & 0.0387 & 374.891 & 0.1337 \\
\hline 72.445 & 0.0004 & 275.565 & 0.0451 & 376.827 & 0.1357 \\
\hline 128.096 & 0.0007 & 278.526 & 0.0474 & 391.284 & 0.1532 \\
\hline 134.391 & 0.0007 & 298.421 & 0.0621 & 392.876 & 0.1553 \\
\hline 151.378 & 0.0008 & 301.189 & 0.0642 & 397.980 & 0.1623 \\
\hline 190.100 & 0.0030 & 322.623 & 0.0819 & 410.957 & 0.1816 \\
\hline 195.833 & 0.0040 & 325.153 & 0.0840 & 414.474 & 0.1879 \\
\hline 211.355 & 0.0085 & 327.582 & 0.0862 & 421.804 & 0.2031 \\
\hline 230.795 & 0.0172 & 348.338 & 0.1056 & 427.262 & 0.2227 \\
\hline
\end{tabular}

Table 224. Test data for specimen 68-140

\begin{tabular}{|c|c|c|c|c|c|}
\hline $\begin{array}{l}\text { Test Number } \\
\text { Aging Temp. } \\
\text { Yield Stress }\end{array}$ & $\begin{array}{l}: 2 \\
: 35 \\
: 17\end{array}$ & $\mathrm{MPa}$ & $\begin{array}{l}\text { Tes } \\
\text { Agi } \\
\text { Ulti }\end{array}$ & $\begin{array}{l}\text { mp. } \\
\text { me } \\
\text { Stress }\end{array}$ & $\begin{array}{l}290^{\circ} \mathrm{C} \\
30,000 \mathrm{~h} \\
479.0 \mathrm{MPa}\end{array}$ \\
\hline $\begin{array}{l}\text { Engr. } \\
\text { Stress } \\
(\mathrm{MPa})\end{array}$ & $\begin{array}{l}\text { Engr. } \\
\text { Strain }\end{array}$ & $\begin{array}{l}\text { Engr. } \\
\text { Stress } \\
(\mathrm{MPa})\end{array}$ & $\begin{array}{l}\text { Engr. } \\
\text { Strain }\end{array}$ & $\begin{array}{l}\text { Engr. } \\
\text { Stress } \\
(\mathrm{MPa}) \\
\end{array}$ & $\begin{array}{l}\text { Engr. } \\
\text { Strain }\end{array}$ \\
\hline 44.260 & 0.0002 & 281.450 & 0.0413 & 416.300 & 0.1427 \\
\hline 48.740 & 0.0002 & 309.740 & 0.0585 & 428.390 & 0.1557 \\
\hline 94.850 & 0.0005 & 313.120 & 0.0607 & 430.280 & 0.1579 \\
\hline 104.690 & 0.0007 & 322.910 & 0.0670 & 435.920 & 0.1645 \\
\hline 161.360 & 0.0025 & 350.440 & 0.0862 & 447.870 & 0.1798 \\
\hline 166.450 & 0.0028 & 353.360 & 0.0884 & 452.540 & 0.1865 \\
\hline 174.850 & 0.0034 & 356.220 & 0.0906 & 461.470 & 0.2023 \\
\hline 223.280 & 0.0125 & 383.360 & 0.1121 & 462.660 & 0.2045 \\
\hline 229.230 & 0.0147 & 385.840 & 0.1143 & 471.640 & 0.2247 \\
\hline 244.580 & 0.0213 & 388.320 & 0.1166 & 473.380 & 0.2293 \\
\hline 270.070 & 0.0348 & 409.670 & 0.1361 & 479.000 & 0.2522 \\
\hline
\end{tabular}


Table 225. Test data for specimen 682-18

$\begin{array}{llll}\text { Test Number } & : \text { MEA Test } & \text { Test Temp. } & : 290^{\circ} \mathrm{C} \\ \text { Aging Temp. } & : 400^{\circ} \mathrm{C} & \text { Aging Time } & : 2,570 \mathrm{~h} \\ \text { Yield Stress } & : 161.3 \mathrm{MPa} & \text { Ultimate Stress } & : 463.1 \mathrm{MPa}\end{array}$

\begin{tabular}{rccccc}
\hline $\begin{array}{c}\text { Engr. } \\
\text { Stress } \\
(\mathrm{MPa})\end{array}$ & $\begin{array}{c}\text { Engr. } \\
\text { Strain }\end{array}$ & $\begin{array}{c}\text { Stress } \\
(\mathrm{MPa})\end{array}$ & $\begin{array}{c}\text { Engr. } \\
\text { Strain }\end{array}$ & $\begin{array}{c}\text { Engr. } \\
\text { Stress } \\
(\mathrm{MPa})\end{array}$ & $\begin{array}{c}\text { Engr. } \\
\text { Strain }\end{array}$ \\
\hline 78.53 & 0.0005 & 213.74 & 0.0143 & 391.21 & 0.1431 \\
102.18 & 0.0007 & 234.35 & 0.0238 & 398.72 & 0.1517 \\
121.76 & 0.0011 & 251.59 & 0.0341 & 409.48 & 0.1605 \\
138.38 & 0.0015 & 262.41 & 0.0440 & 415.34 & 0.1693 \\
149.13 & 0.0022 & 280.27 & 0.0538 & 424.17 & 0.1782 \\
160.17 & 0.0030 & 292.41 & 0.0631 & 428.65 & 0.1872 \\
169.82 & 0.0038 & 305.30 & 0.0723 & 434.78 & 0.1962 \\
178.09 & 0.0049 & 318.40 & 0.0815 & 441.47 & 0.2052 \\
184.37 & 0.0060 & 329.98 & 0.0904 & 445.13 & 0.2151 \\
189.95 & 0.0071 & 341.36 & 0.0993 & 449.74 & 0.2250 \\
196.02 & 0.0084 & 353.49 & 0.1083 & 454.57 & 0.2341 \\
200.57 & 0.0095 & 363.15 & 0.1170 & 457.95 & 0.2459 \\
202.09 & 0.0108 & 374.52 & 0.1257 & 459.47 & 0.2573 \\
207.19 & 0.0119 & 382.59 & 0.1344 & 461.88 & 0.2716 \\
209.95 & 0.0131 & & & & \\
\hline
\end{tabular}

Table 226. Test data for specimen 682-24

$\begin{array}{llll}\text { Test Number } & : 076 & \text { Test Temp. } & : 290^{\circ} \mathrm{C} \\ \text { Aging Temp. } & : 400^{\circ} \mathrm{C} & \text { Aging Time } & : 10,000 \mathrm{~h} \\ \text { Yield Stress } & : 176.7 \mathrm{MPa} & \text { Ultimate Stress } & : 475.6 \mathrm{MPa}\end{array}$

\begin{tabular}{cccccc}
\hline $\begin{array}{c}\text { Engr. } \\
\text { Stress } \\
(\mathrm{MPa})\end{array}$ & $\begin{array}{c}\text { Engr. } \\
\text { Strain }\end{array}$ & $\begin{array}{c}\text { Engr. } \\
\text { Stress } \\
(\mathrm{MPa})\end{array}$ & $\begin{array}{c}\text { Engr. } \\
\text { Strain }\end{array}$ & $\begin{array}{c}\text { Engr. } \\
\text { Stress } \\
(\mathrm{MPa})\end{array}$ & $\begin{array}{c}\text { Engr. } \\
\text { Strain }\end{array}$ \\
\hline & & & & & \\
144.63 & 0.0011 & 300.68 & 0.0386 & 425.46 & 0.1590 \\
164.92 & 0.0019 & 305.23 & 0.0420 & 429.79 & 0.1651 \\
171.17 & 0.0021 & 310.63 & 0.0467 & 441.10 & 0.1832 \\
183.88 & 0.0027 & 318.15 & 0.0528 & 445.55 & 0.1926 \\
212.61 & 0.0042 & 343.22 & 0.0750 & 447.79 & 0.1962 \\
228.63 & 0.0049 & 353.13 & 0.0838 & 450.39 & 0.2017 \\
237.41 & 0.0057 & 358.48 & 0.0887 & 452.39 & 0.2057 \\
241.90 & 0.0066 & 364.49 & 0.0942 & 454.68 & 0.2100 \\
245.26 & 0.0072 & 373.56 & 0.0998 & 458.35 & 0.2196 \\
247.63 & 0.0082 & 379.16 & 0.1047 & 468.65 & 0.2491 \\
250.89 & 0.0092 & 383.98 & 0.1103 & 470.46 & 0.2544 \\
269.81 & 0.0176 & 386.76 & 0.1132 & 471.52 & 0.2588 \\
272.29 & 0.0191 & 406.62 & 0.1355 & 472.54 & 0.2637 \\
274.96 & 0.0208 & 409.80 & 0.1389 & 473.75 & 0.2683 \\
278.12 & 0.0226 & 412.29 & 0.1418 & 475.31 & 0.2727 \\
280.74 & 0.0243 & 414.92 & 0.1455 & 475.15 & 0.2776 \\
284.99 & 0.0270 & 420.54 & 0.1523 & & \\
\hline
\end{tabular}


Table 227. Test data for specimen 68-120

$\begin{array}{llll}\text { Test Number } & : 077 & \text { Test Temp. } & : 290^{\circ} \mathrm{C} \\ \text { Aging Temp. } & : 400^{\circ} \mathrm{C} & \text { Aging Time } & : 10,000 \mathrm{~h} \\ \text { Yield Stress } & : 193.9 \mathrm{MPa} & \text { Ultimate Stress } & : 484.4 \mathrm{MPa}\end{array}$

\begin{tabular}{cccccc}
\hline $\begin{array}{c}\text { Engr. } \\
\text { Stress } \\
(\mathrm{MPa})\end{array}$ & $\begin{array}{c}\text { Engr. } \\
\text { Strain }\end{array}$ & $\begin{array}{c}\text { Engr. } \\
\text { Stress } \\
(\mathrm{MPa})\end{array}$ & $\begin{array}{c}\text { Engr. } \\
\text { Strain }\end{array}$ & $\begin{array}{c}\text { Engr. } \\
\text { Stress } \\
(\mathrm{MPa})\end{array}$ & $\begin{array}{c}\text { Engr. } \\
\text { Strain }\end{array}$ \\
\hline & & & & & \\
159.5000 & 0.0019 & 328.6900 & 0.0532 & 441.4700 & 0.1506 \\
170.9400 & 0.0024 & 335.1200 & 0.0583 & 444.3000 & 0.1544 \\
184.2900 & 0.0030 & 340.7600 & 0.0628 & 446.9200 & 0.1575 \\
196.4600 & 0.0037 & 347.3400 & 0.0678 & 449.5000 & 0.1611 \\
211.0100 & 0.0044 & 351.5400 & 0.0707 & 454.1700 & 0.1679 \\
244.7000 & 0.0069 & 356.3400 & 0.0743 & 456.3100 & 0.1711 \\
249.3900 & 0.0079 & 377.4600 & 0.0898 & 461.4800 & 0.1796 \\
253.9800 & 0.0094 & 383.3000 & 0.0947 & 464.0600 & 0.1838 \\
260.6400 & 0.0116 & 389.3400 & 0.0972 & 465.8000 & 0.1872 \\
264.7900 & 0.0132 & 395.5800 & 0.1024 & 469.8900 & 0.1956 \\
268.4900 & 0.0149 & 400.4300 & 0.1063 & 477.4800 & 0.2170 \\
272.1000 & 0.0166 & 403.7500 & 0.1096 & 478.8400 & 0.2212 \\
276.5000 & 0.0187 & 407.4600 & 0.1127 & 480.1400 & 0.2260 \\
279.4100 & 0.0202 & 410.4800 & 0.1153 & 481.0000 & 0.2300 \\
287.7600 & 0.0249 & 413.3500 & 0.1179 & 482.7700 & 0.2389 \\
296.3100 & 0.0302 & 415.9300 & 0.1204 & 483.3800 & 0.2432 \\
303.0300 & 0.0350 & 422.1200 & 0.1258 & 484.0900 & 0.2479 \\
323.0100 & 0.0488 & 427.5200 & 0.1313 & 484.2600 & 0.2515 \\
\hline
\end{tabular}

Table 228. Test data for specimen 681-06

\begin{tabular}{|c|c|c|c|c|c|}
\hline $\begin{array}{l}\text { Test Number } \\
\text { Aging Temp. } \\
\text { Yield Stress }\end{array}$ & $\begin{array}{l}: M \\
: 45 \\
: 16\end{array}$ & & $\begin{array}{l}\text { Tes } \\
\text { Agi } \\
\text { Ulti }\end{array}$ & $\begin{array}{l}\text { np. } \\
\text { ne } \\
\text { Stress }\end{array}$ & $\begin{array}{l}290^{\circ} \mathrm{C} \\
2,570 \mathrm{~h} \\
486.8 \mathrm{MPa}\end{array}$ \\
\hline $\begin{array}{l}\text { Engr. } \\
\text { Stress } \\
\text { (MPa) }\end{array}$ & $\begin{array}{l}\text { Engr. } \\
\text { Strain }\end{array}$ & $\begin{array}{l}\text { Engr. } \\
\text { Stress } \\
\text { (MPa) }\end{array}$ & $\begin{array}{l}\text { Engr. } \\
\text { Strain }\end{array}$ & $\begin{array}{l}\text { Engr. } \\
\text { Stress } \\
(\mathrm{MPa})\end{array}$ & $\begin{array}{l}\text { Engr. } \\
\text { Strain }\end{array}$ \\
\hline 42.27 & 0.0002 & 238.56 & 0.0108 & 400.72 & 0.1097 \\
\hline 66.60 & 0.0002 & 242.83 & 0.0120 & 408.24 & 0.1165 \\
\hline 89.70 & 0.0004 & 246.00 & 0.0131 & 414.37 & 0.1233 \\
\hline 111.70 & 0.0006 & 263.93 & 0.0193 & 423.41 & 0.1301 \\
\hline 132.17 & 0.0011 & 278.13 & 0.0265 & 430.09 & 0.1368 \\
\hline 147.75 & 0.0017 & 292.61 & 0.0338 & 436.23 & 0.1436 \\
\hline 162.10 & 0.0023 & 304.54 & 0.0409 & 449.19 & 0.1569 \\
\hline 175.26 & 0.0031 & 317.71 & 0.0478 & 451.88 & 0.1635 \\
\hline 188.09 & 0.0039 & 326.40 & 0.0549 & 458.64 & 0.1701 \\
\hline 199.05 & 0.0047 & 337.71 & 0.0619 & 463.12 & 0.1768 \\
\hline 208.70 & 0.0057 & 349.15 & 0.0690 & 467.88 & 0.1832 \\
\hline 215.87 & 0.0066 & 357.29 & 0.0758 & 471.81 & 0.1898 \\
\hline 222.49 & 0.0077 & 365.70 & 0.0827 & 475.05 & 0.1961 \\
\hline 227.66 & 0.0086 & 384.31 & 0.0962 & 479.53 & 0.2024 \\
\hline 233.18 & 0.0098 & 394.31 & 0.1030 & 482.36 & 0.2078 \\
\hline
\end{tabular}


Table 229. Test data for specimen 682-09

$\begin{array}{llll}\text { Test Number } & : \text { MEA Test } & \text { Test Temp. } & : 290^{\circ} \mathrm{C} \\ \text { Aging Temp. } & : 450^{\circ} \mathrm{C} & \text { Aging Time } & : 2,570 \mathrm{~h} \\ \text { Yield Stress } & : 191.6 \mathrm{MPa} & \text { Ultimate Stress } & : 503.8 \mathrm{MPa}\end{array}$

\begin{tabular}{rccccc}
\hline $\begin{array}{c}\text { Engr. } \\
\text { Stress } \\
(\mathrm{MPa})\end{array}$ & $\begin{array}{c}\text { Engr. } \\
\text { Strain }\end{array}$ & $\begin{array}{c}\text { Stress } \\
(\mathrm{MPa})\end{array}$ & $\begin{array}{c}\text { Engr. } \\
\text { Strain }\end{array}$ & $\begin{array}{c}\text { Engr. } \\
\text { Stress } \\
(\mathrm{MPa})\end{array}$ & $\begin{array}{c}\text { Engr. } \\
\text { Strain }\end{array}$ \\
\hline & & & & & \\
21.24 & 0.0002 & 230.91 & 0.0075 & 417.82 & 0.1099 \\
38.68 & 0.0003 & 238.21 & 0.0083 & 428.58 & 0.1199 \\
59.36 & 0.0005 & 242.76 & 0.0094 & 436.51 & 0.1302 \\
80.88 & 0.0006 & 248.56 & 0.0104 & 446.57 & 0.1404 \\
104.32 & 0.0008 & 253.04 & 0.0115 & 452.78 & 0.1508 \\
122.24 & 0.0011 & 279.79 & 0.0203 & 459.81 & 0.1614 \\
143.34 & 0.0015 & 302.27 & 0.0303 & 466.57 & 0.1721 \\
156.99 & 0.0020 & 320.95 & 0.0403 & 472.64 & 0.1829 \\
171.96 & 0.0025 & 336.74 & 0.0503 & 479.12 & 0.1939 \\
185.06 & 0.0032 & 352.60 & 0.0603 & 481.32 & 0.2052 \\
196.36 & 0.0039 & 369.77 & 0.0704 & 488.08 & 0.2167 \\
206.70 & 0.0048 & 381.42 & 0.0802 & 490.84 & 0.2277 \\
217.46 & 0.0056 & 393.97 & 0.0900 & 493.60 & 0.2409 \\
224.91 & 0.0065 & 404.31 & 0.0998 & 498.28 & 0.2515 \\
\hline
\end{tabular}

Table 230. Test data for specimen 733-40

\begin{tabular}{|c|c|c|c|c|c|}
\hline $\begin{array}{l}\text { Test Number } \\
\text { Aging Temp. } \\
\text { Yield Stress }\end{array}$ & $\begin{array}{l}: 0 \\
: U 1 \\
: 25\end{array}$ & & & $\begin{array}{l}\text { np. } \\
\text { ne } \\
\text { Stress }\end{array}$ & $\begin{array}{l}25^{\circ} \mathrm{C} \\
557.0 \mathrm{MPa}\end{array}$ \\
\hline $\begin{array}{l}\text { Engr. } \\
\text { Stress } \\
(\mathrm{MPa})\end{array}$ & $\begin{array}{l}\text { Engr. } \\
\text { Strain }\end{array}$ & $\begin{array}{l}\text { Engr. } \\
\text { Stress } \\
\text { (MPa) }\end{array}$ & $\begin{array}{l}\text { Engr. } \\
\text { Strain }\end{array}$ & $\begin{array}{l}\text { Engr. } \\
\text { Stress } \\
(\mathrm{MPa}) \\
\end{array}$ & $\begin{array}{l}\text { Engr. } \\
\text { Strain }\end{array}$ \\
\hline 63.5814 & 0.0003 & 262.4600 & 0.0084 & 462.4630 & 0.2270 \\
\hline 84.9001 & 0.0005 & 268.5910 & 0.0105 & 476.4350 & 0.2399 \\
\hline 106.8720 & 0.0009 & 275.6970 & 0.0138 & 480.1400 & 0.2456 \\
\hline 124.6350 & 0.0013 & 281.1460 & 0.0173 & 484.6510 & 0.2495 \\
\hline 152.2530 & 0.0018 & 287.8630 & 0.0215 & 492.9110 & 0.2665 \\
\hline 166.9750 & 0.0023 & 295.3100 & 0.0271 & 501.5690 & 0.2853 \\
\hline 185.7380 & 0.0028 & 308.0140 & 0.0382 & 506.0760 & 0.2975 \\
\hline 202.8910 & 0.0032 & 325.7820 & 0.0544 & 515.0850 & 0.3156 \\
\hline 209.3630 & 0.0033 & 342.4300 & 0.0703 & 520.9320 & 0.3343 \\
\hline 221.3840 & 0.0035 & 360.2990 & 0.0893 & 529.8790 & 0.3558 \\
\hline 229.2450 & 0.0036 & 380.5990 & 0.1106 & 537.4600 & 0.3760 \\
\hline 240.0750 & 0.0043 & 398.3240 & 0.1313 & 543.7160 & 0.3972 \\
\hline 248.1560 & 0.0050 & 417.7070 & 0.1569 & 550.2940 & 0.4162 \\
\hline 253.6530 & 0.0059 & 436.4070 & 0.1842 & 554.0220 & 0.4328 \\
\hline 257.4490 & 0.0069 & 452.0410 & 0.2093 & 556.9630 & 0.4463 \\
\hline
\end{tabular}


Table 231. Test data for specimen 733-41

\begin{tabular}{|c|c|c|c|c|c|}
\hline $\begin{array}{l}\text { Test Number } \\
\text { Aging Temp. } \\
\text { Yield Stress }\end{array}$ & $\begin{array}{l}: 0 \\
: U \\
: 24\end{array}$ & & $\begin{array}{l}\text { Tes } \\
\text { Agi } \\
\text { Ulti }\end{array}$ & $\begin{array}{l}\text { np. } \\
\text { ne } \\
\text { Stress }\end{array}$ & $\begin{array}{l}25^{\circ} \mathrm{C} \\
- \\
530.8 \mathrm{MPa}\end{array}$ \\
\hline $\begin{array}{l}\text { Engr. } \\
\text { Stress } \\
(\mathrm{MPa})\end{array}$ & $\begin{array}{l}\text { Engr. } \\
\text { Strain }\end{array}$ & $\begin{array}{l}\text { Engr. } \\
\text { Stress } \\
(\mathrm{MPa})\end{array}$ & $\begin{array}{l}\text { Engr. } \\
\text { Strain }\end{array}$ & $\begin{array}{l}\text { Engr. } \\
\text { Stress } \\
(\mathrm{MPa}) \\
\end{array}$ & $\begin{array}{l}\text { Engr. } \\
\text { Strain } \\
\end{array}$ \\
\hline $\begin{array}{r}57.54 \\
103.56 \\
147.78 \\
194.54 \\
205.22 \\
229.48 \\
248.07 \\
255.24 \\
262.66 \\
267.73 \\
275.69 \\
287.00 \\
297.14\end{array}$ & $\begin{array}{l}0.0003 \\
0.0011 \\
0.0021 \\
0.0038 \\
0.0039 \\
0.0045 \\
0.0055 \\
0.0063 \\
0.0075 \\
0.0090 \\
0.0125 \\
0.0201 \\
0.0284\end{array}$ & $\begin{array}{l}315.80 \\
333.74 \\
351.77 \\
368.00 \\
383.59 \\
399.42 \\
402.92 \\
426.19 \\
447.50 \\
437.57 \\
461.90 \\
472.78\end{array}$ & $\begin{array}{l}0.0467 \\
0.0648 \\
0.0837 \\
0.1007 \\
0.1184 \\
0.1376 \\
0.1403 \\
0.1725 \\
0.2064 \\
0.1907 \\
0.2318 \\
0.2550\end{array}$ & $\begin{array}{l}481.10 \\
489.91 \\
503.35 \\
509.95 \\
514.83 \\
520.18 \\
523.68 \\
527.32 \\
528.83 \\
530.67 \\
530.78 \\
530.79\end{array}$ & $\begin{array}{l}0.2757 \\
0.2954 \\
0.3317 \\
0.3536 \\
0.3748 \\
0.3932 \\
0.4139 \\
0.4321 \\
0.4459 \\
0.4654 \\
0.4808 \\
0.5001\end{array}$ \\
\hline
\end{tabular}

Table 232. Test data for specimen 734-23

\begin{tabular}{|c|c|c|c|c|c|}
\hline $\begin{array}{l}\text { Test Number } \\
\text { Aging Temp. } \\
\text { Yield Stress }\end{array}$ & $\begin{array}{l}: 2 \\
: 32 \\
: 22\end{array}$ & & $\begin{array}{l}\text { Tes } \\
\text { Agi } \\
\text { Ulti }\end{array}$ & $\begin{array}{l}\text { np. } \\
\text { ne } \\
\text { Stress }\end{array}$ & $\begin{array}{l}25^{\circ} \mathrm{C} \\
10,000 \mathrm{~h} \\
475.4 \mathrm{MPa}\end{array}$ \\
\hline $\begin{array}{l}\text { Engr. } \\
\text { Stress } \\
\text { (MPa) }\end{array}$ & $\begin{array}{l}\text { Engr. } \\
\text { Strain }\end{array}$ & $\begin{array}{l}\text { Engr. } \\
\text { Stress } \\
\text { (MPa) } \\
\end{array}$ & $\begin{array}{l}\text { Engr. } \\
\text { Strain }\end{array}$ & $\begin{array}{l}\text { Engr. } \\
\text { Stress } \\
(\mathrm{MPa})\end{array}$ & $\begin{array}{l}\text { Engr. } \\
\text { Strain }\end{array}$ \\
\hline 35.201 & 0.0002 & 316.982 & 0.0882 & 415.842 & 0.2432 \\
\hline 50.230 & 0.0004 & 318.460 & 0.0900 & 424.156 & 0.2605 \\
\hline 109.948 & 0.0011 & 327.099 & 0.1011 & 436.441 & 0.2900 \\
\hline 171.163 & 0.0019 & 344.669 & 0.1252 & 443.083 & 0.3083 \\
\hline 231.938 & 0.0065 & 352.387 & 0.1363 & 450.399 & 0.3307 \\
\hline 261.579 & 0.0293 & 357.424 & 0.1437 & 454.902 & 0.3459 \\
\hline 263.135 & 0.0311 & 371.837 & 0.1662 & 457.632 & 0.3558 \\
\hline 264.870 & 0.0328 & 377.470 & 0.1756 & 465.020 & 0.3876 \\
\hline 291.185 & 0.0584 & 378.464 & 0.1775 & 467.415 & 0.3998 \\
\hline 292.756 & 0.0600 & 401.099 & 0.2160 & 472.976 & 0.4350 \\
\hline 300.484 & 0.0681 & 405.002 & 0.2226 & 475.332 & 0.4714 \\
\hline
\end{tabular}


Table 233. Test data for specimen 734-24

\begin{tabular}{|c|c|c|c|c|c|}
\hline $\begin{array}{l}\text { Test Number } \\
\text { Aging Temp. } \\
\text { Yield Stress }\end{array}$ & $\begin{array}{l}: 2 \\
: 32 \\
: 22\end{array}$ & & $\begin{array}{l}\text { Tes } \\
\text { Agir } \\
\text { Ulti }\end{array}$ & $\begin{array}{l}\text { np. } \\
\text { ne } \\
\text { Stress }\end{array}$ & $\begin{array}{l}25^{\circ} \mathrm{C} \\
10,000 \mathrm{~h} \\
521.4 \mathrm{MPa}\end{array}$ \\
\hline $\begin{array}{l}\text { Engr. } \\
\text { Stress } \\
(\mathrm{MPa})\end{array}$ & $\begin{array}{l}\text { Engr. } \\
\text { Strain }\end{array}$ & $\begin{array}{l}\text { Engr. } \\
\text { Stress } \\
(\mathrm{MPa})\end{array}$ & $\begin{array}{l}\text { Engr. } \\
\text { Strain }\end{array}$ & $\begin{array}{l}\text { Engr. } \\
\text { Stress } \\
(\mathrm{MPa}) \\
\end{array}$ & $\begin{array}{l}\text { Engr. } \\
\text { Strain }\end{array}$ \\
\hline 21.163 & 0.0001 & 373.504 & 0.1373 & 473.833 & 0.3118 \\
\hline 92.275 & 0.0006 & 374.976 & 0.1393 & 478.661 & 0.3253 \\
\hline 164.513 & 0.0011 & 376.803 & 0.1413 & 494.476 & 0.3783 \\
\hline 176.243 & 0.0012 & 410.308 & 0.1858 & 498.945 & 0.3933 \\
\hline 226.308 & 0.0032 & 411.421 & 0.1879 & 503.802 & 0.4166 \\
\hline 257.628 & 0.0160 & 418.736 & 0.1988 & 508.854 & 0.4450 \\
\hline 260.197 & 0.0182 & 438.544 & 0.2322 & 512.788 & 0.4711 \\
\hline 274.920 & 0.0315 & 445.014 & 0.2446 & 516.091 & 0.4978 \\
\hline 320.321 & 0.0777 & 453.704 & 0.2621 & 518.131 & 0.5150 \\
\hline 322.313 & 0.0799 & 462.894 & 0.2834 & 519.151 & 0.5309 \\
\hline 324.377 & 0.0820 & 473.060 & 0.3095 & 521.240 & 0.5848 \\
\hline
\end{tabular}

Table 234. Test data for specimen 732-25

\begin{tabular}{|c|c|c|c|c|c|}
\hline $\begin{array}{l}\text { Test Number } \\
\text { Aging Temp. } \\
\text { Yield Stress }\end{array}$ & $\begin{array}{l}: 0 \\
: 35 \\
: 24\end{array}$ & & $\begin{array}{l}\text { Tes } \\
\text { Agi } \\
\text { Ulti }\end{array}$ & $\begin{array}{l}\text { np. } \\
\text { ne } \\
\text { Stress }\end{array}$ & $\begin{array}{l}25^{\circ} \mathrm{C} \\
2,570 \mathrm{~h} \\
526.5 \mathrm{MPa}\end{array}$ \\
\hline $\begin{array}{l}\text { Engr. } \\
\text { Stress } \\
\text { (MPa) }\end{array}$ & $\begin{array}{l}\text { Engr. } \\
\text { Strain }\end{array}$ & $\begin{array}{l}\text { Engr. } \\
\text { Stress } \\
\text { (MPa) }\end{array}$ & $\begin{array}{l}\text { Engr. } \\
\text { Strain }\end{array}$ & $\begin{array}{l}\text { Engr. } \\
\text { Stress } \\
(\mathrm{MPa}) \\
\end{array}$ & $\begin{array}{l}\text { Engr. } \\
\text { Strain }\end{array}$ \\
\hline 36.15 & 0.0001 & 288.81 & 0.0229 & 483.08 & 0.2457 \\
\hline 60.31 & 0.0003 & 302.72 & 0.0340 & 486.34 & 0.2590 \\
\hline 84.51 & 0.0006 & 323.55 & 0.0524 & 490.23 & 0.2692 \\
\hline 108.59 & 0.0011 & 342.47 & 0.0694 & 494.65 & 0.2836 \\
\hline 132.31 & 0.0016 & 358.96 & 0.0860 & 500.81 & 0.3004 \\
\hline 156.59 & 0.0021 & 375.53 & 0.1021 & 504.35 & 0.3079 \\
\hline 180.62 & 0.0028 & 391.90 & 0.1188 & 507.79 & 0.3226 \\
\hline 203.99 & 0.0034 & 407.09 & 0.1365 & 513.05 & 0.3378 \\
\hline 221.44 & 0.0038 & 421.20 & 0.1537 & 515.33 & 0.3490 \\
\hline 240.27 & 0.0047 & 432.99 & 0.1692 & 518.65 & 0.3610 \\
\hline 243.07 & 0.0049 & 445.48 & 0.1872 & 519.90 & 0.3632 \\
\hline 253.14 & 0.0063 & 457.09 & 0.2055 & 522.92 & 0.3819 \\
\hline 260.80 & 0.0082 & 466.51 & 0.2220 & 524.52 & 0.4021 \\
\hline 268.76 & 0.0111 & 476.07 & 0.2395 & 526.23 & 0.4151 \\
\hline 275.87 & 0.0145 & 478.09 & 0.2429 & 526.46 & 0.4309 \\
\hline
\end{tabular}


Table 235. Test data for specimen 732-26

\begin{tabular}{|c|c|c|c|c|c|}
\hline $\begin{array}{l}\text { Test Number } \\
\text { Aging Temp. } \\
\text { Yield Stress }\end{array}$ & $\begin{array}{l}: 0 \\
: 35 \\
: 24\end{array}$ & & $\begin{array}{l}\text { Tes } \\
\text { Agi } \\
\text { Ulti }\end{array}$ & $\begin{array}{l}\text { np. } \\
\text { ne } \\
\text { Stress }\end{array}$ & $\begin{array}{l}25^{\circ} \mathrm{C} \\
2,570 \mathrm{~h} \\
528.4 \mathrm{MPa}\end{array}$ \\
\hline $\begin{array}{l}\text { Engr. } \\
\text { Stress } \\
(\mathrm{MPa})\end{array}$ & $\begin{array}{l}\text { Engr. } \\
\text { Strain }\end{array}$ & $\begin{array}{l}\text { Engr. } \\
\text { Stress } \\
\text { (MPa) }\end{array}$ & $\begin{array}{l}\text { Engr. } \\
\text { Strain }\end{array}$ & $\begin{array}{l}\text { Engr. } \\
\text { Stress } \\
(\mathrm{MPa}) \\
\end{array}$ & $\begin{array}{l}\text { Engr. } \\
\text { Strain }\end{array}$ \\
\hline 33.1610 & 0.0001 & 286.5200 & 0.0188 & 470.6100 & 0.2373 \\
\hline 58.0510 & 0.0003 & 292.8000 & 0.0228 & 479.3800 & 0.2556 \\
\hline 82.5420 & 0.0006 & 300.7600 & 0.0282 & 481.5200 & 0.2604 \\
\hline 106.4300 & 0.0011 & 307.1600 & 0.0331 & 486.1000 & 0.2614 \\
\hline 129.9400 & 0.0016 & 324.4700 & 0.0480 & 487.8900 & 0.2723 \\
\hline 154.9900 & 0.0022 & 341.3800 & 0.0640 & 494.8400 & 0.2897 \\
\hline 178.0200 & 0.0028 & 358.3900 & 0.0813 & 501.2900 & 0.3075 \\
\hline 206.4600 & 0.0037 & 374.8500 & 0.0982 & 508.0900 & 0.3266 \\
\hline 209.3700 & 0.0037 & 389.7700 & 0.1143 & 514.6400 & 0.3459 \\
\hline 223.7100 & 0.0041 & 403.2300 & 0.1309 & 518.8800 & 0.3648 \\
\hline 237.0900 & 0.0045 & 414.9900 & 0.1455 & 519.5500 & 0.3694 \\
\hline 244.5600 & 0.0052 & 425.4800 & 0.1603 & 522.3200 & 0.3719 \\
\hline 251.4300 & 0.0060 & 435.6200 & 0.1751 & 525.2200 & 0.3906 \\
\hline 257.1300 & 0.0070 & 445.9400 & 0.1919 & 526.4100 & 0.4006 \\
\hline 261.7100 & 0.0082 & 455.4300 & 0.2085 & 527.8400 & 0.4115 \\
\hline 267.7700 & 0.0100 & 462.6900 & 0.2210 & 528.4200 & 0.4296 \\
\hline 275.7600 & 0.0135 & & & & \\
\hline
\end{tabular}

Table 236. Test data for specimen 734-06

\begin{tabular}{|c|c|c|c|c|c|}
\hline $\begin{array}{l}\text { Test Number } \\
\text { Aging Temp. } \\
\text { Yield Stress }\end{array}$ & $\begin{array}{l}: 2 \\
: 35 \\
: 22\end{array}$ & & $\begin{array}{l}\text { Tes } \\
\text { Agir } \\
\text { Ulti }\end{array}$ & $\begin{array}{l}\text { np. } \\
\text { ne } \\
\text { Stress }\end{array}$ & $\begin{array}{l}25^{\circ} \mathrm{C} \\
10,000 \mathrm{~h} \\
536.9 \mathrm{MPa}\end{array}$ \\
\hline $\begin{array}{l}\text { Engr. } \\
\text { Stress } \\
\text { (MPa) }\end{array}$ & $\begin{array}{l}\text { Engr. } \\
\text { Strain }\end{array}$ & $\begin{array}{l}\text { Engr. } \\
\text { Stress } \\
\text { (MPa) }\end{array}$ & $\begin{array}{l}\text { Engr. } \\
\text { Strain }\end{array}$ & $\begin{array}{l}\text { Engr. } \\
\text { Stress } \\
(\mathrm{MPa}) \\
\end{array}$ & $\begin{array}{l}\text { Engr. } \\
\text { Strain }\end{array}$ \\
\hline 18.238 & 0.0001 & 318.152 & 0.0474 & 451.977 & 0.1875 \\
\hline 42.648 & 0.0004 & 346.750 & 0.0746 & 472.198 & 0.2156 \\
\hline 53.881 & 0.0005 & 348.942 & 0.0768 & 480.488 & 0.2285 \\
\hline 179.881 & 0.0022 & 376.168 & 0.1078 & 495.178 & 0.2547 \\
\hline 190.256 & 0.0024 & 378.054 & 0.1101 & 503.226 & 0.2711 \\
\hline 199.656 & 0.0028 & 380.449 & 0.1123 & 514.473 & 0.2981 \\
\hline 250.900 & 0.0076 & 410.366 & 0.1407 & 515.879 & 0.3023 \\
\hline 257.241 & 0.0091 & 412.257 & 0.1427 & 524.799 & 0.3300 \\
\hline 280.837 & 0.0188 & 422.414 & 0.1531 & 526.932 & 0.3386 \\
\hline 303.807 & 0.0353 & 448.831 & 0.1834 & 533.283 & 0.3696 \\
\hline 306.289 & 0.0373 & 450.275 & 0.1854 & 536.822 & 0.4116 \\
\hline
\end{tabular}


Table 237. Test data for specimen 734-07

\begin{tabular}{|c|c|c|c|c|c|}
\hline $\begin{array}{l}\text { Test Number } \\
\text { Aging Temp. } \\
\text { Yield Stress }\end{array}$ & $\begin{array}{l}: 2 \\
: 35 \\
: 21\end{array}$ & & $\begin{array}{l}\text { Tes } \\
\text { Agir } \\
\text { Ulti }\end{array}$ & $\begin{array}{l}\text { mp. } \\
\text { me } \\
\text { Stress }\end{array}$ & $\begin{array}{l}25^{\circ} \mathrm{C} \\
10,000 \mathrm{~h} \\
529.3 \mathrm{MPa}\end{array}$ \\
\hline $\begin{array}{l}\text { Engr. } \\
\text { Stress } \\
(\mathrm{MPa})\end{array}$ & $\begin{array}{l}\text { Engr. } \\
\text { Strain }\end{array}$ & $\begin{array}{l}\text { Engr. } \\
\text { Stress } \\
(\mathrm{MPa})\end{array}$ & $\begin{array}{l}\text { Engr. } \\
\text { Strain }\end{array}$ & $\begin{array}{l}\text { Engr. } \\
\text { Stress } \\
\text { (MPa) }\end{array}$ & $\begin{array}{l}\text { Engr. } \\
\text { Strain }\end{array}$ \\
\hline 39.655 & 0.0002 & 332.413 & 0.0708 & 490.091 & 0.3044 \\
\hline 55.337 & 0.0003 & 373.667 & 0.1109 & 502.862 & 0.3496 \\
\hline 67.588 & 0.0004 & 383.268 & 0.1215 & 503.489 & 0.3519 \\
\hline 123.036 & 0.0007 & 416.276 & 0.1618 & 513.658 & 0.4004 \\
\hline 133.957 & 0.0007 & 419.481 & 0.1660 & 514.574 & 0.4050 \\
\hline 146.791 & 0.0008 & 443.578 & 0.2024 & 518.959 & 0.4332 \\
\hline 210.418 & 0.0024 & 452.379 & 0.2175 & 521.610 & 0.4522 \\
\hline 217.917 & 0.0031 & 465.184 & 0.2424 & 526.237 & 0.4960 \\
\hline 256.709 & 0.0134 & 470.317 & 0.2534 & 527.056 & 0.5068 \\
\hline 274.839 & 0.0246 & 473.247 & 0.2600 & 527.249 & 0.5092 \\
\hline 317.530 & 0.0577 & 486.091 & 0.2932 & 533.659 & 0.4116 \\
\hline
\end{tabular}

Table 238. Test data for specimen 73-119

\begin{tabular}{|c|c|c|c|c|c|}
\hline $\begin{array}{l}\text { Test Number } \\
\text { Aging Temp. } \\
\text { Yield Stress }\end{array}$ & & & $\begin{array}{l}\text { Tes } \\
\text { Agir } \\
\text { Ulti }\end{array}$ & $\begin{array}{l}\text { p. } \\
\text { he } \\
\text { Stress }\end{array}$ & $\begin{array}{l}25^{\circ} \mathrm{C} \\
10,000 \mathrm{~h} \\
488.1 \mathrm{MPa}\end{array}$ \\
\hline $\begin{array}{l}\text { Engr. } \\
\text { Stress } \\
\text { (MPa) }\end{array}$ & $\begin{array}{l}\text { Engr. } \\
\text { Strain }\end{array}$ & $\begin{array}{l}\text { Engr. } \\
\text { Stress } \\
(\mathrm{MPa}) \\
\end{array}$ & $\begin{array}{l}\text { Engr. } \\
\text { Strain }\end{array}$ & $\begin{array}{l}\text { Engr. } \\
\text { Stress } \\
\text { (MPa) } \\
\end{array}$ & $\begin{array}{l}\text { Engr. } \\
\text { Strain }\end{array}$ \\
\hline $\begin{array}{r}81.198 \\
160.878 \\
198.210 \\
206.442 \\
245.229 \\
249.388 \\
253.026 \\
277.109 \\
279.943\end{array}$ & $\begin{array}{l}0.0005 \\
0.0012 \\
0.0023 \\
0.0029 \\
0.0116 \\
0.0138 \\
0.0160 \\
0.0338 \\
0.0361\end{array}$ & $\begin{array}{l}317.202 \\
319.709 \\
337.189 \\
347.960 \\
350.139 \\
369.571 \\
379.942 \\
382.053 \\
397.620\end{array}$ & $\begin{array}{l}0.0694 \\
0.0717 \\
0.0889 \\
0.0999 \\
0.1022 \\
0.1225 \\
0.1339 \\
0.1363 \\
0.1544\end{array}$ & $\begin{array}{l}428.975 \\
430.599 \\
443.887 \\
459.285 \\
465.825 \\
472.187 \\
483.440 \\
485.320 \\
488.115\end{array}$ & $\begin{array}{l}0.1950 \\
0.1973 \\
0.2178 \\
0.2402 \\
0.2586 \\
0.2741 \\
0.3045 \\
0.3168 \\
0.3497\end{array}$ \\
\hline
\end{tabular}


Table 239. Test data for specimen 732-16

$\begin{array}{llll}\text { Test Number } & : 012 & \text { Test Temp. } & : 25^{\circ} \mathrm{C} \\ \text { Aging Temp. } & : 400^{\circ} \mathrm{C} & \text { Aging Time } & : 2,570 \mathrm{~h} \\ \text { Yield Stress } & : 240.8 \mathrm{MPa} & \text { Ultimate Stress } & : 541.8 \mathrm{MPa}\end{array}$

\begin{tabular}{rccccc}
\hline $\begin{array}{c}\text { Engr. } \\
\text { Stress } \\
(\mathrm{MPa})\end{array}$ & $\begin{array}{c}\text { Engr. } \\
\text { Strain }\end{array}$ & $\begin{array}{c}\text { Engr. } \\
\text { Stress } \\
(\mathrm{MPa})\end{array}$ & $\begin{array}{c}\text { Engr. } \\
\text { Strain }\end{array}$ & $\begin{array}{c}\text { Engr. } \\
\text { Stress } \\
(\mathrm{MPa})\end{array}$ & $\begin{array}{c}\text { Engr. } \\
\text { Strain }\end{array}$ \\
\hline & & & & & \\
30.76 & 0.0000 & 333.00 & 0.0585 & 491.68 & 0.2327 \\
51.55 & 0.0002 & 340.74 & 0.0655 & 494.49 & 0.2408 \\
82.38 & 0.0006 & 349.35 & 0.0726 & 500.65 & 0.2515 \\
119.05 & 0.0013 & 363.37 & 0.0864 & 509.64 & 0.2700 \\
151.04 & 0.0020 & 376.95 & 0.0984 & 517.34 & 0.2907 \\
176.61 & 0.0027 & 386.90 & 0.1080 & 521.02 & 0.2948 \\
193.73 & 0.0033 & 399.61 & 0.1203 & 524.73 & 0.3088 \\
211.94 & 0.0036 & 413.26 & 0.1342 & 528.09 & 0.3203 \\
240.09 & 0.0049 & 424.21 & 0.1462 & 532.52 & 0.3385 \\
257.88 & 0.0071 & 433.32 & 0.1567 & 533.30 & 0.3501 \\
269.13 & 0.0098 & 445.81 & 0.1677 & 536.02 & 0.3669 \\
277.83 & 0.0138 & 459.01 & 0.1844 & 537.79 & 0.3805 \\
285.55 & 0.0181 & 474.02 & 0.2054 & 540.14 & 0.3980 \\
291.91 & 0.0223 & 481.76 & 0.2167 & 540.42 & 0.4137 \\
302.71 & 0.0311 & 485.41 & 0.2212 & 540.81 & 0.4314 \\
314.19 & 0.0413 & 489.04 & 0.2305 & 541.81 & 0.4492 \\
\hline
\end{tabular}

Table 240. Test data for specimen 732-17

\begin{tabular}{|c|c|c|c|c|c|}
\hline $\begin{array}{l}\text { Test Number } \\
\text { Aging Temp. } \\
\text { Yield Stress }\end{array}$ & $\begin{array}{l}: 0 \\
: 40 \\
: 25\end{array}$ & & $\begin{array}{l}\text { Tes } \\
\text { Agi } \\
\text { Ulti }\end{array}$ & $\begin{array}{l}\text { np. } \\
\text { ne } \\
\text { Stress }\end{array}$ & $\begin{array}{l}25^{\circ} \mathrm{C} \\
2,570 \mathrm{~h} \\
555.7 \mathrm{MPa}\end{array}$ \\
\hline $\begin{array}{l}\text { Engr. } \\
\text { Stress } \\
\text { (MPa) }\end{array}$ & $\begin{array}{l}\text { Engr. } \\
\text { Strain }\end{array}$ & $\begin{array}{l}\text { Engr. } \\
\text { Stress } \\
(\mathrm{MPa})\end{array}$ & $\begin{array}{l}\text { Engr. } \\
\text { Strain }\end{array}$ & $\begin{array}{l}\text { Engr. } \\
\text { Stress } \\
(\mathrm{MPa}) \\
\end{array}$ & $\begin{array}{l}\text { Engr. } \\
\text { Strain }\end{array}$ \\
\hline 20.02 & 0.0000 & 315.60 & 0.0374 & 506.90 & 0.2655 \\
\hline 42.34 & 0.0001 & 333.72 & 0.0542 & 522.51 & 0.2973 \\
\hline 87.99 & 0.0008 & 349.17 & 0.0684 & 530.96 & 0.3220 \\
\hline 125.42 & 0.0016 & 366.36 & 0.0851 & 534.58 & 0.3360 \\
\hline 159.13 & 0.0025 & 383.00 & 0.0995 & 538.98 & 0.3462 \\
\hline 181.55 & 0.0031 & 397.17 & 0.1132 & 540.32 & 0.3555 \\
\hline 208.85 & 0.0041 & 410.44 & 0.1262 & 543.40 & 0.3729 \\
\hline 238.65 & 0.0050 & 426.68 & 0.1429 & 546.91 & 0.3854 \\
\hline 251.97 & 0.0059 & 437.78 & 0.1552 & 548.39 & 0.3946 \\
\hline 256.64 & 0.0065 & 450.76 & 0.1706 & 549.97 & 0.4048 \\
\hline 264.81 & 0.0080 & 458.38 & 0.1802 & 551.73 & 0.4110 \\
\hline 270.67 & 0.0094 & 466.46 & 0.1907 & 552.43 & 0.4234 \\
\hline 281.79 & 0.0113 & 465.84 & 0.1959 & 554.68 & 0.4394 \\
\hline 292.09 & 0.0171 & 476.96 & 0.2130 & 554.91 & 0.4499 \\
\hline 303.46 & 0.0264 & 491.28 & 0.2365 & 555.72 & 0.4638 \\
\hline
\end{tabular}


Table 241. Test data for specimen 732-15

\begin{tabular}{|c|c|c|c|c|c|}
\hline $\begin{array}{l}\text { Test Number } \\
\text { Aging Temp. } \\
\text { Yield Stress }\end{array}$ & $\begin{array}{l}: 2 \\
: 40 \\
: 23\end{array}$ & & $\begin{array}{l}\text { Tes } \\
\text { Agir } \\
\text { Ulti }\end{array}$ & $\begin{array}{l}\text { mp. } \\
\text { me } \\
\text { Stress }\end{array}$ & $\begin{array}{l}25^{\circ} \mathrm{C} \\
10,000 \mathrm{~h} \\
563.3 \mathrm{MPa}\end{array}$ \\
\hline $\begin{array}{l}\text { Engr. } \\
\text { Stress } \\
(\mathrm{MPa})\end{array}$ & $\begin{array}{l}\text { Engr. } \\
\text { Strain }\end{array}$ & $\begin{array}{l}\text { Engr. } \\
\text { Stress } \\
(\mathrm{MPa})\end{array}$ & $\begin{array}{l}\text { Engr. } \\
\text { Strain }\end{array}$ & $\begin{array}{l}\text { Engr. } \\
\text { Stress } \\
\text { (MPa) }\end{array}$ & $\begin{array}{l}\text { Engr. } \\
\text { Strain }\end{array}$ \\
\hline 29.582 & 0.0001 & 379.611 & 0.0953 & 515.150 & 0.2914 \\
\hline 56.208 & 0.0003 & 395.718 & 0.1109 & 525.892 & 0.3231 \\
\hline 79.423 & 0.0004 & 397.992 & 0.1131 & 530.537 & 0.3389 \\
\hline 161.338 & 0.0009 & 428.919 & 0.1465 & 531.311 & 0.3411 \\
\hline 172.776 & 0.0010 & 445.501 & 0.1663 & 538.423 & 0.3695 \\
\hline 236.484 & 0.0034 & 447.199 & 0.1684 & 542.004 & 0.3849 \\
\hline 275.366 & 0.0136 & 482.960 & 0.2218 & 548.632 & 0.4181 \\
\hline 301.010 & 0.0291 & 493.861 & 0.2420 & 551.826 & 0.4360 \\
\hline 337.284 & 0.0572 & 495.071 & 0.2443 & 556.858 & 0.4692 \\
\hline 340.018 & 0.0596 & 509.925 & 0.2780 & 558.600 & 0.4821 \\
\hline 360.175 & 0.0774 & 514.328 & 0.2891 & 563.196 & 0.5341 \\
\hline
\end{tabular}

Table 242. Test data for specimen 732-22

\begin{tabular}{|c|c|c|c|c|c|}
\hline $\begin{array}{l}\text { Test Number } \\
\text { Aging Temp. } \\
\text { Yield Stress }\end{array}$ & $\begin{array}{l}: 22 \\
: 40 \\
: 21\end{array}$ & $\mathrm{~Pa}$ & $\begin{array}{l}\text { Tes } \\
\text { Agi } \\
\text { Ulti }\end{array}$ & $\begin{array}{l}\text { np. } \\
\text { me } \\
\text { Stress }\end{array}$ & $\begin{array}{l}25^{\circ} \mathrm{C} \\
10,000 \mathrm{~h} \\
530.3 \mathrm{MPa}\end{array}$ \\
\hline $\begin{array}{l}\text { Engr. } \\
\text { Stress } \\
\text { (MPa) }\end{array}$ & $\begin{array}{l}\text { Engr. } \\
\text { Strain }\end{array}$ & $\begin{array}{l}\text { Engr. } \\
\text { Stress } \\
\text { (MPa) } \\
\end{array}$ & $\begin{array}{l}\text { Engr. } \\
\text { Strain }\end{array}$ & $\begin{array}{l}\text { Engr. } \\
\text { Stress } \\
(\mathrm{MPa}) \\
\end{array}$ & $\begin{array}{l}\text { Engr. } \\
\text { Strain }\end{array}$ \\
\hline 53.560 & 0.0005 & 325.970 & 0.0621 & 455.794 & 0.2132 \\
\hline 77.759 & 0.0006 & 336.761 & 0.0723 & 478.769 & 0.2544 \\
\hline 155.999 & 0.0012 & 355.620 & 0.0910 & 485.473 & 0.2687 \\
\hline 176.921 & 0.0015 & 357.644 & 0.0930 & 496.895 & 0.2963 \\
\hline 205.216 & 0.0026 & 359.630 & 0.0950 & 500.654 & 0.3067 \\
\hline 221.357 & 0.0041 & 383.033 & 0.1196 & 504.896 & 0.3192 \\
\hline 251.508 & 0.0088 & 395.834 & 0.1338 & 515.499 & 0.3572 \\
\hline 297.723 & 0.0366 & 416.534 & 0.1584 & 518.969 & 0.3723 \\
\hline 300.224 & 0.0388 & 424.443 & 0.1684 & 524.848 & 0.4059 \\
\hline 302.653 & 0.0409 & 435.186 & 0.1829 & 530.343 & 0.4710 \\
\hline
\end{tabular}


Table 243. Test data for specimen 73-109

\begin{tabular}{|c|c|c|c|c|c|}
\hline $\begin{array}{l}\text { Test Number } \\
\text { Aging Temp. } \\
\text { Yield Stress }\end{array}$ & $\begin{array}{l}: 2 \\
: 40 \\
: 22\end{array}$ & $\mathrm{MPa}$ & $\begin{array}{l}\text { Tes } \\
\text { Agir } \\
\text { Ulti }\end{array}$ & $\begin{array}{l}\text { np. } \\
\text { ne } \\
\text { Stress }\end{array}$ & $\begin{array}{l}25^{\circ} \mathrm{C} \\
10,000 \mathrm{~h} \\
534.0 \mathrm{MPa}\end{array}$ \\
\hline $\begin{array}{l}\text { Engr. } \\
\text { Stress } \\
(\mathrm{MPa})\end{array}$ & $\begin{array}{l}\text { Engr. } \\
\text { Strain }\end{array}$ & $\begin{array}{l}\text { Engr. } \\
\text { Stress } \\
(\mathrm{MPa})\end{array}$ & $\begin{array}{l}\text { Engr. } \\
\text { Strain }\end{array}$ & $\begin{array}{l}\text { Engr. } \\
\text { Stress } \\
(\mathrm{MPa}) \\
\end{array}$ & $\begin{array}{l}\text { Engr. } \\
\text { Strain }\end{array}$ \\
\hline 32.850 & 0.0001 & 336.327 & 0.0891 & 468.932 & 0.2571 \\
\hline 59.574 & 0.0003 & 337.961 & 0.0911 & 482.822 & 0.2865 \\
\hline 104.921 & 0.0006 & 350.675 & 0.1055 & 489.521 & 0.3036 \\
\hline 127.785 & 0.0008 & 377.216 & 0.1322 & 500.221 & 0.3337 \\
\hline 191.731 & 0.0016 & 390.451 & 0.1468 & 502.823 & 0.3424 \\
\hline 199.177 & 0.0019 & 392.393 & 0.1489 & 510.534 & 0.3706 \\
\hline 205.563 & 0.0020 & 429.614 & 0.1941 & 512.655 & 0.3793 \\
\hline 241.531 & 0.0071 & 431.108 & 0.1962 & 513.667 & 0.3837 \\
\hline 275.601 & 0.0261 & 432.646 & 0.1982 & 526.150 & 0.4512 \\
\hline 293.674 & 0.0435 & 459.852 & 0.2403 & 530.439 & 0.4881 \\
\hline 306.523 & 0.0566 & 461.086 & 0.2424 & 534.006 & 0.5508 \\
\hline
\end{tabular}

Table 244. Test data for specimen 731-04

\begin{tabular}{|c|c|c|c|c|c|}
\hline $\begin{array}{l}\text { Test Number } \\
\text { Aging Temp. } \\
\text { Yield Stress }\end{array}$ & $\begin{array}{l}: 0 \\
: 46 \\
: 25\end{array}$ & $\mathrm{MPa}$ & $\begin{array}{l}\text { Tes } \\
\text { Agi } \\
\text { Ulti }\end{array}$ & $\begin{array}{l}\text { np. } \\
\text { me } \\
\text { Stress }\end{array}$ & $\begin{array}{l}25^{\circ} \mathrm{C} \\
2,570 \mathrm{~h} \\
493.1 \mathrm{MPa}\end{array}$ \\
\hline $\begin{array}{l}\text { Engr. } \\
\text { Stress } \\
\text { (MPa) }\end{array}$ & $\begin{array}{l}\text { Engr. } \\
\text { Strain }\end{array}$ & $\begin{array}{l}\text { Engr. } \\
\text { Stress } \\
\text { (MPa) }\end{array}$ & $\begin{array}{l}\text { Engr. } \\
\text { Strain }\end{array}$ & $\begin{array}{l}\text { Engr. } \\
\text { Stress } \\
(\mathrm{MPa})\end{array}$ & $\begin{array}{l}\text { Engr. } \\
\text { Strain }\end{array}$ \\
\hline 8.29 & 0.0001 & 272.92 & 0.0172 & 435.18 & 0.1900 \\
\hline 22.58 & 0.0007 & 283.53 & 0.0235 & 445.30 & 0.2054 \\
\hline 35.00 & 0.0011 & 289.53 & 0.0277 & 450.97 & 0.2155 \\
\hline 53.14 & 0.0016 & 296.27 & 0.0325 & 458.77 & 0.2295 \\
\hline 82.16 & 0.0024 & 299.32 & 0.0357 & 465.85 & 0.2439 \\
\hline 95.14 & 0.0028 & 311.78 & 0.0465 & 473.01 & 0.2584 \\
\hline 119.59 & 0.0035 & 320.91 & 0.0554 & 475.11 & 0.2631 \\
\hline 159.98 & 0.0048 & 334.02 & 0.0679 & 477.20 & 0.2672 \\
\hline 174.77 & 0.0054 & 347.89 & 0.0828 & 478.09 & 0.2679 \\
\hline 187.55 & 0.0060 & 361.71 & 0.0964 & 478.90 & 0.2758 \\
\hline 203.69 & 0.0064 & 375.19 & 0.1112 & 482.31 & 0.2831 \\
\hline 240.20 & 0.0077 & 387.00 & 0.1248 & 483.18 & 0.2866 \\
\hline 251.80 & 0.0113 & 398.53 & 0.1388 & 488.03 & 0.2992 \\
\hline 260.52 & 0.0134 & 406.89 & 0.1494 & 492.21 & 0.3149 \\
\hline 266.20 & 0.0148 & 416.00 & 0.1617 & 493.08 & 0.3271 \\
\hline 271.89 & 0.0167 & 425.66 & 0.1752 & & \\
\hline
\end{tabular}


Table 245. Test data for specimen 731-05

$\begin{array}{llll}\text { Test Number } & : 011 & \text { Test Temp. } & : 25^{\circ} \mathrm{C} \\ \text { Aging Temp. } & : 450^{\circ} \mathrm{C} & \text { Aging Time } & : 2,570 \mathrm{~h} \\ \text { Yield Stress } & : 234.0 \mathrm{MPa} & \text { Ultimate Stress } & : 570.4 \mathrm{MPa}\end{array}$

\begin{tabular}{rccccc}
\hline $\begin{array}{c}\text { Engr. } \\
\text { Stress } \\
(\mathrm{MPa})\end{array}$ & $\begin{array}{c}\text { Engr. } \\
\text { Strain }\end{array}$ & $\begin{array}{c}\text { Engr. } \\
\text { Stress } \\
(\mathrm{MPa})\end{array}$ & $\begin{array}{c}\text { Engr. } \\
\text { Strain }\end{array}$ & $\begin{array}{c}\text { Engr. } \\
\text { Stress } \\
(\mathrm{MPa})\end{array}$ & $\begin{array}{c}\text { Engr. } \\
\text { Strain }\end{array}$ \\
\hline & & & & & \\
39.16 & 0.0001 & 319.71 & 0.0487 & 512.44 & 0.3145 \\
74.25 & 0.0005 & 335.35 & 0.0634 & 523.58 & 0.3431 \\
107.29 & 0.0011 & 345.23 & 0.0725 & 530.14 & 0.3669 \\
151.01 & 0.0022 & 362.62 & 0.0908 & 536.91 & 0.3889 \\
187.59 & 0.0034 & 379.33 & 0.1076 & 542.07 & 0.4098 \\
201.62 & 0.0039 & 393.56 & 0.1227 & 547.56 & 0.4384 \\
213.94 & 0.0042 & 406.91 & 0.1385 & 553.22 & 0.4654 \\
233.52 & 0.0048 & 418.10 & 0.1514 & 555.89 & 0.4896 \\
244.49 & 0.0060 & 428.97 & 0.1654 & 561.52 & 0.5219 \\
253.92 & 0.0074 & 439.08 & 0.1790 & 564.74 & 0.5496 \\
262.56 & 0.0091 & 447.35 & 0.1907 & 567.28 & 0.5770 \\
270.46 & 0.0116 & 457.55 & 0.2041 & 569.40 & 0.6049 \\
278.53 & 0.0153 & 464.72 & 0.2167 & 568.50 & 0.6194 \\
283.34 & 0.0181 & 479.35 & 0.2410 & 569.29 & 0.6316 \\
294.38 & 0.0260 & 490.43 & 0.2626 & 570.00 & 0.6451 \\
309.61 & 0.0396 & 503.95 & 0.2905 & 570.41 & 0.6604 \\
\hline
\end{tabular}

Table 246. Test data for specimen 733-42

\begin{tabular}{|c|c|c|c|c|c|}
\hline $\begin{array}{l}\text { Test Number } \\
\text { Aging Temp. } \\
\text { Yield Stress }\end{array}$ & $\begin{array}{l}: 0 \\
: U 1 \\
: 13\end{array}$ & $\mathrm{~Pa}$ & $\begin{array}{l}\text { Tes } \\
\text { Agir } \\
\text { Ultir }\end{array}$ & $\begin{array}{l}\text { np. } \\
\text { ne } \\
\text { Stress }\end{array}$ & $\begin{array}{l}290^{\circ} \mathrm{C} \\
- \\
366.6 \mathrm{MPa}\end{array}$ \\
\hline $\begin{array}{l}\text { Engr. } \\
\text { Stress } \\
\text { (MPa) }\end{array}$ & $\begin{array}{l}\text { Engr. } \\
\text { Strain }\end{array}$ & $\begin{array}{l}\text { Engr. } \\
\text { Stress } \\
(\mathrm{MPa}) \\
\end{array}$ & $\begin{array}{l}\text { Engr. } \\
\text { Strain }\end{array}$ & $\begin{array}{l}\text { Engr. } \\
\text { Stress } \\
(\mathrm{MPa}) \\
\end{array}$ & $\begin{array}{l}\text { Engr. } \\
\text { Strain }\end{array}$ \\
\hline 36.89 & 0.0001 & 212.64 & 0.0724 & 363.51 & 0.3141 \\
\hline 81.24 & 0.0006 & 224.78 & 0.0881 & 366.21 & 0.3275 \\
\hline 109.37 & 0.0012 & 234.48 & 0.0987 & 365.23 & 0.3426 \\
\hline 119.43 & 0.0015 & 244.10 & 0.1098 & 361.24 & 0.3507 \\
\hline 126.92 & 0.0019 & 256.89 & 0.1250 & 356.19 & 0.3575 \\
\hline 130.73 & 0.0021 & 269.42 & 0.1402 & 348.37 & 0.3646 \\
\hline 136.31 & 0.0025 & 282.96 & 0.1576 & 339.58 & 0.3712 \\
\hline 140.16 & 0.0029 & 295.72 & 0.1751 & 328.57 & 0.3781 \\
\hline 145.85 & 0.0037 & 307.50 & 0.1927 & 311.87 & 0.3865 \\
\hline 151.22 & 0.0054 & 317.37 & 0.2092 & 297.48 & 0.3923 \\
\hline 162.25 & 0.0128 & 328.94 & 0.2295 & 281.67 & 0.3979 \\
\hline 174.94 & 0.0265 & 338.01 & 0.2474 & 269.39 & 0.4014 \\
\hline 191.15 & 0.0478 & 345.53 & 0.2629 & 253.78 & 0.4040 \\
\hline 201.33 & 0.0605 & 356.49 & 0.2925 & 234.47 & 0.4047 \\
\hline
\end{tabular}


Table 247. Test data for specimen 734-40

\begin{tabular}{|c|c|c|c|c|c|}
\hline $\begin{array}{l}\text { Test Number } \\
\text { Aging Temp. } \\
\text { Yield Stress }\end{array}$ & $\begin{array}{l}: 0 \\
: U 1 \\
: 13\end{array}$ & & $\begin{array}{l}\text { Tes } \\
\text { Agi } \\
\text { Ulti }\end{array}$ & $\begin{array}{l}\text { np. } \\
\text { ne } \\
\text { Stress }\end{array}$ & $\begin{array}{l}290^{\circ} \mathrm{C} \\
- \\
365.0 \mathrm{MPa}\end{array}$ \\
\hline $\begin{array}{l}\text { Engr. } \\
\text { Stress } \\
(\mathrm{MPa}) \\
\end{array}$ & $\begin{array}{l}\text { Engr. } \\
\text { Strain }\end{array}$ & $\begin{array}{l}\text { Engr. } \\
\text { Stress } \\
(\mathrm{MPa}) \\
\end{array}$ & $\begin{array}{l}\text { Engr. } \\
\text { Strain }\end{array}$ & $\begin{array}{l}\text { Engr. } \\
\text { Stress } \\
(\mathrm{MPa}) \\
\end{array}$ & $\begin{array}{l}\text { Engr. } \\
\text { Strain }\end{array}$ \\
\hline 29.51 & 0.0001 & 143.94 & 0.0052 & 263.42 & 0.1246 \\
\hline 45.17 & 0.0003 & 146.52 & 0.0059 & 276.04 & 0.1384 \\
\hline 55.34 & 0.0004 & 147.73 & 0.0063 & 287.41 & 0.1516 \\
\hline 66.93 & 0.0006 & 149.18 & 0.0069 & 300.80 & 0.1673 \\
\hline 80.09 & 0.0008 & 151.89 & 0.0082 & 309.67 & 0.1788 \\
\hline 91.18 & 0.0011 & 156.16 & 0.0109 & 317.74 & 0.1894 \\
\hline 102.23 & 0.0014 & 163.03 & 0.0162 & 321.70 & 0.1945 \\
\hline 109.98 & 0.0017 & 169.47 & 0.0225 & 328.21 & 0.2046 \\
\hline 116.46 & 0.0019 & 175.20 & 0.0282 & 333.18 & 0.2119 \\
\hline 120.34 & 0.0021 & 183.59 & 0.0377 & 340.54 & 0.2247 \\
\hline 123.08 & 0.0023 & 193.33 & 0.0489 & 349.87 & 0.2417 \\
\hline 127.10 & 0.0026 & 200.62 & 0.0567 & 357.93 & 0.2578 \\
\hline 130.18 & 0.0029 & 206.60 & 0.0636 & 361.61 & 0.2689 \\
\hline 133.99 & 0.0033 & 214.43 & 0.0721 & 362.49 & 0.2742 \\
\hline 137.27 & 0.0037 & 226.27 & 0.0856 & 364.09 & 0.2765 \\
\hline 139.41 & 0.0041 & 241.51 & 0.1016 & 364.97 & 0.2775 \\
\hline 141.80 & 0.0046 & 251.03 & 0.1112 & & \\
\hline
\end{tabular}

Table 248. Test data for specimen 734-19

$\begin{array}{llll}\text { Test Number } & : 338 & \text { Test Temp. } & : 290^{\circ} \mathrm{C} \\ \text { Aging Temp. } & : 320^{\circ} \mathrm{C} & \text { Aging Time } & : 10,000 \mathrm{~h} \\ \text { Yield Stress } & : 130.4 \mathrm{MPa} & \text { Ultimate Stress } & : 354.0 \mathrm{MPa}\end{array}$

\begin{tabular}{rccccc}
\hline $\begin{array}{c}\text { Engr. } \\
\text { Stress } \\
(\mathrm{MPa})\end{array}$ & $\begin{array}{c}\text { Engr. } \\
\text { Strain }\end{array}$ & $\begin{array}{c}\text { Engr. } \\
\text { Stress } \\
(\mathrm{MPa})\end{array}$ & $\begin{array}{c}\text { Engr. } \\
\text { Strain }\end{array}$ & $\begin{array}{c}\text { Engr. } \\
\text { Stress } \\
(\mathrm{MPa})\end{array}$ & $\begin{array}{c}\text { Engr. } \\
\text { Strain }\end{array}$ \\
\hline & & & & & \\
15.3600 & 0.0001 & 183.1900 & 0.0425 & 299.4700 & 0.1677 \\
69.6300 & 0.0003 & 185.4900 & 0.0447 & 302.7600 & 0.1720 \\
104.8700 & 0.0007 & 194.5700 & 0.0535 & 307.6400 & 0.1787 \\
110.7800 & 0.0010 & 199.0200 & 0.0580 & 310.7900 & 0.1831 \\
127.6700 & 0.0022 & 205.7200 & 0.0646 & 318.4200 & 0.1942 \\
132.8700 & 0.0032 & 207.9000 & 0.0668 & 321.8800 & 0.2013 \\
138.5800 & 0.0051 & 214.4600 & 0.0733 & 327.5000 & 0.2104 \\
142.7900 & 0.0071 & 225.2300 & 0.0842 & 332.8600 & 0.2210 \\
155.3400 & 0.0164 & 235.9500 & 0.0952 & 339.4200 & 0.2327 \\
157.4800 & 0.0184 & 240.1900 & 0.0996 & 342.1600 & 0.2417 \\
164.7700 & 0.0248 & 248.4200 & 0.1082 & 343.9100 & 0.2441 \\
167.1800 & 0.0272 & 258.5700 & 0.1192 & 346.2900 & 0.2511 \\
169.5200 & 0.0294 & 268.5200 & 0.1302 & 350.5000 & 0.2631 \\
171.5200 & 0.0314 & 278.0600 & 0.1412 & 352.3400 & 0.2722 \\
173.8500 & 0.0337 & 285.5100 & 0.1500 & 353.9600 & 0.2833 \\
180.8700 & 0.0403 & 292.5200 & 0.1588 & & \\
\hline
\end{tabular}


Table 249. Test data for specimen 734-22

\begin{tabular}{|c|c|c|c|c|c|}
\hline $\begin{array}{l}\text { Test Number } \\
\text { Aging Temp. } \\
\text { Yield Stress }\end{array}$ & $\begin{array}{l}: 3 \\
: 32 \\
: 13\end{array}$ & & $\begin{array}{l}\text { Tes } \\
\text { Agi } \\
\text { Ulti }\end{array}$ & $\begin{array}{l}\text { np. } \\
\text { ne } \\
\text { Stress }\end{array}$ & $\begin{array}{l}290^{\circ} \mathrm{C} \\
10,000 \mathrm{~h} \\
365.2 \mathrm{MPa}\end{array}$ \\
\hline $\begin{array}{l}\text { Engr. } \\
\text { Stress } \\
\text { (MPa) }\end{array}$ & $\begin{array}{l}\text { Engr. } \\
\text { Strain }\end{array}$ & $\begin{array}{l}\text { Engr. } \\
\text { Stress } \\
(\mathrm{MPa})\end{array}$ & $\begin{array}{l}\text { Engr. } \\
\text { Strain }\end{array}$ & $\begin{array}{l}\text { Engr. } \\
\text { Stress } \\
(\mathrm{MPa}) \\
\end{array}$ & $\begin{array}{l}\text { Engr. } \\
\text { Strain }\end{array}$ \\
\hline 25.2600 & 0.0001 & 186.8700 & 0.0573 & 294.7900 & 0.2073 \\
\hline 74.7300 & 0.0002 & 188.6900 & 0.0595 & 298.8700 & 0.2118 \\
\hline 116.3100 & 0.0008 & 190.5500 & 0.0617 & 304.4800 & 0.2211 \\
\hline 122.9900 & 0.0012 & 197.7900 & 0.0707 & 311.3200 & 0.2363 \\
\hline 138.9000 & 0.0053 & 201.4500 & 0.0750 & 317.7300 & 0.2477 \\
\hline 142.2500 & 0.0075 & 208.6400 & 0.0840 & 321.0300 & 0.2518 \\
\hline 145.0700 & 0.0096 & 212.1700 & 0.0884 & 323.9500 & 0.2606 \\
\hline 154.4700 & 0.0190 & 219.2000 & 0.0971 & 329.1500 & 0.2736 \\
\hline 156.1900 & 0.0210 & 222.6800 & 0.1016 & 334.0800 & 0.2822 \\
\hline 158.3200 & 0.0233 & 227.6500 & 0.1080 & 338.1800 & 0.2961 \\
\hline 160.2700 & 0.0257 & 229.3300 & 0.1100 & 342.3000 & 0.3068 \\
\hline 162.3500 & 0.0280 & 239.5200 & 0.1233 & 346.3900 & 0.3221 \\
\hline 164.2800 & 0.0304 & 255.9800 & 0.1453 & 349.3300 & 0.3283 \\
\hline 171.9400 & 0.0394 & 265.4000 & 0.1586 & 356.7800 & 0.3503 \\
\hline 173.8900 & 0.0418 & 275.3500 & 0.1723 & 360.9500 & 0.3657 \\
\hline 175.7700 & 0.0440 & 284.1800 & 0.1855 & 360.1700 & 0.3720 \\
\hline 177.5900 & 0.0462 & 289.4300 & 0.1940 & 365.2000 & 0.3851 \\
\hline
\end{tabular}

Table 250. Test data for specimen 732-27

\begin{tabular}{|c|c|c|c|c|c|}
\hline $\begin{array}{l}\text { Test Number } \\
\text { Aging Temp. } \\
\text { Yield Stress }\end{array}$ & $\begin{array}{l}: 0 \\
: 35 \\
: 13\end{array}$ & & $\begin{array}{l}\text { Tes } \\
\text { Agi } \\
\text { Ulti }\end{array}$ & $\begin{array}{l}\text { np. } \\
\text { ne } \\
\text { Stress }\end{array}$ & $\begin{array}{l}290^{\circ} \mathrm{C} \\
2570 \mathrm{~h} \\
419.8 \mathrm{MPa}\end{array}$ \\
\hline $\begin{array}{l}\text { Engr. } \\
\text { Stress } \\
(\mathrm{MPa})\end{array}$ & $\begin{array}{l}\text { Engr. } \\
\text { Strain }\end{array}$ & $\begin{array}{l}\text { Engr. } \\
\text { Stress } \\
\text { (MPa) }\end{array}$ & $\begin{array}{l}\text { Engr. } \\
\text { Strain }\end{array}$ & $\begin{array}{l}\text { Engr. } \\
\text { Stress } \\
(\mathrm{MPa}) \\
\end{array}$ & $\begin{array}{l}\text { Engr. } \\
\text { Strain }\end{array}$ \\
\hline 54.670 & 0.004 & 150.050 & 0.014 & 276.170 & 0.152 \\
\hline 62.100 & 0.004 & 155.060 & 0.016 & 344.910 & 0.255 \\
\hline 102.100 & 0.007 & 161.050 & 0.018 & 356.690 & 0.276 \\
\hline 113.460 & 0.008 & 165.610 & 0.021 & 392.930 & 0.360 \\
\hline 122.810 & 0.009 & 171.140 & 0.025 & 407.250 & 0.407 \\
\hline 128.860 & 0.010 & 197.890 & 0.053 & 416.120 & 0.446 \\
\hline 140.410 & 0.012 & 205.220 & 0.062 & 418.900 & 0.460 \\
\hline 145.130 & 0.013 & 260.650 & 0.131 & 419.840 & 0.505 \\
\hline
\end{tabular}


Table 251. Test data for specimen 734-08

\begin{tabular}{|c|c|c|c|c|c|}
\hline $\begin{array}{l}\text { Test Number } \\
\text { Aging Temp. } \\
\text { Yield Stress }\end{array}$ & $\begin{array}{l}: 3 \\
: 35 \\
: 12\end{array}$ & & $\begin{array}{l}\text { Tes } \\
\text { Agi } \\
\text { Ulti }\end{array}$ & $\begin{array}{l}\text { np. } \\
\text { ne } \\
\text { Stress }\end{array}$ & $\begin{array}{l}290^{\circ} \mathrm{C} \\
10,000 \mathrm{~h} \\
377.7 \mathrm{MPa}\end{array}$ \\
\hline $\begin{array}{l}\text { Engr. } \\
\text { Stress } \\
\text { (MPa) }\end{array}$ & $\begin{array}{l}\text { Engr. } \\
\text { Strain }\end{array}$ & $\begin{array}{l}\text { Engr. } \\
\text { Stress } \\
\text { (MPa) }\end{array}$ & $\begin{array}{l}\text { Engr. } \\
\text { Strain }\end{array}$ & $\begin{array}{l}\text { Engr. } \\
\text { Stress } \\
(\mathrm{MPa}) \\
\end{array}$ & $\begin{array}{l}\text { Engr. } \\
\text { Strain }\end{array}$ \\
\hline 14.990 & 0.000 & 201.880 & 0.054 & 300.510 & 0.159 \\
\hline 53.340 & 0.000 & 220.140 & 0.072 & 307.360 & 0.168 \\
\hline 81.630 & 0.001 & 222.370 & 0.074 & 320.220 & 0.186 \\
\hline 109.700 & 0.001 & 231.220 & 0.083 & 326.300 & 0.195 \\
\hline 122.980 & 0.002 & 248.340 & 0.100 & 340.890 & 0.220 \\
\hline 139.960 & 0.006 & 250.480 & 0.102 & 342.320 & 0.222 \\
\hline 162.010 & 0.019 & 256.640 & 0.109 & 355.150 & 0.248 \\
\hline 169.910 & 0.025 & 272.720 & 0.126 & 356.480 & 0.251 \\
\hline 172.570 & 0.028 & 274.650 & 0.128 & 366.090 & 0.278 \\
\hline 192.480 & 0.045 & 280.390 & 0.135 & 374.820 & 0.304 \\
\hline 194.840 & 0.048 & 293.380 & 0.150 & 377.640 & 0.329 \\
\hline
\end{tabular}

Table 252. Test data for specimen 734-09

\begin{tabular}{|c|c|c|c|c|c|}
\hline $\begin{array}{l}\text { Test Number } \\
\text { Aging Temp. } \\
\text { Yield Stress }\end{array}$ & $\begin{array}{l}: 3 \\
: 35 \\
: 13\end{array}$ & $\mathrm{~Pa}$ & $\begin{array}{l}\text { Tes } \\
\text { Agi } \\
\text { Ulti }\end{array}$ & $\begin{array}{l}\text { np. } \\
\text { ne } \\
\text { Stress }\end{array}$ & $\begin{array}{l}290^{\circ} \mathrm{C} \\
10,000 \mathrm{~h} \\
419.8 \mathrm{MPa}\end{array}$ \\
\hline $\begin{array}{l}\text { Engr. } \\
\text { Stress } \\
\text { (MPa) }\end{array}$ & $\begin{array}{l}\text { Engr. } \\
\text { Strain }\end{array}$ & $\begin{array}{l}\text { Engr. } \\
\text { Stress } \\
\text { (MPa) }\end{array}$ & $\begin{array}{l}\text { Engr. } \\
\text { Strain }\end{array}$ & $\begin{array}{l}\text { Engr. } \\
\text { Stress } \\
(\mathrm{MPa})\end{array}$ & $\begin{array}{l}\text { Engr. } \\
\text { Strain }\end{array}$ \\
\hline 69.570 & 0.000 & 227.650 & 0.035 & 326.370 & 0.107 \\
\hline 91.750 & 0.000 & 230.960 & 0.037 & 331.810 & 0.111 \\
\hline 123.330 & 0.000 & 249.940 & 0.051 & 352.110 & 0.128 \\
\hline 131.620 & 0.000 & 253.160 & 0.053 & 356.880 & 0.132 \\
\hline 143.360 & 0.001 & 262.310 & 0.059 & 372.750 & 0.148 \\
\hline 150.760 & 0.001 & 274.630 & 0.068 & 379.020 & 0.154 \\
\hline 156.890 & 0.001 & 277.660 & 0.070 & 390.760 & 0.167 \\
\hline 188.280 & 0.010 & 280.660 & 0.072 & 392.550 & 0.170 \\
\hline 192.590 & 0.013 & 298.340 & 0.085 & 406.350 & 0.190 \\
\hline 196.580 & 0.015 & 301.230 & 0.087 & 414.750 & 0.206 \\
\hline 217.920 & 0.029 & 309.760 & 0.094 & 417.400 & 0.221 \\
\hline
\end{tabular}

Table 253. Test data for specimen 73-120

$\begin{array}{llll}\text { Test Number } & : 335 & \text { Test Temp. } & : 290^{\circ} \mathrm{C} \\ \text { Aging Temp. } & : 350^{\circ} \mathrm{C} & \text { Aging Time } & : 10,000 \mathrm{~h} \\ \text { Yield Stress } & : 136.7 \mathrm{MPa} & \text { Ultimate Stress } & : 386.4 \mathrm{MPa}\end{array}$

Experimental Data Not Available. 
Table 254. Test data for specimen 732-18

\begin{tabular}{|c|c|c|c|c|c|}
\hline $\begin{array}{l}\text { Test Number } \\
\text { Material Type } \\
\text { Aging Temp. } \\
\text { Yield Stress }\end{array}$ & $\begin{array}{l}: 0 \\
: \mathrm{CF} \\
: 35 \\
: 12\end{array}$ & & $\begin{array}{l}\text { Tes } \\
\text { He } \\
\text { Agi } \\
\text { Ulti }\end{array}$ & $\begin{array}{l}\text { np. } \\
\text { mber } \\
\text { me } \\
\text { Stress }\end{array}$ & $\begin{array}{l}290^{\circ} \mathrm{C} \\
73 \\
2,570 \mathrm{~h} \\
444.1 \mathrm{MPa}\end{array}$ \\
\hline $\begin{array}{l}\text { Engr. } \\
\text { Stress } \\
\text { (MPa) }\end{array}$ & $\begin{array}{l}\text { Engr. } \\
\text { Strain }\end{array}$ & $\begin{array}{l}\text { Engr. } \\
\text { Stress } \\
(\mathrm{MPa})\end{array}$ & $\begin{array}{l}\text { Engr. } \\
\text { Strain }\end{array}$ & $\begin{array}{l}\text { Engr. } \\
\text { Stress } \\
(\mathrm{MPa}) \\
\end{array}$ & $\begin{array}{l}\text { Engr. } \\
\text { Strain }\end{array}$ \\
\hline 32.15 & 0.0001 & 189.17 & 0.0034 & 36551 & 0.1686 \\
\hline 47.62 & 0.0001 & 189.26 & 0.0037 & 378.04 & 0.1841 \\
\hline 64.14 & 0.0003 & 189.77 & 0.0042 & 390.04 & 0.2007 \\
\hline 85.81 & 0.0005 & 203.66 & 0.0128 & 400.67 & 0.2173 \\
\hline 109.85 & 0.0008 & 216.66 & 0.0234 & 411.57 & 0.2361 \\
\hline 137.33 & 0.0013 & 233.49 & 0.0387 & 420.82 & 0.2548 \\
\hline 160.82 & 0.0018 & 254.17 & 0.0579 & 428.34 & 0.2732 \\
\hline 176.05 & 0.0022 & 270.95 & 0.0727 & 433.46 & 0.2884 \\
\hline 189.27 & 0.0026 & 288.48 & 0.0899 & 435.29 & 0.2929 \\
\hline 189.36 & 0.0028 & 308.44 & 0.1079 & 436.81 & 0.2991 \\
\hline 189.25 & 0.0029 & 322.78 & 0.1219 & 439.73 & 0.3110 \\
\hline 189.19 & 0.0031 & 337.39 & 0.1368 & 441.12 & 0.3270 \\
\hline 189.23 & 0.0032 & 352.49 & 0.1533 & & \\
\hline
\end{tabular}

Table 255. Test data for specimen 732-23

\begin{tabular}{|c|c|c|c|c|c|}
\hline $\begin{array}{l}\text { Test Number } \\
\text { Aging Temp. } \\
\text { Yield Stress }\end{array}$ & $\begin{array}{l}: 2 \\
: 4 c \\
: 13\end{array}$ & & $\begin{array}{l}\text { Tes } \\
\text { Agi } \\
\text { Ulti }\end{array}$ & $\begin{array}{l}\mathrm{np} . \\
\text { me } \\
\text { Stress }\end{array}$ & $\begin{array}{l}290^{\circ} \mathrm{C} \\
10,000 \mathrm{~h} \\
408.5 \mathrm{MPa}\end{array}$ \\
\hline $\begin{array}{l}\text { Engr. } \\
\text { Stress } \\
(\mathrm{MPa})\end{array}$ & $\begin{array}{l}\text { Engr. } \\
\text { Strain }\end{array}$ & $\begin{array}{l}\text { Engr. } \\
\text { Stress } \\
(\mathrm{MPa})\end{array}$ & $\begin{array}{l}\text { Engr. } \\
\text { Strain }\end{array}$ & $\begin{array}{l}\text { Engr. } \\
\text { Stress } \\
(\mathrm{MPa}) \\
\end{array}$ & $\begin{array}{l}\text { Engr. } \\
\text { Strain }\end{array}$ \\
\hline 39.952 & 0.0002 & 224.072 & 0.0589 & 319.338 & 0.1596 \\
\hline 41.205 & 0.0002 & 225.843 & 0.0609 & 324.781 & 0.1661 \\
\hline 76.550 & 0.0003 & 241.520 & 0.0761 & 337.125 & 0.1817 \\
\hline 105.553 & 0.0006 & 248.507 & 0.0830 & 345.640 & 0.1930 \\
\hline 133.524 & 0.0020 & 250.679 & 0.0851 & 365.740 & 0.2249 \\
\hline 138.580 & 0.0027 & 265.926 & 0.1006 & 371.130 & 0.2342 \\
\hline 161.457 & 0.0080 & 276.716 & 0.1120 & 387.910 & 0.2681 \\
\hline 184.842 & 0.0236 & 278.772 & 0.1142 & 388.747 & 0.2702 \\
\hline 195.375 & 0.0325 & 294.879 & 0.1318 & 399.140 & 0.2986 \\
\hline 197.891 & 0.0349 & 304.673 & 0.1424 & 403.988 & 0.3157 \\
\hline 214.836 & 0.0502 & 317.504 & 0.1575 & 408.483 & 0.3442 \\
\hline
\end{tabular}


Table 256. Test data for specimen 732-24

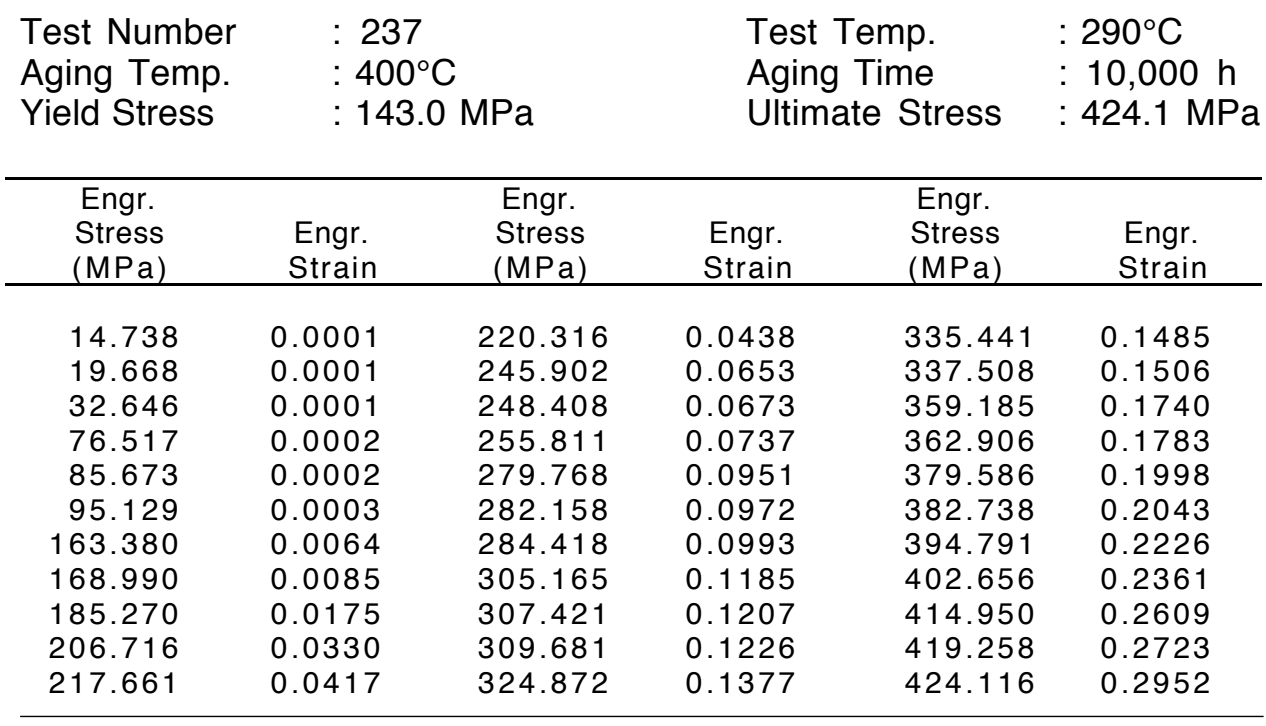

Table 257. Test data for specimen 73-110

\begin{tabular}{|c|c|c|c|c|c|}
\hline $\begin{array}{l}\text { Test Number } \\
\text { Aging Temp. } \\
\text { Yield Stress }\end{array}$ & $\begin{array}{l}: 2 \\
: 40 \\
: 13\end{array}$ & $\mathrm{MPa}$ & $\begin{array}{l}\text { Tes } \\
\text { Agi } \\
\text { Ulti }\end{array}$ & $\begin{array}{l}\text { mp. } \\
\text { me } \\
\text { Stress }\end{array}$ & $\begin{array}{l}290^{\circ} \mathrm{C} \\
10,000 \mathrm{~h} \\
395.8 \mathrm{MPa}\end{array}$ \\
\hline $\begin{array}{l}\text { Engr. } \\
\text { Stress } \\
(\mathrm{MPa})\end{array}$ & $\begin{array}{l}\text { Engr. } \\
\text { Strain }\end{array}$ & $\begin{array}{l}\text { Engr. } \\
\text { Stress } \\
(\mathrm{MPa})\end{array}$ & $\begin{array}{l}\text { Engr. } \\
\text { Strain }\end{array}$ & $\begin{array}{l}\text { Engr. } \\
\text { Stress } \\
(\mathrm{MPa}) \\
\end{array}$ & $\begin{array}{l}\text { Engr. } \\
\text { Strain }\end{array}$ \\
\hline 42.550 & 0.0002 & 238.341 & 0.0716 & 331.449 & 0.1747 \\
\hline 59.248 & 0.0002 & 256.103 & 0.0890 & 332.988 & 0.1769 \\
\hline 71.576 & 0.0002 & 268.974 & 0.1021 & 349.763 & 0.2022 \\
\hline 153.928 & 0.0059 & 271.016 & 0.1045 & 351.137 & 0.2047 \\
\hline 159.463 & 0.0078 & 289.712 & 0.1244 & 364.327 & 0.2276 \\
\hline 164.147 & 0.0101 & 291.676 & 0.1265 & 365.565 & 0.2299 \\
\hline 202.531 & 0.0389 & 293.907 & 0.1290 & 371.159 & 0.2410 \\
\hline 205.178 & 0.0412 & 314.548 & 0.1532 & 378.871 & 0.2583 \\
\hline 207.679 & 0.0435 & 316.387 & 0.1554 & 388.790 & 0.2863 \\
\hline 226.980 & 0.0609 & 318.104 & 0.1577 & 390.460 & 0.2910 \\
\hline 236.091 & 0.0696 & 329.683 & 0.1726 & 395.782 & 0.3195 \\
\hline
\end{tabular}


Table 258. Test data for specimen 731-06

\begin{tabular}{|c|c|c|c|c|c|}
\hline $\begin{array}{l}\text { Test Number } \\
\text { Aging Temp. } \\
\text { Yield Stress }\end{array}$ & $\begin{array}{l}: 0 \\
: 45 \\
: 14\end{array}$ & & $\begin{array}{l}\text { Tes } \\
\text { Agi } \\
\text { Ulti }\end{array}$ & $\begin{array}{l}\text { np. } \\
\text { me } \\
\text { Stress }\end{array}$ & $\begin{array}{l}290^{\circ} \mathrm{C} \\
2,570 \mathrm{~h} \\
411.2 \mathrm{MPa}\end{array}$ \\
\hline $\begin{array}{l}\text { Engr. } \\
\text { Stress } \\
(\mathrm{MPa})\end{array}$ & $\begin{array}{l}\text { Engr. } \\
\text { Strain }\end{array}$ & $\begin{array}{l}\text { Engr. } \\
\text { Stress } \\
(\mathrm{MPa})\end{array}$ & $\begin{array}{l}\text { Engr. } \\
\text { Strain }\end{array}$ & $\begin{array}{l}\text { Engr. } \\
\text { Stress } \\
(\mathrm{MPa}) \\
\end{array}$ & $\begin{array}{l}\text { Engr. } \\
\text { Strain }\end{array}$ \\
\hline 29.02 & 0.0001 & 186.38 & 0.0130 & 332.89 & 0.1288 \\
\hline 65.85 & 0.0004 & 190.12 & 0.0151 & 350.86 & 0.1449 \\
\hline 105.44 & 0.0010 & 195.94 & 0.0187 & 361.90 & 0.1562 \\
\hline 119.94 & 0.0013 & 200.74 & 0.0219 & 372.27 & 0.1668 \\
\hline 132.67 & 0.0018 & 205.77 & 0.0259 & 384.53 & 0.1808 \\
\hline 141.35 & 0.0024 & 215.21 & 0.0333 & 390.69 & 0.1887 \\
\hline 149.35 & 0.0031 & 226.34 & 0.0425 & 394.06 & 0.1937 \\
\hline 157.58 & 0.0041 & 239.92 & 0.0534 & 396.56 & 0.1981 \\
\hline 162.67 & 0.0050 & 254.04 & 0.0643 & 400.20 & 0.2041 \\
\hline 167.37 & 0.0061 & 268.83 & 0.0754 & 405.92 & 0.2139 \\
\hline 171.76 & 0.0073 & 280.80 & 0.0860 & 409.11 & 0.2204 \\
\hline 174.93 & 0.0083 & 292.82 & 0.0954 & 410.07 & 0.2234 \\
\hline 177.95 & 0.0094 & 305.56 & 0.1056 & 411.19 & 0.2260 \\
\hline 181.65 & 0.0109 & 319.21 & 0.1168 & & \\
\hline
\end{tabular}

Table 259. Test data for specimen 732-09

\begin{tabular}{|c|c|c|c|c|c|}
\hline $\begin{array}{l}\text { Test Number } \\
\text { Aging Temp. } \\
\text { Yield Stress }\end{array}$ & $\begin{array}{l}: 0 \\
: 45 \\
: 13\end{array}$ & & $\begin{array}{l}\text { Tes } \\
\text { Agi } \\
\text { Ulti }\end{array}$ & $\begin{array}{l}\text { ne. } \\
\text { Stress }\end{array}$ & $\begin{array}{l}290^{\circ} \mathrm{C} \\
2,570 \mathrm{~h} \\
436.1 \mathrm{MPa}\end{array}$ \\
\hline $\begin{array}{l}\text { Engr. } \\
\text { Stress } \\
(\mathrm{MPa})\end{array}$ & $\begin{array}{l}\text { Engr. } \\
\text { Strain }\end{array}$ & $\begin{array}{l}\text { Engr. } \\
\text { Stress } \\
\text { (MPa) }\end{array}$ & $\begin{array}{l}\text { Engr. } \\
\text { Strain }\end{array}$ & $\begin{array}{l}\text { Engr. } \\
\text { Stress } \\
\text { (MPa) }\end{array}$ & $\begin{array}{l}\text { Engr. } \\
\text { Strain }\end{array}$ \\
\hline 37.5850 & 0.0001 & 171.4200 & 0.0100 & 381.2500 & 0.2277 \\
\hline 69.3680 & 0.0003 & 176.4600 & 0.0126 & 391.7700 & 0.2460 \\
\hline 102.0400 & 0.0008 & 186.5000 & 0.0189 & 396.7800 & 0.2542 \\
\hline 114.3600 & 0.0011 & 197.4700 & 0.0279 & 405.0100 & 0.2717 \\
\hline 124.7100 & 0.0014 & 211.5200 & 0.0413 & 410.7000 & 0.2872 \\
\hline 129.8600 & 0.0017 & 229.1000 & 0.0578 & 417.4200 & 0.3029 \\
\hline 134.2700 & 0.0020 & 246.5600 & 0.0739 & 423.5200 & 0.3173 \\
\hline 137.9400 & 0.0023 & 267.6000 & 0.0945 & 427.7300 & 0.3357 \\
\hline 141.2700 & 0.0027 & 288.8900 & 0.1150 & 429.2200 & 0.3470 \\
\hline 145.0300 & 0.0032 & 309.2100 & 0.1353 & 431.7900 & 0.3501 \\
\hline 147.6700 & 0.0035 & 330.8000 & 0.1595 & 433.2200 & 0.3636 \\
\hline 150.5000 & 0.0039 & 344.6900 & 0.1765 & 432.2100 & 0.3687 \\
\hline 154.3100 & 0.0046 & 357.7500 & 0.1931 & 435.9300 & 0.3796 \\
\hline 160.1600 & 0.0059 & 369.5400 & 0.2100 & 436.0800 & 0.3807 \\
\hline 166.7300 & 0.0079 & & & & \\
\hline
\end{tabular}


Table 260. Test data for specimen 205-26

\begin{tabular}{|c|c|c|c|c|c|}
\hline $\begin{array}{l}\text { Test Number } \\
\text { Material Type } \\
\text { Aging Temp. } \\
\text { Yield Stress }\end{array}$ & $\begin{array}{l}: 0 \\
: \mathrm{CF} \\
: 4 C \\
: 24\end{array}$ & & $\begin{array}{l}\text { Tes } \\
\text { Hec } \\
\text { Agi } \\
\text { Ulti }\end{array}$ & $\begin{array}{l}\text { mp. } \\
\text { mber } \\
\text { me } \\
\text { Stress }\end{array}$ & $\begin{array}{l}25^{\circ} \mathrm{C} \\
205 \\
18,000 \mathrm{~h} \\
670.6 \mathrm{MPa}\end{array}$ \\
\hline $\begin{array}{l}\text { Engr. } \\
\text { Stress } \\
(\mathrm{MPa}) \\
\end{array}$ & $\begin{array}{l}\text { Engr. } \\
\text { Strain }\end{array}$ & $\begin{array}{l}\text { Engr. } \\
\text { Stress } \\
(\mathrm{MPa})\end{array}$ & $\begin{array}{l}\text { Engr. } \\
\text { Strain }\end{array}$ & $\begin{array}{l}\text { Engr. } \\
\text { Stress } \\
(\mathrm{MPa}) \\
\end{array}$ & $\begin{array}{l}\text { Engr. } \\
\text { Strain }\end{array}$ \\
\hline 191.30 & 0.0016 & 431.56 & 0.0324 & 600.09 & 0.1400 \\
\hline 216.73 & 0.0018 & 445.23 & 0.0383 & 607.97 & 0.1484 \\
\hline 234.10 & 0.0020 & 458.65 & 0.0452 & 621.82 & 0.1670 \\
\hline 254.13 & 0.0026 & 474.03 & 0.0537 & 624.86 & 0.1723 \\
\hline 276.36 & 0.0037 & 482.16 & 0.0583 & 628.61 & 0.1762 \\
\hline 297.46 & 0.0048 & 501.75 & 0.0695 & 630.48 & 0.1811 \\
\hline 308.01 & 0.0055 & 513.98 & 0.0769 & 636.90 & 0.1895 \\
\hline 321.36 & 0.0066 & 528.91 & 0.0863 & 640.61 & 0.1953 \\
\hline 332.49 & 0.0079 & 535.40 & 0.0904 & 642.88 & 0.1995 \\
\hline 344.52 & 0.0094 & 546.34 & 0.0979 & 643.48 & 0.2041 \\
\hline 356.19 & 0.0111 & 556.40 & 0.1026 & 645.47 & 0.2076 \\
\hline 365.26 & 0.0126 & 564.22 & 0.1085 & 647.37 & 0.2111 \\
\hline 374.73 & 0.0144 & 568.76 & 0.1122 & 648.22 & 0.2179 \\
\hline 382.19 & 0.0161 & 573.68 & 0.1160 & 651.78 & 0.2214 \\
\hline 389.35 & 0.0179 & 578.41 & 0.1201 & 659.84 & 0.2267 \\
\hline 397.58 & 0.0202 & 586.31 & 0.1274 & 659.19 & 0.2298 \\
\hline 410.97 & 0.0244 & 590.64 & 0.1318 & 660.80 & 0.2337 \\
\hline 424.59 & 0.0293 & 596.05 & 0.1360 & 663.88 & 0.2517 \\
\hline
\end{tabular}

Table 261. Test data for specimen 205-27

\begin{tabular}{|c|c|c|c|c|c|}
\hline $\begin{array}{l}\text { Test Number } \\
\text { Material Type } \\
\text { Aging Temp. } \\
\text { Yield Stress }\end{array}$ & $\begin{array}{l}: 1 \\
: \mathrm{CF} \\
: 40 \\
: 25\end{array}$ & $\mathrm{MPa}$ & $\begin{array}{l}\text { Tes } \\
\text { Hea } \\
\text { Agi } \\
\text { Ulti }\end{array}$ & $\begin{array}{l}\text { mp. } \\
\text { mber } \\
\text { me } \\
\text { Stress }\end{array}$ & $\begin{array}{l}25^{\circ} \mathrm{C} \\
205 \\
18,000 \mathrm{~h} \\
642.4 \mathrm{MPa}\end{array}$ \\
\hline $\begin{array}{l}\text { Engr. } \\
\text { Stress } \\
(\mathrm{MPa}) \\
\end{array}$ & $\begin{array}{l}\text { Engr. } \\
\text { Strain }\end{array}$ & $\begin{array}{l}\text { Engr. } \\
\text { Stress } \\
(\mathrm{MPa}) \\
\end{array}$ & $\begin{array}{l}\text { Engr. } \\
\text { Strain }\end{array}$ & $\begin{array}{l}\text { Engr. } \\
\text { Stress } \\
\text { (MPa) } \\
\end{array}$ & $\begin{array}{l}\text { Engr. } \\
\text { Strain }\end{array}$ \\
\hline 17.540 & 0.0001 & 368.255 & 0.0196 & 572.446 & 0.1578 \\
\hline 30.976 & 0.0002 & 410.249 & 0.0349 & 590.300 & 0.1827 \\
\hline 41.902 & 0.0002 & 427.740 & 0.0433 & 592.913 & 0.1868 \\
\hline 53.175 & 0.0003 & 457.991 & 0.0600 & 607.912 & 0.2140 \\
\hline 122.255 & 0.0006 & 461.189 & 0.0621 & 614.783 & 0.2288 \\
\hline 134.240 & 0.0007 & 489.417 & 0.0807 & 620.492 & 0.2434 \\
\hline 193.178 & 0.0011 & 492.320 & 0.0827 & 625.766 & 0.2584 \\
\hline 269.980 & 0.0044 & 513.803 & 0.0992 & 628.863 & 0.2691 \\
\hline 278.196 & 0.0051 & 542.786 & 0.1251 & 635.540 & 0.2974 \\
\hline 285.976 & 0.0058 & 544.818 & 0.1271 & 637.911 & 0.3108 \\
\hline 362.193 & 0.0180 & 570.946 & 0.1557 & 642.363 & 0.3658 \\
\hline
\end{tabular}


Table 262. Test data for specimen 205-30

\begin{tabular}{|c|c|c|c|c|c|}
\hline $\begin{array}{l}\text { Test Number } \\
\text { Material Type } \\
\text { Aging Temp. } \\
\text { Yield Stress }\end{array}$ & $\begin{array}{l}: 0 \\
: \mathrm{CF} \\
: 4 C \\
: 24\end{array}$ & & $\begin{array}{l}\text { Tes } \\
\text { Hec } \\
\text { Agi } \\
\text { Ulti }\end{array}$ & $\begin{array}{l}\text { np. } \\
\text { mber } \\
\text { me } \\
\text { Stress }\end{array}$ & $\begin{array}{l}25^{\circ} \mathrm{C} \\
205 \\
18,000 \mathrm{~h} \\
646.1 \mathrm{MPa}\end{array}$ \\
\hline $\begin{array}{l}\text { Engr. } \\
\text { Stress } \\
(\mathrm{MPa}) \\
\end{array}$ & $\begin{array}{l}\text { Engr. } \\
\text { Strain }\end{array}$ & $\begin{array}{l}\text { Engr. } \\
\text { Stress } \\
(\mathrm{MPa}) \\
\end{array}$ & $\begin{array}{l}\text { Engr. } \\
\text { Strain }\end{array}$ & $\begin{array}{l}\text { Engr. } \\
\text { Stress } \\
(\mathrm{MPa}) \\
\end{array}$ & $\begin{array}{l}\text { Engr. } \\
\text { Strain }\end{array}$ \\
\hline 206.46 & 0.0017 & 300.74 & 0.0206 & 589.99 & 0.1599 \\
\hline 232.03 & 0.0019 & 425.81 & 0.0304 & 596.92 & 0.1690 \\
\hline 241.03 & 0.0022 & 439.16 & 0.0364 & 600.23 & 0.1746 \\
\hline 265.57 & 0.0029 & 454.41 & 0.0444 & 608.70 & 0.1898 \\
\hline 273.64 & 0.0034 & 467.23 & 0.0515 & 610.94 & 0.1934 \\
\hline 294.30 & 0.0046 & 473.59 & 0.0623 & 616.00 & 0.2039 \\
\hline 305.86 & 0.0052 & 485.56 & 0.0715 & 618.42 & 0.2101 \\
\hline 316.30 & 0.0061 & 514.52 & 0.0911 & 623.86 & 0.2235 \\
\hline 326.24 & 0.0070 & 533.89 & 0.1031 & 627.25 & 0.2341 \\
\hline 335.74 & 0.0082 & 539.57 & 0.1075 & 630.15 & 0.2444 \\
\hline 344.85 & 0.0094 & 552.28 & 0.1185 & 632.95 & 0.2549 \\
\hline 351.41 & 0.0104 & 557.75 & 0.1240 & 633.81 & 0.2596 \\
\hline 360.08 & 0.0118 & 562.05 & 0.1280 & 634.96 & 0.2659 \\
\hline 368.89 & 0.0132 & 567.33 & 0.1334 & 636.11 & 0.2725 \\
\hline 376.70 & 0.0154 & 572.12 & 0.1384 & 637.06 & 0.2802 \\
\hline 384.83 & 0.0172 & 575.73 & 0.1421 & 638.20 & 0.2865 \\
\hline 390.75 & 0.0186 & 582.66 & 0.1506 & 639.67 & 0.3103 \\
\hline
\end{tabular}

Table 263. Test data for specimen 205-25

\begin{tabular}{|c|c|c|c|c|c|}
\hline $\begin{array}{l}\text { Test Number } \\
\text { Material Type } \\
\text { Aging Temp. } \\
\text { Yield Stress }\end{array}$ & $\begin{array}{l}: 0 \\
: \mathrm{CF} \\
: 40 \\
: 17\end{array}$ & & $\begin{array}{l}\text { Tes } \\
\text { Hea } \\
\text { Agi } \\
\text { Ulti }\end{array}$ & $\begin{array}{l}\text { np. } \\
\text { nber } \\
\text { ne } \\
\text { Stress }\end{array}$ & $\begin{array}{l}290^{\circ} \mathrm{C} \\
205 \\
18,000 \mathrm{~h} \\
505.9 \mathrm{MPa}\end{array}$ \\
\hline $\begin{array}{l}\text { Engr. } \\
\text { Stress } \\
(\mathrm{MPa}) \\
\end{array}$ & $\begin{array}{l}\text { Engr. } \\
\text { Strain }\end{array}$ & $\begin{array}{l}\text { Engr. } \\
\text { Stress } \\
(\mathrm{MPa}) \\
\end{array}$ & $\begin{array}{l}\text { Engr. } \\
\text { Strain }\end{array}$ & $\begin{array}{l}\text { Engr. } \\
\text { Stress } \\
(\mathrm{MPa})\end{array}$ & $\begin{array}{l}\text { Engr. } \\
\text { Strain }\end{array}$ \\
\hline 73.83 & 0.0002 & 263.54 & 0.0096 & 427.35 & 0.0939 \\
\hline 105.52 & 0.0006 & 274.92 & 0.0124 & 446.96 & 0.1061 \\
\hline 127.98 & 0.0009 & 285.57 & 0.0155 & 462.80 & 0.1199 \\
\hline 141.76 & 0.0012 & 294.26 & 0.0185 & 473.64 & 0.1309 \\
\hline 156.10 & 0.0016 & 301.80 & 0.0212 & 482.97 & 0.1419 \\
\hline 167.01 & 0.0020 & 317.07 & 0.0277 & 483.46 & 0.1437 \\
\hline 176.70 & 0.0024 & 343.30 & 0.0410 & 485.46 & 0.1484 \\
\hline 191.30 & 0.0033 & 363.02 & 0.0523 & 494.14 & 0.1610 \\
\hline 202.85 & 0.0039 & 379.90 & 0.0624 & 500.51 & 0.1719 \\
\hline 226.10 & 0.0048 & 395.39 & 0.0723 & 504.16 & 0.1812 \\
\hline 240.81 & 0.0057 & 414.01 & 0.0844 & 505.94 & 0.1900 \\
\hline 251.80 & 0.0072 & 432.47 & 0.0972 & & \\
\hline
\end{tabular}


Table 264. Test data for specimen 205-28

\begin{tabular}{|c|c|c|c|c|c|}
\hline $\begin{array}{l}\text { Test Number } \\
\text { Material Type } \\
\text { Aging Temp. } \\
\text { Yield Stress }\end{array}$ & $\begin{array}{l}: 0 \\
: \mathrm{CF} \\
: 40 \\
: 17\end{array}$ & $\mathrm{MPa}$ & $\begin{array}{l}\text { Tes } \\
\text { Hea } \\
\text { Agi } \\
\text { Ulti }\end{array}$ & $\begin{array}{l}\text { np. } \\
\text { nber } \\
\text { ne } \\
\text { Stress }\end{array}$ & $\begin{array}{l}290^{\circ} \mathrm{C} \\
205 \\
18,000 \mathrm{~h} \\
507.8 \mathrm{MPa}\end{array}$ \\
\hline $\begin{array}{l}\text { Engr. } \\
\text { Stress } \\
\text { (MPa) }\end{array}$ & $\begin{array}{l}\text { Engr. } \\
\text { Strain }\end{array}$ & $\begin{array}{l}\text { Engr. } \\
\text { Stress } \\
\text { (MPa) }\end{array}$ & $\begin{array}{l}\text { Engr. } \\
\text { Strain }\end{array}$ & $\begin{array}{l}\text { Engr. } \\
\text { Stress } \\
(\mathrm{MPa}) \\
\end{array}$ & $\begin{array}{l}\text { Engr. } \\
\text { Strain }\end{array}$ \\
\hline 58.95 & 0.0001 & 249.60 & 0.0078 & 437.50 & 0.1023 \\
\hline 97.97 & 0.0005 & 272.90 & 0.0135 & 445.27 & 0.1084 \\
\hline 127.29 & 0.0009 & 282.70 & 0.0168 & 456.48 & 0.1183 \\
\hline 150.45 & 0.0014 & 296.26 & 0.0216 & 464.98 & 0.1266 \\
\hline 161.64 & 0.0018 & 302.94 & 0.0243 & 473.76 & 0.1359 \\
\hline 175.49 & 0.0024 & 313.87 & 0.0292 & 480.32 & 0.1442 \\
\hline 183.44 & 0.0029 & 324.21 & 0.0344 & 488.44 & 0.1558 \\
\hline 191.23 & 0.0035 & 334.40 & 0.0395 & 488.76 & 0.1597 \\
\hline 198.38 & 0.0041 & 345.57 & 0.0459 & 492.46 & 0.1664 \\
\hline 204.58 & 0.0044 & 357.23 & 0.0525 & 495.10 & 0.1720 \\
\hline 207.90 & 0.0045 & 376.94 & 0.0642 & 499.73 & 0.1812 \\
\hline 212.41 & 0.0048 & 388.54 & 0.0713 & 503.65 & 0.1911 \\
\hline 217.47 & 0.0050 & 402.99 & 0.0806 & 506.79 & 0.2007 \\
\hline 224.91 & 0.0052 & 414.19 & 0.0880 & 507.79 & 0.2125 \\
\hline 231.66 & 0.0055 & 426.47 & 0.0961 & & \\
\hline
\end{tabular}

Table 265. Test data for specimen 205-29

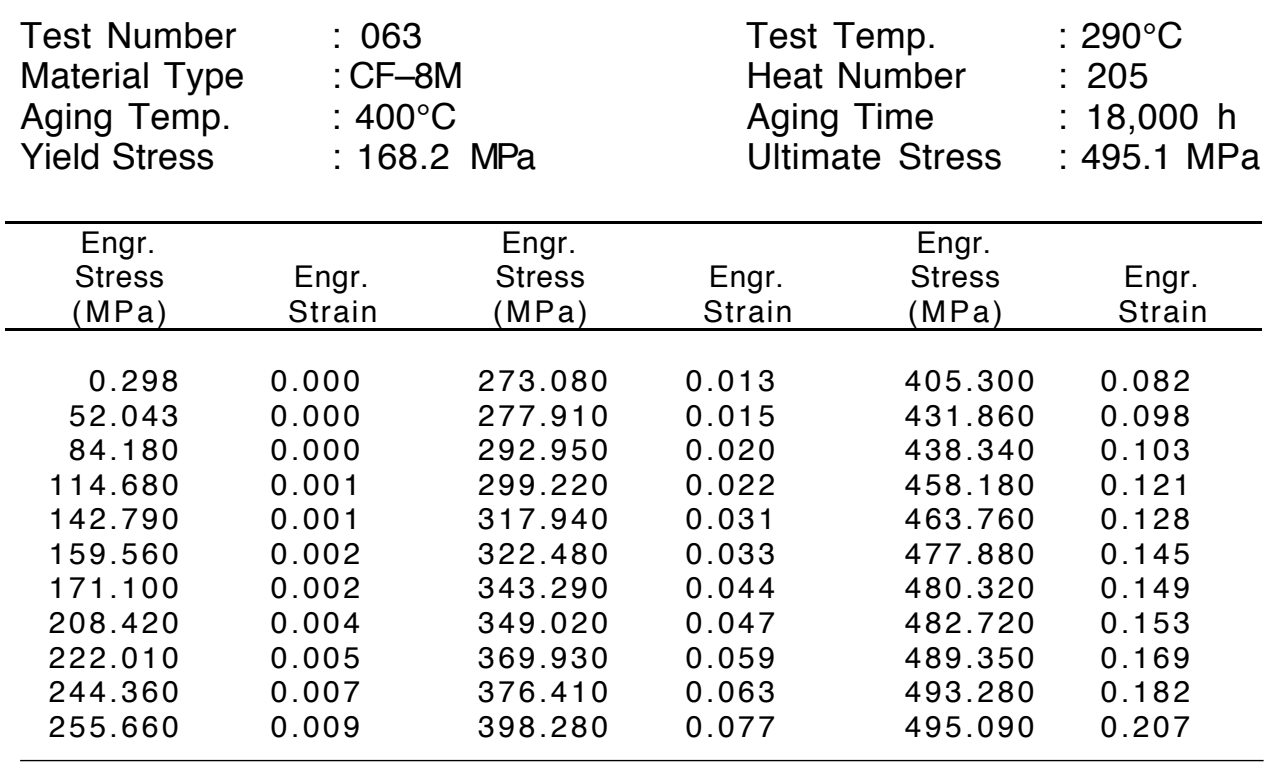


Table 266. Test data for specimen 743-40

\begin{tabular}{|c|c|c|c|c|c|}
\hline $\begin{array}{l}\text { Test Number } \\
\text { Aging Temp. } \\
\text { Yield Stress }\end{array}$ & $\begin{array}{l}: 0 \\
: U 1 \\
: 27\end{array}$ & & $\begin{array}{l}\text { Tes } \\
\text { Agi } \\
\text { Ulti }\end{array}$ & $\begin{array}{l}\text { np. } \\
\text { ne } \\
\text { Stress }\end{array}$ & $\begin{array}{l}25^{\circ} \mathrm{C} \\
- \\
542.4 \mathrm{MPa}\end{array}$ \\
\hline $\begin{array}{l}\text { Engr. } \\
\text { Stress } \\
(\mathrm{MPa})\end{array}$ & $\begin{array}{l}\text { Engr. } \\
\text { Strain }\end{array}$ & $\begin{array}{l}\text { Engr. } \\
\text { Stress } \\
(\mathrm{MPa})\end{array}$ & $\begin{array}{l}\text { Engr. } \\
\text { Strain }\end{array}$ & $\begin{array}{l}\text { Engr. } \\
\text { Stress } \\
(\mathrm{MPa}) \\
\end{array}$ & $\begin{array}{l}\text { Engr. } \\
\text { Strain }\end{array}$ \\
\hline $\begin{array}{r}64.02 \\
79.92 \\
102.27 \\
134.03 \\
163.90 \\
201.03 \\
222.64 \\
233.57\end{array}$ & $\begin{array}{l}0.0001 \\
0.0002 \\
0.0004 \\
0.0007 \\
0.0013 \\
0.0019 \\
0.0020 \\
0.0021\end{array}$ & $\begin{array}{l}242.65 \\
251.00 \\
262.07 \\
271.34 \\
279.30 \\
289.45 \\
293.02 \\
300.97\end{array}$ & $\begin{array}{l}0.0024 \\
0.0026 \\
0.0030 \\
0.0035 \\
0.0040 \\
0.0049 \\
0.0053 \\
0.0064\end{array}$ & $\begin{array}{l}305.32 \\
307.13 \\
311.09 \\
319.74 \\
329.32 \\
333.38 \\
336.51 \\
337.73\end{array}$ & $\begin{array}{l}0.0073 \\
0.0079 \\
0.0090 \\
0.0129 \\
0.0187 \\
0.0216 \\
0.0241 \\
0.0248\end{array}$ \\
\hline
\end{tabular}

Table 267. Test data for specimen 743-41

\begin{tabular}{|c|c|c|c|c|c|}
\hline $\begin{array}{l}\text { Test Number } \\
\text { Aging Temp. } \\
\text { Yield Stress }\end{array}$ & $\begin{array}{l}: 0 \\
: U 1 \\
: 27\end{array}$ & & $\begin{array}{l}\text { Tes } \\
\text { Agi } \\
\text { Ulti }\end{array}$ & $\begin{array}{l}\text { np. } \\
\text { me } \\
\text { Stress }\end{array}$ & $\begin{array}{l}25^{\circ} \mathrm{C} \\
- \\
531.3 \mathrm{MPa}\end{array}$ \\
\hline $\begin{array}{l}\text { Engr. } \\
\text { Stress } \\
(\mathrm{MPa}) \\
\end{array}$ & $\begin{array}{l}\text { Engr. } \\
\text { Strain }\end{array}$ & $\begin{array}{l}\text { Engr. } \\
\text { Stress } \\
\text { (MPa) }\end{array}$ & $\begin{array}{l}\text { Engr. } \\
\text { Strain }\end{array}$ & $\begin{array}{l}\text { Engr. } \\
\text { Stress } \\
(\mathrm{MPa}) \\
\end{array}$ & $\begin{array}{l}\text { Engr. } \\
\text { Strain }\end{array}$ \\
\hline 61.35 & 0.0001 & 332.46 & 0.0220 & 492.73 & 0.2468 \\
\hline 99.28 & 0.0004 & 351.27 & 0.0381 & 500.24 & 0.2679 \\
\hline 147.04 & 0.0009 & 366.49 & 0.0532 & 505.80 & 0.2888 \\
\hline 194.95 & 0.0017 & 383.14 & 0.0703 & 511.08 & 0.3097 \\
\hline 216.27 & 0.0020 & 396.74 & 0.0853 & 516.24 & 0.3324 \\
\hline 230.83 & 0.0021 & 411.77 & 0.0990 & 520.44 & 0.3547 \\
\hline 248.01 & 0.0026 & 426.53 & 0.1172 & 523.26 & 0.3782 \\
\hline 267.12 & 0.0036 & 437.88 & 0.1324 & 525.92 & 0.3995 \\
\hline 275.88 & 0.0041 & 451.84 & 0.1530 & 528.28 & 0.4215 \\
\hline 286.63 & 0.0051 & 461.57 & 0.1698 & 529.56 & 0.4442 \\
\hline 293.24 & 0.0060 & 471.00 & 0.1881 & 529.97 & 0.4676 \\
\hline 299.88 & 0.0074 & 478.94 & 0.2046 & 530.62 & 0.4873 \\
\hline 305.46 & 0.0087 & 478.90 & 0.2059 & 531.29 & 0.5134 \\
\hline 313.12 & 0.0111 & 480.54 & 0.2136 & & \\
\hline 323.90 & 0.0167 & 485.31 & 0.2275 & & \\
\hline
\end{tabular}


Table 268. Test data for specimen 743-36

\begin{tabular}{|c|c|c|c|c|c|}
\hline $\begin{array}{l}\text { Test Number } \\
\text { Aging Temp. } \\
\text { Yield Stress }\end{array}$ & $\begin{array}{l}: 2 \\
: 29 \\
: 28\end{array}$ & & $\begin{array}{l}\text { Tes } \\
\text { Agir } \\
\text { Ultir }\end{array}$ & $\begin{array}{l}\text { mp. } \\
\text { me } \\
\text { Stress }\end{array}$ & $\begin{array}{l}25^{\circ} \mathrm{C} \\
30,000 \mathrm{~h} \\
555.6 \mathrm{MPa}\end{array}$ \\
\hline $\begin{array}{l}\text { Engr. } \\
\text { Stress } \\
(\mathrm{MPa})\end{array}$ & $\begin{array}{l}\text { Engr. } \\
\text { Strain }\end{array}$ & $\begin{array}{l}\text { Engr. } \\
\text { Stress } \\
(\mathrm{MPa})\end{array}$ & $\begin{array}{l}\text { Engr. } \\
\text { Strain }\end{array}$ & $\begin{array}{l}\text { Engr. } \\
\text { Stress } \\
\text { (MPa) }\end{array}$ & $\begin{array}{l}\text { Engr. } \\
\text { Strain }\end{array}$ \\
\hline 16.597 & 0.0001 & 355.981 & 0.0222 & 497.284 & 0.1267 \\
\hline 60.766 & 0.0005 & 369.842 & 0.0290 & 502.649 & 0.1330 \\
\hline 71.086 & 0.0006 & 401.412 & 0.0474 & 517.135 & 0.1519 \\
\hline 150.844 & 0.0012 & 405.065 & 0.0497 & 521.329 & 0.1579 \\
\hline 162.520 & 0.0013 & 415.478 & 0.0566 & 531.181 & 0.1744 \\
\hline 219.047 & 0.0019 & 441.064 & 0.0751 & 534.400 & 0.1804 \\
\hline 246.267 & 0.0025 & 443.932 & 0.0774 & 535.376 & 0.1825 \\
\hline 278.189 & 0.0039 & 452.506 & 0.0844 & 542.887 & 0.1990 \\
\hline 299.878 & 0.0059 & 473.669 & 0.1029 & 546.057 & 0.2074 \\
\hline 305.062 & 0.0066 & 476.161 & 0.1052 & 553.665 & 0.2382 \\
\hline 351.221 & 0.0199 & 491.090 & 0.1201 & 555.519 & 0.2562 \\
\hline
\end{tabular}

Table 269. Test data for specimen 744-34

\begin{tabular}{|c|c|c|c|c|c|}
\hline $\begin{array}{l}\text { Test Number } \\
\text { Aging Temp. } \\
\text { Yield Stress }\end{array}$ & $\begin{array}{l}: 2 \\
: 25 \\
: 25\end{array}$ & $\mathrm{~Pa}$ & $\begin{array}{l}\text { Tes } \\
\text { Agi } \\
\text { Ulti }\end{array}$ & $\begin{array}{l}\text { mp. } \\
\text { me } \\
\text { Stress }\end{array}$ & $\begin{array}{l}25^{\circ} \mathrm{C} \\
30,000 \mathrm{~h} \\
601.2 \mathrm{MPa}\end{array}$ \\
\hline $\begin{array}{l}\text { Engr. } \\
\text { Stress } \\
\text { (MPa) }\end{array}$ & $\begin{array}{l}\text { Engr. } \\
\text { Strain }\end{array}$ & $\begin{array}{l}\text { Engr. } \\
\text { Stress } \\
\text { (MPa) }\end{array}$ & $\begin{array}{l}\text { Engr. } \\
\text { Strain }\end{array}$ & $\begin{array}{l}\text { Engr. } \\
\text { Stress } \\
(\mathrm{MPa})\end{array}$ & $\begin{array}{l}\text { Engr. } \\
\text { Strain }\end{array}$ \\
\hline 31.273 & 0.0002 & 371.929 & 0.0256 & 537.570 & 0.1376 \\
\hline 63.195 & 0.0005 & 406.314 & 0.0433 & 557.323 & 0.1615 \\
\hline 73.871 & 0.0005 & 418.093 & 0.0500 & 561.810 & 0.1681 \\
\hline 171.674 & 0.0012 & 421.946 & 0.0522 & 572.540 & 0.1859 \\
\hline 209.941 & 0.0015 & 452.155 & 0.0706 & 577.223 & 0.1950 \\
\hline 221.739 & 0.0016 & 465.602 & 0.0795 & 588.099 & 0.2203 \\
\hline 288.211 & 0.0037 & 496.211 & 0.1012 & 590.684 & 0.2279 \\
\hline 305.594 & 0.0051 & 504.454 & 0.1077 & 596.293 & 0.2465 \\
\hline 311.705 & 0.0058 & 507.234 & 0.1098 & 599.219 & 0.2609 \\
\hline 357.263 & 0.0188 & 528.987 & 0.1290 & 601.267 & 0.2845 \\
\hline
\end{tabular}


Table 270. Test data for specimen 74-135

\begin{tabular}{|c|c|c|c|c|c|}
\hline $\begin{array}{l}\text { Test Number } \\
\text { Aging Temp. } \\
\text { Yield Stress }\end{array}$ & $\begin{array}{l}2 \\
29 \\
25\end{array}$ & $\mathrm{MPa}$ & $\begin{array}{l}\text { Tes } \\
\text { Agi } \\
\text { Ulti }\end{array}$ & $\begin{array}{l}\text { np. } \\
\text { ne } \\
\text { Stress }\end{array}$ & $\begin{array}{l}25^{\circ} \mathrm{C} \\
30,000 \mathrm{~h} \\
570.4 \mathrm{MPa}\end{array}$ \\
\hline $\begin{array}{l}\text { Engr. } \\
\text { Stress } \\
\text { (MPa) }\end{array}$ & $\begin{array}{l}\text { Engr. } \\
\text { Strain }\end{array}$ & $\begin{array}{l}\text { Engr. } \\
\text { Stress } \\
\text { (MPa) }\end{array}$ & $\begin{array}{l}\text { Engr. } \\
\text { Strain }\end{array}$ & $\begin{array}{l}\text { Engr. } \\
\text { Stress } \\
(\mathrm{MPa}) \\
\end{array}$ & $\begin{array}{l}\text { Engr. } \\
\text { Strain }\end{array}$ \\
\hline 14.921 & 0.0000 & 370.692 & 0.0406 & 515.735 & 0.1776 \\
\hline 27.968 & 0.0001 & 373.988 & 0.0428 & 521.325 & 0.1878 \\
\hline 49.482 & 0.0002 & 414.154 & 0.0705 & 535.977 & 0.2189 \\
\hline 59.892 & 0.0003 & 424.868 & 0.0788 & 540.700 & 0.2313 \\
\hline 185.372 & 0.0012 & 427.547 & 0.0808 & 548.315 & 0.2547 \\
\hline 196.019 & 0.0014 & 460.084 & 0.1089 & 550.821 & 0.2634 \\
\hline 240.021 & 0.0025 & 462.277 & 0.1109 & 554.146 & 0.2764 \\
\hline 284.784 & 0.0058 & 472.191 & 0.1208 & 561.279 & 0.3117 \\
\hline 290.833 & 0.0066 & 495.011 & 0.1470 & 563.110 & 0.3231 \\
\hline 319.094 & 0.0136 & 496.505 & 0.1490 & 567.207 & 0.3552 \\
\hline 352.060 & 0.0295 & 497.999 & 0.1511 & 570.243 & 0.4211 \\
\hline
\end{tabular}

Table 271. Test data for specimen 744-21

\begin{tabular}{|c|c|c|c|c|c|}
\hline $\begin{array}{l}\text { Test Number } \\
\text { Aging Temp. } \\
\text { Yield Stress }\end{array}$ & $\begin{array}{l}: 1 \\
: 32 \\
: 27\end{array}$ & $\mathrm{MPa}$ & $\begin{array}{l}\text { Tes } \\
\text { Agi } \\
\text { Ulti }\end{array}$ & $\begin{array}{l}\text { mp. } \\
\text { me } \\
\text { Stress }\end{array}$ & $\begin{array}{l}25^{\circ} \mathrm{C} \\
10,000 \mathrm{~h} \\
551.0 \mathrm{MPa}\end{array}$ \\
\hline $\begin{array}{l}\text { Engr. } \\
\text { Stress } \\
(\mathrm{MPa}) \\
\end{array}$ & $\begin{array}{l}\text { Engr. } \\
\text { Strain }\end{array}$ & $\begin{array}{l}\text { Engr. } \\
\text { Stress } \\
(\mathrm{MPa}) \\
\end{array}$ & $\begin{array}{l}\text { Engr. } \\
\text { Strain }\end{array}$ & $\begin{array}{l}\text { Engr. } \\
\text { Stress } \\
(\mathrm{MPa}) \\
\end{array}$ & $\begin{array}{l}\text { Engr. } \\
\text { Strain }\end{array}$ \\
\hline 27.489 & 0.0002 & 286.173 & 0.0058 & 364.179 & 0.0283 \\
\hline 37.730 & 0.0003 & 293.457 & 0.0065 & 367.404 & 0.0303 \\
\hline 47.986 & 0.0003 & 300.299 & 0.0073 & 373.795 & 0.0343 \\
\hline 122.563 & 0.0009 & 306.491 & 0.0080 & 376.932 & 0.0364 \\
\hline 237.999 & 0.0027 & 324.277 & 0.0106 & 382.669 & 0.0403 \\
\hline 243.982 & 0.0030 & 335.371 & 0.0141 & 385.515 & 0.0423 \\
\hline 249.457 & 0.0033 & 340.342 & 0.0160 & 390.936 & 0.0463 \\
\hline 254.859 & 0.0035 & 345.598 & 0.0184 & 393.729 & 0.0483 \\
\hline 260.129 & 0.0039 & 353.516 & 0.0223 & 398.627 & 0.0523 \\
\hline 265.337 & 0.0042 & 357.381 & 0.0243 & 401.342 & 0.0543 \\
\hline 270.191 & 0.0045 & 360.906 & 0.0263 & 406.283 & 0.0583 \\
\hline
\end{tabular}


Table 272. Test data for specimen 744-25

\begin{tabular}{|c|c|c|c|c|c|}
\hline $\begin{array}{l}\text { Test Number } \\
\text { Aging Temp. } \\
\text { Yield Stress }\end{array}$ & $\begin{array}{l}: 18 \\
: 32 \\
: 27\end{array}$ & $\mathrm{MPa}$ & $\begin{array}{l}\text { Tes } \\
\text { Agi } \\
\text { Ulti }\end{array}$ & $\begin{array}{l}\mathrm{np} . \\
\text { me } \\
\text { Stress }\end{array}$ & $\begin{array}{l}25^{\circ} \mathrm{C} \\
10,000 \mathrm{~h} \\
565.3 \mathrm{MPa}\end{array}$ \\
\hline $\begin{array}{l}\text { Engr. } \\
\text { Stress } \\
\text { (MPa) }\end{array}$ & $\begin{array}{l}\text { Engr. } \\
\text { Strain }\end{array}$ & $\begin{array}{l}\text { Engr. } \\
\text { Stress } \\
\text { (MPa) }\end{array}$ & $\begin{array}{l}\text { Engr. } \\
\text { Strain }\end{array}$ & $\begin{array}{l}\text { Engr. } \\
\text { Stress } \\
\text { (MPa) }\end{array}$ & $\begin{array}{l}\text { Engr. } \\
\text { Strain }\end{array}$ \\
\hline 15.191 & 0.0001 & 427.065 & 0.0904 & 508.494 & 0.2718 \\
\hline 29.109 & 0.0002 & 432.025 & 0.0968 & 519.386 & 0.3129 \\
\hline 40.396 & 0.0002 & 450.401 & 0.1246 & 519.916 & 0.3151 \\
\hline 147.623 & 0.0008 & 454.965 & 0.1332 & 520.301 & 0.3174 \\
\hline 160.206 & 0.0009 & 469.091 & 0.1610 & 536.446 & 0.3909 \\
\hline 206.921 & 0.0013 & 470.001 & 0.1631 & 537.121 & 0.3931 \\
\hline 216.853 & 0.0015 & 484.397 & 0.1973 & 544.109 & 0.4321 \\
\hline 284.604 & 0.0045 & 485.264 & 0.1995 & 545.700 & 0.4408 \\
\hline 368.997 & 0.0329 & 496.156 & 0.2309 & 546.181 & 0.4429 \\
\hline 377.315 & 0.0393 & 505.506 & 0.2610 & 553.266 & 0.4910 \\
\hline 379.985 & 0.0414 & 506.084 & 0.2632 & 561.941 & 0.5884 \\
\hline
\end{tabular}

Table 273. Test data for specimen 742-42

\begin{tabular}{|c|c|c|c|c|c|}
\hline $\begin{array}{l}\text { Test Number } \\
\text { Aging Temp. } \\
\text { Yield Stress }\end{array}$ & $\begin{array}{l}: 16 \\
: 32 \\
: 31\end{array}$ & $\mathrm{~Pa}$ & $\begin{array}{l}\text { Tes } \\
\text { Agi } \\
\text { Ulti }\end{array}$ & $\begin{array}{l}\text { np. } \\
\text { ne } \\
\text { Stress }\end{array}$ & $\begin{array}{l}25^{\circ} \mathrm{C} \\
30,000 \mathrm{~h} \\
589.9 \mathrm{MPa}\end{array}$ \\
\hline $\begin{array}{l}\text { Engr. } \\
\text { Stress } \\
\text { (MPa) }\end{array}$ & $\begin{array}{l}\text { Engr. } \\
\text { Strain }\end{array}$ & $\begin{array}{l}\text { Engr. } \\
\text { Stress } \\
\text { (MPa) }\end{array}$ & $\begin{array}{l}\text { Engr. } \\
\text { Strain }\end{array}$ & $\begin{array}{l}\text { Engr. } \\
\text { Stress } \\
(\mathrm{MPa})\end{array}$ & $\begin{array}{l}\text { Engr. } \\
\text { Strain }\end{array}$ \\
\hline $\begin{array}{r}0.00 \\
253.26 \\
305.40 \\
323.58 \\
340.03 \\
353.80\end{array}$ & $\begin{array}{l}0.0000 \\
0.0028 \\
0.0048 \\
0.0059 \\
0.0075 \\
0.0096\end{array}$ & $\begin{array}{l}370.90 \\
387.53 \\
401.24 \\
418.04 \\
437.59 \\
467.62\end{array}$ & $\begin{array}{l}0.0137 \\
0.0193 \\
0.0261 \\
0.0357 \\
0.0489 \\
0.0714\end{array}$ & $\begin{array}{l}488.09 \\
510.31 \\
544.67 \\
564.75 \\
589.10 \\
589.93\end{array}$ & $\begin{array}{l}0.0889 \\
0.1124 \\
0.1599 \\
0.2042 \\
0.3110 \\
0.3449\end{array}$ \\
\hline
\end{tabular}

Table 274. Test data for specimen 744-39

\begin{tabular}{|c|c|c|c|c|c|}
\hline $\begin{array}{l}\text { Test Number } \\
\text { Aging Temp. } \\
\text { Yield Stress }\end{array}$ & $\begin{array}{l}: 1 \\
: 32 \\
: 32\end{array}$ & $\mathrm{~Pa}$ & $\begin{array}{l}\text { Tes } \\
\text { Agi } \\
\text { Ulti }\end{array}$ & $\begin{array}{l}\text { mp. } \\
\text { me } \\
\text { Stress }\end{array}$ & $\begin{array}{l}25^{\circ} \mathrm{C} \\
30,000 \mathrm{~h} \\
663.6 \mathrm{MPa}\end{array}$ \\
\hline $\begin{array}{l}\text { Engr. } \\
\text { Stress } \\
\text { (MPa) }\end{array}$ & $\begin{array}{l}\text { Engr. } \\
\text { Strain }\end{array}$ & $\begin{array}{l}\text { Engr. } \\
\text { Stress } \\
\text { (MPa) }\end{array}$ & $\begin{array}{l}\text { Engr. } \\
\text { Strain }\end{array}$ & $\begin{array}{l}\text { Engr. } \\
\text { Stress } \\
\text { (MPa) }\end{array}$ & $\begin{array}{l}\text { Engr. } \\
\text { Strain }\end{array}$ \\
\hline $\begin{array}{r}0.00 \\
299.42 \\
341.87 \\
363.93 \\
380.44 \\
393.51\end{array}$ & $\begin{array}{l}0.0000 \\
0.0040 \\
0.0062 \\
0.0082 \\
0.0101 \\
0.0123\end{array}$ & $\begin{array}{l}407.90 \\
423.94 \\
437.85 \\
455.83 \\
495.61\end{array}$ & $\begin{array}{l}0.0153 \\
0.0196 \\
0.0246 \\
0.0321 \\
0.0539\end{array}$ & $\begin{array}{l}560.82 \\
602.75 \\
640.72 \\
666.18 \\
674.39\end{array}$ & $\begin{array}{l}0.0989 \\
0.1356 \\
0.1874 \\
0.2490 \\
0.2943\end{array}$ \\
\hline
\end{tabular}


Table 275. Test data for specimen 74-245

\begin{tabular}{|c|c|c|c|c|c|}
\hline $\begin{array}{l}\text { Test Number } \\
\text { Aging Temp. } \\
\text { Yield Stress }\end{array}$ & $\begin{array}{l}: 1 \\
: 32 \\
: 27\end{array}$ & & $\begin{array}{l}\text { Tes } \\
\text { Agi } \\
\text { Ulti }\end{array}$ & $\begin{array}{l}\text { ne. } \\
\text { Stress }\end{array}$ & $\begin{array}{l}25^{\circ} \mathrm{C} \\
30,000 \mathrm{~h} \\
617.0 \mathrm{MPa}\end{array}$ \\
\hline $\begin{array}{l}\text { Engr. } \\
\text { Stress } \\
(\mathrm{MPa})\end{array}$ & $\begin{array}{l}\text { Engr. } \\
\text { Strain }\end{array}$ & $\begin{array}{l}\text { Engr. } \\
\text { Stress } \\
\text { (MPa) }\end{array}$ & $\begin{array}{l}\text { Engr. } \\
\text { Strain }\end{array}$ & $\begin{array}{l}\text { Engr. } \\
\text { Stress } \\
\text { (MPa) }\end{array}$ & $\begin{array}{l}\text { Engr. } \\
\text { Strain }\end{array}$ \\
\hline $\begin{array}{r}0.000 \\
234.550 \\
290.390 \\
309.100 \\
330.350 \\
343.310 \\
355.420 \\
365.500\end{array}$ & $\begin{array}{l}0.0000 \\
0.0022 \\
0.0044 \\
0.0055 \\
0.0075 \\
0.0094 \\
0.0117 \\
0.0144\end{array}$ & $\begin{array}{l}376.120 \\
389.090 \\
408.500 \\
430.310 \\
452.460 \\
469.450 \\
488.080 \\
489.760\end{array}$ & $\begin{array}{l}0.0179 \\
0.0231 \\
0.0333 \\
0.0458 \\
0.0606 \\
0.0731 \\
0.0873 \\
0.0890\end{array}$ & $\begin{array}{l}514.960 \\
539.480 \\
559.900 \\
578.040 \\
602.140 \\
615.080 \\
613.660 \\
616.980\end{array}$ & $\begin{array}{l}0.1082 \\
0.1312 \\
0.1572 \\
0.1860 \\
0.2383 \\
0.2834 \\
0.2839 \\
0.3221\end{array}$ \\
\hline
\end{tabular}

Table 276. Test data for specimen 741-28

\begin{tabular}{|c|c|c|c|c|c|}
\hline $\begin{array}{l}\text { Test Number } \\
\text { Aging Temp. } \\
\text { Yield Stress }\end{array}$ & $\begin{array}{l}: 2 \\
: 32 \\
: 30\end{array}$ & & $\begin{array}{l}\text { Tes } \\
\text { Agi } \\
\text { Ulti }\end{array}$ & $\begin{array}{l}\text { np. } \\
\text { ne } \\
\text { Stress }\end{array}$ & $\begin{array}{l}25^{\circ} \mathrm{C} \\
50,000 \mathrm{~h} \\
668.1 \mathrm{MPa}\end{array}$ \\
\hline $\begin{array}{l}\text { Engr. } \\
\text { Stress } \\
\text { (MPa) }\end{array}$ & $\begin{array}{l}\text { Engr. } \\
\text { Strain }\end{array}$ & $\begin{array}{l}\text { Engr. } \\
\text { Stress } \\
\text { (MPa) }\end{array}$ & $\begin{array}{l}\text { Engr. } \\
\text { Strain }\end{array}$ & $\begin{array}{l}\text { Engr. } \\
\text { Stress } \\
(\mathrm{MPa})\end{array}$ & $\begin{array}{l}\text { Engr. } \\
\text { Strain }\end{array}$ \\
\hline 12.454 & 0.0001 & 443.067 & 0.0303 & 609.894 & 0.1800 \\
\hline 16.807 & 0.0000 & 448.216 & 0.0325 & 618.831 & 0.1961 \\
\hline 64.526 & 0.0003 & 493.025 & 0.0574 & 624.126 & 0.2062 \\
\hline 86.700 & 0.0004 & 504.731 & 0.0654 & 628.109 & 0.2143 \\
\hline 254.804 & 0.0017 & 507.986 & 0.0677 & 638.893 & 0.2393 \\
\hline 263.999 & 0.0018 & 537.567 & 0.0915 & 641.953 & 0.2475 \\
\hline 272.616 & 0.0021 & 548.302 & 0.1016 & 646.470 & 0.2599 \\
\hline 359.680 & 0.0076 & 550.488 & 0.1036 & 655.942 & 0.2910 \\
\hline 365.363 & 0.0083 & 582.547 & 0.1399 & 659.682 & 0.3056 \\
\hline 370.541 & 0.0090 & 590.173 & 0.1499 & 665.317 & 0.3351 \\
\hline 437.782 & 0.0280 & 602.414 & 0.1679 & 668.037 & 0.3674 \\
\hline
\end{tabular}


Table 277. Test data for specimen 741-29

\begin{tabular}{|c|c|c|c|c|c|}
\hline $\begin{array}{l}\text { Test Number } \\
\text { Aging Temp. } \\
\text { Yield Stress }\end{array}$ & $\begin{array}{l}: 2 \\
: 32 \\
: 30\end{array}$ & & $\begin{array}{l}\text { Tes } \\
\text { Agir } \\
\text { Ulti }\end{array}$ & $\begin{array}{l}\text { mp. } \\
\text { me } \\
\text { Stress }\end{array}$ & $\begin{array}{l}25^{\circ} \mathrm{C} \\
50,000 \mathrm{~h} \\
638.1 \mathrm{MPa}\end{array}$ \\
\hline $\begin{array}{l}\text { Engr. } \\
\text { Stress } \\
(\mathrm{MPa})\end{array}$ & $\begin{array}{l}\text { Engr. } \\
\text { Strain }\end{array}$ & $\begin{array}{l}\text { Engr. } \\
\text { Stress } \\
(\mathrm{MPa})\end{array}$ & $\begin{array}{l}\text { Engr. } \\
\text { Strain }\end{array}$ & $\begin{array}{l}\text { Engr. } \\
\text { Stress } \\
\text { (MPa) }\end{array}$ & $\begin{array}{l}\text { Engr. } \\
\text { Strain }\end{array}$ \\
\hline 20.328 & 0.0001 & 459.563 & 0.0498 & 583.828 & 0.1908 \\
\hline 39.274 & 0.0001 & 479.887 & 0.0646 & 589.322 & 0.2019 \\
\hline 96.456 & 0.0003 & 482.716 & 0.0667 & 601.708 & 0.2302 \\
\hline 230.596 & 0.0011 & 485.319 & 0.0688 & 602.576 & 0.2323 \\
\hline 241.127 & 0.0012 & 522.525 & 0.1029 & 605.901 & 0.2408 \\
\hline 250.722 & 0.0014 & 524.501 & 0.1051 & 617.661 & 0.2756 \\
\hline 346.080 & 0.0063 & 536.260 & 0.1184 & 620.793 & 0.2869 \\
\hline 351.940 & 0.0070 & 554.767 & 0.1425 & 626.818 & 0.3111 \\
\hline 390.453 & 0.0150 & 556.261 & 0.1447 & 630.480 & 0.3286 \\
\hline 428.097 & 0.0310 & 564.984 & 0.1579 & 633.469 & 0.3461 \\
\hline 431.982 & 0.0330 & 582.575 & 0.1886 & 637.902 & 0.4001 \\
\hline
\end{tabular}

Table 278. Test data for specimen 74-230

\begin{tabular}{|c|c|c|c|c|c|}
\hline $\begin{array}{l}\text { Test Number } \\
\text { Aging Temp. } \\
\text { Yield Stress }\end{array}$ & $\begin{array}{l}: 2 \\
: 32 \\
: 25\end{array}$ & & $\begin{array}{l}\text { Tes } \\
\text { Agi } \\
\text { Ulti }\end{array}$ & $\begin{array}{l}\text { mp. } \\
\text { me } \\
\text { Stress }\end{array}$ & $\begin{array}{l}25^{\circ} \mathrm{C} \\
50,000 \mathrm{~h} \\
631.6 \mathrm{MPa}\end{array}$ \\
\hline $\begin{array}{l}\text { Engr. } \\
\text { Stress } \\
\text { (MPa) }\end{array}$ & $\begin{array}{l}\text { Engr. } \\
\text { Strain }\end{array}$ & $\begin{array}{l}\text { Engr. } \\
\text { Stress } \\
\text { (MPa) }\end{array}$ & $\begin{array}{l}\text { Engr. } \\
\text { Strain }\end{array}$ & $\begin{array}{l}\text { Engr. } \\
\text { Stress } \\
(\mathrm{MPa}) \\
\end{array}$ & $\begin{array}{l}\text { Engr. } \\
\text { Strain }\end{array}$ \\
\hline 17.452 & 0.0001 & 394.430 & 0.0148 & 548.051 & 0.1129 \\
\hline 58.657 & 0.0002 & 400.309 & 0.0165 & 572.727 & 0.1417 \\
\hline 69.006 & 0.0002 & 422.208 & 0.0246 & 574.421 & 0.1439 \\
\hline 90.537 & 0.0004 & 460.964 & 0.0447 & 586.662 & 0.1619 \\
\hline 102.198 & 0.0004 & 478.615 & 0.0559 & 594.791 & 0.1756 \\
\hline 196.364 & 0.0010 & 481.876 & 0.0581 & 600.694 & 0.1871 \\
\hline 207.686 & 0.0011 & 509.102 & 0.0779 & 609.064 & 0.2057 \\
\hline 257.914 & 0.0020 & 511.908 & 0.0801 & 612.741 & 0.2150 \\
\hline 315.230 & 0.0043 & 514.521 & 0.0823 & 619.612 & 0.2365 \\
\hline 320.857 & 0.0047 & 543.842 & 0.1085 & 622.709 & 0.2484 \\
\hline 354.954 & 0.0076 & 545.922 & 0.1107 & 631.611 & 0.3220 \\
\hline
\end{tabular}


Table 279. Test data for specimen 742-25

\begin{tabular}{|c|c|c|c|c|c|}
\hline $\begin{array}{l}\text { Test Number } \\
\text { Aging Temp. } \\
\text { Yield Stress }\end{array}$ & $\begin{array}{l}: 0 \\
: 35 \\
: 26\end{array}$ & & $\begin{array}{l}\text { Tes } \\
\text { Agi } \\
\text { Ulti }\end{array}$ & $\begin{array}{l}\text { np. } \\
\text { ne } \\
\text { Stress }\end{array}$ & $\begin{array}{l}25^{\circ} \mathrm{C} \\
2,570 \mathrm{~h} \\
575.1 \mathrm{MPa}\end{array}$ \\
\hline $\begin{array}{l}\text { Engr. } \\
\text { Stress } \\
\text { (MPa) }\end{array}$ & $\begin{array}{l}\text { Engr. } \\
\text { Strain }\end{array}$ & $\begin{array}{l}\text { Engr. } \\
\text { Stress } \\
(\mathrm{MPa})\end{array}$ & $\begin{array}{l}\text { Engr. } \\
\text { Strain }\end{array}$ & $\begin{array}{l}\text { Engr. } \\
\text { Stress } \\
(\mathrm{MPa}) \\
\end{array}$ & $\begin{array}{l}\text { Engr. } \\
\text { Strain }\end{array}$ \\
\hline 103.90 & 0.0003 & 337.39 & 0.0109 & 517.03 & 0.1733 \\
\hline 147.70 & 0.0009 & 348.85 & 0.0146 & 533.05 & 0.2090 \\
\hline 192.53 & 0.0017 & 368.48 & 0.0230 & 545.09 & 0.2437 \\
\hline 223.79 & 0.0019 & 392.33 & 0.0380 & 552.02 & 0.2650 \\
\hline 235.31 & 0.0021 & 415.14 & 0.0562 & 555.85 & 0.2854 \\
\hline 258.16 & 0.0028 & 438.00 & 0.0767 & 564.23 & 0.3238 \\
\hline 273.43 & 0.0034 & 456.49 & 0.0934 & 567.11 & 0.3468 \\
\hline 283.93 & 0.0041 & 471.29 & 0.1059 & 571.27 & 0.3814 \\
\hline 294.13 & 0.0048 & 478.28 & 0.1134 & 573.37 & 0.4057 \\
\hline 304.25 & 0.0055 & 478.15 & 0.1162 & 573.85 & 0.4243 \\
\hline 310.71 & 0.0062 & 493.35 & 0.1355 & 574.58 & 0.4486 \\
\hline 322.71 & 0.0079 & 506.11 & 0.1544 & 575.08 & 0.4701 \\
\hline 329.47 & 0.0091 & & & & \\
\hline
\end{tabular}

Table 280. Test data for specimen 742-26

\begin{tabular}{|c|c|c|c|c|c|}
\hline $\begin{array}{l}\text { Test Number } \\
\text { Aging Temp. } \\
\text { Yield Stress }\end{array}$ & $\begin{array}{l}: 0 \\
: 35 \\
: 26\end{array}$ & & $\begin{array}{l}\text { Tes } \\
\text { Agir } \\
\text { Ultir }\end{array}$ & $\begin{array}{l}\text { mp. } \\
\text { me } \\
\text { Stress }\end{array}$ & $\begin{array}{l}25^{\circ} \mathrm{C} \\
2,570 \mathrm{~h} \\
536.8 \mathrm{MPa}\end{array}$ \\
\hline $\begin{array}{l}\text { Engr. } \\
\text { Stress } \\
(\mathrm{MPa}) \\
\end{array}$ & $\begin{array}{l}\text { Engr. } \\
\text { Strain }\end{array}$ & $\begin{array}{l}\text { Engr. } \\
\text { Stress } \\
\text { (MPa) } \\
\end{array}$ & $\begin{array}{l}\text { Engr. } \\
\text { Strain }\end{array}$ & $\begin{array}{l}\text { Engr. } \\
\text { Stress } \\
(\mathrm{MPa}) \\
\end{array}$ & $\begin{array}{l}\text { Engr. } \\
\text { Strain }\end{array}$ \\
\hline 47.90 & 0.0000 & 293.22 & 0.0064 & 465.58 & 0.1573 \\
\hline 93.90 & 0.0003 & 299.93 & 0.0074 & 464.49 & 0.1640 \\
\hline 139.66 & 0.0009 & 310.53 & 0.0106 & 481.61 & 0.1941 \\
\hline 173.46 & 0.0014 & 319.42 & 0.0144 & 498.93 & 0.2307 \\
\hline 209.25 & 0.0020 & 332.15 & 0.0219 & 511.09 & 0.2647 \\
\hline 222.43 & 0.0021 & 346.29 & 0.0334 & 514.61 & 0.2804 \\
\hline 239.01 & 0.0024 & 367.51 & 0.0524 & 523.32 & 0.3161 \\
\hline 251.46 & 0.0029 & 386.79 & 0.0705 & 530.14 & 0.3543 \\
\hline 270.91 & 0.0041 & 405.10 & 0.0885 & 532.31 & 0.3751 \\
\hline 281.95 & 0.0050 & 427.53 & 0.1091 & 535.15 & 0.4148 \\
\hline 285.06 & 0.0053 & 451.26 & 0.1376 & 536.71 & 0.4528 \\
\hline 289.33 & 0.0058 & 461.36 & 0.1511 & 536.81 & 0.4799 \\
\hline
\end{tabular}


Table 281. Test data for specimen 744-07

\begin{tabular}{|c|c|c|c|c|c|}
\hline $\begin{array}{l}\text { Test Number } \\
\text { Aging Temp. } \\
\text { Yield Stress }\end{array}$ & $\begin{array}{l}: 1 \\
: 35 \\
: 28\end{array}$ & & $\begin{array}{l}\text { Tes } \\
\text { Agir } \\
\text { Ulti }\end{array}$ & $\begin{array}{l}\text { mp. } \\
\text { me } \\
\text { Stress }\end{array}$ & $\begin{array}{l}25^{\circ} \mathrm{C} \\
10,000 \mathrm{~h} \\
606.1 \mathrm{MPa}\end{array}$ \\
\hline $\begin{array}{l}\text { Engr. } \\
\text { Stress } \\
(\mathrm{MPa})\end{array}$ & $\begin{array}{l}\text { Engr. } \\
\text { Strain }\end{array}$ & $\begin{array}{l}\text { Engr. } \\
\text { Stress } \\
(\mathrm{MPa})\end{array}$ & $\begin{array}{l}\text { Engr. } \\
\text { Strain }\end{array}$ & $\begin{array}{l}\text { Engr. } \\
\text { Stress } \\
\text { (MPa) }\end{array}$ & $\begin{array}{l}\text { Engr. } \\
\text { Strain }\end{array}$ \\
\hline 33.818 & 0.0003 & 408.357 & 0.0401 & 527.097 & 0.1875 \\
\hline 44.883 & 0.0004 & 411.123 & 0.0421 & 540.350 & 0.2185 \\
\hline 55.857 & 0.0004 & 437.230 & 0.0635 & 541.314 & 0.2207 \\
\hline 77.949 & 0.0006 & 464.449 & 0.0909 & 547.194 & 0.2363 \\
\hline 89.356 & 0.0006 & 473.573 & 0.1014 & 561.218 & 0.2788 \\
\hline 232.092 & 0.0020 & 480.546 & 0.1100 & 566.519 & 0.2966 \\
\hline 249.235 & 0.0024 & 489.602 & 0.1222 & 581.893 & 0.3571 \\
\hline 298.291 & 0.0048 & 490.999 & 0.1244 & 585.170 & 0.3727 \\
\hline 303.130 & 0.0051 & 515.096 & 0.1633 & 595.580 & 0.4292 \\
\hline 311.135 & 0.0057 & 516.205 & 0.1655 & 602.809 & 0.4833 \\
\hline 405.306 & 0.0380 & 517.265 & 0.1677 & 605.942 & 0.5303 \\
\hline
\end{tabular}

Table 282. Test data for specimen 744-08

\begin{tabular}{|c|c|c|c|c|c|}
\hline $\begin{array}{l}\text { Test Number } \\
\text { Aging Temp. } \\
\text { Yield Stress }\end{array}$ & $\begin{array}{l}: 1 \\
: 35 \\
: 27\end{array}$ & $\mathrm{~Pa}$ & $\begin{array}{l}\text { Tes } \\
\text { Agi } \\
\text { Ulti }\end{array}$ & $\begin{array}{l}\text { mp. } \\
\text { me } \\
\text { Stress }\end{array}$ & $\begin{array}{l}25^{\circ} \mathrm{C} \\
10,000 \mathrm{~h} \\
619.2 \mathrm{MPa}\end{array}$ \\
\hline $\begin{array}{l}\text { Engr. } \\
\text { Stress } \\
(\mathrm{MPa}) \\
\end{array}$ & $\begin{array}{l}\text { Engr. } \\
\text { Strain }\end{array}$ & $\begin{array}{l}\text { Engr. } \\
\text { Stress } \\
\text { (MPa) }\end{array}$ & $\begin{array}{l}\text { Engr. } \\
\text { Strain }\end{array}$ & $\begin{array}{l}\text { Engr. } \\
\text { Stress } \\
(\mathrm{MPa}) \\
\end{array}$ & $\begin{array}{l}\text { Engr. } \\
\text { Strain }\end{array}$ \\
\hline 21.425 & 0.0001 & 402.243 & 0.0303 & 565.723 & 0.1827 \\
\hline 34.320 & 0.0002 & 406.424 & 0.0325 & 578.255 & 0.2080 \\
\hline 87.225 & 0.0005 & 475.349 & 0.0774 & 579.174 & 0.2103 \\
\hline 98.087 & 0.0006 & 488.694 & 0.0883 & 580.142 & 0.2125 \\
\hline 109.579 & 0.0006 & 491.016 & 0.0905 & 596.206 & 0.2543 \\
\hline 204.584 & 0.0013 & 493.774 & 0.0927 & 599.787 & 0.2654 \\
\hline 268.360 & 0.0031 & 533.692 & 0.1349 & 602.932 & 0.2763 \\
\hline 274.404 & 0.0034 & 535.579 & 0.1371 & 603.609 & 0.2784 \\
\hline 326.839 & 0.0073 & 537.418 & 0.1394 & 610.577 & 0.3059 \\
\hline 331.895 & 0.0080 & 562.965 & 0.1781 & 614.786 & 0.3266 \\
\hline 383.160 & 0.0217 & 564.465 & 0.1804 & 619.238 & 0.3648 \\
\hline
\end{tabular}


Table 283. Test data for specimen 74-119

\begin{tabular}{|c|c|c|c|c|c|}
\hline $\begin{array}{l}\text { Test Number } \\
\text { Aging Temp. } \\
\text { Yield Stress }\end{array}$ & $\begin{array}{l}: 1 \\
: 35 \\
: 26\end{array}$ & & $\begin{array}{l}\text { Tes } \\
\text { Agir } \\
\text { Ultir }\end{array}$ & $\begin{array}{l}\text { np. } \\
\text { ne } \\
\text { Stress }\end{array}$ & $\begin{array}{l}25^{\circ} \mathrm{C} \\
10,000 \mathrm{~h} \\
587.8 \mathrm{MPa}\end{array}$ \\
\hline $\begin{array}{l}\text { Engr. } \\
\text { Stress } \\
\text { (MPa) }\end{array}$ & $\begin{array}{l}\text { Engr. } \\
\text { Strain }\end{array}$ & $\begin{array}{l}\text { Engr. } \\
\text { Stress } \\
\text { (MPa) }\end{array}$ & $\begin{array}{l}\text { Engr. } \\
\text { Strain }\end{array}$ & $\begin{array}{l}\text { Engr. } \\
\text { Stress } \\
(\mathrm{MPa}) \\
\end{array}$ & $\begin{array}{l}\text { Engr. } \\
\text { Strain }\end{array}$ \\
\hline 13.981 & 0.0001 & 326.357 & 0.0097 & 499.928 & 0.1205 \\
\hline 26.410 & 0.0002 & 369.254 & 0.0227 & 501.758 & 0.1225 \\
\hline 78.205 & 0.0006 & 373.821 & 0.0247 & 520.740 & 0.1453 \\
\hline 88.876 & 0.0006 & 390.471 & 0.0329 & 522.426 & 0.1473 \\
\hline 99.701 & 0.0007 & 394.411 & 0.0350 & 525.365 & 0.1516 \\
\hline 111.803 & 0.0008 & 420.986 & 0.0516 & 545.743 & 0.1826 \\
\hline 123.221 & 0.0009 & 432.745 & 0.0599 & 564.725 & 0.2208 \\
\hline 212.906 & 0.0020 & 437.534 & 0.0635 & 572.192 & 0.2407 \\
\hline 244.838 & 0.0029 & 440.256 & 0.0656 & 578.166 & 0.2603 \\
\hline 295.288 & 0.0058 & 467.669 & 0.0886 & 584.815 & 0.2938 \\
\hline 302.346 & 0.0064 & 472.477 & 0.0927 & 587.561 & 0.3302 \\
\hline
\end{tabular}

Table 284. Test data for specimen 743-13

\begin{tabular}{|c|c|c|c|c|c|}
\hline $\begin{array}{l}\text { Test Number } \\
\text { Aging Temp. } \\
\text { Yield Stress }\end{array}$ & & & $\begin{array}{l}\text { Tes } \\
\text { Agi } \\
\text { Ulti }\end{array}$ & $\begin{array}{l}\text { mp. } \\
\text { me } \\
\text { Stress }\end{array}$ & $\begin{array}{l}25^{\circ} \mathrm{C} \\
30,000 \mathrm{~h} \\
647.2 \mathrm{MPa}\end{array}$ \\
\hline $\begin{array}{l}\text { Engr. } \\
\text { Stress } \\
(\mathrm{MPa})\end{array}$ & $\begin{array}{l}\text { Engr. } \\
\text { Strain }\end{array}$ & $\begin{array}{l}\text { Engr. } \\
\text { Stress } \\
\text { (MPa) }\end{array}$ & $\begin{array}{l}\text { Engr. } \\
\text { Strain }\end{array}$ & $\begin{array}{l}\text { Engr. } \\
\text { Stress } \\
(\mathrm{MPa}) \\
\end{array}$ & $\begin{array}{l}\text { Engr. } \\
\text { Strain }\end{array}$ \\
\hline 12.849 & 0.0001 & 428.458 & 0.0438 & 589.825 & 0.2019 \\
\hline 66.522 & 0.0006 & 482.969 & 0.0799 & 594.161 & 0.2105 \\
\hline 77.323 & 0.0006 & 500.361 & 0.0936 & 606.349 & 0.2378 \\
\hline 229.079 & 0.0023 & 502.914 & 0.0959 & 610.204 & 0.2479 \\
\hline 236.103 & 0.0026 & 537.746 & 0.1288 & 621.525 & 0.2825 \\
\hline 242.964 & 0.0028 & 539.673 & 0.1311 & 624.416 & 0.2929 \\
\hline 325.196 & 0.0084 & 541.889 & 0.1335 & 631.498 & 0.3242 \\
\hline 331.050 & 0.0091 & 568.627 & 0.1671 & 633.810 & 0.3369 \\
\hline 366.093 & 0.0159 & 570.313 & 0.1694 & 639.350 & 0.3694 \\
\hline 402.582 & 0.0300 & 577.395 & 0.1804 & 642.675 & 0.4003 \\
\hline 424.396 & 0.0414 & 588.813 & 0.1999 & 646.866 & 0.4810 \\
\hline
\end{tabular}


Table 285. Test data for specimen 743-14

\begin{tabular}{|c|c|c|c|c|c|}
\hline $\begin{array}{l}\text { Test Number } \\
\text { Aging Temp. } \\
\text { Yield Stress }\end{array}$ & $\begin{array}{l}: 1 \\
: 35 \\
: 26\end{array}$ & & $\begin{array}{l}\text { Tes } \\
\text { Agir } \\
\text { Ulti }\end{array}$ & $\begin{array}{l}\text { mp. } \\
\text { me } \\
\text { Stress }\end{array}$ & $\begin{array}{l}25^{\circ} \mathrm{C} \\
30,000 \mathrm{~h} \\
613.5 \mathrm{MPa}\end{array}$ \\
\hline $\begin{array}{l}\text { Engr. } \\
\text { Stress } \\
(\mathrm{MPa})\end{array}$ & $\begin{array}{l}\text { Engr. } \\
\text { Strain }\end{array}$ & $\begin{array}{l}\text { Engr. } \\
\text { Stress } \\
(\mathrm{MPa})\end{array}$ & $\begin{array}{l}\text { Engr. } \\
\text { Strain }\end{array}$ & $\begin{array}{l}\text { Engr. } \\
\text { Stress } \\
\text { (MPa) }\end{array}$ & $\begin{array}{l}\text { Engr. } \\
\text { Strain }\end{array}$ \\
\hline 9.666 & 0.0000 & 391.837 & 0.0241 & 545.340 & 0.1374 \\
\hline 21.484 & 0.0001 & 396.762 & 0.0261 & 547.040 & 0.1394 \\
\hline 61.626 & 0.0004 & 444.860 & 0.0513 & 555.395 & 0.1498 \\
\hline 83.460 & 0.0006 & 448.149 & 0.0534 & 565.741 & 0.1643 \\
\hline 205.648 & 0.0018 & 451.252 & 0.0554 & 567.101 & 0.1664 \\
\hline 214.581 & 0.0020 & 474.641 & 0.0716 & 572.639 & 0.1748 \\
\hline 222.882 & 0.0022 & 487.732 & 0.0817 & 583.617 & 0.1943 \\
\hline 291.051 & 0.0055 & 490.257 & 0.0837 & 588.911 & 0.2050 \\
\hline 295.908 & 0.0058 & 517.896 & 0.1080 & 599.015 & 0.2288 \\
\hline 304.272 & 0.0065 & 519.888 & 0.1101 & 603.435 & 0.2420 \\
\hline 364.849 & 0.0154 & 530.574 & 0.1210 & 613.490 & 0.2991 \\
\hline
\end{tabular}

Table 286. Test data for specimen 74-130

\begin{tabular}{|c|c|c|c|c|c|}
\hline $\begin{array}{l}\text { Test Number } \\
\text { Aging Temp. } \\
\text { Yield Stress }\end{array}$ & $\begin{array}{l}: 1 \\
: 35 \\
: 27\end{array}$ & & $\begin{array}{l}\text { Tes } \\
\text { Agi } \\
\text { Ulti }\end{array}$ & $\begin{array}{l}\text { mp. } \\
\text { me } \\
\text { Stress }\end{array}$ & $\begin{array}{l}25^{\circ} \mathrm{C} \\
30,000 \mathrm{~h} \\
624.8 \mathrm{MPa}\end{array}$ \\
\hline $\begin{array}{l}\text { Engr. } \\
\text { Stress } \\
\text { (MPa) }\end{array}$ & $\begin{array}{l}\text { Engr. } \\
\text { Strain }\end{array}$ & $\begin{array}{l}\text { Engr. } \\
\text { Stress } \\
\text { (MPa) } \\
\end{array}$ & $\begin{array}{l}\text { Engr. } \\
\text { Strain }\end{array}$ & $\begin{array}{l}\text { Engr. } \\
\text { Stress } \\
(\mathrm{MPa}) \\
\end{array}$ & $\begin{array}{l}\text { Engr. } \\
\text { Strain }\end{array}$ \\
\hline 16.372 & 0.0001 & 394.609 & 0.0265 & 559.656 & 0.1374 \\
\hline 39.398 & 0.0003 & 413.752 & 0.0345 & 563.230 & 0.1416 \\
\hline 93.048 & 0.0007 & 418.046 & 0.0365 & 581.786 & 0.1646 \\
\hline 116.548 & 0.0009 & 462.364 & 0.0601 & 583.303 & 0.1667 \\
\hline 163.079 & 0.0013 & 465.737 & 0.0621 & 592.900 & 0.1816 \\
\hline 242.531 & 0.0026 & 469.022 & 0.0641 & 599.166 & 0.1923 \\
\hline 249.728 & 0.0029 & 501.786 & 0.0862 & 603.279 & 0.2009 \\
\hline 273.365 & 0.0041 & 507.025 & 0.0902 & 611.749 & 0.2207 \\
\hline 325.777 & 0.0090 & 517.404 & 0.0983 & 615.519 & 0.2312 \\
\hline 331.133 & 0.0098 & 541.198 & 0.1189 & 620.660 & 0.2517 \\
\hline 355.240 & 0.0143 & 551.774 & 0.1291 & 624.674 & 0.2907 \\
\hline
\end{tabular}


Table 287. Test data for specimen 742-16

\begin{tabular}{|c|c|c|c|c|c|}
\hline $\begin{array}{l}\text { Test Number } \\
\text { Aging Temp. } \\
\text { Yield Stress }\end{array}$ & $\begin{array}{l}: 0 \\
: 44 \\
: 26\end{array}$ & & $\begin{array}{l}\text { Tes } \\
\text { Agi } \\
\text { Ulti }\end{array}$ & $\begin{array}{l}\text { np. } \\
\text { ne } \\
\text { Stress }\end{array}$ & $\begin{array}{l}25^{\circ} \mathrm{C} \\
2,570 \mathrm{~h} \\
602.3 \mathrm{MPa}\end{array}$ \\
\hline $\begin{array}{l}\text { Engr. } \\
\text { Stress } \\
\text { (MPa) }\end{array}$ & $\begin{array}{l}\text { Engr. } \\
\text { Strain }\end{array}$ & $\begin{array}{l}\text { Engr. } \\
\text { Stress } \\
(\mathrm{MPa})\end{array}$ & $\begin{array}{l}\text { Engr. } \\
\text { Strain }\end{array}$ & $\begin{array}{l}\text { Engr. } \\
\text { Stress } \\
(\mathrm{MPa}) \\
\end{array}$ & $\begin{array}{l}\text { Engr. } \\
\text { Strain }\end{array}$ \\
\hline 48.46 & 0.0000 & 328.25 & 0.0076 & 477.92 & 0.0846 \\
\hline 92.80 & 0.0003 & 335.89 & 0.0085 & 498.19 & 0.0996 \\
\hline 129.82 & 0.0008 & 342.28 & 0.0094 & 518.24 & 0.1178 \\
\hline 162.57 & 0.0012 & 351.81 & 0.0111 & 536.80 & 0.1375 \\
\hline 188.33 & 0.0018 & 360.03 & 0.0131 & 550.27 & 0.1560 \\
\hline 215.98 & 0.0021 & 374.07 & 0.0178 & 563.34 & 0.1751 \\
\hline 229.75 & 0.0022 & 385.15 & 0.0224 & 575.95 & 0.1972 \\
\hline 253.17 & 0.0029 & 399.45 & 0.0299 & 584.19 & 0.2167 \\
\hline 264.85 & 0.0033 & 416.92 & 0.0367 & 591.43 & 0.2375 \\
\hline 285.26 & 0.0044 & 433.97 & 0.0488 & 597.46 & 0.2601 \\
\hline 303.68 & 0.0054 & 450.14 & 0.0609 & 600.29 & 0.2797 \\
\hline 311.15 & 0.0059 & 461.65 & 0.0695 & 602.13 & 0.2970 \\
\hline 320.93 & 0.0068 & 476.65 & 0.0815 & 602.32 & 0.3098 \\
\hline
\end{tabular}

Table 288. Test data for specimen 742-17

\begin{tabular}{|c|c|c|c|c|c|}
\hline $\begin{array}{l}\text { Test Number } \\
\text { Aging Temp. } \\
\text { Yield Stress }\end{array}$ & $\begin{array}{l}: 0 \\
: 4 C \\
: 26\end{array}$ & & $\begin{array}{l}\text { Tes } \\
\text { Agir } \\
\text { Ulti }\end{array}$ & $\begin{array}{l}\text { np. } \\
\text { me } \\
\text { Stress }\end{array}$ & $\begin{array}{l}25^{\circ} \mathrm{C} \\
2,570 \mathrm{~h} \\
579.7 \mathrm{MPa}\end{array}$ \\
\hline $\begin{array}{l}\text { Engr. } \\
\text { Stress } \\
(\mathrm{MPa}) \\
\end{array}$ & $\begin{array}{l}\text { Engr. } \\
\text { Strain }\end{array}$ & $\begin{array}{l}\text { Engr. } \\
\text { Stress } \\
\text { (MPa) }\end{array}$ & $\begin{array}{l}\text { Engr. } \\
\text { Strain }\end{array}$ & $\begin{array}{l}\text { Engr. } \\
\text { Stress } \\
(\mathrm{MPa}) \\
\end{array}$ & $\begin{array}{l}\text { Engr. } \\
\text { Strain }\end{array}$ \\
\hline 43.97 & 0.0000 & 311.68 & 0.0075 & 456.70 & 0.0887 \\
\hline 91.10 & 0.0004 & 320.87 & 0.0088 & 479.34 & 0.1045 \\
\hline 141.86 & 0.0010 & 328.31 & 0.0101 & 501.87 & 0.1240 \\
\hline 188.56 & 0.0019 & 339.56 & 0.0131 & 533.88 & 0.1580 \\
\hline 222.89 & 0.0024 & 349.86 & 0.0168 & 548.18 & 0.1772 \\
\hline 239.20 & 0.0027 & 358.75 & 0.0203 & 559.53 & 0.1963 \\
\hline 248.53 & 0.0031 & 380.05 & 0.0313 & 572.39 & 0.2259 \\
\hline 262.01 & 0.0036 & 403.69 & 0.0466 & 577.56 & 0.2451 \\
\hline 277.78 & 0.0045 & 427.01 & 0.0628 & 579.42 & 0.2695 \\
\hline 294.96 & 0.0057 & 446.97 & 0.0775 & 579.69 & 0.2911 \\
\hline 305.05 & 0.0067 & 457.64 & 0.0851 & & \\
\hline
\end{tabular}


Table 289. Test data for specimen 742-22

\begin{tabular}{|c|c|c|c|c|c|}
\hline $\begin{array}{l}\text { Test Number } \\
\text { Aging Temp. } \\
\text { Yield Stress }\end{array}$ & $\begin{array}{l}: 1 \\
: 4 c \\
: 26\end{array}$ & & $\begin{array}{l}\text { Tes } \\
\text { Agir } \\
\text { Ulti }\end{array}$ & $\begin{array}{l}\text { np. } \\
\text { ne } \\
\text { Stress }\end{array}$ & $\begin{array}{l}25^{\circ} \mathrm{C} \\
10,000 \mathrm{~h} \\
626.3 \mathrm{MPa}\end{array}$ \\
\hline $\begin{array}{l}\text { Engr. } \\
\text { Stress } \\
(\mathrm{MPa})\end{array}$ & $\begin{array}{l}\text { Engr. } \\
\text { Strain }\end{array}$ & $\begin{array}{l}\text { Engr. } \\
\text { Stress } \\
(\mathrm{MPa})\end{array}$ & $\begin{array}{l}\text { Engr. } \\
\text { Strain }\end{array}$ & $\begin{array}{l}\text { Engr. } \\
\text { Stress } \\
(\mathrm{MPa}) \\
\end{array}$ & $\begin{array}{l}\text { Engr. } \\
\text { Strain }\end{array}$ \\
\hline 29.307 & 0.0001 & 450.635 & 0.0601 & 555.718 & 0.1472 \\
\hline 44.895 & 0.0002 & 453.035 & 0.0617 & 561.320 & 0.1533 \\
\hline 151.596 & 0.0009 & 473.476 & 0.0760 & 571.992 & 0.1650 \\
\hline 162.621 & 0.0010 & 475.586 & 0.0776 & 573.344 & 0.1664 \\
\hline 184.346 & 0.0012 & 477.702 & 0.0792 & 588.411 & 0.1857 \\
\hline 280.332 & 0.0042 & 496.322 & 0.0935 & 596.910 & 0.1974 \\
\hline 285.417 & 0.0045 & 513.175 & 0.1071 & 601.111 & 0.2041 \\
\hline 400.235 & 0.0334 & 515.010 & 0.1086 & 603.042 & 0.2075 \\
\hline 423.110 & 0.0425 & 523.509 & 0.1155 & 608.403 & 0.2175 \\
\hline 425.679 & 0.0441 & 526.841 & 0.1185 & 618.737 & 0.2431 \\
\hline 448.433 & 0.0585 & 552.869 & 0.1441 & 626.125 & 0.3040 \\
\hline
\end{tabular}

Table 290. Test data for specimen 742-23

\begin{tabular}{|c|c|c|c|c|c|}
\hline $\begin{array}{l}\text { Test Number } \\
\text { Aging Temp. } \\
\text { Yield Stress }\end{array}$ & $\begin{array}{l}: 1 \\
: 40 \\
: 26\end{array}$ & & $\begin{array}{l}\text { Tes } \\
\text { Agi } \\
\text { Ulti }\end{array}$ & $\begin{array}{l}\text { np. } \\
\text { ne } \\
\text { Stress }\end{array}$ & $\begin{array}{l}25^{\circ} \mathrm{C} \\
10,000 \mathrm{~h} \\
624.3 \mathrm{MPa}\end{array}$ \\
\hline $\begin{array}{l}\text { Engr. } \\
\text { Stress } \\
(\mathrm{MPa})\end{array}$ & $\begin{array}{l}\text { Engr. } \\
\text { Strain }\end{array}$ & $\begin{array}{l}\text { Engr. } \\
\text { Stress } \\
(\mathrm{MPa})\end{array}$ & $\begin{array}{l}\text { Engr. } \\
\text { Strain }\end{array}$ & $\begin{array}{l}\text { Engr. } \\
\text { Stress } \\
(\mathrm{MPa}) \\
\end{array}$ & $\begin{array}{l}\text { Engr. } \\
\text { Strain }\end{array}$ \\
\hline 7.838 & 0.0001 & 452.588 & 0.0609 & 545.063 & 0.1425 \\
\hline 18.933 & 0.0001 & 458.157 & 0.0646 & 566.546 & 0.1703 \\
\hline 60.733 & 0.0005 & 461.162 & 0.0670 & 578.207 & 0.1885 \\
\hline 160.862 & 0.0012 & 473.171 & 0.0759 & 593.351 & 0.2171 \\
\hline 171.527 & 0.0013 & 475.804 & 0.0779 & 595.238 & 0.2212 \\
\hline 256.719 & 0.0033 & 478.895 & 0.0802 & 599.835 & 0.2324 \\
\hline 262.796 & 0.0036 & 527.837 & 0.1238 & 612.851 & 0.2728 \\
\hline 331.949 & 0.0091 & 529.870 & 0.1261 & 615.947 & 0.2859 \\
\hline 354.187 & 0.0131 & 532.289 & 0.1285 & 616.383 & 0.2881 \\
\hline 359.040 & 0.0143 & 540.902 & 0.1378 & 621.270 & 0.3192 \\
\hline 364.377 & 0.0158 & 542.934 & 0.1401 & 624.221 & 0.3676 \\
\hline
\end{tabular}


Table 291. Test data for specimen 74-110

\begin{tabular}{|c|c|c|c|c|c|}
\hline $\begin{array}{l}\text { Test Number } \\
\text { Aging Temp. } \\
\text { Yield Stress }\end{array}$ & $\begin{array}{l}: 18 \\
: 40 \\
: 25\end{array}$ & $\mathrm{~Pa}$ & $\begin{array}{l}\text { Tes } \\
\text { Agir } \\
\text { Ultir }\end{array}$ & $\begin{array}{l}\text { mp. } \\
\text { me } \\
\text { Stress }\end{array}$ & $\begin{array}{l}25^{\circ} \mathrm{C} \\
10,000 \mathrm{~h} \\
607.9 \mathrm{MPa}\end{array}$ \\
\hline $\begin{array}{l}\text { Engr. } \\
\text { Stress } \\
(\mathrm{MPa})\end{array}$ & $\begin{array}{l}\text { Engr. } \\
\text { Strain } \\
\end{array}$ & $\begin{array}{l}\text { Engr. } \\
\text { Stress } \\
(\mathrm{MPa})\end{array}$ & $\begin{array}{l}\text { Engr. } \\
\text { Strain }\end{array}$ & $\begin{array}{l}\text { Engr. } \\
\text { Stress } \\
\text { (MPa) }\end{array}$ & $\begin{array}{l}\text { Engr. } \\
\text { Strain }\end{array}$ \\
\hline 27.251 & 0.0003 & 269.609 & 0.0045 & 417.156 & 0.0544 \\
\hline 36.763 & 0.0003 & 299.651 & 0.0073 & 420.523 & 0.0568 \\
\hline 95.948 & 0.0007 & 305.830 & 0.0080 & 505.387 & 0.1330 \\
\hline 106.593 & 0.0008 & 311.627 & 0.0088 & 507.661 & 0.1354 \\
\hline 129.649 & 0.0010 & 362.388 & 0.0226 & 515.644 & 0.1446 \\
\hline 140.526 & 0.0011 & 367.439 & 0.0249 & 518.789 & 0.1486 \\
\hline 183.265 & 0.0015 & 372.278 & 0.0271 & 525.515 & 0.1571 \\
\hline 192.898 & 0.0016 & 388.531 & 0.0361 & 527.160 & 0.1592 \\
\hline 201.932 & 0.0018 & 392.619 & 0.0384 & 541.579 & 0.1797 \\
\hline 259.569 & 0.0039 & 396.330 & 0.0407 & 548.450 & 0.1900 \\
\hline 264.765 & 0.0042 & 413.894 & 0.0521 & 554.159 & 0.1985 \\
\hline
\end{tabular}

Table 292. Test data for specimen 741-04

\begin{tabular}{|c|c|c|c|c|c|}
\hline $\begin{array}{l}\text { Test Number } \\
\text { Aging Temp. } \\
\text { Yield Stress }\end{array}$ & & $\mathrm{Pa}$ & $\begin{array}{l}\text { Tes } \\
\text { Agi } \\
\text { Ulti }\end{array}$ & $\begin{array}{l}\text { np. } \\
\text { me } \\
\text { Stress }\end{array}$ & $\begin{array}{l}25^{\circ} \mathrm{C} \\
2,570 \mathrm{~h} \\
620.5 \mathrm{MPa}\end{array}$ \\
\hline $\begin{array}{l}\text { Engr. } \\
\text { Stress } \\
(\mathrm{MPa}) \\
\end{array}$ & $\begin{array}{l}\text { Engr. } \\
\text { Strain }\end{array}$ & $\begin{array}{l}\text { Engr. } \\
\text { Stress } \\
(\mathrm{MPa}) \\
\end{array}$ & $\begin{array}{l}\text { Engr. } \\
\text { Strain }\end{array}$ & $\begin{array}{l}\text { Engr. } \\
\text { Stress } \\
(\mathrm{MPa}) \\
\end{array}$ & $\begin{array}{l}\text { Engr. } \\
\text { Strain }\end{array}$ \\
\hline 57.76 & 0.0001 & 336.51 & 0.0097 & 505.01 & 0.0943 \\
\hline 84.35 & 0.0003 & 349.61 & 0.0118 & 526.11 & 0.1091 \\
\hline 108.16 & 0.0005 & 361.78 & 0.0143 & 544.66 & 0.1268 \\
\hline 132.20 & 0.0008 & 375.38 & 0.0179 & 561.54 & 0.1466 \\
\hline 156.26 & 0.0012 & 389.25 & 0.0226 & 575.34 & 0.1645 \\
\hline 180.26 & 0.0016 & 400.36 & 0.0268 & 593.78 & 0.1967 \\
\hline 199.16 & 0.0020 & 410.97 & 0.0316 & 605.06 & 0.2220 \\
\hline 216.72 & 0.0021 & 425.03 & 0.0385 & 614.36 & 0.2574 \\
\hline 226.06 & 0.0022 & 449.49 & 0.0533 & 617.97 & 0.2783 \\
\hline 240.32 & 0.0026 & 464.93 & 0.0634 & 620.32 & 0.2996 \\
\hline 328.50 & 0.0086 & 480.00 & 0.0742 & 620.54 & 0.3100 \\
\hline 332.62 & 0.0092 & 481.96 & 0.0770 & 620.52 & 0.3306 \\
\hline
\end{tabular}


Table 293. Test data for specimen 741-05

\begin{tabular}{|c|c|c|c|c|c|}
\hline $\begin{array}{l}\text { Test Number } \\
\text { Aging Temp. } \\
\text { Yield Stress }\end{array}$ & & & $\begin{array}{l}\text { Tes } \\
\text { Agi } \\
\text { Ulti }\end{array}$ & $\begin{array}{l}\text { np. } \\
\text { ne } \\
\text { Stress }\end{array}$ & $\begin{array}{l}25^{\circ} \mathrm{C} \\
2,570 \mathrm{~h} \\
616.7 \mathrm{MPa}\end{array}$ \\
\hline $\begin{array}{l}\text { Engr. } \\
\text { Stress } \\
\text { (MPa) }\end{array}$ & $\begin{array}{l}\text { Engr. } \\
\text { Strain }\end{array}$ & $\begin{array}{l}\text { Engr. } \\
\text { Stress } \\
(\mathrm{MPa})\end{array}$ & $\begin{array}{l}\text { Engr. } \\
\text { Strain }\end{array}$ & $\begin{array}{l}\text { Engr. } \\
\text { Stress } \\
(\mathrm{MPa}) \\
\end{array}$ & $\begin{array}{l}\text { Engr. } \\
\text { Strain }\end{array}$ \\
\hline 46.28 & 0.0000 & 348.16 & 0.0102 & 551.58 & 0.1684 \\
\hline 65.38 & 0.0001 & 355.90 & 0.0114 & 560.05 & 0.1884 \\
\hline 88.01 & 0.0003 & 366.76 & 0.0135 & 569.32 & 0.2109 \\
\hline 107.09 & 0.0004 & 373.19 & 0.0152 & 576.12 & 0.2301 \\
\hline 133.95 & 0.0008 & 385.18 & 0.0188 & 583.73 & 0.2527 \\
\hline 165.32 & 0.0012 & 395.22 & 0.0225 & 593.51 & 0.2900 \\
\hline 192.16 & 0.0017 & 409.99 & 0.0289 & 598.13 & 0.3141 \\
\hline 209.14 & 0.0019 & 424.77 & 0.0375 & 601.79 & 0.3379 \\
\hline 224.96 & 0.0020 & 443.70 & 0.0500 & 604.62 & 0.3558 \\
\hline 239.70 & 0.0024 & 462.41 & 0.0649 & 607.18 & 0.3778 \\
\hline 249.73 & 0.0027 & 478.24 & 0.0784 & 610.03 & 0.4046 \\
\hline 264.87 & 0.0035 & 481.27 & 0.0823 & 614.29 & 0.4488 \\
\hline 282.59 & 0.0043 & 497.88 & 0.0978 & 615.70 & 0.4714 \\
\hline 301.23 & 0.0054 & 512.65 & 0.1115 & 616.36 & 0.4978 \\
\hline 319.14 & 0.0069 & 526.86 & 0.1293 & 616.73 & 0.5243 \\
\hline 328.20 & 0.0077 & 540.49 & 0.1494 & & \\
\hline
\end{tabular}

Table 294. Test data for specimen 744-40

\begin{tabular}{|c|c|c|c|c|c|}
\hline $\begin{array}{l}\text { Test Number } \\
\text { Aging Temp. } \\
\text { Yield Stress }\end{array}$ & $\begin{array}{l}: 04 \\
: \text { Un } \\
: 17\end{array}$ & $\mathrm{~Pa}$ & $\begin{array}{l}\text { Tes } \\
\text { Agir } \\
\text { Ulti }\end{array}$ & $\begin{array}{l}\text { np. } \\
\text { ne } \\
\text { Stress }\end{array}$ & $\begin{array}{l}290^{\circ} \mathrm{C} \\
- \\
412.3 \mathrm{MPa}\end{array}$ \\
\hline $\begin{array}{l}\text { Engr. } \\
\text { Stress } \\
(\mathrm{MPa}) \\
\end{array}$ & $\begin{array}{l}\text { Engr. } \\
\text { Strain } \\
\end{array}$ & $\begin{array}{l}\text { Engr. } \\
\text { Stress } \\
\text { (MPa) }\end{array}$ & $\begin{array}{l}\text { Engr. } \\
\text { Strain }\end{array}$ & $\begin{array}{l}\text { Engr. } \\
\text { Stress } \\
(\mathrm{MPa}) \\
\end{array}$ & $\begin{array}{l}\text { Engr. } \\
\text { Strain }\end{array}$ \\
\hline 42.91 & 0.0000 & 170.80 & 0.0018 & 295.56 & 0.0639 \\
\hline 51.82 & 0.0000 & 173.86 & 0.0019 & 312.41 & 0.0797 \\
\hline 60.94 & 0.0001 & 176.18 & 0.0021 & 328.07 & 0.0942 \\
\hline 76.80 & 0.0002 & 178.69 & 0.0023 & 343.16 & 0.1080 \\
\hline 89.56 & 0.0002 & 181.06 & 0.0026 & 359.03 & 0.1244 \\
\hline 103.75 & 0.0004 & 187.92 & 0.0036 & 373.28 & 0.1419 \\
\hline 114.14 & 0.0005 & 192.66 & 0.0045 & 385.86 & 0.1599 \\
\hline 127.29 & 0.0006 & 196.21 & 0.0054 & 396.80 & 0.1766 \\
\hline 139.22 & 0.0008 & 202.49 & 0.0068 & 404.05 & 0.1924 \\
\hline 148.28 & 0.0010 & 211.24 & 0.0080 & 409.30 & 0.2065 \\
\hline 156.71 & 0.0012 & 221.04 & 0.0096 & 409.26 & 0.2067 \\
\hline 160.85 & 0.0013 & 236.13 & 0.0154 & 407.78 & 0.2071 \\
\hline 164.30 & 0.0015 & 256.44 & 0.0282 & 407.95 & 0.2098 \\
\hline 167.80 & 0.0016 & 276.94 & 0.0463 & 412.30 & 0.2131 \\
\hline
\end{tabular}


Table 295. Test data for specimen 743-42

\begin{tabular}{|c|c|c|c|c|c|}
\hline $\begin{array}{l}\text { Test Number } \\
\text { Aging Temp. } \\
\text { Yield Stress }\end{array}$ & $\begin{array}{l}: 0 \\
: \text { Ur } \\
: 16\end{array}$ & $\mathrm{~Pa}$ & $\begin{array}{l}\text { Tes } \\
\text { Agi } \\
\text { Ulti }\end{array}$ & $\begin{array}{l}\text { np. } \\
\text { ne } \\
\text { Stress }\end{array}$ & $\begin{array}{l}290^{\circ} \mathrm{C} \\
- \\
442.8 \mathrm{MPa}\end{array}$ \\
\hline $\begin{array}{l}\text { Engr. } \\
\text { Stress } \\
\text { (MPa) }\end{array}$ & $\begin{array}{l}\text { Engr. } \\
\text { Strain }\end{array}$ & $\begin{array}{l}\text { Engr. } \\
\text { Stress } \\
(\mathrm{MPa})\end{array}$ & $\begin{array}{l}\text { Engr. } \\
\text { Strain }\end{array}$ & $\begin{array}{l}\text { Engr. } \\
\text { Stress } \\
(\mathrm{MPa}) \\
\end{array}$ & $\begin{array}{l}\text { Engr. } \\
\text { Strain }\end{array}$ \\
\hline 57.58 & 0.0000 & 176.62 & 0.0027 & 358.71 & 0.1229 \\
\hline 88.11 & 0.0002 & 179.68 & 0.0031 & 369.82 & 0.1363 \\
\hline 123.54 & 0.0006 & 183.55 & 0.0038 & 380.25 & 0.1489 \\
\hline 129.27 & 0.0007 & 190.19 & 0.0055 & 387.68 & 0.1592 \\
\hline 144.82 & 0.0010 & 201.80 & 0.0095 & 398.32 & 0.1743 \\
\hline 155.49 & 0.0013 & 216.24 & 0.0120 & 404.50 & 0.1845 \\
\hline 161.27 & 0.0015 & 231.45 & 0.0149 & 414.05 & 0.2025 \\
\hline 163.02 & 0.0016 & 243.78 & 0.0204 & 421.87 & 0.2191 \\
\hline 165.82 & 0.0017 & 263.18 & 0.0361 & 428.54 & 0.2361 \\
\hline 167.32 & 0.0018 & 280.88 & 0.0519 & 430.64 & 0.2405 \\
\hline 168.82 & 0.0019 & 297.27 & 0.0669 & 432.81 & 0.2501 \\
\hline 170.87 & 0.0021 & 313.32 & 0.0814 & 438.13 & 0.2694 \\
\hline 172.67 & 0.0023 & 330.31 & 0.0967 & 441.81 & 0.2893 \\
\hline 174.92 & 0.0024 & 342.43 & 0.1062 & 442.81 & 0.3044 \\
\hline
\end{tabular}

Table 296. Test data for specimen 744-35

\begin{tabular}{|c|c|c|c|c|c|}
\hline $\begin{array}{l}\text { Test Number } \\
\text { Aging Temp. } \\
\text { Yield Stress }\end{array}$ & $\begin{array}{l}: 25 \\
: 29 \\
: 19\end{array}$ & & $\begin{array}{l}\text { Tes } \\
\text { Agir } \\
\text { Ulti }\end{array}$ & $\begin{array}{l}\text { np. } \\
\text { ne } \\
\text { Stress }\end{array}$ & $\begin{array}{l}290^{\circ} \mathrm{C} \\
30,000 \mathrm{~h} \\
424.7 \mathrm{MPa}\end{array}$ \\
\hline $\begin{array}{l}\text { Engr. } \\
\text { Stress } \\
(\mathrm{MPa})\end{array}$ & $\begin{array}{l}\text { Engr. } \\
\text { Strain }\end{array}$ & $\begin{array}{l}\text { Engr. } \\
\text { Stress } \\
\text { (MPa) }\end{array}$ & $\begin{array}{l}\text { Engr. } \\
\text { Strain }\end{array}$ & $\begin{array}{l}\text { Engr. } \\
\text { Stress } \\
(\mathrm{MPa}) \\
\end{array}$ & $\begin{array}{l}\text { Engr. } \\
\text { Strain }\end{array}$ \\
\hline 46.281 & 0.0003 & 262.187 & 0.0618 & 364.863 & 0.1762 \\
\hline 66.334 & 0.0002 & 264.606 & 0.0641 & 365.439 & 0.1783 \\
\hline 76.556 & 0.0002 & 271.916 & 0.0708 & 367.945 & 0.1805 \\
\hline 126.358 & 0.0005 & 290.609 & 0.0885 & 380.079 & 0.2008 \\
\hline 147.294 & 0.0007 & 292.808 & 0.0906 & 386.896 & 0.2127 \\
\hline 187.269 & 0.0068 & 299.241 & 0.0972 & 399.391 & 0.2392 \\
\hline 192.034 & 0.0089 & 315.622 & 0.1147 & 404.331 & 0.2483 \\
\hline 196.248 & 0.0110 & 318.192 & 0.1168 & 409.803 & 0.2664 \\
\hline 224.796 & 0.0309 & 327.131 & 0.1259 & 413.412 & 0.2731 \\
\hline 233.199 & 0.0378 & 338.928 & 0.1429 & 422.166 & 0.3093 \\
\hline 241.553 & 0.0442 & 347.272 & 0.1512 & 424.697 & 0.3363 \\
\hline
\end{tabular}


Table 297. Test data for specimen 744-36

\begin{tabular}{|c|c|c|c|c|c|}
\hline $\begin{array}{l}\text { Test Number } \\
\text { Aging Temp. } \\
\text { Yield Stress }\end{array}$ & $\begin{array}{l}: 2 \\
: 25 \\
: 16\end{array}$ & & $\begin{array}{l}\text { Tes } \\
\text { Agi } \\
\text { Ulti }\end{array}$ & $\begin{array}{l}\text { np. } \\
\text { me } \\
\text { Stress }\end{array}$ & $\begin{array}{l}290^{\circ} \mathrm{C} \\
30,000 \mathrm{~h} \\
424.1 \mathrm{MPa}\end{array}$ \\
\hline $\begin{array}{l}\text { Engr. } \\
\text { Stress } \\
\text { (MPa) }\end{array}$ & $\begin{array}{l}\text { Engr. } \\
\text { Strain }\end{array}$ & $\begin{array}{l}\text { Engr. } \\
\text { Stress } \\
(\mathrm{MPa})\end{array}$ & $\begin{array}{l}\text { Engr. } \\
\text { Strain }\end{array}$ & $\begin{array}{l}\text { Engr. } \\
\text { Stress } \\
\text { (MPa) }\end{array}$ & $\begin{array}{l}\text { Engr. } \\
\text { Strain }\end{array}$ \\
\hline 46.895 & 0.0003 & 259.236 & 0.0618 & 346.882 & 0.1505 \\
\hline 52.240 & 0.0003 & 262.299 & 0.0644 & 357.597 & 0.1659 \\
\hline 113.615 & 0.0004 & 270.063 & 0.0713 & 363.639 & 0.1726 \\
\hline 134.258 & 0.0006 & 289.170 & 0.0885 & 377.679 & 0.1989 \\
\hline 139.842 & 0.0008 & 291.882 & 0.0911 & 381.990 & 0.2011 \\
\hline 187.211 & 0.0095 & 298.514 & 0.0973 & 394.875 & 0.2292 \\
\hline 191.322 & 0.0117 & 315.744 & 0.1148 & 398.825 & 0.2378 \\
\hline 205.060 & 0.0200 & 317.348 & 0.1172 & 407.730 & 0.2597 \\
\hline 223.611 & 0.0333 & 323.405 & 0.1240 & 411.407 & 0.2684 \\
\hline 234.886 & 0.0422 & 338.879 & 0.1410 & 417.099 & 0.2932 \\
\hline 237.695 & 0.0442 & 341.474 & 0.1430 & 423.994 & 0.3209 \\
\hline
\end{tabular}

Table 298. Test data for specimen 74-263

\begin{tabular}{|c|c|c|c|c|c|}
\hline $\begin{array}{l}\text { Test Number } \\
\text { Aging Temp. } \\
\text { Yield Stress }\end{array}$ & $\begin{array}{l}2 \\
: 25 \\
: 17\end{array}$ & $\mathrm{MPa}$ & $\begin{array}{l}\text { Tes } \\
\text { Agi } \\
\text { Ulti }\end{array}$ & $\begin{array}{l}\text { np. } \\
\text { me } \\
\text { Stress }\end{array}$ & $\begin{array}{l}290^{\circ} \mathrm{C} \\
30,000 \mathrm{~h} \\
436.3 \mathrm{MPa}\end{array}$ \\
\hline $\begin{array}{l}\text { Engr. } \\
\text { Stress } \\
(\mathrm{MPa})\end{array}$ & $\begin{array}{l}\text { Engr. } \\
\text { Strain }\end{array}$ & $\begin{array}{l}\text { Engr. } \\
\text { Stress } \\
\text { (MPa) }\end{array}$ & $\begin{array}{l}\text { Engr. } \\
\text { Strain }\end{array}$ & $\begin{array}{l}\text { Engr. } \\
\text { Stress } \\
(\mathrm{MPa}) \\
\end{array}$ & $\begin{array}{l}\text { Engr. } \\
\text { Strain }\end{array}$ \\
\hline 38.320 & 0.0002 & 198.728 & 0.0073 & 336.859 & 0.0937 \\
\hline 40.152 & 0.0002 & 226.304 & 0.0196 & 358.528 & 0.1117 \\
\hline 62.734 & 0.0003 & 230.316 & 0.0219 & 367.223 & 0.1200 \\
\hline 72.026 & 0.0005 & 241.648 & 0.0284 & 386.259 & 0.1384 \\
\hline 101.958 & 0.0007 & 262.365 & 0.0413 & 393.259 & 0.1455 \\
\hline 112.620 & 0.0008 & 272.381 & 0.0478 & 405.966 & 0.1595 \\
\hline 140.380 & 0.0014 & 275.514 & 0.0500 & 410.075 & 0.1671 \\
\hline 148.405 & 0.0016 & 294.507 & 0.0628 & 423.768 & 0.1850 \\
\hline 155.550 & 0.0019 & 304.683 & 0.0697 & 423.171 & 0.1873 \\
\hline 181.372 & 0.0036 & 307.758 & 0.0718 & 430.695 & 0.2054 \\
\hline 193.190 & 0.0056 & 326.420 & 0.0852 & 436.330 & 0.2253 \\
\hline
\end{tabular}


Table 299. Test data for specimen 744-26

$\begin{array}{llll}\text { Test Number } & : 336 & \text { Test Temp. } & : 290^{\circ} \mathrm{C} \\ \text { Aging Temp. } & : 320^{\circ} \mathrm{C} & \text { Aging Time } & : 10,000 \mathrm{~h} \\ \text { Yield Stress } & : 192.7 \mathrm{MPa} & \text { Ultimate Stress } & : 440.1 \mathrm{MPa}\end{array}$

\begin{tabular}{cccccc}
\hline $\begin{array}{c}\text { Engr. } \\
\text { Stress } \\
(\mathrm{MPa})\end{array}$ & $\begin{array}{c}\text { Engr. } \\
\text { Strain }\end{array}$ & $\begin{array}{c}\text { Engr. } \\
\text { Stress } \\
(\mathrm{MPa})\end{array}$ & $\begin{array}{c}\text { Engr. } \\
\text { Strain }\end{array}$ & $\begin{array}{c}\text { Engr. } \\
\text { Stress } \\
(\mathrm{MPa})\end{array}$ & $\begin{array}{c}\text { Engr. } \\
\text { Strain }\end{array}$ \\
\hline & & & & & \\
106.11 & 0.0001 & 280.42 & 0.0515 & 375.61 & 0.1395 \\
142.17 & 0.0003 & 289.10 & 0.0580 & 380.92 & 0.1462 \\
171.87 & 0.0012 & 291.95 & 0.0603 & 384.35 & 0.1506 \\
196.70 & 0.0039 & 294.72 & 0.0624 & 392.74 & 0.1617 \\
201.56 & 0.0049 & 297.52 & 0.0646 & 398.63 & 0.1706 \\
206.97 & 0.0065 & 305.61 & 0.0713 & 405.55 & 0.1817 \\
212.68 & 0.0087 & 308.19 & 0.0735 & 410.35 & 0.1902 \\
226.58 & 0.0154 & 310.94 & 0.0756 & 416.01 & 0.2021 \\
230.71 & 0.0177 & 318.65 & 0.0822 & 417.15 & 0.2043 \\
234.62 & 0.0201 & 326.10 & 0.0889 & 419.29 & 0.2110 \\
238.39 & 0.0224 & 335.70 & 0.0976 & 425.77 & 0.2248 \\
248.94 & 0.0292 & 342.64 & 0.1043 & 428.38 & 0.2313 \\
252.34 & 0.0314 & 351.49 & 0.1130 & 431.64 & 0.2402 \\
262.02 & 0.0381 & 357.75 & 0.1196 & 433.42 & 0.2488 \\
265.25 & 0.0405 & 365.93 & 0.1285 & 435.67 & 0.2581 \\
277.41 & 0.0493 & 371.80 & 0.1352 & 436.29 & 0.2603 \\
\hline
\end{tabular}

Table 300. Test data for specimen 744-27

\begin{tabular}{|c|c|c|c|c|c|}
\hline $\begin{array}{l}\text { Test Number } \\
\text { Aging Temp. } \\
\text { Yield Stress }\end{array}$ & $\begin{array}{l}: 3 \\
: 32 \\
: 18\end{array}$ & & $\begin{array}{l}\text { Tes } \\
\text { Agi } \\
\text { Ulti }\end{array}$ & $\begin{array}{l}\text { np. } \\
\text { ne } \\
\text { Stress }\end{array}$ & $\begin{array}{l}290^{\circ} \mathrm{C} \\
10,000 \mathrm{~h} \\
420.6 \mathrm{MPa}\end{array}$ \\
\hline $\begin{array}{l}\text { Engr. } \\
\text { Stress } \\
\text { (MPa) }\end{array}$ & $\begin{array}{l}\text { Engr. } \\
\text { Strain }\end{array}$ & $\begin{array}{l}\text { Engr. } \\
\text { Stress } \\
(\mathrm{MPa})\end{array}$ & $\begin{array}{l}\text { Engr. } \\
\text { Strain }\end{array}$ & $\begin{array}{l}\text { Engr. } \\
\text { Stress } \\
(\mathrm{MPa}) \\
\end{array}$ & $\begin{array}{l}\text { Engr. } \\
\text { Strain }\end{array}$ \\
\hline 16.87 & 0.0001 & 258.45 & 0.0532 & 376.49 & 0.1870 \\
\hline 86.83 & 0.0003 & 268.36 & 0.0621 & 382.10 & 0.1956 \\
\hline 140.52 & 0.0005 & 280.12 & 0.0731 & 387.60 & 0.2043 \\
\hline 160.25 & 0.0010 & 291.47 & 0.0841 & 391.28 & 0.2136 \\
\hline 172.60 & 0.0017 & 296.00 & 0.0885 & 393.48 & 0.2160 \\
\hline 190.12 & 0.0046 & 304.61 & 0.0974 & 398.73 & 0.2277 \\
\hline 195.82 & 0.0068 & 315.12 & 0.1084 & 404.59 & 0.2395 \\
\hline 200.48 & 0.0089 & 321.17 & 0.1150 & 406.19 & 0.2485 \\
\hline 210.77 & 0.0150 & 327.10 & 0.1216 & 411.17 & 0.2577 \\
\hline 214.15 & 0.0173 & 331.05 & 0.1259 & 411.35 & 0.2601 \\
\hline 216.89 & 0.0193 & 336.75 & 0.1326 & 414.90 & 0.2698 \\
\hline 226.19 & 0.0262 & 338.58 & 0.1347 & 415.08 & 0.2720 \\
\hline 232.02 & 0.0308 & 347.41 & 0.1456 & 416.85 & 0.2789 \\
\hline 240.32 & 0.0376 & 355.92 & 0.1564 & 418.48 & 0.2880 \\
\hline 245.63 & 0.0422 & 362.16 & 0.1650 & 419.34 & 0.2900 \\
\hline 253.46 & 0.0488 & 369.80 & 0.1759 & 420.58 & 0.2969 \\
\hline
\end{tabular}


Table 301. Test data for specimen 742-40

\begin{tabular}{|c|c|c|c|c|c|}
\hline $\begin{array}{l}\text { Test Number } \\
\text { Aging Temp. } \\
\text { Yield Stress }\end{array}$ & $\begin{array}{l}: 1 \\
: 32 \\
: 15\end{array}$ & & $\begin{array}{l}\text { Tes } \\
\text { Agi } \\
\text { Ulti }\end{array}$ & $\begin{array}{l}\text { mp. } \\
\text { me } \\
\text { Stress }\end{array}$ & $\begin{array}{l}290^{\circ} \mathrm{C} \\
30,000 \mathrm{~h} \\
452.7 \mathrm{MPa}\end{array}$ \\
\hline $\begin{array}{l}\text { Engr. } \\
\text { Stress } \\
\text { (MPa) }\end{array}$ & $\begin{array}{l}\text { Engr. } \\
\text { Strain }\end{array}$ & $\begin{array}{l}\text { Engr. } \\
\text { Stress } \\
\text { (MPa) }\end{array}$ & $\begin{array}{l}\text { Engr. } \\
\text { Strain }\end{array}$ & $\begin{array}{l}\text { Engr. } \\
\text { Stress } \\
(\mathrm{MPa})\end{array}$ & $\begin{array}{l}\text { Engr. } \\
\text { Strain }\end{array}$ \\
\hline $\begin{array}{r}0.00 \\
78.74 \\
145.35 \\
183.04 \\
208.12 \\
236.95 \\
264.70\end{array}$ & $\begin{array}{l}0.0000 \\
0.0002 \\
0.0010 \\
0.0027 \\
0.0055 \\
0.0154 \\
0.0321\end{array}$ & $\begin{array}{l}286.83 \\
347.63 \\
392.11 \\
426.74 \\
447.13 \\
452.68\end{array}$ & $\begin{array}{l}0.0490 \\
0.0980 \\
0.1374 \\
0.1873 \\
0.2286 \\
0.2669\end{array}$ & & \\
\hline
\end{tabular}

Table 302. Test data for specimen 742-41

\begin{tabular}{|c|c|c|c|c|c|}
\hline $\begin{array}{l}\text { Test Number } \\
\text { Aging Temp. } \\
\text { Yield Stress }\end{array}$ & $\begin{array}{l}: 1 \\
: 32 \\
: 15\end{array}$ & & $\begin{array}{l}\text { Tes } \\
\text { Agir } \\
\text { Ulti }\end{array}$ & $\begin{array}{l}\text { mp. } \\
\text { me } \\
\text { Stress }\end{array}$ & $\begin{array}{l}290^{\circ} \mathrm{C} \\
30,000 \mathrm{~h} \\
483.3 \mathrm{MPa}\end{array}$ \\
\hline $\begin{array}{l}\text { Engr. } \\
\text { Stress } \\
(\mathrm{MPa})\end{array}$ & $\begin{array}{l}\text { Engr. } \\
\text { Strain }\end{array}$ & $\begin{array}{l}\text { Engr. } \\
\text { Stress } \\
(\mathrm{MPa})\end{array}$ & $\begin{array}{l}\text { Engr. } \\
\text { Strain }\end{array}$ & $\begin{array}{l}\text { Engr. } \\
\text { Stress } \\
\text { (MPa) } \\
\end{array}$ & $\begin{array}{l}\text { Engr. } \\
\text { Strain }\end{array}$ \\
\hline 0.00 & 0.0000 & 236.23 & 0.0083 & 335.07 & 0.0561 \\
\hline 43.48 & 0.0001 & 241.02 & 0.0093 & 346.32 & 0.0638 \\
\hline 74.85 & 0.0004 & 246.71 & 0.0107 & 359.87 & 0.0727 \\
\hline 111.79 & 0.0008 & 251.05 & 0.0119 & 368.82 & 0.0789 \\
\hline 133.01 & 0.0012 & 254.74 & 0.0131 & 381.42 & 0.0877 \\
\hline 146.65 & 0.0014 & 259.29 & 0.0147 & 394.19 & 0.0970 \\
\hline 155.17 & 0.0017 & 263.38 & 0.0163 & 410.61 & 0.1074 \\
\hline 162.99 & 0.0019 & 266.98 & 0.0178 & 422.27 & 0.1173 \\
\hline 171.50 & 0.0022 & 271.22 & 0.0195 & 434.15 & 0.1290 \\
\hline 180.07 & 0.0025 & 276.26 & 0.0218 & 445.44 & 0.1413 \\
\hline 188.89 & 0.0030 & 280.21 & 0.0237 & 454.93 & 0.1531 \\
\hline 195.31 & 0.0033 & 286.20 & 0.0270 & 461.77 & 0.1633 \\
\hline 204.43 & 0.0039 & 293.15 & 0.0307 & 472.59 & 0.1816 \\
\hline 210.66 & 0.0045 & 303.88 & 0.0366 & 481.07 & 0.2002 \\
\hline 218.08 & 0.0053 & 314.33 & 0.0430 & 483.35 & 0.2125 \\
\hline 221.83 & 0.0058 & 320.48 & 0.0468 & 483.13 & 0.2179 \\
\hline 228.90 & 0.0068 & 327.32 & 0.0511 & & \\
\hline
\end{tabular}


Table 303. Test data for specimen 74-246

\begin{tabular}{|c|c|c|c|c|c|}
\hline $\begin{array}{l}\text { Test Number } \\
\text { Aging Temp. } \\
\text { Yield Stress }\end{array}$ & $\begin{array}{l}: 1 \\
: 32 \\
: 18\end{array}$ & & $\begin{array}{l}\text { Tes } \\
\text { Agi } \\
\text { Ulti }\end{array}$ & $\begin{array}{l}\text { np. } \\
\text { ne } \\
\text { Stress }\end{array}$ & $\begin{array}{l}290^{\circ} \mathrm{C} \\
30,000 \mathrm{~h} \\
481.7 \mathrm{MPa}\end{array}$ \\
\hline $\begin{array}{l}\text { Engr. } \\
\text { Stress } \\
(\mathrm{MPa}) \\
\end{array}$ & $\begin{array}{l}\text { Engr. } \\
\text { Strain }\end{array}$ & $\begin{array}{l}\text { Engr. } \\
\text { Stress } \\
(\mathrm{MPa})\end{array}$ & $\begin{array}{l}\text { Engr. } \\
\text { Strain }\end{array}$ & $\begin{array}{l}\text { Engr. } \\
\text { Stress } \\
(\mathrm{MPa})\end{array}$ & $\begin{array}{l}\text { Engr. } \\
\text { Strain }\end{array}$ \\
\hline 0.00 & 0.0000 & 232.55 & 0.0097 & 363.33 & 0.0877 \\
\hline 39.37 & 0.0001 & 236.37 & 0.0108 & 383.42 & 0.1008 \\
\hline 72.32 & 0.0003 & 241.09 & 0.0123 & 403.82 & 0.1187 \\
\hline 106.72 & 0.0008 & 244.95 & 0.0138 & 415.39 & 0.1301 \\
\hline 136.63 & 0.0014 & 249.46 & 0.0157 & 422.87 & 0.1381 \\
\hline 145.89 & 0.0017 & 256.79 & 0.0187 & 430.44 & 0.1468 \\
\hline 164.40 & 0.0023 & 264.17 & 0.0222 & 438.15 & 0.1570 \\
\hline 175.54 & 0.0028 & 273.09 & 0.0270 & 444.12 & 0.1663 \\
\hline 180.37 & 0.0031 & 280.12 & 0.0308 & 451.56 & 0.1776 \\
\hline 193.85 & 0.0039 & 292.59 & 0.0389 & 458.30 & 0.1904 \\
\hline 200.01 & 0.0045 & 299.76 & 0.0434 & 466.64 & 0.2072 \\
\hline 204.99 & 0.0048 & 306.59 & 0.0478 & 466.05 & 0.2076 \\
\hline 210.01 & 0.0054 & 314.15 & 0.0535 & 469.41 & 0.2174 \\
\hline 215.08 & 0.0061 & 322.12 & 0.0588 & 473.03 & 0.2277 \\
\hline 221.88 & 0.0072 & 331.36 & 0.0655 & 476.73 & 0.2426 \\
\hline 225.01 & 0.0080 & 340.36 & 0.0714 & 480.22 & 0.2616 \\
\hline 229.03 & 0.0088 & 350.68 & 0.0789 & 481.67 & 0.2752 \\
\hline
\end{tabular}

Table 304. Test data for specimen 742-28

\begin{tabular}{|c|c|c|c|c|c|}
\hline $\begin{array}{l}\text { Test Number } \\
\text { Aging Temp. } \\
\text { Yield Stress }\end{array}$ & $\begin{array}{l}: 3 \\
: 32 \\
: 20\end{array}$ & & $\begin{array}{l}\text { Tes } \\
\text { Agir } \\
\text { Ulti }\end{array}$ & $\begin{array}{l}\text { mp. } \\
\text { me } \\
\text { Stress }\end{array}$ & $\begin{array}{l}290^{\circ} \mathrm{C} \\
50,000 \mathrm{~h} \\
473.3 \mathrm{MPa}\end{array}$ \\
\hline $\begin{array}{l}\text { Engr. } \\
\text { Stress } \\
(\mathrm{MPa})\end{array}$ & $\begin{array}{l}\text { Engr. } \\
\text { Strain }\end{array}$ & $\begin{array}{l}\text { Engr. } \\
\text { Stress } \\
\text { (MPa) }\end{array}$ & $\begin{array}{l}\text { Engr. } \\
\text { Strain }\end{array}$ & $\begin{array}{l}\text { Engr. } \\
\text { Stress } \\
\text { (MPa) }\end{array}$ & $\begin{array}{l}\text { Engr. } \\
\text { Strain }\end{array}$ \\
\hline 17.080 & 0.0001 & 300.370 & 0.0347 & 406.440 & 0.1224 \\
\hline 30.040 & 0.0002 & 310.690 & 0.0414 & 421.070 & 0.1400 \\
\hline 50.360 & 0.0003 & 313.980 & 0.0436 & 426.140 & 0.1467 \\
\hline 90.470 & 0.0006 & 338.650 & 0.0612 & 427.780 & 0.1489 \\
\hline 100.950 & 0.0007 & 347.250 & 0.0678 & 439.520 & 0.1666 \\
\hline 171.860 & 0.0018 & 350.000 & 0.0699 & 444.480 & 0.1755 \\
\hline 190.750 & 0.0026 & 370.940 & 0.0875 & 452.860 & 0.1932 \\
\hline 196.240 & 0.0029 & 378.240 & 0.0939 & 455.930 & 0.2025 \\
\hline 245.040 & 0.0087 & 380.670 & 0.0961 & 462.940 & 0.2261 \\
\hline 262.330 & 0.0145 & 398.320 & 0.1137 & 469.300 & 0.2473 \\
\hline 267.560 & 0.0167 & 404.400 & 0.1202 & 473.290 & 0.2721 \\
\hline
\end{tabular}


Table 305. Test data for specimen 742-29

\begin{tabular}{|c|c|c|c|c|c|}
\hline $\begin{array}{l}\text { Test Number } \\
\text { Aging Temp. } \\
\text { Yield Stress }\end{array}$ & $\begin{array}{l}: 3 \\
: 32 \\
: 2\end{array}$ & $\Lambda \mathrm{Pa}$ & $\begin{array}{l}\text { Tes } \\
\text { Ag } \\
\text { Ult }\end{array}$ & $\begin{array}{l}\mathrm{np} . \\
\text { me } \\
\text { Stress }\end{array}$ & $\begin{array}{l}290^{\circ} \mathrm{C} \\
50,000 \mathrm{~h} \\
474.4 \mathrm{MPa}\end{array}$ \\
\hline $\begin{array}{l}\text { Engr. } \\
\text { Stress } \\
(\mathrm{MPa})\end{array}$ & $\begin{array}{l}\text { Engr. } \\
\text { Strain }\end{array}$ & $\begin{array}{l}\text { Engr. } \\
\text { Stress } \\
(\mathrm{MPa})\end{array}$ & $\begin{array}{l}\text { Engr. } \\
\text { Strain }\end{array}$ & $\begin{array}{l}\text { Engr. } \\
\text { Stress } \\
\text { (MPa) }\end{array}$ & $\begin{array}{l}\text { Engr. } \\
\text { Strain }\end{array}$ \\
\hline 27.120 & 0.0002 & 268.040 & 0.0128 & 408.120 & 0.1026 \\
\hline 44.370 & 0.0002 & 272.980 & 0.0146 & 410.450 & 0.1047 \\
\hline 55.480 & 0.0002 & 309.550 & 0.0322 & 417.250 & 0.1114 \\
\hline 86.410 & 0.0003 & 313.520 & 0.0345 & 429.960 & 0.1246 \\
\hline 130.880 & 0.0005 & 324.890 & 0.0411 & 437.570 & 0.1335 \\
\hline 140.790 & 0.0006 & 352.490 & 0.0588 & 450.860 & 0.1513 \\
\hline 150.030 & 0.0007 & 355.710 & 0.0610 & 453.750 & 0.1559 \\
\hline 192.710 & 0.0018 & 365.020 & 0.0675 & 461.870 & 0.1691 \\
\hline 198.660 & 0.0020 & 382.350 & 0.0805 & 465.270 & 0.1759 \\
\hline 214.220 & 0.0029 & 390.510 & 0.0871 & 470.750 & 0.1892 \\
\hline 251.920 & 0.0080 & 393.070 & 0.0892 & 474.310 & 0.2089 \\
\hline
\end{tabular}

Table 306. Test data for specimen 74-130

\begin{tabular}{|c|c|c|c|c|c|}
\hline $\begin{array}{l}\text { Test Number } \\
\text { Aging Temp. } \\
\text { Yield Stress }\end{array}$ & $\begin{array}{l}: 3 \\
: 32 \\
: 23\end{array}$ & & $\begin{array}{l}\text { Tes } \\
\text { Agi } \\
\text { Ulti }\end{array}$ & $\begin{array}{l}\text { np. } \\
\text { me } \\
\text { Stress }\end{array}$ & $\begin{array}{l}290^{\circ} \mathrm{C} \\
50,000 \mathrm{~h} \\
517.7 \mathrm{MPa}\end{array}$ \\
\hline $\begin{array}{l}\text { Engr. } \\
\text { Stress } \\
\text { (MPa) }\end{array}$ & $\begin{array}{l}\text { Engr. } \\
\text { Strain }\end{array}$ & $\begin{array}{l}\text { Engr. } \\
\text { Stress } \\
\text { (MPa) }\end{array}$ & $\begin{array}{l}\text { Engr. } \\
\text { Strain }\end{array}$ & $\begin{array}{l}\text { Engr. } \\
\text { Stress } \\
(\mathrm{MPa}) \\
\end{array}$ & $\begin{array}{l}\text { Engr. } \\
\text { Strain }\end{array}$ \\
\hline 21.010 & 0.0001 & 304.520 & 0.0274 & 436.450 & 0.1378 \\
\hline 23.740 & 0.0001 & 308.170 & 0.0297 & 451.880 & 0.1554 \\
\hline 96.150 & 0.0002 & 334.460 & 0.0476 & 456.960 & 0.1620 \\
\hline 108.410 & 0.0003 & 343.580 & 0.0541 & 459.100 & 0.1643 \\
\hline 160.820 & 0.0006 & 346.580 & 0.0564 & 477.670 & 0.1908 \\
\hline 169.310 & 0.0006 & 368.940 & 0.0739 & 482.190 & 0.1984 \\
\hline 192.110 & 0.0012 & 379.530 & 0.0827 & 492.940 & 0.2170 \\
\hline 229.530 & 0.0026 & 401.270 & 0.1025 & 497.070 & 0.2283 \\
\hline 248.700 & 0.0042 & 408.440 & 0.1091 & 505.340 & 0.2467 \\
\hline 253.750 & 0.0050 & 411.000 & 0.1114 & 508.980 & 0.2537 \\
\hline 293.090 & 0.0205 & 428.040 & 0.1289 & 517.560 & 0.2835 \\
\hline
\end{tabular}


Table 307. Test data for specimen 742-27

\begin{tabular}{|c|c|c|c|c|c|}
\hline $\begin{array}{l}\text { Test Number } \\
\text { Aging Temp. } \\
\text { Yield Stress }\end{array}$ & $\begin{array}{l}: 0 \\
: 35 \\
: 17\end{array}$ & & $\begin{array}{l}\text { Tes } \\
\text { Agi } \\
\text { Ulti }\end{array}$ & $\begin{array}{l}\text { mp. } \\
\text { me } \\
\text { Stress }\end{array}$ & $\begin{array}{l}290^{\circ} \mathrm{C} \\
2,570 \mathrm{~h} \\
454.3 \mathrm{MPa}\end{array}$ \\
\hline $\begin{array}{l}\text { Engr. } \\
\text { Stress } \\
(\mathrm{MPa}) \\
\end{array}$ & $\begin{array}{l}\text { Engr. } \\
\text { Strain }\end{array}$ & $\begin{array}{l}\text { Engr. } \\
\text { Stress } \\
\text { (MPa) }\end{array}$ & $\begin{array}{l}\text { Engr. } \\
\text { Strain }\end{array}$ & $\begin{array}{l}\text { Engr. } \\
\text { Stress } \\
(\mathrm{MPa}) \\
\end{array}$ & $\begin{array}{l}\text { Engr. } \\
\text { Strain }\end{array}$ \\
\hline 46.34 & 0.0000 & 236.55 & 0.0078 & 423.15 & 0.1632 \\
\hline 83.52 & 0.0002 & 247.28 & 0.0121 & 428.95 & 0.1728 \\
\hline 109.38 & 0.0005 & 259.81 & 0.0193 & 434.04 & 0.1824 \\
\hline 131.30 & 0.0008 & 275.30 & 0.0296 & 438.59 & 0.1926 \\
\hline 148.35 & 0.0011 & 292.41 & 0.0421 & 441.87 & 0.2008 \\
\hline 163.85 & 0.0016 & 308.93 & 0.0550 & 445.91 & 0.2120 \\
\hline 171.51 & 0.0020 & 326.09 & 0.0691 & 448.80 & 0.2181 \\
\hline 179.31 & 0.0024 & 342.45 & 0.0820 & 448.87 & 0.2225 \\
\hline 186.66 & 0.0029 & 358.21 & 0.0947 & 449.19 & 0.2264 \\
\hline 192.52 & 0.0034 & 371.39 & 0.1038 & 451.04 & 0.2320 \\
\hline 199.12 & 0.0042 & 385.51 & 0.1169 & 452.69 & 0.2384 \\
\hline 208.25 & 0.0049 & 396.91 & 0.1286 & 454.29 & 0.2435 \\
\hline 218.27 & 0.0057 & 405.83 & 0.1390 & 454.13 & 0.2457 \\
\hline 222.08 & 0.0061 & 412.48 & 0.1477 & 454.26 & 0.2553 \\
\hline 228.37 & 0.0067 & 417.64 & 0.1549 & & \\
\hline
\end{tabular}

Table 308. Test data for specimen 744-06

\begin{tabular}{|c|c|c|c|c|c|}
\hline $\begin{array}{l}\text { Test Number } \\
\text { Aging Temp. } \\
\text { Yield Stress }\end{array}$ & $\begin{array}{l}: 3 \\
: 35 \\
: 18\end{array}$ & & $\begin{array}{l}\text { Tes } \\
\text { Agir } \\
\text { Ulti }\end{array}$ & $\begin{array}{l}\text { np. } \\
\text { ne } \\
\text { Stress }\end{array}$ & $\begin{array}{l}290^{\circ} \mathrm{C} \\
10,000 \mathrm{~h} \\
448.0 \mathrm{MPa}\end{array}$ \\
\hline $\begin{array}{l}\text { Engr. } \\
\text { Stress } \\
\text { (MPa) }\end{array}$ & $\begin{array}{l}\text { Engr. } \\
\text { Strain }\end{array}$ & $\begin{array}{l}\text { Engr. } \\
\text { Stress } \\
(\mathrm{MPa})\end{array}$ & $\begin{array}{l}\text { Engr. } \\
\text { Strain }\end{array}$ & $\begin{array}{l}\text { Engr. } \\
\text { Stress } \\
(\mathrm{MPa}) \\
\end{array}$ & $\begin{array}{l}\text { Engr. } \\
\text { Strain }\end{array}$ \\
\hline 14.800 & 0.0001 & 267.360 & 0.0351 & 371.110 & 0.1235 \\
\hline 30.150 & 0.0002 & 270.580 & 0.0374 & 373.180 & 0.1257 \\
\hline 41.190 & 0.0002 & 294.990 & 0.0551 & 385.350 & 0.1391 \\
\hline 62.670 & 0.0003 & 297.820 & 0.0573 & 389.150 & 0.1435 \\
\hline 149.200 & 0.0010 & 300.720 & 0.0596 & 391.090 & 0.1457 \\
\hline 155.050 & 0.0012 & 322.170 & 0.0772 & 406.950 & 0.1657 \\
\hline 160.640 & 0.0014 & 324.720 & 0.0794 & 413.350 & 0.1745 \\
\hline 211.060 & 0.0067 & 327.270 & 0.0816 & 426.950 & 0.1964 \\
\hline 215.910 & 0.0080 & 349.090 & 0.1013 & 430.730 & 0.2034 \\
\hline 232.460 & 0.0143 & 351.270 & 0.1036 & 442.920 & 0.2301 \\
\hline 257.030 & 0.0283 & 368.970 & 0.1212 & 447.860 & 0.2504 \\
\hline
\end{tabular}


Table 309. Test data for specimen 744-09

\begin{tabular}{|c|c|c|c|c|c|}
\hline $\begin{array}{l}\text { Test Number } \\
\text { Aging Temp. } \\
\text { Yield Stress }\end{array}$ & $\begin{array}{l}: 3 \\
: 35 \\
: 19\end{array}$ & $\mathrm{MPa}$ & $\begin{array}{l}\text { Tes } \\
\text { Agi } \\
\text { Ulti }\end{array}$ & $\begin{array}{l}\text { np. } \\
\text { ne } \\
\text { Stress }\end{array}$ & $\begin{array}{l}290^{\circ} \mathrm{C} \\
10,000 \mathrm{~h} \\
507.2 \mathrm{MPa}\end{array}$ \\
\hline $\begin{array}{l}\text { Engr. } \\
\text { Stress } \\
\text { (MPa) }\end{array}$ & $\begin{array}{l}\text { Engr. } \\
\text { Strain }\end{array}$ & $\begin{array}{l}\text { Engr. } \\
\text { Stress } \\
\text { (MPa) }\end{array}$ & $\begin{array}{l}\text { Engr. } \\
\text { Strain }\end{array}$ & $\begin{array}{l}\text { Engr. } \\
\text { Stress } \\
(\mathrm{MPa}) \\
\end{array}$ & $\begin{array}{l}\text { Engr. } \\
\text { Strain }\end{array}$ \\
\hline 13.390 & 0.0001 & 282.940 & 0.0302 & 414.890 & 0.1218 \\
\hline 39.590 & 0.0001 & 287.000 & 0.0325 & 428.640 & 0.1349 \\
\hline 72.350 & 0.0002 & 298.920 & 0.0391 & 430.800 & 0.1371 \\
\hline 116.650 & 0.0003 & 321.040 & 0.0521 & 448.850 & 0.1570 \\
\hline 135.280 & 0.0004 & 324.530 & 0.0544 & 450.790 & 0.1591 \\
\hline 173.970 & 0.0015 & 334.960 & 0.0608 & 456.070 & 0.1657 \\
\hline 179.350 & 0.0017 & 354.370 & 0.0738 & 472.140 & 0.1880 \\
\hline 200.100 & 0.0030 & 357.470 & 0.0761 & 476.340 & 0.1956 \\
\hline 233.810 & 0.0087 & 366.680 & 0.0825 & 484.930 & 0.2117 \\
\hline 239.340 & 0.0104 & 383.880 & 0.0956 & 496.410 & 0.2385 \\
\hline 244.390 & 0.0122 & 394.820 & 0.1042 & 507.070 & 0.2726 \\
\hline
\end{tabular}

Table 310. Test data for specimen 74-120

$\begin{array}{llll}\text { Test Number } & : 332 & \text { Test Temp. } & : 290^{\circ} \mathrm{C} \\ \text { Aging Temp. } & : 350^{\circ} \mathrm{C} & \text { Aging Time } & : 10,000 \mathrm{~h} \\ \text { Yield Stress } & : 176.6 \mathrm{MPa} & \text { Ultimate Stress } & : 453.8 \mathrm{MPa}\end{array}$

Experimental Data Not Available.

Table 311. Test data for specimen 744-18

\begin{tabular}{|c|c|c|c|c|c|}
\hline $\begin{array}{l}\text { Test Number } \\
\text { Aging Temp. } \\
\text { Yield Stress }\end{array}$ & $\begin{array}{l}: 23 \\
: 35 \\
: 22\end{array}$ & & $\begin{array}{l}\text { Tes } \\
\text { Agir } \\
\text { Ulti }\end{array}$ & $\begin{array}{l}\text { np. } \\
\text { ne } \\
\text { Stress }\end{array}$ & $\begin{array}{l}290^{\circ} \mathrm{C} \\
30,000 \mathrm{~h} \\
510.5 \mathrm{MPa}\end{array}$ \\
\hline $\begin{array}{l}\text { Engr. } \\
\text { Stress } \\
\text { (MPa) }\end{array}$ & $\begin{array}{l}\text { Engr. } \\
\text { Strain }\end{array}$ & $\begin{array}{l}\text { Engr. } \\
\text { Stress } \\
\text { (MPa) }\end{array}$ & $\begin{array}{l}\text { Engr. } \\
\text { Strain }\end{array}$ & $\begin{array}{l}\text { Engr. } \\
\text { Stress } \\
(\mathrm{MPa}) \\
\end{array}$ & $\begin{array}{l}\text { Engr. } \\
\text { Strain }\end{array}$ \\
\hline 8.442 & 0.0001 & 230.833 & 0.0083 & 350.463 & 0.0605 \\
\hline 20.800 & 0.0001 & 239.849 & 0.0102 & 370.195 & 0.0727 \\
\hline 41.886 & 0.0002 & 253.007 & 0.0138 & 379.351 & 0.0787 \\
\hline 53.131 & 0.0002 & 254.654 & 0.0143 & 413.145 & 0.1031 \\
\hline 129.137 & 0.0006 & 256.199 & 0.0148 & 428.086 & 0.1154 \\
\hline 137.525 & 0.0007 & 281.982 & 0.0247 & 431.846 & 0.1185 \\
\hline 144.719 & 0.0008 & 284.363 & 0.0258 & 457.804 & 0.1431 \\
\hline 199.449 & 0.0040 & 286.699 & 0.0268 & 469.929 & 0.1570 \\
\hline 203.403 & 0.0044 & 308.091 & 0.0372 & 490.996 & 0.1861 \\
\hline 207.119 & 0.0047 & 327.4 & 0.0474 & 493.424 & 0.1901 \\
\hline 228.390 & 0.0079 & 331.087 & 0.0493 & 510.474 & 0.2396 \\
\hline
\end{tabular}


Table 312. Test data for specimen 743-15

\begin{tabular}{|c|c|c|c|c|c|}
\hline $\begin{array}{l}\text { Test Number } \\
\text { Aging Temp. } \\
\text { Yield Stress }\end{array}$ & $\begin{array}{l}: 2 \\
: 35 \\
: 18\end{array}$ & & $\begin{array}{l}\text { Tes } \\
\text { Agi } \\
\text { Ulti }\end{array}$ & $\begin{array}{l}\mathrm{mp.} \\
\text { me } \\
\text { Stress }\end{array}$ & $\begin{array}{l}290^{\circ} \mathrm{C} \\
30,000 \mathrm{~h} \\
503.8 \mathrm{MPa}\end{array}$ \\
\hline $\begin{array}{l}\text { Engr. } \\
\text { Stress } \\
\text { (MPa) }\end{array}$ & $\begin{array}{l}\text { Engr. } \\
\text { Strain }\end{array}$ & $\begin{array}{l}\text { Engr. } \\
\text { Stress } \\
\text { (MPa) }\end{array}$ & $\begin{array}{l}\text { Engr. } \\
\text { Strain }\end{array}$ & $\begin{array}{l}\text { Engr. } \\
\text { Stress } \\
\text { (MPa) }\end{array}$ & $\begin{array}{l}\text { Engr. } \\
\text { Strain }\end{array}$ \\
\hline 40.729 & 0.0002 & 259.018 & 0.0213 & 423.949 & 0.1307 \\
\hline 60.887 & 0.0002 & 264.040 & 0.0234 & 426.338 & 0.1329 \\
\hline 79.952 & 0.0003 & 294.467 & 0.0387 & 428.087 & 0.1351 \\
\hline 90.021 & 0.0004 & 306.148 & 0.0451 & 449.406 & 0.1571 \\
\hline 121.225 & 0.0005 & 309.889 & 0.0474 & 454.807 & 0.1638 \\
\hline 129.808 & 0.0006 & 349.228 & 0.0715 & 465.867 & 0.1780 \\
\hline 137.497 & 0.0007 & 364.990 & 0.0822 & 474.926 & 0.1915 \\
\hline 196.295 & 0.0043 & 368.007 & 0.0845 & 484.068 & 0.2092 \\
\hline 201.881 & 0.0050 & 384.862 & 0.0980 & 488.503 & 0.2187 \\
\hline 207.112 & 0.0058 & 398.452 & 0.1086 & 497.634 & 0.2428 \\
\hline 242.294 & 0.0146 & 401.080 & 0.1108 & 503.755 & 0.2803 \\
\hline
\end{tabular}

Table 313. Test data for specimen 74-270

\begin{tabular}{|c|c|c|c|c|c|}
\hline $\begin{array}{l}\text { Test Number } \\
\text { Aging Temp. } \\
\text { Yield Stress }\end{array}$ & $\begin{array}{l}: 2 \\
: 35 \\
: 18\end{array}$ & $\mathrm{MPa}$ & $\begin{array}{l}\text { Tes } \\
\text { Agi } \\
\text { Ulti }\end{array}$ & $\begin{array}{l}\text { mp. } \\
\text { me } \\
\text { Stress }\end{array}$ & $\begin{array}{l}290^{\circ} \mathrm{C} \\
30,000 \mathrm{~h} \\
495.0 \mathrm{MPa}\end{array}$ \\
\hline $\begin{array}{l}\text { Engr. } \\
\text { Stress } \\
(\mathrm{MPa})\end{array}$ & $\begin{array}{l}\text { Engr. } \\
\text { Strain }\end{array}$ & $\begin{array}{l}\text { Engr. } \\
\text { Stress } \\
(\mathrm{MPa})\end{array}$ & $\begin{array}{l}\text { Engr. } \\
\text { Strain }\end{array}$ & $\begin{array}{l}\text { Engr. } \\
\text { Stress } \\
(\mathrm{MPa}) \\
\end{array}$ & $\begin{array}{l}\text { Engr. } \\
\text { Strain }\end{array}$ \\
\hline 41.840 & 0.0002 & 295.090 & 0.0383 & 412.530 & 0.1235 \\
\hline 57.710 & 0.0002 & 306.420 & 0.0447 & 429.420 & 0.1407 \\
\hline 78.370 & 0.0002 & 310.070 & 0.0469 & 435.740 & 0.1475 \\
\hline 117.510 & 0.0004 & 340.570 & 0.0664 & 437.600 & 0.1496 \\
\hline 139.570 & 0.0006 & 343.810 & 0.0685 & 454.760 & 0.1712 \\
\hline 192.540 & 0.0037 & 353.080 & 0.0749 & 459.890 & 0.1780 \\
\hline 198.850 & 0.0044 & 376.600 & 0.0924 & 472.920 & 0.2007 \\
\hline 216.710 & 0.0069 & 379.270 & 0.0945 & 481.740 & 0.2187 \\
\hline 249.270 & 0.0161 & 387.770 & 0.1013 & 484.770 & 0.2257 \\
\hline 265.150 & 0.0228 & 403.140 & 0.1149 & 491.050 & 0.2437 \\
\hline 269.810 & 0.0251 & 410.190 & 0.1213 & 495.010 & 0.2637 \\
\hline
\end{tabular}


Table 314. Test data for specimen 742-18

\begin{tabular}{|c|c|c|c|c|c|}
\hline $\begin{array}{l}\text { Test Number } \\
\text { Aging Temp. } \\
\text { Yield Stress }\end{array}$ & $\begin{array}{l}: 05 \\
: 40 \\
: 16\end{array}$ & & $\begin{array}{l}\text { Tes } \\
\text { Agir } \\
\text { Ultir }\end{array}$ & $\begin{array}{l}\text { mp. } \\
\text { me } \\
\text { Stress }\end{array}$ & $\begin{array}{l}290^{\circ} \mathrm{C} \\
2,570 \mathrm{~h} \\
485.1 \mathrm{MPa}\end{array}$ \\
\hline $\begin{array}{l}\text { Engr. } \\
\text { Stress } \\
(\mathrm{MPa})\end{array}$ & $\begin{array}{l}\text { Engr. } \\
\text { Strain } \\
\end{array}$ & $\begin{array}{l}\text { Engr. } \\
\text { Stress } \\
(\mathrm{MPa})\end{array}$ & $\begin{array}{l}\text { Engr. } \\
\text { Strain }\end{array}$ & $\begin{array}{l}\text { Engr. } \\
\text { Stress } \\
\text { (MPa) }\end{array}$ & $\begin{array}{l}\text { Engr. } \\
\text { Strain }\end{array}$ \\
\hline 46.986 & 0.0000 & 183.780 & 0.0024 & 254.680 & 0.0105 \\
\hline 69.838 & 0.0001 & 187.960 & 0.0027 & 261.500 & 0.0132 \\
\hline 86.240 & 0.0002 & 194.900 & 0.0032 & 270.170 & 0.0171 \\
\hline 100.320 & 0.0003 & 196.620 & 0.0033 & 328.240 & 0.0545 \\
\hline 130.710 & 0.0007 & 197.700 & 0.0034 & 340.230 & 0.0635 \\
\hline 137.260 & 0.0008 & 205.710 & 0.0038 & 356.170 & 0.0754 \\
\hline 141.250 & 0.0009 & 209.150 & 0.0039 & 411.380 & 0.1169 \\
\hline 157.350 & 0.0013 & 212.740 & 0.0040 & 424.790 & 0.1300 \\
\hline 161.240 & 0.0015 & 226.360 & 0.0046 & 458.500 & 0.1721 \\
\hline 165.070 & 0.0016 & 229.900 & 0.0048 & 464.560 & 0.1816 \\
\hline 179.840 & 0.0022 & 233.920 & 0.0051 & 485.050 & 0.2446 \\
\hline
\end{tabular}

Table 315. Test data for specimen 742-15

\begin{tabular}{|c|c|c|c|c|c|}
\hline $\begin{array}{l}\text { Test Number } \\
\text { Aging Temp. } \\
\text { Yield Stress }\end{array}$ & $\begin{array}{l}: 2 \\
: 40 \\
: 17\end{array}$ & $\mathrm{MPa}$ & $\begin{array}{l}\text { Tes } \\
\text { Agi } \\
\text { Ulti }\end{array}$ & $\begin{array}{l}\text { mp. } \\
\text { me } \\
\text { Stress }\end{array}$ & $\begin{array}{l}290^{\circ} \mathrm{C} \\
10,000 \mathrm{~h} \\
516.0 \mathrm{MPa}\end{array}$ \\
\hline $\begin{array}{l}\text { Engr. } \\
\text { Stress } \\
(\mathrm{MPa})\end{array}$ & $\begin{array}{l}\text { Engr. } \\
\text { Strain }\end{array}$ & $\begin{array}{l}\text { Engr. } \\
\text { Stress } \\
(\mathrm{MPa})\end{array}$ & $\begin{array}{l}\text { Engr. } \\
\text { Strain }\end{array}$ & $\begin{array}{l}\text { Engr. } \\
\text { Stress } \\
(\mathrm{MPa}) \\
\end{array}$ & $\begin{array}{l}\text { Engr. } \\
\text { Strain }\end{array}$ \\
\hline 42.830 & 0.0002 & 298.520 & 0.0375 & 433.390 & 0.1405 \\
\hline 63.120 & 0.0002 & 326.950 & 0.0553 & 435.520 & 0.1427 \\
\hline 102.860 & 0.0006 & 336.860 & 0.0618 & 452.420 & 0.1611 \\
\hline 142.950 & 0.0013 & 340.150 & 0.0638 & 459.900 & 0.1700 \\
\hline 163.120 & 0.0020 & 364.040 & 0.0814 & 476.920 & 0.1929 \\
\hline 168.930 & 0.0024 & 373.410 & 0.0880 & 481.150 & 0.2003 \\
\hline 220.410 & 0.0068 & 376.230 & 0.0902 & 492.650 & 0.2206 \\
\hline 238.050 & 0.0101 & 397.550 & 0.1076 & 501.160 & 0.2393 \\
\hline 243.040 & 0.0116 & 405.280 & 0.1142 & 504.160 & 0.2465 \\
\hline 282.680 & 0.0285 & 407.810 & 0.1163 & 512.700 & 0.2740 \\
\hline 294.720 & 0.0355 & 426.540 & 0.1338 & 515.990 & 0.3002 \\
\hline
\end{tabular}


Table 316. Test data for specimen 742-24

\begin{tabular}{|c|c|c|c|c|c|}
\hline $\begin{array}{l}\text { Test Number } \\
\text { Aging Temp. } \\
\text { Yield Stress }\end{array}$ & $\begin{array}{l}: 2 \\
: 4 C \\
: 18\end{array}$ & & $\begin{array}{l}\text { Tes } \\
\text { Agi } \\
\text { Ulti }\end{array}$ & $\begin{array}{l}\text { mp. } \\
\text { me } \\
\text { Stress }\end{array}$ & $\begin{array}{l}290^{\circ} \mathrm{C} \\
10,000 \mathrm{~h} \\
509.1 \mathrm{MPa}\end{array}$ \\
\hline $\begin{array}{l}\text { Engr. } \\
\text { Stress } \\
\text { (MPa) }\end{array}$ & $\begin{array}{l}\text { Engr. } \\
\text { Strain }\end{array}$ & $\begin{array}{l}\text { Engr. } \\
\text { Stress } \\
\text { (MPa) }\end{array}$ & $\begin{array}{l}\text { Engr. } \\
\text { Strain }\end{array}$ & $\begin{array}{l}\text { Engr. } \\
\text { Stress } \\
(\mathrm{MPa})\end{array}$ & $\begin{array}{l}\text { Engr. } \\
\text { Strain }\end{array}$ \\
\hline 40.328 & 0.0002 & 285.582 & 0.0325 & 402.677 & 0.1060 \\
\hline 73.263 & 0.0003 & 289.792 & 0.0347 & 421.982 & 0.1212 \\
\hline 97.634 & 0.0004 & 301.985 & 0.0412 & 424.537 & 0.1233 \\
\hline 130.710 & 0.0006 & 324.783 & 0.0540 & 439.691 & 0.1364 \\
\hline 145.143 & 0.0010 & 328.407 & 0.0562 & 442.124 & 0.1386 \\
\hline 172.470 & 0.0020 & 339.095 & 0.0627 & 444.447 & 0.1409 \\
\hline 194.911 & 0.0038 & 359.479 & 0.0757 & 466.379 & 0.1628 \\
\hline 201.229 & 0.0045 & 369.238 & 0.0821 & 472.485 & 0.1697 \\
\hline 235.006 & 0.0111 & 372.374 & 0.0843 & 489.163 & 0.1910 \\
\hline 240.667 & 0.0127 & 391.548 & 0.0976 & 498.985 & 0.2086 \\
\hline 257.307 & 0.0189 & 394.408 & 0.0996 & 508.323 & 0.2346 \\
\hline
\end{tabular}

Table 317. Test data for specimen 74-109

\begin{tabular}{|c|c|c|c|c|c|}
\hline $\begin{array}{l}\text { Test Number } \\
\text { Aging Temp. } \\
\text { Yield Stress }\end{array}$ & $\begin{array}{l}: 2 \\
: 4 C \\
: 17\end{array}$ & & $\begin{array}{l}\text { Tes } \\
\text { Agi } \\
\text { Ulti }\end{array}$ & $\begin{array}{l}\text { mp. } \\
\text { me } \\
\text { Stress }\end{array}$ & $\begin{array}{l}290^{\circ} \mathrm{C} \\
10000 \mathrm{~h} \\
476.8 \mathrm{MPa}\end{array}$ \\
\hline $\begin{array}{l}\text { Engr. } \\
\text { Stress } \\
\text { (MPa) }\end{array}$ & $\begin{array}{l}\text { Engr. } \\
\text { Strain }\end{array}$ & $\begin{array}{l}\text { Engr. } \\
\text { Stress } \\
\text { (MPa) } \\
\end{array}$ & $\begin{array}{l}\text { Engr. } \\
\text { Strain }\end{array}$ & $\begin{array}{l}\text { Engr. } \\
\text { Stress } \\
(\mathrm{MPa}) \\
\end{array}$ & $\begin{array}{l}\text { Engr. } \\
\text { Strain } \\
\end{array}$ \\
\hline 41.960 & 0.0002 & 278.190 & 0.0388 & 401.560 & 0.1428 \\
\hline 45.770 & 0.0002 & 302.930 & 0.0558 & 416.700 & 0.1600 \\
\hline 61.070 & 0.0001 & 312.370 & 0.0626 & 422.110 & 0.1665 \\
\hline 101.970 & 0.0004 & 315.120 & 0.0648 & 423.850 & 0.1688 \\
\hline 125.570 & 0.0008 & 336.710 & 0.0817 & 436.970 & 0.1866 \\
\hline 147.750 & 0.0015 & 344.530 & 0.0880 & 441.460 & 0.1932 \\
\hline 204.990 & 0.0065 & 346.960 & 0.0904 & 452.320 & 0.2130 \\
\hline 222.950 & 0.0107 & 366.630 & 0.1077 & 460.950 & 0.2303 \\
\hline 228.350 & 0.0123 & 373.610 & 0.1143 & 464.570 & 0.2390 \\
\hline 264.110 & 0.0298 & 375.920 & 0.1164 & 471.980 & 0.2601 \\
\hline 274.860 & 0.0365 & 393.400 & 0.1339 & 476.750 & 0.2852 \\
\hline
\end{tabular}


Table 318. Test data for specimen 741-06

\begin{tabular}{|c|c|c|c|c|c|}
\hline $\begin{array}{l}\text { Test Number } \\
\text { Aging Temp. } \\
\text { Yield Stress }\end{array}$ & $\begin{array}{l}: 0 \\
: 45 \\
: 17\end{array}$ & & $\begin{array}{l}\text { Tes } \\
\text { Agi } \\
\text { Ulti }\end{array}$ & $\begin{array}{l}\text { np. } \\
\text { ne } \\
\text { Stress }\end{array}$ & $\begin{array}{l}290^{\circ} \mathrm{C} \\
2,570 \mathrm{~h} \\
500.7 \mathrm{MPa}\end{array}$ \\
\hline $\begin{array}{l}\text { Engr. } \\
\text { Stress } \\
(\mathrm{MPa})\end{array}$ & $\begin{array}{l}\text { Engr. } \\
\text { Strain }\end{array}$ & $\begin{array}{l}\text { Engr. } \\
\text { Stress } \\
(\mathrm{MPa})\end{array}$ & $\begin{array}{l}\text { Engr. } \\
\text { Strain }\end{array}$ & $\begin{array}{l}\text { Engr. } \\
\text { Stress } \\
(\mathrm{MPa}) \\
\end{array}$ & $\begin{array}{l}\text { Engr. } \\
\text { Strain }\end{array}$ \\
\hline 43.97 & 0.0000 & 206.82 & 0.0035 & 446.50 & 0.1035 \\
\hline 66.29 & 0.0001 & 215.45 & 0.0037 & 455.21 & 0.1108 \\
\hline 83.95 & 0.0002 & 220.15 & 0.0039 & 465.41 & 0.1194 \\
\hline 99.14 & 0.0003 & 229.32 & 0.0041 & 472.49 & 0.1267 \\
\hline 107.85 & 0.0004 & 237.45 & 0.0044 & 478.40 & 0.1338 \\
\hline 120.05 & 0.0006 & 249.54 & 0.0058 & 482.94 & 0.1390 \\
\hline 127.18 & 0.0007 & 254.97 & 0.0067 & 483.13 & 0.1428 \\
\hline 136.08 & 0.0008 & 263.92 & 0.0084 & 483.42 & 0.1449 \\
\hline 149.54 & 0.0011 & 326.74 & 0.0309 & 485.26 & 0.1473 \\
\hline 155.78 & 0.0013 & 344.39 & 0.0399 & 487.52 & 0.1511 \\
\hline 174.93 & 0.0020 & 367.85 & 0.0534 & 490.45 & 0.1554 \\
\hline 185.04 & 0.0025 & 392.09 & 0.0683 & 493.15 & 0.1603 \\
\hline 196.53 & 0.0031 & 419.63 & 0.0864 & 495.99 & 0.1664 \\
\hline 202.46 & 0.0033 & 434.98 & 0.0972 & & \\
\hline
\end{tabular}

Table 319. Test data for specimen 742-09

\begin{tabular}{|c|c|c|c|c|c|}
\hline $\begin{array}{l}\text { Test Number } \\
\text { Aging Temp. } \\
\text { Yield Stress }\end{array}$ & $\begin{array}{l}: 0 \\
: 45 \\
: 17\end{array}$ & & $\begin{array}{l}\text { Tes } \\
\text { Agir } \\
\text { Ultir }\end{array}$ & $\begin{array}{l}\text { ne. } \\
\text { Stress }\end{array}$ & $\begin{array}{l}290^{\circ} \mathrm{C} \\
2,570 \mathrm{~h} \\
485.2 \mathrm{MPa}\end{array}$ \\
\hline $\begin{array}{l}\text { Engr. } \\
\text { Stress } \\
(\mathrm{MPa})\end{array}$ & $\begin{array}{l}\text { Engr. } \\
\text { Strain }\end{array}$ & $\begin{array}{l}\text { Engr. } \\
\text { Stress } \\
(\mathrm{MPa})\end{array}$ & $\begin{array}{l}\text { Engr. } \\
\text { Strain }\end{array}$ & $\begin{array}{l}\text { Engr. } \\
\text { Stress } \\
\text { (MPa) }\end{array}$ & $\begin{array}{l}\text { Engr. } \\
\text { Strain }\end{array}$ \\
\hline 45.5000 & 0.0000 & 209.8600 & 0.0038 & 361.8700 & 0.0570 \\
\hline 87.0190 & 0.0003 & 213.2400 & 0.0039 & 374.6400 & 0.0656 \\
\hline 113.9700 & 0.0005 & 220.2400 & 0.0042 & 387.9400 & 0.0744 \\
\hline 129.9500 & 0.0008 & 226.1700 & 0.0043 & 399.1400 & 0.0823 \\
\hline 138.3100 & 0.0009 & 234.0500 & 0.0046 & 410.2100 & 0.0908 \\
\hline 148.3300 & 0.0012 & 243.7800 & 0.0056 & 418.6900 & 0.0972 \\
\hline 153.3400 & 0.0013 & 256.2800 & 0.0078 & 439.2100 & 0.1128 \\
\hline 160.6600 & 0.0015 & 270.6000 & 0.0112 & 453.8700 & 0.1281 \\
\hline 168.4700 & 0.0019 & 284.8100 & 0.0157 & 465.9400 & 0.1432 \\
\hline 173.2300 & 0.0020 & 291.2200 & 0.0181 & 475.7700 & 0.1594 \\
\hline 181.0000 & 0.0024 & 299.0400 & 0.0211 & 482.4100 & 0.1741 \\
\hline 185.1700 & 0.0026 & 311.2500 & 0.0270 & 484.5700 & 0.1784 \\
\hline 194.0500 & 0.0031 & 322.6900 & 0.0327 & 485.1600 & 0.1813 \\
\hline 199.9300 & 0.0035 & 337.8300 & 0.0416 & 484.9300 & 0.1905 \\
\hline 205.9000 & 0.0037 & 349.0300 & 0.0487 & 485.1000 & 0.1967 \\
\hline
\end{tabular}


Table 320. Test data for specimen 753-40

\begin{tabular}{|c|c|c|c|c|c|}
\hline $\begin{array}{l}\text { Test Number } \\
\text { Aging Temp. } \\
\text { Yield Stress }\end{array}$ & $\begin{array}{l}: 0 \\
: \text { Ur } \\
: 31\end{array}$ & & $\begin{array}{l}\text { Tes } \\
\text { Agi } \\
\text { Ulti }\end{array}$ & $\begin{array}{l}\text { np. } \\
\text { ne } \\
\text { Stress }\end{array}$ & $\begin{array}{l}25^{\circ} \mathrm{C} \\
- \\
610.4 \mathrm{MPa}\end{array}$ \\
\hline $\begin{array}{l}\text { Engr. } \\
\text { Stress } \\
\text { (MPa) }\end{array}$ & $\begin{array}{l}\text { Engr. } \\
\text { Strain }\end{array}$ & $\begin{array}{l}\text { Engr. } \\
\text { Stress } \\
(\mathrm{MPa}) \\
\end{array}$ & $\begin{array}{l}\text { Engr. } \\
\text { Strain }\end{array}$ & $\begin{array}{l}\text { Engr. } \\
\text { Stress } \\
(\mathrm{MPa}) \\
\end{array}$ & $\begin{array}{l}\text { Engr. } \\
\text { Strain }\end{array}$ \\
\hline 77.16 & 0.0001 & 365.32 & 0.0062 & 489.21 & 0.0761 \\
\hline 118.01 & 0.0003 & 371.61 & 0.0069 & 507.80 & 0.0934 \\
\hline 160.83 & 0.0007 & 381.23 & 0.0083 & 527.54 & 0.1132 \\
\hline 197.95 & 0.0012 & 388.16 & 0.0099 & 548.51 & 0.1418 \\
\hline 209.02 & 0.0014 & 394.15 & 0.0114 & 559.04 & 0.1605 \\
\hline 227.05 & 0.0016 & 400.38 & 0.0133 & 574.58 & 0.1971 \\
\hline 235.44 & 0.0016 & 412.09 & 0.0179 & 587.16 & 0.2345 \\
\hline 243.44 & 0.0017 & 423.05 & 0.0231 & 594.90 & 0.2637 \\
\hline 279.36 & 0.0023 & 440.90 & 0.0341 & 601.22 & 0.2945 \\
\hline 306.14 & 0.0029 & 464.33 & 0.0519 & 606.19 & 0.3291 \\
\hline 328.63 & 0.0037 & 480.06 & 0.0650 & 609.41 & 0.3687 \\
\hline 343.73 & 0.0045 & 487.62 & 0.0719 & 610.35 & 0.4031 \\
\hline 357.23 & 0.0054 & & & & \\
\hline
\end{tabular}

Table 321. Test data for specimen 753-41

\begin{tabular}{|c|c|c|c|c|c|}
\hline $\begin{array}{l}\text { Test Number } \\
\text { Aging Temp. } \\
\text { Yield Stress }\end{array}$ & $\begin{array}{l}: 03 \\
: U n \\
: 33\end{array}$ & & $\begin{array}{l}\text { Tes } \\
\text { Agi } \\
\text { Ulti }\end{array}$ & $\begin{array}{l}\text { np. } \\
\text { me } \\
\text { Stress }\end{array}$ & $\begin{array}{l}25^{\circ} \mathrm{C} \\
- \\
589.8 \mathrm{MPa}\end{array}$ \\
\hline $\begin{array}{l}\text { Engr. } \\
\text { Stress } \\
\text { (MPa) } \\
\end{array}$ & $\begin{array}{l}\text { Engr. } \\
\text { Strain }\end{array}$ & $\begin{array}{l}\text { Engr. } \\
\text { Stress } \\
\text { (MPa) } \\
\end{array}$ & $\begin{array}{l}\text { Engr. } \\
\text { Strain }\end{array}$ & $\begin{array}{l}\text { Engr. } \\
\text { Stress } \\
(\mathrm{MPa}) \\
\end{array}$ & $\begin{array}{l}\text { Engr. } \\
\text { Strain } \\
\end{array}$ \\
\hline $\begin{array}{r}68.82 \\
111.68 \\
156.27 \\
189.39 \\
204.14 \\
213.28 \\
226.29 \\
243.89 \\
288.66 \\
312.30 \\
327.04 \\
338.88 \\
344.82\end{array}$ & $\begin{array}{l}0.0001 \\
0.0004 \\
0.0008 \\
0.0013 \\
0.0015 \\
0.0016 \\
0.0017 \\
0.0019 \\
0.0027 \\
0.0035 \\
0.0040 \\
0.0047 \\
0.0051\end{array}$ & $\begin{array}{l}353.02 \\
362.44 \\
370.87 \\
379.64 \\
395.01 \\
406.69 \\
422.55 \\
441.60 \\
463.10 \\
479.22 \\
482.62 \\
484.99\end{array}$ & $\begin{array}{l}0.0056 \\
0.0067 \\
0.0080 \\
0.0096 \\
0.0135 \\
0.0182 \\
0.0259 \\
0.0382 \\
0.0548 \\
0.0683 \\
0.0712 \\
0.0744\end{array}$ & $\begin{array}{l}503.22 \\
512.45 \\
525.70 \\
536.89 \\
553.88 \\
566.90 \\
576.29 \\
583.13 \\
587.81 \\
589.38 \\
589.78 \\
589.79\end{array}$ & $\begin{array}{l}0.0919 \\
0.0990 \\
0.1146 \\
0.1304 \\
0.1595 \\
0.1937 \\
0.2257 \\
0.2586 \\
0.2923 \\
0.3107 \\
0.3362 \\
0.3585\end{array}$ \\
\hline
\end{tabular}


Table 322. Test data for specimen 753-30

\begin{tabular}{|c|c|c|c|c|c|}
\hline $\begin{array}{l}\text { Test Number } \\
\text { Aging Temp. } \\
\text { Yield Stress }\end{array}$ & $\begin{array}{l}: 20 \\
: 29 \\
: 31\end{array}$ & $\mathrm{~Pa}$ & $\begin{array}{l}\text { Tes } \\
\text { Agi } \\
\text { Ulti }\end{array}$ & $\begin{array}{l}\text { np. } \\
\text { ne } \\
\text { Stress }\end{array}$ & $\begin{array}{l}25^{\circ} \mathrm{C} \\
30,000 \mathrm{~h} \\
609.0 \mathrm{MPa}\end{array}$ \\
\hline $\begin{array}{l}\text { Engr. } \\
\text { Stress } \\
\text { (MPa) } \\
\end{array}$ & $\begin{array}{l}\text { Engr. } \\
\text { Strain }\end{array}$ & $\begin{array}{l}\text { Engr. } \\
\text { Stress } \\
(\mathrm{MPa}) \\
\end{array}$ & $\begin{array}{l}\text { Engr. } \\
\text { Strain }\end{array}$ & $\begin{array}{l}\text { Engr. } \\
\text { Stress } \\
(\mathrm{MPa}) \\
\end{array}$ & $\begin{array}{l}\text { Engr. } \\
\text { Strain }\end{array}$ \\
\hline 18.907 & 0.0002 & 395.921 & 0.0176 & 562.345 & 0.1509 \\
\hline 42.475 & 0.0005 & 440.565 & 0.0385 & 571.550 & 0.1668 \\
\hline 96.150 & 0.0008 & 459.322 & 0.0498 & 572.611 & 0.1688 \\
\hline 192.666 & 0.0014 & 462.817 & 0.0520 & 583.648 & 0.1931 \\
\hline 204.985 & 0.0015 & 499.739 & 0.0794 & 584.419 & 0.1951 \\
\hline 249.778 & 0.0020 & 502.294 & 0.0817 & 590.347 & 0.2114 \\
\hline 306.663 & 0.0036 & 505.185 & 0.0840 & 594.202 & 0.2237 \\
\hline 323.363 & 0.0045 & 533.090 & 0.1115 & 598.685 & 0.2400 \\
\hline 331.797 & 0.0051 & 535.115 & 0.1138 & 602.637 & 0.2583 \\
\hline 368.503 & 0.0099 & 544.947 & 0.1256 & 607.022 & 0.2849 \\
\hline 390.731 & 0.0157 & 557.140 & 0.1425 & 609.047 & 0.3146 \\
\hline
\end{tabular}

Table 323. Test data for specimen 754-28

\begin{tabular}{|c|c|c|c|c|c|}
\hline $\begin{array}{l}\text { Test Number } \\
\text { Aging Temp. } \\
\text { Yield Stress }\end{array}$ & $\begin{array}{l}: 2 \\
: 25 \\
: 32\end{array}$ & & $\begin{array}{l}\text { Tes } \\
\text { Agi } \\
\text { Ulti }\end{array}$ & $\begin{array}{l}\text { np. } \\
\text { ne } \\
\text { Stress }\end{array}$ & $\begin{array}{l}25^{\circ} \mathrm{C} \\
30,000 \mathrm{~h} \\
619.2 \mathrm{MPa}\end{array}$ \\
\hline $\begin{array}{l}\text { Engr. } \\
\text { Stress } \\
\text { (MPa) }\end{array}$ & $\begin{array}{l}\text { Engr. } \\
\text { Strain }\end{array}$ & $\begin{array}{l}\text { Engr. } \\
\text { Stress } \\
(\mathrm{MPa})\end{array}$ & $\begin{array}{l}\text { Engr. } \\
\text { Strain }\end{array}$ & $\begin{array}{l}\text { Engr. } \\
\text { Stress } \\
(\mathrm{MPa}) \\
\end{array}$ & $\begin{array}{l}\text { Engr. } \\
\text { Strain }\end{array}$ \\
\hline 12.251 & 0.0001 & 419.960 & 0.0218 & 568.133 & 0.1450 \\
\hline 34.804 & 0.0002 & 465.888 & 0.0456 & 569.396 & 0.1471 \\
\hline 88.596 & 0.0006 & 469.108 & 0.0476 & 583.531 & 0.1745 \\
\hline 99.875 & 0.0006 & 472.698 & 0.0499 & 584.552 & 0.1766 \\
\hline 174.477 & 0.0011 & 510.280 & 0.0778 & 585.426 & 0.1787 \\
\hline 186.781 & 0.0012 & 512.563 & 0.0798 & 598.930 & 0.2154 \\
\hline 264.141 & 0.0022 & 517.081 & 0.0838 & 602.573 & 0.2285 \\
\hline 298.523 & 0.0031 & 539.085 & 0.1060 & 609.762 & 0.2594 \\
\hline 306.052 & 0.0033 & 541.028 & 0.1081 & 613.162 & 0.2794 \\
\hline 368.704 & 0.0080 & 544.623 & 0.1121 & 615.300 & 0.2953 \\
\hline 393.258 & 0.0128 & 560.021 & 0.1325 & 619.088 & 0.3533 \\
\hline
\end{tabular}


Table 324. Test data for specimen 75-135

\begin{tabular}{|c|c|c|c|c|c|}
\hline $\begin{array}{l}\text { Test Number } \\
\text { Aging Temp. } \\
\text { Yield Stress }\end{array}$ & $\begin{array}{l}: 2 \\
: 25 \\
: 31\end{array}$ & & $\begin{array}{l}\text { Tes } \\
\text { Agi } \\
\text { Ulti }\end{array}$ & $\begin{array}{l}\mathrm{np} . \\
\text { me } \\
\text { Stress }\end{array}$ & $\begin{array}{l}25^{\circ} \mathrm{C} \\
30,000 \mathrm{~h} \\
630.4 \mathrm{MPa}\end{array}$ \\
\hline $\begin{array}{l}\text { Engr. } \\
\text { Stress } \\
\text { (MPa) }\end{array}$ & $\begin{array}{l}\text { Engr. } \\
\text { Strain }\end{array}$ & $\begin{array}{l}\text { Engr. } \\
\text { Stress } \\
\text { (MPa) }\end{array}$ & $\begin{array}{l}\text { Engr. } \\
\text { Strain }\end{array}$ & $\begin{array}{l}\text { Engr. } \\
\text { Stress } \\
\text { (MPa) }\end{array}$ & $\begin{array}{l}\text { Engr. } \\
\text { Strain }\end{array}$ \\
\hline 16.131 & 0.0001 & 424.177 & 0.0274 & 572.696 & 0.1450 \\
\hline 39.509 & 0.0003 & 460.190 & 0.0458 & 574.110 & 0.1473 \\
\hline 81.564 & 0.0006 & 464.179 & 0.0481 & 589.813 & 0.1742 \\
\hline 140.433 & 0.0011 & 478.702 & 0.0572 & 593.763 & 0.1823 \\
\hline 260.844 & 0.0026 & 505.059 & 0.0754 & 606.978 & 0.2168 \\
\hline 269.748 & 0.0029 & 507.839 & 0.0776 & 616.731 & 0.2518 \\
\hline 313.876 & 0.0044 & 510.862 & 0.0799 & 617.365 & 0.2539 \\
\hline 338.668 & 0.0060 & 538.951 & 0.1047 & 622.388 & 0.2787 \\
\hline 346.046 & 0.0067 & 541.292 & 0.1070 & 622.680 & 0.2808 \\
\hline 397.464 & 0.0171 & 551.045 & 0.1176 & 628.922 & 0.3303 \\
\hline 418.774 & 0.0251 & 571.038 & 0.1426 & 630.288 & 0.3659 \\
\hline
\end{tabular}

Table 325. Test data for specimen 754-21

\begin{tabular}{|c|c|c|c|c|c|}
\hline $\begin{array}{l}\text { Test Number } \\
\text { Aging Temp. } \\
\text { Yield Stress }\end{array}$ & $\begin{array}{l}: 13 \\
: 32 \\
: 35\end{array}$ & $\mathrm{~Pa}$ & $\begin{array}{l}\text { Tes } \\
\text { Agi } \\
\text { Ulti }\end{array}$ & $\begin{array}{l}\text { np. } \\
\text { ne } \\
\text { Stress }\end{array}$ & $\begin{array}{l}: 25^{\circ} \mathrm{C} \\
10,000 \mathrm{~h} \\
: 693.7 \mathrm{MPa}\end{array}$ \\
\hline $\begin{array}{l}\text { Engr. } \\
\text { Stress } \\
\text { (MPa) }\end{array}$ & $\begin{array}{l}\text { Engr. } \\
\text { Strain }\end{array}$ & $\begin{array}{l}\text { Engr. } \\
\text { Stress } \\
\text { (MPa) }\end{array}$ & $\begin{array}{l}\text { Engr. } \\
\text { Strain }\end{array}$ & $\begin{array}{l}\text { Engr. } \\
\text { Stress } \\
(\mathrm{MPa})\end{array}$ & $\begin{array}{l}\text { Engr. } \\
\text { Strain }\end{array}$ \\
\hline $\begin{array}{r}0.000 \\
48.641 \\
208.470 \\
343.960 \\
388.060 \\
413.870\end{array}$ & $\begin{array}{l}0.0000 \\
0.0002 \\
0.0026 \\
0.0061 \\
0.0092 \\
0.0123\end{array}$ & $\begin{array}{l}436.450 \\
459.850 \\
471.710 \\
486.140 \\
524.430 \\
560.710\end{array}$ & $\begin{array}{l}0.0171 \\
0.0241 \\
0.0283 \\
0.0365 \\
0.0578 \\
0.0819\end{array}$ & $\begin{array}{l}593.610 \\
592.740 \\
634.930 \\
663.180 \\
685.700 \\
693.660\end{array}$ & $\begin{array}{l}0.1044 \\
0.1047 \\
0.1531 \\
0.2118 \\
0.2947 \\
0.3958\end{array}$ \\
\hline
\end{tabular}

Table 326. Test data for specimen 754-25

\begin{tabular}{|c|c|c|c|c|c|}
\hline $\begin{array}{l}\text { Test Number } \\
\text { Aging Temp. } \\
\text { Yield Stress }\end{array}$ & $\begin{array}{l}: 1 \\
: 32 \\
: 4 C\end{array}$ & & $\begin{array}{l}\text { Tes } \\
\text { Agi } \\
\text { Ulti }\end{array}$ & $\begin{array}{l}\text { ne. } \\
\text { Stress }\end{array}$ & $\begin{array}{l}25^{\circ} \mathrm{C} \\
10,000 \mathrm{~h} \\
706.0 \mathrm{MPa}\end{array}$ \\
\hline $\begin{array}{l}\text { Engr. } \\
\text { Stress } \\
\text { (MPa) }\end{array}$ & $\begin{array}{l}\text { Engr. } \\
\text { Strain }\end{array}$ & $\begin{array}{l}\text { Engr. } \\
\text { Stress } \\
(\mathrm{MPa}) \\
\end{array}$ & $\begin{array}{l}\text { Engr. } \\
\text { Strain }\end{array}$ & $\begin{array}{l}\text { Engr. } \\
\text { Stress } \\
\text { (MPa) }\end{array}$ & $\begin{array}{l}\text { Engr. } \\
\text { Strain }\end{array}$ \\
\hline $\begin{array}{r}0.000 \\
34.659 \\
206.780 \\
353.210 \\
392.300 \\
427.650\end{array}$ & $\begin{array}{l}0.0000 \\
0.0001 \\
0.0022 \\
0.0040 \\
0.0058 \\
0.0089\end{array}$ & $\begin{array}{l}451.910 \\
473.520 \\
488.760 \\
537.850 \\
570.150 \\
603.730\end{array}$ & $\begin{array}{l}0.0129 \\
0.0182 \\
0.0244 \\
0.0511 \\
0.0733 \\
0.0980\end{array}$ & $\begin{array}{l}637.060 \\
665.550 \\
678.530 \\
688.120 \\
703.330 \\
705.940\end{array}$ & $\begin{array}{l}0.1280 \\
0.1697 \\
0.1957 \\
0.2220 \\
0.2905 \\
0.3728\end{array}$ \\
\hline
\end{tabular}


Table 327. Test data for specimen 752-41

\begin{tabular}{|c|c|c|c|c|c|}
\hline $\begin{array}{l}\text { Test Number } \\
\text { Aging Temp. } \\
\text { Yield Stress }\end{array}$ & $\begin{array}{l}: 16 \\
: 32 \\
: 35\end{array}$ & $\mathrm{MPa}$ & $\begin{array}{l}\text { Tes } \\
\text { Agi } \\
\text { Ulti }\end{array}$ & $\begin{array}{l}\text { np. } \\
\text { ne } \\
\text { Stress }\end{array}$ & $\begin{array}{l}25^{\circ} \mathrm{C} \\
30,000 \mathrm{~h} \\
726.8 \mathrm{MPa}\end{array}$ \\
\hline $\begin{array}{l}\text { Engr. } \\
\text { Stress } \\
\text { (MPa) }\end{array}$ & $\begin{array}{l}\text { Engr. } \\
\text { Strain }\end{array}$ & $\begin{array}{l}\text { Engr. } \\
\text { Stress } \\
\text { (MPa) }\end{array}$ & $\begin{array}{l}\text { Engr. } \\
\text { Strain }\end{array}$ & $\begin{array}{l}\text { Engr. } \\
\text { Stress } \\
(\mathrm{MPa}) \\
\end{array}$ & $\begin{array}{l}\text { Engr. } \\
\text { Strain }\end{array}$ \\
\hline 68.878 & 0.0001 & 433.740 & 0.0099 & 641.470 & 0.1087 \\
\hline 104.730 & 0.0003 & 455.510 & 0.0132 & 653.230 & 0.1205 \\
\hline 148.220 & 0.0008 & 469.720 & 0.0158 & 668.750 & 0.1401 \\
\hline 177.710 & 0.0010 & 486.150 & 0.0202 & 678.970 & 0.1539 \\
\hline 196.030 & 0.0012 & 487.380 & 0.0217 & 689.900 & 0.1705 \\
\hline 219.070 & 0.0016 & 524.100 & 0.0358 & 699.930 & 0.1906 \\
\hline 254.830 & 0.0022 & 551.170 & 0.0499 & 709.200 & 0.2176 \\
\hline 287.150 & 0.0029 & 574.600 & 0.0646 & 717.080 & 0.2421 \\
\hline 308.750 & 0.0034 & 595.730 & 0.0779 & 718.110 & 0.2454 \\
\hline 354.750 & 0.0049 & 610.280 & 0.0877 & 726.010 & 0.2752 \\
\hline 400.620 & 0.0073 & 622.420 & 0.0961 & 726.850 & 0.3031 \\
\hline
\end{tabular}

Table 328. Test data for specimen 754-39

\begin{tabular}{|c|c|c|c|c|c|}
\hline $\begin{array}{l}\text { Test Number } \\
\text { Aging Temp. } \\
\text { Yield Stress }\end{array}$ & $\begin{array}{l}: 1 \\
: 32 \\
: 35\end{array}$ & & $\begin{array}{l}\text { Tes } \\
\text { Agi } \\
\text { Ulti }\end{array}$ & $\begin{array}{l}\text { np. } \\
\text { ne } \\
\text { Stress }\end{array}$ & $\begin{array}{l}25^{\circ} \mathrm{C} \\
30,000 \mathrm{~h} \\
722.5 \mathrm{MPa}\end{array}$ \\
\hline $\begin{array}{l}\text { Engr. } \\
\text { Stress } \\
(\mathrm{MPa}) \\
\end{array}$ & $\begin{array}{l}\text { Engr. } \\
\text { Strain }\end{array}$ & $\begin{array}{l}\text { Engr. } \\
\text { Stress } \\
(\mathrm{MPa}) \\
\end{array}$ & $\begin{array}{l}\text { Engr. } \\
\text { Strain }\end{array}$ & $\begin{array}{l}\text { Engr. } \\
\text { Stress } \\
(\mathrm{MPa}) \\
\end{array}$ & $\begin{array}{l}\text { Engr. } \\
\text { Strain }\end{array}$ \\
\hline 0.000 & 0.0000 & 487.210 & 0.0239 & 668.850 & 0.1412 \\
\hline 361.240 & 0.0060 & 509.070 & 0.0321 & 692.810 & 0.1789 \\
\hline 396.640 & 0.0083 & 539.540 & 0.0467 & 710.940 & 0.2040 \\
\hline 423.810 & 0.0109 & 579.740 & 0.0698 & 720.130 & 0.2679 \\
\hline 442.600 & 0.0133 & 617.330 & 0.0945 & 722.540 & 0.2900 \\
\hline 467.520 & 0.0184 & 652.490 & 0.1225 & & \\
\hline
\end{tabular}

Table 329. Test data for specimen 75-245

\begin{tabular}{|c|c|c|c|c|c|}
\hline $\begin{array}{l}\text { Test Number } \\
\text { Aging Temp. } \\
\text { Yield Stress }\end{array}$ & $\begin{array}{l}: 16 \\
: 32 \\
: 32\end{array}$ & & $\begin{array}{l}\text { Tes } \\
\text { Agi } \\
\text { Ulti }\end{array}$ & $\begin{array}{l}\mathrm{mp} . \\
\text { me } \\
\text { Stress }\end{array}$ & $\begin{array}{l}25^{\circ} \mathrm{C} \\
30,000 \mathrm{~h} \\
691.3 \mathrm{MPa}\end{array}$ \\
\hline $\begin{array}{l}\text { Engr. } \\
\text { Stress } \\
\text { (MPa) }\end{array}$ & $\begin{array}{l}\text { Engr. } \\
\text { Strain }\end{array}$ & $\begin{array}{l}\text { Engr. } \\
\text { Stress } \\
\text { (MPa) }\end{array}$ & $\begin{array}{l}\text { Engr. } \\
\text { Strain }\end{array}$ & $\begin{array}{l}\text { Engr. } \\
\text { Stress } \\
\text { (MPa) }\end{array}$ & $\begin{array}{l}\text { Engr. } \\
\text { Strain }\end{array}$ \\
\hline $\begin{array}{r}0.0000 \\
250.0600 \\
337.1400 \\
388.7400 \\
405.0500 \\
422.8200\end{array}$ & $\begin{array}{l}0.0000 \\
0.0023 \\
0.0052 \\
0.0087 \\
0.0106 \\
0.0134\end{array}$ & $\begin{array}{l}441.7100 \\
463.3100 \\
475.7100 \\
485.2900 \\
535.5200 \\
582.0500\end{array}$ & $\begin{array}{l}0.0176 \\
0.0247 \\
0.0293 \\
0.0331 \\
0.0616 \\
0.0937\end{array}$ & $\begin{array}{l}630.8700 \\
661.2200 \\
683.6300 \\
691.2700\end{array}$ & $\begin{array}{l}0.1369 \\
0.1873 \\
0.2586 \\
0.3612\end{array}$ \\
\hline
\end{tabular}


Table 330. Test data for specimen 751-28

\begin{tabular}{|c|c|c|c|c|c|}
\hline $\begin{array}{l}\text { Test Number } \\
\text { Aging Temp. } \\
\text { Yield Stress }\end{array}$ & $\begin{array}{l}: 0 \\
: 32 \\
: 33\end{array}$ & & $\begin{array}{l}\text { Tes } \\
\text { Agir } \\
\text { Ulti }\end{array}$ & $\begin{array}{l}\text { np. } \\
\text { ne } \\
\text { Stress }\end{array}$ & $\begin{array}{l}25^{\circ} \mathrm{C} \\
50,000 \mathrm{~h} \\
689.5 \mathrm{MPa}\end{array}$ \\
\hline $\begin{array}{l}\text { Engr. } \\
\text { Stress } \\
\text { (MPa) }\end{array}$ & $\begin{array}{l}\text { Engr. } \\
\text { Strain }\end{array}$ & $\begin{array}{l}\text { Engr. } \\
\text { Stress } \\
\text { (MPa) }\end{array}$ & $\begin{array}{l}\text { Engr. } \\
\text { Strain }\end{array}$ & $\begin{array}{l}\text { Engr. } \\
\text { Stress } \\
(\mathrm{MPa})\end{array}$ & $\begin{array}{l}\text { Engr. } \\
\text { Strain }\end{array}$ \\
\hline 68.817 & 0.000 & 338.880 & 0.005 & 479.220 & 0.068 \\
\hline 111.680 & 0.000 & 344.820 & 0.005 & 484.990 & 0.074 \\
\hline 156.270 & 0.001 & 353.020 & 0.006 & 503.220 & 0.092 \\
\hline 189.390 & 0.001 & 362.440 & 0.007 & 512.450 & 0.099 \\
\hline 204.140 & 0.002 & 370.870 & 0.008 & 525.700 & 0.115 \\
\hline 213.280 & 0.002 & 379.640 & 0.010 & 536.890 & 0.130 \\
\hline 226.290 & 0.002 & 395.010 & 0.014 & 553.880 & 0.160 \\
\hline 243.890 & 0.002 & 406.690 & 0.018 & 566.900 & 0.194 \\
\hline 288.660 & 0.003 & 422.550 & 0.026 & 576.290 & 0.226 \\
\hline 312.300 & 0.003 & 441.600 & 0.038 & 583.130 & 0.259 \\
\hline 327.040 & 0.004 & 463.100 & 0.055 & 589.790 & 0.359 \\
\hline
\end{tabular}

Table 331. Test data for specimen 751-29

\begin{tabular}{|c|c|c|c|c|c|}
\hline $\begin{array}{l}\text { Test Number } \\
\text { Aging Temp. } \\
\text { Yield Stress }\end{array}$ & & $\mathrm{MPa}$ & $\begin{array}{l}\text { Tes } \\
\text { Agir } \\
\text { Ultir }\end{array}$ & $\begin{array}{l}\mathrm{mp} . \\
\text { me } \\
\text { Stress }\end{array}$ & $\begin{array}{l}25^{\circ} \mathrm{C} \\
50,000 \mathrm{~h} \\
729.8 \mathrm{MPa}\end{array}$ \\
\hline $\begin{array}{l}\text { Engr. } \\
\text { Stress } \\
\text { (MPa) }\end{array}$ & $\begin{array}{l}\text { Engr. } \\
\text { Strain }\end{array}$ & $\begin{array}{l}\text { Engr. } \\
\text { Stress } \\
\text { (MPa) }\end{array}$ & $\begin{array}{l}\text { Engr. } \\
\text { Strain }\end{array}$ & $\begin{array}{l}\text { Engr. } \\
\text { Stress } \\
\text { (MPa) }\end{array}$ & $\begin{array}{l}\text { Engr. } \\
\text { Strain }\end{array}$ \\
\hline 54.600 & 0.0000 & 445.340 & 0093 & 60.670 & 0.1059 \\
\hline 102.720 & 0.0002 & 451.700 & 0.0101 & 677.290 & 0.1230 \\
\hline 152.200 & 0.0005 & 511.560 & 0.0228 & 686.150 & 0.1336 \\
\hline 190.280 & 0.0007 & 527.310 & 0.0279 & 690.990 & 0.1401 \\
\hline 239.960 & 0.0010 & 544.120 & 0.0342 & 698.550 & 0.1508 \\
\hline 251.660 & 0.0012 & 568.390 & 0.0448 & 702.650 & 0.1573 \\
\hline 283.680 & 0.0017 & 585.260 & 0.0533 & 708.900 & 0.1682 \\
\hline 353.810 & 0.0035 & 603.970 & 0.0638 & 717.000 & 0.1856 \\
\hline 361.280 & 0.0038 & 614.160 & 0.0701 & 723.610 & 0.2034 \\
\hline 382.340 & 0.0047 & 638.240 & 0.0870 & 725.590 & 0.2101 \\
\hline 417.260 & 0.0068 & 651.250 & 0.0975 & 729.710 & 0.2397 \\
\hline
\end{tabular}


Table 332 Test data for specimen 75-230

\begin{tabular}{|c|c|c|c|c|c|}
\hline $\begin{array}{l}\text { Test Number } \\
\text { Aging Temp. } \\
\text { Yield Stress }\end{array}$ & $\begin{array}{l}: 2 \\
: 32 \\
: 37\end{array}$ & & $\begin{array}{l}\text { Tes } \\
\text { Agi } \\
\text { Ulti }\end{array}$ & $\begin{array}{l}\text { mp. } \\
\text { me } \\
\text { Stress }\end{array}$ & $\begin{array}{l}25^{\circ} \mathrm{C} \\
50,000 \mathrm{~h} \\
759.5 \mathrm{MPa}\end{array}$ \\
\hline $\begin{array}{l}\text { Engr. } \\
\text { Stress } \\
\text { (MPa) }\end{array}$ & $\begin{array}{l}\text { Engr. } \\
\text { Strain }\end{array}$ & $\begin{array}{l}\text { Engr. } \\
\text { Stress } \\
\text { (MPa) }\end{array}$ & $\begin{array}{l}\text { Engr. } \\
\text { Strain }\end{array}$ & $\begin{array}{l}\text { Engr. } \\
\text { Stress } \\
(\mathrm{MPa})\end{array}$ & $\begin{array}{l}\text { Engr. } \\
\text { Strain }\end{array}$ \\
\hline 22.710 & 0.0001 & 508.940 & 0.0237 & 714.680 & 0.1461 \\
\hline 28.190 & 0.0001 & 567.950 & 0.0329 & 722.910 & 0.1596 \\
\hline 43.390 & 0.0002 & 587.160 & 0.0413 & 726.700 & 0.1664 \\
\hline 77.140 & 0.0003 & 619.600 & 0.0584 & 731.290 & 0.1755 \\
\hline 135.460 & 0.0005 & 636.700 & 0.0692 & 737.430 & 0.1891 \\
\hline 148.340 & 0.0006 & 639.880 & 0.0713 & 738.490 & 0.1914 \\
\hline 238.630 & 0.0012 & 662.680 & 0.0885 & 742.130 & 0.2006 \\
\hline 307.310 & 0.0020 & 665.240 & 0.0907 & 748.060 & 0.2190 \\
\hline 364.410 & 0.0033 & 692.360 & 0.1171 & 751.520 & 0.2313 \\
\hline 429.700 & 0.0060 & 698.030 & 0.1237 & 754.230 & 0.2429 \\
\hline 505.220 & 0.0142 & 708.340 & 0.1371 & 759.480 & 0.2856 \\
\hline
\end{tabular}

Table 333. Test data for specimen 752-25

\begin{tabular}{|c|c|c|c|c|c|}
\hline $\begin{array}{l}\text { Test Number } \\
\text { Aging Temp. } \\
\text { Yield Stress }\end{array}$ & $\begin{array}{l}: 0 \\
: 35 \\
: 34\end{array}$ & & $\begin{array}{l}\text { Tes } \\
\text { Agi } \\
\text { Ulti }\end{array}$ & $\begin{array}{l}\text { np. } \\
\text { me } \\
\text { Stress }\end{array}$ & $\begin{array}{l}: 25^{\circ} \mathrm{C} \\
2,570 \mathrm{~h} \\
: 667.8 \mathrm{MPa}\end{array}$ \\
\hline $\begin{array}{l}\text { Engr. } \\
\text { Stress } \\
\text { (MPa) }\end{array}$ & $\begin{array}{l}\text { Engr. } \\
\text { Strain }\end{array}$ & $\begin{array}{l}\text { Engr. } \\
\text { Stress } \\
\text { (MPa) }\end{array}$ & $\begin{array}{l}\text { Engr. } \\
\text { Strain }\end{array}$ & $\begin{array}{l}\text { Engr. } \\
\text { Stress } \\
\text { (MPa) }\end{array}$ & $\begin{array}{l}\text { Engr. } \\
\text { Strain }\end{array}$ \\
\hline 73.49 & 00001 & 38117 & 00061 & 54388 & 00696 \\
\hline 119.13 & 0.0004 & 397.04 & 0.0075 & 566.03 & 0.0871 \\
\hline 154.98 & 0.0007 & 407.83 & 0.0088 & 588.31 & 0.1036 \\
\hline 188.94 & 0.0011 & 419.41 & 0.0104 & 606.86 & 0.1220 \\
\hline 199.93 & 0.0013 & 425.20 & 0.0112 & 623.27 & 0.1447 \\
\hline 205.64 & 0.0013 & 433.30 & 0.0130 & 639.85 & 0.1730 \\
\hline 227.79 & 0.0015 & 442.18 & 0.0152 & 649.10 & 0.1938 \\
\hline 239.21 & 0.0017 & 457.15 & 0.0196 & 657.41 & 0.2192 \\
\hline 263.77 & 0.0020 & 471.75 & 0.0253 & 661.75 & 0.2383 \\
\hline 297.97 & 0.0027 & 484.27 & 0.0310 & 666.43 & 0.2619 \\
\hline 325.15 & 0.0035 & 488.93 & 0.0350 & 667.26 & 0.2736 \\
\hline 346.41 & 0.0043 & 515.86 & 0.0508 & 667.75 & 0.2930 \\
\hline 364.99 & 0.0052 & & & & \\
\hline
\end{tabular}


Table 334. Test data for specimen 752-26

\begin{tabular}{|c|c|c|c|c|c|}
\hline $\begin{array}{l}\text { Test Number } \\
\text { Aging Temp. } \\
\text { Yield Stress }\end{array}$ & $\begin{array}{l}: 0 \\
: 35 \\
: 34\end{array}$ & & $\begin{array}{l}\text { Tes } \\
\text { Agi } \\
\text { Ulti }\end{array}$ & $\begin{array}{l}\text { np. } \\
\text { me } \\
\text { Stress }\end{array}$ & $\begin{array}{l}25^{\circ} \mathrm{C} \\
2,570 \mathrm{~h} \\
688.9 \mathrm{MPa}\end{array}$ \\
\hline $\begin{array}{l}\text { Engr. } \\
\text { Stress } \\
(\mathrm{MPa}) \\
\end{array}$ & $\begin{array}{l}\text { Engr. } \\
\text { Strain }\end{array}$ & $\begin{array}{l}\text { Engr. } \\
\text { Stress } \\
\text { (MPa) }\end{array}$ & $\begin{array}{l}\text { Engr. } \\
\text { Strain }\end{array}$ & $\begin{array}{l}\text { Engr. } \\
\text { Stress } \\
(\mathrm{MPa})\end{array}$ & $\begin{array}{l}\text { Engr. } \\
\text { Strain }\end{array}$ \\
\hline 59.65 & 0.0000 & 404.74 & 0.0071 & 570.33 & 0.0760 \\
\hline 93.85 & 0.0002 & 415.16 & 0.0081 & 592.85 & 0.0929 \\
\hline 130.88 & 0.0005 & 420.91 & 0.0086 & 611.04 & 0.1059 \\
\hline 168.71 & 0.0009 & 432.01 & 0.0100 & 625.94 & 0.1218 \\
\hline 199.47 & 0.0013 & 441.47 & 0.0114 & 642.65 & 0.1434 \\
\hline 207.97 & 0.0014 & 451.91 & 0.0133 & 651.15 & 0.1567 \\
\hline 220.14 & 0.0015 & 461.64 & 0.0157 & 660.27 & 0.1739 \\
\hline 234.09 & 0.0016 & 475.87 & 0.0199 & 668.44 & 0.1924 \\
\hline 251.98 & 0.0019 & 486.96 & 0.0241 & 676.12 & 0.2154 \\
\hline 277.03 & 0.0023 & 488.94 & 0.0264 & 680.67 & 0.2334 \\
\hline 310.62 & 0.0030 & 510.17 & 0.0365 & 684.79 & 0.2525 \\
\hline 334.08 & 0.0037 & 539.13 & 0.0540 & 688.70 & 0.2790 \\
\hline 353.25 & 0.0043 & 548.02 & 0.0599 & 688.85 & 0.2967 \\
\hline 386.38 & 0.0058 & & & & \\
\hline
\end{tabular}

Table 335. Test data for specimen 754-06

\begin{tabular}{|c|c|c|c|c|c|}
\hline $\begin{array}{l}\text { Test Number } \\
\text { Aging Temp. } \\
\text { Yield Stress }\end{array}$ & $\begin{array}{l}: 1 \\
: 35 \\
: 33\end{array}$ & $\mathrm{~Pa}$ & $\begin{array}{l}\text { Tes } \\
\text { Agi } \\
\text { Ulti }\end{array}$ & $\begin{array}{l}\text { np. } \\
\text { ne } \\
\text { Stress }\end{array}$ & $\begin{array}{l}25^{\circ} \mathrm{C} \\
10,000 \mathrm{~h} \\
741.1 \mathrm{MPa}\end{array}$ \\
\hline $\begin{array}{l}\text { Engr. } \\
\text { Stress } \\
\text { (MPa) }\end{array}$ & $\begin{array}{l}\text { Engr. } \\
\text { Strain }\end{array}$ & $\begin{array}{l}\text { Engr. } \\
\text { Stress } \\
\text { (MPa) }\end{array}$ & $\begin{array}{l}\text { Engr. } \\
\text { Strain }\end{array}$ & $\begin{array}{l}\text { Engr. } \\
\text { Stress } \\
(\mathrm{MPa}) \\
\end{array}$ & $\begin{array}{l}\text { Engr. } \\
\text { Strain }\end{array}$ \\
\hline 52.922 & 0.0003 & 454.660 & 0.0134 & 671.190 & 0.1332 \\
\hline 204.610 & 0.0023 & 480.910 & 0.0183 & 670.790 & 0.1333 \\
\hline 226.170 & 0.0025 & 495.550 & 0.0249 & 701.930 & 0.1747 \\
\hline 346.910 & 0.0054 & 541.000 & 0.0434 & 725.090 & 0.2325 \\
\hline 406.130 & 0.0081 & 596.460 & 0.0744 & 739.370 & 0.2914 \\
\hline 434.270 & 0.0109 & 645.880 & 0.1070 & 741.080 & 0.3489 \\
\hline
\end{tabular}


Table 336. Test data for specimen 754-07

\begin{tabular}{|c|c|c|c|c|c|}
\hline $\begin{array}{l}\text { Test Number } \\
\text { Aging Temp. } \\
\text { Yield Stress }\end{array}$ & $\begin{array}{l}: 1 \\
: 35 \\
: 35\end{array}$ & & $\begin{array}{l}\text { Tes } \\
\text { Agi } \\
\text { Ulti }\end{array}$ & $\begin{array}{l}\text { ne. } \\
\text { Stress }\end{array}$ & $\begin{array}{l}25^{\circ} \mathrm{C} \\
10,000 \mathrm{~h} \\
728.5 \mathrm{MPa}\end{array}$ \\
\hline $\begin{array}{l}\text { Engr. } \\
\text { Stress } \\
\text { (MPa) }\end{array}$ & $\begin{array}{l}\text { Engr. } \\
\text { Strain }\end{array}$ & $\begin{array}{l}\text { Engr. } \\
\text { Stress } \\
(\mathrm{MPa})\end{array}$ & $\begin{array}{l}\text { Engr. } \\
\text { Strain }\end{array}$ & $\begin{array}{l}\text { Engr. } \\
\text { Stress } \\
\text { (MPa) }\end{array}$ & $\begin{array}{l}\text { Engr. } \\
\text { Strain }\end{array}$ \\
\hline $\begin{array}{r}38.296 \\
205.090 \\
227.300 \\
343.640 \\
402.370 \\
430.080\end{array}$ & $\begin{array}{l}0.0001 \\
0.0020 \\
0.0022 \\
0.0048 \\
0.0085 \\
0.0111\end{array}$ & $\begin{array}{l}454.290 \\
474.210 \\
488.150 \\
528.390 \\
564.520 \\
607.110 \\
636.250\end{array}$ & $\begin{array}{l}0.0144 \\
0.0182 \\
0.0231 \\
0.0389 \\
0.0576 \\
0.0840 \\
0.1036\end{array}$ & $\begin{array}{l}663.470 \\
681.580 \\
702.230 \\
719.020 \\
719.220 \\
727.900 \\
728.490\end{array}$ & $\begin{array}{l}0.1291 \\
0.1546 \\
0.1908 \\
0.2465 \\
0.2463 \\
0.2976 \\
0.2975\end{array}$ \\
\hline
\end{tabular}

Table 337. Test data for specimen 754-08

\begin{tabular}{|c|c|c|c|c|c|}
\hline $\begin{array}{l}\text { Test Number } \\
\text { Aging Temp. } \\
\text { Yield Stress }\end{array}$ & $\begin{array}{l}: 2 \\
: 35 \\
: 30\end{array}$ & & $\begin{array}{l}\text { Tes } \\
\text { Agir } \\
\text { Ulti }\end{array}$ & $\begin{array}{l}\text { np. } \\
\text { ne } \\
\text { Stress }\end{array}$ & $\begin{array}{l}25^{\circ} \mathrm{C} \\
10,000 \mathrm{~h} \\
718.8 \mathrm{MPa}\end{array}$ \\
\hline $\begin{array}{l}\text { Engr. } \\
\text { Stress } \\
(\mathrm{MPa})\end{array}$ & $\begin{array}{l}\text { Engr. } \\
\text { Strain }\end{array}$ & $\begin{array}{l}\text { Engr. } \\
\text { Stress } \\
(\mathrm{MPa})\end{array}$ & $\begin{array}{l}\text { Engr. } \\
\text { Strain }\end{array}$ & $\begin{array}{l}\text { Engr. } \\
\text { Stress } \\
(\mathrm{MPa}) \\
\end{array}$ & $\begin{array}{l}\text { Engr. } \\
\text { Strain }\end{array}$ \\
\hline 33.260 & 0.0002 & 396.930 & 0.0083 & 662.250 & 0.1479 \\
\hline 45.180 & 0.0002 & 404.440 & 0.0090 & 672.080 & 0.1628 \\
\hline 61.780 & 0.0003 & 429.060 & 0.0121 & 680.680 & 0.1779 \\
\hline 110.540 & 0.0005 & 482.310 & 0.0246 & 686.900 & 0.1909 \\
\hline 122.510 & 0.0006 & 577.070 & 0.0696 & 696.420 & 0.2154 \\
\hline 134.590 & 0.0007 & 580.460 & 0.0716 & 706.660 & 0.2489 \\
\hline 208.410 & 0.0013 & 603.710 & 0.0883 & 707.070 & 0.2511 \\
\hline 279.680 & 0.0027 & 606.410 & 0.0904 & 710.910 & 0.2691 \\
\hline 313.330 & 0.0037 & 621.680 & 0.1030 & 711.590 & 0.2736 \\
\hline 320.770 & 0.0040 & 641.880 & 0.1236 & 715.230 & 0.2964 \\
\hline 373.210 & 0.0065 & 651.140 & 0.1329 & 718.730 & 0.3560 \\
\hline
\end{tabular}


Table 338. Test data for specimen 75-119

\begin{tabular}{|c|c|c|c|c|c|}
\hline $\begin{array}{l}\text { Test Number } \\
\text { Aging Temp. } \\
\text { Yield Stress }\end{array}$ & $\begin{array}{l}: 14 \\
: 35 \\
: 35\end{array}$ & & $\begin{array}{l}\text { Tes } \\
\text { Agi } \\
\text { Ulti }\end{array}$ & $\begin{array}{l}\text { ne. } \\
\text { Stress }\end{array}$ & $\begin{array}{l}25^{\circ} \mathrm{C} \\
10,000 \mathrm{~h} \\
742.8 \mathrm{MPa}\end{array}$ \\
\hline $\begin{array}{l}\text { Engr. } \\
\text { Stress } \\
\text { (MPa) }\end{array}$ & $\begin{array}{l}\text { Engr. } \\
\text { Strain }\end{array}$ & $\begin{array}{l}\text { Engr. } \\
\text { Stress } \\
\text { (MPa) }\end{array}$ & $\begin{array}{l}\text { Engr. } \\
\text { Strain }\end{array}$ & $\begin{array}{l}\text { Engr. } \\
\text { Stress } \\
(\mathrm{MPa}) \\
\end{array}$ & $\begin{array}{l}\text { Engr. } \\
\text { Strain }\end{array}$ \\
\hline $\begin{array}{r}57.819 \\
199.560 \\
218.060 \\
320.720 \\
407.600 \\
440.510\end{array}$ & $\begin{array}{l}0.0009 \\
0.0034 \\
0.0039 \\
0.0063 \\
0.0109 \\
0.0136\end{array}$ & $\begin{array}{l}464.680 \\
479.370 \\
488.860 \\
513.370 \\
542.610 \\
581.790\end{array}$ & $\begin{array}{l}0.0168 \\
0.0197 \\
0.0235 \\
0.0316 \\
0.0440 \\
0.0654\end{array}$ & $\begin{array}{l}647.710 \\
677.920 \\
703.990 \\
721.850 \\
735.150 \\
742.780\end{array}$ & $\begin{array}{l}0.1080 \\
0.1408 \\
0.1833 \\
0.2267 \\
0.2859 \\
0.3565\end{array}$ \\
\hline
\end{tabular}

Table 339. Test data for specimen 753-10

\begin{tabular}{|c|c|c|c|c|c|}
\hline $\begin{array}{l}\text { Test Number } \\
\text { Aging Temp. } \\
\text { Yield Stress }\end{array}$ & $\begin{array}{l}: 2 \\
: 35 \\
: 3\end{array}$ & $\mathrm{MPa}$ & $\begin{array}{l}\text { Tes } \\
\text { Agir } \\
\text { Ulti }\end{array}$ & $\begin{array}{l}\text { np. } \\
\text { me } \\
\text { Stress }\end{array}$ & $\begin{array}{l}25^{\circ} \mathrm{C} \\
30,000 \mathrm{~h} \\
766.4 \mathrm{MPa}\end{array}$ \\
\hline $\begin{array}{l}\text { Engr. } \\
\text { Stress } \\
(\mathrm{MPa}) \\
\end{array}$ & $\begin{array}{l}\text { Engr. } \\
\text { Strain }\end{array}$ & $\begin{array}{l}\text { Engr. } \\
\text { Stress } \\
(\mathrm{MPa}) \\
\end{array}$ & $\begin{array}{l}\text { Engr. } \\
\text { Strain }\end{array}$ & $\begin{array}{l}\text { Engr. } \\
\text { Stress } \\
(\mathrm{MPa}) \\
\end{array}$ & $\begin{array}{l}\text { Engr. } \\
\text { Strain }\end{array}$ \\
\hline 15.470 & 0.0001 & 257.140 & 0.0021 & 685.390 & 0.1009 \\
\hline 17.770 & 0.0001 & 340.180 & 0.0046 & 707.290 & 0.1215 \\
\hline 30.840 & 0.0002 & 374.850 & 0.0060 & 722.630 & 0.1398 \\
\hline 41.740 & 0.0002 & 387.210 & 0.0067 & 731.250 & 0.1521 \\
\hline 63.970 & 0.0004 & 393.270 & 0.0070 & 753.540 & 0.1986 \\
\hline 74.670 & 0.0004 & 415.140 & 0.0083 & 757.040 & 0.2095 \\
\hline 85.580 & 0.0005 & 575.470 & 0.0389 & 757.370 & 0.2116 \\
\hline 119.960 & 0.0007 & 580.430 & 0.0408 & 761.310 & 0.2277 \\
\hline 131.950 & 0.0007 & 621.310 & 0.0591 & 763.880 & 0.2412 \\
\hline 144.250 & 0.0008 & 642.270 & 0.0707 & 765.570 & 0.2594 \\
\hline 181.040 & 0.0011 & 672.290 & 0.0906 & 766.280 & 0.2711 \\
\hline
\end{tabular}


Table 340. Test data for specimen 753-11

\begin{tabular}{|c|c|c|c|c|c|}
\hline $\begin{array}{l}\text { Test Number } \\
\text { Aging Temp. } \\
\text { Yield Stress }\end{array}$ & $\begin{array}{l}: 2 \\
: 35 \\
: 3\end{array}$ & & $\begin{array}{l}\text { Tes } \\
\text { Agir } \\
\text { Ulti }\end{array}$ & $\begin{array}{l}\text { np. } \\
\text { ne } \\
\text { Stress }\end{array}$ & $\begin{array}{l}25^{\circ} \mathrm{C} \\
30,000 \mathrm{~h} \\
748.2 \mathrm{MPa}\end{array}$ \\
\hline $\begin{array}{l}\text { Engr. } \\
\text { Stress } \\
(\mathrm{MPa})\end{array}$ & $\begin{array}{l}\text { Engr. } \\
\text { Strain }\end{array}$ & $\begin{array}{l}\text { Engr. } \\
\text { Stress } \\
(\mathrm{MPa})\end{array}$ & $\begin{array}{l}\text { Engr. } \\
\text { Strain }\end{array}$ & $\begin{array}{l}\text { Engr. } \\
\text { Stress } \\
(\mathrm{MPa}) \\
\end{array}$ & $\begin{array}{l}\text { Engr. } \\
\text { Strain }\end{array}$ \\
\hline 11.730 & 0.0001 & 251.070 & 0.0019 & 582.880 & 0.0449 \\
\hline 17.140 & 0.0001 & 291.190 & 0.0026 & 611.890 & 0.0589 \\
\hline 29.920 & 0.0002 & 300.100 & 0.0028 & 615.570 & 0.0609 \\
\hline 40.310 & 0.0003 & 308.730 & 0.0031 & 636.160 & 0.0729 \\
\hline 50.870 & 0.0004 & 341.120 & 0.0041 & 648.570 & 0.0810 \\
\hline 61.090 & 0.0005 & 348.440 & 0.0043 & 689.120 & 0.1136 \\
\hline 71.580 & 0.0005 & 376.080 & 0.0055 & 697.880 & 0.1223 \\
\hline 82.390 & 0.0006 & 416.970 & 0.0077 & 710.000 & 0.1369 \\
\hline 104.540 & 0.0007 & 446.100 & 0.0100 & 739.800 & 0.1898 \\
\hline 228.180 & 0.0016 & 502.770 & 0.0185 & 746.470 & 0.2142 \\
\hline 239.940 & 0.0017 & 526.130 & 0.0243 & 747.650 & 0.2233 \\
\hline
\end{tabular}

Table 341. Test data for specimen 75-130

\begin{tabular}{|c|c|c|c|c|c|}
\hline $\begin{array}{l}\text { Test Number } \\
\text { Aging Temp. } \\
\text { Yield Stress }\end{array}$ & $\begin{array}{l}: 2 \\
: 35 \\
: 3 c\end{array}$ & & $\begin{array}{l}\text { Tes } \\
\text { Agi } \\
\text { Ulti }\end{array}$ & $\begin{array}{l}\text { mp. } \\
\text { me } \\
\text { Stress }\end{array}$ & $\begin{array}{l}25^{\circ} \mathrm{C} \\
30,000 \mathrm{~h} \\
711.0 \mathrm{MPa}\end{array}$ \\
\hline $\begin{array}{l}\text { Engr. } \\
\text { Stress } \\
(\mathrm{MPa}) \\
\end{array}$ & $\begin{array}{l}\text { Engr. } \\
\text { Strain }\end{array}$ & $\begin{array}{l}\text { Engr. } \\
\text { Stress } \\
(\mathrm{MPa}) \\
\end{array}$ & $\begin{array}{l}\text { Engr. } \\
\text { Strain }\end{array}$ & $\begin{array}{l}\text { Engr. } \\
\text { Stress } \\
(\mathrm{MPa}) \\
\end{array}$ & $\begin{array}{l}\text { Engr. } \\
\text { Strain }\end{array}$ \\
\hline 14.680 & 0.0001 & 260.450 & 0.0025 & 562.070 & 0.0527 \\
\hline 27.100 & 0.0001 & 268.520 & 0.0027 & 587.950 & 0.0680 \\
\hline 57.420 & 0.0003 & 321.250 & 0.0044 & 602.990 & 0.0783 \\
\hline 67.750 & 0.0004 & 328.040 & 0.0047 & 605.990 & 0.0803 \\
\hline 112.940 & 0.0006 & 371.970 & 0.0066 & 635.380 & 0.1046 \\
\hline 124.280 & 0.0007 & 406.490 & 0.0088 & 675.220 & 0.1519 \\
\hline 182.390 & 0.0011 & 414.230 & 0.0094 & 686.970 & 0.1727 \\
\hline 193.660 & 0.0012 & 439.280 & 0.0120 & 692.150 & 0.1833 \\
\hline 204.490 & 0.0013 & 452.530 & 0.0140 & 695.610 & 0.1918 \\
\hline 214.600 & 0.0015 & 486.850 & 0.0217 & 710.190 & 0.2560 \\
\hline 251.850 & 0.0022 & 549.230 & 0.0461 & 710.880 & 0.2695 \\
\hline
\end{tabular}


Table 342. Test data for specimen 752-16

\begin{tabular}{|c|c|c|c|c|c|}
\hline $\begin{array}{l}\text { Test Number } \\
\text { Aging Temp. } \\
\text { Yield Stress }\end{array}$ & $\begin{array}{l}: 0 \\
: 40 \\
: 32\end{array}$ & & $\begin{array}{l}\text { Tes } \\
\text { Agi } \\
\text { Ulti }\end{array}$ & $\begin{array}{l}\text { np. } \\
\text { ne } \\
\text { Stress }\end{array}$ & $\begin{array}{l}25^{\circ} \mathrm{C} \\
2,570 \mathrm{~h} \\
703.6 \mathrm{MPa}\end{array}$ \\
\hline $\begin{array}{l}\text { Engr. } \\
\text { Stress } \\
\text { (MPa) }\end{array}$ & $\begin{array}{l}\text { Engr. } \\
\text { Strain }\end{array}$ & $\begin{array}{l}\text { Engr. } \\
\text { Stress } \\
(\mathrm{MPa})\end{array}$ & $\begin{array}{l}\text { Engr. } \\
\text { Strain }\end{array}$ & $\begin{array}{l}\text { Engr. } \\
\text { Stress } \\
(\mathrm{MPa}) \\
\end{array}$ & $\begin{array}{l}\text { Engr. } \\
\text { Strain }\end{array}$ \\
\hline 76.17 & 0.0001 & 396.10 & 0.0074 & 568.50 & 0.0724 \\
\hline 116.51 & 0.0003 & 403.19 & 0.0080 & 591.38 & 0.0877 \\
\hline 152.92 & 0.0007 & 415.13 & 0.0094 & 613.10 & 0.1019 \\
\hline 195.20 & 0.0013 & 427.70 & 0.0111 & 631.87 & 0.1188 \\
\hline 202.49 & 0.0014 & 438.06 & 0.0130 & 651.39 & 0.1405 \\
\hline 217.08 & 0.0015 & 447.88 & 0.0148 & 663.74 & 0.1569 \\
\hline 227.52 & 0.0016 & 464.22 & 0.0190 & 674.85 & 0.1763 \\
\hline 254.96 & 0.0019 & 469.79 & 0.0207 & 686.51 & 0.2011 \\
\hline 293.55 & 0.0027 & 469.75 & 0.0211 & 695.94 & 0.2289 \\
\hline 318.58 & 0.0035 & 482.68 & 0.0255 & 702.18 & 0.2627 \\
\hline 354.25 & 0.0049 & 487.15 & 0.0283 & 703.57 & 0.2788 \\
\hline 374.46 & 0.0059 & 508.36 & 0.0376 & 703.58 & 0.3047 \\
\hline 387.87 & 0.0068 & 538.21 & 0.0535 & & \\
\hline
\end{tabular}

Table 343. Test data for specimen 752-17

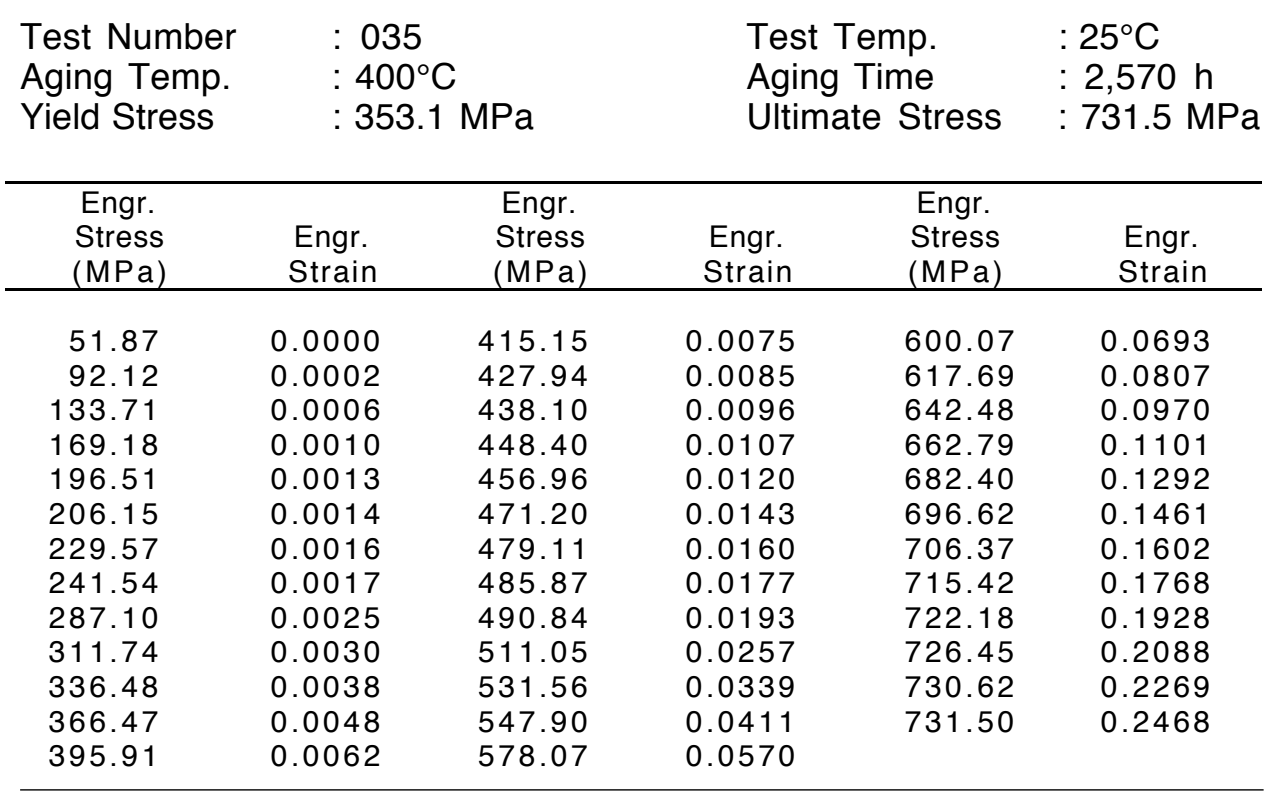


Table 344. Test data for specimen 752-15

\begin{tabular}{|c|c|c|c|c|c|}
\hline $\begin{array}{l}\text { Test Number } \\
\text { Aging Temp. } \\
\text { Yield Stress }\end{array}$ & $\begin{array}{l}: 1 \\
: 40 \\
: 4 C\end{array}$ & & $\begin{array}{l}\text { Tes } \\
\text { Agi } \\
\text { Ulti }\end{array}$ & $\begin{array}{l}\mathrm{mp} . \\
\text { me } \\
\text { Stress }\end{array}$ & $\begin{array}{l}25^{\circ} \mathrm{C} \\
10,000 \mathrm{~h} \\
777.1 \mathrm{MPa}\end{array}$ \\
\hline $\begin{array}{l}\text { Engr. } \\
\text { Stress } \\
\text { (MPa) } \\
\end{array}$ & $\begin{array}{l}\text { Engr. } \\
\text { Strain }\end{array}$ & $\begin{array}{l}\text { Engr. } \\
\text { Stress } \\
\text { (MPa) } \\
\end{array}$ & $\begin{array}{l}\text { Engr. } \\
\text { Strain }\end{array}$ & $\begin{array}{l}\text { Engr. } \\
\text { Stress } \\
(\mathrm{MPa}) \\
\end{array}$ & $\begin{array}{l}\text { Engr. } \\
\text { Strain } \\
\end{array}$ \\
\hline $\begin{array}{l}196.500 \\
234.030 \\
364.040 \\
416.570 \\
452.570\end{array}$ & $\begin{array}{l}0.0023 \\
0.0029 \\
0.0064 \\
0.0089 \\
0.0120\end{array}$ & $\begin{array}{l}476.870 \\
497.610 \\
542.590 \\
592.130 \\
636.250 \\
683.480\end{array}$ & $\begin{array}{l}0.0145 \\
0.0192 \\
0.0308 \\
0.0526 \\
0.0761 \\
0.1051\end{array}$ & $\begin{array}{l}712.470 \\
736.930 \\
761.290 \\
774.830 \\
777.060 \\
777.060\end{array}$ & $\begin{array}{l}0.1307 \\
0.1608 \\
0.2061 \\
0.2507 \\
0.2910 \\
0.3241\end{array}$ \\
\hline
\end{tabular}

Table 345. Test data for specimen 752-22

\begin{tabular}{|c|c|c|c|c|c|}
\hline $\begin{array}{l}\text { Test Number } \\
\text { Aging Temp. } \\
\text { Yield Stress }\end{array}$ & $\begin{array}{l}: 1 \\
: 40 \\
: 36\end{array}$ & $\mathrm{~Pa}$ & $\begin{array}{l}\text { Tes } \\
\text { Agi } \\
\text { Ulti }\end{array}$ & $\begin{array}{l}\text { ne. } \\
\text { ne } \\
\text { Stress }\end{array}$ & $\begin{array}{l}25^{\circ} \mathrm{C} \\
10,000 \mathrm{~h} \\
749.1 \mathrm{MPa}\end{array}$ \\
\hline $\begin{array}{l}\text { Engr. } \\
\text { Stress } \\
\text { (MPa) }\end{array}$ & $\begin{array}{l}\text { Engr. } \\
\text { Strain }\end{array}$ & $\begin{array}{l}\text { Engr. } \\
\text { Stress } \\
\text { (MPa) }\end{array}$ & $\begin{array}{l}\text { Engr. } \\
\text { Strain }\end{array}$ & $\begin{array}{l}\text { Engr. } \\
\text { Stress } \\
\text { (MPa) }\end{array}$ & $\begin{array}{l}\text { Engr. } \\
\text { Strain }\end{array}$ \\
\hline $\begin{array}{r}48.437 \\
136.060 \\
170.050 \\
218.750 \\
218.750 \\
260.200\end{array}$ & $\begin{array}{l}0.0001 \\
0.0010 \\
0.0014 \\
0.0022 \\
0.0023 \\
0.0025\end{array}$ & $\begin{array}{l}457.530 \\
476.050 \\
496.530 \\
527.000 \\
585.350 \\
634.610\end{array}$ & $\begin{array}{l}0.0062 \\
0.0065 \\
0.0081 \\
0.0091 \\
0.0315 \\
0.0630\end{array}$ & $\begin{array}{l}673.900 \\
705.860 \\
726.020 \\
742.570 \\
748.660 \\
748.960 \\
749.070\end{array}$ & $\begin{array}{l}0.0911 \\
0.1202 \\
0.1470 \\
0.1859 \\
0.2244 \\
0.2250 \\
0.2516\end{array}$ \\
\hline
\end{tabular}


Table 346. Test data for specimen 752-23

\begin{tabular}{|c|c|c|c|c|c|}
\hline $\begin{array}{l}\text { Test Number } \\
\text { Aging Temp. } \\
\text { Yield Stress }\end{array}$ & $\begin{array}{l}: 2 \\
: 40 \\
: 33\end{array}$ & & $\begin{array}{l}\text { Tes } \\
\text { Agir } \\
\text { Ulti }\end{array}$ & $\begin{array}{l}\mathrm{np} . \\
\text { me } \\
\text { Stress }\end{array}$ & $\begin{array}{l}25^{\circ} \mathrm{C} \\
10,000 \mathrm{~h} \\
726.6 \mathrm{MPa}\end{array}$ \\
\hline $\begin{array}{l}\text { Engr. } \\
\text { Stress } \\
\text { (MPa) } \\
\end{array}$ & $\begin{array}{c}\text { Engr. } \\
\text { Strain }\end{array}$ & $\begin{array}{l}\text { Engr. } \\
\text { Stress } \\
\text { (MPa) } \\
\end{array}$ & $\begin{array}{l}\text { Engr. } \\
\text { Strain } \\
\end{array}$ & $\begin{array}{l}\text { Engr. } \\
\text { Stress } \\
\text { (MPa) } \\
\end{array}$ & $\begin{array}{l}\text { Engr. } \\
\text { Strain }\end{array}$ \\
\hline 22.240 & 0.0001 & 201.840 & 0.0011 & 407.290 & 0.0070 \\
\hline 34.880 & 0.0002 & 214.420 & 0.0013 & 634.090 & 0.0772 \\
\hline 51.080 & 0.0003 & 271.880 & 0.0020 & 646.910 & 0.0855 \\
\hline 76.780 & 0.0004 & 291.410 & 0.0024 & 672.620 & 0.1043 \\
\hline 88.920 & 0.0005 & 318.200 & 0.0031 & 682.600 & 0.1128 \\
\hline 100.790 & 0.0005 & 334.380 & 0.0036 & 706.530 & 0.1391 \\
\hline 112.790 & 0.0006 & 342.270 & 0.0039 & 708.140 & 0.1413 \\
\hline 137.610 & 0.0007 & 349.800 & 0.0042 & 714.060 & 0.1505 \\
\hline 150.940 & 0.0008 & 390.310 & 0.0060 & 722.490 & 0.1687 \\
\hline 163.660 & 0.0009 & 396.200 & 0.0063 & 724.850 & 0.1775 \\
\hline 176.280 & 0.0010 & 401.980 & 0.0067 & 725.730 & 0.1816 \\
\hline
\end{tabular}

Table 347. Test data for specimen 75-109

\begin{tabular}{|c|c|c|c|c|c|}
\hline $\begin{array}{l}\text { Test Number } \\
\text { Aging Temp. } \\
\text { Yield Stress }\end{array}$ & $\begin{array}{l}: 1 \\
: 40 \\
: 35\end{array}$ & $\mathrm{~Pa}$ & $\begin{array}{l}\text { Tes } \\
\text { Agi } \\
\text { Ulti }\end{array}$ & $\begin{array}{l}\text { np. } \\
\text { ne } \\
\text { Stress }\end{array}$ & $\begin{array}{l}: 25^{\circ} \mathrm{C} \\
: 10,000 \mathrm{~h} \\
: 736.8 \mathrm{MPa}\end{array}$ \\
\hline $\begin{array}{l}\text { Engr. } \\
\text { Stress } \\
\text { (MPa) }\end{array}$ & $\begin{array}{l}\text { Engr. } \\
\text { Strain }\end{array}$ & $\begin{array}{l}\text { Engr. } \\
\text { Stress } \\
\text { (MPa) }\end{array}$ & $\begin{array}{l}\text { Engr. } \\
\text { Strain }\end{array}$ & $\begin{array}{l}\text { Engr. } \\
\text { Stress } \\
(\mathrm{MPa}) \\
\end{array}$ & $\begin{array}{l}\text { Engr. } \\
\text { Strain }\end{array}$ \\
\hline $\begin{array}{r}48.892 \\
199.300 \\
219.940 \\
348.950 \\
403.120 \\
428.950 \\
452.430\end{array}$ & $\begin{array}{l}0.0012 \\
0.0051 \\
0.0054 \\
0.0102 \\
0.0139 \\
0.0168 \\
0.0204\end{array}$ & $\begin{array}{l}472.220 \\
478.010 \\
489.830 \\
520.200 \\
551.920 \\
552.110 \\
600.380\end{array}$ & $\begin{array}{l}0.0247 \\
0.0261 \\
0.0315 \\
0.0433 \\
0.0594 \\
0.0591 \\
0.0858\end{array}$ & $\begin{array}{l}643.790 \\
676.920 \\
702.560 \\
719.440 \\
730.490 \\
736.790 \\
736.850\end{array}$ & $\begin{array}{l}0.1140 \\
0.1447 \\
0.1779 \\
0.2148 \\
0.2542 \\
0.2928 \\
0.3318\end{array}$ \\
\hline
\end{tabular}


Table 348. Test data for specimen 751-04

\begin{tabular}{|c|c|c|c|c|c|}
\hline $\begin{array}{l}\text { Test Number } \\
\text { Aging Temp. } \\
\text { Yield Stress }\end{array}$ & $\begin{array}{l}: 0 \\
: 45 \\
: 31\end{array}$ & & $\begin{array}{l}\text { Tes } \\
\text { Agir } \\
\text { Ulti }\end{array}$ & $\begin{array}{l}\text { mp. } \\
\text { me } \\
\text { Stress }\end{array}$ & $\begin{array}{l}25^{\circ} \mathrm{C} \\
2,570 \mathrm{~h} \\
721.2 \mathrm{MPa}\end{array}$ \\
\hline $\begin{array}{l}\text { Engr. } \\
\text { Stress } \\
\text { (MPa) } \\
\end{array}$ & $\begin{array}{l}\text { Engr. } \\
\text { Strain }\end{array}$ & $\begin{array}{l}\text { Engr. } \\
\text { Stress } \\
(\mathrm{MPa}) \\
\end{array}$ & $\begin{array}{l}\text { Engr. } \\
\text { Strain }\end{array}$ & $\begin{array}{l}\text { Engr. } \\
\text { Stress } \\
\text { (MPa) } \\
\end{array}$ & $\begin{array}{l}\text { Engr. } \\
\text { Strain }\end{array}$ \\
\hline 48.54 & 0.0000 & 408.53 & 0.0077 & 606.25 & 0.0714 \\
\hline 90.43 & 0.0002 & 425.74 & 0.0089 & 630.29 & 0.0880 \\
\hline 134.83 & 0.0005 & 443.64 & 0.0105 & 650.99 & 0.1013 \\
\hline 179.28 & 0.0010 & 459.28 & 0.0123 & 666.13 & 0.1154 \\
\hline 201.61 & 0.0014 & 473.69 & 0.0144 & 681.92 & 0.1326 \\
\hline 218.76 & 0.0016 & 480.11 & 0.0155 & 695.37 & 0.1515 \\
\hline 226.90 & 0.0017 & 489.23 & 0.0181 & 704.74 & 0.1687 \\
\hline 262.63 & 0.0023 & 508.22 & 0.0229 & 712.42 & 0.1847 \\
\hline 302.11 & 0.0032 & 537.36 & 0.0333 & 717.21 & 0.1999 \\
\hline 341.04 & 0.0045 & 560.99 & 0.0451 & 720.61 & 0.2197 \\
\hline 382.40 & 0.0063 & 582.85 & 0.0569 & 721.16 & 0.2289 \\
\hline
\end{tabular}

Table 349. Test data for specimen 751-05

\begin{tabular}{|c|c|c|c|c|c|}
\hline $\begin{array}{l}\text { Test Number } \\
\text { Aging Temp. } \\
\text { Yield Stress }\end{array}$ & $\begin{array}{l}: 0 \\
: 45 \\
: 31\end{array}$ & & $\begin{array}{l}\text { Tes } \\
\text { Agi } \\
\text { Ulti }\end{array}$ & $\begin{array}{l}\text { np. } \\
\text { ne } \\
\text { Stress }\end{array}$ & $\begin{array}{l}25^{\circ} \mathrm{C} \\
2,570 \mathrm{~h} \\
746.4 \mathrm{MPa}\end{array}$ \\
\hline $\begin{array}{l}\text { Engr. } \\
\text { Stress } \\
\text { (MPa) }\end{array}$ & $\begin{array}{l}\text { Engr. } \\
\text { Strain }\end{array}$ & $\begin{array}{l}\text { Engr. } \\
\text { Stress } \\
(\mathrm{MPa})\end{array}$ & $\begin{array}{l}\text { Engr. } \\
\text { Strain }\end{array}$ & $\begin{array}{l}\text { Engr. } \\
\text { Stress } \\
(\mathrm{MPa}) \\
\end{array}$ & $\begin{array}{l}\text { Engr. } \\
\text { Strain }\end{array}$ \\
\hline 69.62 & 0.0001 & 444.52 & 0.0099 & 618.84 & 0.0725 \\
\hline 107.39 & 0.0003 & 461.24 & 0.0115 & 644.55 & 0.0900 \\
\hline 147.01 & 0.0006 & 476.58 & 0.0133 & 665.59 & 0.1043 \\
\hline 189.06 & 0.0012 & 485.24 & 0.0147 & 682.15 & 0.1204 \\
\hline 205.23 & 0.0014 & 491.81 & 0.0160 & 707.31 & 0.1507 \\
\hline 230.62 & 0.0017 & 505.54 & 0.0191 & 716.41 & 0.1659 \\
\hline 253.21 & 0.0020 & 514.49 & 0.0214 & 728.36 & 0.1919 \\
\hline 292.87 & 0.0027 & 525.23 & 0.0245 & 734.97 & 0.2082 \\
\hline 338.76 & 0.0042 & 540.84 & 0.0298 & 739.68 & 0.2245 \\
\hline 384.84 & 0.0059 & 560.52 & 0.0386 & 743.29 & 0.2445 \\
\hline 393.36 & 0.0063 & 580.00 & 0.0488 & 745.32 & 0.2634 \\
\hline 422.32 & 0.0080 & 598.87 & 0.0595 & 746.35 & 0.2819 \\
\hline 433.57 & 0.0089 & & & & \\
\hline
\end{tabular}


Table 350. Test data for specimen 754-40

\begin{tabular}{|c|c|c|c|c|c|}
\hline $\begin{array}{l}\text { Test Number } \\
\text { Aging Temp. } \\
\text { Yield Stress }\end{array}$ & $\begin{array}{l}: 0 \\
: U r \\
: 15\end{array}$ & & $\begin{array}{l}\text { Tes } \\
\text { Agi } \\
\text { Ulti }\end{array}$ & $\begin{array}{l}\text { mp. } \\
\text { me } \\
\text { Stress }\end{array}$ & $\begin{array}{l}290^{\circ} \mathrm{C} \\
-470.8 \mathrm{MPa}\end{array}$ \\
\hline $\begin{array}{l}\text { Engr. } \\
\text { Stress } \\
(\mathrm{MPa})\end{array}$ & $\begin{array}{l}\text { Engr. } \\
\text { Strain }\end{array}$ & $\begin{array}{l}\text { Engr. } \\
\text { Stress } \\
(\mathrm{MPa})\end{array}$ & $\begin{array}{l}\text { Engr. } \\
\text { Strain }\end{array}$ & $\begin{array}{l}\text { Engr. } \\
\text { Stress } \\
(\mathrm{MPa})\end{array}$ & $\begin{array}{l}\text { Engr. } \\
\text { Strain }\end{array}$ \\
\hline 46.18 & 0.0000 & 232.42 & 0.0037 & 321.07 & 0.0442 \\
\hline 89.11 & 0.0003 & 235.69 & 0.0040 & 341.04 & 0.0590 \\
\hline 123.96 & 0.0006 & 238.81 & 0.0045 & 358.98 & 0.0725 \\
\hline 145.21 & 0.0010 & 241.44 & 0.0049 & 378.49 & 0.0875 \\
\hline 168.03 & 0.0015 & 244.76 & 0.0056 & 388.33 & 0.0953 \\
\hline 181.49 & 0.0018 & 248.03 & 0.0065 & 401.73 & 0.1046 \\
\hline 186.97 & 0.0020 & 251.20 & 0.0074 & 416.71 & 0.1194 \\
\hline 191.35 & 0.0022 & 255.12 & 0.0087 & 431.07 & 0.1357 \\
\hline 194.07 & 0.0024 & 259.39 & 0.0103 & 440.38 & 0.1474 \\
\hline 200.63 & 0.0026 & 267.13 & 0.0130 & 450.70 & 0.1642 \\
\hline 209.03 & 0.0028 & 271.85 & 0.0150 & 458.00 & 0.1774 \\
\hline 214.22 & 0.0030 & 277.26 & 0.0177 & 463.66 & 0.1900 \\
\hline 220.40 & 0.0032 & 282.13 & 0.0203 & 468.28 & 0.1987 \\
\hline 226.04 & 0.0034 & 293.96 & 0.0268 & 469.36 & 0.2018 \\
\hline 228.27 & 0.0035 & 308.43 & 0.0357 & 470.76 & 0.2125 \\
\hline
\end{tabular}

Table 351. Test data for specimen 753-42

\begin{tabular}{|c|c|c|c|c|c|}
\hline $\begin{array}{l}\text { Test Number } \\
\text { Aging Temp. } \\
\text { Yield Stress }\end{array}$ & $\begin{array}{l}: 0 \\
: \text { Ur } \\
: 1 S\end{array}$ & & $\begin{array}{l}\text { Tes } \\
\text { Agi } \\
\text { Ulti }\end{array}$ & $\begin{array}{l}\text { np. } \\
\text { ne } \\
\text { Stress }\end{array}$ & $\begin{array}{l}290^{\circ} \mathrm{C} \\
- \\
474.8 \mathrm{MPa}\end{array}$ \\
\hline $\begin{array}{l}\text { Engr. } \\
\text { Stress } \\
(\mathrm{MPa}) \\
\end{array}$ & $\begin{array}{l}\text { Engr. } \\
\text { Strain }\end{array}$ & $\begin{array}{l}\text { Engr. } \\
\text { Stress } \\
\text { (MPa) }\end{array}$ & $\begin{array}{l}\text { Engr. } \\
\text { Strain }\end{array}$ & $\begin{array}{l}\text { Engr. } \\
\text { Stress } \\
(\mathrm{MPa}) \\
\end{array}$ & $\begin{array}{l}\text { Engr. } \\
\text { Strain }\end{array}$ \\
\hline 44.3440 & 0.00 & 222.08 & 0.0034 & 398.27 & 0.1090 \\
\hline 83.0220 & 0.0002 & 230.60 & 0.0039 & 406.65 & 0.1179 \\
\hline 116.82 & 0.0005 & 236.84 & 0.0045 & 412.76 & 0.1236 \\
\hline 134.36 & 0.0007 & 241.51 & 0.0055 & 417.75 & 0.1304 \\
\hline 149.20 & 0.0009 & 246.82 & 0.0071 & 429.08 & 0.1449 \\
\hline 157.04 & 0.0011 & 257.83 & 0.0110 & 435.04 & 0.1534 \\
\hline 167.23 & 0.0013 & 267.57 & 0.0153 & 448.85 & 0.1758 \\
\hline 175.47 & 0.0015 & 275.63 & 0.0197 & 457.06 & 0.1903 \\
\hline 181.93 & 0.0017 & 285.57 & 0.0256 & 461.73 & 0.2050 \\
\hline 188.24 & 0.0019 & 303.24 & 0.0371 & 464.70 & 0.2151 \\
\hline 191.40 & 0.0021 & 323.45 & 0.0513 & 469.08 & 0.2283 \\
\hline 195.44 & 0.0023 & 339.98 & 0.0633 & 473.01 & 0.2433 \\
\hline 200.38 & 0.0026 & 353.00 & 0.0738 & 474.09 & 0.2465 \\
\hline 208.54 & 0.0029 & 363.78 & 0.0821 & 472.18 & 0.2507 \\
\hline 217.28 & 0.0032 & 375.41 & 0.0914 & 472.42 & 0.2565 \\
\hline
\end{tabular}


Table 352. Test data for specimen 754-29

\begin{tabular}{|c|c|c|c|c|c|}
\hline $\begin{array}{l}\text { Test Number } \\
\text { Aging Temp. } \\
\text { Yield Stress }\end{array}$ & $\begin{array}{l}: 2 \\
: 29 \\
: 19\end{array}$ & & $\begin{array}{l}\text { Tes } \\
\text { Agi } \\
\text { Ulti }\end{array}$ & $\begin{array}{l}\text { np. } \\
\text { me } \\
\text { Stress }\end{array}$ & $\begin{array}{l}290^{\circ} \mathrm{C} \\
30,000 \mathrm{~h} \\
528.3 \mathrm{MPa}\end{array}$ \\
\hline $\begin{array}{l}\text { Engr. } \\
\text { Stress } \\
\text { (MPa) }\end{array}$ & $\begin{array}{l}\text { Engr. } \\
\text { Strain }\end{array}$ & $\begin{array}{l}\text { Engr. } \\
\text { Stress } \\
\text { (MPa) }\end{array}$ & $\begin{array}{l}\text { Engr. } \\
\text { Strain }\end{array}$ & $\begin{array}{l}\text { Engr. } \\
\text { Stress } \\
(\mathrm{MPa})\end{array}$ & $\begin{array}{l}\text { Engr. } \\
\text { Strain }\end{array}$ \\
\hline 36.412 & 0.0002 & 179.423 & 0.0025 & 441.862 & 0.1347 \\
\hline 41.148 & 0.0002 & 210.220 & 0.0040 & 451.291 & 0.1433 \\
\hline 57.528 & 0.0002 & 249.736 & 0.0243 & 466.427 & 0.1593 \\
\hline 70.250 & 0.0002 & 274.563 & 0.0351 & 468.035 & 0.1617 \\
\hline 90.632 & 0.0004 & 300.745 & 0.0478 & 476.025 & 0.1705 \\
\hline 100.911 & 0.0004 & 305.263 & 0.0503 & 489.641 & 0.1880 \\
\hline 111.112 & 0.0005 & 337.575 & 0.0668 & 499.259 & 0.2017 \\
\hline 121.536 & 0.0006 & 375.916 & 0.0883 & 505.088 & 0.2109 \\
\hline 130.814 & 0.0007 & 379.132 & 0.0904 & 514.852 & 0.2408 \\
\hline 148.456 & 0.0012 & 407.233 & 0.1086 & 521.458 & 0.2536 \\
\hline 156.695 & 0.0013 & 421.315 & 0.1174 & 528.307 & 0.2792 \\
\hline
\end{tabular}

Table 353. Test data for specimen 754-30

\begin{tabular}{|c|c|c|c|c|c|}
\hline $\begin{array}{l}\text { Test Number } \\
\text { Aging Temp. } \\
\text { Yield Stress }\end{array}$ & $\begin{array}{l}: 2 \\
: 2 c \\
: 2 C\end{array}$ & & $\begin{array}{l}\text { Tes } \\
\text { Agi } \\
\text { Ulti }\end{array}$ & $\begin{array}{l}\text { mp. } \\
\text { me } \\
\text { Stress }\end{array}$ & $\begin{array}{l}290^{\circ} \mathrm{C} \\
30,000 \mathrm{~h} \\
495.3 \mathrm{MPa}\end{array}$ \\
\hline $\begin{array}{l}\text { Engr. } \\
\text { Stress } \\
\text { (MPa) }\end{array}$ & $\begin{array}{l}\text { Engr. } \\
\text { Strain }\end{array}$ & $\begin{array}{l}\text { Engr. } \\
\text { Stress } \\
\text { (MPa) } \\
\end{array}$ & $\begin{array}{l}\text { Engr. } \\
\text { Strain }\end{array}$ & $\begin{array}{l}\text { Engr. } \\
\text { Stress } \\
(\mathrm{MPa}) \\
\end{array}$ & $\begin{array}{l}\text { Engr. } \\
\text { Strain }\end{array}$ \\
\hline 45.680 & 0.0003 & 223.970 & 0.0065 & 437.350 & 0.1377 \\
\hline 51.210 & 0.0003 & 239.970 & 0.0109 & 457.490 & 0.1616 \\
\hline 77.100 & 0.0003 & 246.020 & 0.0132 & 469.940 & 0.1789 \\
\hline 87.660 & 0.0003 & 266.990 & 0.0223 & 470.880 & 0.1812 \\
\hline 131.440 & 0.0005 & 288.840 & 0.0333 & 478.480 & 0.1979 \\
\hline 159.370 & 0.0008 & 317.470 & 0.0486 & 480.510 & 0.2001 \\
\hline 167.100 & 0.0011 & 354.290 & 0.0707 & 483.830 & 0.2023 \\
\hline 186.600 & 0.0017 & 381.810 & 0.0883 & 489.030 & 0.2185 \\
\hline 191.790 & 0.0020 & 394.310 & 0.0971 & 490.780 & 0.2207 \\
\hline 211.650 & 0.0039 & 404.210 & 0.1068 & 494.300 & 0.2384 \\
\hline 218.380 & 0.0050 & 425.420 & 0.1247 & 495.230 & 0.2454 \\
\hline
\end{tabular}


Table 354. Test data for specimen 75-236

\begin{tabular}{|c|c|c|c|c|c|}
\hline $\begin{array}{l}\text { Test Number } \\
\text { Aging Temp. } \\
\text { Yield Stress }\end{array}$ & $\begin{array}{l}: 25 \\
: 29 \\
: 21\end{array}$ & & $\begin{array}{l}\text { Tes } \\
\text { Agir } \\
\text { Ultir }\end{array}$ & $\begin{array}{l}\text { np. } \\
\text { ne } \\
\text { Stress }\end{array}$ & $\begin{array}{l}290^{\circ} \mathrm{C} \\
30,000 \mathrm{~h} \\
486.2 \mathrm{MPa}\end{array}$ \\
\hline $\begin{array}{l}\text { Engr. } \\
\text { Stress } \\
(\mathrm{MPa})\end{array}$ & $\begin{array}{l}\text { Engr. } \\
\text { Strain } \\
\end{array}$ & $\begin{array}{l}\text { Engr. } \\
\text { Stress } \\
(\mathrm{MPa})\end{array}$ & $\begin{array}{l}\text { Engr. } \\
\text { Strain }\end{array}$ & $\begin{array}{l}\text { Engr. } \\
\text { Stress } \\
(\mathrm{MPa}) \\
\end{array}$ & $\begin{array}{l}\text { Engr. } \\
\text { Strain }\end{array}$ \\
\hline 36.892 & 0.0002 & 255.878 & 0.0141 & 361.326 & 0.0738 \\
\hline 37.892 & 0.0002 & 261.540 & 0.0166 & 382.851 & 0.0889 \\
\hline 94.768 & 0.0006 & 289.771 & 0.0303 & 404.074 & 0.1067 \\
\hline 105.428 & 0.0007 & 294.023 & 0.0325 & 421.601 & 0.1244 \\
\hline 166.972 & 0.0018 & 298.105 & 0.0347 & 424.542 & 0.1268 \\
\hline 175.525 & 0.0020 & 317.777 & 0.0457 & 437.309 & 0.1402 \\
\hline 183.733 & 0.0023 & 321.576 & 0.0480 & 450.461 & 0.1679 \\
\hline 217.030 & 0.0043 & 335.938 & 0.0567 & 454.177 & 0.1704 \\
\hline 223.160 & 0.0052 & 339.600 & 0.0588 & 467.364 & 0.1903 \\
\hline 228.276 & 0.0061 & 343.106 & 0.0609 & 477.248 & 0.2153 \\
\hline 251.084 & 0.0121 & 359.107 & 0.0716 & 484.505 & 0.2478 \\
\hline
\end{tabular}

Table 355. Test data for specimen 754-26

\begin{tabular}{|c|c|c|c|c|c|}
\hline $\begin{array}{l}\text { Test Number } \\
\text { Aging Temp. } \\
\text { Yield Stress }\end{array}$ & & $\mathrm{MPa}$ & $\begin{array}{l}\text { Tes } \\
\text { Agi } \\
\text { Ulti }\end{array}$ & $\begin{array}{l}\text { mp. } \\
\text { me } \\
\text { Stress }\end{array}$ & $\begin{array}{l}290^{\circ} \mathrm{C} \\
10,000 \mathrm{~h} \\
538.0 \mathrm{MPa}\end{array}$ \\
\hline $\begin{array}{l}\text { Engr. } \\
\text { Stress } \\
(\mathrm{MPa})\end{array}$ & $\begin{array}{l}\text { Engr. } \\
\text { Strain }\end{array}$ & $\begin{array}{l}\text { Engr. } \\
\text { Stress } \\
(\mathrm{MPa})\end{array}$ & $\begin{array}{l}\text { Engr. } \\
\text { Strain }\end{array}$ & $\begin{array}{l}\text { Engr. } \\
\text { Stress } \\
(\mathrm{MPa}) \\
\end{array}$ & $\begin{array}{l}\text { Engr. } \\
\text { Strain }\end{array}$ \\
\hline 162.940 & 0.0011 & 288.440 & 0.0100 & 496.920 & 0.1370 \\
\hline 170.480 & 0.0013 & 294.330 & 0.0114 & 499.300 & 0.1401 \\
\hline 208.430 & 0.0024 & 323.730 & 0.0215 & 514.560 & 0.1594 \\
\hline 213.960 & 0.0025 & 347.960 & 0.0328 & 519.350 & 0.1694 \\
\hline 219.180 & 0.0026 & 366.250 & 0.0426 & 520.010 & 0.1712 \\
\hline 225.250 & 0.0027 & 397.430 & 0.0605 & 524.370 & 0.1805 \\
\hline 234.270 & 0.0028 & 411.880 & 0.0697 & 527.510 & 0.1911 \\
\hline 265.920 & 0.0055 & 427.730 & 0.0796 & 531.100 & 0.1985 \\
\hline 272.200 & 0.0065 & 431.270 & 0.0820 & 532.860 & 0.2008 \\
\hline 278.730 & 0.0076 & 465.900 & 0.1042 & 533.870 & 0.2045 \\
\hline 283.340 & 0.0087 & 482.360 & 0.1211 & 538.030 & 0.2252 \\
\hline
\end{tabular}


Table 356. Test data for specimen 754-27

\begin{tabular}{|c|c|c|c|c|c|}
\hline $\begin{array}{l}\text { Test Number } \\
\text { Aging Temp. } \\
\text { Yield Stress }\end{array}$ & $\begin{array}{l}: 0 \\
: 32 \\
: 21\end{array}$ & & $\begin{array}{l}\text { Tes } \\
\text { Agi } \\
\text { Ulti }\end{array}$ & $\begin{array}{l}\text { p. } \\
\text { ne } \\
\text { Stress }\end{array}$ & $\begin{array}{l}290^{\circ} \mathrm{C} \\
10,000 \mathrm{~h} \\
534.3 \mathrm{MPa}\end{array}$ \\
\hline $\begin{array}{l}\text { Engr. } \\
\text { Stress } \\
\text { (MPa) }\end{array}$ & $\begin{array}{l}\text { Engr. } \\
\text { Strain }\end{array}$ & $\begin{array}{l}\text { Engr. } \\
\text { Stress } \\
(\mathrm{MPa}) \\
\end{array}$ & $\begin{array}{l}\text { Engr. } \\
\text { Strain }\end{array}$ & $\begin{array}{l}\text { Engr. } \\
\text { Stress } \\
\text { (MPa) }\end{array}$ & $\begin{array}{l}\text { Engr. } \\
\text { Strain }\end{array}$ \\
\hline $\begin{array}{l}177.540 \\
183.830 \\
188.850 \\
194.850 \\
200.550 \\
221.550 \\
226.910 \\
235.910 \\
245.150 \\
262.310 \\
266.490\end{array}$ & $\begin{array}{l}0.0016 \\
0.0018 \\
0.0019 \\
0.0021 \\
0.0023 \\
0.0027 \\
0.0027 \\
0.0029 \\
0.0034 \\
0.0047 \\
0.0053\end{array}$ & $\begin{array}{l}286.010 \\
321.270 \\
324.520 \\
337.700 \\
341.680 \\
356.970 \\
360.900 \\
364.890 \\
387.120 \\
409.150 \\
413.040\end{array}$ & $\begin{array}{l}0.0088 \\
0.0196 \\
0.0210 \\
0.0281 \\
0.0300 \\
0.0381 \\
0.0402 \\
0.0425 \\
0.0555 \\
0.0698 \\
0.0723\end{array}$ & $\begin{array}{l}424.200 \\
431.780 \\
444.420 \\
467.830 \\
471.420 \\
483.540 \\
492.980 \\
494.170 \\
502.840 \\
509.540 \\
521.010\end{array}$ & $\begin{array}{l}0.0791 \\
0.0848 \\
0.0932 \\
0.1091 \\
0.1126 \\
0.1269 \\
0.1391 \\
0.1410 \\
0.1533 \\
0.1658 \\
0.1840\end{array}$ \\
\hline
\end{tabular}

Table 357. Test data for specimen 752-40

\begin{tabular}{|c|c|c|c|c|c|}
\hline $\begin{array}{l}\text { Test Number } \\
\text { Aging Temp. } \\
\text { Yield Stress }\end{array}$ & & $\mathrm{Pa}$ & $\begin{array}{l}\text { Tes } \\
\text { Agir } \\
\text { Ultir }\end{array}$ & $\begin{array}{l}\text { p. } \\
\text { ne } \\
\text { Stress }\end{array}$ & $\begin{array}{l}290^{\circ} \mathrm{C} \\
30,000 \mathrm{~h} \\
589.3 \mathrm{MPa}\end{array}$ \\
\hline $\begin{array}{l}\text { Engr. } \\
\text { Stress } \\
\text { (MPa) }\end{array}$ & $\begin{array}{l}\text { Engr. } \\
\text { Strain }\end{array}$ & $\begin{array}{l}\text { Engr. } \\
\text { Stress } \\
\text { (MPa) }\end{array}$ & $\begin{array}{l}\text { Engr. } \\
\text { Strain }\end{array}$ & $\begin{array}{l}\text { Engr. } \\
\text { Stress } \\
\text { (MPa) }\end{array}$ & $\begin{array}{l}\text { Engr. } \\
\text { Strain }\end{array}$ \\
\hline $\begin{array}{r}93.421 \\
166.240 \\
213.040 \\
245.550 \\
265.980 \\
284.890 \\
300.400 \\
313.740\end{array}$ & $\begin{array}{l}0.0002 \\
0.0010 \\
0.0021 \\
0.0034 \\
0.0046 \\
0.0060 \\
0.0081 \\
0.0102\end{array}$ & $\begin{array}{l}326.590 \\
339.400 \\
352.160 \\
361.640 \\
371.940 \\
390.000 \\
408.280 \\
420.580\end{array}$ & $\begin{array}{l}0.0128 \\
0.0162 \\
0.0199 \\
0.0232 \\
0.0271 \\
0.0347 \\
0.0434 \\
0.0495\end{array}$ & $\begin{array}{l}475.750 \\
492.070 \\
516.690 \\
543.400 \\
559.930 \\
574.500 \\
583.160 \\
588.990\end{array}$ & $\begin{array}{l}0.0805 \\
0.0906 \\
0.1042 \\
0.1291 \\
0.1493 \\
0.1689 \\
0.1895 \\
0.2076\end{array}$ \\
\hline
\end{tabular}


Table 358. Test data for specimen 752-42

\begin{tabular}{|c|c|c|c|c|c|}
\hline $\begin{array}{l}\text { Test Number } \\
\text { Aging Temp. } \\
\text { Yield Stress }\end{array}$ & $\begin{array}{l}: 1 \\
: 32 \\
: 26\end{array}$ & & $\begin{array}{l}\text { Tes } \\
\text { Agi } \\
\text { Ulti }\end{array}$ & $\begin{array}{l}\text { ne. } \\
\text { Stress }\end{array}$ & $\begin{array}{l}290^{\circ} \mathrm{C} \\
30,000 \mathrm{~h} \\
575.8 \mathrm{MPa}\end{array}$ \\
\hline $\begin{array}{l}\text { Engr. } \\
\text { Stress } \\
\text { (MPa) }\end{array}$ & $\begin{array}{l}\text { Engr. } \\
\text { Strain }\end{array}$ & $\begin{array}{l}\text { Engr. } \\
\text { Stress } \\
(\mathrm{MPa}) \\
\end{array}$ & $\begin{array}{l}\text { Engr. } \\
\text { Strain }\end{array}$ & $\begin{array}{l}\text { Engr. } \\
\text { Stress } \\
\text { (MPa) }\end{array}$ & $\begin{array}{l}\text { Engr. } \\
\text { Strain }\end{array}$ \\
\hline $\begin{array}{l}230.660 \\
265.350 \\
284.650 \\
297.110 \\
311.170 \\
322.820 \\
334.870 \\
348.320\end{array}$ & $\begin{array}{l}0.0030 \\
0.0048 \\
0.0062 \\
0.0078 \\
0.0098 \\
0.0120 \\
0.0150 \\
0.0189\end{array}$ & $\begin{array}{l}369.990 \\
381.830 \\
404.550 \\
430.020 \\
459.230 \\
476.450 \\
494.220 \\
507.540\end{array}$ & $\begin{array}{l}0.0264 \\
0.0310 \\
0.0411 \\
0.0540 \\
0.0703 \\
0.0810 \\
0.0927 \\
0.0988\end{array}$ & $\begin{array}{l}518.740 \\
532.150 \\
543.340 \\
552.940 \\
562.120 \\
569.480 \\
573.540 \\
575.810\end{array}$ & $\begin{array}{l}0.1080 \\
0.1200 \\
0.1316 \\
0.1419 \\
0.1553 \\
0.1705 \\
0.1820 \\
0.1904\end{array}$ \\
\hline
\end{tabular}

Table 359. Test data for specimen 75-246

\begin{tabular}{|c|c|c|c|c|c|}
\hline $\begin{array}{l}\text { Test Number } \\
\text { Aging Temp. } \\
\text { Yield Stress }\end{array}$ & $\begin{array}{l}: 1 \\
: 32 \\
: 21\end{array}$ & & $\begin{array}{l}\text { Tes } \\
\text { Agi } \\
\text { Ulti }\end{array}$ & $\begin{array}{l}\text { np. } \\
\text { me } \\
\text { Stress }\end{array}$ & $\begin{array}{l}290^{\circ} \mathrm{C} \\
30,000 \mathrm{~h} \\
521.8 \mathrm{MPa}\end{array}$ \\
\hline $\begin{array}{l}\text { Engr. } \\
\text { Stress } \\
\text { (MPa) }\end{array}$ & $\begin{array}{l}\text { Engr. } \\
\text { Strain }\end{array}$ & $\begin{array}{l}\text { Engr. } \\
\text { Stress } \\
\text { (MPa) }\end{array}$ & $\begin{array}{l}\text { Engr. } \\
\text { Strain }\end{array}$ & $\begin{array}{l}\text { Engr. } \\
\text { Stress } \\
(\mathrm{MPa})\end{array}$ & $\begin{array}{l}\text { Engr. } \\
\text { Strain }\end{array}$ \\
\hline 46.497 & 0.0000 & 258.800 & 0.0065 & 421.490 & 0.0760 \\
\hline 77.711 & 0.0002 & 266.280 & 0.0075 & 427.680 & 0.0800 \\
\hline 132.720 & 0.0009 & 285.060 & 0.0110 & 451.050 & 0.0965 \\
\hline 155.130 & 0.0013 & 291.140 & 0.0126 & 460.800 & 0.1019 \\
\hline 166.430 & 0.0015 & 313.720 & 0.0192 & 475.560 & 0.1176 \\
\hline 189.580 & 0.0021 & 323.000 & 0.0225 & 479.520 & 0.1228 \\
\hline 203.470 & 0.0027 & 337.710 & 0.0289 & 490.390 & 0.1379 \\
\hline 212.740 & 0.0031 & 342.940 & 0.0302 & 495.670 & 0.1478 \\
\hline 222.690 & 0.0036 & 377.670 & 0.0497 & 502.420 & 0.1582 \\
\hline 229.870 & 0.0041 & 390.640 & 0.0570 & 511.320 & 0.1738 \\
\hline 238.280 & 0.0046 & 402.720 & 0.0640 & 521.810 & 0.2189 \\
\hline
\end{tabular}


Table 360. Test data for specimen 752-28

\begin{tabular}{|c|c|c|c|c|c|}
\hline $\begin{array}{l}\text { Test Number } \\
\text { Aging Temp. } \\
\text { Yield Stress }\end{array}$ & $\begin{array}{l}: 3 \\
: 32 \\
: 25\end{array}$ & & $\begin{array}{l}\text { Tes } \\
\text { Agir } \\
\text { Ulti }\end{array}$ & $\begin{array}{l}\text { mp. } \\
\text { me } \\
\text { Stress }\end{array}$ & $\begin{array}{l}290^{\circ} \mathrm{C} \\
50,000 \mathrm{~h} \\
587.9 \mathrm{MPa}\end{array}$ \\
\hline $\begin{array}{l}\text { Engr. } \\
\text { Stress } \\
(\mathrm{MPa})\end{array}$ & $\begin{array}{l}\text { Engr. } \\
\text { Strain }\end{array}$ & $\begin{array}{l}\text { Engr. } \\
\text { Stress } \\
(\mathrm{MPa})\end{array}$ & $\begin{array}{l}\text { Engr. } \\
\text { Strain }\end{array}$ & $\begin{array}{l}\text { Engr. } \\
\text { Stress } \\
\text { (MPa) }\end{array}$ & $\begin{array}{l}\text { Engr. } \\
\text { Strain }\end{array}$ \\
\hline 25.970 & 0.0001 & 298.060 & 0.0063 & 508.970 & 0.0947 \\
\hline 30.490 & 0.0001 & 314.610 & 0.0087 & 517.340 & 0.1013 \\
\hline 45.790 & 0.0002 & 321.370 & 0.0100 & 532.600 & 0.1145 \\
\hline 69.930 & 0.0002 & 348.700 & 0.0166 & 539.410 & 0.1211 \\
\hline 94.410 & 0.0002 & 354.740 & 0.0184 & 555.440 & 0.1387 \\
\hline 106.620 & 0.0003 & 371.160 & 0.0239 & 560.630 & 0.1453 \\
\hline 119.350 & 0.0003 & 408.340 & 0.0382 & 569.760 & 0.1585 \\
\hline 152.680 & 0.0005 & 413.440 & 0.0405 & 571.100 & 0.1608 \\
\hline 172.700 & 0.0006 & 458.240 & 0.0624 & 579.490 & 0.1762 \\
\hline 181.970 & 0.0007 & 487.300 & 0.0796 & 582.490 & 0.1829 \\
\hline 243.170 & 0.0023 & 490.590 & 0.0818 & 587.910 & 0.2074 \\
\hline
\end{tabular}

Table 361. Test data for specimen 752-29

\begin{tabular}{|c|c|c|c|c|c|}
\hline $\begin{array}{l}\text { Test Number } \\
\text { Aging Temp. } \\
\text { Yield Stress }\end{array}$ & $\begin{array}{l}: 3 \\
: 32 \\
: 26\end{array}$ & & $\begin{array}{l}\text { Tes } \\
\text { Agi } \\
\text { Ulti }\end{array}$ & $\begin{array}{l}\text { mp. } \\
\text { me } \\
\text { Stress }\end{array}$ & $\begin{array}{l}290^{\circ} \mathrm{C} \\
50,000 \mathrm{~h} \\
581.7 \mathrm{MPa}\end{array}$ \\
\hline $\begin{array}{l}\text { Engr. } \\
\text { Stress } \\
\text { (MPa) }\end{array}$ & $\begin{array}{l}\text { Engr. } \\
\text { Strain }\end{array}$ & $\begin{array}{l}\text { Engr. } \\
\text { Stress } \\
\text { (MPa) }\end{array}$ & $\begin{array}{l}\text { Engr. } \\
\text { Strain }\end{array}$ & $\begin{array}{l}\text { Engr. } \\
\text { Stress } \\
(\mathrm{MPa}) \\
\end{array}$ & $\begin{array}{l}\text { Engr. } \\
\text { Strain }\end{array}$ \\
\hline 10.470 & 0.0001 & 198.390 & 0.0012 & 461.350 & 0.0630 \\
\hline 15.080 & 0.0001 & 245.470 & 0.0025 & 486.660 & 0.0781 \\
\hline 28.910 & 0.0002 & 264.430 & 0.0033 & 490.150 & 0.0803 \\
\hline 40.310 & 0.0002 & 303.070 & 0.0064 & 517.200 & 0.0996 \\
\hline 52.150 & 0.0002 & 314.860 & 0.0081 & 537.160 & 0.1170 \\
\hline 86.080 & 0.0004 & 331.430 & 0.0111 & 543.650 & 0.1238 \\
\hline 98.690 & 0.0005 & 359.010 & 0.0189 & 557.110 & 0.1391 \\
\hline 123.080 & 0.0005 & 364.680 & 0.0209 & 562.080 & 0.1456 \\
\hline 169.210 & 0.0008 & 402.960 & 0.0351 & 570.670 & 0.1588 \\
\hline 179.540 & 0.0010 & 418.210 & 0.0416 & 574.430 & 0.1655 \\
\hline 189.190 & 0.0011 & 449.620 & 0.0566 & 581.620 & 0.1876 \\
\hline
\end{tabular}


Table 362. Test data for specimen 75-130

\begin{tabular}{|c|c|c|c|c|c|}
\hline $\begin{array}{l}\text { Test Number } \\
\text { Aging Temp. } \\
\text { Yield Stress }\end{array}$ & $\begin{array}{l}: 3 \\
: 32 \\
: 23\end{array}$ & $\mathrm{MPa}$ & $\begin{array}{l}\text { Tes } \\
\text { Agir } \\
\text { Ulti }\end{array}$ & $\begin{array}{l}\mathrm{np} . \\
\text { me } \\
\text { Stress }\end{array}$ & $\begin{array}{l}290^{\circ} \mathrm{C} \\
50,000 \mathrm{~h} \\
557.8 \mathrm{MPa}\end{array}$ \\
\hline $\begin{array}{l}\text { Engr. } \\
\text { Stress } \\
(\mathrm{MPa})\end{array}$ & $\begin{array}{l}\text { Engr. } \\
\text { Strain }\end{array}$ & $\begin{array}{l}\text { Engr. } \\
\text { Stress } \\
(\mathrm{MPa})\end{array}$ & $\begin{array}{l}\text { Engr. } \\
\text { Strain }\end{array}$ & $\begin{array}{l}\text { Engr. } \\
\text { Stress } \\
(\mathrm{MPa}) \\
\end{array}$ & $\begin{array}{l}\text { Engr. } \\
\text { Strain }\end{array}$ \\
\hline 14.610 & 0.0001 & 250.740 & 0.0037 & 501.370 & 0.1157 \\
\hline 15.780 & 0.0001 & 255.690 & 0.0041 & 508.070 & 0.1223 \\
\hline 29.460 & 0.0002 & 277.600 & 0.0063 & 520.080 & 0.1354 \\
\hline 63.210 & 0.0002 & 334.040 & 0.0190 & 525.390 & 0.1421 \\
\hline 73.560 & 0.0002 & 339.410 & 0.0208 & 536.540 & 0.1575 \\
\hline 107.350 & 0.0003 & 366.210 & 0.0312 & 540.710 & 0.1642 \\
\hline 118.460 & 0.0005 & 390.590 & 0.0421 & 548.990 & 0.1796 \\
\hline 167.810 & 0.0008 & 431.340 & 0.0637 & 551.730 & 0.1862 \\
\hline 176.640 & 0.0010 & 455.150 & 0.0787 & 553.380 & 0.1906 \\
\hline 200.580 & 0.0016 & 473.310 & 0.0918 & 556.490 & 0.2026 \\
\hline 221.710 & 0.0023 & 484.180 & 0.1004 & 557.780 & 0.2119 \\
\hline
\end{tabular}

Table 363. Test data for specimen 752-27

\begin{tabular}{|c|c|c|c|c|c|}
\hline $\begin{array}{l}\text { Test Number } \\
\text { Aging Temp. } \\
\text { Yield Stress }\end{array}$ & $\begin{array}{l}: 0 \\
: 35 \\
: 2 C\end{array}$ & & $\begin{array}{l}\text { Tes } \\
\text { Agi } \\
\text { Ulti }\end{array}$ & $\begin{array}{l}\text { np. } \\
\text { me } \\
\text { Stress }\end{array}$ & $\begin{array}{l}290^{\circ} \mathrm{C} \\
2,570 \mathrm{~h} \\
528.8 \mathrm{MPa}\end{array}$ \\
\hline $\begin{array}{l}\text { Engr. } \\
\text { Stress } \\
\text { (MPa) }\end{array}$ & $\begin{array}{l}\text { Engr. } \\
\text { Strain }\end{array}$ & $\begin{array}{l}\text { Engr. } \\
\text { Stress } \\
\text { (MPa) }\end{array}$ & $\begin{array}{l}\text { Engr. } \\
\text { Strain }\end{array}$ & $\begin{array}{l}\text { Engr. } \\
\text { Stress } \\
(\mathrm{MPa})\end{array}$ & $\begin{array}{l}\text { Engr. } \\
\text { Strain }\end{array}$ \\
\hline 64.52 & 0.0001 & 245.45 & 0.0031 & 396.80 & 0.0576 \\
\hline 91.54 & 0.0002 & 249.86 & 0.0033 & 409.19 & 0.0657 \\
\hline 122.15 & 0.0005 & 253.92 & 0.0035 & 423.78 & 0.0752 \\
\hline 133.99 & 0.0006 & 257.98 & 0.0038 & 435.61 & 0.0833 \\
\hline 145.78 & 0.0008 & 262.88 & 0.0042 & 443.82 & 0.0893 \\
\hline 156.83 & 0.0010 & 269.37 & 0.0050 & 454.85 & 0.0971 \\
\hline 164.93 & 0.0012 & 275.47 & 0.0058 & 464.08 & 0.1022 \\
\hline 179.61 & 0.0015 & 279.51 & 0.0064 & 473.90 & 0.1108 \\
\hline 183.09 & 0.0016 & 285.42 & 0.0074 & 484.10 & 0.1240 \\
\hline 194.92 & 0.0019 & 289.31 & 0.0081 & 498.83 & 0.1407 \\
\hline 198.02 & 0.0020 & 297.00 & 0.0098 & 506.89 & 0.1525 \\
\hline 210.50 & 0.0022 & 341.65 & 0.0265 & 514.21 & 0.1641 \\
\hline 217.85 & 0.0023 & 352.90 & 0.0323 & 520.25 & 0.1745 \\
\hline 224.33 & 0.0024 & 363.57 & 0.0379 & 522.82 & 0.1817 \\
\hline 232.13 & 0.0025 & 375.14 & 0.0446 & 527.72 & 0.1995 \\
\hline 240.16 & 0.0028 & 387.46 & 0.0519 & 528.85 & 0.2102 \\
\hline
\end{tabular}


Table 364. Test data for specimen 754-09

\begin{tabular}{|c|c|c|c|c|c|}
\hline $\begin{array}{l}\text { Test Number } \\
\text { Aging Temp. } \\
\text { Yield Stress }\end{array}$ & $\begin{array}{l}: 0 \\
: 35 \\
: 21\end{array}$ & $\mathrm{MPa}$ & $\begin{array}{l}\text { Tes } \\
\text { Agir } \\
\text { Ulti }\end{array}$ & $\begin{array}{l}\mathrm{np} . \\
\text { me } \\
\text { Stress }\end{array}$ & $\begin{array}{l}290^{\circ} \mathrm{C} \\
10,000 \mathrm{~h} \\
614.1 \mathrm{MPa}\end{array}$ \\
\hline $\begin{array}{l}\text { Engr. } \\
\text { Stress } \\
(\mathrm{MPa})\end{array}$ & $\begin{array}{l}\text { Engr. } \\
\text { Strain }\end{array}$ & $\begin{array}{l}\text { Engr. } \\
\text { Stress } \\
(\mathrm{MPa})\end{array}$ & $\begin{array}{l}\text { Engr. } \\
\text { Strain }\end{array}$ & $\begin{array}{l}\text { Engr. } \\
\text { Stress } \\
(\mathrm{MPa}) \\
\end{array}$ & $\begin{array}{l}\text { Engr. } \\
\text { Strain }\end{array}$ \\
\hline 170.910 & 0.0014 & 279.750 & 0.0047 & 482.940 & 0.0737 \\
\hline 185.290 & 0.0017 & 284.780 & 0.0050 & 532.470 & 0.1042 \\
\hline 192.190 & 0.0020 & 289.860 & 0.0055 & 546.750 & 0.1160 \\
\hline 201.790 & 0.0021 & 295.770 & 0.0060 & 560.960 & 0.1292 \\
\hline 208.390 & 0.0022 & 317.070 & 0.0085 & 563.880 & 0.1318 \\
\hline 213.970 & 0.0023 & 324.180 & 0.0097 & 574.620 & 0.1440 \\
\hline 219.980 & 0.0024 & 360.220 & 0.0183 & 590.390 & 0.1657 \\
\hline 256.250 & 0.0034 & 376.650 & 0.0229 & 597.080 & 0.1776 \\
\hline 261.960 & 0.0037 & 395.680 & 0.0301 & 598.230 & 0.1805 \\
\hline 267.730 & 0.0040 & 414.460 & 0.0377 & 605.840 & 0.1992 \\
\hline 271.970 & 0.0042 & 443.340 & 0.0512 & 607.370 & 0.2014 \\
\hline
\end{tabular}

Table 365. Test data for specimen 75-120

$\begin{array}{llll}\text { Test Number } & : 087 & \text { Test Temp. } & : 290^{\circ} \mathrm{C} \\ \text { Aging Temp. } & : 350^{\circ} \mathrm{C} & \text { Aging Time } & : 10,000 \mathrm{~h} \\ \text { Yield Stress } & : 209.9 \mathrm{MPa} & \text { Ultimate Stress } & : 595.6 \mathrm{MPa}\end{array}$

Experimental Data Not Available.

Table 366. Test data for specimen 753-12

\begin{tabular}{|c|c|c|c|c|c|}
\hline $\begin{array}{l}\text { Test Number } \\
\text { Aging Temp. } \\
\text { Yield Stress }\end{array}$ & $\begin{array}{l}: 26 \\
: 35 \\
: 26\end{array}$ & & $\begin{array}{l}\text { Tes } \\
\text { Agi } \\
\text { Ulti }\end{array}$ & $\begin{array}{l}\text { np. } \\
\text { ne } \\
\text { Stress }\end{array}$ & $\begin{array}{l}290^{\circ} \mathrm{C} \\
30,000 \mathrm{~h} \\
615.9 \mathrm{MPa}\end{array}$ \\
\hline $\begin{array}{l}\text { Engr. } \\
\text { Stress } \\
(\mathrm{MPa})\end{array}$ & $\begin{array}{l}\text { Engr. } \\
\text { Strain }\end{array}$ & $\begin{array}{l}\text { Engr. } \\
\text { Stress } \\
(\mathrm{MPa})\end{array}$ & $\begin{array}{l}\text { Engr. } \\
\text { Strain }\end{array}$ & $\begin{array}{l}\text { Engr. } \\
\text { Stress } \\
(\mathrm{MPa}) \\
\end{array}$ & $\begin{array}{l}\text { Engr. } \\
\text { Strain }\end{array}$ \\
\hline 42860 & 0.0002 & 242480 & 0.0018 & 462670 & 0.0549 \\
\hline 46.130 & 0.0002 & 260.210 & 0.0026 & 477.470 & 0.0620 \\
\hline 62.250 & 0.0002 & 265.620 & 0.0030 & 496.720 & 0.0730 \\
\hline 73.840 & 0.0002 & 284.460 & 0.0040 & 511.520 & 0.0817 \\
\hline 84.920 & 0.0002 & 292.560 & 0.0049 & 573.080 & 0.1317 \\
\hline 96.220 & 0.0002 & 313.220 & 0.0069 & 587.290 & 0.1487 \\
\hline 107.810 & 0.0002 & 342.890 & 0.0119 & 588.900 & 0.1509 \\
\hline 155.230 & 0.0003 & 370.950 & 0.0193 & 599.960 & 0.1681 \\
\hline 166.210 & 0.0004 & 387.840 & 0.0242 & 612.080 & 0.1955 \\
\hline 176.320 & 0.0004 & 419.920 & 0.0358 & 615.790 & 0.2114 \\
\hline 201.610 & 0.0007 & 445.240 & 0.0466 & & \\
\hline
\end{tabular}


Table 367. Test data for specimen 754-12

\begin{tabular}{|c|c|c|c|c|c|}
\hline $\begin{array}{l}\text { Test Number } \\
\text { Aging Temp. } \\
\text { Yield Stress }\end{array}$ & $\begin{array}{l}: 2 \\
: 35 \\
: 22\end{array}$ & & $\begin{array}{l}\text { Tes } \\
\text { Agir } \\
\text { Ultir }\end{array}$ & $\begin{array}{l}\mathrm{np} . \\
\text { me } \\
\text { Stress }\end{array}$ & $\begin{array}{l}290^{\circ} \mathrm{C} \\
30,000 \mathrm{~h} \\
613.8 \mathrm{MPa}\end{array}$ \\
\hline $\begin{array}{l}\text { Engr. } \\
\text { Stress } \\
(\mathrm{MPa})\end{array}$ & $\begin{array}{l}\text { Engr. } \\
\text { Strain }\end{array}$ & $\begin{array}{l}\text { Engr. } \\
\text { Stress } \\
(\mathrm{MPa})\end{array}$ & $\begin{array}{l}\text { Engr. } \\
\text { Strain }\end{array}$ & $\begin{array}{l}\text { Engr. } \\
\text { Stress } \\
(\mathrm{MPa}) \\
\end{array}$ & $\begin{array}{l}\text { Engr. } \\
\text { Strain }\end{array}$ \\
\hline 41.500 & 0.0002 & 240.490 & 0.0038 & 482.190 & 0.0713 \\
\hline 48.630 & 0.0002 & 246.130 & 0.0041 & 533.550 & 0.1036 \\
\hline 63.550 & 0.0002 & 261.530 & 0.0052 & 552.940 & 0.1191 \\
\hline 73.690 & 0.0002 & 298.400 & 0.0089 & 560.220 & 0.1258 \\
\hline 84.200 & 0.0003 & 315.180 & 0.0114 & 573.040 & 0.1390 \\
\hline 94.900 & 0.0003 & 320.280 & 0.0122 & 578.930 & 0.1456 \\
\hline 106.280 & 0.0004 & 339.060 & 0.0162 & 587.510 & 0.1566 \\
\hline 117.700 & 0.0005 & 344.600 & 0.0174 & 592.150 & 0.1633 \\
\hline 129.430 & 0.0006 & 436.660 & 0.0493 & 602.110 & 0.1809 \\
\hline 158.620 & 0.0008 & 441.610 & 0.0513 & 609.860 & 0.2006 \\
\hline 167.080 & 0.0011 & 478.120 & 0.0692 & 613.560 & 0.2163 \\
\hline
\end{tabular}

Table 368. Test data for specimen 75-270

\begin{tabular}{|c|c|c|c|c|c|}
\hline $\begin{array}{l}\text { Test Number } \\
\text { Aging Temp. } \\
\text { Yield Stress }\end{array}$ & $\begin{array}{l}: 2 \\
: 35 \\
: 20\end{array}$ & & $\begin{array}{l}\text { Tes } \\
\text { Agir } \\
\text { Ultir }\end{array}$ & $\begin{array}{l}\mathrm{mp} . \\
\text { me } \\
\text { Stress }\end{array}$ & $\begin{array}{l}290^{\circ} \mathrm{C} \\
30,000 \mathrm{~h} \\
584.6 \mathrm{MPa}\end{array}$ \\
\hline $\begin{array}{l}\text { Engr. } \\
\text { Stress } \\
\text { (MPa) }\end{array}$ & $\begin{array}{l}\text { Engr. } \\
\text { Strain }\end{array}$ & $\begin{array}{l}\text { Engr. } \\
\text { Stress } \\
\text { (MPa) }\end{array}$ & $\begin{array}{l}\text { Engr. } \\
\text { Strain }\end{array}$ & $\begin{array}{l}\text { Engr. } \\
\text { Stress } \\
\text { (MPa) }\end{array}$ & $\begin{array}{l}\text { Engr. } \\
\text { Strain }\end{array}$ \\
\hline 20.400 & 0.0001 & 178.270 & 0.0019 & 450.340 & 0.0725 \\
\hline 25.300 & 0.0001 & 185.100 & 0.0021 & 472.740 & 0.0868 \\
\hline 39.620 & 0.0002 & 210.620 & 0.0032 & 482.240 & 0.0933 \\
\hline 51.040 & 0.0002 & 222.360 & 0.0038 & 496.940 & 0.1041 \\
\hline 63.230 & 0.0003 & 243.480 & 0.0052 & 517.630 & 0.1218 \\
\hline 74.150 & 0.0003 & 252.020 & 0.0060 & 520.020 & 0.1241 \\
\hline 84.850 & 0.0003 & 291.630 & 0.0108 & 546.690 & 0.1528 \\
\hline 95.840 & 0.0004 & 296.780 & 0.0117 & 553.470 & 0.1616 \\
\hline 107.200 & 0.0005 & 303.530 & 0.0130 & 565.240 & 0.1790 \\
\hline 118.220 & 0.0006 & 343.790 & 0.0238 & 579.400 & 0.2094 \\
\hline 128.710 & 0.0007 & 431.440 & 0.0618 & 584.560 & 0.2344 \\
\hline
\end{tabular}


Table 369. Test data for specimen 752-18

\begin{tabular}{|c|c|c|c|c|c|}
\hline $\begin{array}{l}\text { Test Number } \\
\text { Aging Temp. } \\
\text { Yield Stress }\end{array}$ & $\begin{array}{l}: 0 \\
: 40 \\
: 20\end{array}$ & & $\begin{array}{l}\text { Tes } \\
\text { Agi } \\
\text { Ulti }\end{array}$ & $\begin{array}{l}\text { mp. } \\
\text { me } \\
\text { Stress }\end{array}$ & $\begin{array}{l}290^{\circ} \mathrm{C} \\
2,570 \mathrm{~h} \\
591.1 \mathrm{MPa}\end{array}$ \\
\hline $\begin{array}{l}\text { Engr. } \\
\text { Stress } \\
\text { (MPa) }\end{array}$ & $\begin{array}{l}\text { Engr. } \\
\text { Strain }\end{array}$ & $\begin{array}{l}\text { Engr. } \\
\text { Stress } \\
\text { (MPa) }\end{array}$ & $\begin{array}{l}\text { Engr. } \\
\text { Strain }\end{array}$ & $\begin{array}{l}\text { Engr. } \\
\text { Stress } \\
(\mathrm{MPa}) \\
\end{array}$ & $\begin{array}{l}\text { Engr. } \\
\text { Strain }\end{array}$ \\
\hline 53.16 & 0.0000 & 276.75 & 0.0050 & 472.83 & 0.0845 \\
\hline 65.69 & 0.0001 & 290.82 & 0.0064 & 482.11 & 0.0904 \\
\hline 83.84 & 0.0001 & 295.84 & 0.0071 & 486.77 & 0.0935 \\
\hline 102.68 & 0.0003 & 303.20 & 0.0082 & 489.76 & 0.0966 \\
\hline 129.64 & 0.0005 & 309.80 & 0.0094 & 509.96 & 0.1090 \\
\hline 153.15 & 0.0009 & 316.91 & 0.0108 & 518.48 & 0.1156 \\
\hline 167.42 & 0.0012 & 323.32 & 0.0121 & 536.20 & 0.1314 \\
\hline 175.48 & 0.0013 & 335.40 & 0.0153 & 547.08 & 0.1430 \\
\hline 188.90 & 0.0017 & 347.52 & 0.0193 & 556.17 & 0.1543 \\
\hline 200.79 & 0.0020 & 361.98 & 0.0245 & 563.58 & 0.1645 \\
\hline 205.86 & 0.0021 & 384.33 & 0.0342 & 572.07 & 0.1788 \\
\hline 219.53 & 0.0024 & 403.70 & 0.0442 & 578.97 & 0.1907 \\
\hline 230.76 & 0.0025 & 420.04 & 0.0531 & 584.98 & 0.2046 \\
\hline 239.27 & 0.0027 & 434.94 & 0.0619 & 588.80 & 0.2162 \\
\hline 249.21 & 0.0032 & 449.84 & 0.0707 & 591.03 & 0.2287 \\
\hline 266.41 & 0.0042 & 460.81 & 0.0770 & 591.11 & 0.2332 \\
\hline
\end{tabular}

Table 370. Test data for specimen 752-24

\begin{tabular}{|c|c|c|c|c|c|}
\hline $\begin{array}{l}\text { Test Number } \\
\text { Aging Temp. } \\
\text { Yield Stress }\end{array}$ & $\begin{array}{l}: 0 \\
: 40 \\
: 2 C\end{array}$ & & $\begin{array}{l}\text { Tes } \\
\text { Agi } \\
\text { Ulti }\end{array}$ & $\begin{array}{l}\mathrm{np} . \\
\text { me } \\
\text { Stress }\end{array}$ & $\begin{array}{l}290^{\circ} \mathrm{C} \\
10,000 \mathrm{~h} \\
630.4 \mathrm{MPa}\end{array}$ \\
\hline $\begin{array}{l}\text { Engr. } \\
\text { Stress } \\
\text { (MPa) }\end{array}$ & $\begin{array}{l}\text { Engr. } \\
\text { Strain }\end{array}$ & $\begin{array}{l}\text { Engr. } \\
\text { Stress } \\
\text { (MPa) }\end{array}$ & $\begin{array}{l}\text { Engr. } \\
\text { Strain }\end{array}$ & $\begin{array}{l}\text { Engr. } \\
\text { Stress } \\
(\mathrm{MPa}) \\
\end{array}$ & $\begin{array}{l}\text { Engr. } \\
\text { Strain } \\
\end{array}$ \\
\hline 166.180 & 0.0012 & 317.100 & 0.0073 & 530.080 & 0.0940 \\
\hline 183.810 & 0.0016 & 334.120 & 0.0091 & 549.340 & 0.1040 \\
\hline 192.600 & 0.0019 & 341.430 & 0.0100 & 562.550 & 0.1153 \\
\hline 201.000 & 0.0020 & 358.330 & 0.0129 & 582.320 & 0.1326 \\
\hline 208.500 & 0.0022 & 396.480 & 0.0230 & 596.330 & 0.1469 \\
\hline 241.190 & 0.0028 & 425.890 & 0.0341 & 599.780 & 0.1520 \\
\hline 249.440 & 0.0031 & 453.060 & 0.0473 & 615.390 & 0.1766 \\
\hline 265.540 & 0.0038 & 459.790 & 0.0509 & 617.680 & 0.1813 \\
\hline 275.700 & 0.0042 & 482.880 & 0.0638 & 624.620 & 0.1961 \\
\hline 300.580 & 0.0059 & 493.570 & 0.0721 & 625.760 & 0.2001 \\
\hline 309.110 & 0.0066 & 509.940 & 0.0813 & 629.660 & 0.2153 \\
\hline
\end{tabular}


Table 371. Test data for specimen 75-110

$\begin{array}{llll}\text { Test Number } & : 081 & \text { Test Temp. } & : 290^{\circ} \mathrm{C} \\ \text { Aging Temp. } & : 400^{\circ} \mathrm{C} & \text { Aging Time } & : 10,000 \mathrm{~h} \\ \text { Yield Stress } & : 206.7 \mathrm{MPa} & \text { Ultimate Stress } & : 593.1 \mathrm{MPa}\end{array}$

\begin{tabular}{cccccc}
\hline $\begin{array}{c}\text { Engr. } \\
\text { Stress } \\
(\mathrm{MPa})\end{array}$ & $\begin{array}{c}\text { Engr. } \\
\text { Strain }\end{array}$ & $\begin{array}{c}\text { Engr. } \\
\text { Stress } \\
(\mathrm{MPa})\end{array}$ & $\begin{array}{c}\text { Engr. } \\
\text { Strain }\end{array}$ & $\begin{array}{c}\text { Engr. } \\
\text { Stress } \\
(\mathrm{MPa})\end{array}$ & $\begin{array}{c}\text { Engr. } \\
\text { Strain }\end{array}$ \\
\hline & & & & & \\
160.190 & 0.0011 & 325.060 & 0.0108 & 486.950 & 0.0865 \\
173.030 & 0.0014 & 335.670 & 0.0127 & 493.300 & 0.0904 \\
181.380 & 0.0016 & 342.500 & 0.0142 & 518.010 & 0.1059 \\
188.460 & 0.0018 & 350.450 & 0.0162 & 546.780 & 0.1307 \\
195.140 & 0.0021 & 371.710 & 0.0223 & 555.630 & 0.1416 \\
203.200 & 0.0022 & 376.380 & 0.0247 & 567.390 & 0.1564 \\
226.540 & 0.0026 & 398.020 & 0.0338 & 569.820 & 0.1600 \\
255.540 & 0.0036 & 430.060 & 0.0497 & 581.550 & 0.1792 \\
263.500 & 0.0042 & 435.800 & 0.0533 & 584.070 & 0.1832 \\
290.530 & 0.0063 & 455.870 & 0.0646 & 589.730 & 0.1987 \\
315.580 & 0.0093 & 469.890 & 0.0733 & 592.600 & 0.2091 \\
\hline
\end{tabular}

Table 372. Test data for specimen 751-06

\begin{tabular}{|c|c|c|c|c|c|}
\hline $\begin{array}{l}\text { Test Number } \\
\text { Aging Temp. } \\
\text { Yield Stress }\end{array}$ & $\begin{array}{l}: 0 \\
: 45 \\
: 21\end{array}$ & $\mathrm{~Pa}$ & & $\begin{array}{l}\text { mp. } \\
\text { me } \\
\text { Stress }\end{array}$ & $\begin{array}{l}290^{\circ} \mathrm{C} \\
2,570 \mathrm{~h} \\
598.2 \mathrm{MPa}\end{array}$ \\
\hline $\begin{array}{l}\text { Engr. } \\
\text { Stress } \\
\text { (MPa) }\end{array}$ & $\begin{array}{l}\text { Engr. } \\
\text { Strain }\end{array}$ & $\begin{array}{l}\text { Engr. } \\
\text { Stress } \\
(\mathrm{MPa})\end{array}$ & $\begin{array}{l}\text { Engr. } \\
\text { Strain }\end{array}$ & $\begin{array}{l}\text { Engr. } \\
\text { Stress } \\
(\mathrm{MPa})\end{array}$ & $\begin{array}{l}\text { Engr. } \\
\text { Strain }\end{array}$ \\
\hline 54.2050 & 0.0000 & 288.9000 & 0.0049 & 469.3600 & 0.0611 \\
\hline 70.7470 & 0.0001 & 301.3900 & 0.0056 & 483.4600 & 0.0707 \\
\hline 92.5100 & 0.0003 & 315.0200 & 0.0067 & 486.9300 & 0.0751 \\
\hline 121.7500 & 0.0006 & 326.3300 & 0.0079 & 506.9400 & 0.0898 \\
\hline 144.3000 & 0.0009 & 338.3800 & 0.0095 & 523.5300 & 0.1002 \\
\hline 167.9700 & 0.0013 & 348.9000 & 0.0111 & 539.3900 & 0.1154 \\
\hline 184.1700 & 0.0017 & 360.4500 & 0.0132 & 554.2400 & 0.1320 \\
\hline 196.9200 & 0.0021 & 370.0800 & 0.0154 & 564.6300 & 0.1454 \\
\hline 207.1000 & 0.0022 & 379.1700 & 0.0177 & 575.8300 & 0.1627 \\
\hline 213.9400 & 0.0023 & 389.4300 & 0.0208 & 583.6300 & 0.1782 \\
\hline 223.4400 & 0.0024 & 401.6600 & 0.0252 & 589.8600 & 0.1940 \\
\hline 232.1500 & 0.0025 & 421.1800 & 0.0336 & 593.0000 & 0.2000 \\
\hline 241.6500 & 0.0028 & 434.8200 & 0.0404 & 593.9100 & 0.2096 \\
\hline 256.4200 & 0.0033 & 441.9700 & 0.0442 & 597.6300 & 0.2170 \\
\hline 274.6700 & 0.0041 & 459.1300 & 0.0546 & 598.2000 & 0.2181 \\
\hline
\end{tabular}


Table 373. Test data for specimen 752-09

\begin{tabular}{|c|c|c|c|c|c|}
\hline $\begin{array}{l}\text { Test Number } \\
\text { Aging Temp. } \\
\text { Yield Stress }\end{array}$ & $\begin{array}{l}: 0 \\
: 45 \\
: 15\end{array}$ & & $\begin{array}{l}\text { Tes } \\
\text { Agi } \\
\text { Ulti }\end{array}$ & $\begin{array}{l}\text { np. } \\
\text { ne } \\
\text { Stress }\end{array}$ & $\begin{array}{l}290^{\circ} \mathrm{C} \\
2,570 \mathrm{~h} \\
606.2 \mathrm{MPa}\end{array}$ \\
\hline $\begin{array}{l}\text { Engr. } \\
\text { Stress } \\
(\mathrm{MPa}) \\
\end{array}$ & $\begin{array}{l}\text { Engr. } \\
\text { Strain }\end{array}$ & $\begin{array}{l}\text { Engr. } \\
\text { Stress } \\
\text { (MPa) }\end{array}$ & $\begin{array}{l}\text { Engr. } \\
\text { Strain }\end{array}$ & $\begin{array}{l}\text { Engr. } \\
\text { Stress } \\
(\mathrm{MPa})\end{array}$ & $\begin{array}{l}\text { Engr. } \\
\text { Strain }\end{array}$ \\
\hline 44.59 & 0.0000 & 243.86 & 0.0035 & 424.61 & 0.0403 \\
\hline 90.76 & 0.0003 & 254.69 & 0.0039 & 438.26 & 0.0469 \\
\hline 125.06 & 0.0006 & 264.50 & 0.0045 & 449.64 & 0.0527 \\
\hline 147.87 & 0.0010 & 274.50 & 0.0052 & 463.48 & 0.0601 \\
\hline 158.56 & 0.0012 & 291.16 & 0.0066 & 479.09 & 0.0689 \\
\hline 170.34 & 0.0015 & 304.61 & 0.0079 & 487.00 & 0.0750 \\
\hline 182.85 & 0.0018 & 318.99 & 0.0097 & 509.92 & 0.0899 \\
\hline 185.86 & 0.0020 & 325.79 & 0.0106 & 526.84 & 0.0996 \\
\hline 193.94 & 0.0022 & 330.57 & 0.0113 & 541.79 & 0.1111 \\
\hline 198.67 & 0.0024 & 345.20 & 0.0142 & 559.48 & 0.1283 \\
\hline 200.89 & 0.0025 & 353.23 & 0.0159 & 573.14 & 0.1451 \\
\hline 209.16 & 0.0027 & 361.90 & 0.0180 & 582.87 & 0.1590 \\
\hline 217.74 & 0.0028 & 379.79 & 0.0232 & 598.18 & 0.1875 \\
\hline 233.70 & 0.0030 & 398.26 & 0.0293 & 603.47 & 0.2025 \\
\hline 237.55 & 0.0032 & 411.17 & 0.0344 & 606.18 & 0.2268 \\
\hline
\end{tabular}




$$
\text { A-182 }
$$


Internal:

O. K. Chopra (25)

T. F. Kassner

C. Malefyt

W. F. Michaud
R. B. Poeppel

W. J. Shack

W. K. Soppet

C. E. Till
P. T. Toben

R. W. Weeks

TIS Files

\section{External:}

NRC, for distribution per R5

ANL Libraries

ANL-E

ANL-W

Manager, Chicago Field Office, DOE

Energy Technology Division Review Committee

H. K. Birnbaum, University of Illinois, Urbana, IL

R. C. Buchanan, University of Cincinnati, Cincinnati, OH

M. S. Dresselhaus, Massachusetts Institute of Technology, Cambridge, MA

B. G. Jones, University of Illinois, Urbana, IL

C.-Y. Li, Cornell U., Ithaca, NY

S.-N. Liu, Fremont, CA

R. E. Smith, Engineering Applied Sciences, Inc., Trafford, PA

D. Atteridge, Battelle Pacific Northwest Laboratory

W. H. Bamford, Westinghouse Electric Corp., Pittsburgh

N. G. Cofie, Nutech, San Jose, CA

A. Cowan, Risley Nuclear Power Development Laboratories, Risley, Warrington, UK

E. L. Creamer, Shell Oil Co., Houston

W. H. Cullen, Materials Engineering Associates, Inc., Lanham, MD

B. J. L. Darlaston, Berkeley Nuclear Laboratories, Berkeley, Gloucestershire, UK

H. Domian, Alliance Research Center, Babcock \& Wilcox Co., Alliance, OH

G. Gage, AEA Technology, Harwell Laboratory, Oxfordshire, UK

J. Gilman, Electric Power Research Institute, Palo Alto, CA

W. Gysel, Georg Fischer, Ltd., Schaffhausen, Switzerland

G. E. Hale, The Welding Institute, Abington, Cambridge, UK

P. Hedgecock, APTECH Engineering Services, Inc., Palo Alto, CA

B. Hemsworth, HM Nuclear Installations Inspectorate, London

J. Jansky, Buro für Technische Beratung, Leonberg, Germany

C. E. Jaske, CC Technologies, Cortest, Columbus, OH

C. Kim, Westinghouse Electric Corp., Pittsburgh

P. M. Lang, Office of Converter Reactor Deployment, U.S. Dept. of Energy, Washington, DC

G. J. Licina, Structural Integrity Associates, San Jose, CA

T. R. Mager, Westinghouse Electric Corp., Pittsburgh

Y. Meyzaud, Framatome, Paris La Defense, France

M. Prager, Materials Properties Council, Inc., New York

P. H. Pumphrey, National Power, Technology and Environment Center, Leatherhead, Surrey, UK 
C. Y. Rieg, Electricité de France, Villeurbanne Cedex, France

V. N. Shah, EG\&G Idaho, Inc., Idaho Falls, Idaho

V. K. Sikka, Oak Ridge National Laboratory

A. Singh, Unical Science \& Technology Division, Brea, CA

G. Slama, Framatome, Paris La Defense, France

G. D. W. Smith, Oxford University, Oxford, UK

H. D. Solomon, General Electric Co., Schenectady, NY

D. M. Stevens, Lynchburg Research Center, Babcock \& Wilcox Co., Lynchburg, VA

L. Taylor, Nuclear Electric plc., Chelsford Rd., Knutsford, Cheshire, UK

J. C. Van Duysen, Electricité de France, Moret-Sur-Loing, France

J. M. Vitek, Oak Ridge National Laboratory

J. Wilks, AMOCO, Naperville, IL 
2. TITLE AND SUBTITLE

Tensile-Property Characterization of Thermally Aged

Cast Stainless Steels

\section{AUTHOR(S)}

W. F. Michaud, P. T. Toben,

W. K. Soppet, and O. K. Chopra

8. PERFORMING ORGANIZATION - NAME AND ADDRESS (If NRC, provide Division, Office or Region, U.S. Nuclear Regulatory Commission, and mailing address; if contractor, provide name and mailing address.)

Argonne National Laboratory

9700 South Cass Avenue

Argonne, IL 60439

9. SPONSORING ORGANIZATION - NAME AND ADDRESS (If NRC, type "Same as above": if contractor, provide NRC Division, Office or Region, U.S. Nuclear Regulatory Commission, and mailing address.)

Division of Engineering

Office of Nuclear Regulatory Research

U. S. Nuclear Regulatory Commission

Washington, DC 20555

10. SUPPLEMENTARY NOTES

11. ABSTRACT (200 words or less)

The effect of thermal aging on tensile properties of cast stainless steels during service in light water reactors has been evaluated. Tensile data for several experimental and commercial heats of cast stainless steels are presented. Thermal aging increases the tensile strength of these steels. The high-C Mo-bearing CF-8M steels are more susceptible to thermal aging than the Mo-free CF-3 or CF-8 steels. A procedure and correlations are presented for predicting the change in tensile flow and yield stresses and engineering stress-vs.-strain curve of cast stainless steel as a function of time and temperature of service. The tensile properties of aged cast stainless steel are estimated from known material information, i.e., chemical composition and the initial tensile strength of the steel. The correlations described in this report may be used for assessing thermal embrittlement of cast stainless steel components.

12. KEY WORDS/DESCRIPTORS (List words or phrases that will assist researchers in locating this report.)

Cast duplex stainless steel

Cast stainless steel

Thermal Embrittlement

Tensile Properties

Flow Stress

Yield Stress

Ramberg-Osgood Equation

Thermal Aging

\begin{tabular}{l} 
13. AVAILABILITY STATEMENT \\
Unlimited \\
\hline \begin{tabular}{l} 
14. SECURITY CLASSIFICATION \\
(This Page) \\
Unclassified \\
$\begin{array}{l}\text { (This Report) } \\
\text { Unclassified }\end{array}$ \\
15. NUMBER OF PAGES \\
254 \\
\hline 16. PRICE
\end{tabular} \\
\end{tabular}

